University of Tennessee Health Science Center

UTHSC Digital Commons

\title{
Transcriptional Regulation of Azole Antifungal Resistance in Candida albicans
}

Teresa T. Liu

University of Tennessee Health Science Center

Follow this and additional works at: https://dc.uthsc.edu/dissertations

Part of the Bacterial Infections and Mycoses Commons, Fungi Commons, and the Pharmaceutical Preparations Commons

\section{Recommended Citation}

Liu, Teresa T. , "Transcriptional Regulation of Azole Antifungal Resistance in Candida albicans" (2008). Theses and Dissertations (ETD). Paper 156. http://dx.doi.org/10.21007/etd.cghs.2008.0186.

This Dissertation is brought to you for free and open access by the College of Graduate Health Sciences at UTHSC Digital Commons. It has been accepted for inclusion in Theses and Dissertations (ETD) by an authorized administrator of UTHSC Digital Commons. For more information, please contact jwelch30@uthsc.edu. 


\title{
Transcriptional Regulation of Azole Antifungal Resistance in Candida albicans
}

\begin{abstract}
Candida albicans is a pathogenic fungi found in the mucosa, gastrointestinal, and urogenital tracts of humans. Oropharyngeal candidiasis (OPC), an opportunistic mucosal infection caused by C. albicans, occurs most frequently in patients infected with human immunodeficiency virus (HIV). OPC is usually treated with azole antifungals, a class of antifungals that target ergosterol biosynthesis, at low doses over long periods of time. This course of treatment allows for the development of azole resistance.
\end{abstract}

Two major mechanisms of azole resistance exist in $\mathrm{C}$. albicans, the up-regulation of genes encoding efflux pumps and the up-regulation of ERG11, a gene encoding the azole drug target lanosterol demethylase. The overexpression of efflux pumps remain the major contributor of azole resistance. The ATP binding cassette (ABC) transporter genes CDR1 and CDR2 have been shown to be regulated by a zinc cluster transcription factor Tac1p. A gain-of-function mutation in the gene encoding this transcription factor is responsible for the overexpression of CDR1 and CDR2. To identify the Tac1p regulon, we analyzed four matched sets of clinical isolates with gain-of-function mutations in TAC1 representing the development of CDR1- and CDR2-mediated azole resistance, using gene expression profiling. We identified genes that were consistently up-regulated coordinately with CDR1 and CDR2, including TAC1 itself. When a resistant strain disrupted for TAC1 was similarly examined, the expression of almost all of these genes returned to levels similar to those in the matched azole-susceptible isolate. Using chromatin Immunoprecipitation microarray (ChIP-chip) analysis, we found genes whose promoters were bound by Tac1p in vivo, including CDR1 and CDR2. Sequence analysis identified genes whose promoters contain the previously reported Tac1p drug-responsive element (DRE; CGGN4CGG), including TAC1. Our results show that Tac1p is constitutively bound to the promoter of its targets, including to its own promoter. They also suggest roles for Tac1p in regulating lipid metabolism (mobilization and trafficking) and oxidative stress response in $\mathrm{C}$. albicans.

Constitutive overexpression of the MDR1 gene, which encodes an efflux pump of the major facilitator superfamily, is another cause of resistance to fluconazole in clinical C. albicans strains. The regulator of MDR1 gene expression has not been identified or characterized. Using genome-wide gene expression profiling, we have identified a gene encoding a zinc cluster transcription factor, designated as MRR1 (multidrug resistance regulator), that was coordinately up-regulated with MDR1 in drug-resistant clinical C. albicans isolates. The disruption of MRR1 in two resistant clinical isolates abrogated MDR1 expression and multidrug resistance. MRR1 alleles were also sequenced and two gain-of-function mutations were identified (P683S and G997V). The integration of the mutated alleles into the genome strain SC5314 resulted in the overexpression of MDR1 and in the increase of drug resistance. Microarray analysis of the resistant isolates, the disruption mutants and the reintegrated gain-of-function mutants allowed us to define a core group of genes regulated by Mrr1p. In addition to conferring multidrug resistance, Mrr1p also regulates genes that encode oxidoreductases that play a role in the response to oxidative stress.

The second major resistance mechanism is the constitutive up-regulation of ERG11. ERG11 encodes lanosterol demethylase, a cytochrome P-450 enzyme that is the target of the azole antifungals. In C. albicans, the zinc cluster transcription factor Upc2p has been shown to regulate the expression of ERG11 and other ergosterol biosynthesis genes. Overexpression of UPC2 increases azole resistance while disruption of UPC2 results in hypersusceptibility. Using genome-wide gene expression profiling, we found UPC2 and ergosterol biosynthesis genes to be coregulated with ERG11 in a fluconazole resistant clinical isolate. Sequence analysis of the susceptible and resistant UPC2 alleles revealed that the resistant isolate contained one UPC2 allele harboring a single nucleotide substitution, resulting in a G648D exchange. Introduction of the mutated UPC2 allele into the genome strain resulted in constitutive up-regulation of ERG11 and increased resistance to fluconazole. By comparing the gene expression profiles of the 
fluconazole resistant isolate and of strains carrying wild-type and mutated UPC2 alleles, we identified target genes that are controlled by Upc2p. We show for the first time that a gain-of-function mutation in UPC2 leads to increased expression of ERG11 and imparts resistance to fluconazole in clinical isolates of C. albicans.

By studying genetically matched sets of azole susceptible and resistant clinical isolates, we identified genes co-regulated with known resistance genes. These core genes specific to particular resistance mechanisms allowed us to identify previously characterized but unknown transcriptional regulators that control this process. Gain-of-function mutations in these trans-regulatory elements are responsible for the development of azole resistance in these clinical isolates. The target genes of each of these transcription factors provide insight into their function in addition to their role in azole resistance.

\section{Document Type}

Dissertation

\section{Degree Name}

Doctor of Philosophy (PhD)

\section{Program}

Interdisciplinary Program

\section{Research Advisor}

P. David Rogers, Pharm.D., Ph.D.

\section{Keywords}

azole resistance, CDR1, CDR2, TAC1, MDR1, MRR1, ERG11, UPC2, transcription factor

\section{Subject Categories}

Bacterial Infections and Mycoses | Chemicals and Drugs | Diseases | Fungi | Medicine and Health Sciences | Organisms | Pharmaceutical Preparations 
TRANSCRIPTIONAL REGULATION OF AZOLE ANTIFUNGAL RESISTANCE IN CANDIDA ALBICANS

\author{
A Dissertation \\ Presented for \\ The Graduate Studies Council \\ The University of Tennessee \\ Health Science Center \\ In Partial Fulfillment \\ Of the Requirements for the Degree \\ Doctor of Philosophy \\ From The University of Tennessee
}

By

Teresa T. Liu

May 2008 
Chapter 2 (C) 2007 by American Society of Microbiology

Chapter 3 (C) 2007 by Morschhäuser et al.

All other material (C) 2008 by Teresa T. Liu 


\section{DEDICATION}

To my parents, Larry and Ginny Liu, for their love and encouragement, and to my husband and best friend, Jeremy Dennis, for keeping me balanced. 


\section{ACKNOWLEDGEMENTS}

First and foremost, I would like to express my sincerest gratitude to my advisor, Dave Rogers, who encouraged and challenged me throughout this journey. The opportunities I was afforded have been incredibly beneficial not only to my current research but have helped me develop relationships with other researchers in the field. I would also like to thank the members of my committee, George Hilliard, Ramin Homayouni, Richard Lee, and Bernd Meibohm. I appreciate the suggestions, guidance, and critical review as I pursued my research. A special thanks to Ramin Homayouni for designing to microarrays used in my research and Lijing $\mathrm{Xu}$ for her assistance in analyzing the data.

I would also like to express my gratitude the members of the Rogers lab, Kathy Barker, Chris Hoehamer, Kelly Caudle, and Qing Zhang, for their friendship and support. A special thanks to Kathy Barker for all she has taught me and her incredible patience as I learned and developed as a scientist. I am also extremely grateful to Joachim Morschhäuser at Universität Würzburg for allowing me to spend a month working in his lab and Martine Raymond at Université de Montréal for her insight and guidance.

Last but not least, I would like to thank my entire family for their love and support throughout this incredible journey. In addition to my wonderful parents who have always believed in me, I would like to thank my family in Memphis. Their encouragement throughout this journey has meant a lot to me. 


\begin{abstract}
Candida albicans is a pathogenic fungi found in the mucosa, gastrointestinal, and urogenital tracts of humans. Oropharyngeal candidiasis (OPC), an opportunistic mucosal infection caused by $C$. albicans, occurs most frequently in patients infected with human immunodeficiency virus (HIV). OPC is usually treated with azole antifungals, a class of antifungals that target ergosterol biosynthesis, at low doses over long periods of time. This course of treatment allows for the development of azole resistance.
\end{abstract}

Two major mechanisms of azole resistance exist in C. albicans, the up-regulation of genes encoding efflux pumps and the up-regulation of ERG11, a gene encoding the azole drug target lanosterol demethylase. The overexpression of efflux pumps remain the major contributor of azole resistance. The ATP binding cassette (ABC) transporter genes $C D R 1$ and $C D R 2$ have been shown to be regulated by a zinc cluster transcription factor Taclp. A gain-of-function mutation in the gene encoding this transcription factor is responsible for the overexpression of $C D R 1$ and $C D R 2$. To identify the Taclp regulon, we analyzed four matched sets of clinical isolates with gain-of-function mutations in $T A C 1$ representing the development of $C D R 1$ - and $C D R 2$-mediated azole resistance, using gene expression profiling. We identified genes that were consistently up-regulated coordinately with $C D R 1$ and $C D R 2$, including $T A C 1$ itself. When a resistant strain disrupted for $T A C 1$ was similarly examined, the expression of almost all of these genes returned to levels similar to those in the matched azole-susceptible isolate. Using chromatin Immunoprecipitation microarray (ChIP-chip) analysis, we found genes whose promoters were bound by Taclp in vivo, including CDR1 and CDR2. Sequence analysis identified genes whose promoters contain the previously reported Taclp drug-responsive element (DRE; $\mathrm{CGGN}_{4} \mathrm{CGG}$ ), including TAC1. Our results show that Tac1p is constitutively bound to the promoter of its targets, including to its own promoter. They also suggest roles for Tac1p in regulating lipid metabolism (mobilization and trafficking) and oxidative stress response in C. albicans.

Constitutive overexpression of the $M D R l$ gene, which encodes an efflux pump of the major facilitator superfamily, is another cause of resistance to fluconazole in clinical C. albicans strains. The regulator of MDRI gene expression has not been identified or characterized. Using genome-wide gene expression profiling, we have identified a gene encoding a zinc cluster transcription factor, designated as MRRI (multidrug resistance regulator), that was coordinately up-regulated with $M D R 1$ in drug-resistant clinical $C$. albicans isolates. The disruption of $M R R l$ in two resistant clinical isolates abrogated $M D R 1$ expression and multidrug resistance. MRR 1 alleles were also sequenced and two gain-of-function mutations were identified (P683S and G997V). The integration of the mutated alleles into the genome strain SC5314 resulted in the overexpression of MDRI and in the increase of drug resistance. Microarray analysis of the resistant isolates, the disruption mutants and the reintegrated gain-of-function mutants allowed us to define a core group of genes regulated by Mrrlp. In addition to conferring multidrug resistance, Mrrlp also regulates genes that encode oxidoreductases that play a role in the response to oxidative stress. 
The second major resistance mechanism is the constitutive up-regulation of ERG11. ERG11 encodes lanosterol demethylase, a cytochrome P-450 enzyme that is the target of the azole antifungals. In $C$. albicans, the zinc cluster transcription factor Upc $2 p$ has been shown to regulate the expression of ERG11 and other ergosterol biosynthesis genes. Overexpression of $U P C 2$ increases azole resistance while disruption of $U P C 2$ results in hypersusceptibility. Using genome-wide gene expression profiling, we found $U P C 2$ and ergosterol biosynthesis genes to be coregulated with ERG11 in a fluconazole resistant clinical isolate. Sequence analysis of the susceptible and resistant UPC2 alleles revealed that the resistant isolate contained one $U P C 2$ allele harboring a single nucleotide substitution, resulting in a G648D exchange. Introduction of the mutated UPC2 allele into the genome strain resulted in constitutive up-regulation of ERG11 and increased resistance to fluconazole. By comparing the gene expression profiles of the fluconazole resistant isolate and of strains carrying wild-type and mutated UPC2 alleles, we identified target genes that are controlled by Upc2p. We show for the first time that a gain-offunction mutation in UPC2 leads to increased expression of ERG11 and imparts resistance to fluconazole in clinical isolates of $C$. albicans.

By studying genetically matched sets of azole susceptible and resistant clinical isolates, we identified genes co-regulated with known resistance genes. These core genes specific to particular resistance mechanisms allowed us to identify previously characterized but unknown transcriptional regulators that control this process. Gain-offunction mutations in these trans-regulatory elements are responsible for the development of azole resistance in these clinical isolates. The target genes of each of these transcription factors provide insight into their function in addition to their role in azole resistance. 


\section{TABLE OF CONTENTS}

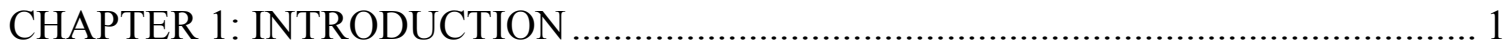

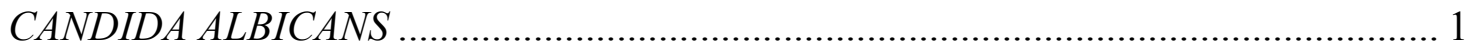

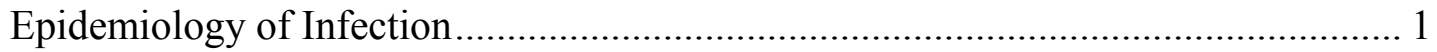

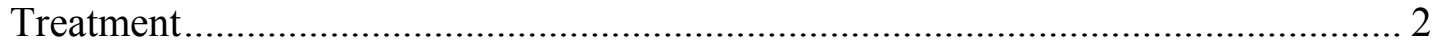

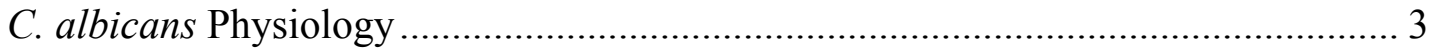

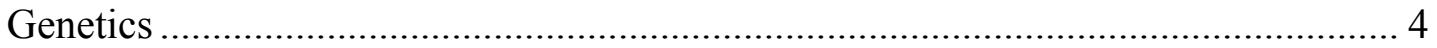

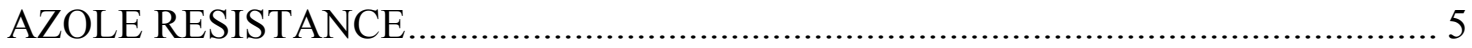

Emergence of Clinical Resistance .................................................................... 5

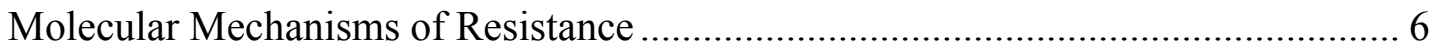

TRANSCRIPTIONAL REGULATORS............................................................... 9

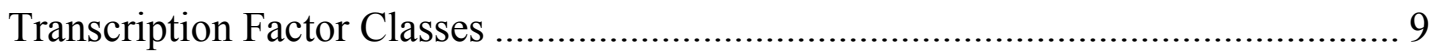

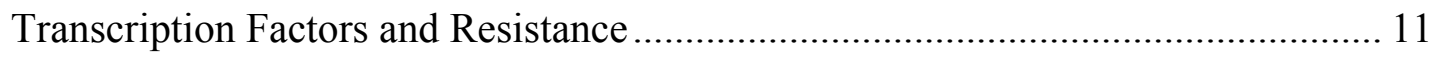

C. albicans Transcription Factors........................................................................ 13

CENTRAL HYPOTHESIS AND RESEARCH OBJECTIVES ................................ 14

CHAPTER 2: IDENTIFICATION OF THE TAC1P REGULON ................................. 16

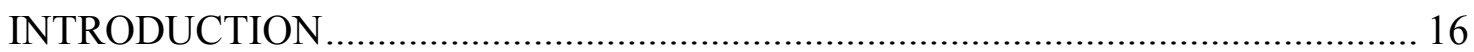

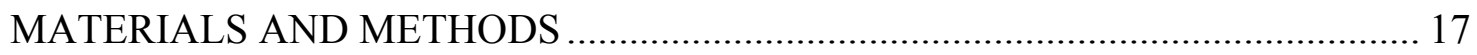

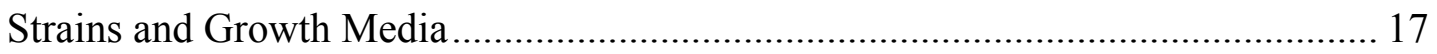

Construction of C. albicans Expression Microarrays............................................ 17

RNA Preparation for Microarrays .................................................................... 19

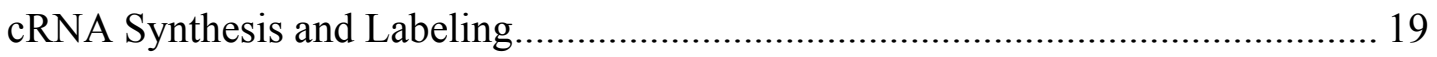

Oligonucleotide Array Hybridization and Analysis ............................................. 19

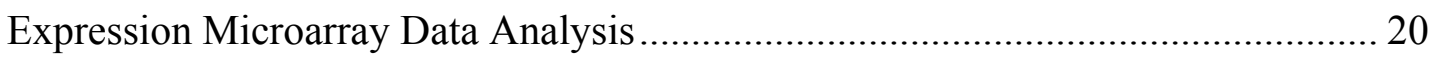

Quantitative Real-Time PCR for Expression Data................................................ 20

Generation of an HA-Tagged Tac1p-Expressing Strain........................................ 22

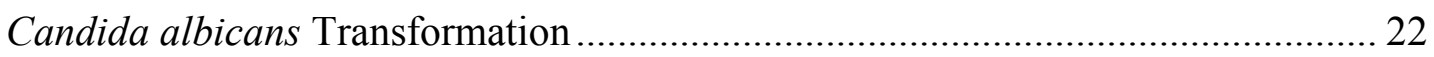

Genomic DNA Isolation and Southern Blot Analysis......................................... 22

Total Protein Preparation and Western Blotting.................................................... 23

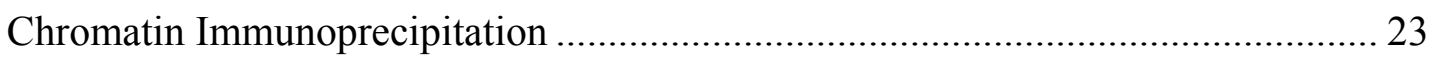

DNA Labeling, Hybridization to Intergenic Microarrays and Data Analysis ........... 24

Quantitative Real-Time PCR Confirmation of the Genome-Wide Location Data.... 24 


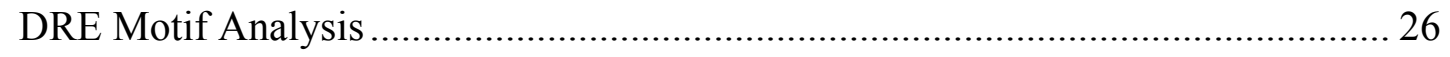

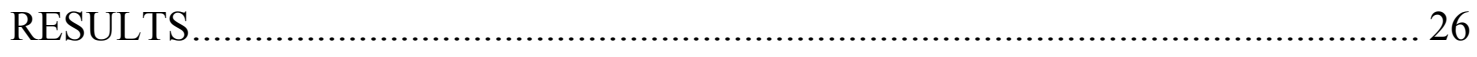

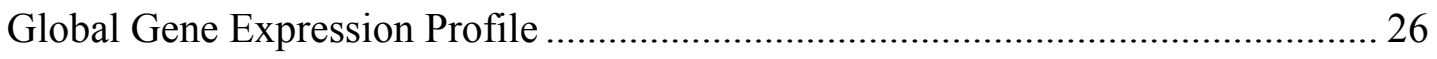

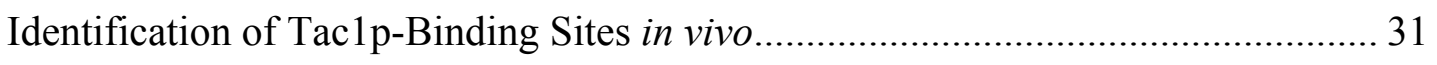

Identification of Potential Tac1p-Binding Motifs ……………………..................... 36

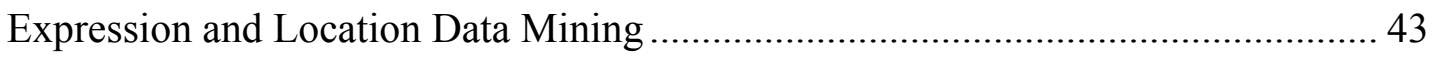

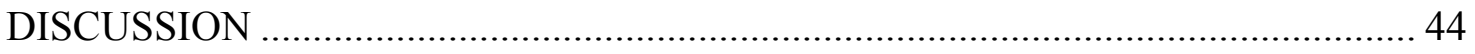

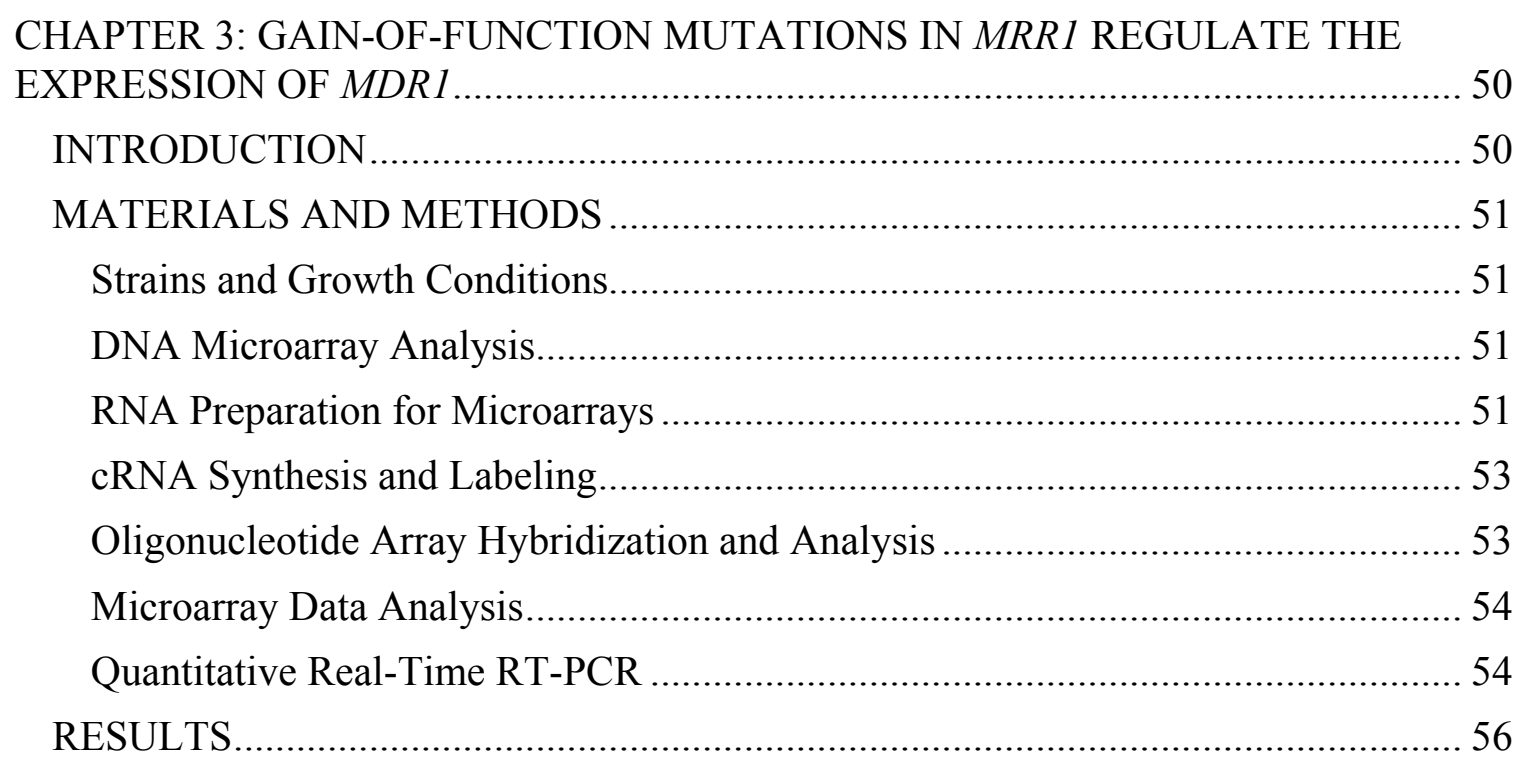

Transcription Factor MRRI Is Coordinately Regulated with MDRl in Resistant

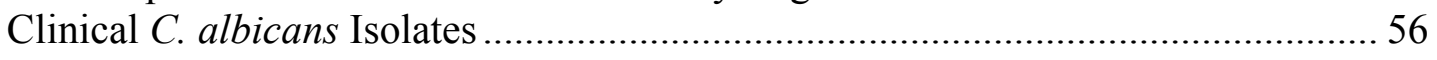

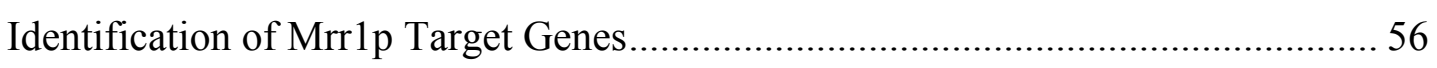

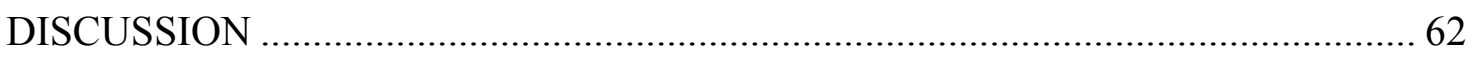

CHAPTER 4: GAIN-OF-FUNCTION MUTATIONS IN UPC2 REGULATE THE EXPRESSION OF ERGOSTEROL BIOSYNTHESIS GENES AND IMPART FLUCONAZOLE RESISTANCE IN A CLINICAL ISOLATE OF CANDIDA

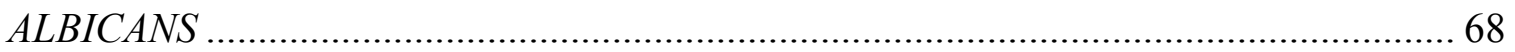

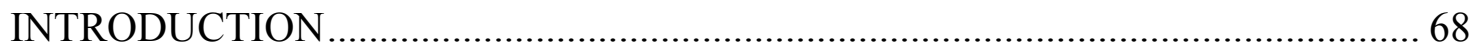

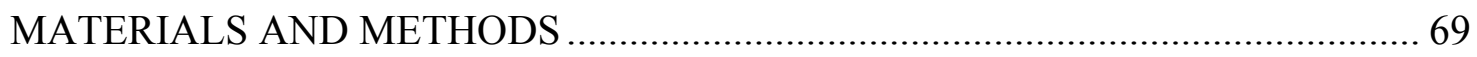

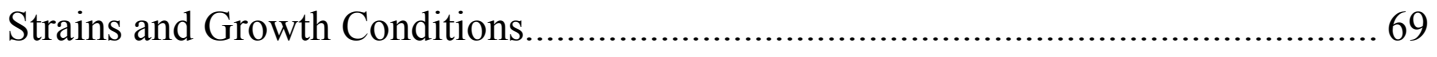

Construction of C. albicans Affymetrix ${ }^{\circledR}$ Expression Arrays ..................................... 69

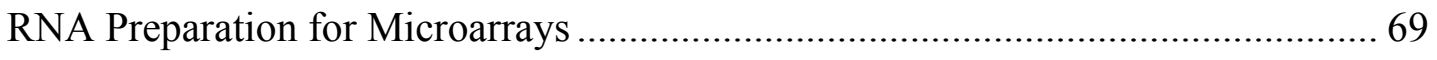

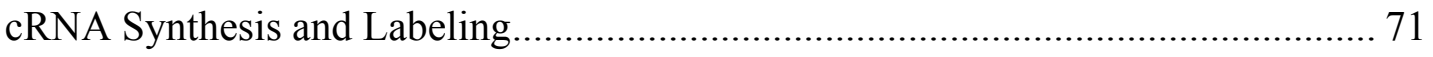

Oligonucleotide Array Hybridization and Analysis .................................................. 71 


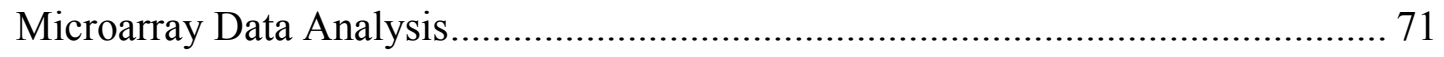

Quantitative Real-Time PCR .......................................................................... 72

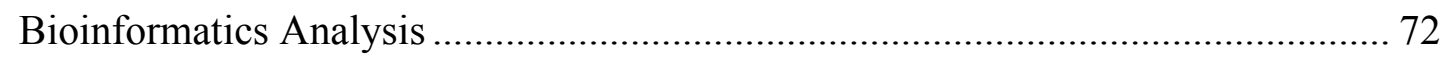

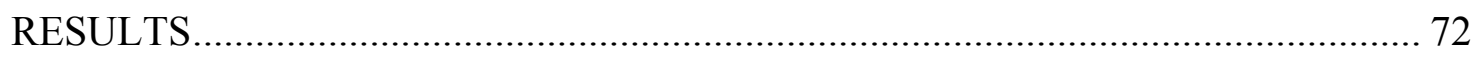

Expression of the Transcription Factor UPC2 Is Coordinately Up-Regulated with ERG11 in Azole Resistant C. albicans Clinical Isolate S2 .................................. 72

Resistant Isolate S2 Contains a Gain-of-Function Mutation in UPC2 ..................... 74

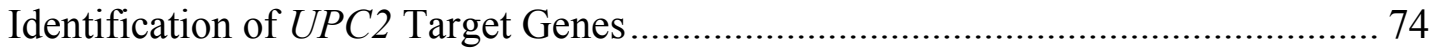

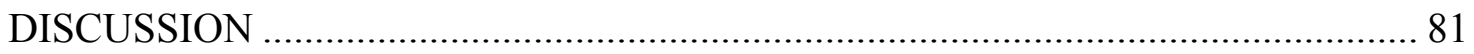

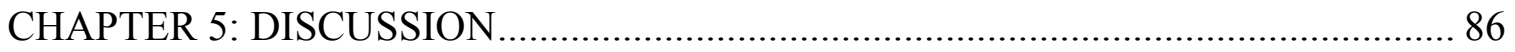

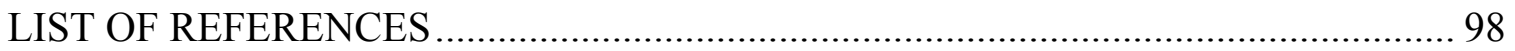

APPENDIX A: TAC1 SUPPLEMENTAL DATA .................................................. 116

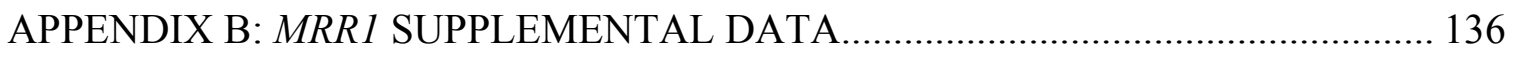

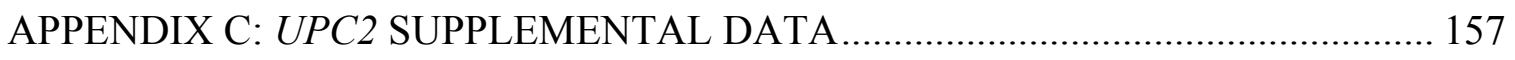

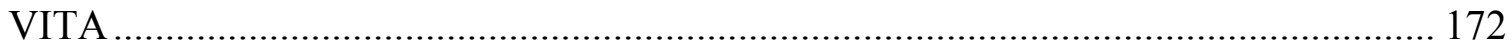




\section{LIST OF TABLES}

Table 2-1. Strains used in this study.................................................................. 18

Table 2-2. Primers used for quantitative real-time PCR expression analysis............ 21

Table 2-3. Primers used for quantitative real-time PCR binding assays.................... 25

Table 2-4. Genes up-regulated in all four matched clinical isolate sets..................... 28

Table 2-5. Genes down-regulated in all four matched clinical isolate sets................ 30

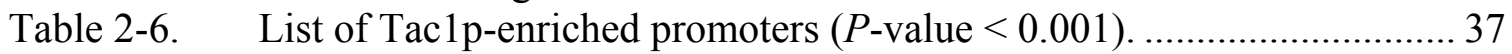

Table 2-7. DRE motifs found in the Tac1p target promoters..................................... 41

Table 3-1. C. albicans strains examined in this study.............................................52

Table 3-2. Primers used for quantitative real-time RT-PCR ..................................... 55

Table 3-3. Genes commonly down-regulated by MRR1 deletion in F5 and G5....... 59

Table 3-4. Identification of Mrrlp target genes in F isolates by DNA microarray

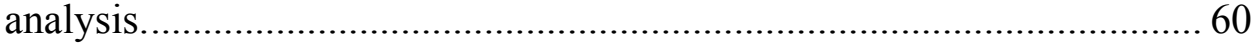

Table 3-5. Identification of Mrrlp target genes in G isolates by DNA microarray

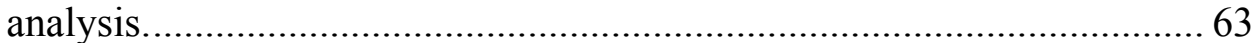

Table 4-1. C. albicans strains used in this study................................................... 70

Table 4-2. Primers used for quantitative real-time PCR analysis. .............................. 73

Table 4-3. Genes up-regulated by the G648D mutation in Upc2p............................ 77

Table 4-4. Genes down-regulated by the G648D mutation in Upc2p....................... 79

Table 4-5. Genes up-regulated in both isolate S2 and transformants expressing the $U P C 2^{\mathrm{S} 2-1}$ allele with SRE motifs in their promoter.................................. 80

Table A-1. Genes up-regulated in at least one matched clinical isolate. .................. 116

Table A-2. Genes down-regulated in at least one matched clinical isolate............... 127

Table B-1. Genes up-regulated in at least one clinical matched isolate overexpressing $M D R 1$

Table B-2. Genes down-regulated in at least one clinical matched isolate

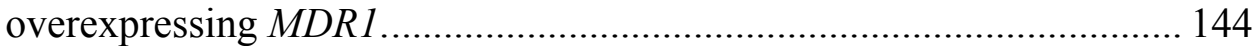

Table B-3. Genes down-regulated in the absence of MRR1 .................................. 152

Table C-1. Genes up-regulated in either resistant isolate $\mathrm{S} 2$ or gain-of-function S2 allele in SC5314

Table C-2. Genes down-regulated in either S2 or gain-of-function S2 allele in

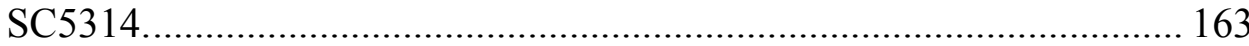

Table C-3. Genes down-regulated in the absence of $U P C 2$................................... 169

Table C-4. Genes up-regulated in the absence of UPC2 ....................................... 170 


\section{LIST OF FIGURES}

Figure 2-1. Quantitative real-time RT-PCR analysis of selected genes differentially expressed in the microarray experiments......................................... 32

Figure 2-2. Chromosomal tagging of Tac 1 p...................................................... 34

Figure 2-3. In vivo enrichment of Taclp binding at the CDR1, PDR 16 and TAC1 promoters using Q-PCR ................................................................. 40

Figure 3-1. Identification of genes that are differentially expressed in MDRI overexpressing clinical C. albicans isolates. ..................................... 57

Figure 3-2. Quantitative real-time PCR analysis of $M D R 1$ and $M R R 1$...................... 65

Figure 4-1. Quantitative real-time PCR for genes of interest................................. 75

Figure 4-2. Susceptibilities to fluconazole and terbinafine of wild-type and gain-offunction mutant UPC2 strains by spot assay. ..................................... 76

Figure 5-1. Proposed model for Tac1p-mediated fluconazole resistance in $C$.

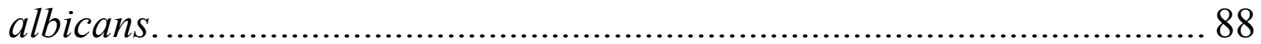

Figure 5-2. Proposed model for Mrr1p-mediated fluconazole resistance in $C$. albicans.

Figure 5-3. Proposed model for Upc2p-mediated fluconazole resistance in $C$. albicans. 


\section{LIST OF ABBREVIATIONS}

5-FOA

$\mathrm{ABC}$

AIDS

AmB

AP-1

ARE

bZip

c-CRD

cDNA

CDR

ChIP

CLSI

CRD

cRNA

$\mathrm{C}_{T}$

DBD

DNA

DRE

GCOS

GEO

GFP

GO

GPI

GST

HA

HAART

HIV

IP

MDR

MFS

MIC

$M L T$

$\mathrm{MM}$

mRNA

NBD

n-CRD

OD

OPC

ORF

PDR

PDRE

PM

Q-PCR
5-Fluoroorotic acid ATP binding cassette Acquired immune deficiency syndrome Amphotericin B Activator protein-1 Activator protein-1 recognition element Basic leucine zipper Carboxyl-terminal cysteine rich domain Complementary deoxyribonucleic acid

Candida drug resistance Chromatin Immunoprecipitation Clinical and Laboratory Standards Institute Cysteine rich domain Complementary ribonucleic acid Cycle threshold DNA binding domain Deoxyribonucleic acid Drug response element GeneChip Operating System Gene Expression Omnibus Green fluorescent protein Gene ontology Glycosylphosphatidylinositol Glutathione $S$-transferase Hemagglutinin Highly active antiretroviral therapy Human immunodeficiency virus Immunoprecipitation Multidrug resistance Major facilitator superfamily Minimum inhibitory concentration Mating type locus Mismatch Messenger ribonucleic acid Nucleotide binding domain Amino-terminal cysteine rich domain

Optical density Oropharyngeal candidiasis Open reading frame Pleiotropic drug resistance Pleiotropic drug resistance element Perfect match Quantitative real-time polymerase chain reaction 
RFLP

$R L U C$

RNA

rRNA

RT-PCR

SBI

$\mathrm{SC}$

SDS

SNP

SRE

SREBP

SV40

TMD

VVC

yAP1

YPD

YRE
Restriction fragment length polymorphism Renilla luciferase

Ribonucleic acid

Ribosomal ribonucleic acid

Reverse transcriptase-polymerase chain reaction Sterol biosynthesis inhibitors

Synthetic complete

Sequence Detection System Single Nucleotide Polymorphism

Sterol response element Sterol response element binding protein

Simian virus 40

Transmembrane domain

Vulvovaginal candidiasis

Yeast activator protein-1

Yeast peptone dextrose

Yeast activator protein-1 recognition element 


\section{CHAPTER 1: INTRODUCTION}

\section{CANDIDA ALBICANS}

\section{Epidemiology of Infection}

Instances of fungal infections have increased dramatically in the past two decades due to the acquired immune deficiency syndrome (AIDS) epidemic, improved life-sustaining technologies, and anti-cancer therapies. Unlike other pathogenic fungi with environmental niches, Candida species are found as a commensal organism in the mucosa, gastrointestinal, and urogenital tracts of $60-80 \%$ of humans (1). The most common infections caused by Candida species are invasive or mucosal infections.

Invasive candidiasis can range in severity from bloodstream infections to infections of one or more organs. Candidemia, the most common form of invasive candidiasis, is the fourth leading cause of nosocomial bloodstream infections in the United States (2). Candidemia usually occurs in patients hospitalized with serious underlying conditions. Risk factors associated with candidemia include neutropenia, cancer chemotherapy, antimicrobial agents, and indwelling catheters (3). Neutropenic patients, patients undergoing cancer chemotherapy, and organ transplant patients are immunosuppressed and cannot fight off opportunistic infections. Use of broad spectrum antimicrobial agents promotes increased colonization of fungal cells due to a decrease in competing flora. Indwelling catheters facilitate candidemia and invasive candidiasis by disrupting the mucocutaneous barrier and allowing direct vascular access. Catheters also provide a surface for the organism to adhere and create a focus for infection. Candidemia has a high rate of morbidity and mortality, 30 to $70 \%$ among cancer patients and 26 to $75 \%$ among non-cancer patients (3).

Mucosal candidiasis occurs mainly in the form of vulvovaginal candidiasis or oropharyngeal candidiasis. In contrast to organs and tissues, mucosal surfaces tolerate the growth of a variety of potentially pathogenic and nonpathogenic indigenous flora (4). Disease occurs when the balance of indigenous flora is disturbed, allowing for an overgrowth of Candida species. Vulvovaginal candidiasis (VVC) occurs most frequently in women of childbearing age with an estimated $75 \%$ of all adult women having had at least one infection. Candida species can be found as a commensal in the vagina in 10 to $25 \%$ of all women (1). Other than pregnancy, infections can also occur due to antibiotic use, immunosuppressive therapy, hormone replacement therapy, or uncontrolled diabetes mellitus (4).

Oropharyngeal candidiasis (OPC) occurs in several patient populations. OPC occurs in elderly patients with dentures due to poor oral hygiene. The dentures provide a surface on which Candida can adhere, and the resolution of the infection occurs with the removal of the dentures (5). OPC occurs most frequently in patients infected with the human immunodeficiency virus (HIV) with an estimated $90 \%$ of patients developing OPC. The occurrences of OPC increase as the $\mathrm{CD}^{+}$cell counts decrease usually to a threshold less than $200 \mathrm{cells} / \mathrm{mm}^{3}$ (6). Although OPC has declined with the introduction of highly active antiretroviral therapy (HAART), the cost of 
drug and the monitoring required makes widespread use of HAART difficult in third world nations where the majority of $\mathrm{HIV}$-infected patients reside $(7,8)$.

OPC is usually classified as pseudomembranous or erythematous. Pseudomembranous OPC is characterized by smooth, white, papular lesions. Erythematous OPC is characterized by diffuse, macular erythema of the palate, oropharynx, buccal mucosa, and dorsal tongue. Hyphae and pseudohyphae found on the mucosal surface are generally associated with pseudomembranous OPC, whereas, hyphae are generally not seen on the mucosal surface in erythematous OPC (6).

In invasive candidiasis, 60 to $75 \%$ of infections are caused by C. albicans (3). In VVC, C. albicans causes 85 to $90 \%$ of infections, whereas in OPC, C. albicans causes 77 to $100 \%$ of infections $(4,6)$. Although the instances of non-albicans species being the causative agent of infection is increasing in both invasive and mucosal candidiasis, C. albicans remains the most prevalent etiological agent of Candida infection.

\section{Treatment}

The treatment of fungal infections is limited to a few classes of antifungal agents. These antifungal compounds must exploit the differences between host and fungal cells; however, this is difficult due to the many similarities between fungal and mammalian cells. They are both eukaryotes with similar organelles, DNA replication, and protein synthesis. A structural difference is the presence of a cell wall in fungal cells. This makes it an optimal target for antifungal agents. The cell wall protects against osmotic pressure and is composed of chitin, mannoproteins, glycosylphosphatidylinositol (GPI)- anchored proteins, and glucans. The echinocandins (caspofungin), the newest class of antifungals, are thought to inhibit $\beta$-1,3-glucan synthase. Most cell wall components are interconnected through $\beta$-1,3-glucans, making it an important component in cell wall structure (9). By inhibiting $\beta$-1,3-glucan synthase, the cell wall destabilizes and the cell is more susceptible to osmotic stress. Caspofungin is fungicidal and effective against a large number of fungal pathogens including Candida. However, it is only available for intravenous administration, and the cost limits the use in developing nations.

Another difference between mammalian and fungal cells is the membrane sterol composition. Sterols regulate the membrane fluidity and integrity of the cell. Mammalian cell membranes consist mainly of cholesterol; fungal cell membranes depend on ergosterol as the major membrane sterol. This difference makes ergosterol a prime target for antifungal drugs. The polyenes target the ergosterol in the plasma membrane directly. The polyenes are fungicidal agents that bind the ergosterol in the membrane forming pores and disrupting the proton gradient (10). Amphotericin B (AmB), the most effective polyene, is still widely used to treat systemic candidiasis. However, high doses of, or long term treatment with, AmB can cause nephrotoxicity. AmB is also administered intravenously, limiting the use of the drug to hospital settings. Newer liposomal formulations of AmB have decreased the toxicity, but the increased cost precludes the widespread use of the drug (11). 
The biosynthesis of ergosterol is another major target of antifungal agents. Three antifungal drug classes function as ergosterol biosynthesis inhibitors, targeting different enzymes of the pathway. The allylamines act as noncompetitive inhibitors of squalene epoxidase encoded by $E R G 1$, causing squalene accumulation and ergosterol depletion. Terbinafine, an allylamine, is used as a topical agent due to its fungicidal activity against dermatophytes; it is fungistatic against Candida species, so it is rarely used to treat Candida infections. The morpholines (fenpropimorph, amorolfine) inhibit two enzymes in the ergosterol biosynthesis pathway, C-14 sterol reductase, encoded by ERG24, and C-8 sterol isomerase, encoded by ERG2. Fenpropimorph is generally used as an agricultural fungicide. Amorolfine, fungicidal against dermatophytes but fungistatic against yeast pathogens, is only used as a topical agent to treat dermatophytes and VVC. The azoles are the largest class of antifungal agents, consisting of two subclasses, triazoles and imidazoles. The azoles inhibit C-14 lanosterol demethylase encoded by ERG11 (5). Lanosterol demethylase is a cytochrome P-450 enzyme with a heme in its active site. The azoles function by binding the iron of the heme through an unhindered nitrogen. This prevents the activation of oxygen necessary for the demethylation of lanosterol (12). The inhibition of lanosterol demethylase causes the accumulation of toxic sterol, $14 \alpha$-methyl-3,6-diol, causing growth arrest (13). Although the azoles are fungistatic, they are most effective against a broad spectrum of fungal pathogens. Ketoconazole, an imidazole, was the first azole to be widely used due to its low toxicity. Fluconazole, a triazole, is now the most widely used azole antifungal agent due to its water solubility, low toxicity, oral administration, and the ability to treat both mucosal and systemic candidiasis $(5,10)$.

\section{C. albicans Physiology}

C. albicans is a dimorphic fungus capable of several cell morphologies. At temperatures less than $35^{\circ} \mathrm{C}$ grown with glucose as a carbon source, C. albicans grows as a blastospore, or yeast cell. Blastospores are characterized as spherical, single cells that undergo mitotic cell division by budding. Budding occurs at one spot on the blastospore, and once nuclear division occurs, a septum is formed between the parent and the daughter cell. The cells separate completely to form two new cells. At temperatures greater than $35^{\circ} \mathrm{C}$ grown in the presence of serum, C. albicans starts to filament. C. albicans can form pseudohyphae and true hyphae under filamentous conditions. Hyphal growth occurs when a blastospore forms a germ tube that continues to extend apically. As the cell grows, mitosis occurs and a septum forms. However, unlike blastospores, the cells do not separate; instead apical growth continues as the hypha continues to elongate. The cell walls of true hyphae are parallel with no constrictions at the septum. Pseudohyphae are differentiated from true hyphae by the constrictions that occur at the septum of pseudohyphae. Pseudohyphae form from blastospores in a process similar to budding. As the daughter cell continues to grow, it takes on a more elongated shape like a hypha. After the septum forms, the cells remain attached. As the cells continue to undergo mitosis, the cells form a chain of blastospores resembling filaments (1).

The change in cell morphology is thought to be a virulence factor of infection. The reversible switch from blastospore to hypha is one method by which $C$. albicans is thought to penetrate the mucosal membrane and invade host tissue. The hyphal form expresses various cell 
wall proteins that allow for better adhesion to epithelial and endothelial cells. Once in the bloodstream, the switch back to yeast cells allows for better dissemination (14).

The morphological changes and the ability to form true hyphae play a large role in the formation of biofilms. Biofilms generally occur on the surfaces of catheters, dentures, or other indwelling devices. Biofilms create a focus of infection in invasive candidiasis and also creates an environment that confers drug resistance (5). The Candida biofilm is a complex, multilayered growth structure. A thin layer of blastospores adhere to the plastics, forming a thin biofilm. The blastospores then start forming a thick hyphal layer and excreting an extracellular matrix of proteins and polysaccharides. The ability to form hyphae allows for thicker biofilms and less penetration of drugs into the extracellular matrix. Biofilms formed by yeast cells alone are less adherent and thinner. Saccharomyces cerevisiae has the ability to form biofilms, but other than a thin basal layer of blastospores, no extracellular matrix is formed. This allows for easier antifungal penetration and less development of antifungal resistance (15).

\section{Genetics}

Genetic manipulation in C. albicans has been difficult because C. albicans is a diploid yeast with no haploid stage in its cell cycle. It has eight chromosomes with 6,354 putative genes (16). It also does not have a conventional sexual cycle. Although there is a mating type locus on chromosome 5 in $C$. albicans, meiotic recombination occurs very infrequently. Instead, reproduction is largely clonal (5).

The initial understanding of the $C$. albicans genome came from studying the better characterized yeast, S. cerevisiae. Although C. albicans and S. cerevisiae diverged 140-841 million years ago, many genes are highly conserved (14). Hybridization to and complementation of $S$. cerevisiae genes were two methods used to characterize functions of genes in C. albicans. Complementation studies in $S$. cerevisiae mutants isolated genes that could restore function; studies in wild-type $S$. cerevisiae isolates found genes that conferred new function. Hybridization studies using $S$. cerevisiae DNA arrays only revealed genes that were most highly conserved between the two species (5). With the sequencing of the C. albicans genome complete, DNA microarrays specific to $C$. albicans are now available. Gene expression patterns under various conditions can now be studied independent of sequence homology to $S$. cerevisiae. Actual $C$. albicans gene expression along with $S$. cerevisiae homology allows for better characterization of cell function and pathways.

Because of the diploid nature of C. albicans, it has historically been very difficult to isolate homozygous recessive mutants. Mutants were generated using chemical mutagens or ultraviolet light and selected on media containing drugs. This method often generated several undefined genomic alterations. Candida, as a genus, also has unconventional codon usage. The CUG codons are predominantly decoded as serines rather than leucines (17). This codon reassignment makes it difficult to use $S$. cerevisiae vectors and reporter constructs that depend on translation of CUG codons to leucine. Once specific auxotrophic strains were developed for $C$. albicans, gene disruptions could be performed more readily. The most frequently used auxotrophic marker used in C. albicans is URA3, a gene that encodes orotidine 5' phosphate 
decarboxylase. The strain CAI4, derived from clinical isolate and genome strain SC5314, has disruptions in both alleles of $U R A 3$ (18). The $U R A 3$ marker allows for easy selection and counterselection of the correct transformants. The URA-blaster method of gene disruption uses the selectable marker URA3. The cassette was constructed by flanking URA3 with direct repeats of bacterial hisG sequences. The 5' and 3' regions of the gene are then cloned on either side of the his $G$ repeats. When the cassette is transformed into a $u \mathrm{ra}^{-}$strain, homologous recombination occurs, replacing one allele of the gene with the cassette. Correct transformants are selected by growth on media lacking uridine. Counterselection then occurs when grown on plates containing 5-fluoroorotic acid (FOA). 5-FOA selects for $\mathrm{ura}^{-}$strains which arise through homologous recombination at the his $G$ repeats. The URA3 gene encodes orotidine 5'-phosphate decarboxylase, a gene required for uracil biosynthesis. When a wild-type strain is grown on 5FOA, the URA3 gene product converts 5-FOA to 5-fluorouracil, a toxic gene product. Because of this conversion, only strains that spontaneously lose the $U R A 3$ gene can grow on the selection media. Once the cassette has been excised from the first allele, a second round of transformations can occur to disrupt the second allele (19).

While auxotrophic strains have allowed for easier gene manipulation, the auxotrophs were derived from either the genome strain SC5314, or strain WO-1. Gene disruption in clinical isolates would be problematic unless the clinical isolate happened to have an auxotrophic marker. Two new methods of gene disruption independent of auxotrophy has allowed for the disruption of genes in any isolates regardless of genetic background. The first method relies on a dominant selection marker that confers resistance to mycophenolic acid fused to a site-specific recombinase FLP from $S$. cerevisiae modified to function in $C$. albicans. The FLP gene is controlled by the $S A P 2$ promoter, so FLP activity only occurs under inducing conditions $(20,21)$. The second method relies on a dominant selection marker that confers resistance to the antibiotic nourseothricin fused to the FLP recombinase under the control of the MAL2 promoter (22). Both these methods effectively disrupt genes in isolates without requiring auxotrophic markers.

\section{AZOLE RESISTANCE}

\section{Emergence of Clinical Resistance}

Clinical resistance occurs when an infection persists or progresses regardless of antifungal treatment. Primary or intrinsic resistance occurs when the organism is resistant before treatment with an antifungal agent. Secondary resistance arises in response to treatment (10). The most common antifungal drug resistance occurs to the azoles due to their widespread use in treating fungal infections. Because of the fungistatic nature of the azoles, it is difficult to eradicate all of the Candida present in the infection particularly in the setting of an immunocompromised host, so relapses occur. The Clinical and Laboratory Standards Institute (CLSI) Subcommittee for Antifungal Susceptibility Testing has established standard breakpoints for fluconazole susceptibility. An isolate is considered sensitive with a minimum inhibitory concentration (MIC) $<8 \mu \mathrm{g} / \mathrm{ml}$; an isolate is resistant with an $\mathrm{MIC} \geq 64 \mu \mathrm{g} / \mathrm{ml}$. An isolate with an MIC of 8 to $64 \mu \mathrm{g} / \mathrm{ml}$ is considered susceptible-dose dependent (23). Infections due to such 
isolates respond to treatment in the same manner as a susceptible strain provided a high enough dosage of fluconazole is administered.

Secondary fluconazole resistance in C. albicans most often occurs in either AIDS patients with OPC or less frequently in immunosuppressed patients with systemic candidiasis. Resistance to fluconazole in systemic candidiasis is usually due to intrinsic azole resistance as a result from infection with C. glabrata or C. krusei. Development of resistance in bloodstream infections by $C$. albicans is rare (8).

Fluconazole resistance in $\mathrm{OPC}$ is due to secondary resistance with resistant $C$. albicans strains recovered from a third of the patients with advanced AIDS. Recurrent OPC occurs most often in patients with $\mathrm{CD}^{+} \mathrm{T}$ cells $<50 / \mathrm{mm}^{3}$ (24). Patients with OPC are usually treated with fluconazole at low doses (100 mg per day) over a long period of time either continuously or intermittently $(8,24)$. When a relapse of OPC occurs, the dose of fluconazole is increased (200, $400,800 \mathrm{mg}$ per day) with a maximum dosage of $800 \mathrm{mg}$ per day. Before the use of HAART, fluconazole prophylaxis decreased the morbidity and mortality attributed to mucosal and invasive fungal infections. With the use of HAART, incidences of OPC have decreased. However, in patients undergoing HAART who develop OPC, resistance has been shown to occur. Of the patients undergoing HAART and fluconazole treatment (continuous or episodic), 36 to $45 \%$ developed fluconazole resistance (25). Although HAART has decreased the incidence of OPC, the cost of therapy in developing nations is too high to make it widely available; poor compliance also limits the effectiveness of HAART. Fluconazole prophylaxis is still necessary in resource-poor regions to control candidiasis, so development of resistance by C. albicans is still problematic.

\section{Molecular Mechanisms of Resistance}

Azole resistance in C. albicans occurs primarily due to the selection of resistance over time rather than replacement by a more resistant strain. Several genotyping techniques are used to determine if an azole resistant strain was derived from a specific azole sensitive parent. Pulsed-field gene electrophoresis (molecular karyotyping), restriction fragment length polymorphism (RFLP), and repetitive marker Ca3 probe-based fingerprinting are three methods used to determine the genetic relationship between a susceptible and a resistant strain. The karyotype and RFLP patterns between C. albicans of different patients are different, suggesting little relationship between strains. However, serially-collected isolates recovered from a single patient typically indicate that the resistant isolate has the same karyotype and RFLP patterns as the previously susceptible isolate $(26,27)$. Using these typing methods to identify matched isolate sets, specific mutations that cause azole resistance can be determined.

There are several known mechanisms by which C. albicans can develop resistance to azoles. A decrease in intracellular accumulation of azoles reduces the amount of drug that comes into contact with its target. Differences in azole accumulation in the cell can be due to the uptake or the efflux of the drug. Using tritium labeled fluconazole, $\left[{ }^{3} \mathrm{H}\right]$ fluconazole, differences in accumulation can be studied. In a susceptible isolate, fluconazole accumulation as a function of concentration does not occur in a linear fashion. This eliminates passive diffusion as a 
mechanism of fluconazole uptake (28). Fluconazole sensitive isolates pretreated with sodium azide and then exposed to fluconazole show little difference in drug accumulation $(28,29)$. Sodium azide, a metabolic inhibitor, interferes with energy dependent pumps by decreasing the amount of ATP generated by the mitochondria. Because accumulation doesn't change, drug import is not energy dependent suggesting that fluconazole enters the cell through facilitated diffusion (28). In resistant isolates, $\left[{ }^{3} \mathrm{H}\right]$ fluconazole accumulation is significantly less than that of the susceptible parent isolate. The resistant isolates pretreated with sodium azide shows increased fluconazole accumulation $(28,29)$. Because fluconazole accumulation increases due to the disruption of energy dependent pumps and drug import seems to be energy independent, increased resistance due to decreased accumulation can be attributed to efflux pumps rather than impaired drug uptake.

One class of efflux pumps found to be overexpressed in resistant isolates of $C$. albicans is the ATP-binding cassette (ABC) transporters. ABC transporters can be found in all organisms, and most transporters are substrate specific. Multidrug resistance $\mathrm{ABC}$ transporters, however, have evolved to transport a wide variety of lipophilic molecules. Prokaryotes have ABC transporters that import and export various substrates; eukaryotic $\mathrm{ABC}$ transporters are predominately exporters (30). All ABC transporters are composed of two six-transmembrane domains (TMD) and two cytoplasmic nucleotide-binding domains (NBD) that contain the ABC. The first and most well characterized ABC multidrug transporter was mammalian P-glycoprotein, responsible for the development of resistance in cancer cells to chemotherapy drugs (31).

In C. albicans, ten efflux pumps have been identified as ABC multidrug transporters. These efflux pumps were named the Candida drug resistance (CDR) family of transporters (10). Of the ten CDR pumps, only two ABC transporters, encoded by $C D R 1$ and $C D R 2$, have been implicated in azole resistance. $C D R 1$ is highly homologous to $P D R 5$, a multidrug transporter in $S$. cerevisiae, and the protein structures are almost identical (32). PDR5 confers cycloheximide, chloramphenicol, and fluconazole resistance as a $p d r 5 \Delta$ double disruption mutant causes hypersensitivity to cycloheximide, chloramphenicol, and fluconazole (33). CDRl was first cloned and characterized for its ability to complement drug resistance in $S$. cerevisiae $(29,32)$. In $C$. albicans clinical matched sets, $C D R 1$ was found to be expressed in both susceptible and resistant isolates. In resistant isolates, the expression of $C D R 1$ increased relative to the MIC of the isolates; the higher the MIC, the more CDRI mRNA was detected $(29,34,35)$. The $C D R 1$ gene was also disrupted in a susceptible strain, CAF4, lacking both alleles of URA3. In the absence of the $\mathrm{ABC}$ transporter, the strain became hypersusceptible to a variety of azoles (36). The overexpression of $C D R 1$ using an inducible promoter in a $c d r 1 \Delta$ double mutant showed resistance to azoles (37).

$C D R 2$, another $\mathrm{ABC}$ transporter efflux pump $84 \%$ identical to $C D R 1$, was also identified by its functional complementation of azole resistance in $S$. cerevisiae. In matched isolates, $C D R 2$ gene expression could not be detected in susceptible isolates. In resistant isolates, the gene is expressed relative to the $\operatorname{MIC}(34,35)$. The expression of $C D R 2$ in a resistant isolate is generally several fold higher than the expression of $C D R 1$ in the same isolates. Disruption of $C D R 2$ in an auxotrophic strain of $C$. albicans showed little change in susceptibility in response to various drugs. A double disruption construct, $c d r 1 \Delta c d r 2 \Delta$, showed hypersusceptibility to azoles. 
However, the lack of baseline expression levels of $C D R 2$ in a susceptible isolate could explain the lack of hypersusceptibility to drugs (38).

The second class of efflux pumps found in Candida is the major facilitator superfamily (MFS) transporters. MFS transporters are energy dependent transporters that rely on the proton motive force generated by the proton gradient across membranes rather than ATP for energy. Like ABC transporters, MFS transporters can be symporters, antiporters, or uniporters, and they can be found in all cells of all organisms (39). The MFS transporters have 12 to 14 transmembrane domains and no $\operatorname{NBD}(10,40)$.

In $C$. albicans, the main MFS transporter contributing to fluconazole resistance is an antiporter multidrug resistance 1 (MDRI), formerly known as BEN1. The Candida MDRI is not to be confused with human $M D R 1$, which is an $\mathrm{ABC}$ transporter. MDR1 was first cloned for its ability to confer resistance in $S$. cerevisiae to benomyl and methotrexate (41). Overexpression of $M D R 1$ in S. cerevisiae under the control of an $A D H 1$ promoter showed resistance to fluconazole but no ketoconazole or itraconazole (29). Unlike the ABC transporters, fluconazole and voriconazole seems to be the only azole substrate for $M D R 1$. Disruption of $M D R 1$ in wild-type isolates showed increased susceptibility to methotrexate, cycloheximide, and 4-nitroquinoline- $N$ oxide. The disruption mutants showed no changes in susceptibility to benomyl or fluconazole $(20,36,42)$. In clinical matched isolates, MDRI mRNA is only detected in resistant isolates; there seems to be no baseline expression of $M D R 1$ in susceptible isolates $(34,43)$. The wild-type disruption mutants not being hypersusceptible to fluconazole are most likely due to the lack of baseline expression of MDR1.

Changes in lanosterol demethylase, the target of azole antifungals, are another mechanism by which resistance develops. Mutations in ERG11 translate into amino acid substitutions that change the three-dimensional structure of the enzyme or have decreased affinity for the azole. Several point mutations have been identified in resistant isolates $(27,44$, 45). One such point mutation causes an arginine to lysine substitution at position 467 . The $\mathrm{R} 467 \mathrm{~K}$ is highly conserved among lanosterol demethylase found in fungi and mammals and is located in the heme binding region of the enzyme $(44,46)$. Using site directed mutagenesis, the R467K mutation was introduced into S. cerevisiae. In the presence of the mutated Erg11p, fluconazole bound to the enzyme with a lower affinity (46). Thus far, there have been thirteen additional point mutations that lead to amino acid substitutions and increased azole MIC have been identified $(27,44)$.

Overexpression of $E R G 11$ can also lead to resistance due to the increased production of lanosterol demethylase overwhelming the azole concentration in the cell. In S. cerevisiae, the overexpression of ERG11 alone is sufficient to confer azole resistance. The ERG11 gene, under the control of an inducible GAL1 promoter, could grow on galactose plates with $128 \mu \mathrm{g} / \mathrm{ml}$ fluconazole (47). In C. albicans, the up-regulation of ERG11 is generally found in conjunction with other resistance mechanisms. Twenty-five percent of resistant isolates recovered from patients overexpressed $E R G 11$ and one of the efflux pumps (27). In response to azole treatment, $E R G 11$ and several other $E R G$ genes were up-regulated $(48,49)$. 


\section{TRANSCRIPTIONAL REGULATORS}

The overexpression of $C D R 1, C D R 2, M D R 1$, and $E R G 11$ can be due to three mechanisms: gene amplification, increased half-life of mRNA, or increased transcription due to promoter mutation or changes in transcriptional activators. Gene amplification occurs when there are more than two copies of a certain gene due to chromosomal duplication. While some isolates have been found to contain trisomies and isochromosomes, many clinical matched sets show overexpression with no chromosomal anomalies (50).

The stability of mRNA also makes an impact on overexpression of resistance genes. A recent study showed that resistant isolates had a slower turnover rate of CDR1 mRNA than susceptible isolates (51). However, mRNA stability alone does not result in the increased mRNA levels in resistant isolates. Nuclear run-on assays performed on susceptible and resistant isolates show an increased level of transcription in the resistant isolates $(34,51)$. In the absence of chromosomal anomalies, the increase in transcription can be attributed to interactions between transcription factors and the promoter.

\section{Transcription Factor Classes}

There are two major classes of transcription factors involved in drug resistance in yeast. The bZip transcription factor class is found exclusively in eukaryotic cells, and the first bZip proteins were purified in mammalian cells in the late 1980's (52). These transcription factors are defined by a highly conserved basic leucine zipper (bZip) motif. The bZip motif is a 60 to 80 amino acid long bipartite $\alpha$-helix that binds the major groove of DNA. The $\mathrm{N}$-terminal portion of the bZip motif contains the DNA binding domain (DBD) consisting of two basic amino acid clusters that are responsible for the binding of DNA. The C-terminal domain is the leucine zipper with leucine residues every seven amino acids (52). It is through the leucine zipper that dimerization occurs $(52,53)$. The activator protein-1 (AP-1) transcription factor family falls into the bZip class of transcription factors. AP-1 transcription factors can be heterodimers consisting of one Fos family and one Jun family of bZip protein or homodimers containing two Jun family proteins. The dimers bind DNA at a specific AP-1 recognition element (ARE), TGA(C/G)TCA (53). This ARE motif is a ubiquitous regulatory element found in promoter and enhancer regions of many genes regulating cell proliferation, differentiation, apoptosis, and oncogenesis.

AP-1 transcription factors have been identified in several fungal species $(54,55)$. These transcription factors are referred to as yeast AP-1 (yAP1) transcription factors. The first yAP-1 transcription factor cloned and characterized was the $S$. cerevisiae $\operatorname{YAPl}(54,56)$. Yaplp was identified for its ability to bind an ARE derived from the Simian virus 40 (SV40) enhancer promoter known to be bound by mammalian AP-1 (56). This yeast promoter motif is called the YAP1 response element (YRE). YAPl homologs were also found in Schizosaccharomyces pombe (PAP1), Kluveromyces lactis (KAP1), and C. albicans (CAP1) (57). The yAP-1 transcription factors have bZip DBDs homologous to that of mammalian AP-1 transcription factors. In addition, yAP-1 transcription factors share two cysteine rich domains (CRD) 3' of the bZip domain. The amino terminal CRD (n-CRD) and the carboxyl terminal CRD (c-CRD) are found in only the yeast bZip transcription factors and play a role in the localization and 
activation of the yAP-1 (58). The yAP-1 transcription factors regulate and mediate the response to oxidative stress and multidrug resistance.

In $S$. cerevisiae, the c-CRD has been implicated as the oxidative stress sensor and the nuclear localization of Yap1p. The c-CRD contains three conserved cysteine residues and a region rich in hydrophobic amino acids that resemble a nuclear export signal $(58,59)$. Studies using a green fluorescent protein (GFP) tagged Yap1p showed that under normal conditions, GFP-Yap1p localizes to the cytoplasm. Nuclear localization occurs when the cells are exposed to oxidative stress such as $\mathrm{H}_{2} \mathrm{O}_{2}$ or diamide. The oxidative stress sensor function was elucidated when the c-CRD domain was fused to a GAL4 DBD and GFP. This fused protein was found in the cytoplasm under unstressed conditions, but under oxidative stress conditions, the GFP was localized to the nucleus (60). This shows that the c-CRD alone has the ability to localize the fused transcription factor to the nucleus in the presence of a stressor. Mutations in or deletion of the c-CRD trapped the GFP-Yaplp in the nucleus independent of oxidative stress $(58,59,60)$. Nuclear localization occurs either through the inhibition of nuclear export or increases in nuclear import. In yeast, Crm1p has been identified as the nuclear export factor that interacts with yAP1 to export the proteins out of the nucleus (61). Disulfide bonds form between the cysteine residues in the presence of $\mathrm{H}_{2} \mathrm{O}_{2}$ or diamide and prevents the interaction between Crm1p and Yap1p (62). Mutations in the c-CRD also prevent the Crm1p-Yaplp interaction. The n-CRD on the other hand determines the response to specific oxidative stresses. In $S$. cerevisiae, disruption of the n-CRD conferred hypersusceptibility to $\mathrm{H}_{2} \mathrm{O}_{2}$ but resistance to diamide (63). This points to the differences in disulfide bond formation between the cysteine residues in response to oxidative stress. Under diamide stress, a disulfide bond forms between two cysteine residues in the c-CRD. In the presence of $\mathrm{H}_{2} \mathrm{O}_{2}$, a disulfide bond forms between a cysteine residue in the c$\mathrm{CRD}$ and a cysteine residue in the $\mathrm{n}-\mathrm{CRD}$. This disulfide bridge does not occur under diamide stress (59).

The zinc finger protein family is the second major class of transcription factors responsible for drug resistance in yeast and are the largest family of transcriptional regulators in eukaryotes (64). The first zinc finger protein was identified in Xenopus in the 1980's (65). This family of transcription factors is characterized as having the common zinc finger motif, consisting of one $\alpha$ helix and a pair of antiparallel $\beta$ strands. It also contains one or more zinc atoms bound by cysteines or histidines stabilizing the domain and contributing to the structure and function of the protein. The majority of zinc finger proteins bind DNA as transcription factors, but some act as mediators of protein/protein interactions, chromatin remodeling, protein chaperoning, lipid binding, stress response, pleiotropic drug resistance (PDR), and zinc sensing (64).

There are three classes of zinc finger proteins. The $\mathrm{Cys}_{2} \mathrm{His}_{2}$ class is the classical zinc finger protein that binds DNA as monomers. These proteins have the zinc finger structure Cys$\mathrm{X}_{2-4}$-Cys- $\mathrm{X}_{12}-\mathrm{His}-\mathrm{X}_{3-5}$-His. The second class of zinc finger proteins is the $\mathrm{Cys}_{4}\left(\mathrm{Cys}-\mathrm{X}_{2}-\mathrm{Cys}_{\mathrm{s}}-\mathrm{X}_{\mathrm{n}^{-}}\right.$ Cys- $\mathrm{X}_{2}-\mathrm{Cys}-\mathrm{X}_{\mathrm{n}}-\mathrm{Cys}-\mathrm{X}_{2}-\mathrm{Cys}-\mathrm{X}_{\mathrm{n}}-\mathrm{Cys}-\mathrm{X}_{2}-\mathrm{Cys}$ ) class of proteins. These zinc finger proteins bind DNA as homodimers or heterodimers. The homodimers bind inverted repeats while the heterodimers bind direct repeats (64). $\mathrm{Cys}_{6}$, the third class of zinc finger proteins, have six cysteines $\left(\mathrm{Cys}-\mathrm{X}_{2}-\mathrm{Cys}-\mathrm{X}_{6}-\mathrm{Cys}-\mathrm{X}_{5-16}-\mathrm{Cys}-\mathrm{X}_{2}-\mathrm{Cys}-\mathrm{X}_{6-8}-\mathrm{Cys}\right)$ bound by two zinc atoms and are exclusive to fungi. These proteins bind DNA as monomers, homodimers, and heterodimers. 
This third class of zinc finger proteins is often referred to as zinc cluster proteins or zinc binuclear proteins $(64,66,67)$.

Zinc cluster transcription factors can be found in both the cytoplasm and the nucleus. The proteins found in the cytoplasm require a nuclear localization signal and importin $\beta$ receptors (64). More commonly, zinc cluster transcription factors remain in the nucleus and are often constitutively bound to the promoter. Zinc cluster proteins contain a DBD, a regulatory domain, and an acidic domain. The DBD consists of the zinc finger motif, the linker, and the dimerization domain (64). The zinc finger motif binds as dimers to CGG nucleotide triplets through major groove interactions. The CGG motifs can be everted, inverted, or direct repeats $(64,66)$. The linker domain of the DBD has no homology between zinc cluster proteins, but this contributes to the specificity of DNA binding (68). A previous study swapped the linker domains of three different zinc cluster transcription factors, and showed that it was the linker that determined the target DNA. The dimerization domain is highly conserved with heptad repeats and resembles the leucine zipper in bZip proteins (64). The regulatory domain, also known as the middle homology domain, spans 80 amino acids and is only found in zinc cluster transcription factors (69). Deletions of this domain render the transcription factor constitutively active. Partial and complete deletions of the regulatory domain in the $S$. cerevisiae transcription factor Leu3p renders it constitutively active (70). The regulatory domain is not highly conserved among zinc cluster proteins. Differences in the regulatory domain may determine the spacing of the CGG motif that the transcription factor binds (69). The acidic domain acts as the activation domain of the protein. Activation of zinc cluster transcription factors occur through phosphorylation or dephosphorylation events (64). There is little homology between the acidic domains of different zinc cluster proteins and the function of the domain differs between proteins (64). In S. cerevisiae, deletion of the acidic domain of Leu3p rendered the transcription factor inactive (70). In the $S$. cerevisiae transcription factor Pdr3p, mutations in this domain results in a gain-of-function of the transcription factor (71).

\section{Transcription Factors and Resistance}

Increased transcription of azole resistance genes in C. albicans could be due to mutations in the promoter region that results in deregulation of the gene (cis-regulation) or mutations in a transcription factor that controls the expression of the gene (trans-regulation). The promoter regions of clinical matched isolates known to overexpress $C D R 1 / C D R 2$ or $M D R 1$ were sequenced $(72,73)$. In the clinical isolates DSY294 (susceptible parent) and DSY296 (resistant), there were no mutations in the promoter regions of the $C D R$ genes (73). In the MDR 1 overexpressing isolates, $\mathrm{F}$ and $\mathrm{G}$, polymorphisms were found between the alleles of the two isolates. However, the same polymorphisms were found in the susceptible and resistant allele of each isolate pair (72). The lack of mutation suggests that the overexpression of the two efflux mechanisms do not occur through a cis-acting mechanism.

In order to determine if a trans-regulatory element was responsible for the up-regulation of the efflux pumps, the wild-type promoter regions were fused to a reporter gene to determine transcriptional activation. The promoters of $C D R 1$ and $C D R 2$ from genome strain SC5314 were fused to Renilla luciferase $(R L U C)$, and the constructs were transformed into a resistant strain 
(2090) derived from the resistant isolate DSY296 (73). A high level of luciferase activity was detected in the cells transformed with the CDR1-RLUC and the CDR2-RLUC constructs even though the promoters were derived from a susceptible isolate. Similarly, to examine the activation of $M D R 1$, the promoter region of MDR1 from wild-type strain CAI4 was fused to GFP. The MDR1-GFP construct was transformed into two resistant isolates, F5 and G5, and their susceptible parent isolates, F2 and G2. There was no fluorescence detected in the susceptible isolates, whereas fluorescence was detected in the resistant isolates (72). The ability for the $C D R$ and $M D R$ promoters from susceptible isolates to be activated when transformed into resistant strains suggests that mutations in transcriptional regulators are responsible for the overexpression of resistance mechanisms.

Regulatory networks and drug resistance have been better characterized in the model organism $S$. cerevisiae. In $S$. cerevisiae, two networks of genes, YAP and pleiotropic drug resistance (PDR), are responsible for multidrug resistance. The YAP network of genes is predominantly responsible for the oxidative stress response. Yap1p, a bZip transcription factor described above, binds the YRE motif T(T/G)A(C/G)TAA. Not only has Yap1p been shown to sense hydrogen peroxide and diamide stress and confer resistance to oxidative stress (62), but it has also been shown to protect against a variety of antimicrobial drugs through the overexpression of $\mathrm{ABC}$ transporters (YCF1) and MFS transporters (FLRl). The overexpression of FLR1 specifically confers resistance to cycloheximide, 4-nitroquinoline $N$-oxide, benomyl, and fluconazole (55). The deletion of YAPl abolished the ability for induction of FLRI by antimicrobial agents (74). Three YRE motifs have been identified in the promoter region of FLR1, and increased activation of Yap $1 \mathrm{p}$ increases the transcription of FLR1 $(55,74)$. Yaplp has also been shown to bind directly to the three YRE motifs, and the deletion of the YREs abolished the induction of FLR1 by fluconazole (74).

The PDR network is characterized by the overexpression of ABC transporters and MFS transporters. The overexpression of the ABC transporter gene, PDR5, confers resistance to several antimicrobial agents including fluconazole. The PDR target genes are controlled by two zinc cluster transcription factors, Pdr1p and Pdr3p. Pdr1p and Pdr3p bind as homodimers or heterodimers to the pleiotropic drug response element (PDRE) motif, TCCGCGGA, with an everted CGG $(64,75)$. The dimers can also bind degenerate PDRE motifs suggesting different specificities for homodimers versus heterodimers (76). At least one PDRE motif has been found in all target genes of Pdr1p and Pdr3p (77). Two PDRE motifs have been found in the promoter region of $P D R 3$, suggesting autoregulation by $P d r 3 p$ and regulation by Pdr1p. Hyperactive $P D R 1$ and $P D R 3$ mutants increase the expression of $P D R 5$ and cause resistance to various drugs $(78,79)$. The up-regulation of PDR1 and $P D R 3$ also increases the expression of PDR3 (71). Deletion of $P D R 1$ and $P D R 3$ genes results in hypersusceptibility to drugs and decreased expression of PDR5 $(79,80)$.

In addition to the efflux mechanisms, the ergosterol biosynthesis pathway is also better characterized in $S$. cerevisiae. In $S$. cerevisiae, ergosterol biosynthesis genes are regulated by two zinc cluster transcription factors Upc2p and Ecm22p. These transcription factors bind a sterol response element (SRE) motif, CTCGTATAAGC (81). The up-regulation of UPC2 and $E C M 22$ causes up-regulation of $E R G 2$ and $E R G 3$. The SRE motif is present in the promoter regions of $E R G 2, E R G 3$, and other ergosterol biosynthesis genes. Deletion of UPC2 causes 
hypersusceptibility to $\mathrm{AmB}$ and ketoconazole while deletion of ECM22 causes

hypersusceptiblity to cycloheximide and calcofluor white $(81,82)$. The disruption mutants also abolish the expression of the $E R G$ genes (81).

\section{C. albicans Transcription Factors}

In $C$. albicans, $C A P 1$ has been identified as a yAP-1 bZip transcription factor closely related to the $S$. cerevisiae YAP1. Functionally, the overexpression of $C A P 1$ in $S$. cerevisiae restores FLR1 expression and resistance to fluconazole (55). The C-terminal region of Cap $1 \mathrm{p}$ and Yaplp, including the c-CRD, are the most highly conserved. As in S. cerevisiae, the activation and nuclear localization occurs through the c-CRD in response to oxidative stress (55, 83). The disruption of the c-CRD of Caplp causes the protein to be constitutively active and remain localized to the nucleus $(83,84)$. To examine the function of Cap1p in C. albicans, the $C A P 1$ gene was deleted in the susceptible wild-type strain CAI4, and a wild-type or a constitutively active $C A P I$ gene was reintroduced into the knockout strain. In the strains with or without $C A P 1$, the cells were unable to respond to fluconazole. However, with the constitutively active Caplp, the strain was resistant to fluconazole, and increased expression of $M D R 1$ was detected (84). FLRI is also homologous to C. albicans MDR1, a MFS transporter gene responsible for fluconazole resistance (55). A YRE motif was found in the promoter region of $M D R 1$ (85). The promoter region of $M D R 1$ was examined in a fluconazole resistant clinical strain disrupted for URA3 (F5U4). In the absence of the YRE, MDRI expression due to benomyl exposure decreased. However, in the absence of the YRE in a resistant isolate, MDR1 is still expressed (86). In another study, Caplp regulation of MDRI is examined in strains with and without $C A P 1$. In the absence of Cap1p, MDR1 gene expression still occurs in response to benomyl (87). These studies indicate that in the absence of both the YRE motif and Caplp, $M D R 1$ gene expression still occurs in response to benomyl and in resistant isolates. This suggests that while Cap $1 \mathrm{p}$ regulates $M D R 1$ expression, another regulator may also function in azole resistance.

The $\mathrm{ABC}$ transporters $C D R 1$ and $C D R 2$ were identified for their ability to complement the PDR5 phenotype in $S$. cerevisiae. However, there are no PDR1/PDR3 homologues in $C$. albicans. The promoter regions of $C D R 1 / C D R 2$ were examined, and a putative drug responsive element (DRE) motif, ACGG(A/T)TATCGGATA, was identified (73). Genes with the DRE in their promoter region were shown to respond in response to oestradiol including $C D R 1 / C D R 2$. In the absence of this motif, the expression of the genes could not be induced by oestradiol, amorolfine, and terbinafine. In response to oestradiol, band shift assays showed specific protein interaction with the DRE (73). Unlike the PDRE, the DRE has two CGG direct repeats separated by four nucleotides. The two CGG motifs in the promoter region points to a zinc cluster transcriptional regulator. $T A C l$ (transcriptional activator of $C D R$ genes), a gene encoding a zinc cluster protein, was identified near the mating type locus (88). The disruption of this gene abolished the expression of $C D R 1 / C D R 2$ in response to fluphenazine. The disruption strains also showed a hypersusceptibility to fluconazole and terbinafine. When a $T A C 1$ allele from a resistant isolate was reintroduced into the disruption strain, resistance to fluconazole was conferred. The specific DRE previously identified was shown to be specifically bound by Tac1p, and the PDRE motif bound by Pdr1p/Pdr3p in S. cerevisiae was not bound by Taclp (88). 
Sequencing of the TAC1 alleles in susceptible and resistant clinical isolates showed an N977D mutation in the C-terminal activation domain (89). While this mutation was found in one allele of $T A C l$ in the susceptible isolate, the resistant isolate carried two copies of this mutated allele. Because zinc cluster transcription factors function as dimers, the hyperactive allele and the wildtype allele are codominant. It is the loss of heterozygosity that causes overexpression of $\mathrm{ABC}$ transporters and increased azole resistance (89).

Ergosterol biosynthesis in S. cerevisiae is controlled by two zinc cluster transcription factors Upc2p and Ecm22p. In C. albicans, Upc2p is the only homolog of ScUpc2p and ScEcm22p. Disruption of UPC2 in a wild-type strain conferred hypersusceptibility to sterol biosynthesis inhibitors (SBI), including fluconazole, itraconazole, ketoconazole, terbinafine, and fenpropimorph $(82,90)$. In the absence of $U P C 2$, there was also no transcription of $E R G$ genes. The reintroduction of wild-type UPC2 restores baseline susceptibility in wild-type strains (90). The overexpression of $U P C 2$ conferred resistance to ketoconazole and fluphenazine (82). The promoter region of the $E R G$ genes in $C$. albicans was examined for the SRE motif found in $S$. cerevisiae, and an SRE motif was found in all known $E R G$ genes and in $U P C 2$ itself $(82,90)$. Using the SRE motif found in ScERG2 and CaERG2, Upc2p was found to bind to both (82).

\section{CENTRAL HYPOTHESIS AND RESEARCH OBJECTIVES}

In $S$. cerevisiae, genes and their function have been better defined and characterized than in C. albicans. Through the use of S. cerevisiae as a model organism, drug efflux mechanisms and ergosterol biosynthesis genes have been identified in C. albicans through homology and complementation. Increased expression of ERG11 in C. albicans increases lanosterol demethylase concentrations in the cell, increasing the amount of enzyme not bound by azole antifungals. Decreased accumulation of azoles in C. albicans due to increased expression of drug efflux pumps also decreases the efficacy of azole treatment. In C. albicans, drug resistance genes have been shown to be controlled by trans-regulatory elements rather than cis-regulatory elements. Several transcriptional regulators have been identified in S. cerevisiae to control drug efflux and sterol biosynthesis. The transcription factors in S. cerevisiae have been shown to be co-regulated with the expression of resistance genes; many are also autoregulated. Importantly, the transcriptional regulators in S. cerevisiae do not have homologs in C. albicans.

I hypothesized that overexpression of resistance genes in C. albicans is due to gain-offunction mutations in transcriptional regulators. With the completion of the C. albicans genome sequencing project, microarray technology can now be used to study drug resistance. By studying matched clinical isolates that overexpress either CDR1/CDR2, MDR1, or ERG11, genes that are co-regulated with these resistance genes can be identified. Among these co-regulated genes, we have identified previously described as well as novel transcriptional regulators.

Taclp, a zinc cluster transcription factor, has been previously identified as the transcriptional regulator of the $\mathrm{ABC}$ transporters $C D R 1$ and $C D R 2$. Gain-of-function mutations in $T A C l$ and loss of heterozygosity contribute to azole resistance. Taclp has also been shown to regulate the expression of genes co-regulated with $C D R 1$ and $C D R 2$. Our first research objective (Chapter 2) was to examine matched clinical isolates known to overexpress $C D R 1 / C D R 2$ by 
gene expression and location profiling in an effort to identify additional genes potentially involved in azole resistance. I hypothesized that a gain-of-function mutation in a transcription factor, now known to be Tac1p (88), regulates the expression of $C D R 1, C D R 2$, and co-regulated genes. Our studies show that like many zinc cluster transcription factors in S. cerevisiae, TAC1 is autoregulated and targets a subset of genes involved in multidrug resistance in C. albicans. Additionally, we identified the Taclp regulon as it pertains to known mutations in $T A C l$ in clinical matched isolates.

In research objective 2 (Chapter 3), we examined clinical matched isolates known to overexpress MDR1. At the time, the transcriptional regulator responsible for $M D R 1$ expression had yet to be identified. I hypothesized that gain-of-function mutations in a transcription factor regulate the expression of $M D R 1$ and $M D R 1$ co-regulated genes. Using expression profiling, we discovered and characterized a previously unidentified zinc cluster transcription factor MRRI that regulates the expression of $M D R I$ in clinical resistant isolates. We also identified a core group of genes regulated specifically by the gain-of-function mutation in MRR1.

In addition to the isolates that overexpress the $C D R$ and/or $M D R I$ efflux pumps, several resistant isolates have been identified with unknown resistance mechanisms. In research objective 3 (Chapter 4), we characterized a clinical matched isolate pair with no previously identified resistance mechanism by gene expression profiling. I hypothesized that a gain-offunction mutation in $U P C 2$ is responsible for the regulation of the $E R G$ genes in azole resistant clinical isolates. In the resistant isolate, several $E R G$ genes were found to be up-regulated. Additionally, UPC2 was also overexpressed. We identified a gain-of-function mutation in UPC2 responsible for the increased expression of $E R G$ genes. Using expression profiling, we defined a set of genes whose expression depended on the mutation found in UPC2. 


\section{CHAPTER 2: IDENTIFICATION OF THE TAC1P REGULON*}

\section{INTRODUCTION}

Several mechanisms of resistance to the azole antifungal agents have been described in $C$. albicans including increased expression of genes encoding multi-drug efflux pumps (91, 92, 93, 94, 95, 96, 97). These include the gene encoding a transporter of the MFS (MDR1) and genes encoding two $\mathrm{ABC}$ transporters $(C D R 1$ and $C D R 2)(91,92,94,95,96)$. Over-expression of these efflux pumps is presumed to prevent accumulation of effective concentrations of the azole antifungal agents within the fungal cell. Among studies examining multiple matched azolesusceptible and -resistant sets of isolates, some isolates only over-express $M D R 1$, whereas others only over-express $C D R 1$ and $C D R 2(91,98)$. These observations suggest that two distinct transcriptional pathways are involved in regulating these efflux pumps.

Previous studies have shown that a wild-type $C D R 1$ promoter fused to a luciferase reporter construct becomes activated when placed in an azole-resistant isolate that over-expresses $C D R 1$ and $C D R 2$ (99). Using this system, it was shown that a conserved DNA sequence element in the $C D R 1$ and $C D R 2$ promoters, named drug response element (DRE; 5'-CGGAA/TATCGG), was necessary for $C D R 1$ constitutive, as well as drug-inducible, transcriptional activation (99). These results suggested that a gain-of-function mutation in a transcription factor was the cause of $C D R 1$ and $C D R 2$ constitutive overexpression in azole-resistant isolates (99). The zinc cluster transcription factor Tac $1 \mathrm{p}$ was recently identified and shown to be responsible for $C D R 1$ and $C D R 2$ transcriptional activation $(89,100)$. An amino acid change from an asparagine to aspartic acid at position 977 (N977D) in Tac1p was able to confer increased expression of CDR1 and $C D R 2$ accompanied by decreased azole susceptibility (89). It was also shown that the DBD of Tac $1 \mathrm{p}$ fused to the glutathione $S$-transferase (GST) protein binds in vitro to the $C D R 1$ and $C D R 2$ DRE (100). Interestingly, increased azole resistance is observed when a gain-of-function mutation is present in both $T A C l$ alleles (89). The TACl locus is on chromosome 5. Homozygosity at the $T A C l$ locus occurs either through mitotic recombination between copies of chromosome 5, or through the presence of extra copies of chromosome 5 harboring a gain-offunction $T A C l$ allele and loss of chromosome 5 with the wild-type $T A C l$ allele (89). Selmecki et al. demonstrated that a specific segmental aneuploidy, consisting of an isochromosome composed of the two left arms of chromosome 5, is associated with azole resistance. Increases and decreases in azole resistance were found to be strongly associated with gain and loss of this isochromosome (50). Recent work by Coste et al. showed that the effect of specific mutations in azole resistance genes located on chromosome 5 (TAC1, ERG11) can be further enhanced by loss-of-heterozygosity and/or addition of extra copies of chromosome 5 (101).

In addition to $C D R 1$ and $C D R 2$, other targets of Tac1p (RTA3, HSP 12, and IFU5) have been identified, all of which contain a putative DRE in their promoter region (100). In a separate study, it was shown that $P D R 16$, encoding a putative phosphatidylinositol transfer protein contributing to clinical azole resistance, is also a target of Tac1p $(102,103)$. We used functional

\footnotetext{
"Liu, T. T., S. Znaidi, K. S. Barker, L. Xu, R. Homayouni, S. Saidane, J. Morschhauser, A. Nantel, M. Raymond and P. D. Rogers. 2007. Genome-wide expression and location analyses of the Candida albicans Tac1p regulon. Eukaryot Cell. 6: 2122-38. Adapted by permission of publisher.
} 
genomic approaches, namely genome-wide expression and location profiling, to identify the Tac $1 p$ regulon. In collaboration with our group, the laboratory of Dr. Martine Raymond at Université de Montréal constructed the chromatin immunoprecipitation (ChIP) microarrays used in the study as well as the triple-HA-tagged Taclp. Our results provide a more comprehensive picture of the molecular effects of Taclp mutations in azole-resistant clinical isolates and suggest other important cellular functions for Tac1p in C. albicans.

\section{MATERIALS AND METHODS}

\section{Strains and Growth Media}

The C. albicans strains used in this study are listed in Table 2-1. The matched clinical isolate sets and strain SZY31 were grown in yeast peptone dextrose (YPD) broth (Sigma-Aldrich, St. Louis, MO) at $30^{\circ} \mathrm{C}$. The CAI4 strain and its epitope-tagged derivatives were grown at $30^{\circ} \mathrm{C}$ in YPD or, under selective conditions, in synthetic complete (SC) medium lacking uracil (104) supplemented or not with $100 \mu \mathrm{g} \cdot \mathrm{ml}^{-1}$ of uridine and $1 \mathrm{mg} \cdot \mathrm{ml}^{-1}$ of 5-fluoroorotic acid (5-FOA, Toronto Research Chemicals, Inc., Toronto, ON). The Escherichia coli DH5 $\alpha$ bacterial strain was used for DNA cloning and maintenance of the plasmid constructs.

\section{Construction of $C$. albicans Expression Microarrays}

The nucleotide sequences corresponding to 6,165 open reading frames (ORFs) for $C$. albicans were downloaded from the Galar Fungail European Consortium (Assembly 6, http://www.pasteur.fr/Galar_Fungail/CandidaDB/). We set out to design two non-overlapping probesets targeting the 3'- 600 bp region of each ORF. Each probeset consisted of 13 'perfect match' 25-bp probes and their corresponding 'mismatch' control probes containing a single mismatch in the center of the oligonuclotide. For ORFs less than $600 \mathrm{bp}$ in length the sequence was divided in two equal segments for subsequent design procedures. Optimum probesets were selected by the Affymetrix design team based on their model which, among other things, considers probe hybridization quality and cross-hybridization potential. Consequently, in some cases, only one probeset was selected for a given ORF. For quality control and normalization purposes, we made 2-3 additional probe sets spanning the entire sequence of the $C$. albicans $18 \mathrm{~S}$ rRNA (GenBank Accession M60302), genes encoding GAPDH, actin and Mdr1p (Bmr1p) in addition to the standard Affymetrix controls (BioB, C, D, cre, DAP, PHE, LYS, THR). In the end, the GeneChip contained 10,736 probe sets including 9 controls, 6,123 unique ORFs and duplicate probe sets for 4,604 ORFs. The duplicate probe sets are made to distinct regions of each particular ORF, thereby allowing 2 independent measurements of the mRNA level for that particular gene. The C. albicans custom Affymetrix NimbleExpress Arrays (CAN04a530004N) were manufactured by NimbleGen Systems (105) per our specification. 
Table 2-1. Strains used in this study.

\begin{tabular}{|c|c|c|c|c|}
\hline Strain & $\begin{array}{c}\text { Parental } \\
\text { strain }\end{array}$ & Genotype or Description & $\begin{array}{c}\text { Fluconazole } \\
\text { MIC } \\
(\mu \mathrm{g} / \mathrm{mL}) \\
\end{array}$ & Reference \\
\hline C43 (DSY294) & & Azole-susceptible clinical isolate & $0.25^{a}$ & $(97)$ \\
\hline C56 (DSY296) & $\mathrm{C} 43$ & Azole-resistant clinical isolate & $128^{a}$ & $(97)$ \\
\hline $\mathrm{Gu} 2$ & & Azole-susceptible clinical isolate & $6.25^{b}$ & $(95)$ \\
\hline Gu5 & $\mathrm{Gu} 2$ & Azole-resistant clinical isolate & $>100^{b}$ & $(95)$ \\
\hline 3 & 2 & Azole-susceptible clinical isolate & $8^{c}$ & $(106)$ \\
\hline 17 & 16 & Azole-resistant clinical isolate & $>64^{c}$ & $(106)$ \\
\hline 5457 & & Azole-susceptible clinical isolate & $\leq 0.5^{d}$ & $(102)$ \\
\hline 5674 & 5457 & Azole-resistant clinical isolate & $32.0^{d}$ & $(102)$ \\
\hline SZY31 & 5674 & tacl $1 \Delta::$ FRT/tacl $\Delta::$ FRT & $0.5^{e}$ & $(103)$ \\
\hline CAI4 & & ura3s::imm434/ura3s::imm434 & $2.0^{e}$ & $(18)$ \\
\hline SZY51 & CAI4 & $T A C 1 / T A C 1:: H A_{3}-U R A 3-H A_{3}$ & ND & This study \\
\hline SZY63 & SZY 51 & $T A C 1 / T A C 1:: H A_{3}$ & $2.0^{e}$ & This study \\
\hline SZY103 & SZY63 & $T A C 1:: H A_{3}$ & $2.0^{e}$ & This study \\
\hline SZY106 & SZY63 & $\mathrm{TACl}: \mathrm{HA}_{3}$ & $2.0^{e}$ & This study \\
\hline SZY91 & SC5314 & $\operatorname{tac} 1 \triangle / T A C 1^{N 972 D}-M P A^{R}-F L P$ & $16.0^{e}$ & $(103)$ \\
\hline
\end{tabular}

${ }^{a}$ Fluconazole concentration yielding at least $90 \%$ growth inhibition after $24 \mathrm{~h}$ of growth compared with the growth of the control (96).

${ }^{b}$ Lowest Fluconazole concentration in which little or no growth was visually detected after $48 \mathrm{~h}$ of growth.

${ }^{c} \mathrm{MIC}_{80}$ according to the CLSI macrodilution reference method or a microdilution modification of the CLSI method $(106,107)$.

${ }^{d} \mathrm{MIC}_{50}$ values, determined as the first concentration of the azole drug able to reduce growth by $50 \%$ as compared with control cells grown in the absence of drug.

${ }^{e} \mathrm{MIC}_{70}$ values. 


\section{RNA Preparation for Microarrays}

The matched clinical isolate sets and strain SZY31 were grown in YPD broth at $30^{\circ} \mathrm{C}$ in a shaking incubator to mid-log phase as described previously (108). The cell pellets were frozen and stored at $-80^{\circ} \mathrm{C}$ prior to RNA preparation. Experiments were repeated independently three times. Total RNA was isolated using the hot SDS-phenol method (109). Frozen cell pellets were suspended in $12 \mathrm{ml}$ of $50 \mathrm{mM}$ sodium acetate $(\mathrm{pH} 5.2), 10 \mathrm{mM}$ EDTA at room temperature, after which $1 \mathrm{ml}$ of $20 \%$ sodium dodecyl sulfate and $12 \mathrm{ml}$ of acid phenol (Fisher Scientific, Waltham, MA) were added. This mixture was incubated $10 \mathrm{~min}$ at $65^{\circ} \mathrm{C}$ with vortexing each minute, cooled on ice for $5 \mathrm{~min}$, and centrifuged for $15 \mathrm{~min}$ at 12,000 x $\mathrm{g}$. Supernatants were transferred to new tubes containing $15 \mathrm{ml}$ of chloroform, mixed and centrifuged at $200 \mathrm{x} g$ for 10 min. The aqueous layer was removed to new tubes, RNA was precipitated with 1 volume isopropanol and 0.1 volume $2 \mathrm{M}$ sodium acetate $(\mathrm{pH}$ 5.0), and then collected by centrifugation at $17,000 \times \mathrm{g}$ for $35 \mathrm{~min}$ at $4^{\circ} \mathrm{C}$. The RNA pellet was suspended in $10 \mathrm{ml}$ of $70 \%$ ethanol, collected again by centrifugation, and suspended in nuclease free water.

\section{cRNA Synthesis and Labeling}

Immediately prior to cDNA synthesis, the purity and concentration of RNA samples were determined from $\mathrm{A}_{260} / \mathrm{A}_{280}$ readings and RNA integrity was determined by capillary electrophoresis using the RNA 6000 Nano Laboratory-on-a-Chip kit and Bioanalyzer 2100 (Agilent Technologies, Santa Clara, CA) as per the manufacturer's instructions. First and second strand cDNA was synthesized from $15 \mu \mathrm{g}$ of total RNA using the SuperScript Double-Stranded cDNA Synthesis Kit (Invitrogen, Carlsbad, CA) and oligo-dT24-T7 primer (PrOligo: SigmaAldrich) according to the manufacturer's instructions. cRNA was synthesized and labeled with biotinylated UTP and CTP by in vitro transcription using the T7 promoter-coupled double stranded cDNA as template and the Bioarray HighYield RNA Transcript Labeling Kit (ENZO Diagnostics, New York, NY). Double-stranded cDNA synthesized from the previous steps was washed twice with $70 \%$ ethanol and suspended in $22 \mu \mathrm{l}$ of RNase-free water. The cDNA was incubated as recommended with reaction buffer, biotin-labeled ribonucleotides, dithtiothreitol, RNase inhibitor mix and T7 RNA polymerase for $5 \mathrm{~h}$ at $37^{\circ} \mathrm{C}$. The labeled cRNA was separated from unincorporated ribonucleotides by passing through a CHROMA SPIN-100 column (Clontech, Mountain View, CA) and ethanol precipitated at $-20^{\circ} \mathrm{C}$ overnight.

\section{Oligonucleotide Array Hybridization and Analysis}

The cRNA pellet was suspended in $10 \mu \mathrm{l}$ of RNase-free water and $10 \mu \mathrm{g}$ was fragmented by ion-mediated hydrolysis at $95^{\circ} \mathrm{C}$ for $35 \mathrm{~min}$ in $200 \mathrm{mM}$ Tris-acetate $(\mathrm{pH} 8.1), 500 \mathrm{mM}$ potassium acetate, $150 \mathrm{mM}$ magnesium acetate. The fragmented cRNA was hybridized for $16 \mathrm{~h}$ at $45^{\circ} \mathrm{C}$ to the $C$. albicans NimbleExpress GeneChip arrays. Arrays were washed at $25^{\circ} \mathrm{C}$ with 6 $\times \mathrm{SSPE}, 0.01 \%$ Tween 20 followed by a stringent wash at $50^{\circ} \mathrm{C}$ with $100 \mathrm{mM} \mathrm{MES,} 0.1 \mathrm{M} \mathrm{NaCl}$, $0.01 \%$ Tween 20 . Hybridizations and washes employed the Affymetrix Fluidics Station 450 using their standard EukGE-WS2v5 protocol. The arrays were then stained with phycoerythreinconjugated streptavidin (Molecular Probes: Invitrogen) and the fluorescence intensities were 
determined using the GCS 3000 high-resolution confocal laser scanner (Affymetrix, Santa Clara, CA). The scanned images were analyzed using software resident in GeneChip Operating System v2.0 (GCOS; Affymetrix). Sample loading and variations in staining were standardized by scaling the average of the fluorescent intensities of all genes on an array to a constant target intensity. The signal intensity for each gene was calculated as the average intensity difference, represented by $[\Sigma(\mathrm{PM}-\mathrm{MM}) /($ number of probe pairs $)]$, where $\mathrm{PM}$ and MM denote perfectmatch and mismatch probes.

\section{Expression Microarray Data Analysis}

The scaled gene expression values from GCOS software were imported into GeneSpring 7.2 software (Agilent Technologies) for preprocessing and data analysis. Probe sets were deleted from subsequent analysis if they were called absent by the Affymetrix criterion and displayed an absolute value below 20 in all experiments. The expression value of each gene was normalized to the median expression of all genes in each chip as well as the median expression for that gene across all chips in the study. Pairwise comparison of gene expression was performed for each matched experiment. Data files of each scanned chip were submitted to the Gene Expression Omnibus database (GEO; www.ncbi.nlm.nih.gov/geo/). The accession number for the series is GSE8727.

\section{Quantitative Real-Time PCR for Expression Data}

An aliquot of the RNA preparations from the samples used in the microarray experiments was saved for quantitative real-time reverse transcription (RT)-PCR follow-up studies. Firststrand cDNAs were synthesized from $2 \mu \mathrm{g}$ of total RNA in a $21-\mu 1$ reaction volume using the SuperScript First-Strand Synthesis System for RT-PCR (Invitrogen) in accordance with the manufacturer's instructions. Quantitative real-time PCRs were performed in triplicate using the 7000 Sequence Detection System (Applied Biosystems, Inc.: ABI, Foster City, CA). Independent PCRs were performed using the same cDNA for both the gene of interest and the 18S rRNA, using the SYBR Green PCR Master Mix (Applied Biosystems, Inc.). Gene-specific primers were designed for the gene of interest and the 18S rRNA using Primer Express software (Applied Biosystems, Inc.) and the Oligo Analysis \& Plotting Tool (QIAGEN, Valencia, CA) and are shown in Table 2-2. The PCR conditions consisted of AmpliTaq Gold activation at $95^{\circ} \mathrm{C}$ for $10 \mathrm{~min}$, followed by 40 cycles of denaturation at $95^{\circ} \mathrm{C}$ for $15 \mathrm{~s}$ and annealing/extension at $60^{\circ} \mathrm{C}$ for $1 \mathrm{~min}$. A dissociation curve was generated at the end of each PCR cycle to verify that a single product was amplified using software provided with the 7000 Sequence Detection System. The change in fluorescence of SYBR Green I dye in every cycle was monitored by the system software, and the threshold cycle $\left(\mathrm{C}_{T}\right)$ above the background for each reaction was calculated. The $\mathrm{C}_{T}$ value of $18 \mathrm{~S}$ rRNA was subtracted from that of the gene of interest to obtain a $\Delta \mathrm{C}_{T}$ value. The $\Delta \mathrm{C}_{T}$ value of an arbitrary calibrator (e.g., untreated sample) was subtracted from the $\Delta \mathrm{C}_{T}$ value of each sample to obtain a $\Delta \Delta \mathrm{C}_{T}$ value. The gene expression level relative to the calibrator was expressed as $2^{-\Delta \Delta \mathrm{CT}}$. Statistical analysis was performed using R software version 2.5.0 (www.r-project.org). Fold enrichment was compared using Student's t-test. The statistical significance threshold was fixed at $\alpha=0.05$. 
Table 2-2. Primers used for quantitative real-time PCR expression analysis.

\begin{tabular}{|c|c|c|}
\hline $\begin{array}{l}\text { Gene } \\
\text { Name }\end{array}$ & Primer Pairs $^{a}$ & Amplicon Size (bp) \\
\hline $18 S$ & $\begin{array}{l}\mathbf{F}, \mathbf{5} \text { '-CACGACGGAGTTTCACAAGA-3' } \\
\text { R, 5'-CGATGGAAGTTTGAGGCAAT-3' }\end{array}$ & 135 \\
\hline CDRI & $\begin{array}{l}\mathbf{F}, \mathbf{5}^{\prime} \text {-ATTCTAAGATGTCGTCGCAAGATG-3' } \\
\mathbf{R}, \mathbf{5}^{\prime} \text {-AGTTCTGGCTAAATTCTGAATGTTTC-3' }\end{array}$ & 140 \\
\hline$C D R 2$ & $\begin{array}{l}\mathbf{F}, \mathbf{5}^{\prime} \text {-TAGTCCATTCAACGGCAACATT-3' } \\
\mathbf{R}, \mathbf{5}^{\prime} \text {-CACCCAGTATTTGGCATTGAAA-3' }\end{array}$ & 76 \\
\hline PDR16 & $\begin{array}{l}\mathbf{F}, \mathbf{5}^{\prime}-\mathrm{CTGCGGGACAAGATTCATTAGC-3'} \\
\mathbf{R}, \mathbf{5}^{\prime} \text {-TTGAGTACCAACAGGATGTGCTTTA-3' }\end{array}$ & 62 \\
\hline$T A C l$ & $\begin{array}{l}\mathbf{F}, \mathbf{5}^{\prime} \text {-TGGCAATGTATTTAGCAGATGAGG-3' } \\
\mathbf{R}, \mathbf{5}^{\prime} \text {-TGCTTGAACTGAGGTGAATTTTG-3' }\end{array}$ & 71 \\
\hline
\end{tabular}

${ }^{a} \mathbf{F}$ : forward, R: reverse 


\section{Generation of an HA-Tagged Tac1p-Expressing Strain}

A DNA fragment overlapping positions -402 to +937 of $C$. albicans URA3 ORF (URA3 marker) was PCR-amplified with Pfu DNA polymerase (Stratagene, La Jolla, CA) from plasmid pCaEXP (110), using primers 5'-ATCATCTCTAGATAAGGATGGTATAAACGGAAAC (introduces an $\mathrm{XbaI}$ site, underlined) and 5'-GCCCCGGGAAGGACCACCTTTGATTG (introduces a SmaI site, underlined). The resulting fragment (1,362 bp) was digested with $X b a I$ and $S m a I$ and used to swap the equivalent $X b a \mathrm{I}-X h o \mathrm{I}$ fragment of plasmid pMPY-3×HA $(1,078$ $\mathrm{bp}$, containing the $S$. cerevisiae URA3 marker) (111), generating plasmid pCaMPY $-3 \times \mathrm{HA} . \mathrm{A}$ TAC1-tagging cassette was amplified from plasmid pCaMPY-3×HA using primers FWD: 5 'ttgattgataattcagctgtaaatttgccatttagtcaatttaataatttacccaattttttctttgacaataatttggggattAGGGAACAAA AGCTGG-3' (the lower case sequence corresponds to positions +2860 to +2943 of the TAC1 ORF) and REV: 5'-

taggaaaaaatatatgaaacaataaatatttacaaagatatacattatacatcgctttcaccaattacaactctttttaacccCTATAGGG CGAATTGG-3' (the lower case sequence corresponds to positions +2947 to +3030 of the TAC1 ORF) which anneal specifically to the in-frame $\mathrm{pCaMPY}-3 \times \mathrm{HA}$ vector sequences $\mathrm{PET}$-up and PET-down (upper case sequences) as described previously (111). The resulting fragment $(1,821$ $\mathrm{bp}$ ), containing the $C$. albicans URA3 marker flanked by direct repeats of the $3 \times \mathrm{HA}$ epitopeencoding sequences and 84 bp of sequences homologous to the 3' end of the TAC1 gene, was used to transform strain CAI4. Counterselection of the URA3 gene was carried out on plates containing 5-FOA as described previously (112) except that uracil was replaced with uridine.

\section{Candida albicans Transformation}

C. albicans transformations were performed as described previously (113) with minor modifications. The cells were grown overnight in $15 \mathrm{ml}$ of YPD medium, diluted to an $\mathrm{OD}_{600}$ of 0.1 in $100 \mathrm{ml}$ of fresh YPD and allowed to grow to an $\mathrm{OD}_{600}$ of 0.4 . The cells were harvested, washed once with $10 \mathrm{ml}$ of sterile water and resuspended in $1 \mathrm{ml}$ of $1 \mathrm{X}$ LiAc solution (113). Cells $(100 \mu \mathrm{l})$ were transferred to a sterile tube containing $6 \mu \mathrm{g}$ of gel-purified PCR- fragment and $100 \mu \mathrm{g}$ of denatured salmon sperm DNA as carrier. A LiAc/40\% PEG solution ( $700 \mu 1$ of $10 \mathrm{mM}$ Tris-HCl pH 7.5, $1 \mathrm{mM}$ EDTA pH 8.0, $100 \mathrm{mM} \mathrm{LiAc,} \mathrm{40 \%} \mathrm{(w/v)} \mathrm{PEG4000)} \mathrm{was} \mathrm{added}$ and the cell suspensions were incubated overnight at $30^{\circ} \mathrm{C}$ with gentle rotation. The cells were heat shocked at $42^{\circ} \mathrm{C}$ for 15 min and plated on SC-ura plates.

\section{Genomic DNA Isolation and Southern Blot Analysis}

C. albicans genomic DNA was prepared as described for $S$. cerevisiae (114). For the Southern hybridization, genomic DNAs (500 ng) were digested to completion with HindIII and EcoRV, electrophoresed on a 1\% agarose gel and transferred to a nylon membrane (Hybond-N; Amersham Biosciences, Piscataway, NJ). Prehybridization, hybridization and washing steps were carried out as previously described (102). The TAC1 probe used consists of a ${ }^{32} \mathrm{P}-$ radiolabeled 927-bp PCR-amplified fragment from SC5314 genomic DNA overlapping positions +2020 to +2946 in the TAC1 ORF. The membrane was exposed to a FUJIFILM Imaging Plate 
screen. The signal was quantified using the Multi Gauge program, version 2.3 (FUJIFILM). The membrane was subsequently exposed to a Kodak XAR film at $-80^{\circ} \mathrm{C}$.

\section{Total Protein Preparation and Western Blotting}

Total protein was prepared from 2 O.D. units of strains CAI4 and SZY63 grown overnight, as described for $S$. cerevisiae (114). Extracts were boiled for $5 \mathrm{~min}$, and $35 \mu 1$ (out of $100 \mu 1$ total) was separated by electrophoresis on an SDS-8\% polyacrylamide gel. Proteins were transferred to a nitrocellulose membrane with a Trans Blot SD Semi-Dry transfer apparatus (BioRad, Hercules, CA) and analyzed with a mouse anti-HA monoclonal antibody (HA-probe (F-7): sc-7392, Santa Cruz Biotechnology Inc., Santa Cruz, CA) at a dilution of 1:2000, using a chemiluminescence detection system under conditions recommended by the manufacturer (Pierce Biotechnology Inc., Rockford, IL).

\section{Chromatin Immunoprecipitation}

Three independent cultures (50 ml each) of strains CAI4 and SZY63 were grown in YPD medium to an $\mathrm{OD}_{600}$ of 0.8 . The cultures were then treated with $1.4 \mathrm{ml}$ of $37 \%$ formaldehyde (final concentration 1\%) to induce protein-DNA cross-links. Cells were incubated 30 min at room temperature with agitation and the cross-linking stopped by adding $1.2 \mathrm{ml}$ of $2.5 \mathrm{M}$ glycine. Cells were harvested, washed 3 times with $40 \mathrm{ml}$ of ice-cold TBS buffer $(20 \mathrm{mM}$ Tris-HCl pH $7.5,150 \mathrm{mM} \mathrm{NaCl}$ ) and snap frozen in liquid nitrogen. Total cell extracts were prepared by adding $0.7 \mathrm{ml}$ of lysis buffer (50 mM HEPES-KOH pH 7.5, $140 \mathrm{mM} \mathrm{NaCl}, 1 \mathrm{mM}$ EDTA, 1\% Triton X-100, 0.1\% Na-deoxycholate, $1 \mathrm{mM}$ PMSF, $1 \mathrm{mM}$ benzamidine, $10 \mu \mathrm{g} / \mathrm{mL}$ aprotinin, 1 $\mathrm{ug} / \mathrm{mL}$ leupeptin, $1 \mathrm{ug} / \mathrm{mL}$ pepstatin), $0.5 \mathrm{ml}$ of acid-washed glass beads (Sigma) and bead beating 4 times for 5 min at highest settings in a mini bead-beater (Biospec Products). Preparation of soluble chromatin fragments was performed by sonicating the extracts 4 times during $20 \mathrm{sec}$ at power 2-3 (output power 8-9 RMS) using Sonic Dismembrator Model 100 sonicator (Fisher Scientific). Immunoprecipitation was performed by incubating the sonicated extracts (from both the tagged SZY63 strain and the untagged parental CAI4 strain) overnight with monoclonal mouse anti-HA antibody (Santa Cruz Biotech) coupled to magnetic beads (Dynabeads Pan Mouse IgG, Dynal Biotech, Brown Deer, WI) at $4^{\circ} \mathrm{C}$. Beads were then washed twice with lysis buffer, twice with lysis buffer supplemented with $360 \mathrm{mM} \mathrm{NaCl}$, twice with wash buffer (10 mM Tris-HCl pH 8.0, $250 \mathrm{mM} \mathrm{LiCl,} \mathrm{0.5 \%} \mathrm{NP40,} \mathrm{0.5 \%} \mathrm{Na-deoxycholate,} 1 \mathrm{mM}$ EDTA) and once with TE buffer (10 mM Tris $\mathrm{pH} 8.0,1 \mathrm{mM}$ EDTA). The cross-links were reversed by incubating the washed beads overnight in $50 \mu 1$ of TE/1\% SDS at $65^{\circ} \mathrm{C}$. The eluted material was then treated with proteinase $\mathrm{K}$, RNase A, extracted twice with phenol:chloroform:isoamyl-alcohol $(25: 24: 1)$ and the DNA precipitated with $100 \%$ ethanol and resuspended in $50 \mu \mathrm{l}$ of TE. $40 \mu \mathrm{l}$ of the immunoprecipitated (IPed) DNA fragments were used for amplification and $\mathrm{Cy} 5 / \mathrm{Cy} 3$ dye labeling prior to hybridization to C. albicans intergenic DNA microarrays. 


\section{DNA Labeling, Hybridization to Intergenic Microarrays and Data Analysis}

Labeling was conducted as described by Drouin et al. (115). Briefly, the IPed DNA fragments were blunted with T4 DNA polymerase and ligated to unidirectional linkers. The DNA was then amplified by LM-PCR in the presence of aminoallyl-modified dUTP. The labeling was carried out post-PCR using monoreactive $\mathrm{Cy}$-dye NHS esters $\left(\mathrm{Cy}^{\mathrm{TM}} 5 / \mathrm{Cy}^{\mathrm{TM}} 3\right.$ MonoReactive Dye Packs, Amersham Biosciences) that react specifically with the aminoallylmodified dUTP (5-(3-aminoallyl)-2' deoxyuridine-5'-triphosphate, Sigma-Aldrich). Both pools of labeled IPed DNA from the tagged SZY63 strain (Cy5) and the untagged parental CAI4 strain (Cy3) were mixed and hybridized to a $C$. albicans intergenic DNA microarray that will be described elsewhere (H. Hogues, H. Lavoie, A. Sellam, M. Mangos, T. Roemer, E. Purisima, A. Nantel and M. Whiteway, submitted for publication). Images of Cy5 and Cy3 fluorescence intensities were generated by scanning arrays using Axon 4000b scanner and were analyzed with GenePix Pro 4.1 software (Molecular Devices, formerly Axon Instruments, Inc, Downington, PA). Normalization of the data was conducted using ArrayPipe 1.7 (116), and replicate slides $(n=3)$ were combined using a weighted average method as described in Ren et al. (117).

\section{Quantitative Real-Time PCR Confirmation of the Genome-Wide Location Data}

Q-PCR was performed with three independent CAI4 and SZY63 ChIP samples prepared as described above, except that the cell cultures were carried out in the presence of DMSO $(0.08 \%)$. Quantification of the DNA recovered from the CAI4 and SZY63 ChIPs was performed using the Quant-iT ${ }^{\mathrm{TM}}$ PicoGreen ${ }^{\circledR}$ dsDNA Assay Kit (Molecular Probes: Invitrogen). A standard curve was prepared using $C$. albicans gDNA quantified by fluorometry and serially diluted in TE buffer $(0,0.1,0.01$, and $0.001 \mathrm{ng} / \mu \mathrm{l})$. The CAI4 and SZY63 ChIP samples $(1 \mu \mathrm{l})$ were resuspended in $49 \mu \mathrm{l}$ of TE buffer. The samples $(50 \mu \mathrm{l})$ were transferred, in duplicate, in a black 96-well plate (Costar 3694, Corning Inc., Corning, NY) and $50 \mu 1$ of the PicoGreen reagent was added. The fluorescence was measured using the Envision luminometer (Perkin-Elmer, Waltham, MA) at an excitation and emission wavelength of 485 and $535 \mathrm{~nm}$, respectively. The DNA concentration of the ChIP samples was an average $500 \mathrm{pg} / \mu \mathrm{l}$, yielding approximately $25 \mathrm{ng}$ of total immunoprecipitated DNA per ChIP sample $(50 \mu \mathrm{l})$.

Q-PCR assays were designed using the Universal ProbeLibrary (Roche Applied Science, formerly Exiqon's ProbeLibray, Indianapolis, IN) and the TaqMan (Integrated DNA Technologies, IDT, Coralville, IA) methodologies. The different primers and probe combinations used for Q-PCR are listed in Table 2-3. The CDR1, PDR16, TAC1 and FUR1 promoter sequences were submitted to the Universal ProbeLibrary, using the web-based ProbeFinder software (version 2.34, Roche Applied Sciences) available on the Roche Applied Science website (www.universalprobelibrary.com). The software assigned optimal PCR specific primer sequences to be combined with the corresponding Universal ProbeLibrary probe for each promoter sequence (Universal ProbeLibrary probes, Roche Diagnostics Corp., Basel, Switzerland). We also used the PrimerQuest tool from the IDT website (http://www.idtdna.com/Scitools/Applications/Primerquest/) to design a custom TaqMan probe for the TAC1 promoter (with its corresponding forward and reverse primers) that binds closer to 
Table 2-3. Primers used for quantitative real-time PCR binding assays.

\begin{tabular}{|c|c|c|}
\hline Promoter & Primers/Probe Sequences 5'-3' & Amplicon Location ${ }^{a}$ \\
\hline CDR1 & $\begin{array}{l}\text { F: GGTGCACACACACACAAACACACA } \\
\text { R: TTGAGCTCCCACTATCCGATCCCTA } \\
\text { P: TaqMan probe }{ }^{b}: \\
\text { CCGCCCTCACTCTGTTCCATACAAAT }\end{array}$ & $-435 \rightarrow-341$ \\
\hline PDR16 & $\begin{array}{l}\text { F: GAAAAGAAAAAGAAATGGCAACAT } \\
\text { R: TCGACACGTCTTTCCATCAC } \\
\text { P: Universal ProbeLibrary }{ }^{\text {p }} \text { probe \# } 77 \text { (cat.no. } \\
\text { 04689003001): GGTGGTGG }\end{array}$ & $-424 \rightarrow-501$ \\
\hline$T A C 1$ & $\begin{array}{l}\text { F: CACGAAGATAAAAATTGTGGTAGC } \\
\text { R: AATTGTGCTGATATTTAATTGTTGGT } \\
\text { P: Universal ProbeLibrary probe \# } 18 \text { (cat.no. } \\
\text { 04686918001) : CAGCAGGA }\end{array}$ & $-1024 \rightarrow-948$ \\
\hline FUR1 & $\begin{array}{l}\text { F: GGTGCTTTTGGGAGAATGAA } \\
\text { R: CTTCCTCAAAACAAAACTGCAA } \\
\text { P: Universal ProbeLibrary probe \# 27(cat.no. } \\
\text { 04687582001) : GCTGCCTG }\end{array}$ & $-987 \rightarrow-913$ \\
\hline$S P S 4^{d}$ & $\begin{array}{l}\text { F: TACAGTTGCCCCAGTCAACA } \\
\text { R: TGTCTTGGAACGGAAACTCA } \\
\text { P: Universal ProbeLibrary probe \# } 15 \text { (cat.no. } \\
\text { 04685148001) : TCCTGCTC }\end{array}$ & $-636 \rightarrow-574$ \\
\hline
\end{tabular}

F: forward, R: reverse, $\mathbf{P}$ : probe.

a position according to the ATG start codon

${ }^{b}$ TaqMan probe (42)

${ }^{c}$ Universal ProbeLibrary probe (64)

dorf 19.7568 
the DRE motif than the Universal ProbeLibrary probe. The $C$. albicans homologue of $S$. cerevisiae SPS4 (orf19.7568) was used as the reference promoter and FURI was used as a control, to perform statistical analyses. The SPS4 and FUR1 genes were selected because i) they were not modulated in our microarray expression study and ii) their promoters were not enriched in the ChIP-chips experiments. A set of probe and primers was designed for each of the SPS4 and FUR1 promoters using the ProbeFinder software (Table 2-3).

Q-PCR reaction mixtures were prepared using the TaqMan Universal PCR Master Mix (Applied Biosystems, Inc.) according to the manufacturer's instructions. For reactions using probes from the Universal ProbeLibrary, $200 \mathrm{pg}$ of ChIP DNA, $250 \mathrm{nM}$ of each forward and reverse primer, $100 \mathrm{nM}$ of the probe, $5 \mu$ of the TaqMan Universal PCR Master Mix and water were combined in a final volume of $10 \mu \mathrm{l}$. Q-PCR reactions using the TaqMan probe were prepared using the same conditions except that the probe and the primers (forward and reverse) were added to a final concentration of $100 \mathrm{nM}$ and $200 \mathrm{nM}$, respectively. Reactions were performed in MicroAmp Optical 384-Well Reaction Plate (Applied Biosystems, Inc.) using an ABI 7900 HT Real-Time PCR instrument with 1 cycle at $95^{\circ} \mathrm{C}$ for $10 \mathrm{~min}$ and 40 cycles at $\left(95^{\circ} \mathrm{C}\right.$ for $15 \mathrm{sec}$ and $60^{\circ} \mathrm{C}$ for $1 \mathrm{~min}$ ). Each biological replicate sample (three) was processed in triplicate.

Data analysis was performed using the Sequence Detection System software (SDS 2.2.2; $\mathrm{ABI})$. For each sample, $\mathrm{C}_{T}$ values were determined using the SDS software. The fold enrichments of the targets (CDR1, PDR16 and TAC1 promoters) were calculated using the relative quantification according to the $2^{-\Delta \Delta \mathrm{Ct}}$ method, where $\Delta \mathrm{CT}=\mathrm{C}_{T \text { target }}-\mathrm{C}_{T \text { reference(SPS4 }}$ promoter) and $\Delta \Delta \mathrm{CT}=\Delta \mathrm{C}_{T}$ test (SZY63 ChIP sample) $-\Delta \mathrm{Ct}$ calibrator (CAI4 ChIP sample) (118). The FUR1 promoter was used as a negative control to confirm the fold enrichment obtained using the $2^{-\Delta \Delta \mathrm{Ct}}$ method. Statistical analysis was performed using the R software (www.r-project.org version 2.5.1) and the $\Delta \Delta C$ T's were compared using Welch's t-test. The statistical significance threshold was fixed at $\alpha=0.001$.

\section{DRE Motif Analysis}

The promoter sequences ( $1.5 \mathrm{~kb}$ upstream of the starting ATG) were retrieved from the Candida Genome Database (http://www.candidagenome.org) and analyzed for the presence of potential DRE motifs using the fuzznuc software from EMBOSS (http://emboss.bioinformatics.nl/).

\section{RESULTS}

\section{Global Gene Expression Profile}

As a means to identify genes that are co-regulated with the $C D R 1$ and $C D R 2$ multidrug efflux pumps and thus potentially new transcriptional targets of Tac1p, we performed gene expression profiling analyses of four matched sets of azole- susceptible and -resistant C. albicans 
clinical isolates in which the acquisition of azole resistance is associated with $C D R 1$ and $C D R 2$ overexpression (Table 2-1). Three of these azole-resistant isolates possess previously defined gain-of-function mutations in the C-terminus of TAC1: a N977D amino acid substitution in isolate C56 (also known as isolate DSY296) (89), a N972D amino acid substitution in isolate 5674 (103), and an A736C amino acid substitution in one allele and L962-N969 deletion in the second allele of TAC1 in isolate 17 (101). Isolate 17 is the last in a series of 17 clinical isolates taken from a single HIV-infected patient $(92,106)$. Relative to the parental isolate 2 , isolate 3 exhibits increased expression of $M D R 1$ whereas isolate 17 exhibits increased expression of $C D R 1$ and $C D R 2$ as well as $M D R 1(92,106)$. We therefore compared isolate 17 to isolate 3 to identify the genes co-regulated with $C D R 1$ and $C D R 2$. The other azole-resistant isolate, Gu5, overexpresses $C D R 1$ and $C D R 2$ and contains a G980E amino acid substitution in Tac1p (unpublished data). The eight strains did not show major chromosomal rearrangements or aneuploidies, as determined by comparative genomic hybridization analyses (data not shown and J. Berman, personal communication).

Three independent RNA samples per strain were hybridized to custom designed Affymetrix C. albicans microarrays and the data analyzed as described in Materials and Methods. Genes were initially considered to be differentially expressed if 1) their average fold change in expression was $\geq 1.5$ for each matched isolate, 2) their expression changed by at least 1.5 -fold in at least 2 of the 3 experiments for each matched isolate, and 3) if the average fold change was statistically significant by t-test. Using these criteria, there were 222 genes up-regulated and 150 genes down-regulated in C56 as compared to C43, 104 genes up-regulated and 63 genes downregulated in Gu5 as compared to $\mathrm{Gu} 2,126$ genes up-regulated and 92 genes down-regulated in 17 as compared to 3, and 327 genes up-regulated and 243 genes down-regulated in 5674 as compared to 5457 (Table A-1 and Table A-2). Genes which met these criteria for all 4 matched isolates and whose average fold change was statistically significant by t-test in at least 2 of the 4 matched isolates were included in a final data set (Table 2-4 and Table 2-5). We found 31 genes to be up-regulated in all four matched clinical isolate sets (Table 2-4) including the known Tac1p targets CDR1, CDR2, IFU5, HSP12 and RTA3 (100). Other up-regulated genes included GPXI (putative glutathione peroxidase), CHK1 (histidine kinase), LCB4 (Sphingoid long-chain base kinase), $N D H 2$ (NADH dehydrogenase), SOU1 (sorbose dehydrogenase), orf19.3047 (transcription cofactor), orf19.4531 (ABC transporter) as well as TACl itself, consistent with our previous proposition that $T A C 1$ transcription is autoactivated, either directly or indirectly, as a consequence of gain-of-function mutations in Tac1p (103). We also found 12 genes to be downregulated in all four matched clinical isolate sets. These included FTRI (iron transporter), IHDI (putative GPI-anchored protein of unknown function), and OPT6 (oligopeptide transporter) all of which encode integral membrane proteins, and SOD5 (superoxide dismutase) which encodes a cell wall protein (Table 2-5). As judged from the expression data, clinical isolate 5674, which carries the Tac1p N972D amino acid substitution, results in the highest impact on gene expression among the isolates tested, as 38 out of the 43 genes modulated in a TAC1-dependent manner had the highest fold modulation in this strain (Table 2-4 and Table 2-5).

In order to determine which of these genes require Tac $1 p$ for their constitutive differential expression in these isolates, the gene expression profile of strain SZY31, a tac $1 \Delta /$ tac $1 \Delta$ derivative of the azole-resistant strain 5674 (Table 2-1), was compared with that of the azole-susceptible parental strain 5457 (85). Genes whose expression was modulated in strain 
Table 2-4. Genes up-regulated in all four matched clinical isolate sets.

\begin{tabular}{|c|c|c|c|c|c|c|c|c|}
\hline $\begin{array}{l}\text { Systematic } \\
\text { Name }^{a}\end{array}$ & $\begin{array}{l}\text { CGD } \\
\text { Name }^{b}\end{array}$ & $\begin{array}{l}\text { CandidaDB } \\
\text { Name }^{c}\end{array}$ & $\begin{array}{l}\text { GO Annotation } \\
\text { (Molecular Function) }^{d}\end{array}$ & $\begin{array}{l}\mathrm{C56} \\
\mathbf{v} \\
\mathrm{C43}\end{array}$ & $\begin{array}{l}\text { Gu5 } \\
\text { v } \\
\text { Gu2 }\end{array}$ & $\begin{array}{l}\mathbf{1 7} \\
\mathbf{v} \\
\mathbf{3}\end{array}$ & $\begin{array}{l}\mathbf{5 6 7 4} \\
\mathbf{v} \\
\mathbf{5 4 5 7}\end{array}$ & $\begin{array}{c}\text { SZY31 } \\
\mathbf{v} \\
\mathbf{5 4 5 7}\end{array}$ \\
\hline orf19.6000 & $C D R 1$ & $C D R 1$ & $\begin{array}{l}\text { Multidrug transporter } \\
\text { activity }\end{array}$ & 1.7 & 3.0 & 2.4 & 4.4 & -1.0 \\
\hline orf19.5958 & $C D R 2$ & $C D R 2$ & Transporter activity & 50.6 & 14.1 & 17.1 & 50.5 & -1.3 \\
\hline orf19.86 & -- & $G P X 1$ & $\begin{array}{l}\text { Glutathione peroxidase } \\
\text { activity }\end{array}$ & 2.3 & 3.0 & 2.3 & 4.0 & -1.2 \\
\hline orf19.896 & CHK1 & $H K 1$ & $\begin{array}{l}\text { Protein histidine kinase } \\
\text { activity }\end{array}$ & 2.5 & 1.9 & 1.8 & 4.5 & 1.3 \\
\hline orf19.3160 & $H S P 12$ & $H S P 12$ & -- & 5.3 & 2.9 & 3.1 & 16.7 & -1.7 \\
\hline orf19.157 & -- & IFA24.3 & -- & 1.5 & 1.7 & 1.6 & 2.1 & 1.1 \\
\hline orf19.2568 & IFU5 & IFU5 & -- & 3.3 & 3.0 & 3.7 & 5.4 & 1.5 \\
\hline orf19.5257 & -- & $L C B 4$ & $\begin{array}{l}\text { D-erythro-sphingosine } \\
\text { kinase activity }\end{array}$ & 2.2 & 1.7 & 2.2 & 4.0 & 1.1 \\
\hline orf19.278 & -- & $M T R$ & -- & 3.1 & 1.8 & 1.9 & 5.2 & -1.1 \\
\hline orf19.5713 & $Y M X 6$ & $\mathrm{NDH} 2$ & $\begin{array}{l}\text { NADH dehydrogenase } \\
\text { activity }\end{array}$ & 5.3 & 3.1 & 2.9 & 15.3 & 1.2 \\
\hline orf19.7218 & $R B E 1$ & PRY2 & -- & 2.1 & 1.8 & 1.7 & 1.7 & 1.2 \\
\hline orf19.23 & RTA3 & $R T A 3$ & $\begin{array}{l}\text { Phospholipid- } \\
\text { translocating ATPase } \\
\text { activity }\end{array}$ & 40.4 & 15.9 & 22.0 & 41.0 & 1.0 \\
\hline orf19.2896 & SOU1 & SOU1 & $\begin{array}{l}\text { Sorbose dehydrogenase } \\
\text { activity }\end{array}$ & 2.0 & 1.5 & 1.5 & 7.0 & -2.0 \\
\hline orf19.3104 & -- & $Y D C 1$ & -- & 3.3 & 1.6 & 2.1 & 5.3 & 1.4 \\
\hline orf19.2726 & -- & IPF10262 & -- & 2.5 & 2.8 & 1.9 & 6.2 & -2.9 \\
\hline orf19.4459 & -- & IPF11849 & -- & 2.0 & 4.1 & 4.3 & 5.0 & -1.5 \\
\hline orf19.2244 & -- & IPF12897 & -- & 1.8 & 1.7 & 1.5 & 5.4 & -1.7 \\
\hline orf19.344 & -- & $I P F 1514$ & -- & 15.5 & 4.7 & 4.0 & 28.9 & 1.9 \\
\hline orf19.4907 & -- & IPF17283 & -- & 3.2 & 1.6 & 2.3 & 2.0 & 1.1 \\
\hline orf19.5313 & -- & IPF19810 & -- & 36.0 & 29.4 & 18.1 & 143.1 & 1.7 \\
\hline orf19.5777 & -- & IPF19961 & -- & 2.0 & 1.7 & 2.2 & 4.2 & -1.3 \\
\hline orf19.6840 & -- & $I P F 3535$ & -- & 1.8 & 2.3 & 1.9 & 2.3 & -1.4 \\
\hline orf19.3047 & SIP3 & IPF3598 & $\begin{array}{l}\text { Transcription cofactor } \\
\text { activity }\end{array}$ & 1.5 & 1.5 & 1.7 & 3.0 & 1.3 \\
\hline orf19.2531 & CSP37 & IPF4991 & $\begin{array}{l}\text { ER to Golgi vesicle- } \\
\text { mediated transport }\end{array}$ & 2.5 & 1.8 & 5.5 & 6.8 & -1.3 \\
\hline orf19.7310 & -- & IPF5981 & -- & 4.9 & 2.7 & 2.0 & 16.8 & 1.2 \\
\hline orf19.1887 & -- & IPF6464 & -- & 2.3 & 1.8 & 1.7 & 4.5 & 1.2 \\
\hline
\end{tabular}


Table 2-4 (continued).

\begin{tabular}{|c|c|c|c|c|c|c|c|c|}
\hline $\begin{array}{l}\text { Systematic } \\
\text { Name }^{a}\end{array}$ & $\begin{array}{l}\text { CGD } \\
\text { Name }^{b}\end{array}$ & $\begin{array}{l}\text { CandidaDB } \\
\text { Name }^{c}\end{array}$ & $\begin{array}{l}\text { GO Annotation } \\
\text { (Molecular Function) }^{d}\end{array}$ & $\begin{array}{c}C 56 \\
v \\
C 43\end{array}$ & $\begin{array}{c}\text { Gu5 } \\
\text { v } \\
\text { Gu2 }\end{array}$ & $\begin{array}{l}17 \\
\mathbf{v} \\
\mathbf{3}\end{array}$ & $\begin{array}{c}\mathbf{5 6 7 4} \\
v \\
5457\end{array}$ & $\begin{array}{c}\text { SZY31 } \\
\mathbf{v} \\
\mathbf{5 4 5 7}\end{array}$ \\
\hline orf19.2790 & - & $I P F 7260$ & $\begin{array}{l}\text { Histone-lysine N- } \\
\text { methyltransferase activity }\end{array}$ & 1.8 & 1.6 & 1.6 & 2.8 & -1.0 \\
\hline orf19.4531 & -- & IPF7530 & ATPase activity & 2.7 & 3.8 & 3.5 & 4.0 & -1.6 \\
\hline orf19.4898 & -- & IPF8950 & -- & 2.4 & 1.6 & 1.8 & 3.4 & 1.1 \\
\hline orf19.3188 & $T A C 1$ & IPF9191.3f & -- & 2.7 & 2.8 & 2.8 & 4.2 & -11.7 \\
\hline orf19.3644 & -- & IPF9683 & -- & 2.5 & 1.5 & 2.1 & 3.4 & 1.1 \\
\hline
\end{tabular}

${ }^{a}$ orf19 nomenclature according to the Assembly 19 version.

${ }^{\boldsymbol{b}}$ Gene name at CGD (http://www.candidagenome.org/).

${ }^{c}$ Gene name at CandidaDB (http://genolist.pasteur.fr/CandidaDB/).

${ }^{d} \mathrm{GO}$ annotation found at CGD (http://www.candidagenome.org/).

Systematic names in bold designate overlap with ChIP-chip experiments.

Gene expression values are represented as fold changes between resistant isolates and susceptible isolates.

Gene expression values in bold designate statistical significance (p-value $<0.05)$ in all three experiments. 
Table 2-5. Genes down-regulated in all four matched clinical isolate sets.

\begin{tabular}{|c|c|c|c|c|c|c|c|c|}
\hline $\begin{array}{l}\text { Systematic } \\
\text { Name }^{a}\end{array}$ & $\begin{array}{l}\text { CGD } \\
\text { Name }^{b}\end{array}$ & $\begin{array}{c}\text { Candida } \\
\text { DB } \\
\text { Name }^{c} \\
\end{array}$ & $\begin{array}{l}\text { GO Annotation } \\
\text { (Molecular Function) }^{d}\end{array}$ & $\begin{array}{l}\mathbf{C 5 6} \\
\mathbf{v} \\
\mathbf{C 4 3} \\
\end{array}$ & $\begin{array}{l}\text { Gu5 } \\
\text { v } \\
\text { Gu2 }\end{array}$ & $\begin{array}{l}17 \\
\mathbf{v} \\
3\end{array}$ & $\begin{array}{l}\mathbf{5 6 7 4} \\
\mathbf{v} \\
\mathbf{5 4 5 7}\end{array}$ & $\begin{array}{c}\text { SZY31 } \\
\mathbf{v} \\
\mathbf{5 4 5 7} \\
\end{array}$ \\
\hline orf19.7219 & FTR1 & FTRl & $\begin{array}{l}\text { Ferrous iron transporter } \\
\text { activity }\end{array}$ & -2.7 & -3.0 & -2.1 & -4.0 & -1.6 \\
\hline orf19.5025 & MET3 & MET3 & $\begin{array}{l}\text { Sulfate } \\
\text { adenylyltransferase } \\
\text { (ATP) activity }\end{array}$ & -2.0 & -7.9 & -1.8 & -2.5 & -1.2 \\
\hline orf19.2947 & SNZ1 & SNZ1 & -- & -3.7 & -1.8 & -1.7 & -2.2 & 1.3 \\
\hline orf19.5760 & IHD1 & IPF10662 & -- & -1.9 & -2.2 & -3.1 & -9.0 & 1.7 \\
\hline orf19.3475 & -- & IPF11725 & -- & -3.9 & -2.1 & -2.7 & -5.8 & -1.2 \\
\hline orf19.4655 & OPT6 & IPF12193 & $\begin{array}{l}\text { Oligopeptide } \\
\text { transporter activity }\end{array}$ & -1.8 & -2.0 & -2.4 & -3.1 & -1.4 \\
\hline orf19.2060 & SOD5 & IPF1222 & $\begin{array}{l}\text { Copper, zinc } \\
\text { superoxide dismutase } \\
\text { activity }\end{array}$ & -2.3 & -8.6 & -17.8 & -26.3 & -1.4 \\
\hline orf19.2059 & -- & $I P F 1228$ & -- & -2.0 & -1.7 & -1.7 & -2.9 & 1.2 \\
\hline orf19.4749 & -- & IPF13921 & -- & -2.3 & -1.8 & -2.4 & -3.0 & -1.4 \\
\hline orf19.670.2 & -- & IPF20159 & -- & -2.3 & -2.0 & -1.7 & -4.9 & -1.9 \\
\hline orf19.6679 & -- & IPF2314 & -- & -1.5 & -2.1 & -1.8 & -2.2 & 1.1 \\
\hline orf19.7561 & $D E F 1$ & IPF946 & -- & -3.4 & -1.5 & -1.6 & -2.8 & -1.4 \\
\hline
\end{tabular}

${ }^{a}$ orf19 nomenclature according to the Assembly 19 version.

${ }^{\boldsymbol{b}}$ Gene name at CGD (http://www.candidagenome.org/).

${ }^{c}$ Gene name at CandidaDB (http://genolist.pasteur.fr/CandidaDB/).

${ }^{d} \mathrm{GO}$ annotation found at CGD (http://www.candidagenome.org/).

Gene expression values are represented as fold changes between resistant isolates and susceptible isolates.

Gene expression values in bold designate statistical significance (p-value $<0.05)$ in all three experiments. 
5674 as compared to strain 5457 and whose expression in strain SZY31 was returned to levels similar to those observed in strain 5457 were determined to be dependent upon Taclp for their up- or down-regulation. As shown in Table 2-4 and Table 2-5, the majority of commonly differentially expressed genes were found to be Tac1p-dependent.

We selected four genes of interest for confirmation of differential expression by real-time RT-PCR. These included CDR1, CDR2, TAC1, and PDR16. PDR16 was selected as it has been shown to be co-regulated with $C D R 1$ and $C D R 2$ in association with azole resistance, yet this is one of a limited number of genes not represented on the microarray used in this study $(102,119)$. As expected, all four genes were confirmed to be up-regulated in all four isolate sets (Figure 2-1). Likewise, up-regulation of all four of these genes in isolate 5674 was ablated in strain SZY31 (Figure 2-1).

\section{Identification of Tac1p-Binding Sites in vivo}

To determine if the $T A C 1$-dependent genes that were differentially expressed in the clinical isolates have their promoter bound in vivo by Tac1p and to identify additional direct targets of Tac1p, we conducted genome-wide location analyses (ChIP-chip), a procedure combining ChIP with hybridization to DNA intergenic microarrays (117). For this purpose, Tac $1 p$ was tagged at its C-terminus with a triple hemaglutinin (HA) epitope, using homologous recombination at the $T A C 1$ chromosomal locus (Figure 2-2). First, we adapted the $S$. cerevisiae PCR epitope-tagging vector pMPY $-3 \times$ HA (111) for its use in $C$. albicans, by substituting the $S$. cerevisiae URA3 marker with the $C$. albicans URA3 gene (see Materials and Methods for details). The resulting vector ( $\mathrm{pCaMPY}-3 \times \mathrm{HA}$ ) contains the $\mathrm{Ca} U R A 3$ marker flanked by direct repeats of the $\mathrm{HA}_{3}$ epitope. The TAC1-tagging cassette was amplified with 100 -bp primers (16 bp of vector sequences and $84 \mathrm{bp}$ from the gene to be tagged), allowing tagging of Taclp at its C-terminus (Figure 2-2A). After transformation of the CAI4 strain and integration of the cassette by homologous recombination, the marker was excised via recombination between the two directly repeated $\mathrm{HA}_{3}$ tags and the excision mutants were selected on 5-FOA medium (Figure 2-2A). The resulting pre-excision (strain SZY51) and post-excision (strain SZY63) strains were characterized by Southern hybridization using HindIII and EcoRV double-digested genomic DNA and the TAC1 probe (Figure 2-2B). As predicted (Figure 2-2B), the probe detected two fragments, one of $3.3 \mathrm{~kb}$ and one of $1.4 \mathrm{~kb}$ in the CAI4 strain, corresponding to the wild-type TAC1-1 (orf19.3188) and TAC1-2 (orf19.10700) alleles, respectively (Figure 2-2C, lane 1). Integration of the $\mathrm{HA}_{3}$-tagging cassette occurred at the TAC1-2 allele in the pre-excision strain SZY 51, as the 1.4-kb band was shifted to the $3.1-\mathrm{kb}$ band (Figure 2-2C, lane 2). Excision of the $\mathrm{CaURA} 3$ marker in strain SZY 63 was confirmed by the presence of the $1.6-\mathrm{kb}$ band (Figure 2-2C, lane 3). To detect the Tac1p-HA $\mathrm{H}_{3}$ fusion protein, Western blotting was performed in strains CAI4 and SZY63, using an anti-HA monoclonal antibody (Figure 2-2D). A signal corresponding to the Tac1p-HA 3 fusion protein was detected in the HA-tagged SZY63 strain but not in strain CAI4 (control) (Figure 2-2D). The size of this band was approximately $120 \mathrm{kDa}$, consistent with the predicted molecular weight of the Tac1p-HA fusion protein $(118 \mathrm{kDa})$. The functionality of the Taclp-HA 3 protein was examined by the Raymond lab and there was change in Tac1p function. 
Figure 2-1. Quantitative real-time RT-PCR analysis of selected genes differentially expressed in the microarray experiments.

(A) Genes differentially expressed in each of the azole resistant clinical isolates as compared to their parent isolates. (B) CDR2 gene expression in each of the azole resistant clinical isolates.

Error bars denote standard error $(n=3)$. Asterisks denote statistical significance by $t$-test ( $p$-value $\leq 0.05)$. 


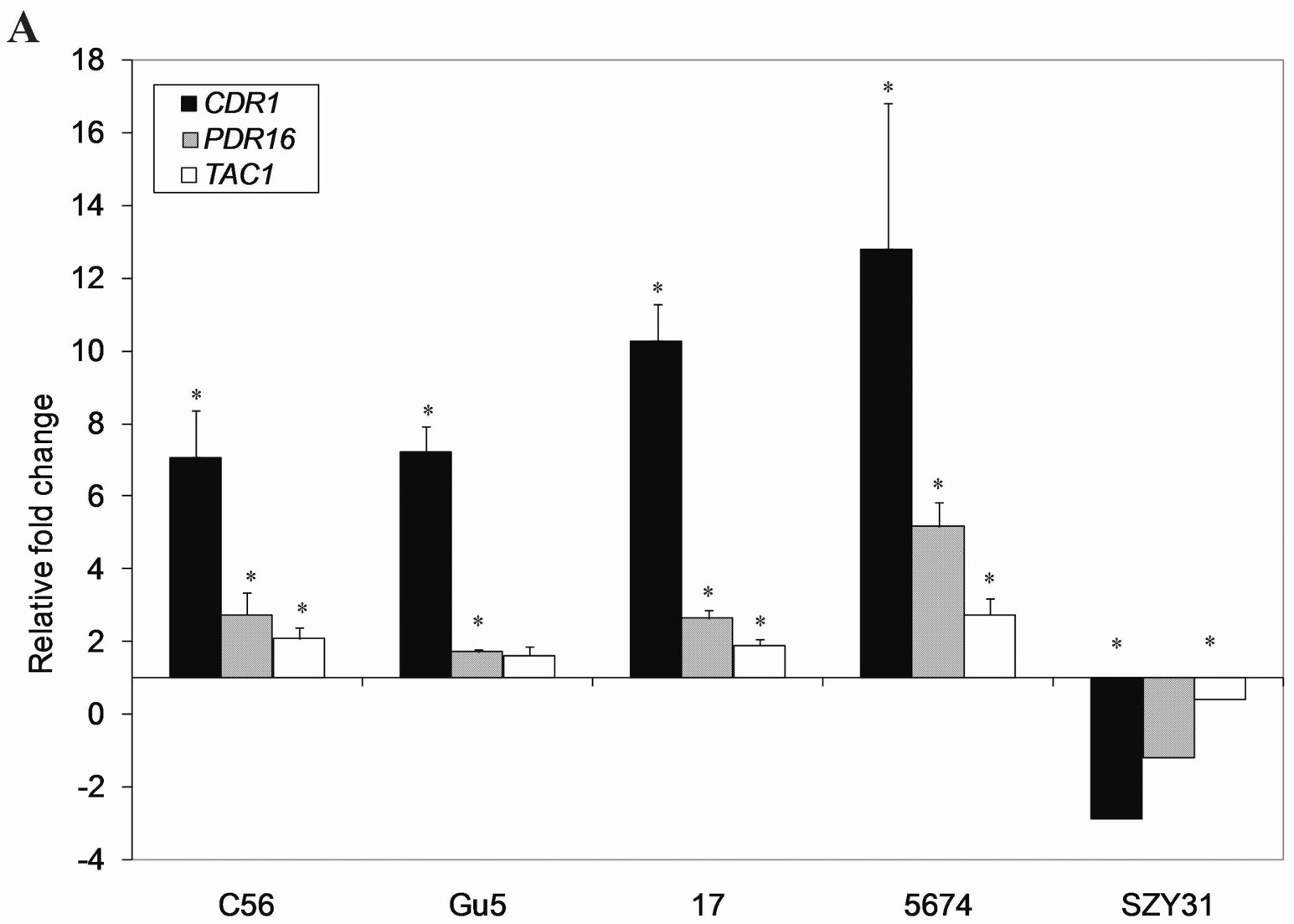

B

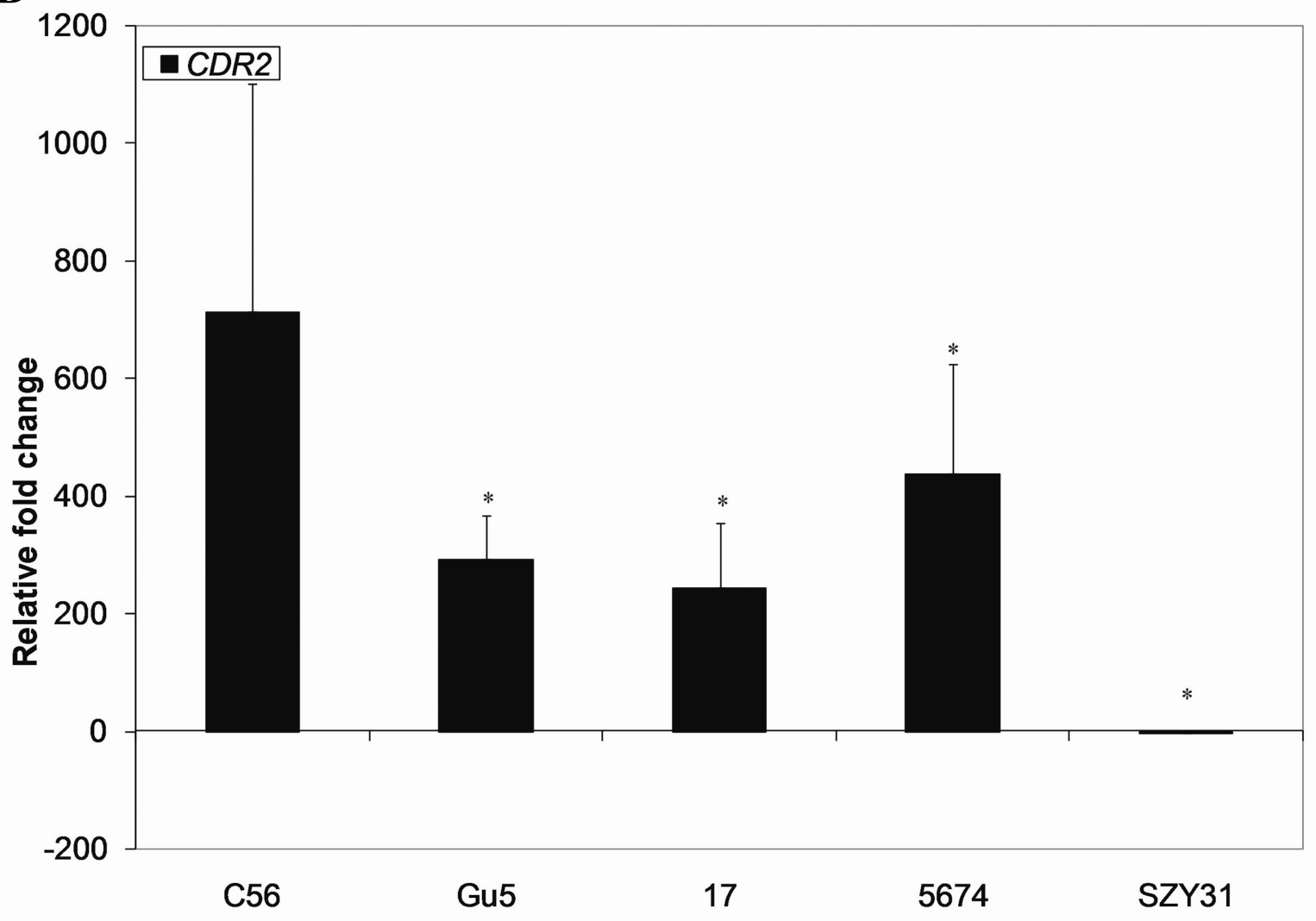


Figure 2-2. Chromosomal tagging of Tac1p.

(A) PCR epitope tagging strategy for C-terminally tagging transcription factor Tac $1 \mathrm{p}$ with the triple HA epitope. Top, 100-nt primers (FWD and REV, see Materials and Methods) are designed such that the 5' 84 bases of the forward (FWD) and reverse primers are homologous to sequences of the TAC1 gene and the 3' 16 bases are complementary and in-frame to unique sequences (open boxes) in the tagging cassette which contains the C. albicans URA3 marker (CaURA3, light gray box) flanked by direct repeats of the $3 \times$ HA-encoding sequences (HA, black boxes). The Tac1p stop codon is indicated by the asterisk. Middle, PCR amplification results in a fragment whose ends include the primer sequences, allowing integration by homologous recombination of the tagging cassette upstream of the TAC1 3' untranslated region (horizontal thick line). The orientation of the TACl ORF (dark gray box) is indicated by the arrow. Bottom, $\mathrm{CaURA3}$ marker excision results in the final product, $T A C 1-H A_{3}$. (B) Schematic representations of the TAC1-1 (orf19.3188) and TAC1-2 (orf19.10700) alleles (gray boxes) in strain CAI4 and sizes of the HindIII, EcoRV double-digested fragments detected by the TACl probe (top, thick line) used for the Southern blot experiment are given for the TAC1-2 allele following integration of the $\mathrm{HA}_{3}$-tagging cassette (open box) and excision of the $\mathrm{Ca} U R A 3$ marker through $\mathrm{HA}_{3}$ recombination. (C) Southern blot of genomic DNA from the CAI4 strain and its URA3-pre(SZY51) and post-excision (SZY63) derivatives, digested with EcoRV and HindIII and hybridized with the $T A C 1$ probe shown in (B). Marker sizes are indicated on the left. (D) Western blot analysis of protein extracts from strains CAI4 and the Tac1p-HA 3 integrant SZY63 with an anti-HA monoclonal antibody. Molecular weights markers are indicated on the left. 
A B
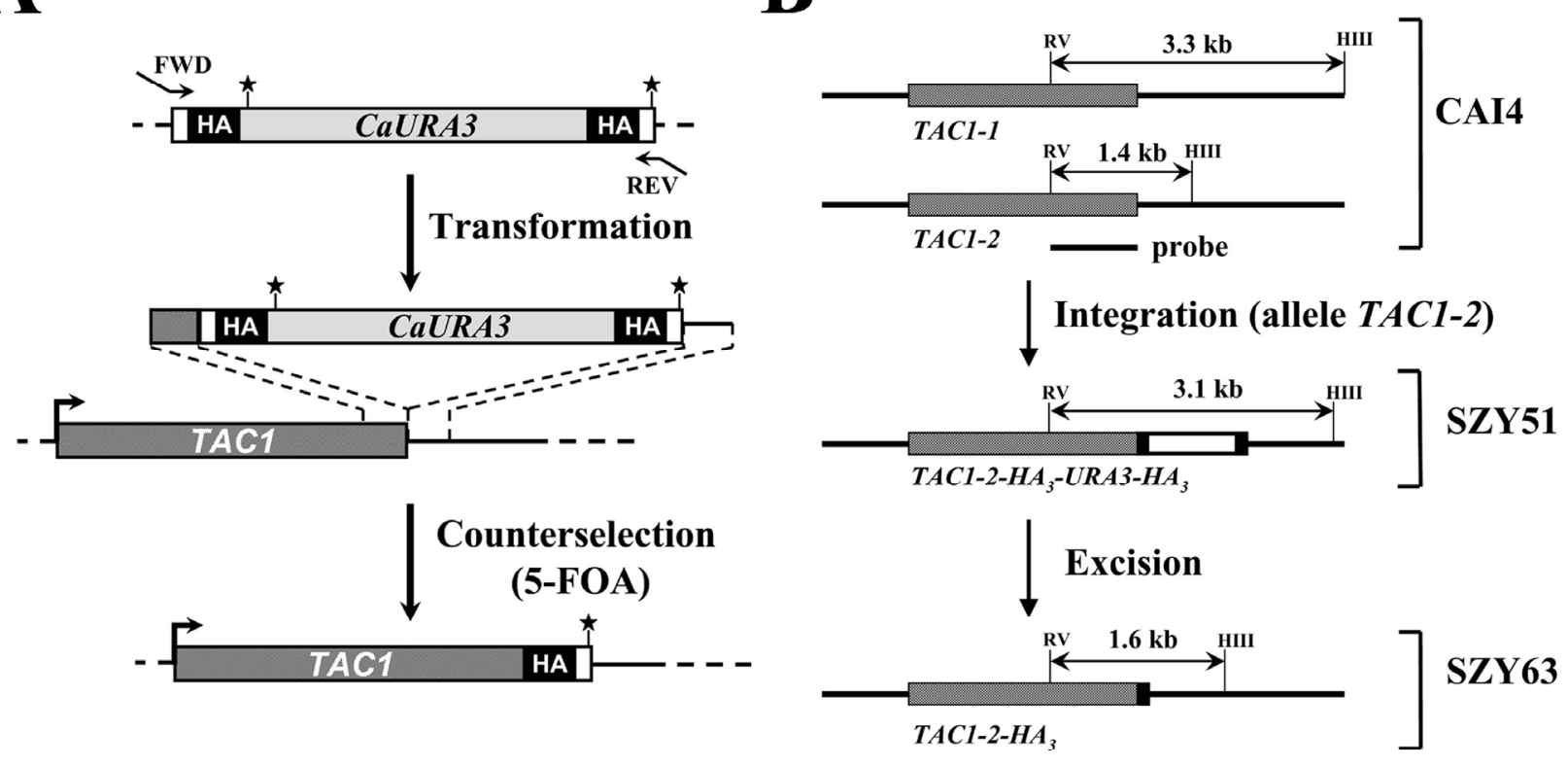

C
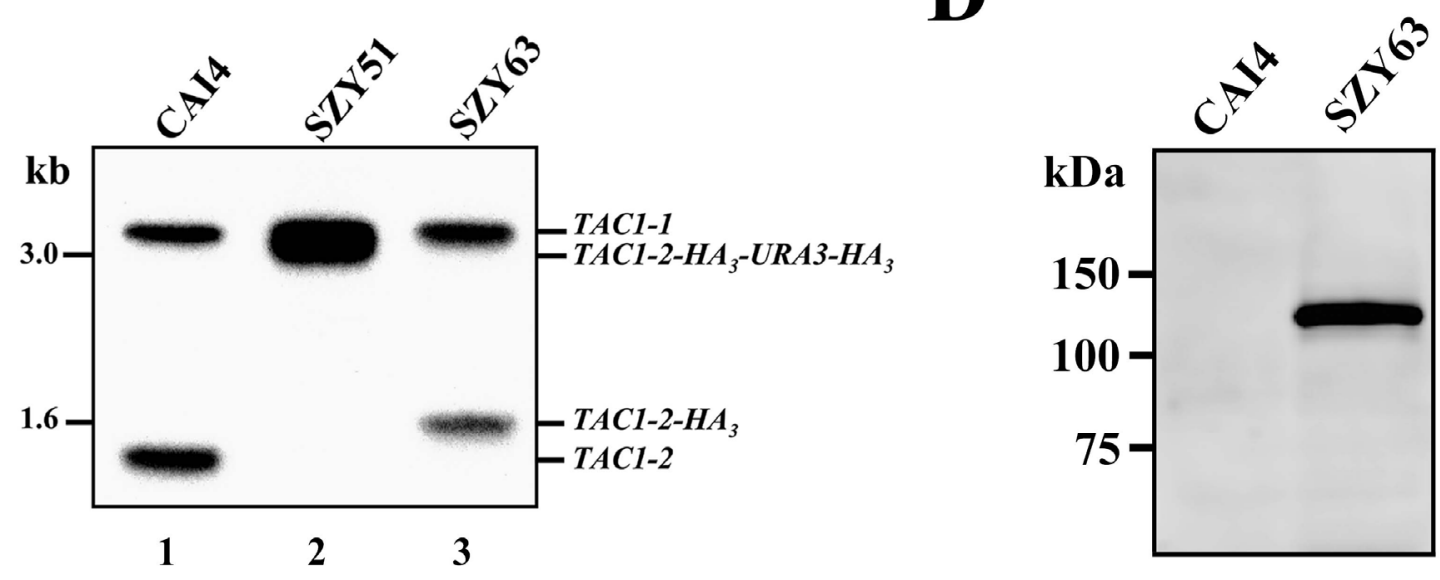
ChIP-chip experiments were conducted using the CAI4 and SZY63 strains and intergenic DNA microarrays containing 70-mer oligonucleotides covering the promoter regions of the $C$. albicans genome. Using a $P$-value $<0.001$ (enriched binding ratio $\geq 1.5$ ), we identified 37 promoters bound by Tac1p (Table 2-6). These included the promoters of genes known to be regulated by Tac1p: CDR1 (10.5), IFU5 (3.0), PDR16 (2.1), RTA3 (1.8) and CDR2 (1.7) (100, $103)$, thus validating our ChIP-chip procedure and showing that Taclp binds in vivo to the promoter of these genes to regulate their expression. HSP12, also known to be regulated by Taclp, had a binding ratio of $1.2(P$-value $=0.09)$ and thus was not significantly bound by Taclp. These experiments also identified several new targets, including genes involved in lipid metabolism, oxidative stress response as well as genes of unknown function. On two occurrences, Taclp binding was found at promoters of two genes located on opposite strands (thus sharing the same probe on the chip): $U G A 33$ (orf19.7317) and $S U C 1$ (orf19.7319) as well as LPE10 (orf19.3455) and KIC2 (orf19.3456) (Table 2-6). While the expression data did not allow to discriminate which one of the $L P E 10$ or KIC2 genes is regulated by Tac1p, we found that $S U C 1$ was up-regulated in a TAC1-dependent manner in 3 out of the 4 clinical isolates tested by expression microarrays Table A-1 while $U G A 33$ expression was unchanged in 3 out of the 4 clinical isolates, suggesting that $S U C 1$, a sucrose uptake zinc cluster regulator (120), is a target of Taclp. The identification of several promoters bound in vivo by Tac1p under non-activating conditions (i.e. in the absence of drugs or gain-of-function mutations) demonstrates that Tac1p is at least constitutively bound to its targets and suggests the possible involvement of post-binding mechanisms for Taclp-mediated transcriptional activation.

Using quantitative real-time PCR, we confirmed the binding of Taclp to the CDR 1 and PDR 16 promoters, with enrichment ratios of $29.1 \pm 4.0$ and $4.1 \pm 0.2$, respectively (Figure 2-3). As a control, we investigated binding of Tac1p to the promoter of the FUR1 gene, which was not enriched in the ChIP-chip experiments nor modulated in the azole-resistant clinical isolates, and found no significant enrichment of that promoter by Q-PCR $(1.1 \pm 0.2)$, confirming the validity of the data obtained in the ChIP-chip experiments (Figure 2-3).

Although our results suggested that the expression of the $T A C 1$ gene is controlled by Tac1p in an autoregulatory loop, the ChIP-chip data showed no enrichment for Tac1p at its own promoter, supporting an indirect auto-regulation mechanism. However, the fact that the TAC1 promoter contains a DRE-like motif located between positions -1051 and -1060 relative to the ATG translation start codon (Table 2-7; see below) and that the TAC1 oligo printed on the intergenic chips is located at position -480 may have prevented us from detecting Taclp binding at the TAC1 promoter. Therefore, we used for Q-PCR a set of TaqMan probe and primers hybridizing close to this DRE motif (Table 2-2). Under these conditions, we observed a strong enrichment of the $T A C 1$ promoter $(179.6 \pm 25.2)$ (Figure 2-3). These results strongly support a direct autoregulatory loop controlling $T A C 1$ expression.

\section{Identification of Potential Tac1p-Binding Motifs}

Tac1p belongs to the fungal-specific family of zinc cluster transcription factors that contain a DBD formed by six cysteines coordinating two zinc atoms (121). Zinc cluster factors can bind as homodimers to CGG triplets with various orientations (everted, inverted, or direct 
Table 2-6. List of Tac1p-enriched promoters $(P$-value $<0.001)$.

\begin{tabular}{|c|c|c|c|c|c|c|c|c|}
\hline $\begin{array}{l}\text { Systematic } \\
\text { Name }^{a}\end{array}$ & $\begin{array}{l}\text { CGD/ } \\
\text { NRC } \\
\text { Name }^{b}\end{array}$ & $\begin{array}{l}\text { Candida } \\
\text { DB Name }\end{array}$ & $\begin{array}{l}\text { S. cerevisiae } \\
\text { Ortholog }^{d}\end{array}$ & GO Terminology ${ }^{e}$ & $\mathbf{B R}^{f}$ & $P^{g}$ & $\begin{array}{c}\mathbf{E} \\
\mathbf{5 6 7 4 /} \\
\mathbf{5 4 5 7} 7^{h}\end{array}$ & $\frac{\mathbf{E}}{\operatorname{SZY31} / 5457^{i}}$ \\
\hline orf19.6000 & $C D R 1$ & $C D R 1$ & PDR5 & Transporter activity & 10.5 & 0.0000 & $4.4^{*}$ & -1.0 \\
\hline orf19.6869 & $A S T 2$ & IPF8041 & $A S T 2$ & Peptidase activity & 4.1 & 0.0000 & 1.6 & 1.9 \\
\hline orf19.769 & IFE 1 & IFE1 & $B D H 2$ & -- & 3.3 & 0.0000 & -- & -- \\
\hline orf19.2568 & IFU5 & IFU5 & $W W M 1$ & -- & 3.0 & 0.0000 & $5.4^{*}$ & 1.5 \\
\hline orf19.86 & $G P X 2$ & $G P X 1$ & HYRl & $\begin{array}{l}\text { Glutathione peroxidase } \\
\text { activity }\end{array}$ & 2.8 & 0.0000 & $4.0^{*}$ & -1.2 \\
\hline orf19.3406 & -- & IPF9898 & $Y H L 008 c$ & -- & 2.5 & 0.0000 & $-2.7^{*}$ & -1.5 \\
\hline orf19.7166 & -- & $I P F 2186$ & YGR110w & -- & 2.8 & 0.0000 & 5.0 & 1.2 \\
\hline orf19.7042 & -- & IPF3080 & -- & -- & 2.1 & 0.0000 & $5.5^{*}$ & 1.3 \\
\hline orf19.93 & -- & $I P F 14895$ & MIC17 & -- & 2.1 & 0.0000 & 1.5 & -1.1 \\
\hline orf19.1027 & PDR16 & PDR17 & PDR16 & $\begin{array}{l}\text { Phosphotidylinositol } \\
\text { transporter activity }\end{array}$ & 2.1 & 0.0000 & $20.0^{j}$ & $1.2^{j}$ \\
\hline orf19.3395 & -- & IPF9483 & YHR048w & -- & 2.0 & 0.0000 & -- & -- \\
\hline orf19.5877 & $A T F 1$ & IPF 1837 & $A T F 1$ & $\begin{array}{l}\text { Alcohol O- } \\
\text { acetyltransferase activity }\end{array}$ & 2.0 & 0.0000 & $2.8^{*}$ & 1.2 \\
\hline orf19.6627 & -- & $I P F 2557$ & -- & -- & 1.9 & 0.0000 & $2.6^{*}$ & -1.0 \\
\hline orf19.23 & $R T A 3$ & $R T A 3$ & $R S B 1$ & $\begin{array}{l}\text { Phospholipid- } \\
\text { translocating ATPase } \\
\text { activity }\end{array}$ & 1.8 & 0.0000 & $41.0^{*}$ & 1.0 \\
\hline orf19.6501 & -- & IPF3931 & YJU3 & -- & 1.8 & 0.0000 & $4.6^{*}$ & 1.1 \\
\hline orf19.5037 & -- & $I P F 17054$ & & -- & 1.8 & 0.0000 & 7.7 & 2.1 \\
\hline orf19.4898 & -- & IPF8950 & FMP52 & -- & 1.8 & 0.0001 & 3.4 & 1.1 \\
\hline orf19.1267 & $C A J 1$ & $I P F 10278$ & CAJ1 & $\begin{array}{l}\text { Chaperone regulator } \\
\text { activity }\end{array}$ & 1.7 & 0.0001 & 2.5 & -1.2 \\
\hline orf19.1887 & -- & IPF6464 & $Y E H 1$ & -- & 1.7 & 0.0001 & 4.5 & 1.2 \\
\hline
\end{tabular}


Table 2-6 (continued).

\begin{tabular}{|c|c|c|c|c|c|c|c|c|}
\hline $\begin{array}{l}\text { Systematic } \\
\text { Name }^{a}\end{array}$ & $\begin{array}{l}\text { CGD/ } \\
\text { NRC } \\
\text { Name }^{b}\end{array}$ & $\begin{array}{l}\text { Candida } \\
\text { DB Name }\end{array}$ & $\begin{array}{l}\text { S. cerevisiae } \\
\text { Ortholog }^{d}\end{array}$ & GO Terminology ${ }^{e}$ & $\mathbf{B R}^{f}$ & $\boldsymbol{P}^{g}$ & $\begin{array}{c}\mathbf{E} \\
\mathbf{5 6 7 4 /} \\
\mathbf{5 4 5 7} 7^{h}\end{array}$ & $\underset{\mathbf{S Z Y 3 1} / 5457^{i}}{\mathbf{E}}$ \\
\hline orf19.691 & GPD2 & GPD2 & $G P D 1$ & $\begin{array}{l}\text { Glycerol-3-phosphate } \\
\text { dehydrogenase (NAD+) } \\
\text { activity }\end{array}$ & 1.7 & 0.0001 & 1.1 & 1.1 \\
\hline orf19.2175 & -- & IPF19998 & $A I F 1$ & Oxioreductase activity & 1.7 & 0.0001 & 3.3 & 1.3 \\
\hline orf19.1444 & ENT2 & IPF17555.3 & ENT2 & $\begin{array}{l}\text { Cytoskeletal adaptor } \\
\text { activity }\end{array}$ & 1.7 & 0.0002 & 1.5 & 1.1 \\
\hline orf19.5958 & $C D R 2$ & $C D R 2$ & PDR5 & Transporter activity & 1.7 & 0.0003 & $50.5^{*}$ & -1.3 \\
\hline orf19.3455 ${ }^{k}$ & $L P E 10^{k}$ & $I P F 9782^{k}$ & LPE10 & $\begin{array}{l}\text { Magnesium ion } \\
\text { transporter activity }\end{array}$ & 1.6 & 0.0003 & 1.7 & -1.7 \\
\hline orf19.1089 & PEX11 & PEX11 & PEX11 & $\begin{array}{l}\text { Peroxisome organization } \\
\text { and biogenesis }\end{array}$ & 1.6 & 0.0003 & $1.8^{*}$ & -2.4 \\
\hline orf19.1665 & MNT1 & $M N T 1$ & KTR1 & $\begin{array}{l}\text { Alpha-1,2- } \\
\text { mannosyltransferase } \\
\text { activity }\end{array}$ & 1.6 & 0.0003 & -- & -- \\
\hline orf19.7306 & -- & IPF5987 & $Y P R 127 w$ & -- & 1.7 & 0.0003 & 2.1 & 1.1 \\
\hline orf19.7319 & $S U C 1^{k}$ & $S U C 1^{k}$ & $M A L 13$ & $\begin{array}{l}\text { Transcription factor } \\
\text { activity }\end{array}$ & 1.6 & 0.0003 & $5.1 *$ & 1.2 \\
\hline orf19.1718 & $Z C F 8$ & IPF19769 & -- & -- & 1.7 & 0.0004 & -1.6 & -1.8 \\
\hline orf19.406 & $E R G 1$ & $E R G 1$ & $E R G 1$ & $\begin{array}{l}\text { Squalene } \\
\text { monooxygenase activity }\end{array}$ & 1.7 & 0.0005 & -1.6 & 1.2 \\
\hline orf19.7603 & -- & IPF660 & $Y M R 244 c-A$ & -- & 1.6 & 0.0005 & -- & -- \\
\hline orf19.5005 & OSM2 & OSM2 & OSM1 & $\begin{array}{l}\text { Fumarate reductase } \\
\text { (NADH) activity }\end{array}$ & 1.6 & 0.0005 & $2.0^{*}$ & 1.2 \\
\hline orf19.577 & -- & IPF7353 & $Y D L 057 c$ & -- & 1.6 & 0.0006 & 2.3 & 1.5 \\
\hline orf19.5257 & $L C B 4$ & $L C B 4$ & $L C B 4$ & $\begin{array}{l}\text { D-erythro-sphingosine } \\
\text { kinase activity }\end{array}$ & 1.6 & 0.0007 & $4.0^{*}$ & 1.1 \\
\hline orf19.5525 & -- & IPF4328 & $Y M R 315 w$ & -- & 1.5 & 0.0008 & $2.3^{*}$ & 1.1 \\
\hline
\end{tabular}


Table 2-6 (continued).

\begin{tabular}{|c|c|c|c|c|c|c|c|c|}
\hline $\begin{array}{l}\text { Systematic } \\
\text { Name }^{a}\end{array}$ & $\begin{array}{c}\text { CGD/ } \\
\text { NRC } \\
\text { Name }^{b}\end{array}$ & $\begin{array}{l}\text { Candida } \\
\text { DB Name }\end{array}$ & $\begin{array}{l}\text { S. cerevisiae } \\
\text { Ortholog }^{d}\end{array}$ & GO Terminology ${ }^{e}$ & $\mathbf{B R}^{f}$ & $\boldsymbol{P}^{g}$ & $\begin{array}{c}\mathrm{E} \\
\mathbf{5 6 7 4 /} \\
\mathbf{5 4 5 7 ^ { h }}\end{array}$ & $\frac{\mathbf{E}}{\operatorname{SZY31} / 5457^{i}}$ \\
\hline orf19.4476 & IFD6 & IFD6 & YPL088w & $\begin{array}{l}\text { Aryl-alcohol } \\
\text { dehydrogenase activity }\end{array}$ & 1.6 & 0.0009 & 2.2 & 1.2 \\
\hline orf19.951 & -- & IPF1548 & -- & -- & 1.6 & 0.0009 & -1.5 & 1.1 \\
\hline
\end{tabular}

${ }^{a}$ orf19 nomenclature according to the Assembly 19 version; orfs in bold are modulated in the four sets of isolates whereas underlined orfs are modulated at least in strain 5674 .

${ }^{b}$ Gene name according to CGD (http://www.candidagenome.org/) or the NRC Candida albicans database (http://candida.bri.nrc.ca/candida/index.cfm?page=CaGeneSearch).

${ }^{c}$ Gene name according to CandidaDB (http://genolist.pasteur.fr/CandidaDB/).

${ }^{d}$ S. cerevisiae ortholog or best hit according to CGD (http://www.candidagenome.org/).

${ }^{e}$ Gene ontology (GO) terminology according to CGD.

${ }^{f}$ Binding ratio. Probes are spotted in duplicate on the ChIP-chip arrays (H. Hogues, H. Lavoie, A. Sellam, M. Mangos, T. Roemer, E. Purisima, A. Nantel and M. Whiteway, submitted for publication). Binding ratio with the most significant $P$-value is shown.

${ }^{g} P$-value of the corresponding binding ratio.

${ }^{h}$ Fold expression of the gene in strain 5674 relative to strain 5457. An asterisk $(*)$ indicates that fold expression is statistically significant (Table A-1).

${ }^{i}$ Fold expression of the gene in strain SZY31 relative to strain 5457.

${ }^{j}$ Value obtained by quantitative real-time RT-PCR.

${ }^{k}$ The intergenic oligonucleotide probe corresponds to a common promoter of two adjacent genes. The orf19.3455 probe is shared by LPE10 and KIC2; the orf19.3719 probe is shared by SUC1 and $U G A 33$. 


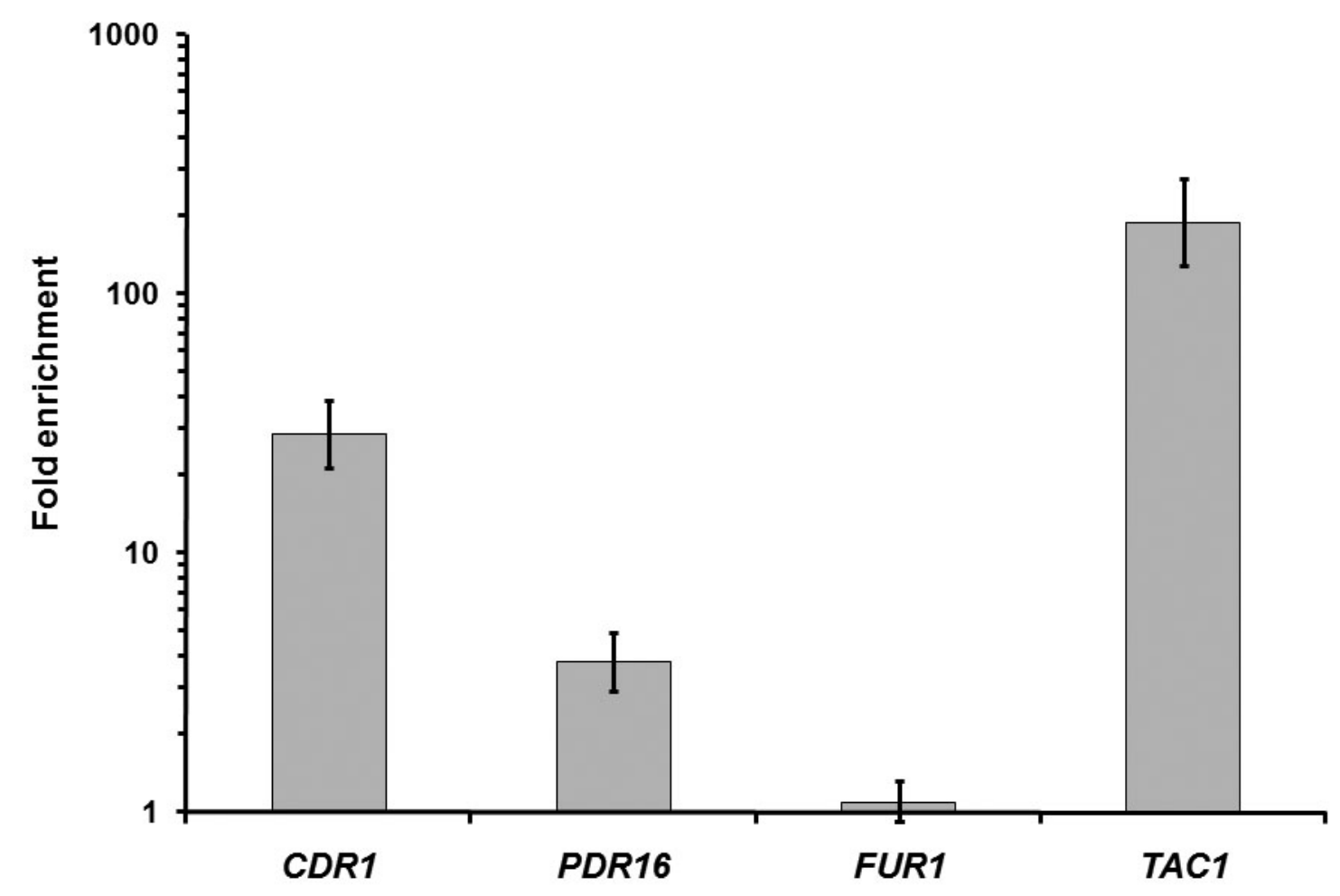

Figure 2-3. In vivo enrichment of Tac1p binding at the CDR1, PDR16 and TAC1 promoters using Q-PCR.

The CAI4 and SZY63 strains were submitted to ChIP (3 biological replicates) and the recovered DNA samples were analyzed by Q-PCR, using Universal ProbeLibrary probes (64) for the PDR16, TAC1, SPS4 and FUR1 promoters or a TaqMan probe (42) for the CDR1 promoter. Fold enrichments are presented in log scale: 3.8 for the $P D R 16$ promoter $(95 \%$ confidence interval: 4.0, 5.0), 28.8 for the $C D R 1$ promoter (95\% confidence interval: $21.4,38.9$ ), 189.3 for the $T A C 1$ promoter $(95 \%$ confidence interval: $128.6,278.6)$ and 1.1 for the $F U R 1$ promoter ( $95 \%$ confidence interval: $0.9,1.3$ ), which was used as a negative control. 
Table 2-7. DRE motifs found in the Tac1p target promoters.

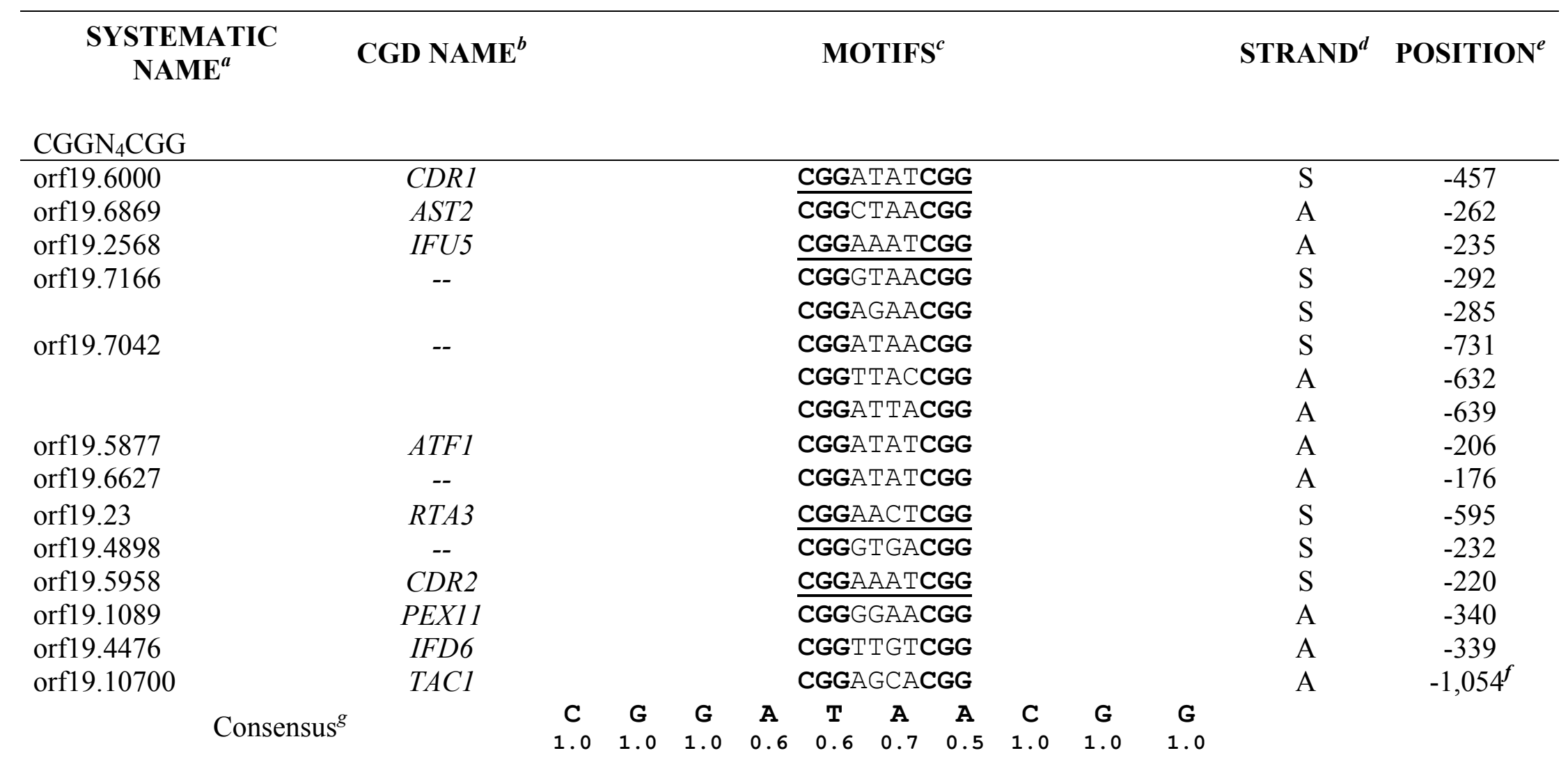


Table 2-7 (continued).

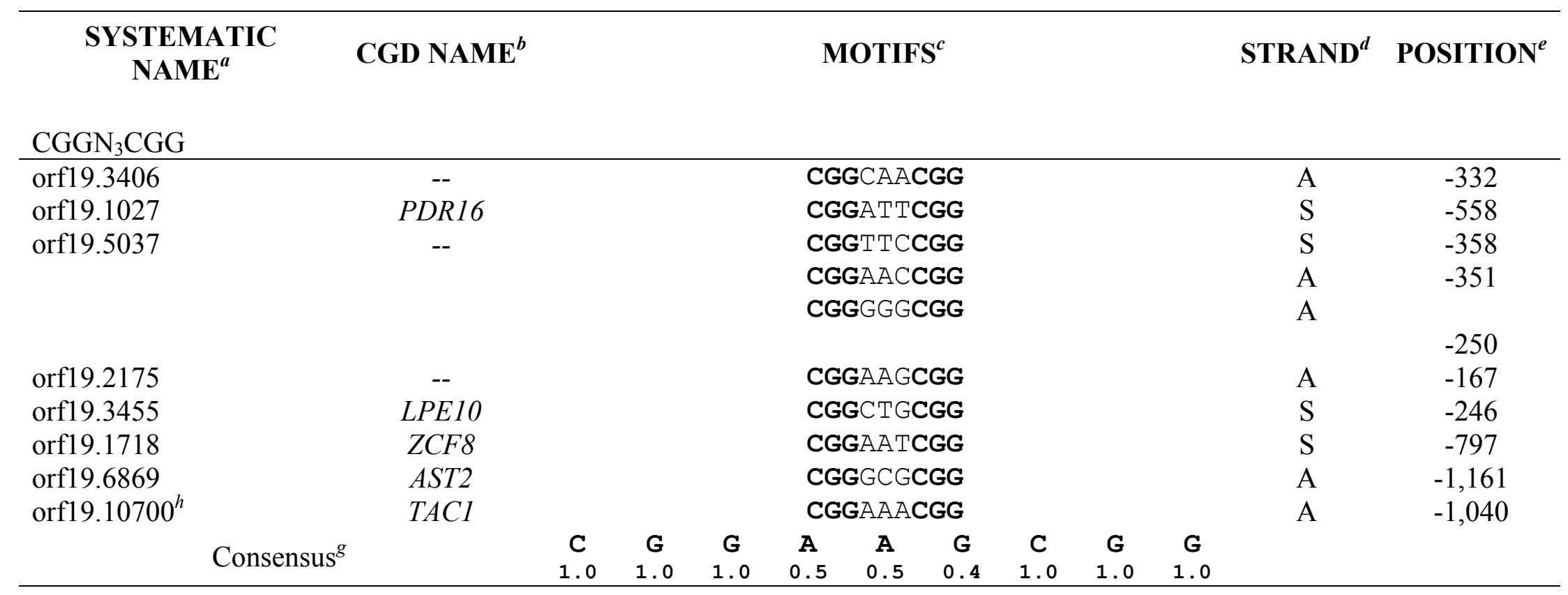

${ }^{a}$ orf19 nomenclature according to the Assembly 19 version.

${ }^{\boldsymbol{b}}$ Gene name at CGD (http://www.candidagenome.org/).

${ }^{c}$ The underlined motifs have been previously described (16)

${ }^{d} \mathrm{~S}$ denotes sense strand and A denotes antisense strand.

${ }^{e}$ Nucleotide position from the ATG translation start site.

${ }^{f}$ Position in contig19.20170, upstream of orf19.10700; position in contig.10170, upstream of orf19.3188 is $-1,051$ due to sequence polymorphisms.

${ }^{g}$ The consensus sequence was obtained using the AlignX program (component of Vector NTI Advance 10.1.1, Invitrogen).

Nucleotide frequency at each position is given below.

${ }^{\boldsymbol{h}}$ Present only in contig.20170 due to a sequence polymorphism (CㅌGAAACGG). 
repeats) and spacing (121). The $C D R 1$ and $C D R 2 \mathrm{DRE}$ consists of a direct CGG repeat with 4 intervening nucleotides (CGGAA/TATCGG), a configuration also found in the promoter of the other Tac1p-regulated genes RTA3 and $\operatorname{IFU5}(99,100)$. We thus looked for a DRE motif in the promoter of the 37 genes identified by the ChIP-chip experiments, using $1.5 \mathrm{~kb}$ of promoter sequence and the motif definition $\mathrm{CGGN}_{4} \mathrm{CGG}$ which allows for complete degeneracy for the 4 nucleotides between the CGG repeats. In addition to $C D R 1, C D R 2, R T A 3$ and IFU5, this analysis identified 8 new genes containing the $\mathrm{CGGN}_{4} \mathrm{CGG}$ sequence: $A S T 2, A T F 1, P E X 11$, IFD 6 and 4 ORFs of unknown function (orf19.7166, orf19.7042, orf19.6627 and orf19.4898), for a total of 15 matches in 12 genes (Table 2-7). As a control, we searched the CGGN $\mathrm{CGG}_{4}$ motif in $1.5 \mathrm{~kb}$ of promoter sequence from 6,068 orfs and found an average of 1.5 match per 37 promoters, yielding a 10-fold enrichment for the presence of the $\mathrm{CGGN}_{4} \mathrm{CGG}$ sequence in the Tac1p-bound promoters. We also searched the 37 enriched promoters for the motif $\mathrm{CGGN}_{3} \mathrm{CGG}$ since our unpublished data indicated that a CGGATTCGG sequence in the $P D R 16$ promoter is involved in its transcriptional activation by Taclp. This analysis identified 7 genes (including PDR16), one of them with three CGGN $_{3}$ CGG motifs (Table 2-7). Searching the 6,068 promoter sequences yielded an average of 2.2 genes per 37 promoters, resulting in a 3.2-fold enrichment for that sequence in the Tac1p-bound promoters.

Finally, we examined whether the $\mathrm{CGGN}_{3-4} \mathrm{CGG}$ motifs appear in the promoter region of the genes whose expression was modulated in all 4 resistant isolates (31 upregulated and 12 down-regulated) compared to their matched parental strains. We found 7 up-regulated genes containing the $\mathrm{CGGN}_{4} \mathrm{CGG}$ motif, 5 of which also identified by the ChIP-chip experiments (CDR1, CDR2, IFU5, RTA3, orf19.4898; the two other ones being $T A C 1$ and orf19.3047) as well as two up-regulated genes with the $\mathrm{CGGN}_{3} \mathrm{CGG}$ motif (TACl and orf19.344) (Table 2-7). Finally, searching the down-regulated genes identified one gene with the $\mathrm{CGGN}_{4} \mathrm{CGG}$ motif (SOD5) and one gene with the $\mathrm{CGGN}_{3} \mathrm{CGG}$ motif (OPT6).

\section{Expression and Location Data Mining}

When merging the expression and location data, we identified 8 genes whose promoters were bound by Tac1p in vivo and which were up-regulated in the four azoleresistant clinical isolates in a TAC1-dependent manner, thus qualifying these genes as bona fide Tac1p targets. These genes were CDR1 and CDR2, GPX1 (putative gluthatione peroxidase), LCB4 (putative sphingosine kinase), RTA3 (putative phospholipid flippase) as well as three genes of unknown function: IFU5, orf19.1887, and orf19.4898 (Table 2-6, in bold, and Table A-1). We also identified 9 genes whose promoters were bound by Tac1p in vivo and that were significantly modulated in a TAC1-dependent manner in at least strain 5674, the isolate displaying the strongest fold change in gene expression. These genes were orf19.5877 (ATF1, alcohol acetyltransferase), orf19.1089 (PEX11, fatty acid oxidation), orf19.5005 (OSM2, fumarate reductase), orf19.7319 (SUC1, sucrose metabolism) as well as 5 ORFs of unknown function (orf19.6627, orf19.7042, orf19.5525, orf19.3406 and orf19.6501) (Table 2-6, underlined, and Table A-1). These results 
suggest that, in addition to its function in azole drug resistance, Tac1p regulates other cellular functions such as lipid metabolism and oxidative stress response.

\section{DISCUSSION}

To identify the Tac1p regulon, we combined genome-wide expression and location analyses, two technologies with complementary strengths and limitations. Expression profiling is extremely valuable since it can identify all of the changes in transcript abundance associated with the perturbation of a specific transcriptional regulator, however it cannot distinguish between direct or indirect effects at individual target promoters. Location profiling is a very powerful tool to identify all the direct targets of a specific transcription factor, however it does not allow the determination of whether the bound factor acts as an activator or a repressor of these targets. Each method also gives rise to false positives and false negatives. Merging the results from the two approaches thus generates data that is most complete and cross-validated.

While merging the expression and location data sets for Tac1p, we found that, out of 37 genes whose promoter was bound by Tac1p, 20 (64\%) did not have their expression modulated (Table 2-6). Possible technical explanations for this limited overlap are that some of these genes did not pass the stringent criteria applied to the expression microarray data or that they represent false positives. However, a biological explanation could be that the expression of the bound promoters is not modulated in response to gainof-function mutations in Tac1p but rather in response to other potential Tac1p-activating signals. We also found that, out of 43 modulated genes, $35(81 \%)$ were not identified in the location profiling experiments, suggesting that these genes are indirect targets of Tac1p (i.e. controlled by Tac1p-regulated transcription factors and/or other transcriptional regulators) or that they are not bound by Tac $1 p$ under non-activating conditions (see below). It is also possible that the detection of Tac1p binding to some of these targets may have been prevented by the fact that their Taclp-binding site is too far from the oligonucleotide sequence printed on the intergenic chip, as we found for $T A C 1$. Lastly, some of these genes could have been missed by using a $P$-value $<0.001$ as the cut-off in the location array experiments. This seems to be the case for the SIP3 gene whose expression was up-regulated in the four azole-resistant isolates in a TAC1dependent manner and which had a binding ratio of 1.6 but a $P$-value of 0.0014 . Taken together, combining the expression and location profiling data allowed us to identify many new genes which unambiguously belong to the Taclp regulon and thereby gain new insights into the biological functions of Taclp.

Previously, we examined the gene expression profiles of matched isolates $2,3,15$, and 17 using an earlier generation microarray (108). In that analysis we identified 5 genes as being co-regulated with CDR1 and CDR2. Two of these, GPX1 and RTA3, were found in the present study to be up-regulated in all four matched isolate sets studied, whereas a third, $E R G 2$, was found to be up-regulated in three of these isolate sets. Likewise, of the 14 genes we previously found to be down-regulated in association with azole resistance in isolate 17, orf19.3475, orf19.2060, and orf19.5760 were found to be 
down-regulated in all four isolate sets, and FET34 was found to be down-regulated in three of the four in this study. Using the same early generation arrays, Coste et al examined the gene expression profiles of isolate C56 as well as strain DSY2926 (isolate C43 expressing a TAC1 allele carrying a gain-of-function mutation) compared to that of their parent isolate C43 (100). They identified 7 genes to be up-regulated in common between these two experimental conditions. In addition to $C D R 1$ and $C D R 2$, these included RTA3 and HSP12, both of which were found to be up-regulated in all four matched isolate sets examined in the present study. In a separate study by the same group, Karababa et al examined the gene expression profiles of isolate C56 compared to parent isolate $\mathrm{C} 43$ as well as strain CAF2-1 exposed to the $C D R 1$ - and $C D R 2$-inducing agent fluphenazine compared to this strain grown in the absence of drug (122). In addition to $C D R 1$ and $C D R 2$, they observed 7 genes to be commonly up-regulated between these two conditions. These included HSP12, GPX1, RTA3, orf19.344, IFU5, orf19.1862 and orf19.7284, all of which were found in the present study to be upregulated in either three or all of the four clinical isolates examined. In this report, we used a more recent generation microarray together with well-characterized sets of azole resistant clinical isolates and a $\operatorname{tac} 1 \Delta / \operatorname{tac} 1 \Delta$ mutant, which allowed us to identify 36 additional genes whose expression is modulated in azole-resistant strains, dependent upon Taclp.

Known Tac1p targets such as CDR1, CDR2, IFU5 and RTA3, are induced in a $T A C 1$-dependent manner upon exposure of the cells to fluphenazine or estradiol or upon expression of a gain-of-function $T A C 1$ allele in a $\operatorname{tac} 1 \Delta / \operatorname{tac} 1 \Delta$ background $(99,100)$. This indicates that the activation of Taclp by such inducers or by gain-of-function mutations is required for Taclp-mediated transcriptional regulation. Our ChIP-chip experiments were done with cells grown in rich media, under uninduced conditions (123). We found that, under these conditions, Tac $1 \mathrm{p}$ binds to its target promoters, indicating that this binding is constitutive or at least partially constitutive since it cannot be excluded that Tac1p binding increases in the presence of an inducer or an activating mutation. Functional characterization of the epitope-tagged Tac1p strain showed that Tac1p binding to its targets is not due to an activating effect of introducing the HA tag at the C-terminus of Tac1p. It was recently reported that the $S$. cerevisiae zinc cluster regulator Pdr1p, which controls the expression of the multidrug transporters PDR5, SNQ2 and YOR1 (124), also binds constitutively to its target promoters in vivo (125). Likewise, the $S$. cerevisiae zinc cluster transcription factor Warlp, which controls the expression of the ABC transporter Pdr12p in response to weak acid stress, has been shown to constitutively bind to the PDR12 promoter in vivo (126). Thus, the mechanisms by which Tac1p activates transcription in response to drugs or to gain-of-function mutations appear to be similar to those already documented for zinc cluster factors in $S$. cerevisiae and most likely involve post-binding mechanisms such as loss of interaction with a repressor protein, as proposed for Upc2p and Ecm 22p (127) or recruitment of coactivator complexes (SAGA, Mediator, SWI/SNF) as shown for Pdr1p (128).

Our analyses of the Tac1p-bound promoters for the presence of a DRE-like motif $\mathrm{CGGN}_{4} \mathrm{CGG}$ allowed us to identify 8 new Tac1p targets, in addition to CDR1, CDR2, RTA3 and IFU5, containing this sequence (Table 2-7). The fact that 25 of the Tac $1 \mathrm{p}-$ 
bound promoters do not contain this motif suggests that Tac1p may recognize additional configurations of the CGG triplets, including monomeric CGG triplets or CGG triplets with different spacing and/or orientations (121). The latest proposition is supported by our unpublished data that a CGGATTCGG sequence in the PDR16 promoter is involved in its transcriptional activation by Tac1p and the enrichment of the CGGN ${ }_{3}$ CGG motif in the Tac1p-bound promoters (Table 2-7). Alternatively, Tac1p may bind upstream or downstream of the DNA sequences analyzed (1.5 kb of upstream sequences). Finally, it is also possible that Tac1p binds to some of its targets indirectly, through its association with other DNA-binding proteins. For instance, the $S$. cerevisiae zinc cluster proteins Rsc30p and Rsc3p, which are part of the chromatin remodelling complex, have been shown to bind indirectly to DNA (129). Whether or not the DRE motifs identified in the Tac1p-bound promoters are functional as well as the sequences/factors mediating Tac1p binding to its target promoters in the absence of a classical DRE remain to be experimentally determined.

We previously showed that strain 5674 overexpresses the $T A C 1$ gene and that introduction of a TAC1 allele carrying the N972D gain-of-function mutation in a tac $1 \Delta /$ tacl $\triangle$ strain leads to the constitutive upregulation of the TAC1 transcript, which suggested that Tac1p is positively autoregulated, directly or indirectly (103). We show in the present study that i) $T A C 1$ is upregulated in three additional azole-resistant isolates in which the Tac1p pathway is activated (Table 2-4); ii) the TAC1 promoter contains a DRE motif, and iii) Taclp binds in vivo to its own promoter (Figure 2-3). Taken together, our findings support the proposition that $T A C 1$ expression is controlled by a direct positive autoregulatory loop. This situation is similar to $S$. cerevisiae $\operatorname{Pdr} 3 \mathrm{p}$, which binds in vivo to two PDRE located in the $P D R 3$ promoter to transactivate its own expression (94). It appears that direct self-regulatory loops are a common feature among zinc cluster transcription factors in yeast, including Pdr3p and Yrrlp (pleiotropic drug resistance), Hap1p (respiration) and Stb5p (pentose phosphate pathway) (121). Studies in budding yeast have shown that transcription factor autoregulation is necessary to respond to environmental stresses. As examples, autoregulation of $P D R 3$ in a $p d r 1 \Delta$ background is crucial for growth on a medium containing cycloheximide (94) and autoregulation of the basic leucine zipper transcription factor Hac1p, which controls the unfolded protein response, is required to protect the cells from prolonged endoplasmic reticulum stress (130). Similarly, autoregulation of C. glabrata AMT1, a copper-sensing transcription factor, is necessary to protect the cells upon exposure to high environmental copper levels (131). Thus, one possible outcome of Tac1p autoregulation would be an amplifiable production of the Tac1p protein necessary for rapid and sustained response to drugs and yet unknown activating signals (123).

Three previously identified Taclp targets (CDR1, CDR2, PDR 16) have been shown to play a role in azole drug resistance $(38,102,132)$. Thus Tac1p confers azole resistance by activating different effectors, each contributing to some extent to the overall azole resistance of the cells. Additional Tac1p targets identified by our studies and coregulated with $C D R 1, C D R 2$ and $P D R 16$ may also play a protective role against toxic injuries. For instance, orf19.4531 is found among the group of genes differentially expressed under the gene ontology (GO) molecular function term "ATPase activity 
coupled to movement of substances", including CDR1, CDR2 and RTA3 (Table 2-4). orf19.4531 encodes a putative $\mathrm{ABC}$ transporter of the PDR subfamily (to which $C D R 1$ and $C D R 2$ belong). Whether this transporter impacts azole resistance, alone or in conjunction with Cdr $1 p$ and $C d r 2 p$, remains to be determined. This efflux pump may also protect the cell from other toxic compounds. As another example, orf19.86 (GPXI), which encodes a putative glutathione peroxidase, is an integral component of the glutathione and glutathione-dependent enzyme system which has been implicated in the resistance of tumor cells to anticancer agents $(133,134)$. Increased activity of this enzyme system is often observed in conjunction with increased activity of the ABC transporter P-glycoprotein in drug-resistant human cancer cells (135).

Although not found among the 37 promoters observed to be bound by Tac1p, $C H K 1$ was among the 31 genes that were consistently co-regulated with CDR1 and $C D R 2$ in all four matched isolate sets. Its up-regulation in these isolates is at least influenced by Tac $1 \mathrm{p}$ as deletion of $T A C 1$ in isolate 5674 reduced its expression to normal levels. Chk1p is a histidine kinase involved in a two-component signaling pathway, along with the response regulator Ssk1p, that regulates cell wall biosynthesis $(136,137)$. Interestingly it was recently shown that strains of $C$. albicans lacking either of these signal transduction proteins are hypersensitive to fluconazole (138). Up-regulation of $C H K 1$ in association with $C D R 1$ - and $C D R 2$-mediated azole resistance suggests that, in addition to its requirement for baseline azole tolerance in azole-susceptible cells, this protein may contribute to azole resistance in clinical isolates. In the related fungal species C. glabrata, ATPase activity of the $\mathrm{ABC}$ transporter Cdrlp and the drug efflux activity of the $\mathrm{ABC}$ transporter Pdh1p (Cdr2p) are regulated by phosphorylation (139, 140). Likewise, phosphorylation has been shown to modulate transcriptional activity of the transcription factor Gal4p (141), and has been suggested to regulate the activity of the transcriptional regulator Pdr3p (142). It is therefore tempting to speculate that Chk1p is involved in the phosphorylation and activity of the efflux pumps Cdr1p and Cdr2p, or possibly Tac $1 p$ itself. Further investigation into the contribution of this signaling pathway to azole resistance will be required to address this question.

Our genome-wide location experiments revealed that Taclp binds to the promoter of a group of genes involved (or predicted to be involved) in lipid metabolism. These genes are CDR1, CDR2, PDR16, RTA3, ATF1, ERG1, LCB4, orf19.6501, orf19.7166, and orf19.1887. With the exception of $E R G 1$, all these genes were significantly upregulated in a TACl-dependent manner at least in one out of the four clinical isolates tested by expression microarrays, strongly suggesting i) that these genes are direct transcriptional targets of Tac1p and ii) a role for Taclp in lipid metabolism. It has been shown that Rsblp, the $S$. cerevisiae orthologue of $C$. albicans Rta3p, plays an essential role in the translocation of long-chain bases across the plasma membrane (143), suggesting a role for Rsblp, and thus potentially Rta3p, in regulating the sphingolipid composition of the plasma membrane. Interestingly, S. cerevisiae LCB4 encodes a major sphingolipid long-chain base kinase required for synthesis of long-chain base phosphates and for the rapid incorporation of long chain bases from the culture medium into sphingolipids (144). We also found that orf19.3104, the orthologue of S. cerevisiae $Y D C 1$ encoding an alkaline dihydroceramidase, is up-regulated in a TACl-dependent 
manner in all four sets of clinical isolates (Table 2-4), although binding of Tac1p to the promoter of this gene was not detected. Ydclp hydrolyzes dihydroceramide to free fatty acid and dihydrosphingosine, the substrate of $L C B 4$ (145). Taken together, these findings suggest a role for Taclp in the synthesis and translocation of sphingolipids into the plasma membrane.

Likewise, C. albicans Cdr1p and Cdr2p have been shown to function as plasma membrane energy-dependent translocators of phospholipids, mediating their in-to-out movement (floppases) (146). Thus, Cdr1p and Cdr2p would act in concert with Rta3p to establish the asymetry of membrane lipids in the plasma membrane. Interestingly, Tac1p binds to the promoter of the $S$. cerevisiae AST1 homologue, orf19.6869 (AST2). S. cerevisiae Ast1p was shown to be required for the raft association and restoration of surface delivery of a mutant of the major plasma membrane proton ATPase, Pma1p, that was mistargeted to the vacuole (147). Elsewhere, it has been shown that Cdrlp is a lipid raft associated protein (148). An attractive model would be that Tac1p contributes to the raft association and proper routing of its target gene-products Cdr1p, Cdr2p and Rta3p to the plasma membrane by regulating AST2 expression. Recent studies have demonstrated that both sphingolipids and sterols are important determinants of surface localization of Cdrlp, as reduced membrane localization of Cdr1p was associated to increased susceptibility of $C$. albicans to ketoconazole in mutants defective in ergosterol biosynthesis (149). Interestingly, we found that Tac1p binding was enriched at the ERG1 (1.7 fold, $P$-value 0.0005$)$ and $E R G 2$ (1.6 fold, $P$-value 0.0011$)$ promoters. Moreover, $E R G 2$ gene expression was significantly up-regulated in a TAC1-dependent manner in isolates C56, Gu5 and 17 (Table A-1), suggesting a role of Tac1p in ergosterol biosynthesis. PDR16, another target of Tac1p, is the functional homologue of the $S$. cerevisiae PDR16, encoding a lipid transfer protein of the Sec14 family $(102,150)$. $S$. cerevisiae Pdr16p was shown to localize to lipid particles and to transport phosphatidylinositol (151). There is strong evidence showing that lipid transfer proteins exchange lipid molecules between organelle membranes and plasma membrane at membrane contact sites (152). Thus, by controlling the expression of the PDR16 gene, Taclp would act as a regulator of phosphatidylinositol transfer within membrane contact sites, further supporting the role of Tac1p in membrane lipid traffic.

Consistent with the potential role of Tac1p in membrane lipid metabolism, we found that the Tac1p targets orf19.7166, orf19.6501 and orf19.1887 encode three putative steryl ester hydrolases or triglycerides lipases as i) a Pfam analysis detected an $\alpha / \beta$ hydrolase fold in their primary sequences (Pfam Entries PF04083.6 for orf19.7166 and PF00561 for orf19.1887 and orf19.6501), ii) their primary sequences contain the lipase consensus sequence motif GXSXG (153) and iii) Kyte-Doolittle hydropathy plots revealed hydrophobic regions in the primary sequences suggesting that these proteins are potentially membrane-anchored to lipid particles (data not shown). Steryl esters and triacylglycerols are neutral lipids stored in lipid particles and serve as an energy source as well as a rapid fatty acid source needed upon lipid depletion conditions (154). The orf19.1887 protein is highly homologous to $S$. cerevisiae Yeh1p, a steryl ester hydrolase localized to lipid particles $(155,156)$, whereas the orf19.7166 and orf19.6501 proteins display moderate homology to the products of the S. cerevisiae YGR110W and YJU3 
genes. Yju3p was shown to be localized in lipid particles as well $(157,158)$. Another Taclp target whose product was shown in S. cerevisiae to be localized in lipid particles is $A T F 1$, encoding an alcohol acetyl transferase (159). This gene was significantly upregulated in a TAC1-dependent manner in strain 5674 (Table A-1). The fact that Atflp localizes to lipid particles and functions in esterification process suggests that it is involved in the metabolism of lipids probably by transferring acetyl groups to free hydroxyl groups of fatty acids. Thus, in addition to be a potential regulator of membrane lipid traffic, Tac1p appears to be an important regulator of lipid mobilization in $C$. albicans, a function needed for rapid restoration of membrane lipids upon lipid depletion conditions (154).

Our studies identified two Tac1p target genes, GPXI and SOD5, which have been shown to be involved in the oxidative stress response. We provide strong evidence that Taclp directly regulates the expression of $G P X 1$, which is implicated in response to oxidative stress (160). Interestingly, although the C. albicans genome encodes two other putative gluthatione peroxidases (encoded by orf19.85 and orf19.87) with high homology to Gpx 1p, only Gpx1p was found to be overproduced upon treatments with diamide or hydrogen peroxide (160), suggesting that, among the three putative gluthatione peroxidases, $G P X 1$ plays an important role in oxidative stress response. It was also shown that $S$. cerevisiae GPXI encodes a phospholipid hydroperoxide glutathione peroxidase which protects the cell against phospholipid hydroperoxides during oxidative stress (161).

SOD5, a copper, zinc superoxide dismutase found in the cell wall of C. albicans, has been shown to be important in protection against osmotic and oxidative stress (162). Since SOD 5 seems to play an important role in this process, it is interesting that the gene is in fact down-regulated in all four azole resistant clinical isolates studied. Martchenko, et al. showed that while deleting SOD5 does not decrease cell viability, susceptibility to hydrogen peroxide under nutrient poor conditions is increased (162). Indeed, we have previously shown that azole resistant isolate 5674 , while resistant to diamide, is hypersusceptible to hydrogen peroxide (163). While this hypersusceptibility to hydrogen peroxide could be explained by the down-regulation of $S O D 5$, resistance to diamide could be attributed to the up-regulation of the $C D R 2$ gene by Tac1p since $C D R 2$ expression was shown to confer diamide resistance in $S$. cerevisiae (163). Taken together, these observations indicate that $T A C 1$ differentially protects the cell against different oxidative stresses. 


\section{CHAPTER 3: GAIN-OF-FUNCTION MUTATIONS IN MRR1 REGULATE THE EXPRESSION OF MDR $1^{\dagger}$}

\section{INTRODUCTION}

Long term treatment of $C$. albicans with azoles results in the development of resistance over time $(27,29,35,43,91,95)$. One mechanism of resistance occurs due to changes in ERG11, the gene that encodes the azole drug target lanosterol demethylase. Mutations that occur in ERG11 result in amino acid substitutions in Erg11p that decrease the binding affinity of the azoles (45). Up-regulation of ERG11 confers resistance by increasing the amount of enzyme in the cell to bind azoles, requiring more drugs to be as efficacious $(27,91)$. Another mechanism of resistance is the up-regulation of genes encoding efflux pumps. $C D R 1$ and $C D R 2$ encode two ABC transporters that are responsible for resistance to azoles $(28,32,35,37,38,73)$. Tac1p, a zinc cluster transcription factor, has been recently identified as the transcriptional regulator of $C D R 1$ and $C D R 2(88,89)$. The up-regulation of $M D R 1$ is also responsible for azole resistance.

MDR1 encodes a MFS transporter that has been implicated in fluconazole resistance in $C$. albicans $(35,39,43,72)$. MDRl was first cloned for its ability to confer resistance to benomyl and methotrexate (41). In clinical matched isolates, MDR 1 expression can only be detected in resistant isolates $(34,43)$. Disruption of $M D R 1$ in wild-type isolates shows no hypersusceptibility to fluconazole, most likely due to the lack of $M D R 1$ expression in susceptible isolates $(20,36,42)$. Disruption of $M D R 1$ in a resistant isolate decreased resistance to fluconazole (21). The promoter region of $M D R 1$ from a susceptible clinical isolate fused to the reporter GFP was transformed into a resistant isolate, and fluorescence was detected pointing to a trans-regulatory element as the regulator of the efflux pump (72).

The bZip transcription factor Cap1p has been implicated in the overexpression of $M D R 1$ in response to benomyl (84). The promoter region of MDRl has been shown to have a YRE motif (85). Sequential disruption of the promoter region of $M D R l$ in a resistant isolate showed that the absence of the YRE motif decreased the expression of $M D R 1$, but the expression was not completely abolished (86). The disruption of CAP1 did not abolish $M D R 1$ expression in response to benomyl (87). This indicates that while Caplp does bind to the promoter of $M D R 1$, the bZip transcription factor is not responsible for azole resistance in clinical isolates.

In this study, we examined three clinical matched isolates known to overexpress $M D R 1$. Using genome-wide expression profiling, we identified genes co-regulated with $M D R 1$, including a zinc cluster transcription factor encoded by $M R R 1$ as the regulator of $M D R 1$ mediated azole resistance. In collaboration with our group, the lab of Joachim Morschhäuser at the Universität Würzburg sequenced $M R R 1$ and constructed the

\footnotetext{
${ }^{\dagger}$ Morschhauser, J., K. S. Barker, T. T. Liu, B. W. J. Bla, R. Homayouni and P. D. Rogers. 2007. The transcription factor Mrrlp controls expression of the MDRl efflux pump and mediates multidrug resistance in Candida albicans. PLoS Pathog. 3: e164. Adapted by permission of authors.
} 
disruption and reintegrated mutants used in this study. In addition, the Morschhäuser lab also performed the drug susceptibility tests that showed MRRl as the regulator of drug induced $M D R 1$ gene expression.

\section{MATERIALS AND METHODS}

\section{Strains and Growth Conditions}

C. albicans strains used in this study are listed in Table 3-1. All strains were stored as frozen stocks with $15 \%$ glycerol at $-80^{\circ} \mathrm{C}$. The strains were routinely grown in YPD medium ( $10 \mathrm{~g}$ yeast extract, $20 \mathrm{~g}$ peptone, $20 \mathrm{~g}$ glucose per liter) at $30^{\circ} \mathrm{C}$.

\section{DNA Microarray Analysis}

The nucleotide sequences corresponding to 6,165 open reading frames (ORFs) for C. albicans were downloaded from the Galar Fungail European Consortium (Assembly 6, http://www.pasteur.fr/Galar_Fungail/CandidaDB/). We set out to design two nonoverlapping probesets targeting the 3'-600 bp region of each ORF. Each probeset consisted of 13 'prefect match' 25-bp probes and their corresponding 'mismatch' control probes containing a single mismatch in the center of the oligonucleotide. For ORFs less than $600 \mathrm{bp}$ in length the sequence was divided in two equal segments for subsequent design procedures. Optimum probesets were selected by the Affymetrix design team based on their model which, among other things, considers probe hybridization quality and cross-hybridization potential. Consequently, in some cases, only one probeset was selected for a given ORF. For quality control and normalization purposes, we made 2-3 additional probe sets spanning the entire sequence of the $C$. albicans $18 \mathrm{~S}$ rRNA (GenBank Accession M60302), genes encoding GAPDH, actin and Mdr1p (Bmr1p) in addition to the standard Affymetrix controls (BioB, C, D, cre, DAP, PHE, LYS, THR). In the end, the GeneChip contained 10,736 probe sets including 9 controls, 6,123 unique ORFs and duplicate probe sets for 4,604 ORFs. The duplicate probe sets are made to distinct regions of the ORF, thereby allowing 2 independent measurements of the mRNA level for that particular gene. The C. albicans custom Affymetrix NimbleExpress Arrays (CAN04a530004N) were manufactured by NimbleGen Systems (105) per our specification.

\section{RNA Preparation for Microarrays}

The matched clinical isolate sets and strain SZY31 were grown in YPD broth at $30^{\circ} \mathrm{C}$ in a shaking incubator to mid-log phase as described previously (108). The cell pellets were frozen and stored at $-80^{\circ} \mathrm{C}$ prior to RNA preparation. Experiments were repeated independently three times. Total RNA was isolated using the hot SDS-phenol 
Table 3-1. C. albicans strains examined in this study.

\begin{tabular}{|c|c|c|c|}
\hline Strain & Parent & $\begin{array}{l}\text { Relevant Characteristics or } \\
\text { Genotype }\end{array}$ & Reference \\
\hline SC5314 & & Wild-type $C$. albicans model strain & (164) \\
\hline CAI4 & SC5314 & ura $3 \Delta::$ imm 434/ura3s::imm434 & (18) \\
\hline F1 & & Fluconazole-susceptible & (43) \\
\hline $\mathrm{F} 2$ & & Fluconazole-susceptible & (43) \\
\hline F5 & & Fluconazole-resistant & (43) \\
\hline G1 & & Fluconazole-susceptible & (43) \\
\hline G2 & & Fluconazole-susceptible & (43) \\
\hline G5 & & Fluconazole-resistant & (43) \\
\hline 5833 & & Fluconazole-susceptible & $(102)$ \\
\hline 6692 & & Fluconazole-resistant & $(102)$ \\
\hline SCMRR1M4A & SC5314 & $m r r 1 \Delta \because: F R T / m r r 1 \Delta: \because F R T$ & this study \\
\hline SCMRR1M4B & SC5314 & $m r r 1 \Delta \because: F R T / m r r 1 \Delta: \because F R T$ & this study \\
\hline F5MRR1M4A & F5 & $\operatorname{mrr} 1 \Delta: \because F R T / m r r 1 \Delta: \because F R T$ & this study \\
\hline F5MRR1M4B & F5 & $m r r 1 \Delta:: F R T / m r r 1 \Delta: \because F R T$ & this study \\
\hline G5MRR1M4A & G5 & $\operatorname{mrr} 1 \Delta: \because F R T / m r r 1 \Delta: \because F R T$ & this study \\
\hline G5MRR1M4B & G5 & $\operatorname{mrr} 1 \Delta:: F R T / m r r 1 \Delta: \because F R T$ & this study \\
\hline SCMRR1M4K2A & SCMRR1M4A & mrrlA /MRR1F2-1-caSAT1 & this study \\
\hline SCMRR1M4K2B & SCMRR1M4B & mrrlA /MRR1F2-1-caSAT1 & this study \\
\hline SCMRR1M4K3A & SCMRR1M4A & mrrlA /MRR1F5-caSAT1 & this study \\
\hline SCMRR1M4K3B & SCMRR1M4B & mrr $1 \Delta / M R R 1 F 5$-caSAT1 & this study \\
\hline SCMRR1M4K4A & SCMRR1M4A & mrrl $1 \Delta / M R R 1 G 2-2-c a S A T 1$ & this study \\
\hline SCMRR1M4K4B & SCMRR1M4B & mrrlA/MRR1G2-2-caSAT1 & this study \\
\hline SCMRR1M4K5A & SCMRR1M4A & mrr $1 \Delta / M R R 1 G 5-c a S A T 1$ & this study \\
\hline SCMRR1M4K5B & SCMRR1M4B & mrr1A/MRRIG5-caSAT1 & this study \\
\hline
\end{tabular}


method (109). Frozen cell pellets were suspended in $12 \mathrm{ml}$ of $50 \mathrm{mM}$ sodium acetate $(\mathrm{pH}$ 5.2), $10 \mathrm{mM}$ EDTA at room temperature, after which $1 \mathrm{ml}$ of $20 \%$ sodium dodecyl sulfate and $12 \mathrm{ml}$ of acid phenol (Fisher Scientific, Waltham, MA) were added. This mixture was incubated $10 \mathrm{~min}$ at $65^{\circ} \mathrm{C}$ with vortexing each minute, cooled on ice for 5 $\mathrm{min}$, and centrifuged for $15 \mathrm{~min}$ at $12,000 \mathrm{x} \mathrm{g}$. Supernatants were transferred to new tubes containing $15 \mathrm{ml}$ of chloroform, mixed and centrifuged at $200 \mathrm{x} g$ for $10 \mathrm{~min}$. The aqueous layer was removed to new tubes, RNA was precipitated with 1 volume isopropanol and 0.1 volume $2 \mathrm{M}$ sodium acetate ( $\mathrm{pH}$ 5.0), and then collected by centrifugation at $17,000 \times \mathrm{g}$ for $35 \mathrm{~min}$ at $4^{\circ} \mathrm{C}$. The RNA pellet was suspended in $10 \mathrm{ml}$ of $70 \%$ ethanol, collected again by centrifugation, and suspended in nuclease free water.

\section{cRNA Synthesis and Labeling}

Immediately prior to cDNA synthesis, the purity and concentration of RNA samples were determined from $\mathrm{A}_{260} / \mathrm{A}_{280}$ readings and RNA integrity was determined by capillary electrophoresis using the RNA 6000 Nano Laboratory-on-a-Chip kit and Bioanalyzer 2100 (Agilent Technologies, Santa Clara, CA) as per the manufacturer's instructions. First and second strand cDNA was synthesized from $15 \mu \mathrm{g}$ of total RNA using the SuperScript Double-Stranded cDNA Synthesis Kit (Invitrogen, Carlsbad, CA) and oligo-dT24-T7 primer (PrOligo: Sigma-Aldrich) according to the manufacturer's instructions. cRNA was synthesized and labeled with biotinylated UTP and CTP by in vitro transcription using the T7 promoter-coupled double stranded cDNA as template and the Bioarray HighYield RNA Transcript Labeling Kit (ENZO Diagnostics, New York, NY). Double-stranded cDNA synthesized from the previous steps was washed twice with $70 \%$ ethanol and suspended in $22 \mu \mathrm{l}$ of RNase-free water. The cDNA was incubated as recommended with reaction buffer, biotin-labeled ribonucleotides, dithtiothreitol, RNase inhibitor mix and T7 RNA polymerase for $5 \mathrm{~h}$ at $37^{\circ} \mathrm{C}$. The labeled cRNA was separated from unincorporated ribonucleotides by passing through a CHROMA SPIN100 column (Clontech, Mountain View, CA) and ethanol precipitated at $-20^{\circ} \mathrm{C}$ overnight.

\section{Oligonucleotide Array Hybridization and Analysis}

The cRNA pellet was suspended in $10 \mu \mathrm{l}$ of RNase-free water and $10 \mu \mathrm{g}$ was fragmented by ion-mediated hydrolysis at $95^{\circ} \mathrm{C}$ for $35 \mathrm{~min}$ in $200 \mathrm{mM}$ Tris-acetate (pH 8.1), $500 \mathrm{mM}$ potassium acetate, $150 \mathrm{mM}$ magnesium acetate. The fragmented cRNA was hybridized for $16 \mathrm{~h}$ at $45^{\circ} \mathrm{C}$ to the C. albicans NimbleExpress GeneChip arrays. Arrays were washed at $25^{\circ} \mathrm{C}$ with $6 \times \mathrm{SSPE}, 0.01 \%$ Tween 20 followed by a stringent wash at $50^{\circ} \mathrm{C}$ with $100 \mathrm{mM}$ MES, $0.1 \mathrm{M} \mathrm{NaCl}, 0.01 \%$ Tween 20.

Hybridizations and washes employed the Affymetrix Fluidics Station 450 using their standard EukGE-WS2v5 protocol. The arrays were then stained with phycoerythreinconjugated streptavidin (Molecular Probes: Invitrogen) and the fluorescence intensities were determined using the GCS 3000 high-resolution confocal laser scanner (Affymetrix, Santa Clara, CA). The scanned images were analyzed using software resident in GeneChip Operating System v2.0 (GCOS; Affymetrix). Sample loading and variations 
in staining were standardized by scaling the average of the fluorescent intensities of all genes on an array to a constant target intensity. The signal intensity for each gene was calculated as the average intensity difference, represented by $[\Sigma(\mathrm{PM}-\mathrm{MM}) /($ number of probe pairs)], where PM and MM denote perfect-match and mismatch probes.

\section{Microarray Data Analysis}

The scaled gene expression values from GCOS software were imported into GeneSpring 7.2 software (Agilent Technologies) for preprocessing and data analysis. Probe sets were deleted from subsequent analysis if they were called absent by the Affymetrix criterion and displayed an absolute value below 20 in all experiments. The expression value of each gene was normalized to the median expression of all genes in each chip as well as the median expression for that gene across all chips in the study. Pairwise comparison of gene expression was performed for each matched experiment. Among direct comparisons between matched clinical isolates genes were considered to be differentially expressed if their change in expression was $\geq 1.5$-fold in three independent experiments.

\section{Quantitative Real-Time RT-PCR}

An aliquot of the RNA preparations from the samples used in the microarray experiments was saved for quantitative real-time reverse transcription (RT)-PCR followup studies. First-strand cDNAs were synthesized from $2 \mu \mathrm{g}$ of total RNA in a $21-\mu 1$ reaction volume using the SuperScript First-Strand Synthesis System for RT-PCR (Invitrogen) in accordance with the manufacturer's instructions. Quantitative real-time PCRs were performed in triplicate using the 7000 Sequence Detection System (Applied Biosystems, Inc.: ABI, Foster City, CA). Independent PCRs were performed using the same cDNA for both the gene of interest and the 18S rRNA, using the SYBR Green PCR Master Mix (Applied Biosystems, Inc.). Gene-specific primers were designed for the gene of interest and the 18S rRNA using Primer Express software (Applied Biosystems, Inc.) and the Oligo Analysis \& Plotting Tool (QIAGEN, Valencia, CA) and are shown in Table 3-2. The PCR conditions consisted of AmpliTaq Gold activation at $95^{\circ} \mathrm{C}$ for 10 min, followed by 40 cycles of denaturation at $95^{\circ} \mathrm{C}$ for $15 \mathrm{~s}$ and annealing/extension at $60^{\circ} \mathrm{C}$ for $1 \mathrm{~min}$. A dissociation curve was generated at the end of each PCR cycle to verify that a single product was amplified using software provided with the 7000 Sequence Detection System. The change in fluorescence of SYBR Green I dye in every cycle was monitored by the system software, and the threshold cycle $\left(\mathrm{C}_{T}\right)$ above the background for each reaction was calculated. The $\mathrm{C}_{T}$ value of $18 \mathrm{~S}$ rRNA was subtracted from that of the gene of interest to obtain a $\Delta \mathrm{C}_{T}$ value. The $\Delta \mathrm{C}_{T}$ value of an arbitrary calibrator (e.g., untreated sample) was subtracted from the $\Delta \mathrm{C}_{T}$ value of each sample to obtain a $\Delta \Delta \mathrm{C}_{T}$ value. The gene expression level relative to the calibrator was expressed as $2^{-\Delta \Delta \mathrm{CT}}$. 
Table 3-2. Primers used for quantitative real-time RT-PCR.

\begin{tabular}{llc}
\hline $\begin{array}{l}\text { Gene } \\
\text { Name }\end{array}$ & Primer Pairs $^{a}$ & Amplicon Size (bp) \\
\hline $18 S$ & $\mathbf{F}, \mathbf{5}^{\prime}$-CACGACGGAGTTTCACAAGA-3' & 135 \\
& $\mathbf{R}, \mathbf{5}^{\prime}$-CGATGGAAGTTTGAGGCAAT-3' & \\
MDRl & $\mathbf{F}, \mathbf{5}^{\prime}$-ACATAAATACTTTGCCCATCCAGAA-3' & 82 \\
& $\mathbf{R}, \mathbf{5}^{\prime}$-AAGAGTTGGTTTGTAATCGGCTAAA-3' & 59 \\
MRR1 & $\mathbf{F , ~ 5 ' - A G T G A A G A A G C C C A A C A G C A A A - 3 ' ~}$ & \\
& $\mathbf{R}, \mathbf{5}^{\prime}$-GACTGCGAACTTGTAAACGGC-3' & \\
\hline
\end{tabular}

${ }^{a} \mathbf{F}$ : forward, $\mathbf{R}$ : reverse 


\section{RESULTS}

\section{Transcription Factor MRR1 Is Coordinately Regulated with MDR1 in Resistant Clinical C. albicans Isolates}

In order to identify the genes coordinately regulated with $M D R 1$, we examined three clinical matched isolates using genome-wide expression profiling (Table 3-1). Previous studies have shown that F1, G1, and 5833 (azole susceptible isolates) do not express baseline $M D R 1$; F5, G5, and 6692 (azole resistant isolates) have been shown to overexpress $M D R 1(43,102)$. Three independent RNA samples were hybridized to custom $C$. albicans Affymetrix microarrays. Genes were considered to be differentially expressed if genes changes 1.5 -fold in all three independent experiments. Using these criteria, there were 111 genes up-regulated and 90 genes down-regulated in F5 compared to F1 (Table B-1 and Table B-2). In G5 compared to G1, there were 194 genes upregulated and 184 genes down-regulated. In 6692 compared to 5833, 66 genes were upregulated and 84 genes were down-regulated. The core genes considered to be coregulated with $M D R 1$ were the genes in common among all three clinical isolates. There were the 21 genes up-regulated including the previously identified genes GRP2, IFD1, IFD4, IFD5, IFD7, and MDRI (Figure 3-1). There were also 7 genes that were downregulated among all three clinical matched isolates (Figure 3-1). Interestingly, one gene encoding a zinc cluster transcription factor, orf19.7372 or IPF1266, was up-regulated in all three resistant clinical isolates. This zinc cluster transcription factor gene was named multidrug resistance regulator, $M R R 1$.

This transcription factor gene was disrupted in the resistant isolates F5 and G5 by the Morschhäuser lab, and genome-wide expression profiling performed on the disruption mutants. In the mrr1 $\Delta$ mutant in F5, 123 genes were down-regulated in the absence of $M R R 1$; in the G5mrr1 $\triangle$ mutant, 94 genes were down-regulated (Table B-3). There were 28 genes commonly down-regulated in the absence of $M R R 1$. Of these genes, MDRl was down-regulated as well as 13 other genes that were up-regulated in all three resistant clinical isolates (Table 3-3). Unlike the disruption of CAP1, the disruption of MRR1 abolished the expression of MDRl.

\section{Identification of Mrr1p Target Genes}

The Morschhäuser lab identified a P683S mutation in one allele of MRR1 in F5 and a G997V mutation in one allele in G5. In order to identify the genes directly under the control of the mutated Mrrlp, the wild-type $M R R 1$ allele $\left(M R R 1^{\mathrm{F} 2-1}\right.$ or $\left.M R R 1^{\mathrm{G} 2-1}\right)$ or the constitutively active mutant allele $\left(M R R 1^{\mathrm{F} 5}\right.$ and $\left.M R R 1^{\mathrm{G} 5}\right)$ was reintegrated into SC5314 with $M R R 1$ disrupted. In the $M R R 1^{\mathrm{F} 5}$ allele compared to $M R R 1^{\mathrm{F} 2-1}$ allele in SC5314, 20 genes were up-regulated specifically due to the P683S (Table 3-4). Of these 20 genes, 16 genes were also up-regulated in the clinical matched isolates. Of the genes that were up-regulated in resistant isolate F5 and up-regulated in SC5314 with $M R R 1^{\mathrm{F} 5}$, 
Figure 3-1. Identification of genes that are differentially expressed in $M D R 1$ overexpressing clinical $C$. albicans isolates.

(A, B) Venn diagrams showing the number of genes that are up-regulated (A) or downregulated (B) in the $M D R 1$ overexpressing, drug-resistant isolates F5, G5, and 6692 as compared with their matched drug-susceptible isolates F1, G1, and 5833, respectively. (C) Heat map showing genes that are up-regulated (106) or down-regulated (105) in all three $M D R I$ overexpressing isolates. Results are from three independent experiments (Exp. 1 to Exp. 3) for each comparison. The genes are ordered according to their relative degree of up- and down-regulation, as indicated by the brightness of the red and green fields. 
A.

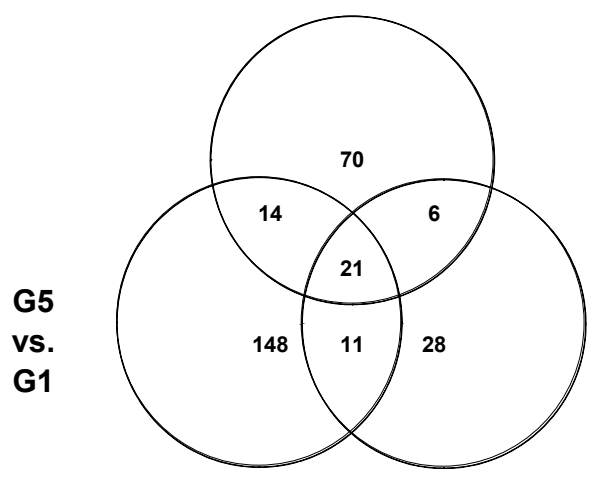

C.
B.

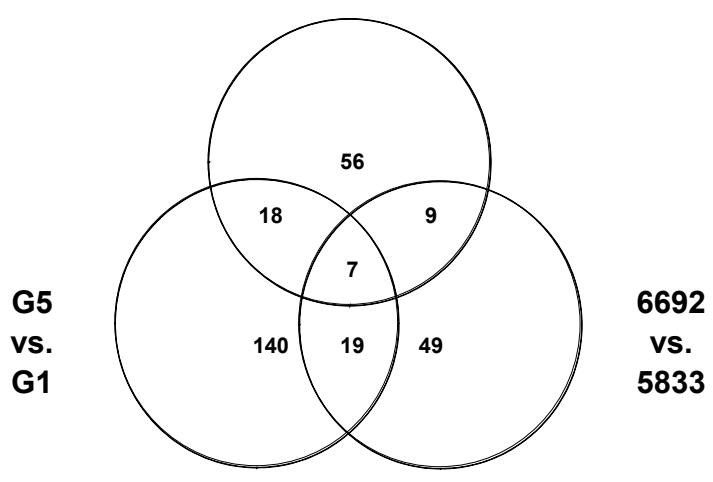

F5 vs F1 $\mathrm{G} 5$ vs $\mathrm{G} 1$

6692 vs 5833

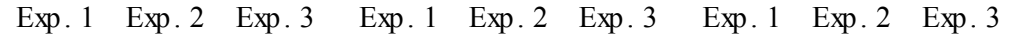

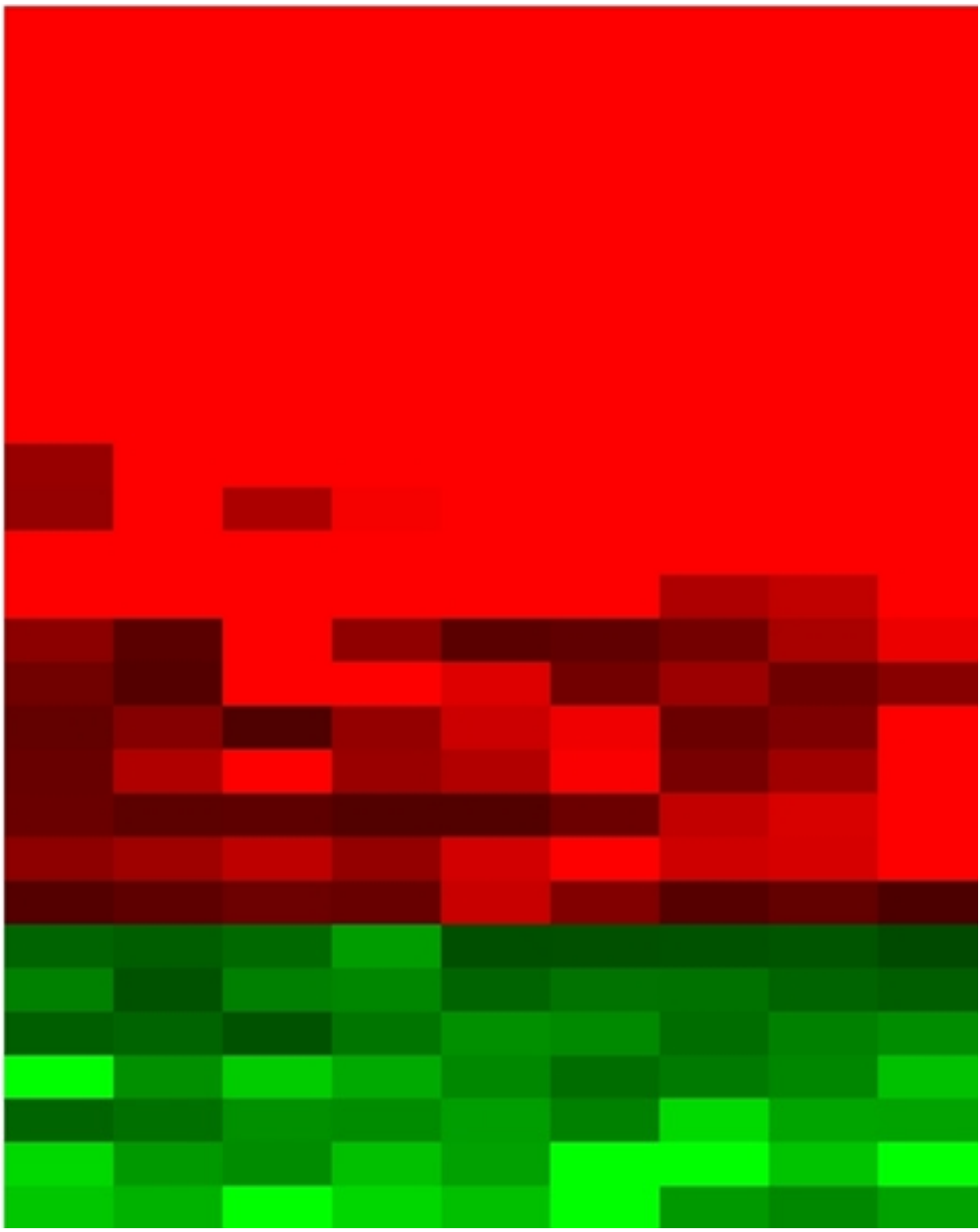

IFD6
IFD5
IFD7
IFD1
IFD4
BMR1c
IPF5987
IPF7817
IPF9538
IPF17186
IPF2186
GRP2
IPF7863
IFD2
MRR1
IPF19538
NDH2
GPX1
IPF1837
IPF9887
DUR1-2
IPF2710.repeat2
IPF9483
SEO2
IPF409
RBT2
FTR1
IPF3634
IPH

orf19.4476

orf19. 1048 orf19. 629

orf19. 1048

orf19.4477

orf19.5604

orf19.7306

orf19.3131

orf19. 271

orf19. 251

orf19.7166

orf19.4309

orf19. 1449

orf19. 771

orf19.7372

orf19.6957.3

orf19.5713

orf19. 86

orf19.5877

orf19. 1240

orf19.780

orf19. 2159

orf19.3395

orf19. 700

orf19. 7502

orf19. 1415

orf19.7219

orf19. 6720 
Table 3-3. Genes commonly down-regulated by MRR1 deletion in F5 and G5.

\begin{tabular}{|c|c|c|c|c|}
\hline Gene Name $^{a}$ & $\begin{array}{l}\text { orf19 } \\
\text { Number }\end{array}$ & GO Annotation (Molecular Function) ${ }^{c}$ & F5 $4 m r r 1$ & G5 $4 m r r 1$ \\
\hline IPF9538 & orf19.271 & 2,4-dienoyl-CoA reductase (NADPH) activity & -282.4 & -17.7 \\
\hline MDR1 & orf19.5604 & Multidrug transporter activity & -46.9 & -126.3 \\
\hline$M R R 1$ & orf19.7372 & $\begin{array}{l}\text { RNA polymerase II transcription factor } \\
\text { activity }\end{array}$ & -50.7 & -44.7 \\
\hline IFD6 & orf19.4476 & Aryl-alcohol dehydrogenase activity & -30.3 & -31.4 \\
\hline IPF5987 & orf19.7306 & -- & -7.4 & -9.8 \\
\hline IFD5 & orf19.1048 & Aryl-alcohol dehydrogenase activity & -13.2 & -4.0 \\
\hline IPF17186 & orf19.251 & -- & -9.2 & -7.0 \\
\hline IPF7863 & orf19.1449 & -- & -11.8 & -3.7 \\
\hline IFD7 & orf19.629 & Aryl-alcohol dehydrogenase activity & -6.7 & -8.1 \\
\hline$H G T 11$ & orf19.4527 & Glucose transporter activity & -5.1 & -5.9 \\
\hline HGT12 & orf19.3668 & Glucose transporter activity & -3.3 & -7.2 \\
\hline IPF525 & orf19.7085 & -- & -6.2 & -2.4 \\
\hline IPF2186 & orf19.7166 & -- & -3.4 & -4.9 \\
\hline IFK3 & orf19.857 & -- & -4.6 & -3.0 \\
\hline IPF19538 & orf19.6957.3 & -- & -2.0 & -5.4 \\
\hline IPF7817 & orf19.3131 & NADPH dehydrogenase activity & -4.4 & -3.0 \\
\hline IFD1 & orf19.1048 & Aryl-alcohol dehydrogenase activity & -2.6 & -4.6 \\
\hline IFD4 & orf19.4477 & Aryl-alcohol dehydrogenase activity & -2.9 & -3.7 \\
\hline$A A F 1$ & orf19.7436 & -- & -3.1 & -3.4 \\
\hline FRE30.53 & orf19.6140 & Ferric-chelate reductase activity & -3.5 & -2.8 \\
\hline FRE30.3 & orf19.6139 & Ferric-chelate reductase activity & -3.2 & -2.9 \\
\hline IFD2 & orf19.771 & Aryl-alcohol dehydrogenase activity & -3.1 & -2.8 \\
\hline IPF1634 & orf19.6578 & Inorganic phosphate transporter activity & -2.4 & -3.3 \\
\hline GRP2 & orf19.4309 & Oxidoreductase activity & -1.9 & -3.4 \\
\hline ARD 8 & orf19.6322 & 2,4-dienoyl-CoA reductase (NADPH) activity & -2.3 & -2.3 \\
\hline FRE7 & orf19.7077 & Ferric-chelate reductase activity & -2.2 & -2.2 \\
\hline GAP6 & orf19.6659 & Amino acid permease activity & -2.1 & -1.7 \\
\hline$A K L 1$ & orf19.5357 & Protein serine/threonine kinase activity & -1.7 & -1.7 \\
\hline
\end{tabular}

${ }^{a}$ Gene name according to CandidaDB (http://genolist.pasteur.fr/CandidaDB/).

${ }^{\boldsymbol{b}}$ orf19 nomenclature according to the Assembly 19 version.

${ }^{c}$ Gene ontology (GO) terminology according to CGD (http://www.candidagenome.org/). Gene expression values are represented as fold changes between disruption strain and resistant parent isolate.

Genes in bold are genes found to be up-regulated in all three clinical matched isolates. 
Table 3-4. Identification of Mrr1p target genes in F isolates by DNA microarray analysis.

Genes that were up-regulated by the P683S mutation in the SC5314 genetic background (MRR ${ }^{\mathrm{F} 5}$ vs. $\left.M R R 1^{\mathrm{F} 2-1}\right)$. The genes are ordered according to their average degree of up-regulation in four repeat experiments performed with two independent transformants (strains A and B). The fold changes in the expression level of these genes in the drug-resistant clinical isolate F5 as compared with a matched susceptible isolate (F5 vs. F1) and in two independently constructed mrr1 $\Delta$ mutants (strains A and B) as compared with their wild-type progenitor F5 (F5mrrl 1 vs. F5) are shown for comparison. Up-regulated genes are highlighted in red, down-regulated genes are highlighted in green, and genes that were not differentially expressed are not highlighted in all pairwise comparisons.

\begin{tabular}{|c|c|c|c|c|c|c|c|c|c|c|c|c|c|}
\hline \multirow{3}{*}{$\begin{array}{l}\text { Gene } \\
\text { Name }\end{array}$} & \multirow{3}{*}{$\begin{array}{c}\text { orf19 } \\
\text { Number }\end{array}$} & \multirow{3}{*}{$\begin{array}{c}\text { GO Annotation } \\
\text { (Molecular Function) }\end{array}$} & \multicolumn{4}{|c|}{$M R R 1^{F 5}$ vs. $M R R 1^{F 2-1}$} & \multicolumn{3}{|c|}{ F5 vs. F1 } & \multicolumn{4}{|c|}{$\mathrm{F} 5 m r r 1 \Delta$ vs. F5 } \\
\hline & & & \multicolumn{2}{|c|}{ Strain A } & \multicolumn{2}{|c|}{ Strain B } & \multirow[b]{2}{*}{ Exp. 1} & \multirow[b]{2}{*}{ Exp. 2} & \multirow[b]{2}{*}{ Exp. 3} & \multicolumn{2}{|c|}{ Strain A } & \multicolumn{2}{|c|}{ Strain B } \\
\hline & & & Exp. 1 & Exp. 2 & Exp. 1 & Exp. 2 & & & & Exp. 1 & Exp. 2 & Exp. 1 & Exp. 2 \\
\hline IFD6 & orf19.4476 & $\begin{array}{l}\text { Aryl-alcohol } \\
\text { dehydrogenase activity }\end{array}$ & 256.4 & 1121.8 & 284.7 & 1147.1 & 965.7 & 1290.0 & 3064.8 & -31.2 & -41.8 & -27.0 & -21.3 \\
\hline IFDI & orf19.1048 & $\begin{array}{l}\text { Aryl-alcohol } \\
\text { dehydrogenase activity }\end{array}$ & 130.7 & 179.8 & 100.4 & 72.1 & 76.2 & 200.7 & 141.5 & -3.3 & -2.1 & -2.6 & -2.6 \\
\hline$B M R 1$ & orf19.5604 & $\begin{array}{l}\text { Multidrug transporter } \\
\text { activity }\end{array}$ & 114.8 & 114.4 & 118.2 & 39.7 & 47.8 & 152.1 & 112.2 & -21.4 & -47.5 & -35.0 & -83.7 \\
\hline IFD7 & orf19.629 & $\begin{array}{l}\text { Aryl-alcohol } \\
\text { dehydrogenase activity }\end{array}$ & 56.3 & 75.9 & 184.2 & 35.3 & 113.1 & 338.2 & 332.4 & -10.5 & -4.3 & -5.8 & -6.0 \\
\hline IFD5 & orf19.1048 & $\begin{array}{l}\text { Aryl-alcohol } \\
\text { dehydrogenase activity }\end{array}$ & 64.8 & 116.6 & 84.5 & 50.4 & 140.8 & 361.9 & 144.3 & -15.0 & -12.2 & -15.8 & -9.6 \\
\hline IFD 4 & orf19.4477 & $\begin{array}{l}\text { Aryl-alcohol } \\
\text { dehydrogenase activity }\end{array}$ & 15.4 & 13.6 & 21.4 & 56.6 & 21.3 & 37.9 & 75.5 & -2.7 & -4.6 & -2.4 & -1.9 \\
\hline IPF5987 & orf19.7306 & -- & 19.6 & 19.3 & 28.3 & 27.6 & 48.6 & 49.5 & 63.3 & -7.4 & -6.4 & -9.0 & -6.9 \\
\hline IPF9538 & orf19.271 & $\begin{array}{l}\text { 2,4-dienoyl-CoA } \\
\text { reductase (NADPH) } \\
\text { activity }\end{array}$ & 16.3 & 24.6 & 5.2 & 6.4 & 108.9 & 279.5 & 131.7 & -164.3 & -548.1 & -280.0 & -137.2 \\
\hline IPF17186 & orf19.251 & -- & 10.4 & 11.9 & 10.2 & 11.2 & 20.7 & 38.9 & 39.7 & -10.9 & -11.0 & -8.7 & -6.2 \\
\hline GRP2 & orf19.4309 & Oxidoreductase activity & 4.2 & 6.9 & 7.0 & 6.5 & 3.0 & 5.3 & 3.4 & -2.1 & -1.9 & -1.8 & -1.8 \\
\hline IPF7863 & orf19.1449 & -- & 3.5 & 3.8 & 3.6 & 3.4 & 6.1 & 9.4 & 9.1 & -16.9 & -6.9 & -15.5 & -8.0 \\
\hline$A L S 10$ & orf19.2355 & $\begin{array}{l}\text { Cell adhesion molecule } \\
\text { binding }\end{array}$ & 2.0 & 3.7 & 2.7 & 1.9 & 1.2 & 1.7 & 1.1 & -14.0 & -27.2 & -1.6 & -1.2 \\
\hline IPF 19538 & orf19.6957.3 & -- & 3.2 & 2.5 & 1.8 & 2.6 & 2.2 & 1.7 & 6.0 & -1.5 & -2.0 & -2.1 & -2.1 \\
\hline
\end{tabular}


Table 3-4 (continued).

\begin{tabular}{|c|c|c|c|c|c|c|c|c|c|c|c|c|c|}
\hline \multirow{3}{*}{$\begin{array}{l}\text { Gene } \\
\text { Name }\end{array}$} & \multirow{3}{*}{$\begin{array}{l}\text { orf19 } \\
\text { Number }\end{array}$} & \multirow{3}{*}{$\begin{array}{c}\text { GO Annotation } \\
\text { (Molecular Function) }\end{array}$} & \multicolumn{4}{|c|}{$M R R 1^{F 5}$ vs. $M R R 1^{F 2-1}$} & \multicolumn{3}{|c|}{ F5 vs. F1 } & \multicolumn{4}{|c|}{ F5mrr1 $1 \Delta$ vs. F5 } \\
\hline & & & \multicolumn{2}{|c|}{ Strain A } & \multicolumn{2}{|c|}{ Strain B } & \multirow[b]{2}{*}{ Exp. 1} & \multirow[b]{2}{*}{ Exp. 2} & \multirow[b]{2}{*}{ Exp. 3} & \multicolumn{2}{|c|}{ Strain A } & \multicolumn{2}{|c|}{ Strain B } \\
\hline & & & Exp. 1 & Exp. 2 & Exp. 1 & Exp. 2 & & & & Exp. 1 & Exp. 2 & Exp. 1 & Exp. 2 \\
\hline ALS3.5eoc & orf19.1816 & $\begin{array}{l}\text { Cell adhesion molecule } \\
\text { binding }\end{array}$ & 2.2 & 3.6 & 2.1 & 1.5 & 1.4 & 1.8 & 1.2 & -43.4 & -22.9 & -1.6 & -1.2 \\
\hline IPF2130 & orf19.7204 & -- & 2.0 & 2.7 & 2.4 & 1.6 & 1.8 & 1.8 & -1.4 & -2.8 & 1.2 & -2.4 & -1.5 \\
\hline IPF7940 & orf19.6608 & -- & 1.9 & 2.2 & 2.1 & 2.0 & 5.4 & 4.3 & 10.3 & -2.2 & -1.9 & 1.1 & 1.0 \\
\hline IPF9377 & orf19.1428 & -- & 2.2 & 1.6 & 1.5 & 2.8 & 1.2 & 1.5 & -1.1 & 1.1 & 1.6 & 1.1 & -4.0 \\
\hline IPF7817 & orf19.3131 & $\begin{array}{l}\text { NADPH dehydrogenase } \\
\text { activity }\end{array}$ & 2.2 & 2.0 & 2.1 & 1.7 & 9.4 & 13.7 & 13.4 & -3.8 & -4.7 & -5.5 & -3.6 \\
\hline DURl,2 & orf19.780 & $\begin{array}{l}\text { Allophanate hydrolase } \\
\text { activity }\end{array}$ & 2.0 & 1.5 & 1.6 & 2.4 & 1.7 & 1.9 & 2.2 & -2.2 & 1.8 & -2.0 & 1.5 \\
\hline IPF2186 & orf19.7166 & -- & 1.5 & 2.0 & 1.9 & 2.0 & 3.0 & 6.0 & 7.3 & -2.9 & -3.5 & -3.7 & -3.4 \\
\hline
\end{tabular}


14 genes were down-regulated in the absence of both alleles of $M R R 1$. In the $M R R 1^{\mathrm{G} 5}$ allele compare to $M R R 1^{\mathrm{G} 2-1}$ allele in SC5314, 27 genes were up-regulated specifically due to the G997V mutation (Table 3-5). Of these 27 genes, 20 genes were up-regulated in resistant isolate G5. Of these 20 genes, 15 genes were down-regulated in the absence of both MRRI alleles. MDRI and MRR1 gene expression was confirmed with real-time RT-PCR (Figure 3-2). Interestingly, 14 genes were up-regulated in all three clinical resistant isolate, up-regulated in both $M R R 1^{\mathrm{F} 5}$ and $M R R 1^{\mathrm{G} 5}$, and down-regulated in the $\Delta m r r l$ disruption mutants. These genes are considered the core genes regulated by Mrr1p.

\section{DISCUSSION}

Increased drug efflux due to the overexpression of efflux pumps is a major mechanism of resistance in C. albicans. The up-regulation of CDR1 and CDR2 results in resistance to azole antifungal agents. Gain-of-function mutations in the gene encoding the zinc cluster transcription factor Tac1p was recently identified as the cause of $C D R 1 / C D R 2$ overexpression $(88,89)$. The expression of the MFS transporter MDR1 is another resistance mechanism found in azole resistant clinical isolates. While the regulator of $M D R l$ was previously unknown, studies have shown that a trans-regulatory element was responsible for the up-regulation of MDRl (72). Previously, the bZip transcription factor Cap1p was identified as the possible transcriptional regulator of MDR1 (84). The promoter region of genes co-regulated with MDR1 as well as the promoter region of $M D R 1$ contained a YRE motif. However, promoter deletion studies performed in azole resistant isolates showed that the deletion of the YRE motif did not abolish the up-regulation of MDR1 $(21,86)$. This suggested that the transcriptional regulator of azole resistance was not limited to CAPI.

Genome-wide expression profiling of clinical matched isolates overexpessing $C D R 1 / C D R 2$ identified $T A C 1$ as a gene co-regulated with the azole resistance genes. Using this technology, we examined three clinical matched isolates that overexpressed $M D R 1$. Of the genes found to be differentially expressed in all three resistant isolates, a gene encoding the zinc cluster transcription factor $M R R 1$ was moderately up-regulated. The degree by which MRRI contributes to azole resistance was studied by looking at gene expression profiles of $M R R 1$ disruption mutants. Unlike the $C A P 1$ disruption mutants, the resistant strains without $M R R 1$ could no longer express $M D R 1$ along with the $M D R 1$ co-regulated genes.

Additionally, in collaboration with our group the Morschhäuser lab demonstrated that the MRRI deletion mutants in resistant isolates were more susceptible to fluconazole, cerulenin, brefeldin A, and diamide. They also showed that the gain-of-function mutation in MRRl was required for constitutive $M D R 1$ expression and resistance to fluconazole, cerulenin, brefeldin A, and diamide. MRRl was also required for the induction of $M D R l$ by benomyl and hydrogen peroxide. In the absence of $C A P 1$, benomyl could still induce $M D R I$ expression; without $M R R 1$ there was no induction by benomyl. This suggests that while Cap1p may play a role in $M D R 1$ gene expression, 
Table 3-5. Identification of Mrr1p target genes in G isolates by DNA microarray analysis.

Genes that were up-regulated by the G997V mutation in the $\mathrm{SC} 5314$ genetic background $\left(M R R 1^{\mathrm{G} 5}\right.$ vs. $\left.M R R 1^{\mathrm{G} 2-1}\right)$. The genes are ordered according to their average degree of up-regulation in four repeat experiments performed with two independent transformants (strains A and B). The fold changes in the expression level of these genes in the drug-resistant clinical isolate G5 as compared with a matched susceptible isolate (G5 vs. G1) and in two independently constructed mrrl $\Delta$ mutants (strains A and B) as compared with their wild-type progenitor G5 (G5mrrl4 vs. G5) are shown for comparison. Up-regulated genes are highlighted in red, down-regulated genes are highlighted in green, and genes that were not differentially expressed are not highlighted in all pairwise comparisons.

\begin{tabular}{|c|c|c|c|c|c|c|c|c|c|c|c|c|c|}
\hline \multirow{3}{*}{$\begin{array}{l}\text { Gene } \\
\text { Name }\end{array}$} & \multirow{3}{*}{$\begin{array}{c}\text { orf19 } \\
\text { Number }\end{array}$} & \multirow{3}{*}{$\begin{array}{c}\text { GO Annotation } \\
\text { (Molecular Function) }\end{array}$} & \multicolumn{4}{|c|}{$M R R 1^{\mathrm{G} 5}$ vs. $M R R 1^{\mathrm{G} 2-1}$} & \multicolumn{3}{|c|}{ G5 vs. G1 } & \multicolumn{4}{|c|}{ G5mrr $1 \Delta$ vs. G5 } \\
\hline & & & \multicolumn{2}{|c|}{ Strain A } & \multicolumn{2}{|c|}{ Strain B } & \multirow[b]{2}{*}{ Exp. 1} & \multirow[b]{2}{*}{ Exp. 2} & \multirow[b]{2}{*}{ Exp. 3} & \multicolumn{2}{|c|}{ Strain A } & \multicolumn{2}{|c|}{ Strain B } \\
\hline & & & Exp. 1 & Exp. 2 & Exp. 1 & Exp. 2 & & & & Exp. 1 & Exp. 2 & Exp. 1 & Exp. 2 \\
\hline IFD6 & orf19.4476 & $\begin{array}{l}\text { Aryl-alcohol } \\
\text { dehydrogenase activity }\end{array}$ & 179.0 & 617.6 & 1559.4 & 1735.9 & 565.4 & 818.4 & 1916.6 & -12.8 & -22.1 & -12.6 & -78.1 \\
\hline IPF9538 & & $\begin{array}{l}\text { 2,4-dienoyl-CoA } \\
\text { reductase (NADPH) } \\
\text { activity }\end{array}$ & 125.7 & 99.9 & 128.9 & 219.6 & 7.8 & 24.5 & 18.1 & -15.3 & -16.6 & -25.5 & -13.3 \\
\hline IFDI & orf19.1048 & $\begin{array}{l}\text { Aryl-alcohol } \\
\text { dehydrogenase activity }\end{array}$ & 135.0 & 123.5 & 175.1 & 139.4 & 151.6 & 195.9 & 299.8 & -6.1 & -5.2 & -4.5 & -2.9 \\
\hline IFD7 & orf19.629 & $\begin{array}{l}\text { Aryl-alcohol } \\
\text { dehydrogenase activity }\end{array}$ & 112.0 & 134.5 & 131.9 & 91.9 & 55.2 & 66.8 & 139.0 & -8.0 & -8.2 & -9.6 & -6.5 \\
\hline$B M R 1$ & orf19.5604 & $\begin{array}{l}\text { Multidrug transporter } \\
\text { activity }\end{array}$ & 103.8 & 73.8 & 135.4 & 68.3 & 116.2 & 181.2 & 210.3 & -168.9 & -149.4 & -143.0 & -43.9 \\
\hline IFD5 & orf19.1048 & $\begin{array}{l}\text { Aryl-alcohol } \\
\text { dehydrogenase activity }\end{array}$ & 59.6 & 82.9 & 92.6 & 141.5 & 137.7 & 155.8 & 201.1 & -4.9 & -4.2 & -4.1 & -2.7 \\
\hline IFD4 & orf19.4477 & $\begin{array}{l}\text { Aryl-alcohol } \\
\text { dehydrogenase activity }\end{array}$ & 29.3 & 44.7 & 21.2 & 31.1 & 81.0 & 115.9 & 149.7 & -4.0 & -4.4 & -3.4 & -2.9 \\
\hline IPF5987 & orf19.7306 & -- & 30.9 & 24.3 & 22.0 & 32.7 & 41.5 & 50.1 & 81.4 & -8.6 & -12.5 & -7.4 & -10.5 \\
\hline IPF17186 & orf19.251 & -- & 15.4 & 12.8 & 14.4 & 13.1 & 9.2 & 14.8 & 14.6 & -5.5 & -9.5 & -5.6 & -7.4 \\
\hline$G R P 2$ & orf19.4309 & Oxidoreductase activity & 6.1 & 5.7 & 5.1 & 7.4 & 4.9 & 6.1 & 5.5 & -3.7 & -3.7 & -3.5 & -2.9 \\
\hline IPF7863 & orf19.1449 & -- & 4.1 & 4.6 & 7.7 & 7.8 & 7.2 & 7.2 & 5.7 & -2.9 & -4.5 & -3.0 & -4.3 \\
\hline IPF11694 & orf19.4355 & $\begin{array}{l}\text { Nicotinamide } \\
\text { mononucleotide } \\
\text { permease activity }\end{array}$ & 2.0 & 4.9 & 3.7 & 10.2 & -1.5 & -1.1 & 1.1 & -2.0 & -3.2 & 1.7 & 1.4 \\
\hline
\end{tabular}


Table 3-5 (continued).

\begin{tabular}{|c|c|c|c|c|c|c|c|c|c|c|c|c|c|}
\hline \multirow{3}{*}{$\begin{array}{l}\text { Gene } \\
\text { Name }\end{array}$} & \multirow{3}{*}{$\begin{array}{c}\text { orf19 } \\
\text { Number }\end{array}$} & \multirow{3}{*}{$\begin{array}{c}\text { GO Annotation } \\
\text { (Molecular Function) }\end{array}$} & \multicolumn{4}{|c|}{$M R R 1^{\mathrm{G} 5}$ vs. $M R R 1^{\mathrm{G} 2-1}$} & \multicolumn{3}{|c|}{ G5 vs. G1 } & \multicolumn{4}{|c|}{ G5mrr1 $1 \Delta$ vs. G5 } \\
\hline & & & \multicolumn{2}{|c|}{ Strain A } & \multicolumn{2}{|c|}{ Strain B } & \multirow[b]{2}{*}{ Exp. 1} & \multirow[b]{2}{*}{ Exp. 2} & \multirow[b]{2}{*}{ Exp. 3} & \multicolumn{2}{|c|}{ Strain A } & \multicolumn{2}{|c|}{ Strain B } \\
\hline & & & Exp. 1 & Exp. 2 & Exp. 1 & Exp. 2 & & & & Exp. 1 & Exp. 2 & Exp. 1 & Exp. 2 \\
\hline IPF19538 & orf19.6957.3 & -- & 3.4 & 4.6 & 5.1 & 5.2 & 8.5 & 4.4 & 2.3 & -7.6 & -6.8 & -4.1 & -3.1 \\
\hline$I P F 2130$ & orf19.7204 & -- & 3.1 & 3.0 & 2.2 & 7.9 & 17.2 & 37.0 & 15.2 & -2.1 & -4.4 & -1.5 & -3.6 \\
\hline IPF7940 & orf19.6608 & -- & 5.5 & 2.3 & 1.6 & 3.9 & 4.3 & 5.2 & 20.2 & -1.2 & -1.9 & -1.2 & -4.9 \\
\hline HST2 & orf19.2580 & $\begin{array}{l}\text { NAD-dependent } \\
\text { histone deacetylase } \\
\text { activity }\end{array}$ & 1.7 & 2.0 & 2.8 & 3.7 & 1.4 & 1.1 & 1.5 & -2.0 & -1.2 & -2.1 & 1.1 \\
\hline DUR1,2 & orf19.780 & $\begin{array}{l}\text { Allophanate } \\
\text { hydrolase activity }\end{array}$ & 2.2 & 3.4 & 1.6 & 3.0 & 2.1 & 4.0 & 2.6 & 1.5 & -1.2 & 1.9 & -1.0 \\
\hline$A L S 10$ & orf19.2355 & $\begin{array}{l}\text { Cell adhesion } \\
\text { molecule binding }\end{array}$ & 2.1 & 3.4 & 1.9 & 2.5 & -1.6 & -2.0 & -2.0 & -1.5 & -1.8 & -1.6 & 1.7 \\
\hline$I P F 2965$ & orf19.4287 & $\begin{array}{l}(\mathrm{R}, \mathrm{R}) \text {-butanediol } \\
\text { dehydrogenase } \\
\text { activity }\end{array}$ & 2.4 & 2.1 & 1.6 & 3.4 & -1.0 & 2.1 & 1.7 & 4.4 & 1.1 & 3.5 & 1.5 \\
\hline$I P F 2186$ & orf19.7166 & -- & 1.9 & 1.8 & 3.0 & 2.9 & 14.0 & 15.8 & 11.7 & -7.0 & -5.1 & -4.8 & -2.7 \\
\hline IPF8976 & orf19.1369 & -- & 2.2 & 1.6 & 1.5 & 4.1 & 6.2 & 7.3 & 4.0 & 1.3 & -1.2 & -1.4 & -1.8 \\
\hline$A L S 3.3 e o c$ & orf19.2355 & $\begin{array}{l}\text { Cell adhesion } \\
\text { molecule binding }\end{array}$ & 2.1 & 2.9 & 1.8 & 2.3 & -1.4 & -1.5 & -1.6 & -1.7 & -2.0 & -1.6 & 1.6 \\
\hline IPF7817 & orf19.3131 & $\begin{array}{l}\text { NADPH } \\
\text { dehydrogenase } \\
\text { activity }\end{array}$ & 2.1 & 1.6 & 3.4 & 1.7 & 7.7 & 11.0 & 8.2 & -3.6 & -2.8 & -3.3 & -2.1 \\
\hline ALS3.5eoc & orf19.1816 & $\begin{array}{l}\text { Cell adhesion } \\
\text { molecule binding }\end{array}$ & 2.0 & 2.8 & 2.0 & 2.0 & -1.1 & -1.3 & -4.9 & -2.0 & -1.0 & -2.0 & 2.1 \\
\hline$G B A 1$ & orf19.4015 & GTPase activity & 1.6 & 1.6 & 1.5 & 3.5 & 41.8 & 21.8 & 20.5 & -1.1 & -1.2 & -1.1 & 1.4 \\
\hline$P D C 12$ & orf19.4608 & $\begin{array}{l}\text { Pyruvate } \\
\text { decarboxylase } \\
\text { activity }\end{array}$ & 1.6 & 1.6 & 1.8 & 2.3 & 1.6 & -1.5 & 1.2 & -1.7 & -1.4 & -4.8 & -2.4 \\
\hline$I P F 7456$ & orf19.2047 & -- & 1.8 & 1.7 & 1.5 & 2.1 & 1.6 & 2.1 & 1.7 & 1.6 & -1.3 & 2.4 & 1.1 \\
\hline
\end{tabular}


Figure 3-2. Quantitative real-time PCR analysis of $M D R 1$ and $M R R 1$.

(A) Expression of MDR1 in the clinical resistant isolates F5, G5, and 6922. (B) Expression of MRR1 in clinical resistant isolates F5, F5, and 6922. (C) Expression of MDR1 in resistant isolate F5 and G5 as well as SC5314 with either P683S or G997V mutant alleles. (D) Expression of MRR1 in resistant isolate F5 and G5 as well as SC5314 with either P683S or G997V mutant alleles. Error bars represent standard errors $(\mathrm{n}=3)$. 
A.

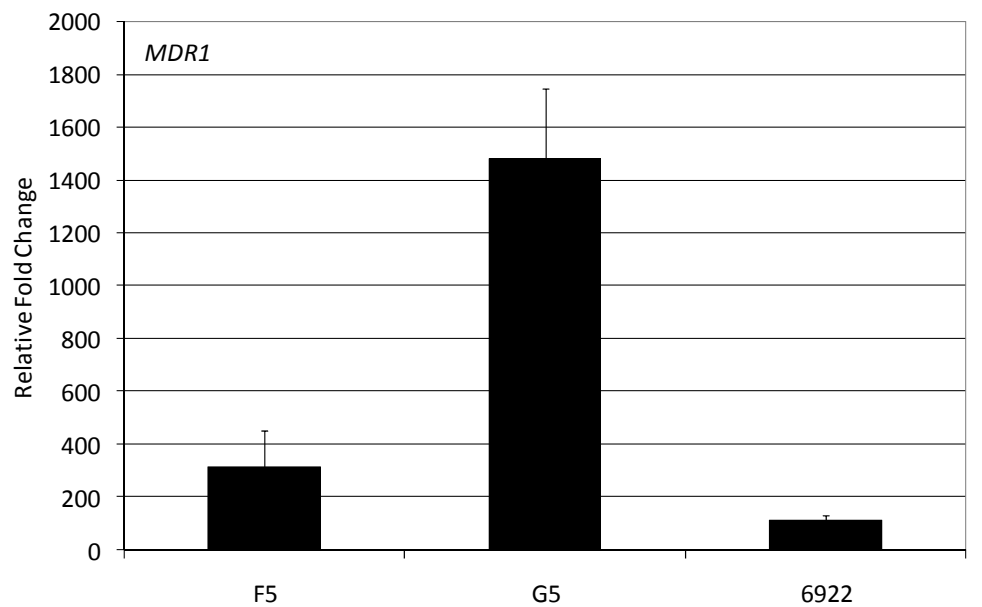

C.

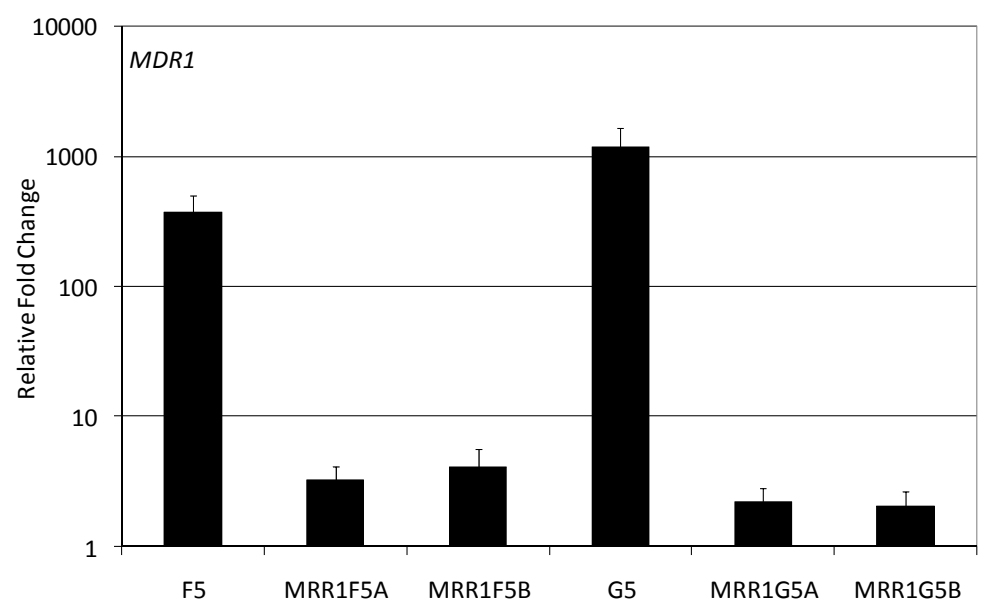

B.

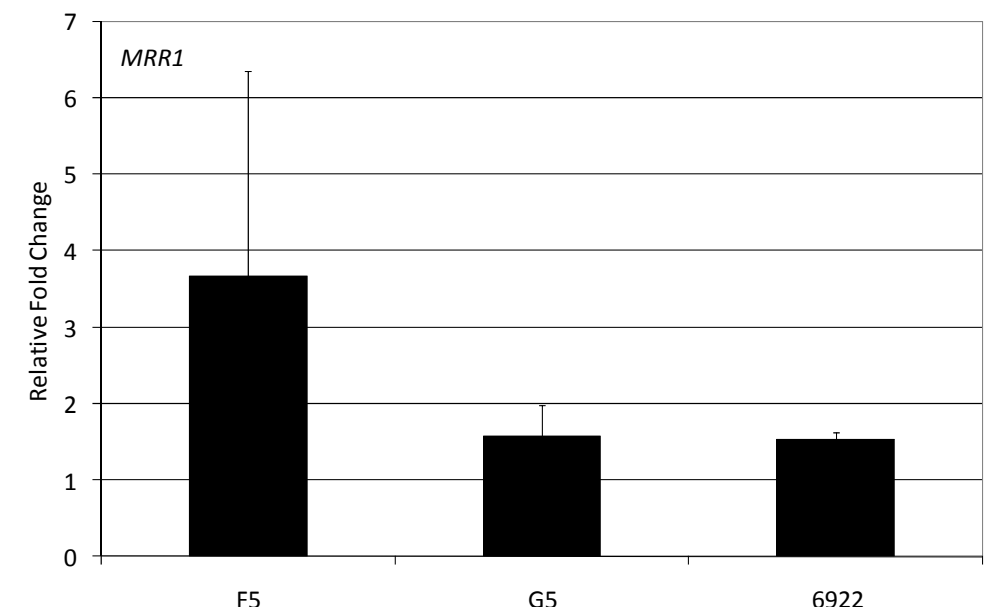

D.

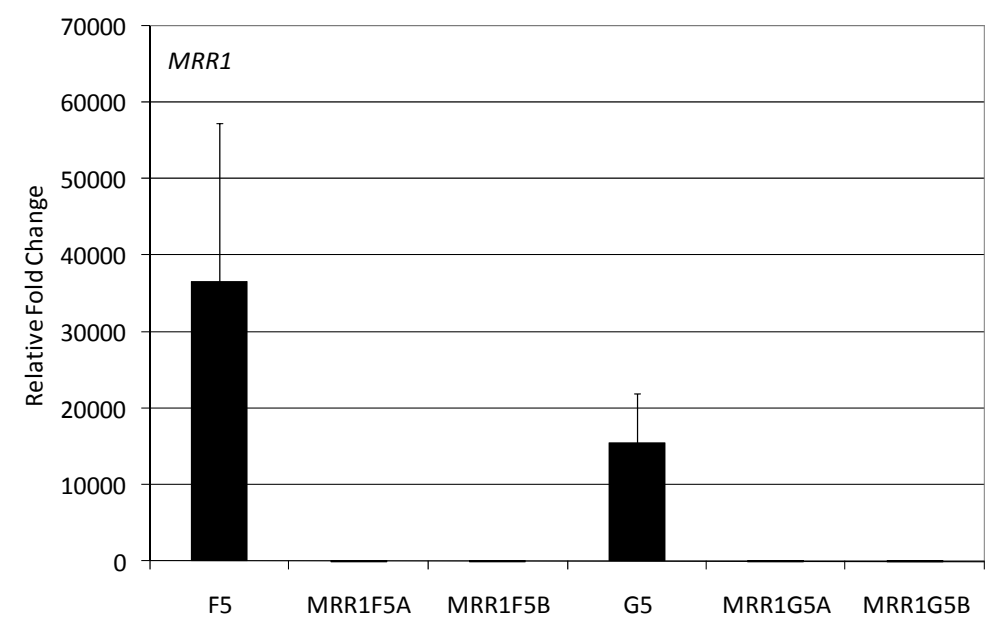


$M R R 1$ is more essential to the regulation of $M D R 1$ and azole resistance.

Examination of the gene expression profiles of the resistant isolates, the disruption mutants, and the MRR1 gain-of-function alleles reintegrated in a wild-type isolates revealed a core group of 14 genes whose expression depend on MRR1. Of the 14 co-regulated genes, six genes have been implicated in the catalysis of an oxidationreduction (redox) reaction. IPF7817 has been shown to be important in the response to oxidative stress. This gene has been shown to be up-regulated in response to hydrogen peroxide in a CAP1 dependent manner (164). GRP2 is another gene that seems to play a role in oxidative stress response. The expression of GRP2 has been shown to be induced by hydrogen peroxide and benomyl $(122,165)$. A YRE motif has also been identified in the promoter region of GRP2 (122). In S. cerevisiae, GRP2 is homologous to ScGRE2 a gene that encodes a stress-induced NADH-dependent methylglyoxal reductase. In $S$. cerevisiae, $S c G R E 2$ is regulated by the zinc cluster transcription factor Pdr1p. The IFD family of genes has also been implicated in oxidative stress. In the presence of hydrogen peroxide or benomyl, the IFD family of genes is up-regulated $(122,165)$. The $S$.

cerevisiae homolog YPLO88w is regulated by the two zinc cluster transcription factors Yrrlp and Yrmlp (166). Because a large portion of the MRR1 core genes respond to oxidative stress, $M R R I$ may play a role in oxidative stress response. Because these oxidoreductase genes also have YRE motifs in their promoter regions, it is possible that $M R R 1$ and $C A P 1$ function in conjunction to respond to oxidative stresses. 


\section{CHAPTER 4: GAIN-OF-FUNCTION MUTATIONS IN UPC2 REGULATE THE EXPRESSION OF ERGOSTEROL BIOSYNTHESIS GENES AND IMPART FLUCONAZOLE RESISTANCE IN A CLINICAL ISOLATE OF CANDIDA ALBICANS}

\section{INTRODUCTION}

The azole antifungals bind to and inhibit the activity of lanosterol demethylase (Erg11p), a key enzyme in the ergosterol biosynthesis pathway (167). Mechanisms of resistance to the azole antifungal agents that have been described for C. albicans include increased expression of the gene encoding the MFS transporter Mdr1p and genes encoding two ABC transporters, Cdr1p and Cdr2p $(29,35,43,95,168)$. Other mechanisms of resistance involve the ERG11 gene itself. Mutations in ERG11 that interfere with the ability of the azole to bind to its target can confer resistance. Furthermore, overexpression of ERG11 leads to increased production of lanosterol demethylase, which can also result in azole resistance $(27,35,43,169,170,171)$.

Constitutive overexpression of $C D R 1$ and $C D R 2$ in azole-resistant clinical isolates has been shown to be due to gain-of-function mutations in the zinc cluster transcription factor Tac $1 \mathrm{p}$ and loss of heterozygosity at the $T A C 1$ locus $(88,89)$. Recently, similar mutations and loss of heterozygosity in the zinc cluster transcription factor Mrrlp was demonstrated to cause constitutive overexpression of MDRl in fluconazole resistant clinical isolates (172). The comparison of gene expression in matched fluconazole-susceptible and -resistant isolates has proved a powerful tool to identify resistance mechanisms of clinical C. albicans isolates. Such studies initially pointed to the involvement of efflux pump overexpression as well as ERG11 overexpression in fluconazole-resistant strains $(29,35)$. More recently, genome-wide transcriptional profiling experiments using DNA microarrays have revealed additional alterations that might be involved in the development of drug resistance $(48,173,174)$. This approach has led to the identification of the transcription factor Mrrlp, which controls expression of the MDRl efflux pump (172).

In the present study we performed genome-wide gene expression profiling of a matched pair of azole-susceptible and -resistant isolates from a series in which no overexpression of $C D R 1 / C D R 2$ or $M D R 1$ in resistant isolates was detected by Northern hybridization in a previous study (95). We observed up-regulation of the gene encoding Upc2p as well as known target genes of this transcription factor. In collaboration with our group, the Morschhäuser lab sequenced the $U P C 2$ genes and described a gain-of-function mutation in $U P C 2$. They also created the disruption and reintegrated UPC2 mutants used in the study. In addition, we show for the first time that a gain-of-function mutation in UPC2 leads to increased expression of ERG11. 


\section{MATERIALS AND METHODS}

\section{Strains and Growth Conditions}

C. albicans strains used in this study are listed in Table 4-1. All strains were stored as frozen stocks with $15 \%$ glycerol at $-80^{\circ} \mathrm{C}$ and subcultured on YPD agar plates $(10 \mathrm{~g}$ yeast extract, $20 \mathrm{~g}$ peptone, $20 \mathrm{~g}$ glucose, $15 \mathrm{~g}$ agar per liter) at $30^{\circ} \mathrm{C}$. For routine growth of the strains YPD liquid medium was used.

\section{Construction of $C$. albicans Affymetrix ${ }^{\circledR}$ Expression Arrays}

We developed a new Affymetrix custom expression array (CAN07, 49-5241 array format with 11 micron features) for $C$. Albicans. The nucleotide sequences corresponding to 6,165 open reading frames (ORFs) for $C$. albicans were downloaded from the Galar Fungail European Consortium (Assembly 6, http://www.pasteur.fr/Galar_Fungail/CandidaDB). We planned to design two separate probe sets for each ORF, each consisting of 11 perfect match and 11 mismatch overlapping 25 bp oligonucleotides, to the 3' 600 bp region. For ORFs less than $600 \mathrm{bp}$ in length the sequence was divided in two equal segments for subsequent design procedures. For quality control and normalization purposes, we made additional control probesets corresponding to GAPDH ( 1 probeset), actin ( 1 probeset) and the 5 ' and middle region of Mdr1p (Bmrlp) transcript in addition to the standard Affymetrix controls (BioB, C, D, cre, DAP, PHE, LYS, THR). The probe selection was performed by the Chip Design group at Affymetrix, Inc. using their proprietary algorithm to calculate probe set scores, which includes a probe quality metric, cross-hybridization penalty and gap penalty. The probe sets were then examined for cross-hybridization against all other sequences in the $C$. albicans genome as well as a number of constitutively expressed genes and rRNA from other common organisms. Consequently, for some target regions we were not able to design high quality probe sets. In the end, the GeneChip contained 10,014 probesets, corresponding to 5,806 unique ORFs. Two probes were represented for 4,208 ORFs, allowing 2 independent measurements of the mRNA level for that particular gene.

\section{RNA Preparation for Microarrays}

Total RNA was isolated using the hot SDS-phenol method (175). Frozen cells were suspended in $12 \mathrm{ml}$ of $50 \mathrm{mM}$ sodium acetate (pH 5.2), $10 \mathrm{mM}$ EDTA at room temperature, after which $1 \mathrm{ml}$ of $20 \%$ sodium dodecyl sulphate and $12 \mathrm{ml}$ of acid phenol (Fisher Scientific) were added. This mixture was incubated $10 \mathrm{~min}$ at $65^{\circ} \mathrm{C}$ with mixing each minute, cooled on ice for $5 \mathrm{~min}$, and centrifuged for $15 \mathrm{~min}$ at $12,000 \mathrm{~g}$. Supernatants were transferred to new tubes containing $15 \mathrm{ml}$ of chloroform, mixed, and centrifuged at $200 \mathrm{~g}$ for $10 \mathrm{~min}$. The aqueous layer was removed to new tubes, RNA was precipitated with 1 volume isopropanol and 0.1 volume 2 $\mathrm{M}$ sodium acetate ( $\mathrm{pH}$ 5.0), and then collected by centrifugation at $17,000 \mathrm{~g}$ for $35 \mathrm{~min}$ at $4{ }^{\circ} \mathrm{C}$. The RNA pellet was suspended in $10 \mathrm{ml}$ of $70 \%$ ethanol, collected again by centrifugation, and suspended in nuclease-free water. 
Table 4-1. C. albicans strains used in this study.

\begin{tabular}{|c|c|c|c|}
\hline Strain & Parent & Relevant characteristics or genotype & Reference \\
\hline S1 & & Susceptible isolate from patient $\mathrm{S}$ & $(95)$ \\
\hline $\mathrm{S} 2$ & & Resistant isolate from patient $\mathrm{S}$ & (95) \\
\hline SC5314 & & Wild-type C. albicans model strain & (176) \\
\hline UPC2M1A & SC5314 & upc2-1 $1 \Delta:: S A T 1-F L I P^{a} / U P C 2-2$ & this study \\
\hline UPC2M1B & SC5314 & UPC2-1/upc2-2A::SAT1-FLIP & this study \\
\hline UPC2M2A & UPC2M1A & upc2-1 $\triangle:: F R T / U P C 2-2$ & this study \\
\hline UPC2M2B & UPC2M1B & $U P C 2-1 / u p c 2-2 \Delta:: F R T$ & this study \\
\hline UPC2M3A & UPC2M2A & 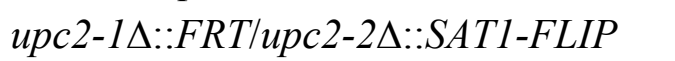 & this study \\
\hline UPC2M3B & UPC2M2B & upc 2-1 $1:: S A T 1-F L I P /$ upc2-2A::FRT & this study \\
\hline UPC2M4A & UPC2M3A & upc2-1 $1 \Delta:: F R T / u p c 2-2 \Delta:: F R T$ & this study \\
\hline UPC2M4B & UPC2M3B & upc $2-1 \Delta::: F R T /$ upc $2-2 \Delta::: F R T$ & this study \\
\hline UPC2M2K21A & UPC2M2A & upc2-1 $1 \Delta:: F R T / U P C 2^{\mathrm{S1-1}}-\mathrm{caSAT1}$ & this study \\
\hline UPC2M2K21B & UPC2M2B & $U P C 2-1^{\mathrm{S} 1-1}-c a S A T 1 / u p c 2-2 \Delta:: F R T$ & this study \\
\hline $\mathrm{UPC} 2 \mathrm{M} 2 \mathrm{~K} 22 \mathrm{~A}$ & $\mathrm{UPC} 2 \mathrm{M} 2 \mathrm{~A}$ & $U P C 2-1^{\mathrm{S} 1-1}-\mathrm{caSAT1} / U P C 2-2$ & this study \\
\hline UPC2M2K22B & UPC2M2B & $U P C 2-1 / U P C 2^{S 1-1}-c a S A T 1$ & this study \\
\hline $\mathrm{UPC} 2 \mathrm{M} 2 \mathrm{~K} 31 \mathrm{~A}$ & $\mathrm{UPC} 2 \mathrm{M} 2 \mathrm{~A}$ & upc2-1 $1 \Delta:: F R T / U P C 2^{\mathrm{S} 2-1}-c a S A T 1$ & this study \\
\hline UPC2M2K31B & UPC2M2B & $U P C 2-1^{\mathrm{S} 2-1}-c a S A T 1 / u p c 2-2 \Delta:: F R T$ & this study \\
\hline UPC2M2K32A & UPC2M2A & $U P C 2-1^{\mathrm{S} 2-1}-c a S A T 1 / U P C 2-2$ & this study \\
\hline $\mathrm{UPC} 2 \mathrm{M} 2 \mathrm{~K} 32 \mathrm{~B}$ & $\mathrm{UPC} 2 \mathrm{M} 2 \mathrm{~B}$ & $U P C 2-1 / U P C 2^{\mathrm{S} 2-1}-c a S A T 1$ & this study \\
\hline
\end{tabular}

${ }^{a}$ SAT1-FLIP denotes the SATI flipper cassette. 


\section{cRNA Synthesis and Labeling}

Immediately prior to cDNA synthesis, the purity and concentration of RNA samples were determined from A260/A280 readings, and RNA integrity was determined by capillary electrophoresis using the RNA 6000 Nano Laboratory-on-a-Chip kit and Bioanalyzer 2100 (Agilent Technologies) as per the manufacturer's instructions. First and second strand cDNA was synthesized from $15 \mu \mathrm{g}$ total RNA using the SuperScript Double-Stranded cDNA Synthesis Kit (Invitrogen) and oligo-dT24-T7 primer (PrOligo) according to the manufacturer's instructions. cRNA was synthesized and labeled with biotinylated UTP and CTP by in vitro transcription using the T7 promoter-coupled double-stranded cDNA as template and the Bioarray HighYield RNA Transcript Labeling Kit (ENZO Diagnostics). Double-stranded cDNA synthesized from the previous steps was washed twice with $70 \%$ ethanol and suspended in $22 \mu 1$ of RNase-free water. The cDNA was incubated as recommended with reaction buffer, biotinlabeled ribonucleotides, dithtiothreitol, RNase inhibitor mix, and T7 RNA polymerase for $5 \mathrm{~h}$ at $37^{\circ} \mathrm{C}$. The labeled cRNA was separated from unincorporated ribonucleotides by passing through a CHROMA SPIN-100 column (Clontech) and ethanol precipitated at $-20{ }^{\circ} \mathrm{C}$ overnight.

\section{Oligonucleotide Array Hybridization and Analysis}

The cRNA pellet was suspended in $10 \mu \mathrm{l}$ of RNase-free water and $10 \mu \mathrm{g}$ was fragmented by ion-mediated hydrolysis at $95^{\circ} \mathrm{C}$ for $35 \mathrm{~min}$ in $200 \mathrm{mM}$ Tris-acetate (pH 8.1), $500 \mathrm{mM}$ potassium acetate, $150 \mathrm{mM}$ magnesium acetate. The fragmented cRNA was hybridized for $16 \mathrm{~h}$ at $45^{\circ} \mathrm{C}$ to the $\mathrm{C}$. albicans NimbleExpress GeneChip arrays. Arrays were washed at $25^{\circ} \mathrm{C}$ with $6 \times \mathrm{SSPE}, 0.01 \%$ Tween 20 followed by a stringent wash at $50{ }^{\circ} \mathrm{C}$ with $100 \mathrm{mM} \mathrm{MES}, 0.1 \mathrm{M}$ $\mathrm{NaCl}, 0.01 \%$ Tween 20. Hybridizations and washes employed the Affymetrix Fluidics Station 450 using their standard EukGE-WS2v5 protocol. The arrays were then stained with phycoerythrein-conjugated streptavidin (Molecular Probes) and the fluorescence intensities were determined using the GCS 3000 high-resolution confocal laser scanner (Affymetrix). The scanned images were analyzed using software resident in GeneChip Operating System v2.0 (Affymetrix). Sample loading and variations in staining were standardized by scaling the average of the fluorescent intensities of all genes on an array to a constant target intensity (250). The signal intensity for each gene was calculated as the average intensity difference, represented by $[\Sigma(\mathrm{PM}-\mathrm{MM}) /($ number of probe pairs) $]$, where PM and MM denote perfect-match and mismatch probes.

\section{Microarray Data Analysis}

The scaled gene expression values from GeneChip Operating System v2.0 software were generated using the MAS5.0 algorithm. The value of each probe set is generated using a Tukey Biweight computation. The algorithm considers the contribution of each match and mismatch probe corrected for the background of the average of the probe set. A weighted mean is then calculated for each probe pair. The raw signal values are then $\log _{2}$ transformed and the array normalized to this value. Probe sets were deleted from subsequent analysis if they were called absent by the Affymetrix criterion. Pairwise comparison of gene expression was performed for 
each matched experiment. Among direct comparisons between matched clinical isolates, genes were considered to be differentially expressed if their change in expression was 1.5 -fold in three independent experiments.

\section{Quantitative Real-Time PCR}

An aliquot of the RNA preparations from the samples used in the microarray experiments was saved for quantitative real-time RT-PCR follow-up studies. First-strand cDNAs were synthesized from $2 \mu \mathrm{g}$ of total RNA in a $21-\mu \mathrm{l}$ reaction volume using the SuperScript FirstStrand Synthesis System for RT-PCR (Invitrogen) in accordance with the manufacturer's instructions. Quantitative real-time PCRs were performed in triplicate using the 7000 Sequence Detection System (Applied Biosystems). Independent PCRs were performed using the same cDNA for both the gene of interest and the 18S rRNA, using the SYBR Green PCR Master Mix (Applied Biosystems). Gene-specific primers were designed for the gene of interest and the 18S rRNA using Primer Express software (Applied Biosystems) and the Oligo Analysis \& Plotting Tool (QIAGEN) and are shown in Table 4-2. The PCR conditions consisted of AmpliTaq Gold activation at $95^{\circ} \mathrm{C}$ for $10 \mathrm{~min}$, followed by 40 cycles of denaturation at $95^{\circ} \mathrm{C}$ for $15 \mathrm{~s}$ and annealing/extension at $60{ }^{\circ} \mathrm{C}$ for $1 \mathrm{~min}$. A dissociation curve was generated at the end of each PCR cycle to verify that a single product was amplified using software provided with the 7000 Sequence Detection System. The change in fluorescence of SYBR Green I dye in every cycle was monitored by the system software, and the threshold cycle $\left(\mathrm{C}_{T}\right)$ above the background for each reaction was calculated. The $\mathrm{C}_{T}$ value of $18 \mathrm{~S}$ rRNA was subtracted from that of the gene of interest to obtain a $\Delta \mathrm{C}_{\mathrm{T}}$ value. The $\Delta \mathrm{C}_{\mathrm{T}}$ value of an arbitrary calibrator (e.g., untreated sample) was subtracted from the $\Delta \mathrm{C}_{\mathrm{T}}$ value of each sample to obtain a $\Delta \Delta \mathrm{C}_{T}$ value. The gene expression level relative to the calibrator was expressed as $2-{ }^{\Delta \Delta C T}$.

\section{Bioinformatics Analysis}

The promoter sequences ( $1 \mathrm{~kb}$ upstream of the start codon) were downloaded from the Candida Genome Database (http://www.candidagenome.org). Using the fuzznuc application from EMBOSS (http://emboss.bioinformatics.nl), the promoter sequences were examined for putative SRE motifs.

\section{RESULTS}

\section{Expression of the Transcription Factor UPC2 Is Coordinately Up-Regulated with ERG11 in Azole Resistant $C$. albicans Clinical Isolate S2}

The $C$. albicans isolates S1 (fluconazole-susceptible) and S2 (fluconazole-resistant) were recovered from an AIDS patient who failed fluconazole therapy for treatment of oropharyngeal candidiasis and are the first two isolates of a series in which no overexpression of the transporter genes $C D R 1 / C D R 2$ and MDR 1 was detected by Northern hybridization (95). In order to identify 
Table 4-2. Primers used for quantitative real-time PCR analysis.

\begin{tabular}{|c|c|c|}
\hline $\begin{array}{l}\text { Gene } \\
\text { Name }\end{array}$ & Primer Pair ${ }^{a}$ & $\begin{array}{c}\text { Amplicon } \\
\text { Size }\end{array}$ \\
\hline \multirow{2}{*}{$18 S$} & F, 5'-CACGACGGAGTTTCACAAGA-3' & 135 \\
\hline & R, 5'-CGATGGAAGTTTGAGGCAAT-3' & \\
\hline \multirow[t]{2}{*}{$C D R 1$} & 'F, 5'-ATTCTAAGATGTCGTCGCAAGATG-3' & 140 \\
\hline & $\mathbf{R}, \mathbf{5}^{\prime}$-AGTTCTGGCTAAATTCTGAATGTTTTC-3' & \\
\hline \multirow[t]{2}{*}{$C D R 2$} & F, 5'-TAGTCCATTCAACGGCAACATT-3' & 76 \\
\hline & R, 5'-CACCCAGTATTTGGCATTGAAA-3' & \\
\hline \multirow[t]{2}{*}{ MDR 1} & F, 5'-ACATAAATACTTTGCCCATCCAGAA-3' & 82 \\
\hline & $\mathbf{R}, \mathbf{5}^{\prime}$-AAGAGTTGGTTTGTAATCGGCTAAA-3' & \\
\hline \multirow[t]{2}{*}{ ERG11 } & F, 5'-TTTAGTTTCTCCAGGTTATGCTCAT-3' & 100 \\
\hline & $\mathbf{R}, \mathbf{5}^{\prime}$-ATTAGCTTTGGCAGCAGCAGTA-3' & \\
\hline \multirow[t]{2}{*}{$U P C 2$} & F, 5'-TCCATCCTTGACCCCTAGTCCT-3' & 52 \\
\hline & R, 5'-CGGCTGAGTTTTGATGTCTTGA-3' & \\
\hline
\end{tabular}

${ }^{a} \mathbf{F}$ : forward, $\mathbf{R}$ : reverse 
genes that are differentially expressed between these two isolates we compared their transcriptional profiles using DNA microarrays. As can be seen in Table C-1 and Table C-2, 138 genes were up-regulated and 173 genes were down-regulated in isolate S2 as compared with S1. Interestingly, among the up-regulated genes were several that are involved in ergosterol biosynthesis (ERG1,ERG3, ERG4, ERG7, ERG24, ERG26, ERG27, POT14, and NCP1) as well as $U P C 2$, which encodes a transcription factor that controls expression of ergosterol biosynthesis genes and induces their expression in the presence of azoles $(82,90,177)$. ERG11, the gene encoding the target of fluconazole, was not found to be differentially expressed between these isolates by microarray analysis. However, real-time RT-PCR analysis revealed increased expression of ERG11 in isolate S2 (Figure 4-1).

\section{Resistant Isolate S2 Contains a Gain-of-Function Mutation in UPC2}

In collaboration with our group, the Morschhäuser lab sequenced the UPC2 alleles in both the resistant isolate $\mathrm{S} 2$ and the susceptible isolate $\mathrm{S} 1$. Two different alleles were found in isolate $\mathrm{S} 1$. Allele one was found to be similar to the UPC2 allele in the wild-type genome strain SC5314; allele two had several mutations, four of which resulted in amino acid substitutions (R68K, I142S, S190N, S228N). Isolate S2 contained the same alleles as S1, with the exception of a G1943A mutation in allele one. This mutation resulted in a G648D amino acid substitution in Upc2p. UPC2 was then disrupted in the wild-type isolate SC5314, and one copy of either wild-type allele one from S1 or gain-of-function mutant allele one from S2 was reintegrated into the disruption strain.

These mutants in the wild-type background were then exposed to the ergosterol biosynthesis inhibitors fluconazole and terbinafine. In the absence of one or both alleles $U P C 2$, the strains were hypersusceptible to fluconazole and terbinafine (Figure 4-2). In the presence of the gain-of-function allele, the strain because resistant to fluconazole and terbinafine. When the wild-type allele was reintegrated into the upc $2 \Delta$ strain, the strain exhibited hypersusceptibility to fluconazole similar to the $U P C 2$ heterozygous disruption strain. In the strain with the wild-type allele reintegrated into a heterozygous $U P C 2$ disruption strain, fluconazole susceptibility returned to the levels found in SC5314.

\section{Identification of UPC2 Target Genes}

In order to identify target genes of Upc $2 \mathrm{p}$, we compared the gene expression profiles of strains expressing either a wild-type $U P C 2$ allele $\left(U P C 2^{\mathrm{S} 1-1}\right)$ or its constitutively active, mutated counterpart (UPC2 ${ }^{\mathrm{S} 2-1}$ ) in the SC5314 background. As shown in Table C-1, 93 genes were consistently up-regulated in the transformants expressing the $U P C 2^{\mathrm{S} 2-1}$ allele. Thirty two of these were also up-regulated in azole-resistant isolate S2 as compared to isolate S1 (Table 4-3). Sixtysix genes were consistently downregulated in the transformants expressing the $U P C 2^{\mathrm{S} 2-1}$ allele. Of these, only eleven were also downregulated in isolate S2 (Table 4-4). These represent the core set of Upc $2 p$ target genes. Among these, 18 genes contained at least one SRE motif within their promoters (Table 4-5). 


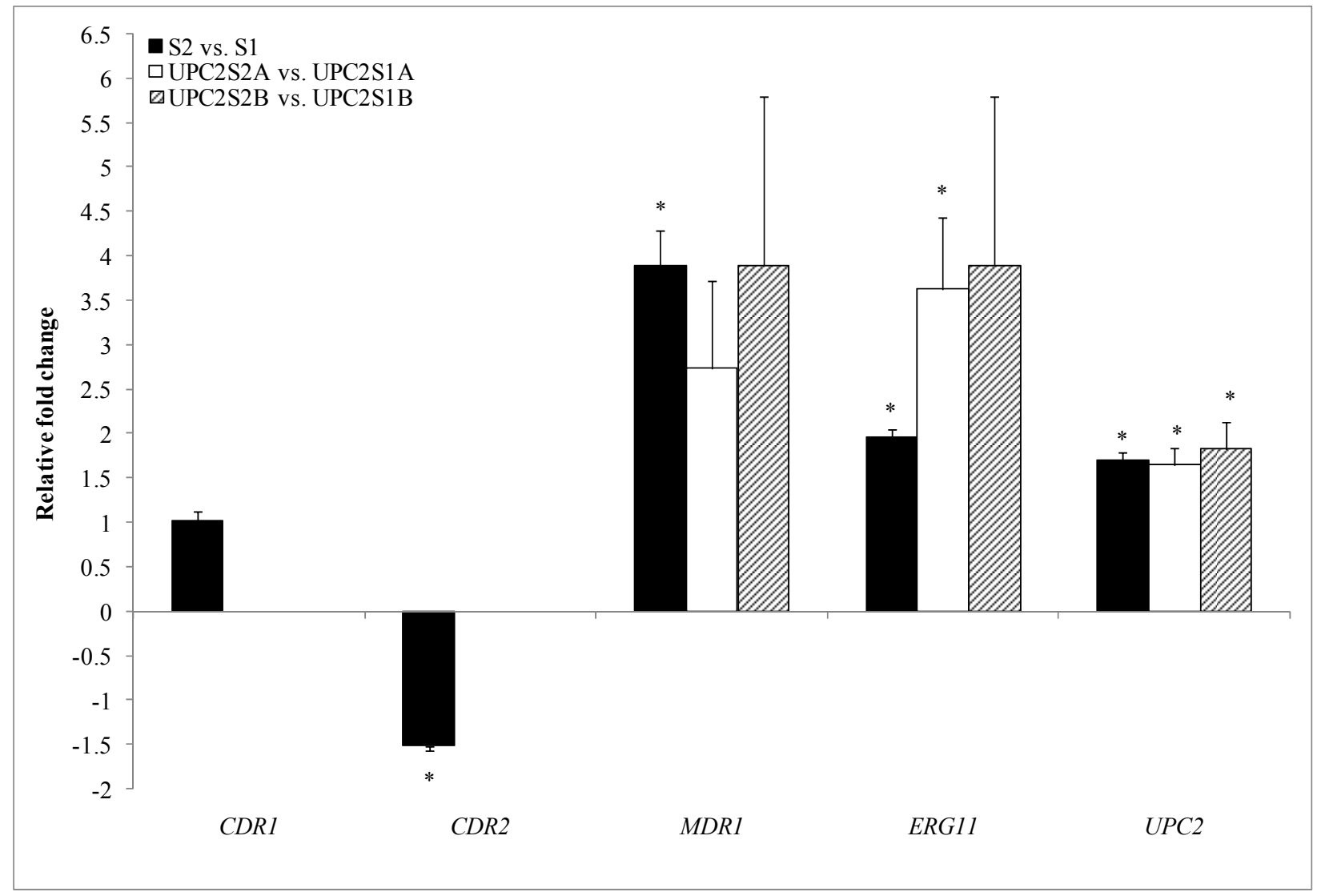

Figure 4-1. Quantitative real-time PCR for genes of interest.

Genes differentially expressed in clinical matched isolate (S2 vs. S1) and two strains with the $U P C 2^{\mathrm{S} 2-1}$ or $U P C 2^{\mathrm{S} 1-1}$ allele in SC5314. Asterisks denote statistical significance by t-test (pvalue $\leq 0.05)$. Error bars represent standard error $(n=3)$. 


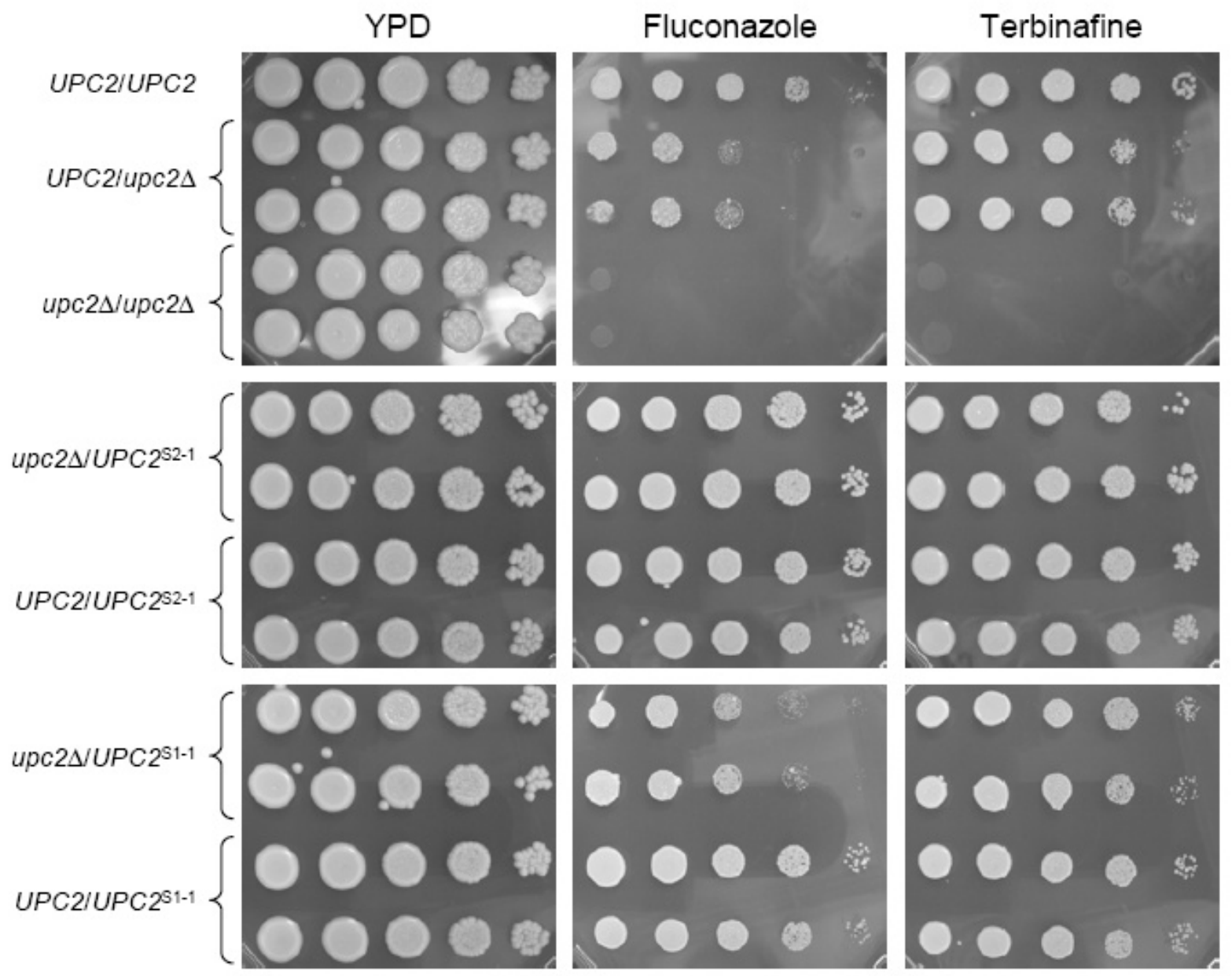

Figure 4-2. Susceptibilities to fluconazole and terbinafine of wild-type and gain-offunction mutant $U P C 2$ strains by spot assay.

All strains were in the SC5314 background. Serial 10-fold dilutions of YPD overnight cultures of the strains were spotted on YPD plates without or with $2 \mu \mathrm{g} \mathrm{ml}^{-1}$ fluconazole or $2 \mu \mathrm{g} \mathrm{ml}^{-1}$ terbinafine and incubated for 2 days at $30^{\circ} \mathrm{C}$. 
Table 4-3. Genes up-regulated by the G648D mutation in Upc2p.

\begin{tabular}{|c|c|c|c|c|c|c|c|c|c|}
\hline \multirow{3}{*}{$\begin{array}{l}\text { CandidaDB } \\
\text { Name }^{a}\end{array}$} & \multirow{3}{*}{$\begin{array}{c}\text { Common } \\
\text { Name }^{b}\end{array}$} & \multirow{3}{*}{$\begin{array}{c}\text { orf19 } \\
\text { Number }^{c}\end{array}$} & \multirow{3}{*}{$\begin{array}{c}\text { GO Annotation (Molecular } \\
\text { Function) }\end{array}$} & & & \multicolumn{4}{|c|}{$U P C 2^{\mathrm{S} 2-1}$ vs. $U P C 2^{\mathrm{S} 1-1}$} \\
\hline & & & & \multicolumn{2}{|c|}{ S2 vs. $\mathbf{S 1}$} & \multicolumn{2}{|c|}{ Strain A } & \multicolumn{2}{|c|}{ Strain B } \\
\hline & & & & Exp. 1 & Exp. 2 & Exp. 1 & Exp. 2 & Exp. 1 & Exp. 2 \\
\hline BMR1 & MDR1 & orf19.5604 & Multidrug transporter activity & 2.1 & 3.7 & 5.4 & 3.5 & 2.5 & 5.1 \\
\hline$D D R 48$ & $D D R 48$ & orf19.4082 & -- & 7.1 & 8.0 & 2.4 & 3.4 & 2.4 & 3.8 \\
\hline ECM3 & ECM3 & orf19.1563 & -- & 1.6 & 1.5 & 1.7 & 4.1 & 2.9 & 3.2 \\
\hline ERG1 & ERG1 & orf19.406 & Squalene monooxygenase activity & 2.4 & 1.6 & 1.5 & 1.7 & 1.6 & 1.8 \\
\hline$E R G 24$ & $E R G 24$ & orf19.1598 & Delta 14-sterol reductase activity & 2.3 & 2.0 & 3.2 & 2.9 & 3.5 & 2.9 \\
\hline ERG26 & $E R G 26$ & orf19.2909 & $\begin{array}{l}\text { C-3 sterol dehydrogenase (C-4 sterol } \\
\text { decarboxylase) activity }\end{array}$ & 2.0 & 1.6 & 2.0 & 2.0 & 1.9 & 2.0 \\
\hline$E R G 27$ & $E R G 27$ & orf19.3240 & 3-keto sterol reductase activity & 2.3 & 2.1 & 2.2 & 3.6 & 3.0 & 2.8 \\
\hline FEN2 & -- & orf19.5535 & Pantothenate transporter activity & 2.2 & 2.2 & 2.3 & 2.7 & 2.7 & 2.8 \\
\hline FTH1 & FTH1 & orf19.4802 & -- & 1.5 & 1.5 & 1.8 & 4.3 & 2.4 & 3.7 \\
\hline$I D P 2$ & $I D P 2$ & orf19.3733 & $\begin{array}{l}\text { Isocitrate dehydrogenase (NADP+) } \\
\text { activity }\end{array}$ & 3.6 & 2.1 & 1.8 & 1.6 & 1.8 & 1.7 \\
\hline IFA24.3 & FGR51 & orf19.156 & -- & 1.8 & 1.9 & 1.7 & 1.7 & 1.6 & 1.7 \\
\hline$N C P 1$ & NCP1 & orf19.2672 & $\begin{array}{l}\text { NADPH-hemoprotein reductase } \\
\text { activity }\end{array}$ & 2.5 & 1.5 & 1.9 & 2.2 & 1.7 & 1.8 \\
\hline QRI8 & -- & orf19.7329 & Ubiquitin-protein ligase activity & 1.5 & 2.0 & 1.6 & 1.7 & 1.5 & 1.7 \\
\hline TEF41 & TEF4 & orf19.2652 & Translation elongation factor activity & 4.1 & 3.4 & 18.8 & 28.9 & 19.0 & 14.9 \\
\hline IPF12101 & $P G A 7$ & orf19.5635 & -- & 1.6 & 2.7 & 5.4 & 8.8 & 6.0 & 9.0 \\
\hline IPF1218 & SOD4 & orf19.2062 & $\begin{array}{l}\text { Copper, zinc superoxide dismutase } \\
\text { activity }\end{array}$ & 2.3 & 2.3 & 2.2 & 1.7 & 1.8 & 1.6 \\
\hline IPF14119 & $D A G 7$ & orf19.4688 & -- & 1.8 & 1.6 & 1.7 & 1.6 & 1.7 & 1.8 \\
\hline IPF 14652 & -- & orf19.2653 & -- & 4.3 & 1.6 & 1.7 & 2.8 & 4.3 & 1.9 \\
\hline IPF18080 & -- & orf19.7357 & -- & 1.9 & 1.6 & 1.5 & 1.8 & 1.6 & 1.6 \\
\hline IPF20010 & -- & orf19.5799 & -- & 2.0 & 2.2 & 2.2 & 2.8 & 1.6 & 2.0 \\
\hline IPF20169 & PGA10 & orf19.5674 & Heme binding & 4.1 & 10.8 & 5.6 & 13.0 & 7.0 & 11.1 \\
\hline IPF2903 & -- & orf19.7456 & -- & 1.8 & 2.3 & 2.0 & 1.7 & 2.2 & 1.6 \\
\hline IPF3352 & -- & orf19.4013 & -- & 2.3 & 2.3 & 2.0 & 2.5 & 1.9 & 2.0 \\
\hline IPF4059 & -- & orf19.1865 & -- & 1.6 & 2.1 & 2.7 & 2.5 & 1.9 & 2.4 \\
\hline IPF4721 & -- & orf19.3737 & -- & 1.5 & 1.6 & 2.3 & 1.5 & 1.8 & 1.6 \\
\hline IPF6298 & -- & orf19.1964 & -- & 3.0 & 1.6 & 1.7 & 1.7 & 2.3 & 1.7 \\
\hline IPF6518 & -- & orf19.1691 & -- & 3.3 & 5.3 & 1.7 & 2.3 & 2.0 & 2.7 \\
\hline IPF7289 & $U P C 2$ & orf19.391 & Transcription factor activity & 1.6 & 2.4 & 2.1 & 2.5 & 2.4 & 2.5 \\
\hline IPF7397 & -- & orf19.1800 & -- & 2.9 & 2.6 & 1.8 & 1.7 & 1.6 & 2.2 \\
\hline
\end{tabular}


Table 4-3 (continued).

\begin{tabular}{|c|c|c|c|c|c|c|c|c|c|}
\hline \multirow{3}{*}{$\begin{array}{l}\text { CandidaDB } \\
\text { Name }^{a}\end{array}$} & \multirow{3}{*}{$\begin{array}{c}\text { Common } \\
\text { Name }^{b}\end{array}$} & \multirow{3}{*}{$\begin{array}{c}\text { orf19 } \\
\text { Number }^{c}\end{array}$} & \multirow{3}{*}{$\begin{array}{c}\text { GO Annotation (Molecular } \\
\text { Function) }\end{array}$} & & & \multicolumn{4}{|c|}{$U P C 2^{\mathrm{S2-1}}$ vs. $U P C 2^{\mathrm{S} 1-1}$} \\
\hline & & & & \multicolumn{2}{|c|}{ S2 vs. S1 } & \multicolumn{2}{|c|}{ Strain A } & \multicolumn{2}{|c|}{ Strain B } \\
\hline & & & & Exp. 1 & Exp. 2 & Exp. 1 & Exp. 2 & Exp. 1 & Exp. 2 \\
\hline IPF7432 & -- & orf19.6219 & -- & 2.0 & 2.3 & 1.9 & 2.4 & 2.1 & 2.2 \\
\hline IPF867 & SET3 & orf19.7221 & $\begin{array}{l}\text { NAD-dependent histone deacetylase } \\
\text { activity }\end{array}$ & 2.1 & 1.7 & 3.6 & 2.9 & 3.2 & 2.4 \\
\hline IPF8970 & -- & orf19.496 & -- & 1.9 & 2.5 & 2.2 & 1.9 & 1.6 & 1.8 \\
\hline
\end{tabular}

${ }^{a}$ Gene name from CandidaDB (http://genolist.pasteur.fr/CandidaDB/).

${ }^{\boldsymbol{b}}$ Gene name from CGD (http://www.candidagenome.org/).

$c$ orf19 nomenclature according to Assembly 19.

${ }^{d}$ Gene Ontology (33) annotation found at CGD (http://www.candidagenome.org/).

Gene expression values are represented as fold changes between resistant isolates and susceptible isolates. 
Table 4-4. Genes down-regulated by the G648D mutation in Upc2p.

\begin{tabular}{|c|c|c|c|c|c|c|c|c|c|}
\hline \multirow{3}{*}{$\begin{array}{c}\text { CandidaDB } \\
\text { Name }^{a}\end{array}$} & \multirow{3}{*}{$\begin{array}{c}\text { Common } \\
\text { Name }^{b} \\
\end{array}$} & \multirow{3}{*}{$\begin{array}{c}\text { orf19 } \\
\text { Number }^{c}\end{array}$} & \multirow[b]{3}{*}{ GO Annotation (Molecular Function) ${ }^{d}$} & & & \multicolumn{4}{|c|}{$U P C 2^{\mathrm{S} 2-1}$ vs. $U P C 2^{\mathrm{S} 1-1}$} \\
\hline & & & & \multicolumn{2}{|c|}{ S2 vs. $\mathbf{S 1}$} & \multicolumn{2}{|c|}{ Strain A } & \multicolumn{2}{|c|}{ Strain B } \\
\hline & & & & Exp. 1 & Exp. 2 & Exp. 1 & Exp. 2 & Exp. 1 & Exp. 2 \\
\hline COQ1 & -- & orf19.7478 & Trans-hexaprenyltranstransferase activity & -2.8 & -2.9 & -1.5 & -2.5 & -2.4 & -3.8 \\
\hline$M A S 2$ & -- & orf19.6295 & $\begin{array}{l}\text { Mitochondrial processing peptidase } \\
\text { activity }\end{array}$ & -1.6 & -1.7 & -1.6 & -2.3 & -2.7 & -2.9 \\
\hline MET14 & MET14 & orf19.946 & Adenylylsulfate kinase activity & -5.6 & -6.9 & -2.3 & -2.9 & -3.5 & -3.7 \\
\hline MET3 & MET3 & orf19.5025 & Sulfate adenylyltransferase (ATP) activity & -4.2 & -3.8 & -3.3 & -4.0 & -3.5 & -5.9 \\
\hline PET127 & -- & orf19.2309 & RNA binding & -1.7 & -1.8 & -2.0 & -4.4 & -7.7 & -5.9 \\
\hline IPF11801 & -- & orf19.2798 & Helicase activity & -1.6 & -1.7 & -2.3 & -2.2 & -2.0 & -1.8 \\
\hline$I P F 14872$ & -- & orf19.4133 & -- & -2.0 & -1.8 & -1.8 & -3.5 & -2.3 & -4.0 \\
\hline IPF20118 & -- & orf19.3846 & -- & -3.8 & -4.4 & -3.6 & -3.1 & -1.7 & -1.6 \\
\hline IPF525 & -- & orf19.7085 & -- & -2.4 & -2.2 & -1.7 & -3.1 & -1.7 & -1.7 \\
\hline IPF6504 & -- & orf19.1544 & -- & -2.1 & -2.0 & -1.8 & -1.5 & -1.7 & -1.6 \\
\hline IPF7334 & -- & orf19.3724 & -- & -2.3 & -2.2 & -1.6 & -3.1 & -1.8 & -3.4 \\
\hline
\end{tabular}

${ }^{a}$ Gene name from CandidaDB (http://genolist.pasteur.fr/CandidaDB/).

${ }^{b}$ Gene name from CGD (http://www.candidagenome.org/).

${ }^{c}$ orf19 nomenclature according to Assembly 19.

${ }^{d}$ Gene Ontology (33) annotation found at CGD (http://www.candidagenome.org/).

Gene expression values are represented as fold changes between resistant isolates and susceptible isolates. 
Table 4-5. Genes up-regulated in both isolate $\mathrm{S} 2$ and transformants expressing the $U P C 2^{\mathrm{S} 2-1}$ allele with SRE motifs in their promoter.

\begin{tabular}{|c|c|c|c|c|}
\hline $\begin{array}{c}\text { CandidaDB } \\
\text { Name }^{a}\end{array}$ & $\begin{array}{c}\text { Common } \\
\text { Name }^{b}\end{array}$ & $\begin{array}{c}\text { orf19 } \\
\text { Number }^{c}\end{array}$ & SRE Motifs & Coordinates \\
\hline DDR48 & $D D R 48$ & orf19.4082 & TGTTCGTATAACT & -661 to -649 \\
\hline ECM3 & ECM3 & orf19.1563 & ATGTATACGATGA & -312 to -300 \\
\hline$E R G 1$ & $E R G 1$ & orf19.406 & TCATCGTATATTT & -376 to -364 \\
\hline \multirow[t]{2}{*}{ ERG11 } & ERG11 & orf19.922 & ATCTATACGACGA & -489 to -477 \\
\hline & & & ATGTCGTATATTC & -235 to -223 \\
\hline$E R G 24$ & $E R G 24$ & orf19.1598 & CGTTATACGACCG & -142 to -130 \\
\hline$E R G 26$ & $E R G 26$ & orf19.2909 & CTCTATACGACAA & -283 to -271 \\
\hline$E R G 27$ & $E R G 27$ & orf19.3240 & GTTTATACGATCG & -154 to -142 \\
\hline QRI8 & -- & orf19.7329 & ATTTCGTATATCT & -367 to -355 \\
\hline TEF41 & TEF4 & orf19.2652 & TGCTATACGATGG & -308 to -296 \\
\hline IPF 18080 & -- & orf19.7357 & CCCTCGTATAGCG & -910 to -898 \\
\hline$I P F 2903$ & -- & orf19.7456 & ATTTATACGACAT & -278 to -266 \\
\hline IPF4059 & -- & orf19.1865 & CGATCGTATATAC & -215 to -203 \\
\hline IPF6518 & -- & orf19.1691 & TTCTATACGACAG & -716 to -704 \\
\hline IPF7289 & $U P C 2$ & orf19.391 & AGCTATACGACTT & -440 to -428 \\
\hline IPF7397 & -- & orf19.1800 & TGATCGTATAGAT & -566 to -554 \\
\hline IPF7432 & -- & orf19.6219 & CACTATACGACGA & -353 to -341 \\
\hline \multirow[t]{3}{*}{$I P F 867$} & SET3 & orf19.7221 & TCGTCGTATAGAC & -544 to -532 \\
\hline & & & TGATCGTATAGAG & -685 to -673 \\
\hline & & & GTCTATACGACCA & -721 to -709 \\
\hline \multirow[t]{2}{*}{ IPF8970 } & -- & orf19.496 & CAGTCGTATACTG & -348 to -336 \\
\hline & & & TATTATACGATGG & -211 to -199 \\
\hline
\end{tabular}

${ }^{a}$ Gene name from CandidaDB (http://genolist.pasteur.fr/CandidaDB/).

${ }^{b}$ Gene name from CGD (http://www.candidagenome.org/).

${ }^{c}$ orf19 nomenclature according to Assembly 19.

The SRE is in bold. 
Disruption of UPC2 in wild-type strain SC5314 also provided the opportunity to identify genes whose base-line expression requires Upc $2 p$ under the growth conditions used in our study. We therefore compared the gene expression profile of strain SC5314 to that of the two independently constructed homozygous upc $2 \Delta$ mutants UPC2M4A and UPC2M4B. Surprisingly only 5 genes were expressed at higher levels in strain SC5314 as compared to the disruption mutants (Table C-3), suggesting that Upc2p is not required for base-line expression of the core target genes listed in Table 4-3 and Table 4-4. Previous studies have shown that disruption of UPC2 in C. albicans results in no baseline changes in expression of $E R G 2, E R G 7, E R G 11$, and $E R G 25(82,90)$. Interestingly disruption of UPC2 in strain SC5314 resulted in up-regulation of 41 genes (Table C-4). This raises the possibility that Upc $2 p$ may also have a role as a transcriptional repressor. Alternatively, this may simply reflect compensatory changes in gene expression.

\section{DISCUSSION}

Zinc cluster transcription factors constitute a large family of transcription factors that is specific to fungi. In $S$. cerevisiae mutations in the zinc cluster transcription factor Pdr1p result in constitutive up-regulation of its target genes, including the $A B C$ transporter Pdr5p, and cause the pleiotropic drug resistance phenotype. Azole-resistant clinical $C$. albicans isolates that overexpress the $\mathrm{ABC}$ transporters $\mathrm{Cdr} 1 \mathrm{p}$ and $\mathrm{Cdr} 2 \mathrm{p}$ have acquired mutations in the zinc cluster transcription factor Tac1p and become homozygous for the mutated $T A C 1$ alleles $(88,89,178)$. Likewise, fluconazole resistant clinical isolates that constitutively overexpress the major facilitator superfamily transporter Mdrlp contain mutations in the zinc cluster transcription factor Mrrlp and have also become homozygous for the mutated MRRI alleles (172). In addition to decreased drug accumulation via efflux pumps, resistance to azoles can occur due to changes in the ergosterol biosynthesis pathway. Point mutations in the ERG11 gene, encoding the drug target lanosterol demethylase, results in amino acid substitutions that decrease the binding affinity of the enzyme to azoles $(27,35,44)$.

Increased transcription of ergosterol biosynthesis genes has also been associated with resistance. Overexpression of ERG11 has been observed in azole-resistant isolates. In $S$. cerevisiae, overexpression of $E R G 11$ alone is sufficient to confer azole resistance. A strain containing the ERG11 gene placed under the control of an inducible GAL1 promoter could grow on galactose plates at fluconazole concentrations of $128 \mu \mathrm{g} / \mathrm{ml}(47)$. The overexpression of ERG11 in serially collected clinical C. albicans isolates coincided with increases in the MIC $(27,168)$. In a series of isolates described by Lopez-Ribot et al, resistant isolate 2307 (MIC $>128 \mu \mathrm{g} / \mathrm{ml}$ ) expressed more ERG11 than both parent isolate $412(\mathrm{MIC}=0.5 \mu \mathrm{g} / \mathrm{ml})$ and isolate $1907(\mathrm{MIC}=8 \mu \mathrm{g} / \mathrm{ml})$. There was increased expression of $C D R 1$ and $C D R 2$ in isolate 1907 compared to parent isolate 412, but the expression of the $\mathrm{ABC}$ transporters were not present in resistant isolate 2307 even though the MIC increases (168). In another study, a clinical resistant $C$. albicans isolate 3795 (MIC $>64$ $\mu \mathrm{g} / \mathrm{ml}$ ) overexpressed $E R G 11$ as compared to the parent isolate 1002 (MIC=0.25 $\mu \mathrm{g} / \mathrm{ml})$. There was no evidence of any other known resistance mechanisms, and there were no point mutations in ERG11 (27). 
Although ERG11 alone can cause azole resistance in C. albicans, the upregulation of ERG11 is often found in conjunction with other resistance mechanisms. In the study by Perea et al., of the twelve patients from which clinical isolates were collected, eleven isolates had point mutations in ERG11 in conjunction with another resistance mechanism. Seven isolates exhibited up-regulation of $C D R 1$ and $C D R 2$, seven isolates exhibited up-regulation of $M D R 1$, and three isolates exhibited up-regulation of both classes of efflux pumps (27).

In this study, we characterized the gene expression profile of the matched clinical isolate pair S1 and S2. The gene expression profile of S2 showed a number of ergosterol biosynthesis genes up-regulated as compared to susceptible parent S1. Transcriptional profiling and real-time RT-PCR showed that $8 E R G$ genes were up-regulated in S2. In addition to the $E R G$ genes, $U P C 2$ was also coordinately regulated. Sequence analysis of the UPC2 alleles identified a G1943A mutation in allele 1 of S2 that was not present in $\mathrm{S} 1$. This resulted in a G648D substitution in Upc2p in S2. Transcriptional profiling of the mutated $U P C 2^{\mathrm{S} 2-1}$ allele expressed in SC5314 as compared to the wild-type UPC2 $2^{\mathrm{S} 1-1}$ allele showed 9 genes up-regulated. UPC2 was also up-regulated in the isolate expressing the $U P C 2^{\mathrm{S} 2-1}$ allele. In total, $12 E R G$ genes were differentially expressed in either $\mathrm{S} 2$ or $U P C 2^{\mathrm{S} 2-1}$ expressed in $\mathrm{SC} 5314$ with four $E R G$ genes up-regulated in both cases.

C. albicans UPC2 is homologous to two zinc cluster transcription factor genes $U P C 2$ and ECM22 in S. cerevisiae. Upc2p and Ecm 22 p both play a role in the regulation of sterol biosynthesis and uptake of sterols from the environment $(81,82,90,179,180)$. Deletion of ScUPC2 causes hypersusceptibility to AmB and ketoconazole while deletion of $S c E C M 22$ causes hypersusceptibility to cycloheximide and calcofluor white $(81,82)$. In addition to drug resistance, $S c U P C 2$ and $S c E C M 22$ are also involved in sterol uptake. Under aerobic conditions, ergosterol is synthesized and no exogenous sterols are imported. Under anaerobic conditions, the heme in lanosterol demethylase cannot be activated and sterol biosynthesis is inhibited. However, in the absence of oxygen, $S$. cerevisiae has the ability to import exogenous sterols. The mutant hyperactive allele, upc2-1, has a G888D mutation that causes exogenous sterol uptake under aerobic conditions. Strains that carry this allele have the ability to take up ten times more exogenous sterols than the wild-type parental strain (181). A G790D mutation was introduced into a wild-type ECM22 allele that duplicated the mutation present in hyperactive allele upc2-1 (180). This mutation resulted in aerobic sterol uptake similar to that of upc2-1. Deletion of ScUPC2 and ScECM22 did not change aerobic sterol uptake, but it decreased the amount of sterol imported under anaerobic condition (179). Sequence alignment showed that the G648D mutation that we identified in one of the $U P C 2$ alleles of C. albicans isolate S2 corresponds exactly to the G888D and G790D mutations that result in hyperactive UPC2 and ECM22 alleles, respectively, in $S$. cerevisiae. It is striking that the same mutation has been selected for in a clinical $C$. albicans isolate during fluconazole therapy. 
In response to azole antifungals (fluconazole, itraconazole, ketoconazole), wildtype $C$. albicans strains overexpress $E R G 11, U P C 2$, and other $E R G$ genes as well as $N C P 1$ and $D D R 48(48,174,182)$. Forced overexpression of $U P C 2$ using a MET3 promoter confers resistance to azoles, whereas disruption of UPC2 in wild-type isolates causes hypersusceptibility to the azoles and other sterol biosynthesis inhibitors such as terbinafine and fenpropimorph $(82,90)$. In response to sterol biosynthesis inhibitors, $U P C 2$ disruption strains show no induction of $E R G$ genes (90). The disruption strain accumulates lower levels of exogenous sterols than the wild-type and the reconstituted strains (90), demonstrating the role of UPC2 in the uptake of exogenous sterols. Strains without $U P C 2$ also show a growth defect under anaerobic conditions (82), presumably due to the inability to take up sterols in the absence of UPC2.

$S$. cerevisiae Upc2p and Ecm $22 \mathrm{p}$ were first identified for their ability to regulate ScERG2 and ScERG3. An 11-bp SRE was identified in the promoters of ScERG2 and $S c E R G 3$ and was found to be bound directly by Upc2p or Ecm $22 \mathrm{p}$. Analysis of the promoter region of other ergosterol biosynthesis genes identified the same SRE motifs (81). Mammalian cells contain sterol regulatory element binding proteins (SREBP) to regulate cholesterol synthesis. There are no homologous SREBPs in yeast, but ScUPC2 and ScECM22 regulate ergosterol biosynthesis. Promoter analysis on C. albicans ERG genes was performed with known $S$. cerevisiae SRE (TCGTATA) (81). Several ERG genes contained the putative SRE motif in their promoters. The DBD of $C$. albicans Upc $2 p$ was purified and used to test direct binding to the SRE found in the promoter region of $S$. cerevisiae and $C$. albicans ERG2. Upc2p could bind the SREs of both $S$. cerevisiae and $C$. albicans, and no binding occurred when the SRE was mutated (82). Of the $12 E R G$ genes up-regulated in $\mathrm{S} 2$ or by $U C P 2^{\mathrm{S} 2-1}$ in SC5314, nine (ERG1, ERG2, ERG5, ERG6, ERG10, ERG11, ERG24, ERG26, and ERG27) have been previously identified to have the $S$. cerevisiae putative SRE core motif, TCGTATA $(82,90)$. In addition, ERG7 and UPC2 were previously identified as having a putative SRE (82). While our analyses did not find an SRE motif in the promoter of $E R G 7$, we did find the TCGTATA motif in UPC2 itself, suggesting this gene may be directly or indirectly autoregulated.

In addition to the $E R G$ genes, UPC2 regulates a host of other genes. One gene of particular interest that was found to be up-regulated by the G648D mutation in Upc2p is MDR1. Constitutive overexpression of this efflux pump leads to fluconazole resistance and represents a major mechanism of resistance in clinical isolates $(27,28,29,35,43,95$, 168). Previous Northern hybridization analyses showed no MDRl up-regulation in isolates of the S series, although isolate S2 itself was not tested in that study (95). However, in the present study, microarray analysis revealed MDRl to be up-regulated in isolate $\mathrm{S} 2$ by between 2- and 4-fold. This observation was confirmed by real-time RTPCR. This is modest in comparison to isolates in which MDRl has been shown to contribute to fluconazole resistance. Such isolates exhibit increased expression of $M D R 1$ on the order of 100-fold. It is therefore unlikely that enhanced expression of MDR 1 contributes significantly to fluconazole resistance in isolate S2. Moreover, as MDR1mediated resistance appears to be specific for fluconazole, but not other ergosterol biosynthesis inhibitors, it is unlikely that it contributes to the UPC2-dependent changes in 
susceptibility to terbinafine observed in the present study. Although we were unable to identify an SRE motif in the promoter region of $M D R 1$, these data suggest that Upc $2 p$ may play a role in the regulation of $M D R 1$ expression.

Another gene that was highly up-regulated by the G648D mutation in Upc2p is $P G A 10$, a gene required for hemoglobin-iron utilization (183). FTH1, a gene homologous to ScFTH1 that encodes a putative high affinity iron transporter, was also overexpressed. Several other iron permease genes were overexpressed 1.5- to 40-fold in either isolate $\mathrm{S} 2$ or strains expressing the $U P C 2^{\mathrm{S} 2-1}$ allele, including FET34, FRE5, FRE10, and FTR1. FRE10 and FTRI were up-regulated in S2 whereas FRP1 was upregulated in transformants expressing the $U P C 2^{\mathrm{S} 2-1}$ allele. FET34 appears to be upregulated in both cases, because FET34 and FET5 are two alleles of FET34 according to the Candida Genome Database (CGD; http://www.candidagenome.org). A previous study determined that under iron deprivation conditions, C. albicans down-regulates $E R G 11$ and is more susceptible to fluconazole, cycloheximide, nystatin, and anisomycin. The down-regulation of ERG11 decreases the amount of membrane ergosterol, causing decreased membrane fluidity and increased drug susceptibility (184). In the present study, the expression of ERG11 seems to be coordinately regulated with the genes that transport iron into cells, even though the iron uptake genes do not contain an SRE in the promoter. The ERG11 gene product, lanosterol demethylase, has a heme molecule in the active site that is required in order for it to bind molecular oxygen. Because iron is central to this heme, up-regulation of ERG11 may necessitate an increase of iron uptake. It is tempting to speculate that differential expression of these iron metabolism genes may be a direct result of altered ergosterol production, and not directly regulated by Upc $2 p$.

In the azole resistant isolate $\mathrm{S} 2$, the most highly up-regulated gene was $D D R 48$ which was also found to have a putative SRE in its promoter region. Previous studies have shown $D D R 48$, a gene associated with cell stress response, to be differentially expressed in resistant isolates overexpressing CDR1, CDR2, and MDR1 $(172,178)$. It has also been found to be overexpressed in response to fluconazole, itraconazole, ketoconazole, $\mathrm{AmB}$, and caspofungin treatment $(48,174,182)$. DDR 48 was also found to be up-regulated under hypoxic conditions (185). Interestingly, $D D R 48$ was also one of the 41 genes up-regulated in the SC5314 upc2 4 /upc $2 \Delta$ disruption mutants. While the biological role of $D D R 48$ requires further study, our data suggest it may be both positively and negatively regulated by Upc2p.

As mentioned above, $S c U P C 2$ has been implicated in the uptake of exogenous sterols. Sterol uptake is mediated by an ABC transporter, ScAUS1 (186). In C. albicans, ScAUS1 is homologous to a number of ABC transporters including CDR1 and CDR2. However, the actual mechanism of sterol uptake in C. albicans is unknown. By microarray analysis we found genes encoding $\mathrm{ABC}$ transporters to be up-regulated by the $U P C 2$ gain-of-function mutation. In the resistant isolate $\mathrm{S} 2$, the $\mathrm{ABC}$ transporter gene $C D R 12$ is up-regulated 1.5- to 3.0-fold, and in SC5314 transformants expressing the $U P C 2^{\mathrm{S} 2-1}$ allele the $\mathrm{ABC}$ transporter gene $C D R 11$ is up-regulated 1.5- to 3.5-fold. According to the CGD, CDR 11 and CDR 12 are two different alleles of the gene CDR11. Because of the homology of CDR11 to SCAUS1, Cdr11p is a strong candidate for the 
transporter that mediates the uptake of exogenous sterols in C. albicans. This hypothesis is currently under investigation. 


\section{CHAPTER 5: DISCUSSION}

With advancements such as highly active antiretroviral therapy (HAART), the morbidity and mortality of Candida infections have decreased. Incidences of OPC have decreased especially in developed countries where newer drugs are available $(187,188)$. However, in developing nations, the cost of the drugs and the long term course of treatment become prohibitive and impractical $(7,8)$. Without effective HAART, HIV patients develop OPC at pre-HAART rates (189). In these situations, OPC is still often treated with azole antifungals, and the development of resistance remains a problem.

Because azoles are fungistatic, the development of resistance can occur, especially when treatment spans a long period of time. In C. albicans, several common resistance mechanisms can develop through antifungal treatment. The azoles bind lanosterol demethylase, a cytochrome P-450 enzyme essential to the synthesis of ergosterol in the cell. One resistance mechanism occurs through mutations in ERG11, the gene that encodes lanosterol demethylase. Several nonsynonymous mutations have been identified in ERG11 that affect drug resistance $(44,45,46)$. Mutations in ERG11 change the conformation or spatial structure of Erg11p, altering the enzyme-drug interaction without necessarily changing the enzyme function. Another mechanism of resistance is the overexpression of the azole target gene $E R G 11(27,43,168)$. By increasing the amount of drug target, fewer enzymes are bound by the drug. In order to achieve the same level of inhibition, the dosage of the drug would need to be increased. In $S$. cerevisiae, the regulation of ergosterol biosynthesis genes occurs through the zinc cluster transcription factors ScUpc2p and ScEcm $22 p(81)$. One homologous gene UPC2 is present in $C$. albicans, and Upc2p regulates ergosterol genes in $C$. albicans $(82,90)$.

The major mechanism of azole resistance involves active efflux of the drug from the cell. The two major classes of efflux pumps in C. albicans are the $\mathrm{ABC}$ transporters and the MFS transporters. The ABC transporters responsible for azole drug resistance are Cdr1p and Cdr2p $(32,35,37,38,51)$. The expression and regulation of CDR 1 and $C D R 2$ have been identified to be determined by the zinc cluster transcription factor Tac1p $(88,89)$. Gain-of-function mutations in TAC1 result in azole resistance. One hyperactive allele of $T A C 1$ is insufficient to cause azole resistance. In high level azole resistance, two hyperactive $T A C 1$ alleles are present due to the loss of heterozygosity. $T A C 1$ is found on chromosome 5 near the mating type locus $(M T L)$. Recent genetic studies have shown that the loss of heterozygosity of the $M T L$ may be the mechanism by which TAC1 alleles become homozygous, but the homozygosity of the $M T L$ is not sufficient in itself to cause resistance (89). The overexpression of the MFS transporter Mdr1p also confers resistance $(21,35,39,43,72,168)$. C. albicans MDR1 is not to be confused with the human $M D R 1$, an $\mathrm{ABC}$ transporter. The substrate also differs between C. albicans Mdr1p and Cdr1p/Cdr2p. The CDR efflux pumps have the ability to transport all azole antifungals; Mdr1p is specific to fluconazole. The bZip transcription factor Cap1p was originally thought to be responsible for the expression of MDRI in resistance. However, deletion of $C A P 1$ in a resistance isolate overexpressing MDRl does 
not abrogate $M D R 1$ gene expression. The deletion of $C A P 1$ also does not prevent the induction of $M D R l$ in response to benomyl exposure $(83,84)$.

In $S$. cerevisiae, transcriptional regulators of drug resistance have been shown to be co-regulated with their target genes as well as autoregulated $(75,76,78)$. As is the case with $S$. cerevisiae, we hypothesized that the azole resistance mechanisms developed in clinical isolates of $C$. albicans is due to gain-of-function mutations in a transcriptional regulator. Using custom Affymetrix microarrays specific to C. albicans, we were able to examine the genome-wide expression profile of clinical matched susceptible and resistant isolates. By studying matched isolates, we were able to identify specific changes that occurred between susceptible and resistant isolates. Because of the precedence of transcription factors being differentially expressed in $S$. cerevisiae, we looked for putative transcriptional regulators in our co-regulated genes.

Our first objective was to better characterize the genes co-regulated with $C D R 1$ and $C D R 2$ using our new microarrays designed from the newly completed $C$. albicans genome sequencing project. Previous studies have shown several genes consistently coregulated with the expression of $C D R 1$ and $C D R 2$. With the new arrays, we confirmed the co-regulation of $C D R 1 / C D R 2$ with previously identified genes. In addition, we also discovered the co-regulation of the zinc cluster transcription factor Tac1p. Although Tac1p had been shown to be the transcriptional regulator of $C D R 1$ and $C D R 2$, it had not been shown to be autoregulated. Using expression profiling of four matched isolates from four different patients in conjunction with a tac $1 \Delta$ mutant in a resistant isolate, we were able to narrow down the list of genes considered to be Tac1p dependent. The development of a Tac1p ChIP-chip assay allowed us to examine the genes whose promoters were directly bound by Taclp. Taclp-HA 3 binding was found to occur in both inducing (mutant TAC1 allele) and noninducing (wild-type TACl allele) conditions. This binding occurred independent of the $\mathrm{HA}_{3}$ tagging as no gain- or loss-of-function mutation was detected by the Raymond lab. These data suggest that Tac1p is constitutively bound to the promoter region of the target genes. The gain-of-function mutation does not increase affinity of binding or act as a nuclear localization signal. Instead, the mutation could prevent the binding of a repressor element or act as an activator of transcription. By utilizing both expression and location profiling, we could identify the co-regulated genes that were directly bound by Tac1p (Figure 5-1).

Interestingly, there were genes that were up-regulated in the presence of the gainof-function allele whose expression was abolished in the absence of TAC1 but whose promoters were not bound by Tac1p. These data suggest the possibility of another transcriptional factor involved in gene regulation. Because Tac $1 \mathrm{p}$ is a zinc cluster transcription factor, it binds to the DRE motif as homo- or hetero-dimers (64). The genes that are dependent on the presence of TAC1 for expression could require a Tac $1 \mathrm{p}$ heterodimer to bind to the promoter motif. Conversely, there were genes whose promoters were bound by Tac1p but the genes were not differentially expressed in resistant isolates. This suggests that Taclp also acts as a transcriptional regulator for other cellular functions. 
Figure 5-1. Proposed model for Tac1p-mediated fluconazole resistance in $C$.

albicans.

(A) Schematic of a fluconazole susceptible isolate expressing baseline levels of Cdr1p. Taclp is constitutively bound to the DRE of target genes, including CDR1,CDR2, and $T A C 1$. The lack of Tac1p-mediated transcription of CDR1, CDR2, TAC1, and other coregulated genes could be due to the inefficient recruitment of an enhancer or the binding of a repressor. (B) Schematic of a fluconazole resistant cell expressing increased levels of $C D R 1, C D R 2, T A C 1$, and co-regulated genes. The gain-of-function mutation (amino acid substitution) results in increased expression of Tac $1 \mathrm{p}$ target genes and fluconazole resistance possibly through preventing the binding of a repressor or through the increased recruitment of enhancers or cofactors. Additional Tac1p target genes may also contribute to fluconazole resistance. 
A.

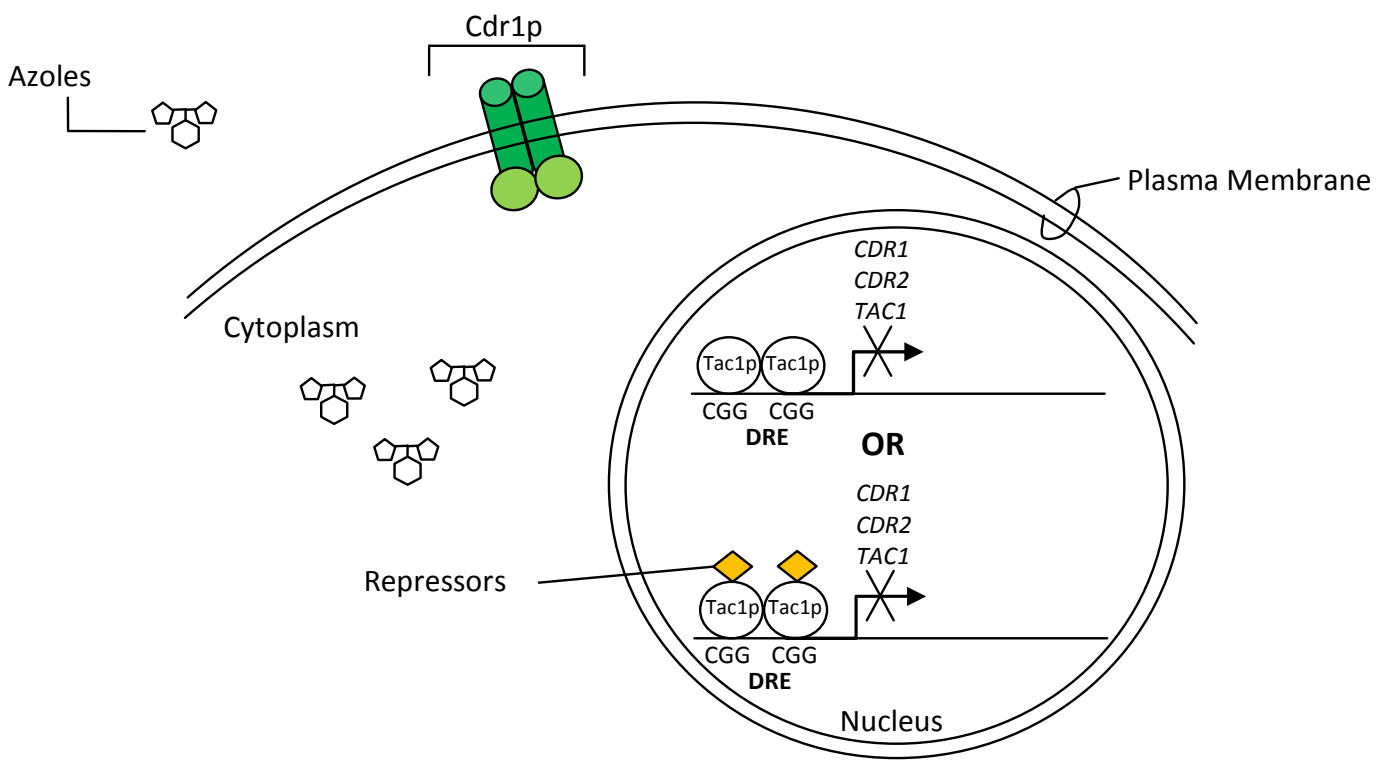

B. 吅

啊 吅

Azoles L

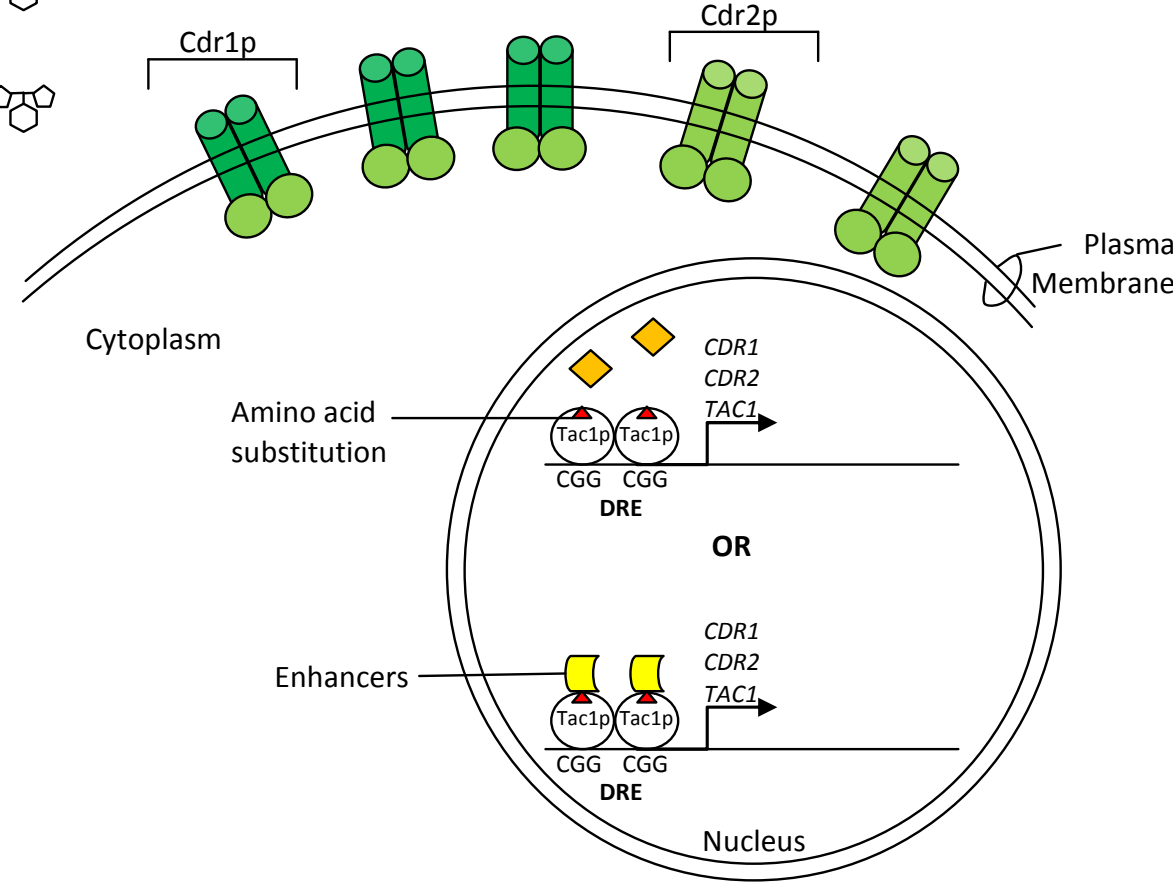


The gain-of-function mutation in $T A C l$ in addition to the constitutive binding of Taclp to the promoter region of its target genes warrants further study. The gain-offunction mutation does not increase binding of Tac1p to the promoter region of target genes. However, the gain-of-function mutation in TAC1 clearly increases the expression of $C D R 1$ and $C D R 2$. The more efficient recruitment of an enhancer or the incompatibility of a repressor due to the mutation could result in the constitutive activation of Taclp. The identification of this cofactor could provide further insight on how C. albicans responds to drug stresses.

Because the identification of transcriptional regulators was possible by gene expression profiling, we next examined three clinical matched isolate pairs known to overexpress $M D R 1$. Our results in Chapter 3 showed a smaller number of genes coregulated with $M D R 1$, with significant overlap between all three resistant isolates. Our experiments confirmed previously identified co-regulated genes. In addition, we found a zinc cluster transcription factor we named $M R R 1$. A gain-of-function mutation was identified in each resistant allele. The deletion of $M R R l$ abrogates the expression of $M D R 1$ in resistant isolates and in response to cerulenin, brefeldin A, hydrogen peroxide, diamide, and fluconazole. Reintegration of gain-of-function MRR1 in wild-type SC5314 restored the overexpression of $M D R 1$ in response to cerulenin, brefeldin A, diamide, and fluconazole. A loss of heterozygosity of $M R R l$ alleles also contributes to resistance. In the fluconazole resistant isolates, both alleles of $M R R I$ carry the gain-of-function mutation. Similar to $T A C 1$, both $M R R 1$ alleles containing the gain-of-function mutation confer higher drug resistance than heterozygous $M R R 1$ alleles.

A large number of genes regulated by $M R R I$ are homologous to $S$. cerevisiae genes implicated in the oxidative stress response. Additionally, these $S$. cerevisiae genes have also been identified as being controlled by Yrrlp and Yrm1p, two zinc cluster transcription factors found in the PDR family of drug resistance. These genes have not been shown to be regulated by $Y A P 1$. The oxidative stress response in C. albicans has been shown to be mediated by $C A P 1$. The resistance to azole antifungal agents depends partly on $C A P 1$, but the disruption of $C A P 1$ does not abrogate the expression of $M D R 1$. Our group, in collaboration with the Morschhäuser lab, has shown that $M R R I$ mediates the hydrogen peroxide and benomyl response through the control of $M D R 1$ gene expression; the disruption of $M R R 1$ completely abolishes $M D R 1$ gene expression in the presence of hydrogen peroxide and benomyl. However, in the absence of $M R R 1$, the strains retain the ability to respond to hydrogen peroxide stress (data not shown). The redundancy of these two transcriptional regulators could be a consequence of the mechanism of transcriptional activation. Cap1p binds promoter regions of genes only if the transcription factor is localized to the nucleus in response to oxidative stress. Mutations in the nuclear localization signal domain can also cause constitutive activation of the Cap1p. The mechanism of MRRl activation by azole antifungals is still unknown. However, a gain-of-function mutation in $M R R 1$ constitutively activates the transcription factor and up-regulates $M D R 1$. The schematics in Figure 5-2 show the possible mechanisms by which Mrrlp transcriptional regulation occurs. 
Figure 5-2. Proposed model for Mrr1p-mediated fluconazole resistance in $C$.

albicans.

(A) Schematic of a fluconazole susceptible isolate with no baseline Mdrlp. The lack of Mrrlp-mediated transcription of $M D R 1, M R R 1$, and co-regulated genes could be due to the cytoplasmic localization of Mrr1p, the inactivation of Mrr1p that prevents binding to the promoter, inefficient recruitment of an enhancer, or the binding of a repressor. (B) Schematic of a fluconazole resistant isolate expressing increased levels of $M D R l$, and coregulated genes. The gain-of-function mutation (amino acid substitution) results in increased expression of Mrrlp target genes and fluconazole resistance possibly through a) increased nuclear localization of Mrrlp, b) by preventing the binding of a repressor or c) through the increased recruitment of enhancers or cofactors. Additional Mrrlp target genes may also contribute to fluconazole resistance. 
A.

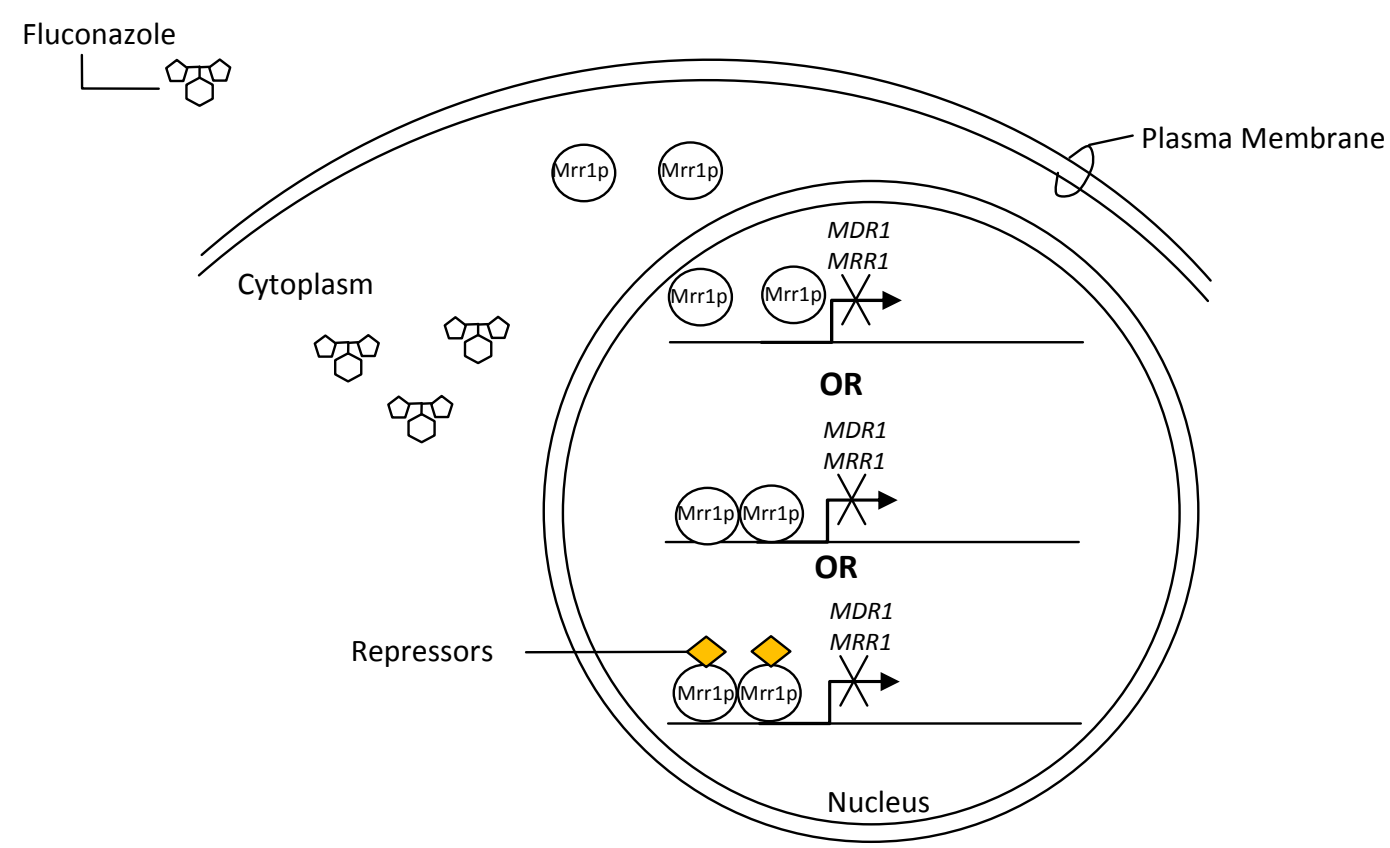

B.

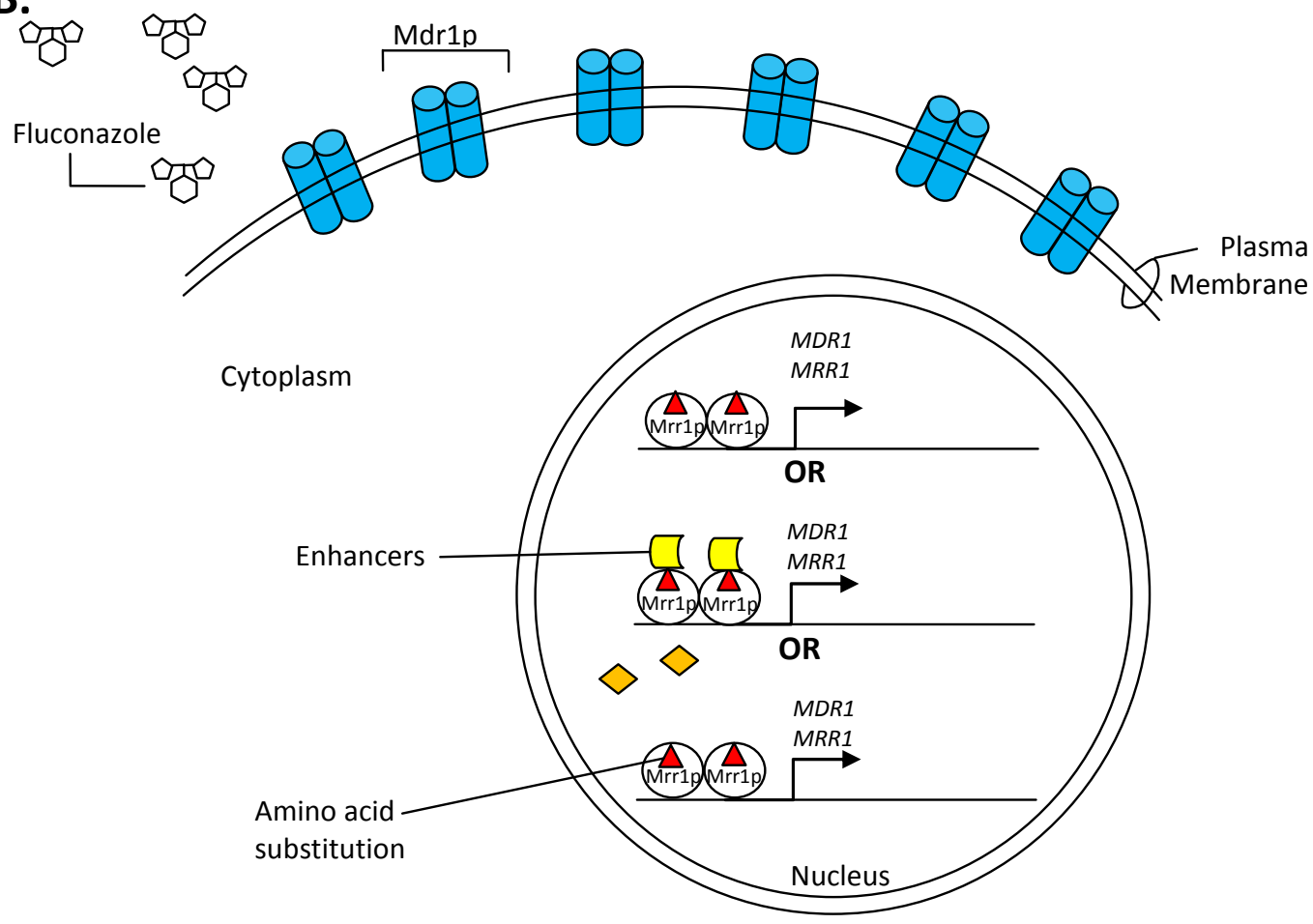


Further study is needed to determine the relationship between the gain-of-function mutation and the activation of MRR1. Mrrlp could behave similar to Tac1p in that it binds constitutively to the promoter of the target genes. However, the gain-of-function mutation could result in increased affinity of the protein to the binding site. Mrrlp location profiling would allow us to study the binding of Mrrlp to promoter regions of $M D R 1$ co-regulated genes. By finding a core set of genes that are bound directly by Mrr1p, a binding motif can also be defined. Another important study would be to examine the interaction between $M R R l$ and $C A P 1$ regulon. As it stands, $M R R l$ seems to be responsible for drug resistance while $C A P 1$ is responsible for the oxidative stress response. However, these two transcription factors have overlapping functions and how they interact requires further study.

In our collection of clinical matched isolate pairs, we had a susceptible and resistant isolate which did not express known resistance genes (CDR1, CDR2, MDR1). Using genome-wide expression profiling, we looked to characterize the resistance mechanisms. Because high level azole resistance is most likely due to gain-of-function mutations in a trans-regulatory element, we examined the gene expression profile for putative transcriptional regulators. The resistant isolate overexpressed a large number of ergosterol biosynthesis genes including ERG11. Additionally, we found UPC2 upregulated in conjunction with the $E R G$ genes (Figure 5-3). Sequencing of the UPC2 alleles identified a gain-of-function mutation identical to the mutation found in $S c U P C 2$ (upc2-1) and ScECM22. The gain-of-function mutation reintegrated in a susceptible isolate conferred fluconazole resistance and increased the expression of $E R G$ genes. The UPC2 disruption strains conferred hypersusceptibility to fluconazole.

Interestingly, fluconazole resistance in clinical isolates as a result of gain-offunction mutations in UPC2 is unlike the gain-of-function mutations in TAC1. One mutant allele of UPC2 is sufficient in conferring fluconazole resistance in this clinical isolate. Loss of heterozygosity in addition to the gain-of-function mutations in the TAC1 alleles contributed to high level azole resistance in fluconazole resistant clinical isolates. One $M R R 1$ allele containing a gain-of-function mutation is also sufficient in conferring azole resistance (Morschhäuser lab, personal communication), although the resistant isolates used in our study demonstrated a loss of heterozygosity and higher levels of azole resistance. It is possible that two mutant UPC2 alleles could confer higher levels of azole resistance; however, we did not examine this possibility at this time.

Another gene of interest identified in this study was the gene encoding the ABC transporter CDR11. In our microarray data, we found the resistant clinical isolate to overexpress the $\mathrm{ABC}$ transporter $\mathrm{Cdr} 12 \mathrm{p}$; in the reintegrated gain-of-function mutant, Cdr11p was up-regulated. According to the newly assembled Candida genome database (CGD), CDR11 and CDR12 are actually two alleles of the CDR11 gene. This ABC transporter may be responsible for uptake of exogenous sterols. In $S$. cerevisiae, the $\mathrm{ABC}$ transporter ScAUS1 has been implicated in the utilization of exogenous sterols under sterol starvation conditions. While sterol uptake in $C$. albicans has not been well characterized, previous studies have shown that sterol uptake is possible (90). The expression of different $C D R 11$ alleles in response to the same UPC2 mutation could be 
Figure 5-3. Proposed model for Upc2p-mediated fluconazole resistance in $C$. albicans.

(A) Schematic of a fluconazole susceptible isolate with baseline ergosterol biosynthesis gene expression. The lack of overexpression of ERG11, UPC2, and other ergosterol biosynthesis genes could be due to the localization of Upc $2 p$ to the membrane of the endoplasmic reticulum (ER) or the binding of a repressor. (B) Schematic of a fluconazole resistant cell expressing increased levels of ERG11, other ergosterol biosynthesis genes, and other Upc $2 p$ target genes. The gain-of-function mutation (amino acid substitution) results in increased expression of Upc $2 p$ target genes and fluconazole resistance possibly through a) increased nuclear localization of $U p c 2 p, b)$ by preventing the binding of a repressor or c) through the increased recruitment of enhancers or cofactors. Additional Upc $2 p$ target genes may also contribute to fluconazole resistance. 
A.

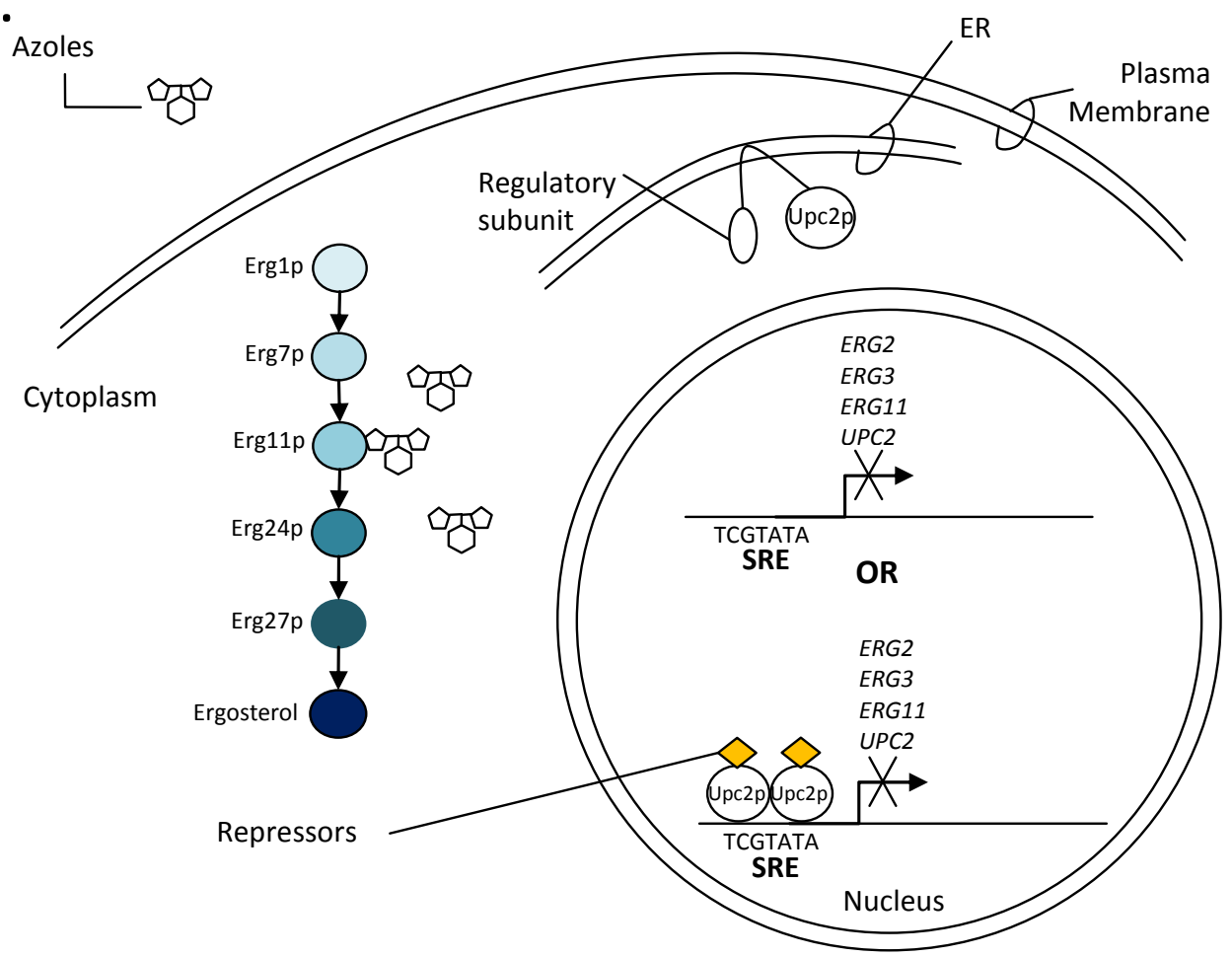

B.

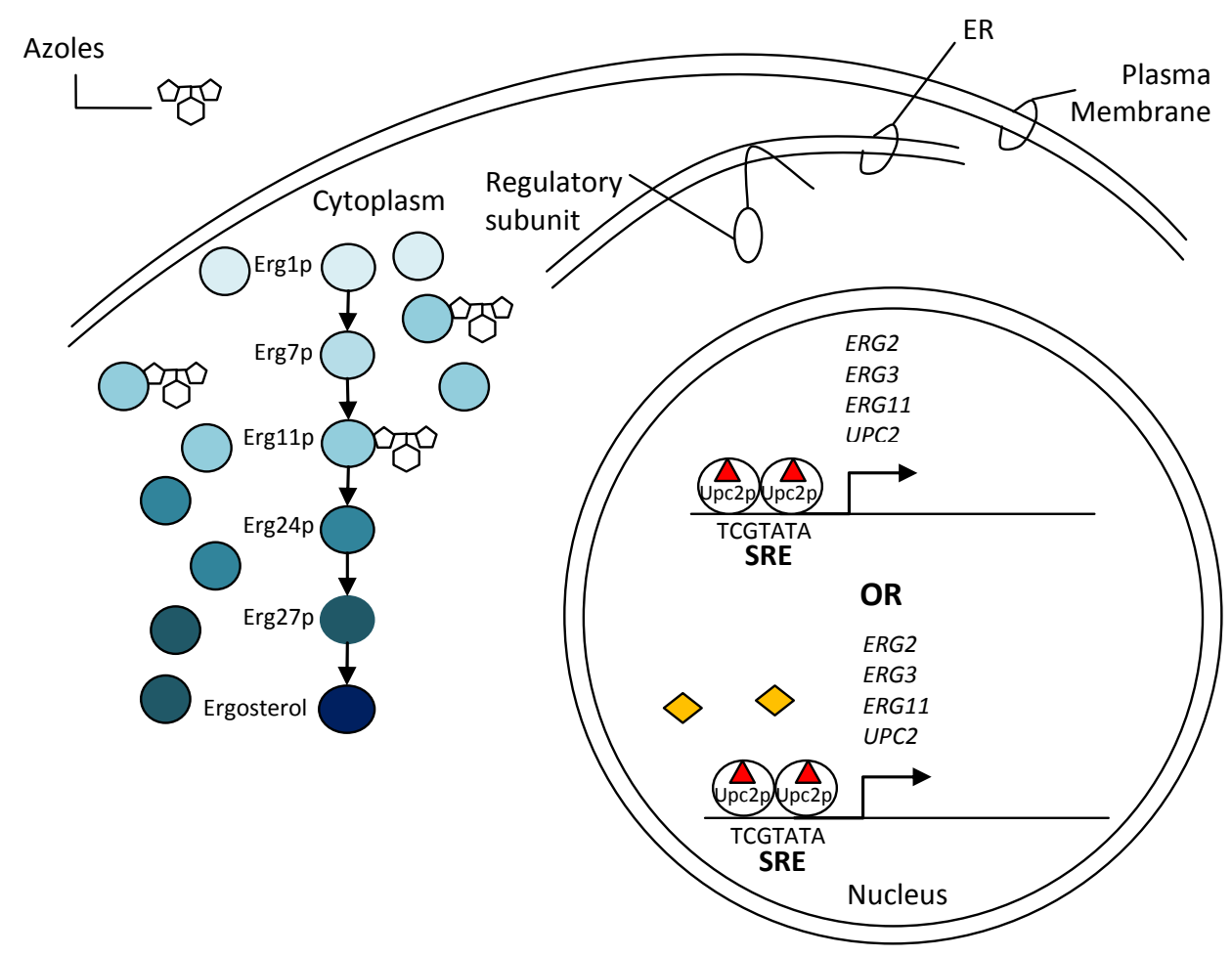


due to the background strain. The resistant clinical isolate and the genome strain SC5314 are not isogenic, so different $C D R 11$ alleles may be present in different $C$. albicans strains.

Further study is necessary to determine if $C D R 11$ is in fact responsible for the uptake of exogenous sterols by $C$. albicans. Because the UPC2 gain-of-function mutation in C. albicans is the same as the UPC2 and ECM22 gain-of-function mutations in $S$. cerevisiae and the gain-of-function mutations in $S$. cerevisiae confers sterol uptake under aerobic conditions, the same phenotype may occur in C. albicans. The loss of heterozygosity of $U P C 2$ alleles in the development of azole resistance also requires further study. Our resistant clinical isolate only contained one gain-of-function mutation allele. Currently, no isolates have been characterized to contain two homozygous gainof-function mutations in UPC2.

By studying matched susceptible and resistant clinical isolates, we were able to identify genes specific to the development of resistance. We successfully identified several gain-of-function mutations in transcriptional regulators of clinical azole resistance, and characterized core genes affected by these specific mutations. We have also identified a previously uncharacterized transcriptional regulator of $M D R 1$. This transcription factor, Mrrlp, was also shown to contain gain-of-function mutations that constitutively activate its target genes conferring high level azole resistance. We also identified gain-of-function mutations in a known transcriptional regulator in a clinical matched set with an undefined resistance mechanism. These results demonstrate the importance of using genome-wide analysis to identify gain-of-function mutations in zinc cluster transcription factors in the development of azole resistance in C. albicans.

The discovery and identification of gain-of-function mutations in the transcriptional regulators of fluconazole resistance, combined with known azole resistance mutations in the ERG11 gene itself, may allow for the development of more efficient diagnostic tools to better predict clinical success of azole therapy against a specific isolate. Theoretically, if all (or most) relevant mutations that confer azole resistance were identified in C. albicans, single nucleotide polymorphism (SNP) detection assays could be developed to quickly identify the presence of these mutations in clinical isolates. If such an assay could be created, it is quite possible that such genetic information would better correlate with clinical outcomes as compared to in vitro susceptibility testing. Moreover, the speed and efficiency with which such assays could be performed would allow for quicker initiation of optimal antifungal therapy. Major challenges will be the ability to first identify the majority of mutations that contribute to azole resistance in the overall clinical isolate population and the current cost of routine use of such technology.

The discovery of the transcription factors that regulate the major resistance mechanisms in C. albicans also has significant therapeutic ramifications. Tac1p, Mrrlp, and $U p c 2 p$ are all zinc cluster transcription factors which are a class of zinc finger proteins unique to fungi. If inhibitors of this fungal-specific class of proteins could be identified, such compounds could be used in combination with the azole antifungals to 
inhibit expression of azole resistance genes, or to prevent the development of azole resistance from the time of initiation of therapy. 


\section{LIST OF REFERENCES}

1. Odds, F. C. 1988. Candida and Candidosis. Bailliere Tindall, London.

2. Edmond, M. B., S. E. Wallace, D. K. McClish, M. A. Pfaller, R. N. Jones and R. P. Wenzel. 1999. Nosocomial bloodstream infections in United States hospitals: a three-year analysis. Clin Infect Dis. 29: 239-44.

3. Pfaller, M. A. and D. J. Diekema. 2007. Epidemiology of invasive candidiasis: a persistent public health problem. Clin Microbiol Rev. 20: 133-63.

4. Fidel, P. L., Jr. and J. D. Sobel. 1996. Immunopathogenesis of recurrent vulvovaginal candidiasis. Clin Microbiol Rev. 9: 335-48.

5. Calderone, R. A. 2002. Candida and Candidiasis. ASM Press, Washington.

6. de Repentigny, L., D. Lewandowski and P. Jolicoeur. 2004. Immunopathogenesis of oropharyngeal candidiasis in human immunodeficiency virus infection. Clin Microbiol Rev. 17: 729-59, table of contents.

7. Darbyshire, J. 2000. Therapeutic interventions in HIV infection - a critical view. Trop Med Int Health. 5: A26-31.

8. Kontoyiannis, D. P. and R. E. Lewis. 2002. Antifungal drug resistance of pathogenic fungi. Lancet. 359: 1135-44.

9. Klis, F. M., P. de Groot and K. Hellingwerf. 2001. Molecular organization of the cell wall of Candida albicans. Med Mycol. 39 Suppl 1: 1-8.

10. White, T. C., K. A. Marr and R. A. Bowden. 1998. Clinical, cellular, and molecular factors that contribute to antifungal drug resistance. Clin Microbiol Rev. 11: $382-402$.

11. Patel, R. 1998. Antifungal agents. Part I. Amphotericin B preparations and flucytosine. Mayo Clin Proc. 73: 1205-25.

12. Joseph-Horne, T. and D. W. Hollomon. 1997. Molecular mechanisms of azole resistance in fungi. FEMS Microbiol Lett. 149: 141-9.

13. Lupetti, A., R. Danesi, M. Campa, M. Del Tacca and S. Kelly. 2002. Molecular basis of resistance to azole antifungals. Trends Mol Med. 8: 76-81.

14. Berman, J. and P. E. Sudbery. 2002. Candida Albicans: a molecular revolution built on lessons from budding yeast. Nat Rev Genet. 3: 918-30. 
15. Chandra, J., D. M. Kuhn, P. K. Mukherjee, L. L. Hoyer, T. McCormick and M. A. Ghannoum. 2001. Biofilm formation by the fungal pathogen Candida albicans: development, architecture, and drug resistance. J Bacteriol. 183: 538594.

16. Braun, B. R., M. van Het Hoog, C. d'Enfert, M. Martchenko, J. Dungan, A. Kuo, D. O. Inglis, M. A. Uhl, H. Hogues, M. Berriman, M. Lorenz, A. Levitin, U. Oberholzer, C. Bachewich, D. Harcus, A. Marcil, D. Dignard, T. Iouk, R. Zito, L. Frangeul, F. Tekaia, K. Rutherford, E. Wang, C. A. Munro, S. Bates, N. A. Gow, L. L. Hoyer, G. Kohler, J. Morschhauser, G. Newport, S. Znaidi, M. Raymond, B. Turcotte, G. Sherlock, M. Costanzo, J. Ihmels, J. Berman, D. Sanglard, N. Agabian, A. P. Mitchell, A. D. Johnson, M. Whiteway and A. Nantel. 2005. A human-curated annotation of the Candida albicans genome. PLoS Genet. 1: 36-57.

17. Santos, M. A., T. Ueda, K. Watanabe and M. F. Tuite. 1997. The non-standard genetic code of Candida spp.: an evolving genetic code or a novel mechanism for adaptation? Mol Microbiol. 26: 423-31.

18. Fonzi, W. A. and M. Y. Irwin. 1993. Isogenic strain construction and gene mapping in Candida albicans. Genetics. 134: 717-28.

19. Magee, P. T., C. Gale, J. Berman and D. Davis. 2003. Molecular genetic and genomic approaches to the study of medically important fungi. Infect Immun. 71: 2299-309.

20. Morschhauser, J., S. Michel and P. Staib. 1999. Sequential gene disruption in Candida albicans by FLP-mediated site-specific recombination. Mol Microbiol. 32: $547-56$.

21. Wirsching, S., S. Michel and J. Morschhauser. 2000. Targeted gene disruption in Candida albicans wild-type strains: the role of the MDR1 gene in fluconazole resistance of clinical Candida albicans isolates. Mol Microbiol. 36: 856-65.

22. Reuss, O., A. Vik, R. Kolter and J. Morschhauser. 2004. The SAT1 flipper, an optimized tool for gene disruption in Candida albicans. Gene. 341: 119-27.

23. Rex, J. H., M. A. Pfaller, J. N. Galgiani, M. S. Bartlett, A. Espinel-Ingroff, M. A. Ghannoum, M. Lancaster, F. C. Odds, M. G. Rinaldi, T. J. Walsh and A. L. Barry. 1997. Development of interpretive breakpoints for antifungal susceptibility testing: conceptual framework and analysis of in vitro-in vivo correlation data for fluconazole, itraconazole, and candida infections. Subcommittee on Antifungal Susceptibility Testing of the National Committee for Clinical Laboratory Standards. Clin Infect Dis. 24: 235-47. 
24. Rex, J. H., M. G. Rinaldi and M. A. Pfaller. 1995. Resistance of Candida species to fluconazole. Antimicrob Agents Chemother. 39: 1-8.

25. Goldman, M., G. A. Cloud, K. D. Wade, A. C. Reboli, C. J. Fichtenbaum, R. Hafner, J. D. Sobel, W. G. Powderly, T. F. Patterson, L. J. Wheat, D. K. Stein, W. E. Dismukes and S. G. Filler. 2005. A randomized study of the use of fluconazole in continuous versus episodic therapy in patients with advanced HIV infection and a history of oropharyngeal candidiasis: AIDS Clinical Trials Group Study 323/Mycoses Study Group Study 40. Clin Infect Dis. 41: 1473-80.

26. Millon, L., A. Manteaux, G. Reboux, C. Drobacheff, M. Monod, T. Barale and Y. Michel-Briand. 1994. Fluconazole-resistant recurrent oral candidiasis in human immunodeficiency virus-positive patients: persistence of Candida albicans strains with the same genotype. J Clin Microbiol. 32: 1115-8.

27. Perea, S., J. L. Lopez-Ribot, W. R. Kirkpatrick, R. K. McAtee, R. A. Santillan, M. Martinez, D. Calabrese, D. Sanglard and T. F. Patterson. 2001. Prevalence of molecular mechanisms of resistance to azole antifungal agents in Candida albicans strains displaying high-level fluconazole resistance isolated from human immunodeficiency virus-infected patients. Antimicrob Agents Chemother. 45: 2676-84.

28. Albertson, G. D., M. Niimi, R. D. Cannon and H. F. Jenkinson. 1996. Multiple efflux mechanisms are involved in Candida albicans fluconazole resistance. Antimicrob Agents Chemother. 40: 2835-41.

29. Sanglard, D., K. Kuchler, F. Ischer, J. L. Pagani, M. Monod and J. Bille. 1995. Mechanisms of resistance to azole antifungal agents in Candida albicans isolates from AIDS patients involve specific multidrug transporters. Antimicrob Agents Chemother. 39: 2378-86.

30. Chang, G. 2003. Multidrug resistance ABC transporters. FEBS Lett. 555: 102-5.

31. Higgins, C. F. 2007. Multiple molecular mechanisms for multidrug resistance transporters. Nature. 446: 749-57.

32. Prasad, R., P. De Wergifosse, A. Goffeau and E. Balzi. 1995. Molecular cloning and characterization of a novel gene of Candida albicans, CDR1, conferring multiple resistance to drugs and antifungals. Curr Genet. 27: 320-9.

33. Balzi, E., M. Wang, S. Leterme, L. Van Dyck and A. Goffeau. 1994. PDR5, a novel yeast multidrug resistance conferring transporter controlled by the transcription regulator PDR1. J Biol Chem. 269: 2206-14.

34. Lyons, C. N. and T. C. White. 2000. Transcriptional analyses of antifungal drug resistance in Candida albicans. Antimicrob Agents Chemother. 44: 2296-303. 
35. White, T. C. 1997. Increased mRNA levels of ERG16, CDR, and MDR1 correlate with increases in azole resistance in Candida albicans isolates from a patient infected with human immunodeficiency virus. Antimicrob Agents Chemother. 41: 1482-7.

36. Sanglard, D., F. Ischer, M. Monod and J. Bille. 1996. Susceptibilities of Candida albicans multidrug transporter mutants to various antifungal agents and other metabolic inhibitors. Antimicrob Agents Chemother. 40: 2300-5.

37. Niimi, M., K. Niimi, Y. Takano, A. R. Holmes, F. J. Fischer, Y. Uehara and R. D. Cannon. 2004. Regulated overexpression of CDR1 in Candida albicans confers multidrug resistance. J Antimicrob Chemother. 54: 999-1006.

38. Sanglard, D., F. Ischer, M. Monod and J. Bille. 1997. Cloning of Candida albicans genes conferring resistance to azole antifungal agents: characterization of $C D R 2$, a new multidrug $A B C$ transporter gene. Microbiology. 143 ( Pt 2): 40516.

39. Pasrija, R., D. Banerjee and R. Prasad. 2007. Structure and function analysis of CaMdr1p, a major facilitator superfamily antifungal efflux transporter protein of Candida albicans: identification of amino acid residues critical for $\mathrm{drug} / \mathrm{H}+$ transport. Eukaryot Cell. 6: 443-53.

40. Akins, R. A. 2005. An update on antifungal targets and mechanisms of resistance in Candida albicans. Med Mycol. 43: 285-318.

41. Fling, M. E., J. Kopf, A. Tamarkin, J. A. Gorman, H. A. Smith and Y. Koltin. 1991. Analysis of a Candida albicans gene that encodes a novel mechanism for resistance to benomyl and methotrexate. Mol Gen Genet. 227: 318-29.

42. Goldway, M., D. Teff, R. Schmidt, A. B. Oppenheim and Y. Koltin. 1995. Multidrug resistance in Candida albicans: disruption of the BENr gene. Antimicrob Agents Chemother. 39: 422-6.

43. Franz, R., S. L. Kelly, D. C. Lamb, D. E. Kelly, M. Ruhnke and J. Morschhauser. 1998. Multiple molecular mechanisms contribute to a stepwise development of fluconazole resistance in clinical Candida albicans strains. Antimicrob Agents Chemother. 42: 3065-72.

44. Sanglard, D., F. Ischer, L. Koymans and J. Bille. 1998. Amino acid substitutions in the cytochrome P-450 lanosterol 14alpha-demethylase (CYP51A1) from azole-resistant Candida albicans clinical isolates contribute to resistance to azole antifungal agents. Antimicrob Agents Chemother. 42: 241-53. 
45. White, T. C. 1997. The presence of an R467K amino acid substitution and loss of allelic variation correlate with an azole-resistant lanosterol 14alpha demethylase in Candida albicans. Antimicrob Agents Chemother. 41: 1488-94.

46. Lamb, D. C., D. E. Kelly, T. C. White and S. L. Kelly. 2000. The R467K amino acid substitution in Candida albicans sterol 14alpha-demethylase causes drug resistance through reduced affinity. Antimicrob Agents Chemother. 44: 63-7.

47. Kontoyiannis, D. P., N. Sagar and K. D. Hirschi. 1999. Overexpression of Erg11p by the regulatable GAL1 promoter confers fluconazole resistance in Saccharomyces cerevisiae. Antimicrob Agents Chemother. 43: 2798-800.

48. De Backer, M. D., T. Ilyina, X. J. Ma, S. Vandoninck, W. H. Luyten and H. Vanden Bossche. 2001. Genomic profiling of the response of Candida albicans to itraconazole treatment using a DNA microarray. Antimicrob Agents Chemother. 45: 1660-70.

49. Henry, K. W., J. T. Nickels and T. D. Edlind. 2000. Upregulation of ERG genes in Candida species by azoles and other sterol biosynthesis inhibitors. Antimicrob Agents Chemother. 44: 2693-700.

50. Selmecki, A., A. Forche and J. Berman. 2006. Aneuploidy and isochromosome formation in drug-resistant Candida albicans. Science. 313: 367-70.

51. Manoharlal, R., N. A. Gaur, S. L. Panwar, J. Morschhauser and R. Prasad. 2008. Transcriptional Activation And Increased mRNA Stability Contribute To Overexpression Of CDR1 In Azole-Resistant Candida Albicans. Antimicrob Agents Chemother.

52. Vinson, C., M. Myakishev, A. Acharya, A. A. Mir, J. R. Moll and M.

Bonovich. 2002. Classification of human B-ZIP proteins based on dimerization properties. Mol Cell Biol. 22: 6321-35.

53. Chinenov, Y. and T. K. Kerppola. 2001. Close encounters of many kinds: FosJun interactions that mediate transcription regulatory specificity. Oncogene. 20: 2438-52.

54. Moye-Rowley, W. S., K. D. Harshman and C. S. Parker. 1989. Yeast YAP1 encodes a novel form of the jun family of transcriptional activator proteins. Genes Dev. 3: 283-92.

55. Alarco, A. M., I. Balan, D. Talibi, N. Mainville and M. Raymond. 1997. AP1mediated multidrug resistance in Saccharomyces cerevisiae requires FLR1 encoding a transporter of the major facilitator superfamily. J Biol Chem. 272: 19304-13. 
56. Harshman, K. D., W. S. Moye-Rowley and C. S. Parker. 1988. Transcriptional activation by the SV40 AP-1 recognition element in yeast is mediated by a factor similar to AP-1 that is distinct from GCN4. Cell. 53: 321-30.

57. Toone, W. M. and N. Jones. 1999. AP-1 transcription factors in yeast. Curr Opin Genet Dev. 9: 55-61.

58. Toone, W. M., B. A. Morgan and N. Jones. 2001. Redox control of AP-1-like factors in yeast and beyond. Oncogene. 20: 2336-46.

59. Moye-Rowley, W. S. 2003. Regulation of the transcriptional response to oxidative stress in fungi: similarities and differences. Eukaryot Cell. 2: 381-9.

60. Kuge, S., N. Jones and A. Nomoto. 1997. Regulation of yAP-1 nuclear localization in response to oxidative stress. EMBO J. 16: 1710-20.

61. Yan, C., L. H. Lee and L. I. Davis. 1998. Crm1p mediates regulated nuclear export of a yeast AP-1-like transcription factor. EMBO J. 17: 7416-29.

62. Kuge, S., M. Arita, A. Murayama, K. Maeta, S. Izawa, Y. Inoue and A. Nomoto. 2001. Regulation of the yeast Yap1p nuclear export signal is mediated by redox signal-induced reversible disulfide bond formation. Mol Cell Biol. 21: 6139-50.

63. Wemmie, J. A., S. M. Steggerda and W. S. Moye-Rowley. 1997. The Saccharomyces cerevisiae AP-1 protein discriminates between oxidative stress elicited by the oxidants H2O2 and diamide. J Biol Chem. 272: 7908-14.

64. MacPherson, S., M. Larochelle and B. Turcotte. 2006. A fungal family of transcriptional regulators: the zinc cluster proteins. Microbiol Mol Biol Rev. 70: 583-604.

65. Miller, J., A. D. McLachlan and A. Klug. 1985. Repetitive zinc-binding domains in the protein transcription factor IIIA from Xenopus oocytes. EMBO J. 4: 1609-14.

66. Akache, B. and B. Turcotte. 2002. New regulators of drug sensitivity in the family of yeast zinc cluster proteins. J Biol Chem. 277: 21254-60.

67. Akache, B., K. Wu and B. Turcotte. 2001. Phenotypic analysis of genes encoding yeast zinc cluster proteins. Nucleic Acids Res. 29: 2181-90.

68. Reece, R. J. and M. Ptashne. 1993. Determinants of binding-site specificity among yeast C6 zinc cluster proteins. Science. 261: 909-11. 
69. Schjerling, P. and S. Holmberg. 1996. Comparative amino acid sequence analysis of the $\mathrm{C} 6$ zinc cluster family of transcriptional regulators. Nucleic Acids Res. 24: 4599-607.

70. Zhou, K. M., Y. L. Bai and G. B. Kohlhaw. 1990. Yeast regulatory protein LEU3: a structure-function analysis. Nucleic Acids Res. 18: 291-8.

71. Nourani, A., D. Papajova, A. Delahodde, C. Jacq and J. Subik. 1997.

Clustered amino acid substitutions in the yeast transcription regulator Pdr3p increase pleiotropic drug resistance and identify a new central regulatory domain. Mol Gen Genet. 256: 397-405.

72. Wirsching, S., S. Michel, G. Kohler and J. Morschhauser. 2000. Activation of the multiple drug resistance gene MDR1 in fluconazole-resistant, clinical Candida albicans strains is caused by mutations in a trans-regulatory factor. $\mathrm{J}$ Bacteriol. 182: 400-4.

73. de Micheli, M., J. Bille, C. Schueller and D. Sanglard. 2002. A common drugresponsive element mediates the upregulation of the Candida albicans $\mathrm{ABC}$ transporters CDR1 and CDR2, two genes involved in antifungal drug resistance. Mol Microbiol. 43: 1197-214.

74. Nguyen, D. T., A. M. Alarco and M. Raymond. 2001. Multiple Yap1p-binding sites mediate induction of the yeast major facilitator FLR1 gene in response to drugs, oxidants, and alkylating agents. J Biol Chem. 276: 1138-45.

75. Mamnun, Y. M., R. Pandjaitan, Y. Mahe, A. Delahodde and K. Kuchler. 2002. The yeast zinc finger regulators Pdr1p and Pdr3p control pleiotropic drug resistance (PDR) as homo- and heterodimers in vivo. Mol Microbiol. 46: 1429-40.

76. Katzmann, D. J., T. C. Hallstrom, Y. Mahe and W. S. Moye-Rowley. 1996. Multiple Pdr1p/Pdr3p binding sites are essential for normal expression of the ATP binding cassette transporter protein-encoding gene PDR5. J Biol Chem. 271: 23049-54.

77. Kolaczkowska, A. and A. Goffeau. 1999. Regulation of pleiotropic drug resistance in yeast. Drug Resist Updat. 2: 403-14.

78. Carvajal, E., H. B. van den Hazel, A. Cybularz-Kolaczkowska, E. Balzi and A. Goffeau. 1997. Molecular and phenotypic characterization of yeast PDR1 mutants that show hyperactive transcription of various $\mathrm{ABC}$ multidrug transporter genes. Mol Gen Genet. 256: 406-15.

79. Meyers, S., W. Schauer, E. Balzi, M. Wagner, A. Goffeau and J. Golin. 1992. Interaction of the yeast pleiotropic drug resistance genes PDR1 and PDR5. Curr Genet. 21: 431-6. 
80. Katzmann, D. J., P. E. Burnett, J. Golin, Y. Mahe and W. S. Moye-Rowley. 1994. Transcriptional control of the yeast PDR5 gene by the PDR3 gene product. Mol Cell Biol. 14: 4653-61.

81. Vik, A. and J. Rine. 2001. Upc2p and Ecm22p, dual regulators of sterol biosynthesis in Saccharomyces cerevisiae. Mol Cell Biol. 21: 6395-405.

82. MacPherson, S., B. Akache, S. Weber, X. De Deken, M. Raymond and B. Turcotte. 2005. Candida albicans zinc cluster protein Upc2p confers resistance to antifungal drugs and is an activator of ergosterol biosynthetic genes. Antimicrob Agents Chemother. 49: 1745-52.

83. Zhang, X., M. De Micheli, S. T. Coleman, D. Sanglard and W. S. MoyeRowley. 2000. Analysis of the oxidative stress regulation of the Candida albicans transcription factor, Cap1p. Mol Microbiol. 36: 618-29.

84. Alarco, A. M. and M. Raymond. 1999. The bZip transcription factor Cap1p is involved in multidrug resistance and oxidative stress response in Candida albicans. J Bacteriol. 181: 700-8.

85. Harry, J. B., B. G. Oliver, J. L. Song, P. M. Silver, J. T. Little, J. Choiniere and T. C. White. 2005. Drug-induced regulation of the MDR1 promoter in Candida albicans. Antimicrob Agents Chemother. 49: 2785-92.

86. Hiller, D., S. Stahl and J. Morschhauser. 2006. Multiple cis-acting sequences mediate upregulation of the MDR1 efflux pump in a fluconazole-resistant clinical Candida albicans isolate. Antimicrob Agents Chemother. 50: 2300-8.

87. Rognon, B., Z. Kozovska, A. T. Coste, G. Pardini and D. Sanglard. 2006. Identification of promoter elements responsible for the regulation of MDR1 from Candida albicans, a major facilitator transporter involved in azole resistance. Microbiology. 152: 3701-22.

88. Coste, A. T., M. Karababa, F. Ischer, J. Bille and D. Sanglard. 2004. TAC1, transcriptional activator of CDR genes, is a new transcription factor involved in the regulation of Candida albicans $\mathrm{ABC}$ transporters CDR1 and CDR2. Eukaryot Cell. 3: 1639-52.

89. Coste, A., V. Turner, F. Ischer, J. Morschhauser, A. Forche, A. Selmecki, J. Berman, J. Bille and D. Sanglard. 2006. A mutation in Tac1p, a transcription factor regulating $C D R 1$ and $C D R 2$, is coupled with loss of heterozygosity at chromosome 5 to mediate antifungal resistance in Candida albicans. Genetics. 172: $2139-56$. 
90. Silver, P. M., B. G. Oliver and T. C. White. 2004. Role of Candida albicans transcription factor Upc2p in drug resistance and sterol metabolism. Eukaryot Cell. 3: 1391-7.

91. Lopez-Ribot, J. L., R. K. McAtee, L. N. Lee, W. R. Kirkpatrick, T. C. White, D. Sanglard and T. F. Patterson. 1998. Distinct patterns of gene expression associated with development of fluconazole resistance in serial Candida albicans isolates from human immunodeficiency virus-infected patients with oropharyngeal candidiasis. Antimicrob. Agents Chemother. 42: 2932-7.

92. White, T. C. 1997. Increased mRNA levels of ERG16, CDR, and MDR1 correlate with increases in azole resistance in Candida albicans isolates from a patient infected with human immunodeficiency virus. Antimicrob. Agents Chemother. 41: 1482-7.

93. White, T. C. 1997. The presence of an R467K amino acid substitution and loss of allelic variation correlate with an azole-resistant lanosterol 14alpha demethylase in Candida albicans. Antimicrob. Agents Chemother. 41: 1488-94.

94. Franz, R., S. L. Kelly, D. C. Lamb, D. E. Kelly, M. Ruhnke and J. Morschhauser. 1998. Multiple molecular mechanisms contribute to a stepwise development of fluconazole resistance in clinical Candida albicans strains. Antimicrob. Agents Chemother. 42: 3065-72.

95. Franz, R., M. Ruhnke and J. Morschhauser. 1999. Molecular aspects of fluconazole resistance development in Candida albicans. Mycoses. 42: 453-8.

96. Sanglard, D., K. Kuchler, F. Ischer, J. L. Pagani, M. Monod and J. Bille. 1995. Mechanisms of resistance to azole antifungal agents in Candida albicans isolates from AIDS patients involve specific multidrug transporters. Antimicrob. Agents Chemother. 39: 2378-86.

97. Sanglard, D., F. Ischer, L. Koymans and J. Bille. 1998. Amino acid substitutions in the cytochrome P-450 lanosterol 14alpha-demethylase (CYP51A1) from azole-resistant Candida albicans clinical isolates contribute to resistance to azole antifungal agents. Antimicrob. Agents Chemother. 42: 241-53.

98. Perea, S., J. L. Lopez-Ribot, W. R. Kirkpatrick, R. K. McAtee, R. A. Santillan, M. Martinez, D. Calabrese, D. Sanglard and T. F. Patterson. 2001. Prevalence of molecular mechanisms of resistance to azole antifungal agents in Candida albicans strains displaying high-level fluconazole resistance isolated from human immunodeficiency virus-infected patients. Antimicrob. Agents Chemother. 45: 2676-84.

99. de Micheli, M., J. Bille, C. Schueller and D. Sanglard. 2002. A common drugresponsive element mediates the upregulation of the Candida albicans $\mathrm{ABC}$ 
transporters $C D R 1$ and $C D R 2$, two genes involved in antifungal drug resistance. Mol. Microbiol. 43: 1197-214.

100. Coste, A. T., M. Karababa, F. Ischer, J. Bille and D. Sanglard. 2004. TAC1, transcriptional activator of CDR genes, is a new transcription factor involved in the regulation of Candida albicans $\mathrm{ABC}$ transporters $C D R 1$ and $C D R 2$. Eukaryot. Cell. 3: 1639-52.

101. Coste, A., A. Selmecki, A. Forche, D. Diogo, M. E. Bougnoux, C. d'Enfert, J. Berman and D. Sanglard. 2007. Genotypic evolution of azole resistance mechanisms in sequential Candida albicans isolates. Eukaryot. Cell doi:10.1128/EC.00151-07.

102. Saidane, S., S. Weber, X. De Deken, G. St-Germain and M. Raymond. 2006. PDR 16-mediated azole resistance in Candida albicans. Mol. Microbiol. 60: 154662.

103. Znaidi, S., X. De Deken, S. Weber, T. Rigby, A. Nantel and M. Raymond. The zinc cluster transcription factor Tac1p regulates PDR16 expression in Candida albicans. Mol. Microbiol., in press.

104. Sherman, F. 1991. Getting started with yeast. Methods Enzymol. 194: 3-21.

105. Albert, T. J., J. Norton, M. Ott, T. Richmond, K. Nuwaysir, E. F. Nuwaysir, K. P. Stengele and R. D. Green. 2003. Light-directed 5'-->3' synthesis of complex oligonucleotide microarrays. Nucleic Acids Res. 31: e35.

106. White, T. C., M. A. Pfaller, M. G. Rinaldi, J. Smith and S. W. Redding. 1997. Stable azole drug resistance associated with a substrain of Candida albicans from an HIV-infected patient. Oral Dis. 3 Suppl 1: S102-9.

107. Pfaller, M. A., C. Grant, V. Morthland and J. Rhine-Chalberg. 1994. Comparative evaluation of alternative methods for broth dilution susceptibility testing of fluconazole against Candida albicans. J. Clin. Microbiol. 32: 506-9.

108. Rogers, P. D. and K. S. Barker. 2003. Genome-wide expression profile analysis reveals coordinately regulated genes associated with stepwise acquisition of azole resistance in Candida albicans clinical isolates. Antimicrob. Agents Chemother. 47: 1220-7.

109. Schmitt, M. E., T. A. Brown and B. L. Trumpower. 1990. A rapid and simple method for preparation of RNA from Saccharomyces cerevisiae. Nucleic Acids Res. 18: 3091-2. 
110. Care, R. S., J. Trevethick, K. M. Binley and P. E. Sudbery. 1999. The MET3 promoter: a new tool for Candida albicans molecular genetics. Mol. Microbiol. 34: $792-8$.

111. Schneider, B. L., W. Seufert, B. Steiner, Q. H. Yang and A. B. Futcher. 1995. Use of polymerase chain reaction epitope tagging for protein tagging in Saccharomyces cerevisiae. Yeast. 11: 1265-74.

112. Boeke, J. D., F. LaCroute and G. R. Fink. 1984. A positive selection for mutants lacking orotidine-5'-phosphate decarboxylase activity in yeast: 5-fluoroorotic acid resistance. Mol. Gen. Genet. 197: 345-6.

113. Ito, H., Y. Fukuda, K. Murata and A. Kimura. 1983. Transformation of intact yeast cells treated with alkali cations. J. Bacteriol. 153: 163-8.

114. Rose, M. D., F. Winston and P. Hieter. 1990. Methods in yeast genetics: a laboratory course manual. Cold Spring Harbor Laboratory Press, Cold Spring Harbor, New York.

115. Drouin, S. and F. Robert. Genome-wide location analysis of chromatinassociated proteins by ChIP on chip: controls matter. Methods, in press.

116. Hokamp, K., F. M. Roche, M. Acab, M. E. Rousseau, B. Kuo, D. Goode, D. Aeschliman, J. Bryan, L. A. Babiuk, R. E. Hancock and F. S. Brinkman. 2004. ArrayPipe: a flexible processing pipeline for microarray data. Nucleic Acids Res. 32: W457-9.

117. Ren, B., F. Robert, J. J. Wyrick, O. Aparicio, E. G. Jennings, I. Simon, J. Zeitlinger, J. Schreiber, N. Hannett, E. Kanin, T. L. Volkert, C. J. Wilson, S. P. Bell and R. A. Young. 2000. Genome-wide location and function of DNA binding proteins. Science. 290: 2306-9.

118. Livak, K. J. and T. D. Schmittgen. 2001. Analysis of relative gene expression data using real-time quantitative PCR and the 2(-Delta Delta C(T)) Method. Methods. 25: 402-8.

119. De Deken, X. and M. Raymond. 2004. Constitutive activation of the PDR16 promoter in a Candida albicans azole-resistant clinical isolate overexpressing $C D R 1$ and $C D R 2$. Antimicrob. Agents Chemother. 48: 2700-3.

120. Kelly, R. and K. J. Kwon-Chung. 1992. A zinc finger protein from Candida albicans is involved in sucrose utilization. J. Bacteriol. 174: 222-32.

121. MacPherson, S., M. Larochelle and B. Turcotte. 2006. A fungal family of transcriptional regulators: the zinc cluster proteins. Microbiol. Mol. Biol. Rev. 70: 583-604. 
122. Karababa, M., A. T. Coste, B. Rognon, J. Bille and D. Sanglard. 2004. Comparison of gene expression profiles of Candida albicans azole-resistant clinical isolates and laboratory strains exposed to drugs inducing multidrug transporters. Antimicrob. Agents Chemother. 48: 3064-79.

123. Lee, T. I., N. J. Rinaldi, F. Robert, D. T. Odom, Z. Bar-Joseph, G. K. Gerber, N. M. Hannett, C. T. Harbison, C. M. Thompson, I. Simon, J. Zeitlinger, E. G. Jennings, H. L. Murray, D. B. Gordon, B. Ren, J. J. Wyrick, J. B. Tagne, T. L. Volkert, E. Fraenkel, D. K. Gifford and R. A. Young. 2002. Transcriptional regulatory networks in Saccharomyces cerevisiae. Science. 298: 799-804.

124. Balzi, E. and A. Goffeau. 1995. Yeast multidrug resistance: the PDR network. J. Bioenerg. Biomembr. 27: 71-6.

125. Fardeau, V., G. Lelandais, A. Oldfield, H. Salin, S. Lemoine, M. Garcia, V. Tanty, S. Le Crom, C. Jacq and F. Devaux. 2007. The central role of PDR1 in the foundation of yeast drug resistance. J. Biol. Chem. 282: 5063-74.

126. Kren, A., Y. M. Mamnun, B. E. Bauer, C. Schuller, H. Wolfger, K. Hatzixanthis, M. Mollapour, C. Gregori, P. Piper and K. Kuchler. 2003. War1p, a novel transcription factor controlling weak acid stress response in yeast. Mol. Cell. Biol. 23: 1775-85.

127. Davies, B. S., H. S. Wang and J. Rine. 2005. Dual activators of the sterol biosynthetic pathway of Saccharomyces cerevisiae: similar activation/regulatory domains but different response mechanisms. Mol. Cell. Biol. 25: 7375-85.

128. Gao, C., L. Wang, E. Milgrom and W. C. Shen. 2004. On the mechanism of constitutive Pdr1 activator-mediated PDR5 transcription in Saccharomyces cerevisiae: evidence for enhanced recruitment of coactivators and altered nucleosome structures. J. Biol. Chem. 279: 42677-86.

129. Angus-Hill, M. L., A. Schlichter, D. Roberts, H. Erdjument-Bromage, P. Tempst and B. R. Cairns. 2001. A Rsc3/Rsc30 zinc cluster dimer reveals novel roles for the chromatin remodeler RSC in gene expression and cell cycle control. Mol. Cell. 7: 741-51.

130. Ogawa, N. and K. Mori. 2004. Autoregulation of the $H A C 1$ gene is required for sustained activation of the yeast unfolded protein response. Genes Cells. 9: 95104.

131. Zhou, P. and D. J. Thiele. 1993. Rapid transcriptional autoregulation of a yeast metalloregulatory transcription factor is essential for high-level copper detoxification. Genes Dev. 7: 1824-35. 
132. Prasad, R., P. De Wergifosse, A. Goffeau and E. Balzi. 1995. Molecular cloning and characterization of a novel gene of Candida albicans, CDR1, conferring multiple resistance to drugs and antifungals. Curr. Genet. 27: 320-9.

133. Black, S. M. and C. R. Wolf. 1991. The role of glutathione-dependent enzymes in drug resistance. Pharmacol. Ther. 51: 139-54.

134. Doroshow, J. H., S. Akman, F. F. Chu and S. Esworthy. 1990. Role of the glutathione-glutathione peroxidase cycle in the cytotoxicity of the anticancer quinones. Pharmacol. Ther. 47: 359-70.

135. Buser, K., F. Joncourt, H. J. Altermatt, M. Bacchi, A. Oberli and T. Cerny. 1997. Breast cancer: pretreatment drug resistance parameters (GSH-system, ATase, P-glycoprotein) in tumor tissue and their correlation with clinical and prognostic characteristics. Ann. Oncol. 8: 335-41.

136. Calera, J. A. and R. Calderone. 1999. Histidine kinase, two-component signal transduction proteins of Candida albicans and the pathogenesis of candidosis. Mycoses. 42 Suppl 2: 49-53.

137. Calera, J. A., G. H. Choi and R. A. Calderone. 1998. Identification of a putative histidine kinase two-component phosphorelay gene (CaHKl) in Candida albicans. Yeast. 14: 665-74.

138. Chauhan, N., M. Kruppa and R. Calderone. 2007. The Ssk1p response regulator and Chk1p histidine kinase mutants of Candida albicans are hypersensitive to fluconazole and voriconazole. Antimicrob. Agents Chemother., in press. (AAC00929-7).

139. Wada, S., M. Niimi, K. Niimi, A. R. Holmes, B. C. Monk, R. D. Cannon and Y. Uehara. 2002. Candida glabrata ATP-binding cassette transporters Cdr1p and Pdh1p expressed in a Saccharomyces cerevisiae strain deficient in membrane transporters show phosphorylation-dependent pumping properties. J. Biol. Chem. 277: 46809-21.

140. Wada, S., K. Tanabe, A. Yamazaki, M. Niimi, Y. Uehara, K. Niimi, E. Lamping, R. D. Cannon and B. C. Monk. 2005. Phosphorylation of Candida glabrata ATP-binding cassette transporter $\mathrm{Cdr} 1 \mathrm{p}$ regulates drug efflux activity and ATPase stability. J. Biol. Chem. 280: 94-103.

141. Sadowski, I., C. Costa and R. Dhanawansa. 1996. Phosphorylation of Ga14p at a single $\mathrm{C}$-terminal residue is necessary for galactose-inducible transcription. Mol. Cell. Biol. 16: 4879-87. 
142. Mamnun, Y. M., R. Pandjaitan, Y. Mahe, A. Delahodde and K. Kuchler. 2002. The yeast zinc finger regulators Pdr1p and Pdr3p control pleiotropic drug resistance (PDR) as homo- and heterodimers in vivo. Mol. Microbiol. 46: 1429-40.

143. Kihara, A. and Y. Igarashi. 2002. Identification and characterization of a Saccharomyces cerevisiae gene, RSB1, involved in sphingoid long-chain base release. J. Biol. Chem. 277: 30048-54.

144. Funato, K., R. Lombardi, B. Vallee and H. Riezman. 2003. Lcb4p is a key regulator of ceramide synthesis from exogenous long chain sphingoid base in Saccharomyces cerevisiae. J. Biol. Chem. 278: 7325-34.

145. Mao, C., R. Xu, A. Bielawska, Z. M. Szulc and L. M. Obeid. 2000. Cloning and characterization of a Saccharomyces cerevisiae alkaline ceramidase with specificity for dihydroceramide. J. Biol. Chem. 275: 31369-78.

146. Smriti, S. Krishnamurthy, B. L. Dixit, C. M. Gupta, S. Milewski and R. Prasad. 2002. ABC transporters Cdr1p, Cdr2p and Cdr3p of a human pathogen Candida albicans are general phospholipid translocators. Yeast. 19: 303-18.

147. Bagnat, M., A. Chang and K. Simons. 2001. Plasma membrane proton ATPase Pmalp requires raft association for surface delivery in yeast. Mol. Biol. Cell. 12: 4129-38.

148. Insenser, M., C. Nombela, G. Molero and C. Gil. 2006. Proteomic analysis of detergent-resistant membranes from Candida albicans. Proteomics. 6 Suppl 1: S74-81.

149. Mukhopadhyay, K., T. Prasad, P. Saini, T. J. Pucadyil, A. Chattopadhyay and R. Prasad. 2004. Membrane sphingolipid-ergosterol interactions are important determinants of multidrug resistance in Candida albicans. Antimicrob. Agents Chemother. 48: 1778-87.

150. Phillips, S. E., P. Vincent, K. E. Rizzieri, G. Schaaf, V. A. Bankaitis and E. A. Gaucher. 2006. The diverse biological functions of phosphatidylinositol transfer proteins in eukaryotes. Crit. Rev. Biochem. Mol. Biol. 41: 21-49.

151. Schnabl, M., O. V. Oskolkova, R. Holic, B. Brezna, H. Pichler, M. Zagorsek, S. D. Kohlwein, F. Paltauf, G. Daum and P. Griac. 2003. Subcellular localization of yeast $\mathrm{Sec} 14$ homologues and their involvement in regulation of phospholipid turnover. Eur. J. Biochem. 270: 3133-45.

152. Holthuis, J. C. and T. P. Levine. 2005. Lipid traffic: floppy drives and a superhighway. Nat. Rev. Mol. Cell Biol. 6: 209-20. 
153. Derewenda, Z. S. and U. Derewenda. 1991. Relationships among serine hydrolases: evidence for a common structural motif in triacylglyceride lipases and esterases. Biochem. Cell Biol. 69: 842-51.

154. Wagner, A. and G. Daum. 2005. Formation and mobilization of neutral lipids in the yeast Saccharomyces cerevisiae. Biochem. Soc. Trans. 33: 1174-7.

155. Koffel, R. and R. Schneiter. 2006. Yeh1 constitutes the major steryl ester hydrolase under heme-deficient conditions in Saccharomyces cerevisiae. Eukaryot. Cell. 5: 1018-25.

156. Koffel, R., R. Tiwari, L. Falquet and R. Schneiter. 2005. The Saccharomyces cerevisiae YLL012/YEH1, YLRO20/YEH2, and TGL1 genes encode a novel family of membrane-anchored lipases that are required for steryl ester hydrolysis. Mol. Cell. Biol. 25: 1655-68.

157. Athenstaedt, K., D. Zweytick, A. Jandrositz, S. D. Kohlwein and G. Daum. 1999. Identification and characterization of major lipid particle proteins of the yeast Saccharomyces cerevisiae. J. Bacteriol. 181: 6441-8.

158. Natter, K., P. Leitner, A. Faschinger, H. Wolinski, S. McCraith, S. Fields and S. D. Kohlwein. 2005. The spatial organization of lipid synthesis in the yeast Saccharomyces cerevisiae derived from large scale green fluorescent protein tagging and high resolution microscopy. Mol. Cell. Proteomics. 4: 662-72.

159. Verstrepen, K. J., S. D. Van Laere, J. Vercammen, G. Derdelinckx, J. P. Dufour, I. S. Pretorius, J. Winderickx, J. M. Thevelein and F. R. Delvaux. 2004. The Saccharomyces cerevisiae alcohol acetyl transferase Atflp is localized in lipid particles. Yeast. 21: 367-77.

160. Kusch, H., S. Engelmann, D. Albrecht, J. Morschhauser and M. Hecker. 2007. Proteomic analysis of the oxidative stress response in Candida albicans. Proteomics. 7: 686-97.

161. Avery, A. M. and S. V. Avery. 2001. Saccharomyces cerevisiae expresses three phospholipid hydroperoxide glutathione peroxidases. J. Biol. Chem. 276: 33730-5.

162. Martchenko, M., A. M. Alarco, D. Harcus and M. Whiteway. 2004. Superoxide dismutases in Candida albicans: transcriptional regulation and functional characterization of the hyphal-induced SOD5 gene. Mol. Biol. Cell. 15: 456-67.

163. Gauthier, C., S. Weber, A. M. Alarco, O. Alqawi, R. Daoud, E. Georges and M. Raymond. 2003. Functional similarities and differences between Candida albicans Cdr1p and Cdr2p transporters. Antimicrob. Agents Chemother. 47: 1543-54. 
164. Wang, Y., Y. Y. Cao, X. M. Jia, Y. B. Cao, P. H. Gao, X. P. Fu, K. Ying, W. S. Chen and Y. Y. Jiang. 2006. Caplp is involved in multiple pathways of oxidative stress response in Candida albicans. Free Radic Biol Med. 40: 1201-9.

165. Enjalbert, B., A. Nantel and M. Whiteway. 2003. Stress-induced gene expression in Candida albicans: absence of a general stress response. Mol Biol Cell. 14: 1460-7.

166. Lucau-Danila, A., T. Delaveau, G. Lelandais, F. Devaux and C. Jacq. 2003. Competitive promoter occupancy by two yeast paralogous transcription factors controlling the multidrug resistance phenomenon. J Biol Chem. 278: 52641-50.

167. Kelly, S. L., A. Arnoldi and D. E. Kelly. 1993. Molecular genetic analysis of azole antifungal mode of action. Biochem Soc Trans. 21: 1034-8.

168. Lopez-Ribot, J. L., R. K. McAtee, L. N. Lee, W. R. Kirkpatrick, T. C. White, D. Sanglard and T. F. Patterson. 1998. Distinct patterns of gene expression associated with development of fluconazole resistance in serial candida albicans isolates from human immunodeficiency virus-infected patients with oropharyngeal candidiasis. Antimicrob Agents Chemother. 42: 2932-7.

169. Morschhauser, J. 2002. The genetic basis of fluconazole resistance development in Candida albicans. Biochim Biophys Acta. 1587: 240-8.

170. Lamb, D. C., D. E. Kelly, W. H. Schunck, A. Z. Shyadehi, M. Akhtar, D. J. Lowe, B. C. Baldwin and S. L. Kelly. 1997. The mutation T315A in Candida albicans sterol 14alpha-demethylase causes reduced enzyme activity and fluconazole resistance through reduced affinity. J Biol Chem. 272: 5682-8.

171. Nakayama, H., N. Nakayama, M. Arisawa and Y. Aoki. 2001. In vitro and in vivo effects of 14alpha-demethylase (ERG11) depletion in Candida glabrata. Antimicrob Agents Chemother. 45: 3037-45.

172. Morschhauser, J., K. S. Barker, T. T. Liu, B. W. J. Bla, R. Homayouni and P. D. Rogers. 2007. The transcription factor Mrrlp controls expression of the MDR1 efflux pump and mediates multidrug resistance in Candida albicans. PLoS Pathog. 3: e164.

173. Barker, K. S., S. Crisp, N. Wiederhold, R. E. Lewis, B. Bareither, J. Eckstein, R. Barbuch, M. Bard and P. D. Rogers. 2004. Genome-wide expression profiling reveals genes associated with amphotericin B and fluconazole resistance in experimentally induced antifungal resistant isolates of Candida albicans. $\mathbf{J}$ Antimicrob Chemother. 54: 376-85. 
174. Liu, T. T., R. E. Lee, K. S. Barker, L. Wei, R. Homayouni and P. D. Rogers. 2005. Genome-wide expression profiling of the response to azole, polyene, echinocandin, and pyrimidine antifungal agents in Candida albicans. Antimicrob Agents Chemother. 49: 2226-36.

175. Schmitt, M. E., T. A. Brown and B. L. Trumpower. 1990. A rapid and simple method for preparation of RNA from Saccharomyces cerevisiae. Nucleic Acids Res. 18: 3091-2.

176. Gillum, A. M., E. Y. Tsay and D. R. Kirsch. 1984. Isolation of the Candida albicans gene for orotidine-5'-phosphate decarboxylase by complementation of S. cerevisiae ura3 and E. coli pyrF mutations. Mol Gen Genet. 198: 179-82.

177. Oliver, B. G., J. L. Song, J. H. Choiniere and T. C. White. 2007. cis-Acting elements within the Candida albicans ERG11 promoter mediate the azole response through transcription factor Upc2p. Eukaryot Cell. 6: 2231-9.

178. Liu, T. T., S. Znaidi, K. S. Barker, L. Xu, R. Homayouni, S. Saidane, J. Morschhauser, A. Nantel, M. Raymond and P. D. Rogers. 2007. Genome-wide expression and location analyses of the Candida albicans Taclp regulon. Eukaryot Cell. 6: 2122-38.

179. Crowley, J. H., F. W. Leak, Jr., K. V. Shianna, S. Tove and L. W. Parks. 1998. A mutation in a purported regulatory gene affects control of sterol uptake in Saccharomyces cerevisiae. J Bacteriol. 180: 4177-83.

180. Shianna, K. V., W. D. Dotson, S. Tove and L. W. Parks. 2001. Identification of a UPC2 homolog in Saccharomyces cerevisiae and its involvement in aerobic sterol uptake. J Bacteriol. 183: 830-4.

181. Leak, F. W., Jr., S. Tove and L. W. Parks. 1999. In yeast, upc2-1 confers a decrease in tolerance to $\mathrm{LiCl}$ and $\mathrm{NaCl}$, which can be suppressed by the P-type ATPase encoded by ENA2. DNA Cell Biol. 18: 133-9.

182. Copping, V. M., C. J. Barelle, B. Hube, N. A. Gow, A. J. Brown and F. C. Odds. 2005. Exposure of Candida albicans to antifungal agents affects expression of SAP2 and SAP9 secreted proteinase genes. J Antimicrob Chemother. 55: 64554.

183. Weissman, Z. and D. Kornitzer. 2004. A family of Candida cell surface haembinding proteins involved in haemin and haemoglobin-iron utilization. Mol Microbiol. 53: 1209-20.

184. Prasad, T., A. Chandra, C. K. Mukhopadhyay and R. Prasad. 2006. Unexpected link between iron and drug resistance of Candida spp.: iron depletion 
enhances membrane fluidity and drug diffusion, leading to drug-susceptible cells. Antimicrob Agents Chemother. 50: 3597-606.

185. Setiadi, E. R., T. Doedt, F. Cottier, C. Noffz and J. F. Ernst. 2006.

Transcriptional response of Candida albicans to hypoxia: linkage of oxygen sensing and Efg1p-regulatory networks. J Mol Biol. 361: 399-411.

186. Wilcox, L. J., D. A. Balderes, B. Wharton, A. H. Tinkelenberg, G. Rao and S. L. Sturley. 2002. Transcriptional profiling identifies two members of the ATPbinding cassette transporter superfamily required for sterol uptake in yeast. J Biol Chem. 277: 32466-72.

187. Aguirre, J. M., M. A. Echebarria, E. Ocina, L. Ribacoba and M. Montejo. 1999. Reduction of HIV-associated oral lesions after highly active antiretroviral therapy. Oral Surg Oral Med Oral Pathol Oral Radiol Endod. 88: 114-5.

188. Revankar, S. G., S. E. Sanche, O. P. Dib, M. Caceres and T. F. Patterson. 1998. Effect of highly active antiretroviral therapy on recurrent oropharyngeal candidiasis in HIV-infected patients. AIDS. 12: 2511-3.

189. Lattif, A. A., U. Banerjee, R. Prasad, A. Biswas, N. Wig, N. Sharma, A. Haque, N. Gupta, N. Z. Baquer and G. Mukhopadhyay. 2004. Susceptibility pattern and molecular type of species-specific Candida in oropharyngeal lesions of Indian human immunodeficiency virus-positive patients. J Clin Microbiol. 42: 1260-2. 
APPENDIX A: TAC1 SUPPLEMENTAL DATA

Table A-1. Genes up-regulated in at least one matched clinical isolate.

\begin{tabular}{|c|c|c|c|c|c|c|c|c|}
\hline $\begin{array}{c}\text { Systematic } \\
\text { Name }^{a}\end{array}$ & $\begin{array}{c}\text { CGD } \\
\text { Name }^{b}\end{array}$ & $\begin{array}{c}\text { CandidaDB } \\
\text { Name }^{c}\end{array}$ & GO Annotation (Molecular Function) ${ }^{d}$ & $\begin{array}{l}\text { C56 vs. } \\
\text { C43 }\end{array}$ & $\begin{array}{c}\text { Gu5 vs. } \\
\text { Gu2 }\end{array}$ & $\begin{array}{c}17 \text { vs. } \\
3\end{array}$ & $\begin{array}{c}\mathbf{5 6 7 4} \text { vs. } \\
\mathbf{5 4 5 7}\end{array}$ & $\begin{array}{c}\text { SZY31 vs. } \\
5457\end{array}$ \\
\hline orf19.6229 & CAT1 & CTA1 & Catalase activity & 1.7 & 1.1 & -1.0 & -1.1 & -1.1 \\
\hline orf19.3682 & CWH8 & CWH8 & Pyrophosphatase activity & 2.0 & 1.1 & -1.0 & 1.1 & -1.1 \\
\hline orf19.7056 & -- & DIP53.exon2 & Amino acid permease activity & 2.1 & -1.0 & 1.2 & 2.6 & 1.1 \\
\hline orf19.781 & -- & DUR31 & Urea transporter activity & 2.0 & -1.4 & -1.0 & 1.4 & 1.6 \\
\hline orf19.5017 & -- & DUR32 & Urea transporter activity & 1.8 & -1.4 & -1.2 & 1.2 & 1.1 \\
\hline orf19.767 & $E R G 3$ & ERG3 & C-5 sterol desaturase activity & 1.5 & 1.1 & 1.4 & -1.2 & 1.0 \\
\hline orf19.1631 & ERG6 & ERG6 & Sterol 24-C-methyltransferase activity & 1.8 & 1.2 & 1.4 & -1.7 & -1.3 \\
\hline orf19.460 & CEK2 & ERK2 & MAP kinase activity & 1.8 & 1.2 & 1.8 & 1.7 & 2.8 \\
\hline orf19.4015 & CAG1 & GBA1 & GTPase activity & 30.8 & 1.1 & -1.3 & 2.3 & 30.8 \\
\hline orf19.1392 & -- & IPF11068 & Protein disulfide isomerase activity & 1.6 & 1.1 & -1.6 & -1.1 & 1.0 \\
\hline orf19.1616 & FGR23 & IPF12262 & -- & 2.3 & 1.3 & 1.1 & 8.4 & 19.6 \\
\hline orf19.3180 & -- & IPF12790 & Inositol-polyphosphate 5-phosphatase activity & 1.9 & 1.0 & 1.4 & 1.5 & 1.2 \\
\hline orf19.2646 & ZCF13 & IPF13024 & Specific RNA polymerase II transcription factor activity & 1.7 & 1.4 & 1.2 & 2.8 & -1.3 \\
\hline orf19.209 & $D F G 10$ & IPF13438 & -- & 1.7 & -1.0 & 1.0 & 1.2 & -1.0 \\
\hline orf19.3380 & $H W P 2$ & IPF14331 & -- & 1.5 & 1.2 & 1.3 & -2.1 & 2.1 \\
\hline orf19.3586 & -- & IPF14773.exon 2 & -- & 1.5 & -1.2 & 1.0 & 1.1 & -1.3 \\
\hline orf19.4134 & -- & IPF14985 & -- & 1.5 & 1.2 & 1.2 & 1.4 & -1.1 \\
\hline orf19.4795 & -- & IPF16501 & -- & 1.8 & 1.0 & -2.1 & 5.9 & 2.5 \\
\hline orf19.720 & GST3 & IPF17507 & Transcription corepressor activity & 1.6 & -1.2 & -1.2 & 2.2 & 1.0 \\
\hline orf19.6469 & -- & IPF19295.3f & -- & 1.8 & -1.0 & -1.1 & -1.1 & 1.9 \\
\hline orf19.5194.1 & -- & IPF20014 & -- & 1.5 & -1.1 & 1.3 & -1.0 & 1.2 \\
\hline orf19.3563 & -- & IPF3189 & -- & 1.8 & -1.3 & -1.1 & 2.5 & 1.1 \\
\hline orf19.7522 & -- & IPF364 & Arylformamidase activity & 1.6 & 1.2 & 1.1 & -1.1 & 1.1 \\
\hline orf19.296 & -- & IPF3833 & -- & 1.6 & -1.1 & 1.4 & 1.3 & 1.7 \\
\hline orf19.538 & -- & IPF4293 & -- & 1.7 & 1.0 & 1.4 & -1.2 & -1.1 \\
\hline orf19.6534 & -- & IPF5158 & -- & 1.7 & 1.0 & 1.0 & 5.3 & 1.1 \\
\hline orf19.5655 & -- & IPF5268.exon2 & -- & 1.7 & -1.2 & 1.1 & 15.0 & 1.7 \\
\hline orf19.7105 & FAR1 & IPF568 & Cyclin-dependent protein kinase inhibitor activity & 16.9 & 1.1 & 1.5 & 1.3 & 12.0 \\
\hline orf19.5952 & -- & IPF65 & -- & 2.0 & -1.5 & -2.0 & 2.5 & -1.5 \\
\hline orf19.6468 & -- & IPF6617.repeat1 & -- & 2.0 & -1.7 & 1.5 & -1.1 & 1.1 \\
\hline orf19.1171 & -- & IPF7635 & -- & 1.8 & -2.0 & 1.2 & 2.8 & 1.3 \\
\hline orf19.5342 & -- & IPF768 & -- & 2.3 & 1.0 & 1.4 & 1.4 & 1.5 \\
\hline orf19.31 & -- & IPF8878 & Structural constituent of cell wall & 2.0 & -1.3 & -1.3 & 1.8 & 2.1 \\
\hline orf19.2836 & -- & IPF9098 & -- & 1.9 & -1.6 & 3.7 & 2.5 & 1.9 \\
\hline orf19.3713 & -- & IPF9211.5f & -- & 1.7 & -1.2 & 1.3 & 7.2 & 1.2 \\
\hline orf19.2494 & STE3 & IPF9230 & Mating-type a-factor pheromone receptor activity & 4.1 & -1.4 & 1.8 & 11.4 & 5.4 \\
\hline-- & -- & IPF9851 & -- & 7.1 & 1.9 & 2.9 & 18.5 & 12.0 \\
\hline
\end{tabular}


Table A-1 (continued).

\begin{tabular}{|c|c|c|c|c|c|c|c|c|}
\hline $\begin{array}{c}\text { Systematic } \\
\text { Name }^{a}\end{array}$ & $\begin{array}{c}\text { CGD } \\
\text { Name }^{b}\end{array}$ & $\begin{array}{c}\text { CandidaDB } \\
\text { Name }^{c}\end{array}$ & GO Annotation (Molecular Function) ${ }^{d}$ & $\begin{array}{c}\text { C56 vs. } \\
\text { C43 }\end{array}$ & $\begin{array}{c}\text { Gu5 vs. } \\
\text { Gu2 }\end{array}$ & $\begin{array}{c}17 \text { vs. } \\
3\end{array}$ & $\begin{array}{c}5674 \text { vs. } \\
5457 \\
\end{array}$ & $\begin{array}{c}\text { SZY31 vs. } \\
5457\end{array}$ \\
\hline orf19.5172 & LIP9 & LIP9.exon2 & Lipase activity & 2.1 & 1.1 & 1.8 & 4.5 & 1.1 \\
\hline orf19.7420 & -- & MED6 & RNA polymerase II transcription mediator activity & 1.7 & -1.0 & 1.2 & 1.1 & -1.1 \\
\hline orf19.3197 & PAPl & PAP12 & RNA binding & 1.8 & -1.0 & 1.2 & 1.1 & 2.1 \\
\hline orf19.4599 & PHO89 & PHO89 & High affinity phosphate transporter activity & 1.6 & -1.6 & -1.6 & -2.3 & -1.1 \\
\hline orf19.3199 & PIKA & PIK1 & 1-phosphatidylinositol 4-kinase activity & 2.0 & 1.1 & -1.1 & 1.3 & 2.3 \\
\hline orf19.756 & SAP7 & SAP7 & Aspartic-type endopeptidase activity & 2.7 & -1.1 & 1.1 & -1.4 & -1.1 \\
\hline orf19.3893 & SCW11 & SCW11.3eoc & Glucan 1,3-beta-glucosidase activity & 1.6 & 1.2 & -1.0 & 1.1 & -1.1 \\
\hline orf19.2179 & SIT1 & SIT1 & Ferrichrome transporter activity & 1.6 & -1.3 & -1.7 & 1.1 & 1.2 \\
\hline orf19.5820 & UGA6 & UGA6.3eoc & L-gamma-aminobutyric acid transporter activity & 3.8 & 2.0 & 2.0 & 2.6 & 1.5 \\
\hline orf19.1163 & -- & IPF13079 & -- & -1.2 & 1.5 & 1.0 & -1.0 & -1.1 \\
\hline orf19.7034 & -- & IPF3069 & -- & -1.1 & 1.6 & 1.2 & 1.1 & 1.7 \\
\hline orf19.4608 & $P D C 12$ & PDC12.exon2 & Pyruvate carboxylase activity & 1.2 & 2.1 & 1.3 & 3.7 & -1.1 \\
\hline orf19.3796 & -- & RNT1 & Ribonuclease III activity & -1.2 & 1.7 & -1.5 & 1.1 & 1.1 \\
\hline orf19.6053 & CIS2 & CIS2 & Protein-glutamine gamma-glutamyltransferase activity & 1.2 & 1.3 & 1.9 & 1.1 & 1.3 \\
\hline orf19.7652 & CKA1 & CKA1 & Protein kinase $\mathrm{CK} 2$ activity & 1.1 & 1.2 & 1.9 & -1.1 & -1.1 \\
\hline orf19.1448.1 & -- & DYN2.3 & -- & -1.0 & 1.1 & 1.7 & 1.3 & -1.5 \\
\hline orf19.5535 & -- & FEN2 & Pantothenate transporter activity & 1.6 & 1.4 & 1.7 & -1.0 & 1.1 \\
\hline orf19.4182 & -- & IPF11898 & -- & 1.1 & 1.1 & 1.5 & -1.2 & -1.1 \\
\hline orf19.3466 & -- & IPF13112 & -- & -1.0 & -1.2 & 1.6 & 2.4 & -1.3 \\
\hline orf19.1804 & -- & IPF14864 & Transporter activity & -1.3 & 1.0 & 1.5 & 1.2 & -1.5 \\
\hline orf19.1177 & -- & IPF15134 & -- & 1.1 & 1.2 & 1.5 & -1.1 & 1.5 \\
\hline orf19.4884 & WORI & IPF15255 & Transcription regulator activity & 2.1 & 2.2 & 2.3 & 2.4 & 1.2 \\
\hline orf19.4159 & -- & IPF15660 & Transporter activity & -1.0 & 1.2 & 1.6 & -1.7 & -1.5 \\
\hline orf19.7468 & -- & $I P F 2511$ & -- & 1.0 & 1.0 & 1.7 & 1.8 & -1.2 \\
\hline orf19.7456 & -- & IPF2903 & -- & 1.3 & 1.5 & 2.2 & 4.4 & 1.1 \\
\hline orf19.5523 & -- & IPF4325 & -- & -1.2 & -1.0 & 1.7 & 1.2 & -1.0 \\
\hline orf19.5276 & -- & IPF4708 & -- & -1.0 & 1.3 & 1.6 & 1.7 & -1.3 \\
\hline orf19.3615 & -- & IPF5192 & -- & 1.0 & 1.2 & 1.5 & 2.1 & 1.1 \\
\hline orf19.1109 & -- & IPF6338 & -- & -1.3 & -1.1 & 1.6 & -1.5 & -1.6 \\
\hline orf19.480 & -- & IPF7097 & -- & 1.0 & 1.0 & 1.5 & -1.0 & -1.4 \\
\hline orf19.2104 & -- & $J A C 1$ & Chaperone binding & -1.1 & 1.0 & 1.6 & -1.1 & -1.1 \\
\hline orf19.6044 & -- & $M O B 2$ & Protein kinase activator activity & 1.0 & 1.1 & 1.5 & -1.2 & -1.0 \\
\hline orf19.6041 & RPO41 & RPO41 & DNA-directed RNA polymerase activity & -1.4 & 1.1 & 1.5 & -2.1 & -1.2 \\
\hline orf19.514 & SNP3 & SNP3 & RNA splicing factor activity, transesterification mechanism & -1.1 & 1.0 & 1.7 & -1.3 & -2.4 \\
\hline orf19.854 & -- & UGA11.exon2 & 4-aminobutyrate transaminase activity & 1.1 & 1.1 & 1.8 & -1.2 & 1.1 \\
\hline orf19.7436 & $A A F 1$ & $A A F 1$ & -- & 1.0 & 1.2 & 1.2 & 1.5 & 1.5 \\
\hline orf19.3417 & $A C F 2$ & $A C F 2$ & Glucan 1,3-beta-glucosidase activity & 1.3 & 1.1 & 1.1 & 1.9 & 1.1 \\
\hline orf19.2608 & ADH5 & ADH5 & Alcohol dehydrogenase activity & 1.3 & 1.2 & 1.4 & 1.7 & 1.2 \\
\hline orf19.4679 & $A G P 2$ & $A G P 2$ & Hydrogen:amino acid symporter activity & 1.4 & 1.3 & 1.4 & 1.8 & -1.1 \\
\hline orf19.5742 & $A L S 9$ & ALS11.3f & -- & 1.5 & 1.3 & 1.1 & 2.7 & 2.9 \\
\hline orf19.7400 & $A L S 7$ & $A L S 7$ & Carbohydrate binding & 1.0 & -1.0 & 1.0 & 1.5 & 1.5 \\
\hline
\end{tabular}


Table A-1 (continued).

\begin{tabular}{|c|c|c|c|c|c|c|c|c|}
\hline $\begin{array}{c}\text { Systematic } \\
\text { Name }^{a}\end{array}$ & $\begin{array}{c}\text { CGD } \\
\text { Name }^{b} \\
\end{array}$ & $\begin{array}{c}\text { CandidaDB } \\
\text { Name }^{c} \\
\end{array}$ & GO Annotation (Molecular Function) ${ }^{d}$ & $\begin{array}{c}\text { C56 vs. } \\
\text { C43 }\end{array}$ & $\begin{array}{c}\text { Gu5 vs. } \\
\text { Gu2 }\end{array}$ & $\begin{array}{c}17 \text { vs. } \\
3 \\
\end{array}$ & $\begin{array}{c}5674 \text { vs. } \\
5457 \\
\end{array}$ & $\begin{array}{c}\text { SZY31 vs. } \\
\mathbf{5 4 5 7} \\
\end{array}$ \\
\hline orf19.4413 & CMD1 & CMD1 & Calcium ion binding & 1.0 & 1.1 & 1.2 & 1.7 & -1.2 \\
\hline orf19.4555 & ALS4 & CPS2.3f & Gly-X carboxypeptidase activity & 1.1 & 1.2 & 1.2 & 1.6 & 1.3 \\
\hline orf19.4610 & -- & CPS2.5f & Gly-X carboxypeptidase activity & 1.2 & -1.0 & 1.2 & 1.9 & 1.5 \\
\hline orf19.6755 & -- & $D L D 2$ & D-lactate dehydrogenase (cytochrome) activity & 1.3 & 1.2 & 1.1 & 2.4 & 1.5 \\
\hline orf19.780 & DUR1,2 & DURI,2 & Allophanate hydrolase activity & -1.1 & 1.1 & 1.1 & 1.9 & 2.8 \\
\hline orf19.5999 & DYN1 & DYN1 & Minus-end-directed microtubule motor activity & 1.1 & 1.1 & 1.0 & 2.9 & 2.7 \\
\hline orf19.7495 & -- & EBP6 & NADPH dehydrogenase activity & -1.1 & -1.2 & 2.3 & 3.5 & -1.0 \\
\hline orf19.5816 & EBP7 & EBP7 & NADPH dehydrogenase activity & 1.2 & 1.1 & -1.1 & 2.4 & 1.4 \\
\hline orf19.7379 & FAA2 & FAA22 & Long-chain-fatty-acid-CoA ligase activity & 1.4 & 1.1 & 1.3 & 5.1 & -1.3 \\
\hline orf19.3653 & FAT1 & FAT1 & Long-chain-fatty-acid-CoA ligase activity & 1.1 & 1.1 & 1.2 & 1.5 & -1.1 \\
\hline orf19.6178 & FBP1 & FBPI & Fructose-bisphosphatase activity & 1.2 & 1.1 & 1.3 & 1.8 & 1.2 \\
\hline orf19.1153 & GAD1 & GAD1 & Glutamate decarboxylase activity & 1.0 & -1.1 & -1.0 & 2.0 & 1.1 \\
\hline orf19.5880 & -- & $G E F 1.3 f$ & Voltage-gated chloride channel activity & 1.3 & 1.2 & 1.1 & 1.6 & 1.6 \\
\hline orf19.734 & GLK1 & GLK1 & Glucokinase activity & 1.2 & 1.2 & 1.1 & 1.5 & 1.2 \\
\hline orf19.4309 & GRP2 & GRP2 & Oxidoreductase activity & -1.1 & 1.2 & 1.3 & 3.1 & 1.4 \\
\hline orf19.5059 & GCS1 & GSH1.exon1 & Glutamate-cysteine ligase activity & 1.0 & 1.0 & 1.3 & 2.7 & 1.4 \\
\hline orf19.7419 & HNT2 & HNT2 & Hydrolase activity & 1.4 & 1.2 & 1.3 & 1.9 & 1.2 \\
\hline orf19.6323 & -- & HPA3 & Histone acetyltransferase activity & 1.4 & 1.3 & 1.4 & 3.0 & 1.1 \\
\hline orf19.1191 & -- & HRD3 & Ubiquitin-protein ligase activity & 1.2 & 1.2 & -1.1 & 1.6 & 1.7 \\
\hline orf19.931 & -- & IFA13 & -- & 1.0 & 1.2 & 1.3 & 2.3 & 1.1 \\
\hline orf19.996 & -- & IFA15 & Adenylate cyclase activity & -1.0 & -1.1 & 1.4 & 5.2 & 2.3 \\
\hline orf19.3919 & -- & IFA23 & Cysteine-type endopeptidase activity & 1.0 & -1.0 & -1.0 & 1.6 & 1.5 \\
\hline orf19.570 & IFF8 & IFF8 & -- & 1.4 & -1.2 & 1.4 & 1.6 & 1.4 \\
\hline orf19.428 & -- & IKSI & Protein kinase activity & 1.2 & -1.1 & -1.0 & 1.6 & 1.1 \\
\hline orf19.4590 & -- & IPF1009 & -- & -1.1 & 1.7 & 1.3 & 2.1 & -1.5 \\
\hline orf19.4575 & -- & IPF1031 & -- & 1.3 & -1.0 & 1.0 & 2.0 & -1.1 \\
\hline orf19.3364 & -- & IPF10394 & -- & -1.1 & -1.3 & 1.1 & 2.6 & -1.3 \\
\hline orf19.424 & TRP99 & IPF10482.exon1 & Alkyl hydroperoxide reductase activity & 1.3 & 1.1 & -1.5 & 1.5 & -1.1 \\
\hline orf19.1287 & -- & IPF10595 & -- & -1.7 & 1.1 & 1.1 & 12.0 & -1.3 \\
\hline orf19.5413 & -- & IPF1104 & -- & 1.1 & 1.1 & 1.2 & 1.6 & -1.1 \\
\hline orf19.1421 & -- & IPF11234 & Ureidoglycolate hydrolase activity & 1.8 & 1.2 & -2.2 & 2.9 & 1.0 \\
\hline orf19.3661 & -- & IPF11332 & Ubiquitin-specific protease activity & 1.1 & 1.1 & 1.3 & 2.0 & 1.1 \\
\hline orf19.5295 & -- & IPF11876 & -- & 1.4 & 1.4 & 2.0 & 4.5 & -1.3 \\
\hline orf19.4234 & -- & IPF11954 & -- & 1.1 & 1.1 & 1.0 & 1.8 & -1.0 \\
\hline orf19.5644 & -- & IPF12127 & -- & 1.1 & 1.4 & 1.2 & 2.0 & 1.5 \\
\hline orf19.3872 & -- & IPF12179 & -- & 1.2 & 1.1 & 1.1 & 1.7 & -1.3 \\
\hline orf19.3508 & -- & IPF12233 & -- & 1.1 & 1.1 & 1.2 & 2.3 & 1.1 \\
\hline orf19.5070 & -- & IPF12312 & -- & 1.2 & 2.9 & 1.7 & 11.0 & 1.3 \\
\hline orf19.7344 & -- & IPF 12537 & -- & 2.0 & -1.4 & 1.5 & 3.3 & 1.8 \\
\hline orf19.2515 & -- & IPF12799 & -- & 1.3 & -1.1 & -1.2 & 1.9 & 1.6 \\
\hline orf19.1162 & -- & IPF13081 & -- & -1.1 & 1.2 & -1.3 & 1.9 & 1.9 \\
\hline
\end{tabular}


Table A-1 (continued).

\begin{tabular}{|c|c|c|c|c|c|c|c|c|}
\hline $\begin{array}{c}\text { Systematic } \\
\text { Name }^{a}\end{array}$ & $\begin{array}{c}\text { CGD } \\
\text { Name }^{b}\end{array}$ & $\begin{array}{c}\text { CandidaDB } \\
\text { Name }^{c}\end{array}$ & GO Annotation (Molecular Function) ${ }^{d}$ & $\begin{array}{l}\text { C56 vs. } \\
\text { C43 }\end{array}$ & $\begin{array}{l}\text { Gu5 vs. } \\
\text { Gu2 }\end{array}$ & $\begin{array}{c}17 \text { vs. } \\
3\end{array}$ & $\begin{array}{c}5674 \text { vs. } \\
5457\end{array}$ & $\begin{array}{c}\text { SZY31 vs. } \\
5457\end{array}$ \\
\hline orf19.1181 & -- & IPF13097 & Phosphatidate phosphatase activity & 3.6 & -1.4 & -1.0 & 11.3 & 14.4 \\
\hline orf19.5729 & FGR17 & IPF 13158 & Transcription factor activity & 1.1 & 1.1 & 1.1 & 2.1 & -1.3 \\
\hline orf19.6420 & PGA13 & IPF1341 & -- & 1.3 & -1.1 & -1.1 & 4.9 & -1.7 \\
\hline orf19.1656 & -- & IPF13675 & Phosphatidylinositol binding & -1.0 & -1.2 & 1.5 & 2.0 & -1.2 \\
\hline orf19.6443 & -- & IPF1380 & Dodecenoyl-CoA delta-isomerase activity & -1.2 & -1.1 & 1.1 & 2.1 & 2.2 \\
\hline orf19.2344 & ASRI & IPF13836 & -- & 2.8 & 1.8 & 1.4 & 18.3 & 1.7 \\
\hline orf19.1120 & FAV2 & IPF13879 & -- & 1.4 & 1.1 & 1.4 & 2.3 & -1.8 \\
\hline orf19.914 & -- & IPF14094.repeat4 & -- & 1.1 & -1.0 & 1.1 & 1.5 & -1.3 \\
\hline orf19.3586 & -- & IPF14773 & -- & 1.4 & -1.1 & -1.2 & 1.8 & 1.1 \\
\hline orf19.4376 & -- & IPF15098 & -- & 1.2 & 1.1 & 1.6 & 3.4 & 1.2 \\
\hline orf19.2607 & -- & IPF15672 & -- & 1.4 & -1.1 & -1.3 & 1.9 & -1.2 \\
\hline orf19.972 & -- & IPF1588 & -- & 1.2 & 1.3 & 1.1 & 1.9 & -1.5 \\
\hline orf19.1946 & -- & IPF16194 & -- & 1.3 & 1.2 & -1.1 & 1.8 & -1.0 \\
\hline orf19.3922 & -- & IPF16228 & Nucleotidase activity & 1.1 & -1.1 & 1.0 & 2.0 & 1.1 \\
\hline orf19.3932 & -- & IPF16253 & -- & 1.2 & 1.2 & -1.0 & 6.4 & -1.2 \\
\hline orf19.5877 & -- & IPF1837 & Alcohol O-acetyltransferase activity & 1.3 & 1.2 & -1.1 & 2.8 & 1.2 \\
\hline orf19.5620 & -- & IPF18418 & -- & 1.3 & 1.5 & 1.1 & 2.3 & -1.2 \\
\hline orf19.5727 & -- & IPF18553 & -- & 1.5 & 1.1 & -1.0 & 2.1 & -1.5 \\
\hline orf19.2737 & -- & IPF18712 & -- & 1.4 & 1.4 & 1.4 & 2.8 & 1.0 \\
\hline orf19.711 & -- & IPF18732 & -- & 1.4 & 1.4 & 1.2 & 3.0 & 1.3 \\
\hline orf19.7317 & UGA33 & IPF1960.5f & Transcription factor activity & 1.3 & -1.1 & 1.1 & 1.6 & 1.0 \\
\hline orf19.7322 & -- & IPF1969 & -- & -1.1 & 1.1 & 1.2 & 1.8 & -1.2 \\
\hline orf19.5517 & -- & IPF20104 & Alcohol dehydrogenase (NADP+) activity & 1.2 & 1.1 & 1.2 & 1.7 & -1.2 \\
\hline orf19.4077 & MITI & IPF2067 & Mannosyltransferase activity & 1.0 & 1.2 & 1.1 & 1.8 & 2.1 \\
\hline orf19.5980 & -- & IPF21 & -- & 1.4 & 1.1 & -1.1 & 2.0 & 1.2 \\
\hline orf19.6662 & -- & $I P F 2287$ & -- & 1.1 & -1.1 & 1.0 & 3.2 & -1.8 \\
\hline orf19.2158 & NAG3 & IPF2710.repeat 2 & Monosaccharide transporter activity & -2.4 & -1.7 & -1.5 & 2.4 & 6.1 \\
\hline orf19.7297 & -- & $I P F 2837$ & -- & 1.1 & 1.2 & -1.1 & 2.1 & 1.1 \\
\hline orf19.3273 & -- & $I P F 285.3 f$ & -- & 1.3 & -1.1 & 1.0 & 2.0 & 1.2 \\
\hline orf19.7281 & -- & IPF2861 & -- & 1.3 & 1.4 & 1.8 & 12.6 & 3.3 \\
\hline orf19.4305.1 & -- & IPF2930 & -- & 1.2 & -1.2 & -1.1 & 2.1 & -1.0 \\
\hline orf19.3282 & -- & IPF300 & -- & 1.0 & 1.1 & 1.3 & 1.5 & -1.2 \\
\hline orf19.4438 & RME1 & IPF3121 & Transcription factor activity & 1.2 & 1.1 & -1.3 & 5.7 & 1.7 \\
\hline orf19.6758 & -- & IPF3484 & Aldo-keto reductase activity & 2.4 & -1.1 & -1.5 & 2.9 & 1.1 \\
\hline orf19.6757 & -- & IPF3485 & Aldo-keto reductase activity & 1.1 & 1.2 & 1.4 & 2.0 & -1.1 \\
\hline orf19.675 & -- & IPF3964 & -- & 1.2 & 1.1 & 1.2 & 4.2 & 2.4 \\
\hline orf19.535 & $R B R 1$ & IPF4299 & -- & 1.3 & -1.1 & 1.3 & 1.7 & 1.3 \\
\hline orf19.2204 & -- & IPF5496 & -- & 1.3 & 1.1 & 1.1 & 1.8 & -1.3 \\
\hline orf19.6325.1 & -- & IPF5730 & -- & 1.3 & 1.1 & 1.1 & 1.6 & -1.8 \\
\hline orf19.5213 & -- & IPF5866 & -- & 1.1 & 1.2 & 1.2 & 2.7 & 1.4 \\
\hline orf19.7631 & -- & IPF610 & -- & 1.1 & -1.1 & 1.0 & 1.7 & -1.1 \\
\hline
\end{tabular}


Table A-1 (continued).

\begin{tabular}{|c|c|c|c|c|c|c|c|c|}
\hline $\begin{array}{c}\text { Systematic } \\
\text { Name }^{a}\end{array}$ & $\begin{array}{c}\text { CGD } \\
\text { Name }^{b}\end{array}$ & $\begin{array}{c}\text { CandidaDB } \\
\text { Name }^{c}\end{array}$ & GO Annotation (Molecular Function) ${ }^{d}$ & $\begin{array}{l}\text { C56 vs. } \\
\text { C43 }\end{array}$ & $\begin{array}{c}\text { Gu5 vs. } \\
\text { Gu2 } \\
\end{array}$ & $\begin{array}{c}17 \text { vs. } \\
3 \\
\end{array}$ & $\begin{array}{c}5674 \text { vs. } \\
5457 \\
\end{array}$ & $\begin{array}{c}\text { SZY31 vs. } \\
5457\end{array}$ \\
\hline orf19.3292 & -- & IPF6231 & Protein-methionine-R-oxide reductase activity & 1.2 & 1.0 & -1.3 & 2.0 & 1.1 \\
\hline orf19.1461 & -- & IPF6257 & -- & 1.2 & -1.1 & 1.0 & 2.5 & 1.1 \\
\hline orf19.6318 & -- & IPF6280 & -- & 1.3 & 1.1 & 1.1 & 1.6 & -1.6 \\
\hline orf19.2724 & -- & IPF6339 & -- & 1.2 & 1.3 & -1.3 & 4.3 & -1.6 \\
\hline orf19.384 & -- & IPF6367 & -- & 1.2 & 1.2 & 1.3 & 1.7 & 1.6 \\
\hline orf19.1573 & -- & IPF6387.3 & -- & 1.2 & 1.1 & -2.3 & 1.6 & -1.1 \\
\hline orf19.6489 & FMP45 & IPF6624 & -- & 1.3 & -1.4 & 1.2 & 4.8 & 2.2 \\
\hline orf19.2762 & $A H P 1$ & IPF6629 & Alkyl hydroperoxide reductase activity & 1.2 & 1.2 & -1.2 & 2.5 & -1.3 \\
\hline orf19.7596 & -- & IPF673 & -- & 1.7 & 1.0 & -2.0 & 15.2 & 1.1 \\
\hline orf19.320 & -- & IPF6755 & -- & 1.4 & -1.1 & 1.3 & 4.6 & 1.0 \\
\hline orf19.3813 & -- & IPF6945.3f & -- & 1.3 & -1.0 & 1.3 & 2.4 & 1.2 \\
\hline orf19.5770 & OPT8 & IPF6990 & Oligopeptide transporter activity & -1.0 & -1.1 & 1.1 & 2.0 & -1.0 \\
\hline orf19.1562 & -- & IPF7141 & -- & 1.2 & 1.4 & 1.4 & 2.3 & 1.2 \\
\hline orf19.1564 & -- & IPF7145 & -- & 1.2 & 1.3 & 2.4 & 1.8 & -1.2 \\
\hline orf19.860 & -- & IPF7647 & -- & 1.2 & 1.3 & 1.4 & 2.4 & 1.6 \\
\hline orf19.2693 & -- & IPF7968 & Transcription corepressor activity & -1.1 & 1.1 & 1.3 & 5.0 & 1.6 \\
\hline orf19.1719 & $S G A 1$ & IPF8402 & Glucan 1,4-alpha-glucosidase activity & 1.1 & 1.0 & 1.6 & 2.4 & -1.4 \\
\hline orf19.6952 & -- & IPF8666 & -- & 1.1 & 1.3 & 1.2 & 1.9 & 1.2 \\
\hline orf19.4903 & -- & IPF8860 & $\mathrm{N}$-acetylglucosaminylphosphatidylinositol deacetylase activity & -1.1 & 1.1 & -1.1 & 1.7 & -1.3 \\
\hline orf19.5943 & -- & IPF91 & -- & 1.2 & 1.1 & -1.0 & 1.8 & 1.2 \\
\hline orf19.4370 & -- & IPF9450 & -- & 1.1 & 1.1 & -1.1 & 2.4 & 1.0 \\
\hline orf19.4911 & -- & IPF9618 & -- & 1.2 & 1.1 & 1.0 & 2.3 & -1.3 \\
\hline orf19.4593.1 & -- & IPF995 & -- & -1.1 & -1.0 & 1.4 & 1.6 & -1.5 \\
\hline orf19.184 & -- & MDM10 & -- & 1.0 & 1.4 & 1.3 & 2.0 & 1.2 \\
\hline orf19.478 & -- & MON1.3 & -- & 1.3 & -1.1 & -1.0 & 1.9 & -2.4 \\
\hline orf19.4044 & MUM2 & MUM2 & -- & 1.1 & 1.3 & 3.8 & 2.4 & 1.5 \\
\hline orf19.5005 & OSM2 & OSM2 & Fumarate reductase (NADH) activity & 1.3 & -1.0 & -1.3 & 2.0 & 1.2 \\
\hline orf19.2769 & -- & PBI2 & Endopeptidase inhibitor activity & 1.3 & 1.2 & 1.1 & 3.3 & -1.2 \\
\hline orf19.4186 & PCT1 & PCT1 & Choline-phosphate cytidylyltransferase activity & -1.2 & 1.3 & -1.2 & 1.9 & 1.2 \\
\hline orf19.2094 & PDR6 & PDR6 & Protein carrier activity & -1.1 & -1.0 & -1.0 & 1.7 & -1.1 \\
\hline orf19.1089 & -- & PEX11 & -- & -1.0 & 1.3 & 1.0 & 1.8 & -2.4 \\
\hline orf19.5285 & PST3 & PST2 & -- & 1.1 & -1.1 & 1.2 & 2.3 & 1.0 \\
\hline orf19.6341 & -- & RIB7 & -- & 1.1 & 1.0 & -1.1 & 2.2 & 1.2 \\
\hline orf19.2087 & -- & SAS2 & Acetyltransferase activity & -1.2 & 1.3 & 1.4 & 1.9 & -1.3 \\
\hline orf19.2770.1 & SOD1 & SOD1.3 & Copper, zinc superoxide dismutase activity & -1.0 & 1.1 & -1.1 & 2.2 & -1.1 \\
\hline orf19.2299 & -- & URMI & -- & -1.1 & 1.0 & 1.0 & 1.7 & 1.6 \\
\hline orf19.5634 & FRP1 & FRE5 & Ferric-chelate reductase activity & 2.6 & 2.6 & 1.6 & 1.7 & -1.2 \\
\hline orf19.2176 & IFM3 & IFM3 & -- & 1.7 & 1.5 & 1.1 & 1.2 & 1.0 \\
\hline orf19.6219 & -- & IPF7432 & -- & 1.8 & 1.5 & 1.1 & -1.1 & 1.1 \\
\hline orf19.1878 & -- & IPF9160 & RNA polymerase II transcription mediator activity & 1.5 & 2.4 & -1.0 & 1.0 & -1.5 \\
\hline
\end{tabular}


Table A-1 (continued).

\begin{tabular}{|c|c|c|c|c|c|c|c|c|}
\hline $\begin{array}{c}\text { Systematic } \\
\text { Name }^{a}\end{array}$ & $\begin{array}{c}\text { CGD } \\
\text { Name }^{b}\end{array}$ & $\begin{array}{c}\text { CandidaDB } \\
\text { Name }^{c}\end{array}$ & GO Annotation (Molecular Function) ${ }^{d}$ & $\begin{array}{l}\text { C56 vs. } \\
\text { C43 }\end{array}$ & $\begin{array}{c}\text { Gu5 vs. } \\
\text { Gu2 } \\
\end{array}$ & $\begin{array}{c}17 \text { vs. } \\
3\end{array}$ & $\begin{array}{c}5674 \text { vs. } \\
5457 \\
\end{array}$ & $\begin{array}{c}\text { SZY31 vs. } \\
5457\end{array}$ \\
\hline orf19.5169 & -- & $A M D 21$ & Amidase activity & 1.8 & -1.5 & 1.5 & 3.5 & 1.3 \\
\hline orf19.393 & -- & APS3 & -- & 1.7 & 1.2 & 1.5 & 1.5 & 1.0 \\
\hline orf19.7053 & GACl & $G A C 1$ & Protein phosphatase type 1 activity & 2.0 & -1.2 & 1.7 & 1.0 & 1.5 \\
\hline orf19.5431 & -- & IPF1129 & -- & 1.7 & 1.1 & 2.2 & 1.2 & 1.1 \\
\hline orf19.7316 & -- & IPF5971 & -- & 1.5 & 1.3 & 1.5 & 1.0 & -1.1 \\
\hline orf19.5362 & $\mathrm{PSO} 2$ & IPF730 & -- & 6.9 & -1.1 & 1.5 & 1.7 & 10.0 \\
\hline orf19.1725 & -- & IPF9336.3eoc & -- & 1.8 & 1.1 & 1.5 & 1.1 & 1.5 \\
\hline orf19.3112 & ZRTI & ZRT1 & Low-affinity zinc ion transporter activity & 1.7 & -1.6 & 1.5 & 3.9 & 1.2 \\
\hline orf19.4774 & $A O X 1$ & $A O X 1$ & Alternative oxidase activity & 2.2 & -1.1 & 1.3 & 3.2 & -1.1 \\
\hline orf19.4788 & ARG5,6 & ARG5,6 & $\mathrm{N}$-acetyl-gamma-glutamyl-phosphate reductase activity & 1.6 & 1.1 & 2.3 & 2.3 & -1.9 \\
\hline orf19.6214 & ATC1 & ATH1 & Alpha, alpha-trehalase activity & 1.8 & 1.3 & 1.1 & 5.1 & 2.6 \\
\hline orf19.2401 & -- & AUT2 & Microtubule binding & 1.5 & -1.2 & 1.1 & 1.7 & 1.1 \\
\hline orf19.5604 & MDRI & $B M R I$ & Multidrug transporter activity & 1.8 & -1.1 & 1.1 & 1.8 & 1.3 \\
\hline orf19.113 & CIPI & CIP1 & -- & 1.5 & 1.2 & 1.4 & 2.2 & 1.7 \\
\hline orf19.6956 & DAL9 & DAL51 & Allantoate transporter activity & 1.9 & 1.1 & 1.2 & 2.9 & 1.6 \\
\hline orf19.3234 & -- & EBP5 & NADPH dehydrogenase activity & 1.8 & 1.0 & 1.2 & 2.5 & -1.1 \\
\hline orf19.6958 & ECM18 & ECM18 & -- & 2.7 & 1.1 & -1.0 & 3.4 & 1.2 \\
\hline orf19.4589 & -- & FMS1 & -- & 1.6 & 1.1 & -1.1 & 1.8 & -1.2 \\
\hline orf19.6995 & -- & FRP7 & -- & 1.7 & 1.3 & 1.7 & 2.2 & 1.5 \\
\hline orf19.313 & DAL4 & FUR4 & Allantoin permease activity & 2.4 & -1.2 & 1.3 & 4.5 & 2.1 \\
\hline orf19.7021 & $G P H 1$ & GPH1 & Glycogen phosphorylase activity & 1.8 & 1.2 & 1.2 & 1.6 & 1.8 \\
\hline orf19.1582 & -- & HOL $5.5 f$ & Transporter activity & 1.5 & -1.3 & 1.3 & 5.3 & 6.2 \\
\hline orf19.1048 & -- & IFDI & Aryl-alcohol dehydrogenase activity & 2.1 & 1.3 & -1.1 & 3.5 & 1.2 \\
\hline orf19.3311 & IFD3 & IFD3 & Aryl-alcohol dehydrogenase activity & 1.7 & -1.1 & 1.3 & 2.1 & 1.2 \\
\hline orf19.1048 & IFD6 & IFD5 & Aryl-alcohol dehydrogenase activity & 1.6 & 1.2 & 1.5 & 3.7 & 1.5 \\
\hline orf19.7472 & IFF4 & IFF4 & -- & 2.6 & 1.3 & 1.2 & 3.0 & -1.1 \\
\hline orf19.5840 & -- & IPF11222 & -- & 1.8 & 1.2 & 1.4 & 2.4 & -1.3 \\
\hline orf19.5210 & -- & IPF11259 & Transcription factor activity & 2.3 & 1.7 & 1.2 & 1.8 & 2.1 \\
\hline orf19.1405 & PHO13 & IPF11589 & 4-nitrophenylphosphatase activity & 1.7 & 1.1 & 1.2 & 1.6 & -1.8 \\
\hline orf19.1152 & -- & IPF11713 & -- & 1.6 & 1.2 & -1.3 & 1.7 & -1.1 \\
\hline orf19.2076 & -- & IPF1197 & -- & 1.7 & 1.1 & 1.1 & 2.3 & 1.6 \\
\hline orf19.1950 & -- & IPF12138 & -- & 1.6 & -1.0 & -1.0 & 3.4 & 1.6 \\
\hline orf19.2064 & -- & IPF1216 & Transcription factor activity & 1.5 & -1.1 & 2.7 & 1.7 & 1.5 \\
\hline orf19.2131 & -- & IPF12244 & -- & 1.6 & 1.1 & 1.4 & 1.7 & 1.2 \\
\hline orf19.4356 & HGT3 & IPF12946 & Glucose transporter activity & 1.5 & 1.2 & -1.0 & 1.8 & 1.3 \\
\hline orf19.6366 & -- & IPF13450 & -- & 1.6 & 2.7 & 2.0 & 10.6 & 1.6 \\
\hline orf19.5925 & -- & IPF138 & -- & 2.4 & 1.1 & -1.0 & 3.0 & 1.7 \\
\hline orf19.5204 & -- & IPF13971 & -- & 1.7 & 1.2 & 1.5 & 2.5 & 1.8 \\
\hline orf 19.4688 & $D A G 7$ & IPF14119 & -- & 2.4 & 1.1 & 1.1 & 1.5 & 1.2 \\
\hline orf19.4132 & -- & IPF14871 & -- & 1.6 & 1.3 & -1.1 & 2.1 & -1.3 \\
\hline
\end{tabular}


Table A-1 (continued).

\begin{tabular}{|c|c|c|c|c|c|c|c|c|}
\hline $\begin{array}{c}\text { Systematic } \\
\text { Name }^{a}\end{array}$ & $\begin{array}{c}\text { CGD } \\
\text { Name }^{b}\end{array}$ & $\begin{array}{c}\text { CandidaDB }^{\text {Name }} \\
\text { Name }^{c}\end{array}$ & GO Annotation (Molecular Function) ${ }^{d}$ & $\begin{array}{l}\text { C56 vs. } \\
\text { C43 }\end{array}$ & $\begin{array}{l}\text { Gu5 vs. } \\
\text { Gu2 }\end{array}$ & $\begin{array}{c}17 \text { vs. } \\
3\end{array}$ & $\begin{array}{c}\mathbf{5 6 7 4} \text { vs. } \\
5457\end{array}$ & $\begin{array}{c}\text { SZY31 vs. } \\
5457\end{array}$ \\
\hline orf19.348 & -- & IPF1505 & -- & 2.6 & 1.2 & 1.2 & 3.7 & 1.8 \\
\hline orf19.3053 & -- & IPF15297 & -- & 1.5 & 1.2 & -1.1 & 1.8 & 1.1 \\
\hline orf19.3910 & -- & IPF15494 & -- & 1.6 & -1.3 & 1.1 & 3.0 & 2.2 \\
\hline orf19.6586 & -- & IPF1617 & _- & 1.5 & 1.1 & 1.2 & 9.9 & -1.0 \\
\hline orf19.69 & -- & IPF17131 & -- & 1.8 & 1.1 & 1.3 & 5.5 & 1.4 \\
\hline orf19.5879 & -- & IPF1839 & Acylglycerone-phosphate reductase activity & 1.6 & 1.0 & 1.4 & 6.0 & -1.0 \\
\hline orf19.4334 & PGA58 & IPF19706 & -- & 2.3 & 1.3 & -1.3 & 1.6 & 1.1 \\
\hline orf19.7288 & -- & IPF 2852 & -- & 1.9 & -1.2 & 1.0 & 2.8 & 2.6 \\
\hline orf19.4439 & -- & IPF3105 & -- & 1.8 & 1.2 & 1.0 & 2.5 & -1.2 \\
\hline orf19.2870 & -- & IPF3250 & -- & 1.5 & -1.4 & -1.2 & 3.3 & -1.0 \\
\hline orf19.3839 & SAP10 & IPF4089 & Aspartic-type signal peptidase activity & 1.6 & 1.0 & -1.3 & 2.5 & 1.6 \\
\hline orf19.5449 & -- & IPF4176 & -- & 1.5 & 1.3 & 1.2 & 3.3 & 1.1 \\
\hline orf19.1632 & -- & IPF4256 & -- & 2.3 & 1.5 & 1.4 & 2.7 & -1.0 \\
\hline orf19.6329 & -- & IPF5723.exon1 & -- & 1.6 & 1.0 & 1.3 & 1.6 & -1.5 \\
\hline orf19.1307 & -- & IPF6678 & _- & 6.9 & -1.4 & -2.3 & 3.5 & 1.2 \\
\hline orf19.4246 & -- & IPF6881 & -- & 1.7 & 1.3 & 1.3 & 2.2 & -1.1 \\
\hline orf19.2966 & -- & IPF7778 & Carboxymethylenebutenolidase activity & 1.6 & 1.2 & 1.3 & 2.0 & 1.2 \\
\hline orf19.6025 & -- & IPF7900 & -- & 1.6 & 1.2 & 1.0 & 2.4 & -1.3 \\
\hline orf19.6869 & -- & IPF8041 & Peptidase activity & 1.6 & -1.1 & 1.6 & 1.6 & 1.9 \\
\hline orf19.4543 & UGA2 & IPF8048 & Succinate-semialdehyde dehydrogenase $[\mathrm{NAD}(\mathrm{P})+]$ activity & 1.8 & 1.4 & 1.1 & 2.7 & 1.1 \\
\hline orf19.7235 & -- & IPF846 & 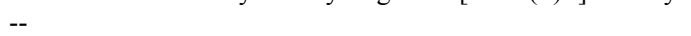 & 1.6 & 1.4 & 1.2 & 2.0 & 1.2 \\
\hline orf19.2302 & -- & IPF8814 & -- & 1.5 & 1.1 & -1.3 & 2.0 & 1.1 \\
\hline orf19.7214 & -- & IPF885 & -- & 2.4 & 1.4 & 1.3 & 4.6 & 1.5 \\
\hline orf19.4905 & -- & IPF8862 & -- & 1.5 & 1.1 & -1.4 & 1.7 & 1.2 \\
\hline orf19.1360 & -- & IPF8914 & -- & 1.8 & 1.4 & -1.7 & 8.2 & 4.1 \\
\hline orf19.7210 & -- & IPF893 & -- & 1.8 & -1.3 & 1.4 & 2.6 & 1.3 \\
\hline orf19.2737 & -- & IPF9167 & -- & 1.6 & 1.3 & 1.4 & 2.3 & 1.2 \\
\hline orf19.5942 & -- & IPF96 & -- & 1.6 & 1.4 & 1.1 & 1.7 & 1.5 \\
\hline orf19.4910 & FGR41 & IPF9616 & -- & 1.5 & 1.2 & 1.3 & 1.6 & 1.1 \\
\hline orf19.3499 & -- & IPF9955 & -- & 2.1 & 1.3 & -1.1 & 4.8 & -1.0 \\
\hline orf19.7447 & JEN1 & JEN1 & Lactate transporter activity & 1.7 & -1.1 & 1.1 & 2.0 & 1.2 \\
\hline orf19.7498 & LEU1 & LEU1 & 3-isopropylmalate dehydratase activity & 1.6 & -1.0 & 1.3 & 1.6 & 1.9 \\
\hline orf19.4584 & PHO114 & PHOI2 & Acid phosphatase activity & 1.5 & -1.2 & 1.1 & 1.9 & -1.1 \\
\hline orf19.1586 & FGR22 & $P L C 3$ & Phosphoinositide phospholipase $\mathrm{C}$ activity & 1.6 & 1.2 & -1.0 & 1.5 & -2.4 \\
\hline orf19.2969 & RAD16 & RAD16 & DNA-dependent ATPase activity & 1.6 & 1.2 & 1.0 & 1.9 & 1.6 \\
\hline orf19.5636 & RBT5 & RBT5 & Heme binding & 2.4 & 1.2 & 1.3 & 3.3 & 1.7 \\
\hline orf19.5714 & $S A P 1$ & $S A P 1$ & Aspartic-type endopeptidase activity & 2.0 & 1.3 & 1.1 & 2.2 & 1.5 \\
\hline orf19.5542 & SAP6 & SAP6 & Aspartic-type endopeptidase activity & 2.1 & 1.1 & 1.1 & 3.6 & 1.2 \\
\hline orf19.6928 & SAP9 & SAP9 & Aspartic-type endopeptidase activity & 1.5 & 1.2 & 1.0 & 1.6 & 1.3 \\
\hline orf19.2812 & -- & SNG2 & -- & 2.7 & -1.3 & 1.3 & 3.6 & 1.2 \\
\hline
\end{tabular}


Table A-1 (continued).

\begin{tabular}{|c|c|c|c|c|c|c|c|c|}
\hline $\begin{array}{c}\text { Systematic } \\
\text { Name }^{a}\end{array}$ & $\begin{array}{c}\text { CGD } \\
\text { Name }^{b}\end{array}$ & $\begin{array}{c}\text { CandidaDB } \\
\text { Name }^{c}\end{array}$ & GO Annotation (Molecular Function) ${ }^{d}$ & $\begin{array}{l}\text { C56 vs. } \\
\text { C43 }\end{array}$ & $\begin{array}{c}\text { Gu5 vs. } \\
\text { Gu2 } \\
\end{array}$ & $\begin{array}{c}17 \text { vs. } \\
3 \\
\end{array}$ & $\begin{array}{c}\mathbf{5 6 7 4} \text { vs. } \\
5457 \\
\end{array}$ & $\begin{array}{c}\text { SZY31 vs. } \\
5457\end{array}$ \\
\hline orf19.2897 & SOU2 & $\mathrm{SOU} 2$ & 2,4-dienoyl-CoA reductase (NADPH) activity & 1.7 & 1.1 & 1.1 & 1.6 & 1.2 \\
\hline orf19.4980 & HSP70 & SSA4 & Unfolded protein binding & 2.1 & 1.1 & 1.5 & 3.1 & 3.2 \\
\hline orf19.7637 & YHB4 & YHB2 & Nitric oxide dioxygenase activity & 1.5 & -1.1 & 1.1 & 1.9 & 1.6 \\
\hline orf19.4476 & -- & IFD6 & Aryl-alcohol dehydrogenase activity & 1.6 & 4.8 & 2.3 & 2.2 & 1.2 \\
\hline orf19.1865 & -- & IPF4059 & -- & 1.2 & 1.8 & 1.6 & -1.4 & -1.1 \\
\hline orf19.6996 & -- & IPF4514 & Alpha-1,3-mannosyltransferase activity & 1.1 & 1.6 & 2.1 & 1.1 & 1.0 \\
\hline orf19.3737 & -- & IPF4721 & -- & 1.3 & 1.5 & 1.9 & 1.1 & -1.2 \\
\hline orf19.2652 & TEF4 & TEF41 & Translation elongation factor activity & 1.3 & 3.7 & 2.9 & -1.1 & -2.4 \\
\hline orf19.3195 & HIPl & GAP7.3eoc & Amino acid permease activity & 1.2 & 1.5 & 1.1 & 1.6 & 1.5 \\
\hline orf19.3133 & GUT2 & GUT2 & Glycerol-3-phosphate dehydrogenase activity & 1.0 & 1.5 & 1.3 & 1.8 & 1.2 \\
\hline orf19.4763 & -- & IPF10916 & -- & -1.1 & 1.5 & 1.0 & 3.7 & -1.3 \\
\hline orf19.1395 & -- & IPF11059 & Inorganic phosphate transporter activity & 1.3 & 1.5 & -1.1 & 9.7 & -1.4 \\
\hline orf19.1698 & -- & IPF13217 & -- & 1.1 & 1.5 & 1.0 & 1.5 & 1.3 \\
\hline orf19.956 & -- & IPF1566 & -- & -1.2 & 1.5 & 1.4 & 1.7 & 1.3 \\
\hline orf19.5145 & SSP96 & IPF18316.3f & -- & -1.0 & 1.6 & -1.4 & 3.4 & 1.5 \\
\hline orf19.909 & STP4 & IPF4553 & Transcription factor activity & -1.0 & 1.9 & 1.0 & 1.9 & -1.5 \\
\hline orf19.2184 & -- & IPF5534 & -- & -1.0 & 1.6 & 1.5 & 1.9 & 1.8 \\
\hline orf19.3338 & -- & IPF7374 & -- & -1.1 & 2.1 & 2.3 & 5.8 & 1.5 \\
\hline orf19.4670 & CAS5 & IPF7414 & Transcription factor activity & 1.3 & 1.6 & 1.5 & 4.2 & 1.9 \\
\hline orf19.1424 & -- & IPF9370 & -- & 1.3 & 1.8 & 1.9 & 2.8 & 1.6 \\
\hline orf19.4478 & -- & $M S D 1$ & Aspartate-tRNA ligase activity & 1.1 & 1.7 & 1.1 & 1.6 & 1.2 \\
\hline orf19.5614 & -- & RNHI & Ribonuclease $\mathrm{H}$ activity & -1.0 & 1.5 & 1.1 & 1.5 & -1.0 \\
\hline orf19.6951 & -- & DPL1 & -- & 1.2 & 1.1 & 1.7 & 2.0 & 1.0 \\
\hline orf19.932 & -- & DRS24 & Phospholipid-translocating ATPase activity & 1.2 & 1.3 & 1.6 & 1.6 & 1.1 \\
\hline orf19.629 & IFD7 & IFD7 & Aryl-alcohol dehydrogenase activity & 5.2 & 1.7 & 2.0 & 1.8 & -1.1 \\
\hline orf19.2574 & -- & IFU4 & -- & 1.2 & 1.0 & 1.5 & 2.2 & 1.8 \\
\hline orf19.1676 & -- & IPF12501 & -- & 1.1 & 1.2 & 1.8 & 1.7 & -1.3 \\
\hline orf19.2910 & $P G A 43$ & IPF13139 & -- & 1.3 & 1.1 & 2.2 & 2.2 & -1.2 \\
\hline orf19.4347 & -- & IPF14273 & Receptor signaling protein serine/threonine kinase activity & -1.8 & -1.1 & 1.5 & 2.1 & 1.2 \\
\hline orf19.3461 & -- & IPF14968 & -- & 1.1 & 1.5 & 2.4 & 2.6 & 1.1 \\
\hline orf19.5612 & -- & IPF15015 & -- & 1.5 & 1.4 & 4.8 & 3.3 & 1.3 \\
\hline orf19.6754 & -- & IPF3490 & -- & -1.1 & 1.3 & 1.6 & 1.7 & -1.1 \\
\hline orf19.3743 & -- & IPF4729 & -- & 1.4 & 1.2 & 1.6 & 2.5 & -1.0 \\
\hline orf19.3826 & -- & IPF5830 & -- & 1.1 & 1.3 & 1.9 & 1.6 & -1.2 \\
\hline orf19.3859 & -- & IPF6600 & -- & 1.1 & 1.3 & 1.7 & 2.0 & 1.2 \\
\hline orf19.5587 & -- & IPF6812 & -- & 1.4 & 1.0 & 1.5 & 3.7 & -1.3 \\
\hline orf19.4539 & -- & IPF8055 & Rho GDP-dissociation inhibitor activity & -1.1 & 1.1 & 2.3 & 2.1 & 1.5 \\
\hline orf19.6220.3 & -- & $M M D 1.3$ & -- & 1.0 & 1.2 & 1.5 & 1.6 & 1.1 \\
\hline orf19.5731 & -- & PAD1 & Carboxy-lyase activity & 1.4 & 1.2 & 2.5 & 3.0 & -2.4 \\
\hline orf19.7389 & REV3 & REV $3.3 f$ & Zeta DNA polymerase activity & 1.0 & 1.1 & 1.7 & 1.7 & -1.0 \\
\hline
\end{tabular}


Table A-1 (continued).

\begin{tabular}{|c|c|c|c|c|c|c|c|c|}
\hline $\begin{array}{c}\text { Systematic } \\
\text { Name }^{a}\end{array}$ & $\begin{array}{c}\text { CGD } \\
\text { Name }^{b}\end{array}$ & $\begin{array}{c}\text { CandidaDB } \\
\text { Name }^{c}\end{array}$ & GO Annotation (Molecular Function) ${ }^{d}$ & $\begin{array}{l}\text { C56 vs. } \\
\text { C43 }\end{array}$ & $\begin{array}{c}\text { Gu5 vs. } \\
\text { Gu2 }\end{array}$ & $\begin{array}{c}17 \text { vs. } \\
3\end{array}$ & $\begin{array}{c}5674 \text { vs. } \\
5457\end{array}$ & $\begin{array}{c}\text { SZY31 vs. } \\
5457\end{array}$ \\
\hline orf19.732 & -- & SOU3 & 2,4-dienoyl-CoA reductase (NADPH) activity & 1.3 & 1.2 & 1.7 & 2.3 & -1.1 \\
\hline orf19.6026 & $E R G 2$ & $E R G 2$ & C-8 sterol isomerase activity & 1.8 & 3.0 & 2.5 & 1.6 & -1.4 \\
\hline orf19.1598 & ERG24 & ERG24 & Delta 14 -sterol reductase activity & 1.6 & 1.8 & 2.1 & -1.2 & -1.0 \\
\hline orf19.3421.1 & -- & IPF17743 & RNA polymerase II transcription mediator activity & 1.6 & 1.6 & 1.9 & 1.2 & -1.5 \\
\hline orf19.2849 & $A Q Y 1$ & $A Q Y 1$ & Water channel activity & 3.6 & 2.4 & 1.7 & 8.4 & 2.4 \\
\hline orf19.3442 & -- & $E B P 2$ & NADPH dehydrogenase activity & 1.7 & 1.5 & 2.1 & 2.4 & 1.4 \\
\hline orf19.4255 & ECM331 & ECM331 & -- & 2.7 & 1.6 & 1.3 & 4.2 & -1.4 \\
\hline orf19.2613 & ECM4 & ECM41.3 & -- & 1.6 & 2.0 & 2.0 & 4.6 & -1.0 \\
\hline orf19.2990 & XOG1 & EXG1 & Glucan 1,3-beta-glucosidase activity & 2.9 & 2.2 & 1.2 & 1.8 & 1.0 \\
\hline orf19.2952 & $E X G 2$ & $E X G 2$ & Glucan 1,3-beta-glucosidase activity & 1.5 & 1.5 & -1.2 & 2.4 & 1.0 \\
\hline orf19.6659 & GAP6 & GAP6 & Amino acid permease activity & 1.6 & 1.5 & 1.2 & 4.0 & 1.1 \\
\hline orf19.4477 & CSHI & IFD4 & Aryl-alcohol dehydrogenase activity & 2.9 & 2.3 & -1.0 & 4.6 & 1.6 \\
\hline orf19.6678 & -- & IFJ5 & -- & 2.1 & 1.5 & 1.2 & 12.3 & 1.6 \\
\hline orf19.2280 & ZCF10 & IPF10079 & Transcription factor activity & 1.6 & 1.8 & 1.5 & 6.1 & 2.1 \\
\hline orf19.5635 & $P G A 7$ & IPF12101 & -- & 3.2 & 1.7 & 1.5 & 2.8 & 1.0 \\
\hline orf19.251 & -- & IPF17186 & -- & 2.0 & 1.8 & 1.2 & 4.2 & 1.1 \\
\hline orf19.2175 & -- & IPF19998 & Oxidoreductase activity & 2.4 & 1.6 & 1.3 & 3.3 & 1.3 \\
\hline orf19.7284 & ASR2 & IPF2857 & -- & 5.2 & 2.3 & 10.5 & 17.6 & 1.5 \\
\hline orf19.7042 & -- & IPF3080 & -- & 2.1 & 1.5 & 1.2 & 5.5 & 1.3 \\
\hline orf19.1862 & -- & IPF4065 & -- & 3.5 & 1.7 & 1.1 & 9.0 & -1.1 \\
\hline orf19.5525 & -- & IPF4328 & -- & 1.5 & 1.6 & 1.2 & 2.3 & 1.1 \\
\hline orf19.2296 & -- & IPF7715 & -- & 2.3 & 1.5 & 1.2 & 4.1 & 1.7 \\
\hline orf19.3131 & -- & IPF7817 & NADPH dehydrogenase activity & 1.7 & 1.8 & 1.4 & 5.1 & 1.4 \\
\hline orf19.2691 & -- & IPF7970 & -- & 1.6 & 1.6 & 1.2 & 4.9 & 3.0 \\
\hline orf19.744 & $G D B 1$ & IPF9740 & 4-alpha-glucanotransferase activity & 1.7 & 1.5 & 1.1 & 3.1 & 1.7 \\
\hline orf19.1240 & -- & IPF9887.3eoc & -- & 1.5 & 1.6 & 1.2 & 2.6 & 1.6 \\
\hline orf19.2285 & -- & IPF9996 & -- & 4.6 & 2.2 & 1.6 & 8.1 & 1.2 \\
\hline orf19.1149 & $M R F 1$ & $M R F 1$ & DNA binding & 1.8 & 1.6 & -1.1 & 8.0 & 1.5 \\
\hline orf19.1655 & PXP2 & PXP2 & Acyl-CoA oxidase activity & 2.1 & 2.1 & 1.1 & 3.0 & 1.2 \\
\hline orf19.6202 & RBT4 & RBT4 & -- & 1.7 & 1.7 & -1.1 & 2.6 & -1.1 \\
\hline orf19.5585 & SAP5 & SAP5 & Aspartic-type endopeptidase activity & 1.8 & 1.5 & -1.1 & 5.4 & -1.0 \\
\hline orf19.2991 & HOL1 & HOL1 & Transporter activity & 2.3 & 1.3 & 1.8 & 2.5 & 1.1 \\
\hline orf19.7585 & INOI & INOI & Inositol-3-phosphate synthase activity & 3.1 & 1.3 & 1.8 & 6.1 & 1.3 \\
\hline orf19.1266 & -- & IPF10280 & -- & 1.6 & 1.4 & 2.0 & 2.2 & 1.1 \\
\hline orf19.1180 & -- & IPF13098 & -- & 1.7 & 1.2 & 1.5 & 1.8 & 2.2 \\
\hline orf19.2296 & -- & IPF14282 & -- & 1.8 & 1.4 & 2.3 & 6.1 & 2.4 \\
\hline orf19.3929 & -- & IPF14603 & -- & 1.9 & 1.0 & 1.9 & 1.9 & -1.1 \\
\hline orf19.347 & RSN1 & IPF1506 & -- & 2.0 & 1.1 & 1.7 & 2.0 & 1.8 \\
\hline orf19.3190 & HAL9 & IPF16067 & Specific RNA polymerase II transcription factor activity & 1.9 & 1.2 & 1.6 & 1.8 & 1.6 \\
\hline orf19.94 & -- & IPF19749 & -- & 2.0 & 1.1 & 1.5 & 2.8 & -1.0 \\
\hline
\end{tabular}


Table A-1 (continued).

\begin{tabular}{|c|c|c|c|c|c|c|c|c|}
\hline $\begin{array}{c}\text { Systematic } \\
\text { Name }^{a}\end{array}$ & $\begin{array}{c}\text { CGD } \\
\text { Name }^{b}\end{array}$ & $\begin{array}{c}\text { CandidaDB } \\
\text { Name }^{c}\end{array}$ & GO Annotation (Molecular Function) ${ }^{d}$ & $\begin{array}{l}\text { C56 vs. } \\
\text { C43 }\end{array}$ & $\begin{array}{c}\text { Gu5 vs. } \\
\text { Gu2 }\end{array}$ & $\begin{array}{c}17 \text { vs. } \\
3\end{array}$ & $\begin{array}{c}5674 \text { vs. } \\
5457\end{array}$ & $\begin{array}{c}\text { SZY31 vs. } \\
5457 \\
\end{array}$ \\
\hline orf19.3846 & -- & IPF20118 & -- & 1.6 & -1.1 & 3.8 & 2.6 & -1.5 \\
\hline orf19.6627 & -- & IPF2557 & -- & 1.7 & 1.2 & 2.2 & 2.6 & -1.0 \\
\hline orf19.3043 & -- & IPF3594 & Triacylglycerol lipase activity & 1.5 & -1.1 & 1.8 & 1.6 & 1.1 \\
\hline orf19.3544 & -- & IPF6438 & -- & 1.6 & 1.3 & 2.8 & 2.7 & 1.6 \\
\hline orf19.4533 & -- & IPF7533 & -- & 1.5 & 1.3 & 1.6 & 1.6 & 1.1 \\
\hline orf19.1395 & -- & IPF7686 & Inorganic phosphate transporter activity & 1.5 & 1.4 & 2.1 & 6.3 & -1.5 \\
\hline orf19.3188 & TACl & IPF9191.5f & -- & 2.5 & 1.5 & 2.4 & 45.4 & -11.7 \\
\hline orf19.3071 & -- & IPF9605 & Protein tyrosine phosphatase activity & 1.5 & 1.0 & 1.5 & 1.7 & 1.5 \\
\hline orf19.5102 & PLB5 & PLB5 & Lysophospholipase activity & 1.5 & 1.3 & 1.6 & 3.6 & 1.1 \\
\hline orf19.2046 & POT1-2 & POT13 & Acetyl-CoA C-acyltransferase activity & 1.5 & 1.3 & 1.6 & 1.7 & 1.2 \\
\hline orf19.7319 & SUC1 & SUC1 & Transcription factor activity & 2.0 & 1.2 & 1.8 & 5.1 & 1.2 \\
\hline orf19.1974 & TFS1 & TFSI & Lipid binding & 1.5 & 1.4 & 1.7 & 2.4 & -1.0 \\
\hline orf19.345 & -- & UGA2 & Succinate-semialdehyde dehydrogenase activity & 1.8 & 1.3 & 1.6 & 4.0 & 3.0 \\
\hline orf19.4082 & DDR48 & DDR48 & 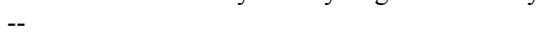 & 1.2 & 2.5 & 2.6 & 2.5 & -1.6 \\
\hline orf19.6445 & ECII & IPF1384 & Dodecenoyl-CoA delta-isomerase activity & 1.3 & 1.8 & 1.6 & 4.4 & 1.2 \\
\hline orf19.354 & -- & IPF1493 & -- & -1.0 & 1.5 & 1.7 & 1.8 & 1.2 \\
\hline orf19.1365 & -- & IPF16016 & -- & 1.2 & 1.6 & 1.6 & 2.1 & -1.2 \\
\hline orf19.5799 & -- & IPF20010 & -- & 1.4 & 1.8 & 1.6 & 1.7 & 1.7 \\
\hline orf19.6770 & -- & IPF2804 & Cytoskeletal adaptor activity & 1.3 & 1.6 & 1.5 & 2.7 & -1.0 \\
\hline orf19.3045 & -- & IPF3597 & Aldehyde dehydrogenase (NAD) activity & 1.4 & 1.6 & 1.6 & 1.6 & 1.2 \\
\hline orf19.6501 & -- & IPF3931 & -- & 2.0 & 3.1 & 7.4 & 4.6 & 1.1 \\
\hline orf19.6734 & TCC1 & IPF6067 & General transcriptional repressor activity & -1.1 & 1.8 & 1.6 & 1.7 & -1.0 \\
\hline orf19.4056 & GAT2 & IPF7666 & Transcription factor activity & 1.2 & 2.1 & 2.3 & 5.4 & -1.7 \\
\hline orf19.2248 & ARE2 & ARE2 & Sterol O-acyltransferase activity & 2.1 & 1.7 & 1.5 & 3.0 & 1.7 \\
\hline orf19.6000 & $C D R 1$ & $C D R 1$ & Multidrug transporter activity & 1.7 & 3.0 & 2.4 & 4.4 & -1.0 \\
\hline orf19.5958 & $C D R 2$ & $C D R 2$ & Transporter activity & 50.6 & 14.1 & 17.1 & 50.5 & -1.3 \\
\hline orf19.86 & -- & $G P X 1$ & Glutathione peroxidase activity & 2.3 & 3.0 & 2.3 & 4.0 & -1.2 \\
\hline orf19.896 & CHKI & HK1 & Protein histidine kinase activity & 2.5 & 1.9 & 1.8 & 4.5 & 1.3 \\
\hline orf19.3160 & HSP 12 & HSP12 & -- & 5.3 & 2.9 & 3.1 & 16.7 & -1.7 \\
\hline orf19.157 & -- & IFA24.3 & -- & 1.5 & 1.7 & 1.6 & 2.1 & 1.1 \\
\hline orf19.2568 & IFU5 & IFU5 & -- & 3.3 & 3.0 & 3.7 & 5.4 & 1.5 \\
\hline orf19.2726 & -- & IPF10262 & -- & 2.5 & 2.8 & 1.9 & 6.2 & -2.9 \\
\hline orf19.1267 & -- & IPF10278 & Chaperone regulator activity & 1.8 & 1.7 & 1.8 & 2.5 & -1.2 \\
\hline orf19.4459 & -- & IPF11849 & -- & 2.0 & 4.1 & 4.3 & 5.0 & -1.5 \\
\hline orf19.2244 & -- & IPF12897 & -- & 1.8 & 1.7 & 1.5 & 5.4 & -1.7 \\
\hline orf19.851 & -- & IPF14030 & -- & 1.7 & 1.9 & 1.9 & 4.0 & -1.3 \\
\hline orf19.344 & -- & IPF1514 & -- & 15.5 & 4.7 & 4.0 & 28.9 & 1.9 \\
\hline orf19.4907 & -- & IPF 17283 & -- & 3.2 & 1.6 & 2.3 & 2.0 & 1.1 \\
\hline orf19.5313 & -- & IPF19810 & -- & 36.0 & 29.4 & 18.1 & 143.1 & 1.7 \\
\hline orf19.5777 & -- & IPF19961 & -- & 2.0 & 1.7 & 2.2 & 4.2 & -1.3 \\
\hline
\end{tabular}


Table A-1 (continued).

\begin{tabular}{|c|c|c|c|c|c|c|c|c|}
\hline $\begin{array}{c}\text { Systematic } \\
\text { Name }^{a}\end{array}$ & $\begin{array}{c}\text { CGD } \\
\text { Name }^{b}\end{array}$ & $\begin{array}{c}\text { CandidaDB } \\
\text { Name }^{c}\end{array}$ & GO Annotation (Molecular Function) ${ }^{d}$ & $\begin{array}{l}\text { C56 vs. } \\
\text { C43 }\end{array}$ & $\begin{array}{c}\text { Gu5 vs. } \\
\text { Gu2 }\end{array}$ & $\begin{array}{c}17 \text { vs. } \\
3\end{array}$ & $\begin{array}{c}\mathbf{5 6 7 4} \text { vs. } \\
\mathbf{5 4 5 7} \\
\end{array}$ & $\begin{array}{c}\text { SZY31 vs. } \\
5457\end{array}$ \\
\hline orf19.7166 & -- & IPF2186 & -- & 4.2 & 2.2 & 1.5 & 5.0 & 1.2 \\
\hline orf19.6840 & -- & IPF3535 & -- & 1.8 & 2.3 & 1.9 & 2.3 & -1.4 \\
\hline orf19.3047 & -- & IPF3598 & Transcription cofactor activity & 1.5 & 1.5 & 1.7 & 3.0 & 1.3 \\
\hline orf19.2531 & CSP37 & IPF4991 & ER to Golgi vesicle-mediated transport & 2.5 & 1.8 & 5.5 & 6.8 & -1.3 \\
\hline orf19.3007.2 & -- & IPF5360.3 & -- & 2.0 & 1.6 & 1.9 & 2.8 & 1.1 \\
\hline orf19.7310 & -- & IPF5981 & -- & 4.9 & 2.7 & 2.0 & 16.8 & 1.2 \\
\hline orf19.1887 & -- & IPF6464 & -- & 2.3 & 1.8 & 1.7 & 4.5 & 1.2 \\
\hline orf19.2790 & -- & IPF7260 & Histone-lysine $\mathrm{N}$-methyltransferase activity & 1.8 & 1.6 & 1.6 & 2.8 & -1.0 \\
\hline orf19.4531 & -- & IPF7530 & ATPase activity & 2.7 & 3.8 & 3.5 & 4.0 & -1.6 \\
\hline orf19.4898 & -- & IPF8950 & -- & 2.4 & 1.6 & 1.8 & 3.4 & 1.1 \\
\hline orf19.3188 & TACl & IPF9191.3f & -- & 2.7 & 2.8 & 2.8 & 4.2 & -11.0 \\
\hline orf19.3644 & -- & IPF9683 & -- & 2.5 & 1.5 & 2.1 & 3.4 & 1.1 \\
\hline orf19.5257 & -- & $L C B 4$ & D-erythro-sphingosine kinase activity & 2.2 & 1.7 & 2.2 & 4.0 & 1.1 \\
\hline orf19.278 & -- & MTR & -- & 3.1 & 1.8 & 1.9 & 5.2 & -1.1 \\
\hline orf19.5713 & $Y M X 6$ & $\mathrm{NDH} 2$ & NADH dehydrogenase activity & 5.3 & 3.1 & 2.9 & 15.3 & 1.3 \\
\hline orf19.7218 & RBE1 & PRY2 & -- & 2.1 & 1.8 & 1.7 & 1.7 & 1.2 \\
\hline orf19.23 & RTA3 & RTA3 & Phospholipid-translocating ATPase activity & 40.4 & 15.9 & 22.0 & 41.0 & 1.0 \\
\hline orf19.2896 & SOU1 & SOU1 & Sorbose dehydrogenase activity & 2.0 & 1.5 & 1.5 & 7.0 & -2.0 \\
\hline orf19.1290 & -- & $X K S 1$ & Xylulokinase activity & 1.6 & 1.7 & 1.6 & 2.7 & 1.6 \\
\hline orf19.3104 & -- & $Y D C 1$ & -- & 3.3 & 1.6 & 2.1 & 5.3 & 1.4 \\
\hline
\end{tabular}

${ }^{a}$ orf19 nomenclature according to the Assembly 19 version.

${ }^{b}$ Gene name at CGD (http://www.candidagenome.org/).

${ }^{c}$ Gene name at CandidaDB (http://genolist.pasteur.fr/CandidaDB/).

${ }^{d} \mathrm{GO}$ annotation found at CGD (http://www.candidagenome.org/).

Gene expression values in bold designate statistical significance $(p$-value $<0.05)$ in all three experiments. 
Table A-2. Genes down-regulated in at least one matched clinical isolate.

\begin{tabular}{|c|c|c|c|c|c|c|c|c|}
\hline $\begin{array}{c}\text { Systematic } \\
\text { Name }^{a}\end{array}$ & $\begin{array}{c}\text { CGD } \\
\text { Name }^{b}\end{array}$ & $\begin{array}{c}\text { CandidaDB } \\
\text { Name }^{c}\end{array}$ & GO Annotation (Molecular Function) ${ }^{d}$ & $\begin{array}{c}\text { C56 vs. } \\
\text { C43 }\end{array}$ & $\begin{array}{c}\text { Gu5 vs. } \\
\text { Gu2 }\end{array}$ & $\begin{array}{c}17 \text { vs. } \\
3\end{array}$ & $\begin{array}{c}\mathbf{5 6 7 4} \text { vs. } \\
5457 \\
\end{array}$ & $\begin{array}{c}\text { SZY31 vs. } \\
5457 \\
\end{array}$ \\
\hline orf19.7043 & -- & ACB1.exon2 & Acyl-CoA binding & -2.0 & 1.6 & 1.9 & 1.4 & 1.1 \\
\hline orf19.798 & -- & $\mathrm{ANC1}$ & General RNA polymerase II transcription factor activity & -1.8 & -1.0 & 1.1 & -1.1 & -2.5 \\
\hline orf19.5345 & -- & DSK2 & Protein binding & -1.5 & -1.0 & 1.2 & -1.1 & 1.6 \\
\hline orf19.4295 & -- & HIR2 & Transcription corepressor activity & -2.3 & 1.1 & 1.7 & 1.0 & 1.2 \\
\hline orf19.4051 & HTS1 & HTS1 & Histidine-tRNA ligase activity & -1.5 & 1.1 & 1.2 & -1.5 & 1.2 \\
\hline orf19.3691 & -- & IPF10716 & -- & -1.5 & -1.0 & 1.3 & 1.1 & 1.1 \\
\hline orf19.2356 & CRZ2 & IPF10795 & Transcription factor activity & -2.2 & 2.1 & -1.4 & 1.4 & 1.3 \\
\hline orf19.2827 & -- & IPF10963 & Structural constituent of cytoskeleton & -2.2 & 1.5 & -1.0 & 5.3 & 3.9 \\
\hline orf19.4658 & -- & IPF11006 & -- & -1.5 & 1.0 & 2.5 & 2.6 & 1.4 \\
\hline orf19.3198 & $O B P A$ & IPF11167 & Oxysterol binding & -85.5 & 1.1 & 1.3 & 2.1 & -1.8 \\
\hline orf19.5241 & SNT1 & IPF12584 & NAD-dependent histone deacetylase activity & -1.5 & 1.1 & 1.3 & -1.2 & 1.2 \\
\hline orf19.7371 & ZCF35 & IPF1264 & Transcription factor activity & -1.7 & 1.8 & 1.3 & 1.1 & -1.6 \\
\hline orf19.2363 & -- & IPF12845 & -- & -1.5 & 1.2 & 1.2 & -1.4 & 1.1 \\
\hline orf19.3551 & -- & IPF13370 & Structural constituent of cytoskeleton & -1.8 & -1.0 & 1.4 & 1.6 & -1.7 \\
\hline orf19.4474 & -- & IPF14379 & -- & -1.8 & -1.7 & 4.2 & -1.1 & 1.1 \\
\hline orf19.4342 & -- & IPF14623 & Specific RNA polymerase II transcription factor activity & -3.2 & 1.3 & 1.1 & -1.0 & -1.7 \\
\hline orf19.954 & -- & IPF1557 & -- & -1.5 & 1.1 & -1.2 & -1.3 & -1.2 \\
\hline orf19.4698 & -- & IPF15977 & -- & -1.8 & -1.0 & 1.2 & -1.9 & -1.2 \\
\hline orf19.5297 & -- & IPF19753 & General RNA polymerase II transcription factor activity & -1.8 & 1.3 & 1.6 & -1.1 & -1.0 \\
\hline orf19.6920 & -- & IPF2195 & -- & -2.2 & -1.2 & -1.3 & 1.1 & 1.3 \\
\hline orf19.3260 & -- & IPF256 & -- & -1.7 & 2.0 & 1.1 & 1.9 & 1.7 \\
\hline orf19.871 & -- & IPF3950 & 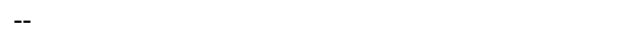 & -2.1 & -1.0 & 1.6 & 1.1 & 1.0 \\
\hline orf19.7506 & -- & IPF 404.5f & Protein binding & -1.7 & 1.2 & 1.1 & -1.2 & -1.0 \\
\hline orf19.6934 & -- & IPF4206 & -- & -1.5 & 1.1 & 2.4 & -1.0 & -1.6 \\
\hline orf19.5908 & TEC1 & IPF4351 & Specific RNA polymerase II transcription factor activity & -1.6 & 1.0 & -1.1 & 1.1 & -1.6 \\
\hline orf19.5910 & -- & IPF4356 & -- & -1.6 & -1.3 & 1.4 & 1.5 & -1.2 \\
\hline orf19.7675 & -- & IPF4955 & Structural constituent of ribosome & -1.6 & 1.2 & 1.2 & -1.5 & 1.0 \\
\hline orf19.2529 & -- & IPF4988 & -- & -1.6 & -1.4 & 1.2 & 1.1 & 1.0 \\
\hline orf19.7256 & -- & IPF5243 & -- & -1.6 & 1.0 & 1.5 & 1.2 & -1.5 \\
\hline orf19.7085 & -- & IPF525 & -- & -1.5 & 1.0 & -1.0 & 1.2 & 1.8 \\
\hline orf19.7314 & $C D G 1$ & IPF5972 & -- & -1.9 & 1.5 & -1.1 & -1.2 & -1.5 \\
\hline orf19.1542 & $H E X 3$ & IPF6497 & -- & -1.7 & -1.0 & -1.3 & -1.1 & -1.1 \\
\hline orf19.7545 & -- & IPF7010.3 & -- & -1.7 & 1.1 & 1.2 & -1.0 & -1.6 \\
\hline orf19.5368 & -- & IPF714 & -- & -1.9 & 1.2 & 4.6 & 1.1 & 1.4 \\
\hline orf19.1449 & -- & IPF7863 & -- & -1.7 & -1.2 & 1.1 & 2.7 & 1.4 \\
\hline orf19.2939 & -- & IPF8114 & -- & -1.8 & -1.3 & -1.4 & 1.2 & -2.8 \\
\hline orf19.3714 & -- & IPF8295 & -- & -1.5 & 1.0 & -1.8 & -1.4 & -1.2 \\
\hline orf19.4066 & -- & IPF9466 & -- & -2.1 & -1.3 & -1.1 & 1.1 & 1.0 \\
\hline orf19.4144 & -- & IPF9825 & -- & -2.1 & 1.1 & 1.3 & 1.5 & 1.0 \\
\hline orf19.585 & -- & MRPL17 & Structural constituent of ribosome & -1.8 & 1.6 & 1.2 & 1.0 & -1.7 \\
\hline orf19.3321 & -- & MRS7 & -- & -1.8 & 1.3 & 1.7 & 1.4 & -1.1 \\
\hline
\end{tabular}


Table A-2 (continued).

\begin{tabular}{|c|c|c|c|c|c|c|c|c|}
\hline $\begin{array}{c}\text { Systematic } \\
\text { Name }^{a}\end{array}$ & $\begin{array}{c}\text { CGD } \\
\text { Name }^{b}\end{array}$ & $\begin{array}{c}\text { CandidaDB } \\
\text { Name }^{c}\end{array}$ & GO Annotation (Molecular Function) ${ }^{d}$ & $\begin{array}{c}\text { C56 vs. } \\
\text { C43 }\end{array}$ & $\begin{array}{c}\text { Gu5 vs. } \\
\text { Gu2 }\end{array}$ & $\begin{array}{c}17 \text { vs. } \\
3\end{array}$ & $\begin{array}{c}\mathbf{5 6 7 4} \text { vs. } \\
5457 \\
\end{array}$ & $\begin{array}{c}\text { SZY31 vs. } \\
5457\end{array}$ \\
\hline orf19.6294 & -- & MYO1 & Microfilament motor activity & -1.6 & 1.0 & 1.7 & 1.1 & -1.2 \\
\hline orf19.3181.1 & -- & NCE11 & -- & -2.7 & -1.1 & 1.3 & -1.0 & -1.5 \\
\hline orf19.7279 & -- & NIT2 & -- & -1.6 & -1.1 & -1.1 & -1.3 & 1.1 \\
\hline orf19.3197 & PAPl & PAP11 & RNA binding & -108.1 & 1.2 & -1.1 & -1.2 & -50.0 \\
\hline orf19.3974 & PUT2 & PUT2 & 1-Pyrroline-5-carboxylate dehydrogenase activity & -1.6 & -1.0 & 1.1 & -1.3 & -1.1 \\
\hline orf19.607 & -- & RAD26 & DNA-dependent ATPase activity & -1.7 & -1.0 & 1.8 & 1.6 & 1.1 \\
\hline orf19.5968 & RDII & RDI1 & Rho GDP-dissociation inhibitor activity & -1.6 & 1.0 & -1.0 & 1.0 & -1.4 \\
\hline orf19.2823 & RFG1 & ROX1 & DNA bending activity & -1.5 & 1.2 & 1.0 & -1.0 & -1.0 \\
\hline orf19.6933 & -- & RRD2 & Protein phosphatase type $2 \mathrm{~A}$ regulator activity & -1.5 & -1.2 & 1.0 & -1.1 & -1.1 \\
\hline orf19.2555 & URA5 & URA5 & Orotate phosphoribosyltransferase activity & -1.5 & 1.1 & 1.1 & -1.3 & -2.0 \\
\hline orf19.1495 & -- & UTR4 & -- & -2.0 & 1.4 & -1.2 & 1.8 & -2.2 \\
\hline orf19.6169 & -- & FRP2 & -- & 1.2 & -1.6 & 1.5 & 2.2 & -1.1 \\
\hline orf19.4570 & -- & IPF1038 & -- & 1.1 & -2.0 & 6.7 & 1.6 & -1.6 \\
\hline orf19.6308 & -- & IPF12227.3f & -- & 1.2 & -1.5 & -1.0 & 1.1 & 1.1 \\
\hline orf19.3905 & -- & IPF17642 & -- & -1.0 & -3.9 & 1.7 & 1.6 & 1.6 \\
\hline orf19.2336 & -- & IPF8682 & -- & 1.7 & -1.7 & 1.4 & 2.8 & -1.2 \\
\hline orf19.2028 & $M X R I$ & MXR1 & Protein-methionine-S-oxide reductase activity & -1.2 & -1.6 & -1.3 & -1.5 & -1.2 \\
\hline orf19.6031 & -- & VPS27 & -- & -1.2 & -1.5 & -1.1 & 1.3 & 1.2 \\
\hline orf19.1816 & $A L S 3$ & ALS3.3eoc & Cell adhesion molecule binding & 1.0 & -1.4 & -2.4 & 1.0 & -1.5 \\
\hline orf19.2419 & -- & DOM34 & -- & 1.1 & 1.1 & -1.7 & -1.1 & -1.1 \\
\hline orf19.3433 & OYE23 & EBP4 & NADPH dehydrogenase activity & 1.0 & 1.0 & -2.0 & -1.7 & 2.0 \\
\hline orf19.3374 & ECE1 & ECE1 & -- & 1.9 & 1.5 & -3.8 & 5.6 & 1.5 \\
\hline orf19.1774 & -- & FDH13.5f & Formate dehydrogenase activity & -1.1 & -1.7 & -1.5 & -1.1 & -1.8 \\
\hline orf19.6947 & -- & GTT1.3 & Glutathione transferase activity & -1.4 & -1.2 & -1.5 & 1.3 & -1.0 \\
\hline orf19.6706 & GYP7 & GYP7 & Rab GTPase activator activity & -1.4 & -1.1 & -1.9 & 1.2 & 1.6 \\
\hline orf19.779 & -- & HAT1 & $\mathrm{H} 3 / \mathrm{H} 4$ histone acetyltransferase activity & -1.3 & 1.1 & -1.6 & 1.0 & -1.0 \\
\hline orf19.1979 & GIT1 & IFN1 & Phospholipid transporter activity & -1.3 & -1.1 & -1.5 & -1.0 & 1.2 \\
\hline orf19.2283 & -- & IPF10071 & -- & 1.1 & -1.1 & -1.5 & 1.1 & -1.2 \\
\hline orf19.1392 & -- & IPF11068 & Protein disulfide isomerase activity & 1.6 & 1.1 & -1.6 & -1.1 & 1.0 \\
\hline orf19.4771 & -- & IPF11667 & -- & -1.4 & -1.4 & -1.7 & -1.4 & 1.2 \\
\hline orf19.4457 & BNI4 & IPF11847 & -- & -1.0 & 1.2 & -1.5 & -1.1 & 1.6 \\
\hline orf19.3222 & -- & IPF162 & -- & -1.2 & -1.1 & -1.6 & -1.0 & 1.9 \\
\hline orf19.2989 & -- & IPF16795 & -- & -1.0 & 1.0 & -1.9 & 1.5 & 2.0 \\
\hline orf19.4997 & KIS2 & IPF1792 & AMP-activated protein kinase activity & -1.0 & -1.0 & -1.6 & -1.1 & 1.5 \\
\hline orf19.730 & -- & IPF20142 & -- & -1.1 & -1.0 & -1.5 & -1.3 & 1.7 \\
\hline orf19.588 & -- & IPF6380 & -- & 1.2 & 1.2 & -1.8 & 1.3 & -1.6 \\
\hline orf19.1573 & -- & IPF6387.3 & -- & 1.2 & 1.1 & -2.3 & 1.6 & -1.1 \\
\hline orf19.6077 & -- & IPF8369 & -- & 1.2 & -1.2 & -1.7 & -1.1 & 1.5 \\
\hline orf19.1187 & $\mathrm{CPH} 2$ & IPF9062 & Double-stranded DNA binding & -1.1 & -1.0 & -1.6 & -1.5 & -1.1 \\
\hline orf19.4374 & -- & IPF9438 & -- & -1.0 & -1.0 & -1.5 & -1.3 & -1.7 \\
\hline orf19.6671 & $L A P 4$ & LAP42 & Aminopeptidase I activity & 1.0 & -1.2 & -1.7 & -1.1 & 1.5 \\
\hline
\end{tabular}


Table A-2 (continued).

\begin{tabular}{|c|c|c|c|c|c|c|c|c|}
\hline $\begin{array}{c}\text { Systematic } \\
\text { Name }^{a}\end{array}$ & $\begin{array}{c}\text { CGD } \\
\text { Name }^{b} \\
\end{array}$ & $\begin{array}{c}\text { CandidaDB } \\
\text { Name }^{c}\end{array}$ & GO Annotation (Molecular Function) ${ }^{d}$ & $\begin{array}{c}\text { C56 vs. } \\
\text { C43 }\end{array}$ & $\begin{array}{c}\text { Gu5 vs. } \\
\text { Gu2 }\end{array}$ & $\begin{array}{c}17 \text { vs. } \\
3\end{array}$ & $\begin{array}{c}5674 \text { vs. } \\
\mathbf{5 4 5 7} \\
\end{array}$ & $\begin{array}{c}\text { SZY31 vs } \\
5457\end{array}$ \\
\hline orf19.3796 & -- & RNT1 & Ribonuclease III activity & -1.2 & 1.7 & -1.5 & 1.1 & 1.1 \\
\hline orf19.5750 & SHM2 & SHM2 & Glycine hydroxymethyltransferase activity & -1.2 & -1.1 & -1.6 & -1.5 & 1.2 \\
\hline orf19.2179 & SIT1 & SIT1 & Ferrichrome transporter activity & 1.6 & -1.3 & -1.7 & 1.1 & 1.2 \\
\hline orf19.6260 & -- & UBP12 & Ubiquitin-specific protease activity & -1.1 & 1.1 & -1.6 & 1.9 & 1.2 \\
\hline orf19.559 & FGR14 & Zorro1a & -- & 1.1 & 1.1 & -2.9 & -1.1 & -1.1 \\
\hline orf19.3331 & $A B C 1$ & $\mathrm{ABC} 1$ & -- & -1.1 & 1.2 & 1.7 & -2.1 & -1.2 \\
\hline orf19.6632 & $A C O 2$ & $\mathrm{ACO} 2$ & Aconitate hydratase activity & -1.2 & -1.0 & 1.1 & -1.9 & 1.1 \\
\hline orf19.3468 & ALG11 & ALG11 & Alpha-1,2-mannosyltransferase activity & 1.3 & -1.0 & -1.3 & -1.9 & 1.0 \\
\hline orf19.7551 & $A L O 1$ & ALO1 & D-arabinono-1,4-lactone oxidase activity & -1.2 & -1.0 & 1.1 & -2.0 & 1.1 \\
\hline orf19.2848 & -- & APG13 & Protein binding & -1.0 & -1.1 & -1.1 & -1.5 & 1.3 \\
\hline orf19.5487 & $C D C 46$ & CDC46 & ATP-dependent DNA helicase activity & 1.1 & -1.2 & 1.1 & -1.7 & 1.1 \\
\hline orf19.5870 & CTP1 & CTP1 & Tricarboxylate carrier activity & -1.2 & -1.0 & 1.0 & -1.7 & -1.3 \\
\hline orf19.4870 & -- & DBP3 & ATP-dependent RNA helicase activity & -1.2 & 1.1 & -1.0 & -1.9 & -1.0 \\
\hline orf19.3393 & -- & DBP9 & ATP-dependent RNA helicase activity & -1.3 & 1.1 & 1.1 & -2.2 & -1.6 \\
\hline orf19.6702 & DED81 & DED81 & ATP binding & -1.1 & -1.0 & -1.1 & -1.9 & 1.2 \\
\hline orf19.5164 & ECM39 & ECM39 & Alpha-1,6-mannosyltransferase activity & 1.0 & 1.0 & -1.2 & -2.3 & 1.7 \\
\hline orf19.7332 & ELF1 & ELF1 & ATP binding & -1.3 & 1.5 & 1.1 & -2.2 & -1.6 \\
\hline orf19.333 & $F C Y 2$ & FCY22 & Cytosine-purine permease activity & -1.3 & 1.1 & -1.3 & -2.0 & 1.3 \\
\hline orf19.7231 & FTR2 & FTR2 & Iron ion binding & -1.2 & 1.1 & -1.1 & -2.2 & 1.0 \\
\hline orf19.481 & $G C D 1$ & GCD1 & Translation initiation factor activity & -1.3 & 1.1 & 1.1 & -1.9 & -1.0 \\
\hline orf19.500 & -- & GCD10 & tRNA methyltransferase activity & -1.2 & 1.2 & -1.2 & -2.2 & -1.3 \\
\hline orf19.2003 & $H N M 1$ & HNM1 & Choline transporter activity & 1.0 & -1.4 & -1.0 & -2.3 & -1.7 \\
\hline orf19.4889 & -- & HOL2 & Transporter activity & -1.3 & -1.1 & -1.2 & -2.6 & -1.2 \\
\hline orf19.3476 & -- & HRR25 & Casein kinase activity & -1.2 & 1.3 & 1.1 & -1.5 & -1.0 \\
\hline orf19.6515 & HSP90 & HSP90 & ATPase activity, coupled & -1.1 & -1.0 & 1.1 & -1.7 & 1.3 \\
\hline orf19.7488 & -- & IMP3 & snoRNA binding & -1.1 & 1.1 & -1.1 & -1.7 & -2.6 \\
\hline orf19.5071 & $N R P 1$ & IPF10092 & -- & -1.1 & 1.1 & -1.0 & -1.8 & -1.2 \\
\hline orf19.5034 & -- & IPF10424 & -- & -1.2 & -1.1 & -1.2 & -1.6 & -1.0 \\
\hline orf19.5987 & -- & IPF11 & -- & -1.1 & 1.1 & -1.1 & -1.6 & -1.1 \\
\hline orf19.4623 & -- & IPF11090.exon2 & -- & -1.3 & 1.1 & -1.1 & -1.6 & 1.2 \\
\hline orf19.51 & -- & IPF11177 & Translation initiation factor activity & -1.4 & 1.1 & 1.2 & -1.9 & -1.5 \\
\hline orf19.1418 & SEC15 & IPF11236.3f & -- & -1.1 & 1.1 & -1.1 & -1.5 & -1.1 \\
\hline orf19.4518 & -- & IPF11424 & -- & 1.0 & 1.1 & -1.1 & -1.6 & -1.0 \\
\hline orf19.6463 & -- & IPF11432 & -- & -1.3 & 1.1 & -1.1 & -1.5 & 1.0 \\
\hline orf19.1646 & -- & IPF11615 & rRNA primary transcript binding & -1.2 & -1.1 & 1.1 & -2.3 & -1.5 \\
\hline orf19.3412 & ATG15 & IPF12033 & Lipase activity & 1.0 & -1.0 & -1.2 & -1.7 & 1.2 \\
\hline orf19.4787 & -- & IPF12091 & ATPase activator activity & -1.1 & 1.3 & 1.1 & -2.0 & -1.2 \\
\hline orf19.2667 & $R P F 1$ & IPF12428 & -- & -1.3 & 1.1 & -1.1 & -1.9 & -1.7 \\
\hline orf19.5238 & -- & IPF12577 & -- & -1.2 & -1.0 & -1.0 & -1.9 & 1.2 \\
\hline orf19.2898 & -- & IPF13465 & -- & 1.1 & 1.0 & -1.2 & -2.3 & 1.5 \\
\hline orf19.2451 & $P G A 45$ & IPF14109 & -- & 1.3 & 1.2 & 1.1 & -2.2 & 1.1 \\
\hline
\end{tabular}


Table A-2 (continued).

\begin{tabular}{|c|c|c|c|c|c|c|c|c|}
\hline $\begin{array}{c}\text { Systematic } \\
\text { Name }^{a}\end{array}$ & $\begin{array}{c}\text { CGD } \\
\text { Name }^{b}\end{array}$ & $\begin{array}{c}\text { CandidaDB } \\
\text { Name }^{c}\end{array}$ & GO Annotation (Molecular Function) ${ }^{d}$ & $\begin{array}{c}\text { C56 vs. } \\
\text { C43 }\end{array}$ & $\begin{array}{c}\text { Gu5 vs. } \\
\text { Gu2 } \\
\end{array}$ & $\begin{array}{c}17 \text { vs. } \\
3\end{array}$ & $\begin{array}{c}\mathbf{5 6 7 4} \text { vs. } \\
\mathbf{5 4 5 7} \\
\end{array}$ & $\begin{array}{c}\text { SZY31 vs. } \\
5457\end{array}$ \\
\hline orf19.2452 & -- & IPF14850 & -- & -1.1 & -1.1 & -1.4 & -3.8 & -1.1 \\
\hline orf19.2108 & SOD6 & IPF 15423 & Copper, zinc superoxide dismutase activity & 1.5 & -1.0 & 1.1 & -1.7 & 1.5 \\
\hline orf19.2256 & -- & IPF15468 & -- & -1.2 & -1.0 & -1.2 & -1.8 & -1.3 \\
\hline orf19.6539 & -- & IPF15737 & ATPase activity & -1.2 & 1.0 & -1.2 & -1.7 & 1.4 \\
\hline orf19.6581 & -- & IPF1629 & 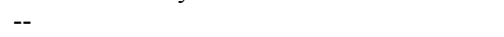 & 1.1 & 1.1 & 1.2 & -1.6 & 1.0 \\
\hline orf19.6566 & -- & IPF1660 & -- & 1.0 & -1.1 & 1.2 & -1.8 & 1.2 \\
\hline orf19.2917 & -- & IPF16748 & -- & -1.2 & 1.9 & -1.0 & -1.8 & -1.4 \\
\hline orf19.2664 & -- & IPF16761 & -- & -1.0 & 1.1 & 1.5 & -1.8 & 1.2 \\
\hline orf19.2143 & -- & IPF17094 & -- & -1.2 & 1.1 & 1.2 & -2.0 & -1.2 \\
\hline orf19.3553 & -- & IPF17139 & -- & -1.3 & 1.2 & -1.4 & -1.8 & -1.5 \\
\hline orf19.6707 & -- & IPF17904.3 & -- & -1.3 & 1.1 & -1.1 & -4.8 & 1.2 \\
\hline orf19.7028 & -- & IPF18125 & -- & 1.2 & -1.0 & -1.1 & -1.7 & 1.9 \\
\hline orf19.5884 & -- & IPF1846 & -- & -1.1 & 1.3 & -1.2 & -1.8 & -1.1 \\
\hline orf19.3159 & UTP20 & IPF18641.exon2 & -- & 1.0 & 1.1 & 1.0 & -2.0 & -1.4 \\
\hline orf19.7154 & -- & IPF1948 & -- & -1.1 & -1.0 & 1.1 & -2.1 & -1.1 \\
\hline orf19.5559 & STE23 & IPF19660 & -- & 1.1 & -1.2 & -1.4 & -1.7 & -1.3 \\
\hline orf19.6789 & -- & IPF19818 & Structural constituent of cytoskeleton & -1.1 & 1.1 & 2.0 & -2.0 & -1.1 \\
\hline orf19.7182 & -- & IPF2166 & -- & -1.2 & 1.1 & -1.1 & -2.1 & -1.0 \\
\hline orf19.6902 & -- & IPF2228 & ATP-dependent RNA helicase activity & -1.3 & -1.1 & -1.1 & -2.8 & 1.2 \\
\hline orf19.7022 & -- & IPF2359 & -- & -1.0 & -1.1 & -1.2 & -1.6 & 1.2 \\
\hline orf19.6818 & -- & IPF2409 & Helicase activity & -1.1 & 1.1 & 1.3 & -2.1 & -1.4 \\
\hline orf19.7424 & -- & IPF2441 & -- & -1.3 & 1.3 & -1.1 & -2.3 & -1.5 \\
\hline orf19.6625 & -- & IPF2560 & -- & -1.1 & -1.2 & -1.1 & -1.6 & -1.3 \\
\hline orf19.3559 & -- & IPF3184.exon1 & -- & -1.3 & -1.0 & -1.1 & -1.7 & -1.4 \\
\hline orf19.3559 & -- & IPF3184.exon2 & -- & -1.0 & 1.0 & 1.0 & -1.8 & -1.6 \\
\hline orf19.4384 & HXT5 & IPF3282 & Fructose transporter activity & 1.2 & 1.0 & -1.1 & -1.6 & -1.3 \\
\hline orf19.7398 & -- & IPF3309.3eoc & -- & -1.2 & 1.2 & -1.4 & -2.0 & -1.4 \\
\hline orf19.7388 & PBS2 & IPF3329 & MAP kinase kinase activity & -1.1 & 1.1 & 1.2 & -2.0 & 1.1 \\
\hline orf19.6752 & -- & IPF3492 & Structural constituent of ribosome & -1.1 & -1.0 & 1.0 & -1.5 & -1.5 \\
\hline orf19.28 & -- & IPF3853 & Transporter activity & -1.2 & -1.2 & -1.2 & -1.9 & -1.3 \\
\hline orf19.768 & SYG1 & IPF3887 & -- & 1.1 & -1.1 & -1.2 & -2.5 & 1.7 \\
\hline orf19.6503 & -- & IPF3928 & -- & -1.3 & 1.5 & 1.0 & -1.8 & 1.2 \\
\hline orf19.671 & PSP1 & IPF3970 & -- & -1.3 & -1.1 & -1.2 & -1.6 & 1.2 \\
\hline orf19.1630 & -- & IPF4253 & -- & -1.2 & 1.1 & -1.0 & -1.6 & 2.2 \\
\hline orf19.1633 & -- & IPF4257 & snoRNA binding & -1.3 & 1.1 & -1.0 & -1.7 & -1.3 \\
\hline orf19.549 & -- & IPF4276 & Structural constituent of ribosome & -1.2 & 1.1 & 1.1 & -1.8 & -1.2 \\
\hline orf19.541 & -- & IPF4290 & -- & -1.2 & -1.4 & -1.3 & -2.1 & 1.0 \\
\hline orf19.5279 & -- & IPF4703 & -- & -1.3 & 1.0 & 1.1 & -1.7 & -1.9 \\
\hline orf19.6276 & -- & IPF4859 & -- & 1.1 & 1.2 & -1.4 & -1.6 & 1.7 \\
\hline orf19.6392 & -- & IPF5129 & -- & 1.0 & 1.3 & 1.2 & -1.7 & -1.2 \\
\hline orf19.5681 & -- & IPF5469 & -- & 1.2 & -1.1 & 1.2 & -1.8 & -1.3 \\
\hline
\end{tabular}


Table A-2 (continued).

\begin{tabular}{|c|c|c|c|c|c|c|c|c|}
\hline $\begin{array}{c}\text { Systematic } \\
\text { Name }^{a}\end{array}$ & $\begin{array}{c}\text { CGD } \\
\text { Name }^{b}\end{array}$ & $\begin{array}{c}\text { CandidaDB } \\
\text { Name }^{c}\end{array}$ & GO Annotation (Molecular Function) ${ }^{d}$ & $\begin{array}{c}\text { C56 vs. } \\
\text { C43 }\end{array}$ & $\begin{array}{l}\text { Gu5 vs. } \\
\text { Gu2 }\end{array}$ & $\begin{array}{c}17 \text { vs. } \\
3\end{array}$ & $\begin{array}{c}\mathbf{5 6 7 4} \text { vs. } \\
\mathbf{5 4 5 7}\end{array}$ & $\begin{array}{c}\text { SZY31 vs. } \\
5457\end{array}$ \\
\hline orf19.7098 & -- & IPF556 & -- & -1.1 & -1.2 & -1.2 & -1.8 & 1.2 \\
\hline orf19.7107 & -- & IPF5615 & -- & -1.1 & 1.7 & -1.1 & -1.9 & -1.0 \\
\hline orf19.4176 & -- & IPF6175 & Structural constituent of ribosome & -1.3 & 1.0 & -1.1 & -2.2 & -1.5 \\
\hline orf19.1971 & -- & IPF6286 & DNA binding & -1.1 & 1.0 & 1.5 & -2.3 & -1.5 \\
\hline orf19.1964 & -- & IPF6298 & -- & -1.1 & 1.3 & 1.1 & -2.3 & -1.3 \\
\hline orf19.7618 & -- & IPF630 & -- & -1.2 & 1.3 & -1.0 & -2.3 & -1.4 \\
\hline orf19.1693 & CAS4 & IPF6521.3eoc & Transcription factor activity & -1.2 & 1.2 & 1.1 & -1.6 & 1.1 \\
\hline orf19.7599 & -- & IPF668 & snoRNA binding & -1.3 & 1.2 & -1.0 & -1.9 & -1.1 \\
\hline orf19.1565 & -- & IPF7147 & tRNA dihydrouridine synthase activity & -1.2 & -1.2 & -1.0 & -1.9 & 1.2 \\
\hline orf19.386 & SAM4 & IPF7279 & Homocysteine S-methyltransferase activity & -1.2 & -1.0 & -1.0 & -2.2 & 1.0 \\
\hline orf19.2447 & -- & IPF7472 & -- & -1.3 & -1.2 & -1.3 & -1.5 & -1.4 \\
\hline orf19.2936 & -- & IPF8110 & -- & -1.0 & -1.3 & -1.1 & -2.3 & -1.7 \\
\hline orf19.3327 & -- & IPF8326 & tRNA methyltransferase activity & -1.2 & 1.1 & 1.0 & -1.8 & -1.7 \\
\hline orf19.3977 & -- & IPF8652 & -- & -1.4 & -1.1 & -1.2 & -1.6 & -1.4 \\
\hline orf19.2487 & -- & IPF9225 & -- & -1.1 & 1.0 & 1.3 & -1.6 & -1.0 \\
\hline orf19.4375 & -- & IPF9435 & -- & -1.2 & 1.1 & -1.1 & -1.8 & -1.2 \\
\hline orf19.6092 & KEL1 & KEL1 & -- & -1.4 & -1.0 & -1.1 & -2.1 & 1.8 \\
\hline orf19.6086 & LEU4 & LEU41 & 2-isopropylmalate synthase activity & 1.2 & 1.2 & -1.1 & -2.0 & 1.2 \\
\hline orf19.3540 & MAK5 & MAK5 & ATP-dependent RNA helicase activity & -1.2 & -1.2 & -1.1 & -1.8 & -1.5 \\
\hline orf19.2551 & MET6 & MET6 & 5-methyltetrahydropteroyltriglutamate-homocysteine S-methyltransferase activity & 1.0 & -1.1 & -1.1 & -1.9 & -1.0 \\
\hline orf19.7383 & $M N N 9$ & MNN9 & Mannosyltransferase activity & -1.0 & 1.2 & 1.2 & -1.7 & -1.1 \\
\hline orf19.1662 & -- & MRP1.3f & Structural constituent of ribosome & -1.3 & 1.2 & 1.1 & -1.5 & -1.4 \\
\hline orf19.185 & -- & MRP51 & Structural constituent of ribosome & -1.2 & 1.1 & -1.1 & -1.8 & -2.7 \\
\hline orf19.3532 & MRPL10 & MRPL10.3 & Structural constituent of ribosome & -1.5 & 1.7 & 1.2 & -2.1 & -1.5 \\
\hline orf19.2019 & -- & MRPL16 & Peptidyltransferase activity & -1.1 & 1.1 & -1.1 & -1.7 & -1.3 \\
\hline orf19.6129 & MRPL8 & MRPL8 & Structural constituent of ribosome & -1.1 & 1.1 & -1.2 & -1.6 & -1.0 \\
\hline orf19.706 & $N M D 3$ & NMD3 & RNA binding & -1.2 & 1.2 & 1.0 & -2.5 & -1.0 \\
\hline orf19.1199 & NOP5 & NOP58 & snoRNA binding & -1.1 & 1.2 & 1.2 & -1.6 & 1.0 \\
\hline orf19.3000 & -- & ORC1 & ATPase activity & -1.0 & 3.1 & 1.1 & -3.2 & -1.1 \\
\hline orf19.2199 & PHO86 & PHO86 & -- & -1.1 & -1.3 & -1.2 & -1.8 & 1.1 \\
\hline orf19.5847 & -- & RET1 & DNA-directed RNA polymerase activity & -1.2 & 1.2 & 1.0 & -2.0 & 1.0 \\
\hline orf19.2320 & -- & RIO1 & Amino acid kinase activity & -1.4 & 1.3 & -1.1 & -2.1 & -1.6 \\
\hline orf19.5420 & -- & RML2 & Structural constituent of ribosome & -1.2 & 1.2 & 1.2 & -1.8 & -1.0 \\
\hline orf19.2594 & -- & RPA43 & DNA-directed RNA polymerase activity & -1.3 & 1.1 & 1.0 & -2.2 & -1.8 \\
\hline orf19.443 & -- & RPC25 & DNA-directed RNA polymerase activity & -1.3 & 1.2 & 1.3 & -1.8 & -1.5 \\
\hline orf19.2830 & -- & RRP9 & snoRNA binding & -1.3 & -1.0 & 1.5 & -1.7 & -1.7 \\
\hline orf19.6355 & -- & RSA2 & -- & -1.3 & 1.2 & -1.1 & -1.9 & -1.2 \\
\hline orf19.24 & RTA2 & RTA2 & Phospholipid-translocating ATPase activity & 1.5 & -1.2 & -1.1 & -2.1 & 1.1 \\
\hline orf19.642 & -- & SAP155 & Protein serine/threonine kinase activity & -1.0 & -1.2 & 1.2 & -2.1 & -1.1 \\
\hline orf19.6558 & -- & SEC231 & GTPase activator activity & -1.2 & 1.4 & 1.1 & -1.7 & 1.4 \\
\hline orf19.5263 & SER33 & SER33 & Phopshoglycerate dehydrogenase activity & -1.0 & -1.2 & -1.1 & -1.7 & 1.2 \\
\hline
\end{tabular}


Table A-2 (continued).

\begin{tabular}{|c|c|c|c|c|c|c|c|c|}
\hline $\begin{array}{c}\text { Systematic } \\
\text { Name }^{a}\end{array}$ & $\begin{array}{c}\text { CGD } \\
\text { Name }^{b} \\
\end{array}$ & $\begin{array}{c}\text { CandidaDB } \\
\text { Name }^{c}\end{array}$ & GO Annotation (Molecular Function) ${ }^{d}$ & $\begin{array}{c}\text { C56 vs. } \\
\text { C43 }\end{array}$ & $\begin{array}{c}\text { Gu5 vs. } \\
\text { Gu2 }\end{array}$ & $\begin{array}{c}17 \text { vs. } \\
3\end{array}$ & $\begin{array}{c}\mathbf{5 6 7 4} \text { vs. } \\
\mathbf{5 4 5 7} \\
\end{array}$ & $\begin{array}{c}\text { SZY31 vs } \\
\mathbf{5 4 5 7} \\
\end{array}$ \\
\hline orf19.5953 & -- & SFP1 & Transcription factor activity & 1.0 & 1.2 & 1.2 & -1.6 & 1.3 \\
\hline orf19.7569 & -- & SIK1 & snoRNA binding & -1.2 & 1.2 & -1.0 & -1.5 & -1.0 \\
\hline orf19.4367 & SMC1 & SMC1 & ATP DNA binding & 1.2 & -1.1 & 1.1 & -1.6 & -1.1 \\
\hline orf19.5407 & -- & SOF1 & snoRNA binding & -1.2 & 1.1 & -1.0 & -1.6 & -1.6 \\
\hline orf19.4960 & -- & SPE4 & -- & -1.2 & 1.0 & 1.0 & -2.5 & -1.2 \\
\hline orf19.1139 & -- & SVL3 & -- & -1.2 & 1.1 & 1.2 & -1.6 & -1.1 \\
\hline orf19.1352 & -- & TIM22 & Protein transporter activity & -1.2 & 1.0 & -1.0 & -1.9 & -1.7 \\
\hline orf19.5143 & -- & TIM54 & Protein transporter activity & -1.3 & 1.2 & 1.5 & -2.0 & 1.2 \\
\hline orf19.1532 & -- & TOM37 & -- & 1.2 & 1.4 & 1.5 & -2.0 & -1.5 \\
\hline orf19.429 & -- & TRF4 & DNA-directed DNA polymerase activity & -1.4 & 1.2 & 1.4 & -2.1 & -1.5 \\
\hline orf19.802 & -- & $\mathrm{UGA} 12.5 \mathrm{f}$ & 4-aminobutyrate transaminase activity & -1.3 & -1.1 & 1.1 & -2.3 & 1.8 \\
\hline orf19.3363 & VTC4 & VTC4 & -- & -1.1 & -1.0 & -1.0 & -1.6 & 1.0 \\
\hline orf19.7036 & -- & WHI2 & -- & -1.1 & -1.1 & 1.3 & -1.5 & 1.1 \\
\hline orf19.3300 & ZPRI & ZPR1 & Transcription factor activity & -1.3 & 1.0 & -1.1 & -1.8 & -1.1 \\
\hline orf19.4773 & $A O X 2$ & AOX2 & Alternative oxidase activity & -2.6 & -2.1 & -1.1 & 1.1 & -2.7 \\
\hline orf19.1078 & $H B R 2$ & IPF14203.5f & -- & -1.6 & -1.5 & -1.1 & -1.1 & -1.2 \\
\hline orf19.2158 & NAG3 & IPF2710.repeat2 & Monosaccharide transporter activity & -2.4 & -1.7 & -1.5 & 2.4 & 6.1 \\
\hline orf19.2948 & SNO1 & SNO1 & Imidazoleglycerol-phosphate synthase activity & -2.6 & -1.6 & -1.4 & -1.2 & 1.3 \\
\hline orf19.7111 & -- & SOD21.5f & Manganese superoxide dismutase activity & -1.6 & -1.8 & -1.1 & 2.3 & 2.0 \\
\hline orf19.7111 & -- & SOD22.3f & Manganese superoxide dismutase activity & -1.6 & -2.4 & 1.3 & 1.0 & 1.9 \\
\hline orf19.5531 & $C D C 37$ & $\mathrm{CDC} 37$ & Unfolded protein binding & -1.7 & -1.0 & -2.4 & -1.0 & -1.2 \\
\hline orf19.3915 & -- & IPF $10029.5 \mathrm{eoc}$ & -- $\quad$ r & -1.8 & -1.3 & -1.5 & -1.4 & 1.5 \\
\hline orf19.2246 & -- & IPF12900 & -- & -1.6 & -1.2 & -1.7 & 1.0 & -1.5 \\
\hline orf19.3050 & $A G E 1$ & IPF3610 & ARF GTPase activator activity & -1.5 & -1.3 & -1.5 & -1.3 & 1.2 \\
\hline orf19.4900 & -- & IPF8854 & Alpha-1,3-mannosyltransferase activity & -1.9 & 1.1 & -1.6 & -1.3 & -1.3 \\
\hline orf19.1769 & -- & PLP2 & -- & -1.9 & -1.0 & -2.0 & -1.4 & -1.0 \\
\hline orf19.5906 & $A D E 2$ & ADE2 & Phosphoribosylaminoimidazole carboxylase activity & -1.5 & -1.3 & -1.3 & -1.8 & 1.6 \\
\hline orf19.5806 & ALD5 & ALD5 & 3-chloroallyl aldehyde dehydrogenase activity & -1.6 & -1.3 & 1.2 & -4.1 & -1.0 \\
\hline orf19.1623 & CAPl & CAP1 & Transcription factor activity & -1.7 & 1.1 & -1.0 & -1.7 & -1.1 \\
\hline orf19.2876 & $C B F 1$ & CBF1 & Centromeric DNA binding & -1.8 & -1.1 & -1.2 & -1.7 & 1.1 \\
\hline orf19.6948 & $\mathrm{CCCl}$ & $\mathrm{CCC} 1$ & -- & -1.8 & -1.4 & -1.0 & -2.3 & -1.2 \\
\hline orf19.1055 & $C D C 3$ & $\mathrm{CDC} 3$ & Nucleotide binding & -1.6 & 1.2 & -1.1 & -1.6 & 1.2 \\
\hline orf19.1996 & CHAl & CHA12 & L-serine ammonia-lyase activity & -5.3 & -1.1 & -1.2 & -2.6 & -1.9 \\
\hline orf19.7478 & -- & COQ1 & Trans-hexaprenyltranstransferase activity & -1.7 & 1.1 & 1.0 & -1.6 & -1.6 \\
\hline orf19.6652 & $D B P 8$ & DBP8 & ATP-dependent RNA helicase activity & -1.5 & 1.2 & -1.1 & -2.1 & -1.1 \\
\hline orf19.7635 & DRS1 & DRS1 & ATP-dependent RNA helicase activity & -1.7 & 1.0 & -1.1 & -2.1 & 1.2 \\
\hline orf19.3670 & GAL1 & GAL1 & Galactokinase activity & -1.6 & -1.1 & -1.3 & -1.8 & 1.3 \\
\hline orf19.385 & $G C V 2$ & GCV2 & Glycine dehydrogenase (decarboxylating) activity & -1.7 & -1.1 & -1.1 & -1.9 & 1.1 \\
\hline orf19.2396 & IFR2 & IFR2 & -- & -1.7 & -1.3 & -1.2 & -1.7 & 1.3 \\
\hline orf19.473 & TPO4 & IPF11142 & -- & -1.5 & -1.4 & -1.1 & -1.7 & 1.6 \\
\hline orf19.512 & -- & IPF11315 & -- & -1.6 & 1.2 & 1.0 & -2.0 & 1.1 \\
\hline
\end{tabular}


Table A-2 (continued).

\begin{tabular}{|c|c|c|c|c|c|c|c|c|}
\hline $\begin{array}{c}\text { Systematic } \\
\text { Name }^{a}\end{array}$ & $\begin{array}{c}\text { CGD } \\
\text { Name }^{b}\end{array}$ & $\begin{array}{c}\text { CandidaDB } \\
\text { Name }^{c}\end{array}$ & GO Annotation (Molecular Function) ${ }^{d}$ & $\begin{array}{l}\text { C56 vs. } \\
\text { C43 }\end{array}$ & $\begin{array}{c}\text { Gu5 vs. } \\
\text { Gu2 }\end{array}$ & $\begin{array}{c}17 \text { vs. } \\
3\end{array}$ & $\begin{array}{c}5674 \text { vs. } \\
5457\end{array}$ & $\begin{array}{c}\text { SZY31 vs. } \\
5457\end{array}$ \\
\hline orf19.1715 & IROI & IPF11363 & -- & -1.7 & -1.0 & -1.2 & -1.7 & 1.3 \\
\hline orf19.4337 & -- & IPF11698 & Monocarboxylic acid transporter activity & -1.6 & -1.3 & -1.3 & -1.6 & 1.8 \\
\hline orf19.2067 & -- & IPF1210 & -- & -1.5 & 1.1 & 1.3 & -1.6 & -1.2 \\
\hline orf19.2185 & -- & IPF12457 & -- & -1.7 & 1.5 & 1.1 & -2.1 & -1.4 \\
\hline orf19.2330 & -- & IPF13361 & snoRNA binding & -1.6 & 1.1 & -1.1 & -1.8 & 1.0 \\
\hline orf19.4746 & JIP5 & IPF14019 & -- & -1.7 & 1.1 & 1.1 & -2.2 & -1.8 \\
\hline orf19.4931 & -- & IPF 15618 & Cysteine-tRNA ligase activity & -1.5 & 1.0 & -1.1 & -1.7 & 1.1 \\
\hline orf19.4751 & -- & IPF16405 & Structural constituent of ribosome & -1.6 & 1.2 & -1.3 & -2.4 & -1.6 \\
\hline orf19.3547 & -- & IPF16996 & -- & -1.6 & 1.2 & -1.1 & -1.8 & -2.1 \\
\hline orf19.2778 & -- & IPF18002 & -- & -1.7 & 1.2 & -1.2 & -1.8 & -1.4 \\
\hline orf19.4929 & -- & IPF19785 & Translation regulator activity & -1.6 & 1.3 & 1.9 & -1.6 & 1.0 \\
\hline orf19.4093 & -- & IPF2093 & -- & -1.5 & 1.1 & -1.1 & -2.5 & 1.0 \\
\hline orf19.4105 & -- & IPF2109 & -- & -2.1 & -1.2 & -1.3 & -1.9 & -1.3 \\
\hline orf19.4835 & -- & IPF4485 & snoRNA binding & -1.8 & 1.1 & 1.1 & -1.9 & -1.1 \\
\hline orf19.6648 & -- & IPF5009 & -- & -1.8 & -1.2 & 1.1 & -1.9 & -1.2 \\
\hline orf19.3448 & -- & IPF5330 & -- & -10.5 & 1.3 & 1.2 & -3.0 & -1.6 \\
\hline orf19.7091 & -- & IPF538 & -- & -1.6 & 1.1 & 1.0 & -1.8 & -1.1 \\
\hline orf 19.5680 & -- & IPF5471 & -- & -1.6 & -1.2 & -1.3 & -1.7 & 1.3 \\
\hline orf19.4805 & -- & IPF6117 & -- & -1.5 & 1.1 & -1.1 & -2.5 & 1.1 \\
\hline orf19.7621 & -- & IPF625 & -- & -1.7 & 1.5 & 2.0 & -1.8 & -1.3 \\
\hline orf19.4191 & -- & IPF7998 & -- & -1.6 & 1.1 & -1.1 & -2.3 & -1.6 \\
\hline orf19.4268 & -- & IPF9525 & snoRNA binding & -1.5 & 1.2 & -1.3 & -1.6 & -1.2 \\
\hline orf19.3406 & -- & IPF9898 & -- & -1.9 & -1.2 & -1.5 & -2.7 & -1.5 \\
\hline orf19.1244 & GYP2 & MDR1 & Rab GTPase activator activity & -1.6 & -1.1 & 1.0 & -1.7 & 1.2 \\
\hline orf19.7384 & -- & NOG1 & GTPase activity & -1.5 & 1.3 & 1.0 & -2.8 & -1.5 \\
\hline orf19.1030 & -- & NPI46 & Peptidyl-prolyl cis-trans isomerase activity & -2.2 & 1.0 & 1.2 & -1.6 & -1.0 \\
\hline orf19.1687 & -- & PRP43 & ATP-dependent RNA helicase activity & -1.5 & 1.3 & -1.0 & -1.6 & -1.1 \\
\hline orf19.4274 & PUT1 & PUT1 & Proline dehydrogenase activity & -2.1 & 1.0 & 1.9 & -1.5 & 1.2 \\
\hline orf19.4593 & $R G A 2$ & RGA2 & Rho GTPase activator activity & -1.7 & 1.1 & 1.1 & -2.0 & -1.3 \\
\hline orf19.3867 & RPL7 & RLP7 & rRNA binding & -1.8 & 1.0 & -1.2 & -1.9 & -1.7 \\
\hline orf19.6041 & RPO41 & RPO41 & DNA-directed RNA polymerase activity & -1.5 & 1.1 & 1.5 & -2.1 & -1.2 \\
\hline orf19.1923 & $R R N 3$ & RRN3 & RNA polymerase I transcription factor activity & -1.7 & 1.6 & 1.6 & -2.2 & -1.0 \\
\hline orf19.1161 & PLD1 & SPO14.5eoc & Phospholipase D activity & -1.6 & 1.3 & 1.0 & -2.4 & 1.6 \\
\hline orf19.6109 & TUP1 & TUP1 & General transcriptional repressor activity & -1.8 & 1.1 & 1.1 & -1.7 & 1.5 \\
\hline orf19.1536 & -- & ZRC1 & $\mathrm{Di}-$, tri-valent inorganic cation transporter activity & -1.6 & 1.0 & -1.0 & -1.7 & -1.1 \\
\hline orf19.1816 & ALS3 & ALS10 & Cell adhesion molecule binding & -1.1 & -1.5 & -3.2 & 1.2 & -1.5 \\
\hline orf19.1816 & ALS3 & ALS3.5eoc & Cell adhesion molecule binding & 1.1 & -1.6 & -2.3 & 1.0 & 1.0 \\
\hline orf19.1264 & CFL2 & CFL2 & Ferric-chelate reductase activity & 1.6 & -2.0 & -1.6 & -1.3 & -1.6 \\
\hline orf19.1587 & $H G T 20$ & IPF13131.3 & Glucose transporter activity & 1.1 & -1.6 & -1.9 & 1.2 & 2.9 \\
\hline orf19.1121 & -- & IPF2329 & -- & -1.4 & -1.7 & -1.5 & -1.1 & -1.3 \\
\hline orf19.6636 & -- & IPF2524 & -- & -1.5 & -1.6 & -1.7 & -1.4 & 1.0 \\
\hline
\end{tabular}


Table A-2 (continued).

\begin{tabular}{|c|c|c|c|c|c|c|c|c|}
\hline $\begin{array}{c}\text { Systematic } \\
\text { Name }^{a}\end{array}$ & $\begin{array}{c}\text { CGD } \\
\text { Name }^{b}\end{array}$ & $\begin{array}{c}\text { CandidaDB } \\
\text { Name }^{c}\end{array}$ & GO Annotation (Molecular Function) ${ }^{d}$ & $\begin{array}{c}\text { C56 vs. } \\
\text { C43 }\end{array}$ & $\begin{array}{c}\text { Gu5 vs. } \\
\text { Gu2 }\end{array}$ & $\begin{array}{c}17 \text { vs. } \\
3\end{array}$ & $\begin{array}{c}\mathbf{5 6 7 4} \text { vs. } \\
\mathbf{5 4 5 7}\end{array}$ & $\begin{array}{c}\text { SZY31 vs. } \\
5457\end{array}$ \\
\hline orf19.6249 & HAKl & IPF9136.5eoc & Potassium ion transporter activity & 1.1 & -3.6 & -3.1 & -1.3 & -1.5 \\
\hline orf19.5591 & ADO1 & ADO1 & Adenosine kinase activity & 1.0 & -1.5 & -1.1 & -2.1 & -1.2 \\
\hline orf19.4099 & ECM17 & ECM17 & Sulfite reductase (NADPH) activity & -1.2 & -1.7 & -1.4 & -2.2 & 1.2 \\
\hline orf19.6570 & NUP & IPF1651 & Nucleoside permease activity & -1.1 & -1.7 & -1.1 & -1.8 & 2.1 \\
\hline orf19.6957.3 & -- & IPF19538 & -- & -1.3 & -1.6 & -1.3 & -1.5 & 1.1 \\
\hline orf19.7046 & MET28 & IPF3087 & Specific RNA polymerase II transcription factor activity & -1.1 & -1.9 & -1.1 & -1.8 & 1.3 \\
\hline orf19.1789.1 & LYS1 & LYS1.5eoc & Saccharopine dehydrogenase (NAD+, L-lysine-forming) activity & -1.2 & -1.5 & -1.4 & -2.4 & 1.0 \\
\hline orf19.3870 & ADE13 & ADE13 & Adenylosuccinate lyase activity & -1.2 & -1.2 & -1.5 & -1.9 & 1.4 \\
\hline orf19.5789 & ADE 8 & ADE8 & Phosphoribosylglycinamide formyltransferase activity & -1.3 & -1.4 & -1.5 & -2.0 & -1.2 \\
\hline orf19.2337 & $A L P 1$ & ALP1 & Basic amino acid transporter activity & -1.1 & -1.5 & -1.8 & -2.2 & -1.0 \\
\hline orf19.6459 & -- & DPP3 & Phosphatidase phosphatase activity & -1.2 & -1.2 & -1.8 & -2.2 & 1.4 \\
\hline orf19.4215 & FET34 & FET5 & Ferroxidase activity & 1.1 & -1.4 & -2.7 & -1.8 & 1.0 \\
\hline orf19.4456 & GAP4 & GAP5 & Amino acid permease activity & 1.0 & -1.1 & -1.5 & -2.9 & -1.0 \\
\hline orf19.7566 & -- & GNP1 & Amino acid permease activity & -1.0 & -1.5 & -1.6 & -2.8 & 1.2 \\
\hline orf19.2020 & HGT6 & HXT61 & Fructose transporter activity & 1.1 & -1.0 & -1.7 & -4.3 & 1.1 \\
\hline orf19.2923 & -- & IPF11448 & -- & -1.1 & -1.2 & -1.6 & -2.5 & 1.3 \\
\hline orf19.1961 & -- & IPF14506 & -- & 1.2 & 1.1 & $-\mathbf{3 . 0}$ & -1.5 & -2.8 \\
\hline orf19.3581 & -- & IPF3227 & -- & 1.0 & 1.0 & -1.9 & -1.7 & -1.7 \\
\hline orf19.6936 & RAD53 & RAD53 & Protein serine/threonine kinase activity & -1.1 & 1.1 & -1.5 & -1.6 & -1.0 \\
\hline orf19.5838 & -- & SER2 & Phosphoserine phosphatase activity & -1.3 & -1.3 & -1.6 & -2.1 & 1.1 \\
\hline orf19.844 & STE11 & STE11 & MAP kinase kinase kinase activity & -1.1 & 1.2 & -1.5 & -1.5 & 1.1 \\
\hline orf19.34 & -- & GIT1 & Phospholipid transporter activity & -2.0 & -2.8 & -2.5 & -1.4 & -1.0 \\
\hline orf19.7370 & -- & IPF1261 & -- & -1.8 & -1.5 & -1.5 & -1.0 & -1.3 \\
\hline orf19.2738 & SUL2 & SUL1 & Sulfate transporter activity & -2.0 & -4.3 & -2.1 & -1.0 & 1.5 \\
\hline orf19.4669 & AAT22 & AAT22 & Aspartate transaminase activity & -1.8 & -1.5 & -1.3 & -2.8 & -1.6 \\
\hline orf19.993 & -- & IPF20135 & -- & -1.5 & -1.5 & -1.3 & -1.7 & 1.1 \\
\hline orf19.847 & -- & IPF3415 & Mitochondrial inner membrane peptidase activity & -1.8 & -1.7 & -1.0 & -1.5 & 1.7 \\
\hline orf19.3040 & -- & IPF3589 & -- & -1.8 & -1.5 & -1.0 & -1.5 & 1.2 \\
\hline orf19.3529 & $A B P 2$ & IPF4463 & -- & -2.8 & -1.5 & 1.0 & -2.0 & -1.2 \\
\hline orf19.1847 & ARO10 & IPF4667 & Carboxy-lyase activity & -3.4 & -1.7 & -1.0 & -2.6 & 1.5 \\
\hline orf19.7422 & -- & LCP5 & -- & -1.5 & -1.9 & -1.1 & -2.0 & 1.0 \\
\hline orf19.5519 & GCV1 & GCV1 & Glycine dehydrogenase (decarboxylating) activity & -1.8 & -1.1 & -1.8 & -1.7 & 1.7 \\
\hline orf19.5392 & NGT1 & IPF1065 & $\mathrm{N}$-acetylglucosamine transporter activity & -1.5 & -1.4 & -1.8 & -2.7 & -1.2 \\
\hline orf19.4779 & -- & IPF12884 & Multidrug transporter activity & -2.2 & -1.1 & -1.7 & -2.2 & 1.3 \\
\hline orf19.4450.1 & -- & IPF6551 & -- & -1.7 & -1.3 & -1.5 & -1.9 & -1.0 \\
\hline orf19.1510 & -- & IPF9282 & -- & -1.6 & -1.4 & -1.9 & -3.4 & 1.7 \\
\hline orf19.3810 & -- & MTD1 & Methylenetetrahydrofolate dehydrogenase (NAD+) activity & -1.5 & -1.4 & -1.9 & -1.9 & 1.6 \\
\hline orf19.3599 & TIF4631 & TIF4631 & Translation initiation factor activity & -1.6 & 1.0 & -1.5 & -1.5 & -1.1 \\
\hline orf19.3122 & $A R R 3$ & ARR3 & Arsenite transporter activity & -1.2 & -1.6 & -1.6 & -1.8 & -1.1 \\
\hline orf19.111 & CAN2 & CAN2 & Basic amino acid transporter activity & -1.0 & -2.1 & -2.5 & -2.2 & 1.1 \\
\hline orf19.4215 & FET34 & FET34.3eoc & Ferroxidase activity & 1.1 & -1.5 & -2.2 & -3.2 & -1.1 \\
\hline
\end{tabular}


Table A-2 (continued).

\begin{tabular}{|c|c|c|c|c|c|c|c|c|}
\hline $\begin{array}{c}\text { Systematic } \\
\text { Name }^{a}\end{array}$ & $\begin{array}{c}\text { CGD } \\
\text { Name }^{b}\end{array}$ & $\begin{array}{c}\text { CandidaDB } \\
\text { Name }^{c}\end{array}$ & GO Annotation (Molecular Function) ${ }^{d}$ & $\begin{array}{l}\text { C56 vs. } \\
\text { C43 }\end{array}$ & $\begin{array}{l}\text { Gu5 vs. } \\
\text { Gu2 }\end{array}$ & $\begin{array}{c}17 \text { vs. } \\
3\end{array}$ & $\begin{array}{l}5674 \text { vs. } \\
5457\end{array}$ & $\begin{array}{c}\text { SZY31 vs. } \\
5457\end{array}$ \\
\hline orf19.1978 & GIT2 & IFN3 & Phospholipid transporter activity & 1.4 & -1.6 & -2.6 & -2.3 & 1.1 \\
\hline orf19.3902 & -- & IPF11503 & -- & 1.2 & -2.6 & -2.3 & -2.2 & 1.6 \\
\hline orf19.2475 & $P G A 26$ & IPF7204 & -- & -1.2 & -2.0 & -1.9 & -4.0 & 1.3 \\
\hline orf19.288 & -- & MET13 & Structural constituent of ribosome & -1.2 & -2.4 & -1.8 & -2.6 & -1.1 \\
\hline orf19.946 & MET14 & MET14 & Adenylylsulfate kinase activity & -1.2 & -2.7 & -2.0 & -2.1 & -2.0 \\
\hline orf19.638 & FDH1 & FDH12 & Formate dehydrogenase activity & -1.6 & -3.8 & -1.8 & -2.4 & -1.5 \\
\hline orf19.7219 & FTRI & FTR1 & Ferrous iron transporter activity & -2.7 & -3.0 & -2.1 & -4.0 & -1.6 \\
\hline orf19.4527 & HGT1 & HGT11 & Glucose transporter activity & -1.7 & -1.5 & -1.6 & -2.5 & -1.1 \\
\hline orf19.2461 & PRN4 & IFS1 & -- & -1.7 & -1.7 & -1.6 & -1.8 & -1.2 \\
\hline orf19.5760 & IHDI & IPF10662 & -- & -1.9 & -2.2 & -3.1 & -9.0 & 1.7 \\
\hline orf19.3475 & -- & IPF11725 & -- & -3.9 & -2.1 & -2.7 & -5.8 & -1.2 \\
\hline orf19.4655 & OPT6 & IPF12193 & Oligopeptide transporter activity & -1.8 & -2.0 & -2.4 & -3.1 & -1.4 \\
\hline orf19.2060 & SOD5 & IPF1222 & Copper, zinc superoxide dismutase activity & -2.3 & -8.6 & -17.8 & -26.3 & -1.4 \\
\hline orf19.2059 & -- & IPF1228 & -- & -2.0 & -1.7 & -1.7 & -2.9 & 1.2 \\
\hline orf19.5673 & OPT7 & IPF12736 & Oligopeptide transporter activity & -1.6 & -1.7 & -1.5 & -1.6 & 1.8 \\
\hline orf19.4749 & -- & IPF13921 & -- & -2.3 & -1.7 & -2.4 & -3.0 & -1.4 \\
\hline orf19.670.2 & -- & IPF20159 & -- & -2.3 & -2.0 & -1.7 & -4.9 & -1.9 \\
\hline orf19.6679 & -- & IPF2314 & -- & -1.5 & -2.1 & -1.8 & -2.2 & 1.1 \\
\hline orf19.7296 & -- & IPF2839 & -- & -1.6 & -3.4 & -2.5 & -5.4 & 1.2 \\
\hline orf19.7561 & $D E F 1$ & IPF946 & -- & -3.4 & -1.5 & -1.6 & -2.8 & -1.4 \\
\hline orf19.2618 & MET2 & MET2 & Homoserine $\mathrm{O}$-acetyltransferase activity & -1.5 & -1.5 & -1.6 & -1.6 & 1.2 \\
\hline orf19.5025 & MET3 & MET3 & Sulfate adenylyltransferase (ATP) activity & -2.0 & -7.9 & -1.8 & -2.5 & -1.2 \\
\hline orf19.700 & SEOI & $\mathrm{SEO} 2$ & Transporter activity & -2.3 & -2.5 & -3.0 & -2.1 & -1.2 \\
\hline orf19.2947 & SNZ1 & SNZ1 & -- & -3.7 & -1.8 & -1.7 & -2.2 & 1.3 \\
\hline
\end{tabular}

${ }^{a}$ orf19 nomenclature according to the Assembly 19 version.

${ }^{\boldsymbol{b}}$ Gene name at CGD (http://www.candidagenome.org/).

${ }^{c}$ Gene name at CandidaDB (http://genolist.pasteur.fr/CandidaDB/).

${ }^{d} \mathrm{GO}$ annotation found at CGD (http://www.candidagenome.org/).

Gene expression values in bold designate statistical significance $(p$-value $<0.05)$ in all three experiments. 


\section{APPENDIX B: MRR1 SUPPLEMENTAL DATA}

Table B-1. Genes up-regulated in at least one clinical matched isolate overexpressing MDR1.

\begin{tabular}{|c|c|c|c|c|c|c|c|c|c|c|c|}
\hline \multirow[b]{2}{*}{ CandidaDB Name $^{a}$} & \multirow[b]{2}{*}{ orf19 Name ${ }^{b}$} & \multirow[b]{2}{*}{ GO Annotation (Molecular Function) ${ }^{c}$} & \multicolumn{3}{|c|}{ F5 vs. F1 } & \multicolumn{3}{|c|}{ G5 vs. G1 } & \multicolumn{3}{|c|}{6692 vs. 5833} \\
\hline & & & 1 & 2 & 3 & 1 & 2 & 3 & 1 & 2 & 3 \\
\hline$A A F 1$ & orf19.7436 & 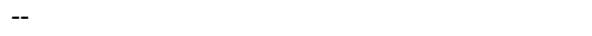 & 1.4 & 1.3 & 1.0 & 1.9 & 2.1 & 2.2 & 1.2 & 1.8 & 1.2 \\
\hline$A A H 1$ & orf19.2251 & Adenine deaminase activity & -2.0 & -1.6 & 1.6 & 1.5 & 1.7 & 2.6 & 1.5 & -1.2 & 3.0 \\
\hline$A C B 1.3 f$ & orf19.7043 & Acyl-CoA binding & -1.0 & -2.3 & 4.9 & 1.6 & 2.3 & 10.6 & 1.7 & -1.1 & 2.0 \\
\hline ADH5 & orf19.2608 & Alcohol dehydrogenase activity & 1.3 & 2.0 & 1.0 & -1.0 & 1.3 & -1.1 & 1.6 & 1.7 & 2.9 \\
\hline ALD4 & orf19.6306 & Aldehyde dehydrogenase (NAD) activity & -1.4 & -1.2 & 1.2 & 1.5 & 1.9 & 1.9 & 1.2 & 1.6 & -1.1 \\
\hline ALS10 & orf19.2355 & Cell adhesion molecule binding & 1.2 & 1.7 & 1.1 & -1.6 & -2.0 & -2.0 & 5.9 & 5.3 & 8.7 \\
\hline ALS10 & orf19.2355 & Cell adhesion molecule binding & 1.3 & 1.8 & 1.2 & -1.4 & -1.5 & -1.6 & 4.9 & 4.8 & 18.0 \\
\hline ALS3.5eoc & orf19.1816 & Cell adhesion molecule binding & 1.4 & 1.8 & 1.2 & -1.1 & -1.3 & -4.9 & 4.1 & 4.3 & 7.9 \\
\hline ALS6 & orf19.7414 & Cell adhesion molecule binding & 1.8 & 2.7 & 3.0 & -8.1 & -10.4 & -8.1 & 1.2 & 1.1 & 2.3 \\
\hline$A M D 21$ & orf19.5169 & Amidase activity & 1.0 & 1.5 & -1.2 & 1.8 & 2.4 & 2.1 & 1.1 & -1.0 & 1.2 \\
\hline ARE2 & orf19.2248 & Sterol O-acyltransferase activity & -1.3 & 2.0 & 1.6 & 1.6 & 2.1 & 1.2 & 1.8 & 1.6 & 3.0 \\
\hline ARO9 & orf19.1237 & Aromatic-amino-acid transaminase activity & 2.2 & 2.3 & 20.0 & -1.1 & 1.0 & 1.2 & -1.0 & 1.0 & -2.5 \\
\hline AUT1 & orf19.6020 & -- & -1.5 & -1.1 & -1.3 & 1.6 & 1.5 & 1.8 & -1.2 & -1.5 & 1.3 \\
\hline$B M R 1$ & orf19.5604 & Multidrug transporter activity & 47.8 & 152.1 & 112.2 & 116.2 & 181.2 & 210.3 & 97.1 & 169.5 & 132.3 \\
\hline CBPI & orf19.7323 & Steroid binding & 1.6 & 2.1 & 1.4 & 1.6 & 2.0 & 1.8 & 1.5 & 1.5 & 1.8 \\
\hline CDR11.5f & orf19.918 & ATP binding & 2.1 & 2.3 & 1.4 & 1.3 & 1.9 & -1.3 & 1.7 & 2.3 & 1.7 \\
\hline CDR12 & orf19.2802 & ATPase activity & 1.7 & 2.3 & 2.0 & 2.4 & 2.6 & 2.7 & 2.1 & 1.6 & -1.3 \\
\hline CFL11 & orf19.701 & Ferric-chelate reductase activity & 1.6 & 1.7 & 1.9 & -1.7 & -1.7 & 1.3 & -4.8 & 1.7 & 1.1 \\
\hline CHA12.3f & orf19.1996 & L-serine ammonia-lyase activity & -1.3 & -1.5 & -1.6 & -1.1 & 1.2 & 1.4 & 1.7 & 1.8 & 2.7 \\
\hline Cirt2 & orf19.3820 & -- & 1.1 & 1.4 & -1.1 & 1.8 & 1.6 & 1.8 & 1.7 & 1.0 & -1.3 \\
\hline CLN2 & orf19.1960 & Cyclin-dependent protein kinase regulator activity & -1.0 & 1.4 & 1.4 & -1.6 & -1.2 & -3.5 & 1.5 & 2.0 & 2.1 \\
\hline CLN21 & orf19.6028 & Cyclin-dependent protein kinase regulator activity & 7.2 & 2.6 & 1.9 & -3.2 & 1.1 & 1.1 & 1.4 & 4.7 & 2.2 \\
\hline CRD1 & orf19.4784 & Copper ion binding & 1.2 & 1.7 & -1.1 & -1.7 & 1.5 & 1.5 & 1.5 & 1.6 & 1.5 \\
\hline CRN1.3f & orf19.6534.2 & Actin filament binding & -1.3 & 1.1 & 1.1 & 1.7 & 1.8 & 2.7 & 1.4 & 1.4 & 1.4 \\
\hline CTR9 & orf19.7067 & RNA polymerase II transcription factor activity & -1.4 & -2.0 & 1.5 & 1.6 & 1.6 & 1.9 & 1.1 & 1.1 & 2.1 \\
\hline DAL1 & orf19.5454 & Allantoinase activity & 1.6 & 1.2 & 2.8 & 2.7 & 2.4 & 2.5 & 2.0 & 1.5 & 4.9 \\
\hline DDR48 & orf19.4082 & -- & 1.9 & 4.3 & 2.3 & -1.4 & -1.1 & 1.0 & -2.2 & -1.2 & -1.9 \\
\hline DFRI & orf19.5142 & Dihydrofolate reductase activity & 1.3 & 1.1 & -1.3 & 1.9 & 1.7 & 2.1 & 1.5 & -1.1 & -1.7 \\
\hline DIP51.5f & orf19.2942 & Amino acid permease activity & 1.7 & 2.5 & 1.6 & 3.0 & -1.0 & -6.3 & -2.0 & -1.3 & 1.0 \\
\hline DLD2 & orf19.6755 & D-lactate dehydrogenase (cytochrome) activity & 1.3 & 1.2 & -1.1 & 2.9 & 2.2 & 1.9 & 1.2 & 2.1 & -1.2 \\
\hline DPB11 & or19.1434 & Epsilon DNA polymerase activity & 1.3 & 1.7 & 1.6 & 1.9 & 1.5 & 2.0 & 1.4 & 1.1 & -3.3 \\
\hline DSK2 & orf19.5345 & Protein binding & 1.3 & -1.2 & -1.3 & 1.5 & 1.8 & 1.7 & 1.3 & 1.1 & 1.0 \\
\hline DUR1,2 & orf19.780 & Allophanate hydrolase activity & 1.7 & 1.9 & 2.2 & 2.1 & 4.0 & 2.6 & 1.7 & 2.0 & 1.5 \\
\hline EBP4 & orf19.3433 & NADPH dehydrogenase activity & 1.1 & 2.4 & 2.8 & 1.8 & 2.5 & 2.0 & 7.9 & 8.0 & 11.4 \\
\hline ECE1 & orf19.3374 & -- & 25.6 & 16.4 & 38.9 & 1.4 & 1.1 & 2.4 & 2.7 & -1.3 & 2.1 \\
\hline ECM29.5f & orf19.6773 & -- & 2.6 & 1.6 & 1.5 & -1.0 & -1.0 & -22.7 & -1.1 & 1.0 & 2.7 \\
\hline$E R G 24$ & orf19.1598 & Delta 14-sterol reductase activity & 1.5 & 1.6 & 1.6 & 1.4 & 1.6 & 1.4 & 1.0 & -1.2 & 1.5 \\
\hline
\end{tabular}


Table B-1 (continued).

\begin{tabular}{|c|c|c|c|c|c|c|c|c|c|c|c|}
\hline \multirow[b]{2}{*}{ CandidaDB Name ${ }^{a}$} & \multirow[b]{2}{*}{ orf19 Name $^{b}$} & \multirow[b]{2}{*}{ GO Annotation (Molecular Function) ${ }^{c}$} & \multicolumn{3}{|c|}{ F5 vs. F1 } & \multicolumn{3}{|c|}{ G5 vs. G1 } & \multicolumn{3}{|c|}{6692 vs. 5833} \\
\hline & & & 1 & 2 & 3 & 1 & 2 & 3 & 1 & 2 & 3 \\
\hline$E R G 26$ & orf19.2909 & C-3 sterol dehydrogenase (C-4 sterol decarboxylase) activity & 1.6 & 1.5 & 2.1 & -1.4 & 1.4 & 1.8 & -1.3 & -1.2 & -1.3 \\
\hline ERG27 & orf19.3240 & 3-keto sterol reducatase activity & 1.5 & 1.6 & 1.9 & 1.1 & 1.2 & 1.1 & 1.0 & -1.1 & -1.4 \\
\hline EXG2 & orf19.2952 & Glucan 1,3-beta-glucosidase activity & 2.2 & 2.4 & 1.8 & 1.9 & 2.0 & 1.6 & 1.3 & 1.5 & -1.0 \\
\hline FAA24 & orf19.7156 & Long-chain-fatty-acid-CoA ligase activity & 1.4 & 2.7 & 1.4 & 2.3 & 1.9 & 3.0 & -1.3 & -1.1 & 2.5 \\
\hline FEN2 & orf19.5535 & Pantothenate transporter activity & 1.5 & 2.1 & 1.5 & 1.7 & 1.4 & 1.1 & -1.1 & -1.0 & -1.4 \\
\hline FET34.3eoc & orf19.4215 & Ferroxidase activity & -2.2 & -1.4 & -1.0 & 1.6 & 1.6 & 4.0 & -1.9 & -2.5 & -1.7 \\
\hline FMS1 & orf19.4589 & 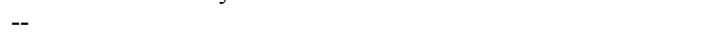 & 1.7 & 1.6 & -1.0 & 2.6 & 2.4 & 2.3 & 1.0 & 1.2 & -1.3 \\
\hline FRE $30.3 f$ & orf19.6139 & Ferric-chelate reductase activity & -1.8 & 1.3 & -2.5 & 1.5 & 1.5 & 1.5 & 1.6 & 1.5 & 1.9 \\
\hline FRE $30.53 f$ & orf19.6140 & Ferric-chelate reductase activity & -1.2 & 1.2 & -1.8 & 2.7 & 1.6 & -5.2 & 1.5 & 2.0 & 1.7 \\
\hline FRE7 & orf19.7077 & Ferric-chelate reductase activity & -1.5 & 1.2 & -2.1 & 1.7 & 1.7 & 2.8 & -1.0 & 1.3 & 2.4 \\
\hline FRP2 & orf19.6169 & -- & 1.7 & 7.4 & 2.5 & 1.1 & -1.5 & -1.5 & -1.8 & 1.3 & -1.4 \\
\hline FTH2 & orf19.3227 & Iron ion transporter activity & 1.5 & 1.9 & 2.3 & -1.2 & 1.2 & 2.1 & 1.2 & 1.3 & 1.4 \\
\hline GAL1 & orf19.3670 & Galactokinase activity & 1.5 & 2.4 & 2.4 & -1.5 & -1.4 & -1.4 & -1.5 & -1.2 & -1.2 \\
\hline GBAl & orf19.4015 & GTPase activity & -1.6 & -1.2 & 1.3 & 41.8 & 21.8 & 20.5 & -1.2 & -1.2 & 1.3 \\
\hline GDI1 & orf19.7261 & Rab GDP-dissociation inhibitor activity & 1.4 & 1.6 & -1.1 & 1.7 & 1.6 & 2.0 & 1.2 & 1.1 & -1.1 \\
\hline GPH1 & orf19.7021 & Glycogen phosphorylase activity & -1.2 & 1.5 & -1.7 & -1.5 & 1.3 & 1.5 & 1.6 & 1.6 & 3.8 \\
\hline GPRI & orf19.1944 & G-protein coupled receptor activity & 2.5 & 1.6 & 1.8 & 1.5 & -1.7 & -1.7 & -1.6 & -1.2 & -1.0 \\
\hline GPXI & orf19.86 & Glutathione peroxidase activity & 2.1 & 3.5 & 5.2 & 3.1 & 3.5 & 4.9 & 2.4 & 3.2 & 8.8 \\
\hline GRP2 & orf19.4309 & Oxidoreductase activity & 3.0 & 5.3 & 3.4 & 4.9 & 6.1 & 5.5 & 7.6 & 8.6 & 18.6 \\
\hline HBSI & orf19.7144 & -- & -1.1 & -1.0 & -1.4 & 1.7 & 1.6 & 1.7 & -1.2 & 1.5 & 1.4 \\
\hline HEMI & orf19.2601 & 5-aminolevulinate synthase activity & 1.7 & 2.2 & 1.8 & -1.4 & 1.1 & -1.7 & -1.2 & 1.1 & 1.1 \\
\hline HGT11 & orf19.4527 & Glucose transporter activity & 3.0 & 4.4 & 1.7 & 1.7 & -1.3 & -1.6 & -2.1 & -1.5 & -2.5 \\
\hline HGT12 & orf19.3668 & Glucose transporter activity & 2.4 & 14.0 & 4.4 & 6.8 & 2.6 & 1.8 & -3.1 & -3.1 & -3.0 \\
\hline HIK1 & orf19.5181 & Osmosensor activity & 1.6 & 1.8 & 1.6 & 3.8 & 1.5 & -1.6 & 1.2 & 1.3 & 2.0 \\
\hline HIK1 & orf19.5181 & Osmosensor activity & 2.5 & 1.8 & 1.8 & 2.5 & 1.4 & -7.0 & -1.2 & 1.5 & 1.7 \\
\hline$H W P 1$ & orf19.1321 & 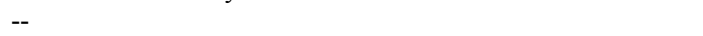 & 1.5 & 3.0 & 1.5 & 1.0 & -1.1 & -1.1 & 3.2 & 1.4 & -1.6 \\
\hline IFA18.53f & orf19.4508 & -- & 2.0 & 1.8 & 1.6 & -1.2 & 1.3 & -1.2 & 1.6 & -1.5 & -2.0 \\
\hline IFA24.3eoc & orf19.156 & -- & 1.1 & 1.4 & 2.2 & 1.7 & 2.1 & 2.8 & -1.1 & -1.4 & 2.3 \\
\hline IFD2 & orf19.771 & Aryl-alcohol dehydrogenase activity & 13.8 & 14.7 & 23.7 & 5.4 & 8.2 & 10.9 & 3.5 & 3.8 & 5.1 \\
\hline IFD4 & orf19.4477 & Aryl-alcohol dehydrogenase activity & 21.3 & 37.9 & 75.5 & 81.0 & 115.9 & 149.7 & 129.0 & 97.7 & 212.5 \\
\hline IFD5 & orf19.1048 & Aryl-alcohol dehydrogenase activity & 76.2 & 200.7 & 141.5 & 151.6 & 195.9 & 299.8 & 263.9 & 333.2 & 247.6 \\
\hline IFD5 & orf19.1048 & Aryl-alcohol dehydrogenase activity & 140.8 & 361.9 & 144.3 & 137.7 & 155.8 & 201.1 & 307.3 & 274.7 & 964.2 \\
\hline IFD6 & orf19.4476 & Aryl-alcohol dehydrogenase activity & 965.7 & 1290.0 & 3064.8 & 565.4 & 818.4 & 1916.6 & 1220.6 & 2580.4 & 2890.9 \\
\hline IFD7 & orf19.629 & Aryl-alcohol dehydrogenase activity & 113.1 & 338.2 & 332.4 & 55.2 & 66.8 & 139.0 & 575.5 & 311.7 & 656.4 \\
\hline IFK2 & orf19.856 & -- & 2.0 & 1.4 & 1.2 & 1.5 & 1.7 & 2.2 & 1.8 & -1.2 & 1.2 \\
\hline IFK2 & orf19.856 & -- & 2.6 & 2.5 & 4.3 & 1.8 & -1.0 & -1.3 & 1.3 & -1.3 & 5.2 \\
\hline IFK3.3f & orf19.857 & -- & 2.0 & 2.2 & 2.2 & 1.1 & 1.3 & 1.8 & -1.4 & -1.4 & 1.5 \\
\hline IFM1 & orf19.5167 & Translation initiation factor activity & 1.8 & -1.3 & -1.0 & 2.6 & 1.7 & 2.7 & 1.2 & -1.1 & 1.5 \\
\hline IFU6.5f & orf19.5167 & -- & 2.2 & 2.8 & 1.8 & 1.5 & 2.0 & 1.6 & -1.3 & 1.3 & -1.4 \\
\hline IFV9.5f & orf19.3144 & -- & 1.7 & 1.7 & 1.6 & 2.6 & -1.1 & -8.1 & -1.0 & 1.1 & 1.9 \\
\hline INOI & orf19.7585 & Inositol-3-phosphate synthase activity & 1.1 & 1.8 & 1.0 & 1.9 & 2.3 & 1.6 & -1.2 & 1.2 & 1.2 \\
\hline
\end{tabular}


Table B-1 (continued).

\begin{tabular}{|c|c|c|c|c|c|c|c|c|c|c|c|}
\hline \multirow[b]{2}{*}{ CandidaDB Name $^{a}$} & \multirow[b]{2}{*}{ orf19 Name $^{b}$} & \multirow[b]{2}{*}{ GO Annotation (Molecular Function) ${ }^{c}$} & \multicolumn{3}{|c|}{ F5 vs. F1 } & \multicolumn{3}{|c|}{ G5 vs. G1 } & \multicolumn{3}{|c|}{6692 vs. 5833} \\
\hline & & & 1 & 2 & 3 & 1 & 2 & 3 & 1 & 2 & 3 \\
\hline IPF1009 & orf19.4590 & (1) & 1.2 & 1.5 & 1.9 & -1.4 & -1.3 & -1.2 & 2.1 & 2.0 & 1.7 \\
\hline IPF10124 & orf19.276 & -- & 1.7 & 1.8 & 1.5 & 1.1 & 1.0 & -1.2 & 1.3 & 1.8 & 4.6 \\
\hline IPF10168 & orf19.643 & -- & 1.9 & 1.5 & 2.0 & 2.0 & 1.5 & 1.5 & -1.3 & -1.1 & 2.5 \\
\hline IPF10262 & orf19.2726 & -- & 1.2 & 1.9 & 8.3 & 1.7 & 2.3 & 1.8 & -1.0 & 1.7 & 2.2 \\
\hline IPF10300 & orf19.2274 & -- & 2.1 & 1.7 & 1.9 & 2.0 & -1.5 & -2.1 & 1.0 & 1.3 & 2.0 \\
\hline IPF10333 & orf19.1835 & Cytoskeletal regulatory protein binding & 1.6 & 1.5 & 1.7 & 1.2 & -1.7 & -1.3 & 1.1 & 2.3 & -1.9 \\
\hline IPF10394 & orf19.3364 & -- & 1.0 & -1.1 & 1.9 & 1.5 & 1.7 & 2.9 & 1.5 & 1.5 & 2.1 \\
\hline IPF10595 & orf19.1287 & -- & -1.7 & -1.4 & -1.1 & -1.3 & 1.0 & 1.6 & 2.1 & 1.7 & 1.7 \\
\hline IPF10735 & orf19.6790 & RNA binding & 1.9 & 2.9 & 5.0 & -1.0 & 1.2 & -1.0 & -1.1 & -1.1 & -1.2 \\
\hline IPF10795 & orf19.2356 & Transcription factor activity & 18.2 & -1.3 & 1.4 & 2.1 & 1.6 & 1.9 & 1.8 & 2.0 & -1.9 \\
\hline IPF11006 & orf19.4658 & 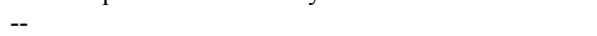 & 1.6 & 1.1 & 1.5 & 3.4 & 2.0 & 4.9 & -1.0 & 1.1 & 3.2 \\
\hline IPF11101 & orf19.4615 & -- & 1.2 & 1.6 & 1.6 & 1.9 & 1.6 & 1.7 & -1.1 & 1.3 & -1.1 \\
\hline IPF11161.3 & orf19.3201 & Transcription factor activity & 1.9 & -1.5 & -2.6 & 4.4 & 2.0 & 2.6 & -1.2 & -1.1 & 1.5 \\
\hline IPF11167 & orf19.3198 & Oxysterol binding & 2.2 & 3.2 & -1.7 & 1.8 & 1.8 & 2.6 & 1.1 & 1.0 & 1.1 \\
\hline IPF1118 & orf19.5422 & -- & -1.5 & 1.0 & -1.2 & 1.0 & 1.2 & -1.8 & 3.4 & 2.4 & 7.3 \\
\hline IPF11222 & orf19.5840 & -- & -1.1 & 1.0 & 1.3 & -1.1 & -1.0 & -1.2 & 2.3 & 2.7 & 4.6 \\
\hline IPF11432 & orf19.6463 & -- & -1.3 & -1.8 & -1.1 & 1.8 & 1.7 & 1.9 & -1.2 & -1.1 & 1.3 \\
\hline IPF11435 & orf19.6461 & -- & -1.1 & 1.4 & 1.5 & 2.6 & 1.6 & 1.5 & 1.5 & -1.1 & -1.1 \\
\hline IPF11694 & orf19.4355 & Nicotinamide mononucleotide permase activity & 1.8 & 9.9 & 8.4 & -1.4 & -1.1 & -1.2 & -2.1 & -2.0 & 1.4 \\
\hline IPF11821 & orf19.6552 & Thiol oxidase activity & -2.0 & -1.4 & 1.3 & 1.9 & 2.0 & 3.2 & 1.4 & -1.1 & 2.3 \\
\hline IPF1264 & orf19.7371 & Transcription factor activity & 3.8 & 3.2 & 2.8 & 2.0 & 1.7 & 1.2 & 1.5 & 2.2 & 2.4 \\
\hline IPF1272 & orf19.7376 & -- & -1.5 & -3.7 & 2.4 & 2.3 & 1.5 & 2.0 & 1.2 & 1.3 & 1.5 \\
\hline IPF12963 & orf19.5094 & -- & 2.6 & 3.3 & 2.8 & -1.3 & 1.4 & -1.4 & 1.7 & 2.1 & 21.4 \\
\hline IPF1321 & orf19.6413 & -- & 1.7 & -1.1 & -1.2 & 4.8 & 2.8 & 1.9 & 1.0 & 1.0 & 1.9 \\
\hline IPF13319 & orf19.740 & Transcriptional activator activity & 2.5 & 1.9 & 2.1 & 1.6 & -1.8 & -1.4 & -1.7 & -1.3 & -1.4 \\
\hline IPF13423 & orf19.4128 & -- & 2.1 & 1.4 & 1.2 & 1.7 & 2.2 & 3.9 & 1.9 & -1.9 & -5.0 \\
\hline IPF13609 & orf19.6526 & -- & -1.8 & -1.3 & 1.1 & 1.8 & 1.6 & 1.8 & 1.3 & 1.3 & 1.8 \\
\hline IPF13723 & orf19.260 & -- & 1.5 & 1.5 & 1.6 & -1.5 & 2.5 & 1.9 & 1.5 & -1.2 & 1.7 \\
\hline IPF13883 & orf19.5502 & -- & -1.3 & -1.4 & -1.6 & 2.3 & 1.9 & 1.7 & 1.4 & 1.4 & -1.9 \\
\hline IPF14030 & orf19.851 & -- & -1.5 & -1.0 & -1.9 & 5.7 & 2.4 & 1.6 & -1.3 & -1.2 & -4.4 \\
\hline IPF14119 & orf19.4688 & -- & -1.6 & -1.2 & 1.4 & 1.2 & 1.2 & -1.2 & 1.8 & 2.1 & 2.6 \\
\hline IPF14379 & orf19.4474 & -- & -1.1 & -4.2 & 3.0 & 1.6 & 1.5 & 2.9 & 1.3 & -1.2 & 1.2 \\
\hline IPF14448 & orf19.5601 & -- & 2.1 & 1.3 & 1.8 & 1.6 & 1.7 & 3.3 & 1.2 & -1.1 & -1.5 \\
\hline IPF14968 & orf19.3461 & -- & 1.7 & 1.5 & 2.0 & 1.6 & 1.1 & 1.4 & -1.1 & 1.3 & -1.3 \\
\hline IPF14981 & orf19.3483 & -- & 5.1 & 1.8 & 2.0 & 3.3 & 1.8 & 3.0 & 1.8 & 1.1 & 1.7 \\
\hline IPF15255 & orf19.4884 & Transcription regulator activity & 2.1 & 3.3 & 1.6 & -1.7 & -3.8 & -1.7 & 1.2 & -2.1 & 1.2 \\
\hline IPF15273 & orf19.1822 & Transcription factor activity & 2.5 & 2.1 & 2.1 & -1.4 & -1.8 & -1.3 & 1.4 & 4.8 & -2.1 \\
\hline IPF15890 & orf19.6349 & Cytoskeletal protein binding & 1.9 & 1.5 & 1.6 & 1.1 & 3.0 & 2.9 & 1.5 & -1.4 & 1.3 \\
\hline IPF1621 & orf19.6585 & 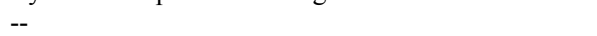 & -1.9 & 1.1 & -1.1 & 2.8 & 2.4 & 2.8 & -1.2 & 1.4 & 5.8 \\
\hline IPF1632 & orf19.6579 & -- & 1.2 & -1.3 & 1.0 & 1.5 & 1.6 & 1.6 & 1.3 & -1.1 & -1.5 \\
\hline IPF16320 & orf19.5131 & -- & -1.1 & 1.1 & 1.0 & 1.6 & 2.6 & 2.2 & 1.5 & 1.3 & -1.1 \\
\hline
\end{tabular}


Table B-1 (continued).

\begin{tabular}{|c|c|c|c|c|c|c|c|c|c|c|c|}
\hline \multirow[b]{2}{*}{ CandidaDB Name ${ }^{a}$} & \multirow[b]{2}{*}{ orf19 Name $^{b}$} & \multirow[b]{2}{*}{ GO Annotation (Molecular Function) ${ }^{c}$} & \multicolumn{3}{|c|}{ F5 vs. F1 } & \multicolumn{3}{|c|}{ G5 vs. G1 } & \multicolumn{3}{|c|}{6692 vs. 5833} \\
\hline & & & 1 & 2 & 3 & $\mathbf{1}$ & 2 & 3 & $\mathbf{1}$ & 2 & $\mathbf{3}$ \\
\hline IPF16323 & orf19.6460 & ATPase activity & 1.7 & -1.8 & 2.3 & 3.1 & 1.6 & 1.6 & 1.7 & -1.8 & -1.2 \\
\hline IPF1651 & orf19.6570 & Nucleoside permease activity & -1.4 & 1.2 & -1.4 & 3.8 & 2.7 & 2.5 & -1.3 & 1.2 & 1.6 \\
\hline IPF1674 & orf19.6559 & -- & -1.0 & -1.1 & -1.8 & 2.1 & 3.2 & 2.7 & 1.5 & -1.0 & -1.3 \\
\hline IPF1674 & orf19.6559 & -- & 1.0 & 1.4 & -1.5 & 1.9 & 2.5 & 3.2 & 1.6 & -1.3 & -1.0 \\
\hline IPF17186 & orf19.251 & -- & 20.7 & 38.9 & 39.7 & 9.2 & 14.8 & 14.6 & 20.8 & 31.4 & 21.0 \\
\hline IPF17255 & orf19.696 & Mating-type alpha-factor pheromone receptor activity & -1.5 & 5.6 & -3.4 & 8.6 & 41.6 & 17.3 & -3.9 & -1.9 & -4.3 \\
\hline IPF18161 & orf19.6587 & -- & -2.1 & -2.4 & 1.1 & 2.6 & 1.6 & 2.0 & 1.2 & 1.1 & -2.0 \\
\hline IPF18316 & orf19.5145 & -- & 1.3 & 1.4 & 1.5 & 4.0 & 2.2 & 1.5 & -1.6 & -1.1 & -1.6 \\
\hline IPF1837 & orf19.5877 & Alcohol O-acetyltransferase activity & 2.1 & 1.9 & 1.9 & 1.7 & 1.6 & 2.1 & 3.8 & 4.3 & 6.0 \\
\hline IPF18385 & orf19.4143 & -- & 4.4 & 3.4 & 1.4 & 1.0 & 2.4 & 1.0 & 1.5 & 1.5 & 3.0 \\
\hline IPF18418 & orf19.5620 & -- & 1.3 & 2.4 & 2.4 & 2.0 & 2.8 & 1.7 & 2.4 & 2.7 & 3.6 \\
\hline IPF1862.5f & orf19.5894 & -- & 1.7 & 1.3 & -1.6 & 2.4 & 1.7 & 2.1 & -7.7 & 1.9 & 1.4 \\
\hline IPF18641.5f & orf19.3159 & -- & 1.9 & 1.9 & 14.6 & -1.5 & -2.2 & -9.5 & -1.1 & -1.2 & 2.0 \\
\hline IPF1899 & orf19.7131 & -- & -1.1 & 1.5 & -1.5 & 2.0 & 1.8 & 2.1 & 1.0 & 1.1 & 2.1 \\
\hline IPF1912 & orf19.7140 & -- & -1.6 & -1.3 & -1.6 & 1.8 & 1.7 & 1.9 & -1.5 & 1.2 & 1.6 \\
\hline IPF19295.5f & orf19.4649 & -- & -1.3 & -1.5 & -2.7 & 1.9 & 3.0 & 2.2 & 1.4 & 1.0 & -1.8 \\
\hline IPF19513 & orf19.6476 & -- & -1.0 & -1.4 & -1.2 & 1.5 & 1.6 & 1.7 & -1.5 & -1.0 & -1.3 \\
\hline IPF19538.3f & orf19.6957.3 & -- & 2.2 & 1.7 & 6.0 & 8.5 & 4.4 & 2.3 & 3.1 & 2.2 & 2.7 \\
\hline IPF19908 & orf19.1344 & -- & 1.2 & 2.7 & 1.8 & 3.2 & 2.2 & 4.2 & -1.6 & -1.5 & -1.0 \\
\hline IPF19997 & orf19.6458.1 & -- & -1.6 & -1.3 & 1.5 & 1.9 & 1.8 & 2.4 & 1.5 & 1.5 & 1.9 \\
\hline IPF19998 & orf19.2175 & Oxidoreductase activity & 1.0 & 1.1 & -1.0 & 1.6 & 1.9 & 1.8 & 1.3 & 1.2 & 1.8 \\
\hline IPF20010 & orf19.5799 & -- & 1.9 & 1.9 & 1.6 & 1.6 & 1.5 & 1.4 & 1.0 & 1.2 & -1.4 \\
\hline IPF20015 & orf19.499 & -- & 1.5 & 1.6 & 1.5 & 1.1 & -1.1 & -1.1 & -1.2 & -1.3 & -1.3 \\
\hline IPF20157 & orf19.6696 & -- & 1.9 & 3.8 & 2.0 & 1.2 & 2.4 & 10.5 & 1.8 & 1.6 & -10.0 \\
\hline IPF2021 & orf19.5245 & -- & 1.6 & 2.2 & 1.6 & -1.3 & -1.2 & -1.4 & -1.3 & 1.4 & 2.2 \\
\hline IPF2050 & orf19.5265 & Structural constituent of cytoskeleton & 1.7 & 1.7 & 2.3 & 1.2 & -1.0 & -1.3 & 1.4 & 1.5 & -1.9 \\
\hline IPF2130 & orf19.7204 & -- & 1.8 & 1.8 & -1.4 & 17.2 & 37.0 & 15.2 & 5.0 & 7.4 & 3.0 \\
\hline IPF2150 & orf19.7193 & -- & -1.1 & 1.2 & -1.1 & 1.7 & 1.8 & 2.8 & -1.3 & 1.1 & -1.3 \\
\hline IPF2170 & orf19.7179 & -- & 1.1 & -1.2 & -1.1 & 3.0 & 1.5 & 2.5 & 2.5 & -1.7 & -1.0 \\
\hline IPF 2175 & orf19.7175 & -- & 1.1 & -1.1 & -1.2 & 1.5 & 1.6 & 1.6 & 1.1 & -1.1 & -1.1 \\
\hline IPF2186 & orf19.7166 & -- & 3.0 & 6.0 & 7.3 & 14.0 & 15.8 & 11.7 & 11.8 & 11.1 & 22.5 \\
\hline IPF2189 & orf19.7165 & -- & 1.1 & -1.5 & -1.1 & 3.1 & 2.5 & 1.9 & 1.2 & 1.3 & -1.0 \\
\hline IPF2190 & orf19.7164 & -- & -1.5 & 1.4 & -1.5 & 1.5 & 1.7 & 2.0 & -1.2 & -1.4 & -1.0 \\
\hline IPF 2228 & orf19.6902 & ATP-dependent RNA helicase activity & -1.2 & -1.8 & -1.0 & 2.1 & 1.5 & 1.6 & -1.0 & 1.0 & 1.7 \\
\hline IPF 2280 & orf19.6658 & -- & 1.1 & 2.6 & -1.0 & 1.9 & 2.0 & 1.5 & -1.2 & 1.1 & -1.3 \\
\hline IPF2361 & orf19.7023 & -- & 1.2 & -1.5 & 1.1 & 1.6 & 2.5 & 1.7 & 1.2 & 1.5 & 2.2 \\
\hline IPF2557 & orf19.6627 & -- & -1.1 & 1.3 & 1.1 & 1.5 & 1.7 & 1.7 & 1.4 & 2.0 & 2.2 \\
\hline IPF2582 & orf19.5504 & -- & -1.3 & -1.7 & -1.1 & 1.8 & 1.6 & 2.8 & 1.2 & 1.4 & 1.8 \\
\hline IPF2905 & orf19.7457 & -- & 1.8 & -1.4 & 1.4 & 1.7 & 2.5 & 2.0 & -1.4 & -1.2 & 1.1 \\
\hline IPF300 & orf19.3282 & -- & 1.0 & -1.3 & 1.2 & 1.5 & 2.2 & 1.7 & -1.1 & 1.4 & 3.6 \\
\hline IPF3014 & orf19.5030 & -- & 2.2 & 1.6 & 2.3 & 1.4 & 1.4 & -1.5 & 1.2 & -1.4 & 3.1 \\
\hline
\end{tabular}


Table B-1 (continued).

\begin{tabular}{|c|c|c|c|c|c|c|c|c|c|c|c|}
\hline \multirow[b]{2}{*}{ CandidaDB Name ${ }^{a}$} & \multirow[b]{2}{*}{ orf19 Name ${ }^{b}$} & \multirow[b]{2}{*}{ GO Annotation (Molecular Function) ${ }^{c}$} & \multicolumn{3}{|c|}{ F5 vs. F1 } & \multicolumn{3}{|c|}{ G5 vs. G1 } & \multicolumn{3}{|c|}{6692 vs. 5833} \\
\hline & & & 1 & 2 & 3 & 1 & 2 & 3 & 1 & 2 & 3 \\
\hline IPF3069 & orf19.7034 & ( & 1.0 & 1.3 & -1.2 & 1.6 & 1.5 & 1.5 & -1.1 & 1.4 & 1.5 \\
\hline IPF3080 & orf19.7042 & -- & 2.3 & 1.0 & 2.7 & 6.2 & 4.7 & 5.5 & 10.7 & 11.3 & 12.2 \\
\hline IPF3081 & orf19.7043 & -- & -1.2 & -1.1 & 1.4 & 1.5 & 2.3 & 2.9 & 1.1 & -1.6 & 1.6 \\
\hline IPF3121 & orf19.4438 & Transcription factor activity & -1.1 & 1.9 & -1.1 & 3.0 & 8.0 & 3.9 & 1.1 & 1.1 & -7.2 \\
\hline IPF3277 & orf19.4384 & Fructose transporter activity & 2.5 & 5.3 & 2.3 & -1.1 & 1.2 & -2.0 & -1.5 & -1.4 & -1.5 \\
\hline IPF3277 & orf19.4384 & Fructose transporter activity & 1.7 & 4.0 & 1.7 & -1.0 & -1.8 & -3.3 & -1.4 & -1.2 & -1.7 \\
\hline IPF3282.3eoc & orf19.4384 & Fructose transporter activity & 1.7 & 4.5 & 1.6 & -1.3 & -1.7 & -2.5 & -1.6 & -1.3 & -1.6 \\
\hline IPF3352 & orf19.4013 & -- & 1.1 & 1.3 & 1.3 & 1.8 & 2.2 & 2.8 & -1.0 & -1.1 & -1.0 \\
\hline IPF3491 & orf19.6753 & -- & 1.0 & 1.3 & 1.3 & 1.8 & 1.7 & 1.5 & -1.1 & 1.1 & -1.1 \\
\hline IPF3535 & orf19.6840 & -- & 1.8 & 2.2 & 4.2 & 2.4 & 1.7 & 1.8 & -1.6 & -1.5 & 1.7 \\
\hline IPF3679 & orf19.889 & Transcription factor activity & 2.3 & 1.5 & 1.8 & 1.0 & 1.0 & -1.0 & -1.6 & -1.6 & -1.1 \\
\hline IPF3836 & orf19.279 & Hydrolase activity, acting on ester bonds & -1.2 & 1.2 & -1.4 & -1.1 & -1.1 & 1.5 & 1.5 & 1.5 & 1.7 \\
\hline IPF3866 & orf19.21 & -- & -1.4 & 1.0 & -1.0 & 2.0 & 1.6 & 1.6 & -1.0 & 1.3 & 1.5 \\
\hline IPF3876 & orf19.775 & -- & 1.9 & 1.8 & 1.1 & 1.5 & 1.9 & 2.9 & 1.6 & 1.0 & 1.3 \\
\hline IPF3927 & orf19.6506 & -- & 1.3 & -1.6 & -1.4 & 1.6 & 1.5 & 3.1 & 1.0 & 1.1 & -1.5 \\
\hline IPF4059 & orf19.1865 & -- & 1.5 & 1.6 & 1.5 & 1.3 & 1.3 & 1.3 & -1.1 & 1.0 & 1.1 \\
\hline IPF 4325 & orf19.5523 & -- & 1.5 & 1.6 & 1.9 & 1.5 & 2.3 & 2.9 & 1.1 & -1.4 & -1.8 \\
\hline IPF448 & orf19.7051 & -- & -1.8 & -1.7 & 1.6 & 2.6 & 2.2 & 1.9 & -1.6 & -1.2 & 6.7 \\
\hline IPF4567.3f & orf19.6525 & -- & -1.1 & -1.1 & -1.2 & 1.9 & 1.5 & 1.9 & -1.3 & -1.1 & -1.1 \\
\hline IPF474 & orf19.7060 & -- & 1.4 & 1.6 & -2.0 & 1.5 & 2.0 & 2.0 & -1.3 & -1.3 & -1.2 \\
\hline IPF480 & orf19.7063 & -- & -1.4 & -1.0 & -1.2 & 2.0 & 1.8 & 1.8 & -1.1 & -1.0 & 1.2 \\
\hline IPF4835 & orf19.5992 & Transcription factor activity & 1.3 & 1.3 & 1.1 & 2.8 & 2.0 & 3.9 & 2.3 & 1.7 & 1.7 \\
\hline IPF4890 & orf19.6263 & Monocarboxylic acid transporter activity & -1.1 & 1.1 & -1.0 & -1.8 & -1.0 & 1.0 & 1.5 & 1.7 & 3.4 \\
\hline IPF511 & orf19.7078 & -- & -1.6 & 1.2 & 1.2 & 1.9 & 1.7 & 1.8 & 1.0 & 1.2 & 1.8 \\
\hline IPF525 & orf19.7085 & -- & 1.3 & 1.4 & 1.0 & 2.5 & 1.7 & 2.3 & 1.5 & 1.4 & -1.6 \\
\hline IPF538 & orf19.7091 & -- & -1.1 & -1.4 & -1.2 & 1.5 & 1.7 & 2.3 & 1.0 & -1.0 & 1.2 \\
\hline IPF539 & orf19.7092 & -- & 1.3 & 1.4 & -1.1 & 1.5 & 1.7 & 2.0 & 1.2 & 1.2 & 1.2 \\
\hline IPF5426 & orf19.6378 & -- & -1.1 & -1.3 & -1.2 & 1.7 & 1.5 & 2.3 & 1.5 & -1.0 & -1.6 \\
\hline IPF553 & orf19.7096 & -- & -1.1 & 1.4 & -1.3 & 1.5 & 1.9 & 1.9 & -1.2 & -1.1 & 1.1 \\
\hline IPF5567 & orf19.5160 & Protein phosphatase regulator activity & 1.0 & -1.4 & 1.1 & 2.0 & 1.7 & 2.4 & 1.3 & 1.0 & 1.4 \\
\hline IPF5625 & orf19.7112 & Ferric-chelate reductase activity & 1.4 & 1.3 & 1.8 & 2.0 & 2.2 & 2.8 & -1.3 & -1.2 & 1.3 \\
\hline IPF568 & orf19.7105 & Cyclin-dependent protein kinase inhibitor activity & 1.3 & 1.1 & -2.0 & 30.5 & 21.8 & 21.0 & -1.1 & 1.1 & -1.5 \\
\hline IPF5723 & orf19.6329 & -- & -1.4 & 1.2 & -1.3 & -2.4 & -1.3 & -1.3 & 1.6 & 1.5 & 3.7 \\
\hline IPF5987 & orf19.7306 & -- & 48.6 & 49.5 & 63.3 & 41.5 & 50.1 & 81.4 & 45.5 & 38.2 & 35.7 \\
\hline IPF6032 & orf19.4607 & -- & 1.6 & 2.0 & 3.3 & 1.7 & 1.6 & 1.4 & 1.5 & 2.0 & 1.6 \\
\hline IPF6041 & orf19.4612 & -- & -1.3 & 1.6 & 1.2 & 1.7 & 2.3 & 2.2 & 2.2 & -1.2 & 3.1 \\
\hline IPF6050 & orf19.6737 & -- & 1.5 & 1.5 & 2.0 & -1.1 & -1.4 & -1.8 & 1.0 & 1.0 & 1.3 \\
\hline IPF610 & orf19.7631 & -- & 1.5 & 1.8 & 1.6 & -1.0 & -1.2 & -1.6 & -1.2 & 1.5 & -1.1 \\
\hline IPF6203 & orf19.4166 & -- & 1.6 & 2.1 & 1.5 & 1.1 & -1.1 & 1.2 & -1.0 & 1.2 & -1.4 \\
\hline IPF6235 & orf19.5372 & -- & 1.0 & 1.2 & -1.1 & 1.9 & 3.4 & 3.4 & -1.0 & 1.1 & 4.5 \\
\hline IPF6238 & orf19.2374 & -- & 1.2 & 1.0 & 1.3 & 2.2 & 3.4 & 2.8 & 1.3 & 1.0 & 3.9 \\
\hline
\end{tabular}


Table B-1 (continued).

\begin{tabular}{|c|c|c|c|c|c|c|c|c|c|c|c|}
\hline \multirow[b]{2}{*}{ CandidaDB Name ${ }^{a}$} & \multirow[b]{2}{*}{ orf19 Name $^{b}$} & \multirow[b]{2}{*}{ GO Annotation (Molecular Function) ${ }^{c}$} & \multicolumn{3}{|c|}{ F5 vs. F1 } & \multicolumn{3}{|c|}{ G5 vs. G1 } & \multicolumn{3}{|c|}{6692 vs. 5833} \\
\hline & & & 1 & 2 & 3 & 1 & 2 & 3 & 1 & 2 & 3 \\
\hline IPF6318 & orf19.1664 & ( & 1.0 & 1.4 & -1.2 & 1.5 & 1.9 & 3.5 & 1.5 & -1.1 & 1.3 \\
\hline IPF643 & orf19.7610 & Protein tyrosine phosphotase activity & 1.3 & -1.1 & 1.2 & -1.3 & 1.1 & 1.8 & 1.9 & 1.7 & 3.0 \\
\hline IPF6444 & orf19.1900 & -- & 1.2 & -1.3 & 1.4 & 2.3 & 3.1 & 1.6 & 1.3 & 1.1 & -1.5 \\
\hline IPF6518 & orf19.1691 & -- & 1.5 & 3.5 & 3.5 & 2.0 & 3.2 & 4.4 & 1.2 & -1.2 & 2.6 \\
\hline IPF7171.3f & orf19.5372 & -- & 1.9 & 2.2 & 1.8 & 2.6 & -1.2 & -1.0 & -1.1 & -1.2 & -2.0 \\
\hline IPF730 & orf19.5362 & -- & -1.1 & -1.1 & -1.9 & 13.3 & 3.7 & 3.9 & 1.1 & -1.1 & -1.5 \\
\hline IPF7397 & orf19.1800 & -- & 2.2 & 1.9 & 2.4 & 1.6 & 1.5 & 1.3 & -1.2 & -1.6 & -2.5 \\
\hline IPF7456 & orf19.2047 & -- & -1.2 & 1.7 & -1.1 & 1.6 & 2.1 & 1.7 & 1.3 & 1.6 & 1.4 \\
\hline IPF7493 & orf19.4682 & Glucose transporter activity & 1.5 & 11.3 & 9.5 & 5.8 & 2.1 & 3.2 & -2.8 & -2.0 & -2.7 \\
\hline IPF7602 & orf19.6837 & -- & -1.1 & 1.1 & -1.3 & -1.8 & -1.0 & 1.1 & 1.6 & 1.8 & 1.9 \\
\hline IPF7666 & orf19.4056 & Transcription factor activity & 2.5 & 3.9 & 3.3 & 2.3 & 1.0 & 1.3 & 3.2 & 2.3 & 3.5 \\
\hline IPF7686 & orf19.1395 & Inorganic phosphate transporter activity & 1.9 & 3.3 & 2.3 & 1.4 & 1.3 & -1.0 & 2.6 & 2.0 & 5.0 \\
\hline IPF7686 & orf19.1395 & Inorganic phosphate transporter activity & 1.7 & 2.3 & 1.9 & -1.2 & 1.2 & 1.4 & -1.1 & 1.1 & -1.0 \\
\hline IPF7770 & orf19.3881 & -- & 1.5 & 1.6 & -1.1 & 1.8 & 1.6 & 2.1 & 1.0 & 1.0 & -1.7 \\
\hline IPF7817 & orf19.3131 & NADPH dehydrogenase activity & 9.4 & 13.7 & 13.4 & 7.7 & 11.0 & 8.2 & 11.8 & 13.3 & 84.2 \\
\hline IPF7863 & orf19.1449 & -- & 6.1 & 9.4 & 9.1 & 7.2 & 7.2 & 5.7 & 6.5 & 9.5 & 5.7 \\
\hline IPF7940 & orf19.6608 & -- & 5.4 & 4.3 & 10.3 & 4.3 & 5.2 & 20.2 & 6.4 & 2.6 & -1.0 \\
\hline IPF7947 & orf19.6602 & -- & -1.5 & -1.2 & 2.1 & 1.6 & 1.5 & 1.9 & 1.3 & -1.1 & 1.6 \\
\hline IPF7968 & orf19.2693 & Transcription corepressor activity & -1.1 & 1.4 & 1.3 & 1.5 & 1.7 & 1.8 & 1.4 & 1.5 & -1.1 \\
\hline IPF8016 & orf19.1336.2 & -- & -1.0 & -1.9 & 1.8 & 1.7 & 1.7 & 4.3 & 2.2 & -1.2 & 13.7 \\
\hline IPF8024 & orf19.1334 & -- & -1.5 & -1.4 & -1.3 & 1.6 & 1.8 & 2.3 & -1.2 & -1.0 & 1.0 \\
\hline IPF8067 & orf19.3697 & -- & 1.6 & 1.4 & 2.6 & 3.0 & 2.4 & 1.7 & 3.5 & 1.5 & 1.2 \\
\hline IPF8147 & orf19.6200 & -- & 2.4 & 8.5 & 1.6 & -2.6 & -1.9 & 3.6 & 2.6 & 1.2 & -1.7 \\
\hline IPF8378 & orf19.6072 & -- & 1.9 & -1.4 & 1.3 & 1.9 & 1.5 & 1.6 & 1.2 & 1.1 & 1.1 \\
\hline IPF8439 & orf19.6498 & -- & 1.3 & -1.0 & -1.5 & 1.7 & 1.8 & 2.7 & -1.5 & -1.1 & 1.2 \\
\hline IPF8591 & orf19.5862 & Arginase activity & 2.5 & 3.1 & 7.0 & 1.0 & 2.0 & 1.9 & 1.8 & -1.3 & -1.2 \\
\hline IPF8627 & orf19.3969 & Specific transcriptional repressor activity & 2.8 & 2.7 & 1.8 & 1.2 & -1.1 & 1.3 & 1.3 & 1.3 & 2.3 \\
\hline IPF867 & orf19.7221 & NAD-dependent histone deacetylase activity & 2.4 & 1.6 & 1.5 & 2.1 & 1.5 & 1.6 & -1.1 & 1.0 & -2.0 \\
\hline IPF8951 & orf19.4897 & -- & 1.7 & 1.3 & 2.1 & 2.1 & 2.1 & 2.3 & 2.0 & 1.6 & -1.1 \\
\hline IPF8970 & orf19.496 & -- & 2.8 & 2.1 & 1.4 & 3.6 & 2.5 & 2.9 & 1.0 & -1.1 & -1.6 \\
\hline IPF8976 & orf19.1369 & -- & 5.2 & 5.7 & 14.9 & 6.2 & 7.3 & 4.0 & 1.2 & 2.3 & -1.2 \\
\hline IPF9370 & orf19.1424 & -- & 1.6 & 2.0 & 2.1 & 1.1 & 1.2 & 1.3 & -1.3 & 1.2 & -4.2 \\
\hline IPF9410 & orf19.6148 & Structural constituent of cytoskeleton & 2.5 & 1.6 & 1.7 & 1.4 & 1.4 & 1.1 & -1.8 & -1.1 & -2.1 \\
\hline IPF9450 & orf19.4370 & -- & -1.1 & 1.1 & -1.0 & 2.3 & 1.6 & 1.9 & 1.4 & 1.9 & 1.3 \\
\hline IPF9496 & orf19.2809 & Carnitine O-acetyltransferase activity & 2.3 & 5.8 & 2.5 & 2.2 & 1.5 & 2.9 & -2.2 & -2.2 & -1.2 \\
\hline IPF9538 & orf19.271 & 2,4-dienoyl-CoA reductase (NADPH) activity & 108.9 & 279.5 & 131.7 & 7.8 & 24.5 & 18.1 & 29.2 & 29.3 & 27.6 \\
\hline IPF9704 & orf19.4882 & General RNA polymerase II transcription factor activity & 1.5 & -1.3 & 1.2 & 1.5 & 1.8 & 2.1 & 1.2 & -1.0 & 1.5 \\
\hline IPF9748 & orf19.746 & -- & 2.8 & -1.2 & 1.5 & 1.5 & 1.8 & 3.8 & 1.5 & -1.5 & -2.1 \\
\hline IPF9828 & orf19.6861 & Protein binding & 1.1 & -2.5 & -1.1 & 1.8 & 1.9 & 3.5 & 1.1 & -1.7 & -2.4 \\
\hline IPF9887 & orf19.1240 & -- & 2.8 & 3.1 & 3.8 & 2.9 & 4.2 & 5.8 & 4.1 & 4.2 & 5.5 \\
\hline IQG1 & orf19.6536 & Cytoskeletal protein binding & -1.0 & -1.0 & 1.2 & 1.7 & 1.8 & 2.8 & 1.1 & 1.2 & 2.7 \\
\hline
\end{tabular}


Table B-1 (continued).

\begin{tabular}{|c|c|c|c|c|c|c|c|c|c|c|c|}
\hline \multirow[b]{2}{*}{ CandidaDB Name ${ }^{a}$} & \multirow[b]{2}{*}{ orf19 Name $^{b}$} & \multirow[b]{2}{*}{ GO Annotation (Molecular Function) ${ }^{c}$} & \multicolumn{3}{|c|}{ F5 vs. F1 } & \multicolumn{3}{|c|}{ G5 vs. G1 } & \multicolumn{3}{|c|}{6692 vs. 5833} \\
\hline & & & 1 & 2 & 3 & 1 & 2 & 3 & 1 & 2 & 3 \\
\hline KAP114 & orf19.7086 & Protein carrier activity & -1.1 & -1.2 & -1.1 & 1.5 & 1.9 & 2.5 & 1.0 & -1.1 & 1.9 \\
\hline KEXI & orf19.7020 & Carboxypeptidase D activity & 1.0 & 1.0 & -1.0 & 1.5 & 1.8 & 2.2 & 1.3 & 1.4 & 3.1 \\
\hline KIN3 & orf19.5325 & Protein kinase activity & -1.3 & -1.2 & -1.1 & 2.0 & 1.8 & 3.6 & 1.4 & 1.4 & -1.1 \\
\hline LPI9 & orf19.6544 & -- & 1.1 & 1.0 & -1.3 & 2.2 & 1.8 & 1.8 & -1.1 & 1.2 & -4.2 \\
\hline LYS21 & orf19.4506 & Homocitrate synthase activity & -2.3 & -2.5 & -1.2 & -1.2 & -1.2 & -1.2 & 1.5 & 2.8 & 3.4 \\
\hline MDM12 & orf19.6900 & -- & 1.4 & -1.2 & -1.1 & 2.0 & 1.7 & 2.3 & 1.3 & -1.3 & 1.2 \\
\hline$M D N 1$ & orf19.4697 & ATPase activity & 2.5 & 1.6 & 3.7 & 2.1 & -1.2 & -1.7 & 1.1 & -1.3 & -1.0 \\
\hline MET13 & orf19.288 & Structural constituent of ribosome & -1.0 & -1.2 & 1.4 & -4.1 & -1.4 & -1.3 & 1.8 & 1.7 & 2.8 \\
\hline$M G M 1$ & orf19.2690 & GTPase activity & 1.1 & -1.1 & 1.4 & 1.7 & 1.5 & 2.2 & 1.4 & 1.1 & 1.5 \\
\hline MRE11 & orf19.6915 & $3^{\prime}-5^{\prime}$ exonuclease activity & 1.2 & -1.3 & -1.2 & 2.0 & 1.9 & 1.7 & -1.0 & 1.0 & 2.1 \\
\hline MRF1 & orf19.1149 & DNA binding & 1.6 & 1.7 & 2.7 & -1.7 & -1.1 & 1.1 & -1.0 & -1.0 & 1.2 \\
\hline MRR1 & orf19.7372 & RNA polymerase II transcription factor activity & 2.8 & 1.8 & 5.7 & 2.9 & 1.8 & 1.9 & 2.3 & 3.4 & 4.7 \\
\hline MSD1 & orf19.4478 & Aspartate-tRNA ligase activity & 1.1 & 1.0 & -1.1 & 1.5 & 1.5 & 1.8 & -1.0 & -1.1 & -1.2 \\
\hline MSK1 & orf19.6533 & Lysine-tRNA ligase activity & -1.1 & -1.0 & -1.2 & 1.5 & 1.6 & 2.4 & 1.0 & 1.0 & -1.3 \\
\hline $\mathrm{NDH} 2$ & orf19.5713 & NADH dehydrogenase activity & 2.0 & 2.6 & 1.6 & 2.9 & 4.0 & 4.7 & 2.1 & 2.5 & 10.9 \\
\hline OPT1 & orf19.2602 & Oligopeptide transporter activity & -1.6 & 1.1 & -2.2 & -4.5 & -2.4 & -4.4 & 1.8 & 2.4 & 3.0 \\
\hline PAP11 & orf19.3197 & RNA binding & 4.7 & -3.8 & -1.5 & 2.2 & 2.3 & 2.5 & -1.3 & 1.5 & 2.2 \\
\hline PET112 & orf19.2494 & -- & 1.2 & 1.4 & -1.4 & 2.4 & 1.5 & 1.7 & 1.4 & 1.1 & 1.3 \\
\hline PET8 & orf19.7082 & Transporter activity & -1.0 & 1.1 & -1.2 & 1.5 & 1.7 & 2.3 & 1.1 & 1.1 & -2.5 \\
\hline PEX17 & orf19.3605 & -- & 2.5 & 1.6 & 1.5 & 1.7 & 1.2 & 1.6 & -1.5 & -1.4 & 1.1 \\
\hline PEX4 & orf19.4041 & Ubiquitin conjugating enzyme activity & -1.0 & 1.8 & -1.5 & 1.5 & 1.5 & 1.8 & -1.6 & -1.1 & -3.6 \\
\hline PGA10 & orf19.5674 & Heme binding & 1.8 & 4.3 & 1.2 & 2.0 & 3.9 & 3.2 & 1.2 & -1.1 & -1.1 \\
\hline PGA26 & orf19.2475 & -- & -3.2 & -1.4 & -1.6 & 2.8 & 6.0 & 2.9 & 1.5 & 1.1 & 1.4 \\
\hline PHRI & orf19.3829 & 1,3-beta-glucanosyltransferase activity & 1.9 & 2.4 & 1.5 & -1.1 & -1.4 & -2.1 & -1.0 & 1.4 & 1.2 \\
\hline PIBI & orf19.7547 & Ubiquitin-protein ligase activity & 1.5 & 1.5 & -1.4 & 1.7 & 2.0 & 2.9 & -1.0 & -1.3 & -1.5 \\
\hline PLB4.3f & orf19.1442 & Lysophospholipase activity & -1.8 & 1.1 & 1.0 & 1.9 & 2.6 & 4.3 & -1.2 & -1.3 & -1.2 \\
\hline POLO & orf19.5373 & -- & 1.4 & 1.3 & 1.4 & 3.0 & 4.2 & 3.9 & -1.0 & -1.0 & 1.7 \\
\hline POL3 & orf19.5182 & Delta DNA polymerase activity & 1.8 & 1.9 & 1.6 & 2.9 & 1.7 & -4.3 & 1.5 & -1.2 & -1.2 \\
\hline POT13 & orf19.2046 & Acetyl-CoA C-acyltransferase activity & 1.1 & 1.8 & 1.4 & 1.5 & 1.5 & 2.2 & -1.1 & -1.0 & -1.1 \\
\hline PRE10 & orf19.6582 & Endopeptidase activity & -1.2 & -1.3 & 1.7 & 1.9 & 1.9 & 2.4 & 1.3 & -1.1 & -1.2 \\
\hline PRE5 & orf19.7178 & Endopeptidase activity & -1.2 & 1.1 & -1.0 & 1.5 & 1.5 & 1.6 & -1.3 & -1.1 & -2.2 \\
\hline$P R Y 2$ & orf19.7218 & -- & 1.1 & 1.1 & -1.1 & 3.4 & 2.7 & 2.6 & -1.3 & 1.1 & -1.3 \\
\hline$P Y C 2.3 f$ & orf19.789 & Pyruvate carboxylase activity & 1.3 & -1.3 & 1.1 & -1.4 & 1.2 & 1.7 & 1.6 & 1.8 & 1.5 \\
\hline PYC2.5f & orf19.789 & Pyruvate carboxylase activity & 2.1 & 1.5 & 4.8 & 1.4 & 1.5 & -5.8 & 1.6 & 1.7 & 2.1 \\
\hline$R B T 1$ & orf19.1327 & -- & -1.2 & 1.2 & -1.2 & -1.4 & -1.3 & -2.3 & 1.8 & 1.5 & 2.1 \\
\hline RBT4 & orf19.6202 & -- & 3.6 & 3.3 & 3.2 & 1.2 & -1.5 & -2.4 & -1.1 & 1.4 & 1.4 \\
\hline RPN4 & orf19.1069 & Transcription factor activity & 2.4 & 2.6 & 2.2 & 2.8 & 2.8 & 2.0 & 1.5 & 1.4 & 1.1 \\
\hline SLA2 & orf19.7201 & Structural constituent of cytoskeleton & -1.6 & -1.5 & -1.2 & 2.0 & 2.2 & 4.1 & 1.4 & 1.1 & 1.2 \\
\hline SNF31 & orf19.7094 & Glucose transporter activity & -1.0 & 14.4 & 3.2 & 1.9 & 1.7 & 3.4 & -3.0 & -1.1 & -1.5 \\
\hline SNZ1 & orf19.2947 & -- & 1.5 & 2.1 & 1.9 & 2.3 & -1.2 & -1.3 & -1.5 & 1.3 & 1.0 \\
\hline SOD21 & orf19.7111 & Manganese superoxide dismutase activity & -1.1 & 2.7 & -2.0 & 2.0 & 2.3 & 1.6 & 3.2 & 2.2 & 2.6 \\
\hline
\end{tabular}


Table B-1 (continued).

\begin{tabular}{|c|c|c|c|c|c|c|c|c|c|c|c|}
\hline \multirow[b]{2}{*}{ CandidaDB Name ${ }^{a}$} & \multirow[b]{2}{*}{ orf19 Name ${ }^{b}$} & \multirow[b]{2}{*}{ GO Annotation (Molecular Function) ${ }^{c}$} & \multicolumn{3}{|c|}{ F5 vs. F1 } & \multicolumn{3}{|c|}{ G5 vs. G1 } & \multicolumn{3}{|c|}{6692 vs. 5833} \\
\hline & & & 1 & 2 & 3 & 1 & 2 & 3 & 1 & 2 & 3 \\
\hline SOD21 & orf19.7111 & Manganese superoxide dismutase activity & -1.0 & 4.8 & -5.0 & 5.9 & 2.6 & 3.1 & 2.9 & 2.3 & 1.7 \\
\hline SOU3 & orf19.732 & 2,4-dienoyl-CoA reductase (NADPH) activity & 1.9 & 2.5 & 1.5 & 2.2 & 2.2 & 2.0 & -1.5 & -1.1 & -1.4 \\
\hline STL2 & orf19.7093 & Glucose transporter activity & 1.4 & 1.9 & -1.1 & 1.5 & 2.0 & 2.2 & -1.3 & -1.1 & -2.4 \\
\hline TAF19 & orf19.5174 & General RNA polymerase II transcription factor activity & 1.0 & 1.1 & 1.2 & 2.0 & 1.8 & 2.1 & -1.2 & -1.0 & -1.5 \\
\hline TEF41 & orf19.2652 & Translation elongation factor activity & 3.4 & 3.9 & 13.6 & 5.0 & 5.2 & 7.0 & -1.1 & -1.5 & -1.7 \\
\hline TERT2 & orf19.5089 & Telomeric template RNA reverse transcriptase activity & 2.0 & 2.5 & 1.4 & 8.1 & 2.7 & 1.7 & -1.6 & -2.1 & -3.2 \\
\hline TES12 & orf19.4122 & -- & 2.4 & 1.5 & 1.7 & -2.8 & -2.1 & -2.0 & 1.2 & -1.1 & -1.4 \\
\hline TIM9 & orf19.6696 & Protein transporter activity & -1.3 & 1.3 & 1.6 & 3.0 & 1.8 & 3.1 & 1.2 & -1.4 & 2.1 \\
\hline TOM72 & orf19.3700 & Protein transporter activity & 1.0 & -1.5 & 1.0 & 1.9 & 1.7 & 1.8 & 1.3 & -1.2 & 3.1 \\
\hline TRP4 & orf19.3099 & Anthranilate phosphoribosyl transferase activity & 1.5 & 1.2 & 1.2 & 1.2 & 1.2 & 2.2 & 1.5 & 1.7 & 1.9 \\
\hline UFD1 & orf19.5833 & -- & -1.5 & 1.0 & -1.1 & 1.5 & 1.5 & 2.0 & -1.1 & 1.0 & -2.1 \\
\hline$U G A 2$ & orf19.345 & Succinate-semialdehyde dehydrogenase activity & 1.8 & 2.9 & 16.0 & 1.2 & 1.0 & -1.1 & 2.4 & 2.5 & 3.4 \\
\hline UGT51 & orf19.2616 & Sterol 3-beta-glucosyltransferase activity & 1.1 & 1.8 & -4.4 & 3.3 & 1.8 & 4.7 & 1.2 & 1.1 & 5.7 \\
\hline YARI & orf19.7160 & -- & -1.6 & -1.9 & 2.2 & 1.6 & 1.7 & 2.5 & 1.6 & -1.0 & 1.1 \\
\hline YOXI & orf19.7017 & -- & 1.5 & 1.5 & 1.7 & 1.3 & 1.2 & -1.1 & 1.3 & 1.9 & 1.9 \\
\hline
\end{tabular}

${ }^{a}$ Gene name at CandidaDB (http://genolist.pasteur.fr/CandidaDB/).

${ }^{b}$ orf19 nomenclature according to the Assembly 19 version.

${ }^{c} \mathrm{GO}$ annotation found at CGD (http://www.candidagenome.org/). 
Table B-2. Genes down-regulated in at least one clinical matched isolate overexpressing MDR1.

\begin{tabular}{|c|c|c|c|c|c|c|c|c|c|c|c|}
\hline \multirow[b]{2}{*}{ CandidaDB Name ${ }^{a}$} & \multirow[b]{2}{*}{ orf19 $\mathrm{Name}^{b}$} & \multirow[b]{2}{*}{ GO Annotation (Molecular Function) ${ }^{c}$} & \multicolumn{3}{|c|}{ F5 vs. F1 } & \multicolumn{3}{|c|}{ G5 vs. G1 } & \multicolumn{3}{|c|}{6692 vs. 5833} \\
\hline & & & $\mathbf{1}$ & 2 & 3 & $\mathbf{1}$ & 2 & 3 & 1 & 2 & 3 \\
\hline AAT1 & orf19.3554 & Aspartate transaminase activity & -1.3 & 1.1 & -1.0 & -1.5 & -1.2 & -1.1 & -2.1 & -1.6 & -1.8 \\
\hline AAT21 & orf19.6287 & Aspartate transaminase activity & -1.4 & 1.1 & -1.2 & -1.9 & -1.6 & -2.0 & -1.4 & 1.0 & 1.1 \\
\hline$A D E 1$ & orf19.7484 & $\begin{array}{l}\text { Phosphoribosylaminoimidazolesuccino carboxamide synthase } \\
\text { activity }\end{array}$ & -1.7 & -1.8 & -1.5 & -1.3 & -1.2 & -1.1 & -2.1 & -1.9 & -4.8 \\
\hline ADE13 & orf19.3870 & Adenylosuccinate lyase activity & -1.7 & -1.7 & -1.8 & -1.4 & -1.2 & -1.1 & -2.2 & -1.9 & -1.8 \\
\hline$A D E 2$ & orf19.5906 & Phosphoribosylaminoimidazole carboxylase activity & -1.3 & -1.3 & -2.2 & -1.1 & -1.3 & -1.4 & -2.1 & -1.7 & -2.3 \\
\hline ADE6 & orf19.6317 & Phosphoribosylformylglycinamidine synthase activity & -1.5 & -1.6 & -1.5 & -1.8 & -1.2 & 1.1 & -1.6 & -1.7 & -1.4 \\
\hline ADE8 & orf19.5789 & Phosphoribosylglycinamide formyltransferase activity & -2.2 & -1.8 & -1.5 & -1.1 & -1.6 & -1.7 & -2.2 & -1.8 & -3.1 \\
\hline$A G P 2$ & orf19.4679 & Hydrogen:amino acid symporter activity & -2.5 & -2.0 & -2.2 & -6.9 & -6.0 & -7.6 & -1.1 & 1.6 & 4.6 \\
\hline AKRI & orf19.4950 & -- & -1.2 & -1.2 & -2.4 & -1.8 & 1.0 & 1.5 & -1.5 & -1.7 & -1.6 \\
\hline$A L P 1$ & orf19.2337 & Basic amino acid transporter activity & -2.8 & -2.3 & -1.9 & -3.3 & -1.6 & -1.6 & 1.0 & -1.1 & -1.0 \\
\hline ALS1 & orf19.5741 & Cell adhesion molecule binding & -1.9 & -1.0 & -2.4 & -2.3 & -2.1 & -3.1 & 1.2 & 1.6 & 1.0 \\
\hline ALS6 & orf19.7414 & Cell adhesion molecule binding & 1.0 & 2.2 & 1.3 & -6.3 & -7.2 & -1.9 & -1.1 & -1.2 & 1.2 \\
\hline$A O X 1$ & orf19.4774 & Alternative oxidase activity & 1.1 & -1.9 & -2.4 & -1.6 & -1.3 & 1.2 & -1.6 & -2.2 & -2.2 \\
\hline AOX2.3f & orf19.4773 & Alternative oxidase activity & 1.4 & 1.6 & 1.7 & -3.2 & -6.3 & -4.9 & -2.0 & -1.6 & 1.3 \\
\hline$A Q Y 1$ & orf19.2849 & Water channel activity & -3.2 & 1.1 & -2.2 & -6.6 & -3.3 & -2.9 & -3.2 & -2.3 & -2.0 \\
\hline$A R O 3$ & orf19.1517 & 3-deoxy-7-phosphoheptulonate synthase activity & -1.4 & 1.2 & -1.4 & -1.5 & -1.5 & -1.7 & -1.0 & 1.9 & 1.8 \\
\hline ARR3 & orf19.3122 & Arsenite transporter activity & -2.1 & -1.5 & -1.5 & -4.5 & -2.0 & -2.1 & -1.5 & -1.4 & -1.9 \\
\hline AURI & orf19.1945 & Inositol phosphoceramide synthase activity & -1.4 & -1.1 & -1.0 & -3.0 & -1.5 & -2.3 & -1.3 & -1.0 & 1.5 \\
\hline AXL2 & orf19.5292 & -- & 1.2 & -1.6 & 3.4 & -2.2 & -1.7 & -1.6 & 1.6 & 1.2 & -2.7 \\
\hline AYR2 & orf19.5615 & Acylglycerone-phosphate reductase activity & -1.8 & -1.1 & -1.7 & -3.1 & -1.5 & -2.1 & -1.3 & -1.2 & -1.5 \\
\hline CAN1 & orf19.97 & L-arginine transporter activity & -1.7 & -1.1 & -1.5 & -2.8 & -1.9 & -2.7 & -1.8 & -2.1 & 1.1 \\
\hline CAN2 & orf19.111 & Basic amino acid transporter activity & -1.6 & 1.0 & -4.0 & -4.9 & -2.8 & -4.9 & -3.7 & -3.0 & -6.3 \\
\hline CAPI & orf19.1623 & Transcription factor activity & 1.2 & 1.1 & -1.0 & -1.9 & -1.6 & -1.8 & -1.2 & 1.2 & -1.1 \\
\hline CAT2 & orf19.4591 & Carnitine O-acetyltransferase activity & -1.0 & 1.0 & -1.3 & 1.1 & -1.4 & -1.6 & -1.7 & -1.9 & -2.5 \\
\hline CFL1 & orf19.1263 & Ferric-chelate reductase activity & -1.5 & -1.5 & -2.0 & -1.4 & 1.1 & -1.5 & -1.7 & -2.4 & -2.4 \\
\hline CPAI & orf19.4630 & Carbamoyl-phosphate synthase activity & -1.8 & -2.0 & -1.9 & -2.4 & -1.3 & -1.7 & -1.4 & -1.1 & -1.3 \\
\hline CPA2 & orf19.3221 & Carbamoyl-phosphate synthase activity & -1.4 & -1.7 & 1.4 & -2.2 & -2.4 & -1.5 & 1.1 & -1.0 & -1.2 \\
\hline CTA1 & orf19.6229 & Catalase activity & -1.9 & 1.6 & -1.8 & -1.6 & -2.0 & -2.5 & -2.3 & -1.3 & -1.2 \\
\hline CTA211.5f & orf19.362 & -- & -1.8 & -1.7 & -1.8 & -2.2 & -1.3 & 1.8 & -1.2 & 1.1 & 17.7 \\
\hline CYB3 & orf19.5000 & L-lactate dehydrogenase activity & 1.4 & 2.9 & 2.5 & 1.2 & 1.0 & -1.1 & -2.4 & -1.9 & -1.7 \\
\hline CYT2 & orf19.4578 & Holocytochrome-c synthase activity & 1.0 & 1.3 & -1.3 & -2.4 & -2.1 & -2.4 & -1.0 & 1.0 & -1.2 \\
\hline$D A K 2$ & orf19.4777 & Glycerone kinase activity & -1.5 & -1.6 & -2.7 & -1.9 & 1.1 & 1.7 & 2.4 & 1.1 & -1.7 \\
\hline$D B P 2$ & orf19.171 & RNA helicase activity & 1.0 & -1.4 & 1.1 & -1.5 & -1.8 & -3.3 & -1.0 & 1.3 & -1.1 \\
\hline DOG2 & orf19.3392 & 2-deoxyglucose-6-phosphatase activity & -1.1 & -1.0 & 1.0 & -1.9 & -1.5 & -2.1 & 1.4 & 1.4 & 1.6 \\
\hline$D P P 1$ & orf19.656 & Phosphatidase phosphatase activity & -1.4 & 1.3 & 1.7 & -2.3 & -1.6 & -1.8 & -1.2 & 1.1 & 1.0 \\
\hline DRS21.5eoc & orf19.783 & Calcium-transporting ATPase activity & -1.1 & -1.2 & -1.3 & -1.6 & -1.2 & -1.1 & -1.8 & -1.5 & -1.6 \\
\hline DUR32 & orf19.5017 & Urea transporter activity & 2.0 & 1.3 & 1.1 & -4.2 & -1.6 & -2.1 & -1.4 & -1.8 & -1.9 \\
\hline DUR33.5eoc & orf19.7205 & Urea transporter activity & -1.6 & 1.1 & 1.0 & -2.3 & -1.8 & -1.9 & 1.3 & -1.1 & -2.2 \\
\hline$E B P 1$ & orf19.125 & NADPH dehydrogenase activity & -5.5 & -4.2 & -2.9 & -4.5 & -2.8 & -2.7 & -2.9 & -2.8 & -3.9 \\
\hline ECM17 & orf19.4099 & Sulfite reductase (NADPH) activity & 1.1 & -1.1 & -1.3 & -1.6 & -1.7 & -2.1 & -1.2 & -1.1 & 1.4 \\
\hline
\end{tabular}


Table B-2 (continued).

\begin{tabular}{|c|c|c|c|c|c|c|c|c|c|c|c|}
\hline \multirow[b]{2}{*}{ CandidaDB Name ${ }^{a}$} & \multirow[b]{2}{*}{ orf19 Name ${ }^{b}$} & \multirow[b]{2}{*}{ GO Annotation (Molecular Function) ${ }^{c}$} & \multicolumn{3}{|c|}{ F5 vs. F1 } & \multicolumn{3}{|c|}{ G5 vs. G1 } & \multicolumn{3}{|c|}{6692 vs. 5833} \\
\hline & & & 1 & 2 & 3 & 1 & 2 & 3 & 1 & 2 & $\mathbf{3}$ \\
\hline ECM22 & orf19.2623 & Transcription factor activity & -2.1 & -1.3 & -2.2 & -2.8 & -2.3 & -2.5 & -1.8 & -1.5 & -1.3 \\
\hline ECM42 & orf19.6500 & Amino-acid N-acetyltransferase activity & -2.2 & -1.8 & -1.8 & 1.2 & 1.4 & 1.7 & 1.1 & 1.1 & -1.0 \\
\hline ENA22 & orf19.6070 & ATPase activity & -1.7 & -1.4 & -2.1 & -2.9 & -1.5 & -1.7 & -1.5 & -1.4 & -1.0 \\
\hline FAA21 & orf19.272 & Long-chain-fatty-acid-CoA ligase activity & 1.6 & 1.1 & 1.5 & -3.0 & -2.4 & -1.8 & -1.4 & -1.1 & 1.6 \\
\hline FCR1 & orf19.6817 & Transcription factor activity & -2.0 & -1.5 & -2.0 & -2.1 & -1.6 & -2.3 & 1.0 & 1.1 & 1.1 \\
\hline FDH12 & orf19.638 & Formate dehydrogenase activity & -1.7 & 1.8 & -2.2 & -2.4 & -3.0 & -2.4 & -2.4 & -2.4 & -1.8 \\
\hline $\mathrm{FDH} 2$ & orf19.1117 & Formate dehydrogenase activity & 1.2 & -4.1 & -12.0 & -2.3 & -7.5 & -4.6 & -2.5 & -1.5 & -2.0 \\
\hline$F D H 3.3 f$ & orf19.1774 & Formate dehydrogenase activity & -1.9 & -1.1 & -2.2 & -2.5 & -4.7 & -4.7 & -3.1 & -3.6 & -4.3 \\
\hline FDH3.5f & orf19.1774 & Formate dehydrogenase activity & 1.4 & -12.4 & -5.1 & -2.6 & -4.6 & -2.1 & -2.3 & -4.4 & -2.8 \\
\hline FET33 & orf19.4211 & Ferroxidase activity & 1.5 & 1.4 & 1.1 & -1.8 & -1.7 & -2.8 & -1.7 & -1.5 & -2.3 \\
\hline FOX2 & orf19.1288 & 3-hydroxyacyl-CoA dehydrogenase activity & -1.2 & 3.8 & -1.5 & -2.0 & -1.9 & -1.8 & -1.9 & -1.4 & -1.4 \\
\hline FTR1 & orf19.7219 & Ferrous iron transporter activity & -4.3 & -3.0 & -2.8 & -3.8 & -3.2 & -5.2 & -5.6 & -3.9 & -5.4 \\
\hline GAD1 & orf19.1153 & Glutamate decarboxylase activity & -1.4 & -1.3 & -1.7 & -2.3 & -1.2 & -1.0 & -1.6 & -1.5 & -2.6 \\
\hline GAL10 & orf19.1153 & UDP-glucose 4-epimerase activity & 1.2 & 2.1 & 1.6 & -2.4 & -1.8 & -1.7 & -1.7 & -1.2 & -2.0 \\
\hline GAL7 & orf19.3675 & UTP:galactose-1-phosphate uridylyltransferase activity & -1.0 & 1.8 & 2.3 & -2.2 & -2.4 & -2.3 & -1.7 & -1.3 & -1.7 \\
\hline GAP4 & orf19.1799 & Amino acid permease activity & -1.6 & -1.6 & -1.6 & -1.5 & -1.1 & -1.3 & -1.2 & -1.2 & 5.0 \\
\hline GCVI & orf19.5519 & Glycine dehydrogenase (decarboxylating) activity & -1.9 & -2.1 & -2.2 & -1.6 & -1.2 & 1.2 & -2.3 & -3.1 & -2.5 \\
\hline$G C V 2$ & orf19.385 & Glycine dehydrogenase (decarboxylating) activity & -1.4 & -1.5 & -2.4 & 1.3 & -1.1 & -1.1 & -2.8 & -1.8 & -2.5 \\
\hline GNP1 & orf19.7566 & Amino acid permease activity & 1.0 & 2.4 & -1.7 & -1.5 & -1.4 & -1.7 & -2.2 & -1.7 & -1.8 \\
\hline GPT1 & orf19.4063 & L-gamma-aminobutyric acid transporter activity & 1.9 & -1.5 & -1.6 & -2.1 & -1.6 & -1.7 & 1.1 & -1.1 & -1.8 \\
\hline GRP4 & orf19.3150 & Oxidoreductase activity & -1.4 & 1.3 & 1.7 & -1.9 & -1.5 & -2.3 & -1.9 & -1.7 & -1.1 \\
\hline GSL22 & orf19.2495 & 1,3-beta-glucan synthase activity & -1.4 & 1.1 & 1.0 & -2.0 & -1.5 & -2.4 & -1.1 & 1.2 & 1.9 \\
\hline GSL23.3f & orf19.3269 & 1,3-beta-glucan synthase activity & -1.0 & -1.1 & -1.2 & -1.9 & -1.6 & -2.2 & -1.1 & -1.1 & -1.3 \\
\hline GTT1 & orf19.6947 & Glutathione transferase activity & -1.5 & 1.2 & 1.0 & -1.8 & -1.5 & -1.7 & -1.1 & 1.1 & -1.3 \\
\hline GYP1 & orf19.3811 & Rab GTPase activator activity & 1.1 & 1.4 & -1.3 & -1.1 & -1.1 & 1.2 & -1.6 & -1.5 & -1.9 \\
\hline HGT11 & orf19.4527 & Glucose transporter activity & 3.1 & 4.4 & 1.7 & 1.8 & -1.2 & -1.5 & -2.3 & -1.5 & -3.0 \\
\hline HGT12 & orf19.3668 & Glucose transporter activity & 2.4 & 14.0 & 4.4 & 6.8 & 2.6 & 1.8 & -3.1 & -3.1 & -3.0 \\
\hline HIPl & orf19.4940 & L-histidine transporter activity & -1.6 & -1.6 & 1.1 & -2.2 & -1.7 & -1.7 & -1.0 & 1.0 & -1.6 \\
\hline HIS3 & orf19.183 & Imidazoleglycerol-phosphate dehydratase activity & 1.3 & -1.2 & 1.7 & -2.0 & -3.0 & -2.9 & -1.1 & -1.1 & 1.7 \\
\hline HNM1 & orf19.2003 & Choline transporter activity & -1.4 & -1.1 & -1.1 & -1.7 & -1.6 & -2.1 & -1.4 & -1.1 & -1.2 \\
\hline HOL1 & orf19.2991 & Transporter activity & -1.2 & -1.1 & -1.6 & -2.2 & -1.7 & -2.2 & 1.3 & 1.5 & 2.1 \\
\hline HSP12 & orf19.3160 & -- & -2.2 & 1.1 & -3.0 & -12.3 & -4.4 & -3.5 & -7.0 & -1.7 & -1.6 \\
\hline HSP31 & orf19.3664 & -- & -2.8 & -2.3 & -2.5 & -1.5 & -1.3 & -1.6 & 1.4 & -1.2 & -3.1 \\
\hline HXT5.3f & orf19.2021 & Fructose transporter activity & -1.6 & -1.2 & -1.6 & -3.8 & -2.5 & -3.1 & -1.6 & -1.1 & -1.3 \\
\hline HXT5.5f & orf19.2021 & Fructose transporter activity & -1.6 & -1.2 & -2.0 & -3.7 & -3.0 & -6.2 & -1.4 & -1.3 & -1.5 \\
\hline IFC3 & orf19.3749 & Oligopeptide transporter activity & -2.1 & -1.6 & -3.0 & -6.7 & -1.4 & 1.0 & -1.2 & -2.1 & -1.7 \\
\hline IFF4 & orf19.7472 & -- & 1.2 & 1.4 & -1.5 & -1.6 & -1.1 & 1.5 & -2.0 & -1.8 & -12.4 \\
\hline IFN1 & orf19.1979 & Phospholipid transporter activity & -3.1 & -1.9 & -1.8 & -2.8 & -1.5 & -1.6 & -1.0 & -1.2 & -1.9 \\
\hline IFN3 & orf19.1978 & Phospholipid transporter activity & -1.5 & -1.4 & -1.5 & -3.9 & -2.0 & -2.0 & -1.1 & 1.1 & -1.1 \\
\hline IFR2 2 & orf19.2396 & -- & -1.8 & -1.6 & -1.8 & -2.0 & -1.5 & -2.4 & -1.1 & -1.1 & 1.1 \\
\hline IFY2.5f & orf19.2158 & Monosaccharide transporter activity & -2.0 & -1.9 & -2.1 & -3.1 & -1.5 & -1.6 & -1.6 & -1.7 & -1.5 \\
\hline
\end{tabular}


Table B-2 (continued).

\begin{tabular}{|c|c|c|c|c|c|c|c|c|c|c|c|}
\hline \multirow[b]{2}{*}{ CandidaDB Name ${ }^{a}$} & \multirow[b]{2}{*}{ orf19 Name ${ }^{b}$} & \multirow[b]{2}{*}{ GO Annotation (Molecular Function) ${ }^{c}$} & \multicolumn{3}{|c|}{ F5 vs. F1 } & \multicolumn{3}{|c|}{ G5 vs. G1 } & \multicolumn{3}{|c|}{6692 vs. 5833} \\
\hline & & & 1 & 2 & 3 & 1 & 2 & 3 & 1 & 2 & $\mathbf{3}$ \\
\hline IPF10029.5eoc & orf19.3915 & 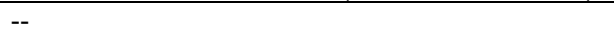 & -1.4 & -1.7 & -1.1 & -1.5 & -1.5 & -5.3 & 1.1 & -1.0 & -1.0 \\
\hline IPF10153 & orf19.2170 & -- & -1.4 & -1.0 & -1.2 & -2.3 & -1.7 & -2.2 & -1.2 & -1.1 & -1.6 \\
\hline IPF10355 & orf19.3809 & Transcription factor activity & 1.5 & 1.1 & -1.3 & 1.5 & 1.2 & -1.6 & -2.0 & -1.6 & -1.8 \\
\hline IPF11105 & orf19.2262 & -- & -2.4 & 1.6 & 1.1 & -1.9 & -1.6 & -1.9 & -2.5 & -1.9 & -2.4 \\
\hline IPF11142 & orf19.473 & -- & -1.5 & -1.9 & -2.4 & -1.8 & -1.2 & -1.5 & -1.1 & 1.1 & -1.1 \\
\hline IPF11448 & orf19.2923 & -- & -1.5 & -1.7 & -1.8 & -2.1 & -1.3 & -1.3 & 1.0 & 1.0 & 1.3 \\
\hline IPF11503 & orf19.3902 & -- & -2.1 & -1.1 & -1.9 & -4.1 & -1.7 & -2.6 & -4.8 & -3.2 & -3.6 \\
\hline IPF11548 & orf19.3854 & Protein kinase activity & 1.0 & -2.4 & 1.0 & -1.6 & -1.9 & -1.6 & -1.1 & -1.3 & -1.4 \\
\hline IPF11713.3f & orf19.1152 & -- & -2.1 & -1.2 & -2.3 & -5.1 & -2.2 & -3.5 & -1.2 & -1.0 & 1.5 \\
\hline IPF11725 & orf19.3475 & -- & -1.3 & 1.0 & -1.1 & -1.9 & -1.6 & -1.8 & -1.1 & -1.2 & 1.1 \\
\hline IPF11759.3f & orf19.4737 & -- & -1.8 & -1.9 & -4.3 & -2.7 & -1.0 & -1.7 & -1.1 & -1.2 & -2.2 \\
\hline IPF11849 & orf19.4559 & -- & 1.1 & 1.4 & 1.4 & -1.2 & -5.5 & -2.1 & -1.5 & -1.5 & -2.8 \\
\hline IPF11858 & orf19.1277 & -- & -1.0 & 1.1 & -1.2 & -2.0 & -1.8 & -2.4 & -1.4 & -1.6 & 1.1 \\
\hline IPF1193 & orf19.2079 & -- & -1.4 & -1.1 & 1.1 & -2.4 & -1.8 & -2.0 & -1.0 & -1.1 & 1.1 \\
\hline IPF12082.3f & orf19.6832 & -- & -1.7 & -2.1 & -2.1 & 1.2 & 1.7 & -1.0 & 1.3 & -1.0 & 1.4 \\
\hline IPF12568 & orf19.3516 & -- & -1.2 & 1.1 & 1.3 & -1.6 & -1.7 & -1.9 & -1.3 & -1.1 & -1.2 \\
\hline IPF 12577 & orf19.5238 & -- & -1.7 & -1.6 & -1.5 & -1.7 & 1.0 & 1.3 & -1.0 & -1.1 & 1.2 \\
\hline IPF12579 & orf19.5239 & -- & -1.1 & -1.9 & 1.0 & -1.7 & -1.0 & -1.2 & -1.9 & -1.6 & -5.3 \\
\hline IPF12758.5f & orf19.334 & -- & -1.2 & 1.4 & -1.3 & -1.9 & -1.7 & -1.7 & -1.8 & -1.4 & -1.5 \\
\hline IPF12887 & orf19.4780 & -- & -2.0 & -1.8 & -2.4 & -1.8 & -1.1 & -1.6 & -1.1 & 1.1 & 1.3 \\
\hline IPF1292 & orf19.7381 & Transcription factor activity & 1.3 & -1.2 & 1.4 & -4.6 & -3.0 & -3.5 & 1.2 & 1.1 & 1.0 \\
\hline IPF13131.3 & orf19.1587 & Glucose transporter activity & -2.0 & -1.2 & -3.4 & -1.9 & -1.7 & -2.0 & -2.9 & -3.8 & -7.4 \\
\hline IPF13377 & orf19.2350 & -- & -2.1 & -1.5 & -2.3 & -1.6 & 1.2 & 1.1 & -1.8 & 1.0 & 1.2 \\
\hline IPF13493 & orf19.5063 & -- & -4.3 & -3.0 & -1.5 & -1.3 & -1.1 & -1.1 & -1.7 & -1.9 & -2.5 \\
\hline IPF13769 & orf19.6209 & Monocarboxylic acid transporter activity & -1.1 & -1.3 & -1.6 & -1.5 & -1.8 & -2.5 & -1.6 & -1.6 & -1.9 \\
\hline IPF1384 & orf19.6445 & Dodecenoyl-CoA delta-isomerase activity & -1.1 & 1.3 & -1.7 & 1.0 & -2.1 & 1.5 & -1.8 & -1.8 & -1.8 \\
\hline IPF13879 & orf19.1120 & -- & 2.2 & 3.5 & 1.3 & -2.0 & -1.5 & -1.5 & 1.2 & 1.3 & 3.9 \\
\hline IPF1390 & orf19.6449 & -- & -1.5 & 2.1 & -1.4 & -1.3 & -1.0 & -2.0 & -4.3 & -1.8 & -2.0 \\
\hline IPF13921 & orf19.4749 & -- & -1.9 & -1.7 & -1.5 & -3.6 & -2.9 & -2.2 & -1.2 & -1.0 & 1.0 \\
\hline IPF13971 & orf19.5204 & -- & -1.3 & 1.0 & -2.6 & -2.2 & -1.5 & -2.8 & -1.5 & -1.2 & -3.1 \\
\hline IPF1401 & orf19.6066 & -- & -1.7 & -1.0 & -1.7 & -1.5 & -1.5 & -1.5 & -1.7 & -1.0 & -1.3 \\
\hline$I P F 14501.5 f$ & orf19.2455 & -- & 1.3 & 1.6 & -1.1 & -1.6 & -1.5 & -2.0 & -1.1 & -1.3 & -1.2 \\
\hline IPF1471 & orf19.304 & -- & -1.5 & -1.5 & -1.2 & -1.6 & -1.5 & -1.7 & 1.1 & 1.1 & -1.3 \\
\hline IPF14744 & orf19.5125 & -- & -1.3 & 2.5 & -1.7 & 1.2 & 1.1 & -1.4 & -3.5 & -2.7 & -1.5 \\
\hline$I P F 14773.5 f$ & orf19.3586 & -- & -1.1 & 1.4 & 1.3 & 1.4 & -1.7 & -5.5 & -1.6 & -1.7 & -2.0 \\
\hline IPF1505 & orf19.348 & -- & -2.9 & -1.3 & -2.1 & -3.2 & -1.5 & -1.7 & -1.0 & -1.2 & -8.9 \\
\hline IPF15051 & orf19.1258 & -- & -1.4 & -2.7 & 1.2 & -2.2 & 1.2 & 1.9 & -2.5 & -1.6 & -2.7 \\
\hline IPF15153 & orf19.5867 & -- & 1.0 & 1.2 & 1.2 & -1.9 & -1.7 & -2.6 & 1.1 & 1.2 & 1.4 \\
\hline IPF15177 & orf19.750 & -- & -1.0 & 1.1 & -1.0 & -1.7 & -1.6 & -2.0 & 1.1 & 1.1 & -1.5 \\
\hline IPF15575 & orf19.1247 & -- & -1.7 & -1.5 & -1.5 & -1.2 & -1.2 & -1.3 & -1.5 & 1.1 & -1.2 \\
\hline IPF15632 & orf19.3469 & -- & -1.7 & -1.2 & -1.8 & -2.2 & -1.6 & -1.5 & -1.4 & -1.1 & -1.2 \\
\hline
\end{tabular}


Table B-2 (continued).

\begin{tabular}{|c|c|c|c|c|c|c|c|c|c|c|c|}
\hline \multirow[b]{2}{*}{ CandidaDB Name ${ }^{a}$} & \multirow[b]{2}{*}{ orf19 Name $^{b}$} & \multirow[b]{2}{*}{ GO Annotation (Molecular Function) ${ }^{c}$} & \multicolumn{3}{|c|}{ F5 vs. F1 } & \multicolumn{3}{|c|}{ G5 vs. G1 } & \multicolumn{3}{|c|}{6692 vs. 5833} \\
\hline & & & 1 & 2 & $\mathbf{3}$ & 1 & 2 & 3 & 1 & 2 & 3 \\
\hline IPF16067 & orf19.3190 & Specific RNA polymerase II transcription factor activity & -1.1 & -1.2 & -1.9 & -1.6 & -1.8 & -2.4 & -2.4 & -1.4 & -1.7 \\
\hline IPF1615.3eoc & orf19.980 & -- & -1.1 & -1.0 & -1.8 & -1.2 & -1.2 & -1.8 & -1.8 & -1.5 & -2.4 \\
\hline IPF16253 & orf19.3932 & -- & -1.5 & -1.3 & -1.5 & -2.1 & -3.3 & -1.7 & -2.8 & -1.7 & 1.4 \\
\hline IPF16752 & orf19.2711 & RNA polymerase II transcription elongation factor activity & -1.3 & -1.6 & 1.1 & -1.6 & -1.5 & -1.7 & 1.1 & 1.0 & -1.2 \\
\hline IPF17050 & orf19.1049 & -- & -2.7 & -8.2 & -5.6 & -11.4 & -2.3 & -17.6 & -1.9 & -2.4 & 1.1 \\
\hline IPF17074 & orf19.2792 & -- & -1.8 & -1.3 & -1.4 & -2.3 & -1.5 & -1.9 & -1.0 & -1.1 & -1.7 \\
\hline IPF17094 & orf19.2143 & -- & -1.2 & -1.1 & -1.3 & -1.5 & -1.6 & -2.7 & -1.2 & 1.2 & 1.1 \\
\hline IPF17190 & orf19.3869 & -- & -1.5 & -1.1 & -1.3 & -2.2 & -2.0 & -1.5 & -1.0 & 1.3 & -1.1 \\
\hline IPF17430.5eoc & orf19.73 & -- & 1.2 & -9.5 & 3.6 & -1.5 & -1.6 & -6.1 & 1.1 & -1.0 & 1.2 \\
\hline IPF17706 & -- & -- & 1.1 & 1.2 & -1.0 & -3.5 & -11.2 & -4.7 & -1.6 & -1.1 & -1.9 \\
\hline IPF18474 & orf19.6610 & Sturctural constituent of cytoskeleton & 1.1 & -1.1 & -1.7 & -1.3 & -1.3 & -1.6 & -1.8 & -1.7 & -1.8 \\
\hline IPF18732 & orf19.711 & -- & 1.4 & 1.5 & -1.2 & -1.3 & 1.1 & 1.0 & -2.4 & -1.7 & -1.9 \\
\hline IPF19749 & orf19.94 & -- & -1.5 & 1.0 & -1.4 & -1.4 & -2.0 & 1.1 & -2.6 & -1.9 & -3.0 \\
\hline IPF19772 & orf19.5602 & -- & -1.1 & -1.5 & -1.2 & -1.3 & -1.1 & 1.1 & -1.5 & -1.8 & -2.0 \\
\hline IPF19814 & orf19.7106 & -- & -1.7 & -1.7 & -1.8 & 1.1 & 1.6 & 1.7 & 1.1 & -1.3 & 1.0 \\
\hline IPF19859 & orf19.3375 & -- & -1.3 & 1.8 & -1.6 & -1.9 & -1.7 & -1.6 & -1.0 & 1.2 & -1.1 \\
\hline IPF20135 & orf19.993 & -- & -2.4 & -1.7 & -1.6 & -1.8 & -1.7 & -2.0 & 1.2 & 1.2 & -1.3 \\
\hline IPF2053 & orf19.5267 & -- & -5.5 & -5.3 & -6.8 & -1.1 & 1.0 & -1.3 & -1.0 & 1.3 & -1.5 \\
\hline IPF2275.5eoc & orf19.6654 & -- & -2.2 & -1.9 & -2.0 & -1.9 & -2.0 & -4.1 & -1.1 & 1.2 & 1.1 \\
\hline IPF2283 & orf19.6660 & -- & -2.6 & -2.4 & -2.4 & -2.8 & -1.5 & -1.7 & 1.2 & -1.4 & 1.9 \\
\hline IPF2319 & orf19.6680 & Transcription factor activity & 1.0 & -1.0 & 1.0 & -1.5 & -1.7 & -1.6 & 1.1 & 1.1 & 1.8 \\
\hline IPF2374 & orf19.6805 & -- & -1.7 & -2.4 & 2.0 & -2.1 & -2.1 & -2.3 & -1.5 & -1.5 & 1.1 \\
\hline IPF2383 & orf19.6808 & -- & -1.7 & -2.4 & -2.2 & -1.2 & 1.1 & 1.1 & -1.1 & -1.4 & -1.9 \\
\hline IPF2522 & orf19.7460 & -- & -1.7 & -1.5 & -2.1 & 1.2 & 1.1 & 1.2 & -1.4 & -1.0 & 1.0 \\
\hline IPF2542 & orf19.6629 & Phospholipase $\mathrm{C}$ activity & -1.3 & -1.6 & 1.1 & -1.6 & -1.5 & -2.0 & -1.0 & -1.1 & 1.0 \\
\hline IPF2645 & orf19.7405 & -- & -1.8 & -1.5 & -1.9 & -1.2 & -1.3 & -1.4 & -1.1 & 1.2 & -1.7 \\
\hline IPF2795 & orf19.6763 & -- & -1.8 & -1.6 & -1.8 & -1.4 & -1.5 & -1.3 & 1.2 & 1.2 & -1.1 \\
\hline IPF2822 & orf19.6781 & Transcription factor activity & -2.4 & -2.4 & -1.1 & -2.2 & -3.1 & -2.2 & 1.5 & -1.4 & -1.4 \\
\hline IPF2839 & orf19.7296 & -- & -2.1 & -1.6 & -3.7 & -1.7 & -1.5 & -1.7 & -1.9 & -1.8 & -5.4 \\
\hline IPF3092 & orf19.4445 & -- & 1.1 & 1.6 & 1.0 & -1.2 & -1.9 & -1.4 & -2.4 & -2.0 & -1.5 \\
\hline IPF3506 & orf19.6741 & -- & -1.6 & -1.3 & -1.1 & -6.4 & -3.6 & -5.0 & 1.1 & 1.5 & 2.8 \\
\hline IPF3539 & orf19.2813 & -- & -2.1 & 7.3 & -1.1 & -1.2 & -1.4 & -7.6 & -2.1 & -2.2 & -1.6 \\
\hline IPF3624 & orf19.6713 & -- & 1.2 & 1.4 & -1.5 & -1.9 & -1.9 & -2.8 & -2.4 & -1.5 & -2.1 \\
\hline IPF3631 & orf19.6718 & -- & -1.3 & -1.7 & -1.1 & -12.6 & -4.5 & -6.2 & 1.8 & -1.3 & 1.2 \\
\hline IPF3634 & orf19.6720 & -- & -3.9 & -3.5 & -5.8 & -4.2 & -3.8 & -6.0 & -3.0 & -2.7 & -3.2 \\
\hline IPF3661 & orf19.899 & -- & -2.5 & -1.6 & -1.9 & -1.3 & 1.1 & -1.0 & -1.4 & -1.1 & -1.8 \\
\hline IPF3937 & orf19.868 & -- & -1.0 & 1.0 & -1.3 & -3.0 & -2.0 & -2.2 & -1.2 & -1.1 & -1.3 \\
\hline IPF4065 & orf19.1862 & -- & -2.9 & -1.6 & -2.1 & -8.3 & -1.9 & -1.9 & 1.3 & 1.5 & -1.1 \\
\hline IPF407 & orf19.7504 & -- & 1.2 & 1.5 & 1.1 & 1.2 & 1.2 & 2.3 & -1.5 & -1.7 & -2.4 \\
\hline IPF409 & orf19.7502 & -- & -7.2 & -2.8 & -4.0 & -3.3 & -2.7 & -2.1 & -2.4 & -2.7 & -3.8 \\
\hline IPF4430 & orf19.2959.1 & -- & -1.6 & -1.0 & -2.4 & -5.0 & -3.0 & -4.6 & -2.2 & -1.5 & 1.7 \\
\hline
\end{tabular}


Table B-2 (continued).

\begin{tabular}{|c|c|c|c|c|c|c|c|c|c|c|c|}
\hline \multirow[b]{2}{*}{ CandidaDB Name ${ }^{a}$} & \multirow[b]{2}{*}{ orf19 Name $^{b}$} & \multirow[b]{2}{*}{ GO Annotation (Molecular Function) ${ }^{c}$} & \multicolumn{3}{|c|}{ F5 vs. F1 } & \multicolumn{3}{|c|}{ G5 vs. G1 } & \multicolumn{3}{|c|}{6692 vs. 5833} \\
\hline & & & 1 & 2 & 3 & 1 & 2 & 3 & 1 & 2 & 3 \\
\hline IPF4537 & orf19.905 & 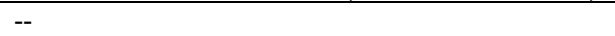 & -2.2 & -1.7 & -1.6 & -2.5 & -1.1 & -1.3 & -1.0 & -1.0 & 1.4 \\
\hline IPF4667 & orf19.1847 & Carboxy-lyase activity & -2.2 & -1.6 & -1.8 & -3.6 & -3.3 & -2.9 & -1.0 & -1.2 & 3.2 \\
\hline IPF4674 & orf19.1852 & -- & -1.4 & -1.1 & -1.4 & -1.5 & -1.5 & -1.9 & -1.4 & 1.0 & -1.1 \\
\hline IPF4704 & orf19.5278 & -- & -1.6 & 1.1 & -1.2 & -1.1 & -1.1 & -1.1 & -1.7 & -1.6 & -2.0 \\
\hline IPF4928 & orf19.7660 & -- & -1.1 & 1.0 & -1.5 & -1.7 & -1.6 & -1.5 & -1.4 & -1.6 & -1.2 \\
\hline IPF4991 & orf19.2531 & -- & -1.5 & 1.1 & -2.1 & -5.6 & -6.5 & -8.3 & -2.3 & -1.8 & 1.1 \\
\hline IPF4999 & orf19.5843 & -- & -1.4 & -2.2 & 1.0 & -6.6 & -6.7 & -7.8 & -1.2 & -1.1 & 1.7 \\
\hline IPF5282 & orf19.5663 & -- & -1.5 & -1.6 & -2.6 & -2.1 & 1.1 & -1.3 & -1.0 & 1.1 & 1.3 \\
\hline IPF5330 & orf19.3448 & -- & -1.9 & -6.0 & -1.8 & -4.1 & -1.7 & 1.9 & 2.1 & -2.1 & 1.7 \\
\hline IPF5353 & orf19.3003 & -- & -1.3 & -1.2 & -1.4 & -1.7 & -1.7 & -1.9 & -1.1 & 1.1 & -1.1 \\
\hline IPF5360 & orf19.3007.2 & -- & 1.1 & 1.0 & -2.0 & -2.9 & -2.0 & -3.1 & -2.2 & -1.9 & -2.0 \\
\hline IPF5453 & orf19.5692 & -- & 1.1 & 1.1 & -1.3 & -2.3 & -1.8 & -1.9 & 1.1 & 1.1 & 1.1 \\
\hline IPF5806 & orf19.815 & -- & -1.4 & 1.0 & 1.2 & -1.7 & -1.8 & -2.1 & -1.1 & 1.2 & 1.2 \\
\hline IPF5849 & orf19.5496 & -- & -2.1 & -1.6 & -1.7 & -2.1 & -1.1 & -1.2 & 1.2 & 1.1 & 2.0 \\
\hline IPF5966 & orf19.6793 & -- & -1.2 & -1.1 & -2.0 & -1.7 & -2.0 & -1.7 & -1.5 & 1.4 & 2.4 \\
\hline IPF5972 & orf19.7314 & -- & -1.3 & 1.0 & -1.3 & -1.6 & -1.7 & -2.2 & -1.5 & -1.1 & -1.8 \\
\hline IPF5988 & orf19.7305 & -- & -3.1 & -2.9 & -3.3 & -8.4 & -5.1 & -5.0 & -1.8 & -1.7 & 1.1 \\
\hline IPF6003 & orf19.1490 & -- & 1.0 & 1.2 & -1.0 & -1.7 & -1.5 & -1.8 & 1.2 & 1.2 & 2.3 \\
\hline IPF6108 & orf19.4811 & -- & -1.8 & -2.4 & -1.5 & 1.0 & -1.1 & -1.2 & 1.0 & 1.0 & 1.2 \\
\hline IPF6181 & orf19.4174 & Nucleoside transporter activity & -1.8 & -1.6 & -2.2 & -2.7 & -1.2 & 1.1 & -1.0 & -1.1 & -1.3 \\
\hline IPF6274 & orf19.6316 & -- & -1.8 & -1.2 & -1.5 & -2.5 & -2.0 & -3.0 & 1.1 & -1.0 & 1.7 \\
\hline IPF6339 & orf19.2724 & -- & -1.0 & 1.0 & -1.8 & -3.3 & -3.8 & -4.3 & -3.2 & -2.3 & -2.4 \\
\hline IPF6459 & orf19.1890 & -- & -1.5 & -1.1 & -1.3 & -2.0 & -1.7 & -1.7 & -1.1 & 1.0 & -1.1 \\
\hline IPF6572 & orf19.6874 & -- & -1.2 & -1.1 & -1.4 & -1.4 & -1.3 & -2.6 & -1.9 & -1.6 & -1.5 \\
\hline IPF6679 & orf19.1306 & -- & -1.6 & -1.3 & -1.4 & -1.1 & -1.2 & -1.1 & -2.4 & -2.1 & -1.7 \\
\hline IPF6881 & orf19.4246 & -- & -1.2 & 1.1 & 1.1 & -2.6 & -1.8 & -1.9 & -1.5 & -1.1 & -1.4 \\
\hline IPF6960 & orf19.6654 & -- & -1.9 & -1.8 & -2.0 & -2.6 & -1.3 & -1.2 & -1.1 & 1.1 & 1.2 \\
\hline IPF7109 & orf19.7350 & -- & 1.0 & 1.1 & -1.3 & -1.9 & -1.6 & -2.3 & -1.5 & -1.2 & -1.8 \\
\hline IPF7405 & orf19.4666 & -- & -2.0 & -1.5 & 1.0 & -2.8 & -2.2 & -1.9 & -1.0 & 1.0 & 1.9 \\
\hline IPF7452 & orf19.2045 & -- & -2.1 & -2.2 & -1.6 & -1.6 & -1.5 & -1.3 & -1.0 & -1.0 & 2.1 \\
\hline IPF7530 & orf19.4531 & ATP binding & -1.3 & -1.6 & 1.1 & -2.4 & -2.5 & -2.1 & -1.4 & -1.0 & -1.1 \\
\hline IPF7616 & orf19.1159 & Homoserine $\mathrm{O}$-acetyltransferase activity & 1.3 & 1.6 & -1.2 & -2.3 & -2.5 & -2.0 & -1.4 & -1.3 & -1.6 \\
\hline IPF7681 & orf19.1397 & Electron carrier activity & -1.2 & 1.0 & 1.3 & -1.8 & -1.8 & -1.9 & 1.1 & 1.0 & 1.4 \\
\hline IPF7711 & orf19.681 & -- & -1.1 & -1.6 & -2.3 & -1.4 & -1.3 & -2.6 & -1.5 & -1.5 & -1.9 \\
\hline IPF7715.3eoc & orf19.2296 & -- & -1.4 & 1.3 & -2.1 & -2.4 & -1.7 & -2.2 & -1.6 & -1.3 & -3.3 \\
\hline IPF7721 & orf19.431 & Transcription factor activity & -1.4 & -1.1 & -1.5 & -1.7 & -1.6 & -2.2 & -1.1 & -1.1 & -1.3 \\
\hline IPF7733 & orf19.4855 & -- & -1.7 & -1.5 & -2.0 & -1.1 & -1.1 & 1.0 & 1.3 & -1.4 & 1.6 \\
\hline IPF7785 & orf19.2962 & -- & -2.4 & -3.6 & 1.5 & -2.0 & -1.1 & 1.2 & -1.5 & -1.5 & -2.0 \\
\hline IPF7999 & orf19.4190 & -- & -1.3 & -1.5 & -1.2 & -1.9 & -2.5 & -2.1 & 1.3 & 1.0 & 1.1 \\
\hline IPF8302 & orf19.6007 & -- & -2.3 & -2.0 & 1.2 & -2.0 & -1.6 & -1.5 & -1.0 & -1.2 & 1.6 \\
\hline IPF8307 & orf19.6005 & Glucose transporter activity & -1.2 & 1.1 & -1.4 & -2.2 & -1.5 & -2.4 & -1.4 & -1.4 & -3.2 \\
\hline
\end{tabular}


Table B-2 (continued).

\begin{tabular}{|c|c|c|c|c|c|c|c|c|c|c|c|}
\hline \multirow[b]{2}{*}{ CandidaDB Name ${ }^{a}$} & \multirow[b]{2}{*}{ orf19 Name $^{b}$} & \multirow[b]{2}{*}{ GO Annotation (Molecular Function) ${ }^{c}$} & \multicolumn{3}{|c|}{ F5 vs. F1 } & \multicolumn{3}{|c|}{ G5 vs. G1 } & \multicolumn{3}{|c|}{6692 vs. 5833} \\
\hline & & & $\mathbf{1}$ & 2 & $\mathbf{3}$ & 1 & 2 & 3 & 1 & 2 & 3 \\
\hline IPF8369 & orf19.6077 & ( & -2.5 & -1.5 & -3.2 & -3.5 & -1.9 & -2.2 & -2.1 & -1.4 & 1.0 \\
\hline IPF8901 & orf19.3758 & -- & -6.2 & -3.4 & -4.1 & -1.6 & -1.5 & -3.5 & 1.2 & -2.1 & -1.3 \\
\hline IPF9057 & orf19.1189 & DNA bending activity & -3.0 & -2.3 & -2.6 & -1.9 & 1.0 & 1.8 & -3.2 & -1.9 & -3.0 \\
\hline IPF9126 & orf19.5537 & -- & 1.1 & -1.2 & -1.6 & -1.8 & -1.6 & -1.6 & 1.3 & 1.4 & 1.8 \\
\hline IPF9132 & orf19.5541 & -- & -1.3 & 1.0 & 1.4 & -2.3 & -1.5 & -2.3 & -1.1 & 1.2 & 1.1 \\
\hline IPF9145 & orf19.6245 & -- & -1.4 & -1.3 & -1.3 & -2.3 & -1.5 & -1.7 & -1.8 & 1.3 & -3.3 \\
\hline IPF9167 & orf19.2737 & -- & -1.5 & 1.3 & -1.7 & -3.1 & -1.7 & -1.8 & -1.2 & -1.2 & -1.3 \\
\hline IPF9167 & orf19.2737 & -- & -1.4 & 1.6 & -1.8 & -1.8 & -1.8 & -1.6 & -1.3 & -1.1 & -2.0 \\
\hline IPF9282 & orf19.1510 & -- & -1.3 & -1.1 & -1.4 & -1.7 & -1.5 & -2.9 & -1.6 & -1.4 & -2.4 \\
\hline IPF9425.3 & orf19.6888 & -- & 2.0 & 1.4 & -1.3 & -1.5 & -1.7 & -2.4 & -1.2 & -1.4 & -1.3 \\
\hline IPF946 & orf19.7561 & -- & 1.6 & 1.2 & -1.2 & -2.1 & -4.5 & -5.5 & 1.1 & 1.2 & -2.6 \\
\hline IPF9483 & orf19.3395 & -- & -2.6 & -1.6 & -2.5 & -2.7 & -2.0 & -2.3 & -2.2 & -2.0 & -1.8 \\
\hline IPF9605 & orf19.3071 & Protein tyrosine phosphatase activity & -2.6 & -1.9 & -1.5 & -1.9 & -1.1 & -1.1 & 1.3 & -1.0 & 1.6 \\
\hline IPF9703.3f & orf19.4880 & -- & -1.1 & -1.3 & -1.6 & -1.8 & -1.2 & 1.0 & -1.9 & -2.1 & -1.8 \\
\hline IPF9706 & orf19.4883 & -- & -4.3 & -1.9 & -2.1 & 1.2 & 1.0 & 1.5 & 1.1 & -1.2 & 1.2 \\
\hline IPF9821.3f & orf19.4142 & -- & -1.3 & -1.7 & -1.1 & -2.5 & -2.0 & -3.0 & 1.0 & 1.6 & -1.4 \\
\hline IPF9898 & orf19.3406 & -- & 1.0 & -1.7 & 1.2 & -1.2 & -1.3 & 1.0 & -1.6 & -1.6 & -1.9 \\
\hline IPF993 & orf19.4595 & -- & -1.5 & -1.6 & -1.5 & -1.9 & 1.3 & 1.6 & 1.5 & 1.1 & -3.1 \\
\hline IPT1.5f & orf19.4769 & Transferase activity & -1.4 & -1.5 & -1.0 & -1.5 & -1.8 & -2.4 & -1.2 & 1.2 & -1.6 \\
\hline KRE2.3f & orf19.4377 & -- & -1.3 & 1.1 & 1.5 & -1.8 & -1.8 & -2.5 & 1.1 & 1.8 & -1.8 \\
\hline KRE2.5f & orf19.4349.6 & -- & -1.4 & -1.2 & 1.9 & -1.6 & -1.6 & -4.6 & -1.0 & 1.7 & 1.5 \\
\hline MAE1 & orf19.3419 & Malate dehydrogenase activity & 1.2 & 1.2 & -1.2 & -1.6 & -1.5 & -1.6 & 1.0 & 1.3 & -1.4 \\
\hline MAL31 & orf19.3981 & Alpha-glucoside:hydrogen symporter activity & -1.2 & 24.1 & 5.6 & -1.9 & -3.3 & -2.5 & -2.8 & -2.0 & -2.3 \\
\hline MEP3 & orf19.1614 & Ammonium transporter activity & -1.4 & -1.5 & -3.1 & -2.6 & -1.1 & -1.6 & -2.1 & -2.5 & -1.6 \\
\hline MET1 & orf19.5811 & Uroporphyrin-III C-methyltransferase activity & -1.2 & -1.1 & -1.6 & -2.9 & -2.9 & -2.2 & -1.2 & 1.1 & -1.0 \\
\hline MET10 & orf19.4076 & Sulfite reductase (NADPH) activity & -1.9 & -2.3 & -1.7 & -1.7 & -1.5 & 1.1 & -1.3 & 1.1 & 1.3 \\
\hline MET14 & orf19.946 & Adenylylsulfate kinase activity & -1.8 & -1.7 & -1.2 & -2.1 & -2.8 & -3.1 & 1.2 & 1.2 & 1.8 \\
\hline MET15 & orf19.5645 & O-acetylhomoserine aminocarboxypropyltransferase activity & -1.2 & 1.2 & -1.5 & -1.8 & -1.7 & -1.8 & -1.1 & 1.0 & -1.3 \\
\hline MET2 & orf19.2618 & Homoserine $\mathrm{O}$-acetyltransferase activity & 1.0 & 1.1 & -1.9 & -1.7 & -1.6 & -1.8 & -1.6 & -1.8 & -2.1 \\
\hline MET3 & orf19.5025 & Sulfate adenylyltransferase (ATP) activity & -1.4 & -1.3 & -1.3 & -7.8 & -7.8 & -8.1 & -1.0 & 1.2 & -1.7 \\
\hline MLS1 & orf19.4833 & Malate synthase activity & -1.5 & 1.1 & -3.2 & -1.5 & 1.2 & 1.1 & -2.2 & -1.7 & -2.4 \\
\hline$M S C 2$ & orf19.3132 & Cation:cation antiporter activity & -1.2 & 1.4 & -1.5 & -1.5 & -1.5 & -2.4 & -1.1 & -1.2 & 1.6 \\
\hline MTD1 & orf19.3810 & Methylenetetrahydrofolate dehydrogenase (NAD+) activity & -1.4 & -1.5 & -2.3 & -1.0 & -1.5 & -2.0 & -3.2 & -2.6 & -3.3 \\
\hline MUP1 & orf19.5280 & L-methionine porter activity & -1.5 & -2.0 & -2.2 & -2.3 & -1.3 & -2.1 & -1.4 & -1.1 & 1.8 \\
\hline MUP3 & orf19.321 & L-methionine porter activity & -2.3 & -1.7 & -1.8 & -2.1 & -1.1 & -1.2 & -1.6 & -1.3 & -1.3 \\
\hline NTA1 & orf19.850 & Protein N-terminal asparagine amidohydrolase activity & -1.0 & -1.4 & -1.3 & -1.5 & -1.6 & -1.6 & 1.4 & -1.1 & -2.9 \\
\hline$O A C 1$ & orf19.7411 & Oxaloacetate carrier activity & -1.0 & -3.4 & -5.1 & -1.7 & -2.7 & -3.5 & -2.0 & -1.5 & -6.3 \\
\hline OPT1 & orf19.2602 & Oligopeptide transporter activity & -1.6 & 1.1 & -2.2 & -4.5 & -2.4 & -4.4 & 1.8 & 2.4 & 3.0 \\
\hline OSM2 & orf19.5005 & Fumarate reductase (NADH) activity & 1.2 & 3.1 & 1.5 & -1.1 & -1.3 & 1.1 & -1.7 & -1.7 & -11.9 \\
\hline PADl & orf19.5731 & Carboxy-lyase activity & 1.2 & 1.0 & -1.1 & -1.7 & -2.0 & -1.8 & -1.2 & 1.0 & -2.2 \\
\hline PAP11 & orf19.3197 & RNA binding & 1.0 & -1.0 & -1.0 & -114.9 & -116.9 & -119.7 & 1.1 & 1.2 & 2.1 \\
\hline
\end{tabular}


Table B-2 (continued).

\begin{tabular}{|c|c|c|c|c|c|c|c|c|c|c|c|}
\hline \multirow[b]{2}{*}{ CandidaDB Name ${ }^{a}$} & \multirow[b]{2}{*}{ orf19 Name $^{b}$} & \multirow[b]{2}{*}{ GO Annotation (Molecular Function) ${ }^{c}$} & \multicolumn{3}{|c|}{ F5 vs. F1 } & \multicolumn{3}{|c|}{ G5 vs. G1 } & \multicolumn{3}{|c|}{6692 vs. 5833} \\
\hline & & & 1 & 2 & 3 & 1 & 2 & 3 & 1 & 2 & 3 \\
\hline PCK1 & orf19.7514 & Phosphoenolpyruvate carboxykinase (ATP) activity & -1.4 & 1.2 & -2.4 & -4.8 & -3.9 & -2.9 & -2.0 & -1.4 & -1.8 \\
\hline PGA13 & orf19.6420 & -- & -2.6 & -1.6 & -2.3 & -1.6 & -1.7 & -3.8 & 2.1 & 2.9 & 4.5 \\
\hline PGA18 & orf19.301 & -- & -1.2 & 1.1 & -1.5 & -1.8 & -1.5 & -2.7 & -1.3 & 1.0 & -1.4 \\
\hline PGA26 & orf19.2475 & -- & -3.3 & -2.2 & -1.8 & 2.8 & 6.5 & 3.2 & 1.5 & 1.1 & 1.5 \\
\hline$P G A 31$ & orf19.5302 & -- & 1.4 & 2.3 & -1.3 & -1.1 & -1.6 & -1.4 & -1.7 & -2.8 & -2.8 \\
\hline PGA35 & orf19.4910 & -- & -2.2 & -1.9 & -2.1 & -1.3 & -1.0 & -1.4 & 1.3 & 1.5 & 1.8 \\
\hline PGA36 & orf19.5760 & -- & 1.0 & 1.3 & -1.6 & -2.3 & -1.9 & -3.0 & -2.0 & -1.6 & -2.2 \\
\hline PGA49 & orf19.4404 & -- & -6.6 & 2.2 & 1.7 & -2.5 & -1.9 & -2.3 & -1.0 & 1.1 & -12.8 \\
\hline PHO87 & orf19.2454 & Inorganic phosphate transporter activity & -1.8 & -1.9 & -1.6 & -2.5 & -1.5 & -1.9 & -1.2 & 1.2 & 2.0 \\
\hline PIK1 & orf19.3199 & 1-phosphotidylinositol 4-kinase activity & 1.1 & -1.2 & -1.4 & -19.1 & -30.5 & -11.5 & -1.0 & -1.0 & 1.4 \\
\hline$P M T 2$ & orf19.6812 & $\begin{array}{l}\text { Dolichyl-phosphate-mannose-protein mannosyltransferase } \\
\text { activity }\end{array}$ & -1.5 & -1.9 & -1.6 & -1.9 & 1.0 & -1.1 & -1.1 & 1.1 & 1.4 \\
\hline POL21.3f & orf19.2669 & Di-, tri-valent inorganic cation transporter activity & -1.8 & -1.7 & -1.5 & -2.4 & 1.0 & -1.1 & -3.1 & -1.5 & 1.6 \\
\hline POL21.5f & orf19.2668 & -- & -1.7 & -1.8 & -1.7 & -3.6 & -1.5 & 2.0 & 1.1 & -1.9 & -2.0 \\
\hline POT12 & orf19.1704 & Acetyl-CoA C-acyltransferase activity & -1.2 & 1.9 & 2.0 & 1.5 & -1.3 & -2.3 & -1.8 & -1.6 & -2.1 \\
\hline POX18.3f & orf19.3330.3 & -- & -1.1 & -2.7 & -1.9 & -1.6 & 1.3 & 1.0 & -1.6 & -2.0 & -6.6 \\
\hline$P R C 3$ & orf19.2474 & -- & -1.9 & -1.5 & -1.5 & -1.9 & 1.1 & 1.5 & 1.4 & 1.7 & -1.4 \\
\hline PRP9 & orf19.3178 & -- & -1.6 & 1.1 & 1.3 & -3.2 & -2.2 & -1.7 & -1.1 & -1.0 & -2.4 \\
\hline PST2 & orf19.5285 & -- & -1.9 & -1.2 & -2.2 & -1.7 & -2.0 & -1.8 & -1.3 & -1.7 & -1.3 \\
\hline PUT2 & orf19.3974 & 1-Pyrroline-5-carboxylate dehydrogenase activity & -1.4 & 1.5 & 1.1 & -1.5 & -1.7 & -1.8 & 1.1 & -1.0 & -1.8 \\
\hline$R B T 2$ & orf19.1415 & Ferric-chelate reductase activity & -2.0 & -2.2 & -2.8 & -2.8 & -3.1 & -2.6 & -4.3 & -3.2 & -3.2 \\
\hline RDH54 & orf19.5367 & DNA topoisomerase activity & -1.5 & -1.5 & -1.7 & -2.0 & -1.3 & 1.3 & 1.6 & 1.0 & 1.1 \\
\hline$R N R 2$ & orf19.5845 & Ribonucleoside-diphosphate reductase activity & -2.5 & -1.4 & -2.0 & 1.3 & -1.2 & -2.0 & -2.3 & -1.5 & -3.8 \\
\hline$R N R 22$ & orf19.1868 & Ribonucleoside-diphosphate reductase activity & -3.0 & -2.2 & -3.3 & -2.2 & 1.4 & -1.3 & 2.8 & 1.1 & -1.2 \\
\hline ROM2 & orf19.906 & Rho-guanyl-nucleotide exchange factor activity & 1.1 & 1.4 & -1.3 & -1.5 & -1.6 & -2.1 & -1.0 & -1.1 & -2.2 \\
\hline RTA2 & orf19.24 & Phospholipid-translocating ATPase activity & -2.0 & -1.9 & -1.7 & -1.2 & -1.1 & -1.1 & -2.6 & -1.6 & -1.3 \\
\hline RTA3 & orf19.23 & Phospholipid-translocating ATPase activity & -3.0 & -2.1 & -1.5 & 1.0 & 1.4 & 2.3 & -2.1 & -1.5 & -1.5 \\
\hline SAP7 & orf19.756 & Aspartic-type endopeptidase activity & -1.0 & -1.3 & -1.1 & -3.0 & -2.2 & -1.8 & -2.1 & -1.6 & -2.4 \\
\hline $\mathrm{SEO} 2$ & orf19.700 & Transporter activity & -1.9 & -2.0 & -1.6 & -2.3 & -2.8 & -2.7 & -2.1 & -2.5 & -2.8 \\
\hline SER2 & orf19.5838 & Phosphoserine phosphatase activity & -2.0 & -2.3 & -1.7 & 1.1 & 1.0 & -1.0 & -2.1 & -1.6 & -3.4 \\
\hline SFT2 & orf19.2342 & -- & -1.8 & -1.6 & -1.7 & -3.1 & -1.3 & 1.1 & -1.0 & -1.3 & 1.2 \\
\hline SHM2 & orf19.5750 & Glycine hydroxymethyltransferase activity & -2.1 & -2.0 & -1.8 & -1.8 & -1.2 & -1.1 & -2.2 & -1.5 & 1.1 \\
\hline SKN1.3 & orf19.7362 & -- & 1.4 & -1.1 & 1.5 & -2.0 & -2.2 & -2.6 & -1.2 & -1.1 & -1.9 \\
\hline$S M F 3$ & orf19.5022 & Manganese ion transporter activity & -1.3 & -1.4 & -1.6 & -1.5 & -1.5 & -2.1 & -1.1 & -1.2 & 1.2 \\
\hline SMP3 & orf19.5792 & Alpha-1,2-mannosyltransferase activity & -1.3 & -1.3 & 1.1 & -1.7 & -1.5 & -2.1 & -1.0 & 1.2 & 1.3 \\
\hline SNA2 & orf19.4132 & -- & -1.9 & -1.3 & -1.0 & -2.5 & -2.1 & -1.7 & -1.2 & 1.0 & -1.2 \\
\hline SOU1 & orf19.2896 & 2,4-dienoyl-CoA reductase (NADPH) activity & -3.2 & -2.3 & -2.4 & -9.2 & -6.6 & -8.5 & -2.1 & -1.2 & -1.2 \\
\hline SSU1 & orf19.7313 & Sulfite transporter activity & -1.1 & 1.1 & 1.1 & -1.5 & -1.5 & -1.4 & -1.7 & -1.7 & -1.5 \\
\hline SSY1 & orf19.814 & Amino acid binding & -2.0 & -1.5 & -2.1 & -2.1 & 1.2 & 1.6 & -1.1 & -1.3 & -1.8 \\
\hline STL1 & orf19.5753 & Glucose transporter activity & -1.7 & -1.6 & -1.7 & -4.5 & -8.7 & -10.8 & -1.7 & -1.1 & 1.4 \\
\hline SUL1 & orf19.2738 & Sulfate transporter activity & -2.5 & -1.8 & -2.8 & -17.9 & -6.0 & -8.1 & -1.4 & -1.0 & -1.5 \\
\hline
\end{tabular}


Table B-2 (continued).

\begin{tabular}{|c|c|c|c|c|c|c|c|c|c|c|c|}
\hline \multirow[b]{2}{*}{ CandidaDB Name ${ }^{a}$} & \multirow[b]{2}{*}{ orf19 Name ${ }^{b}$} & \multirow[b]{2}{*}{ GO Annotation (Molecular Function) ${ }^{c}$} & \multicolumn{3}{|c|}{ F5 vs. F1 } & \multicolumn{3}{|c|}{ G5 vs. G1 } & \multicolumn{3}{|c|}{6692 vs. 5833} \\
\hline & & & $\mathbf{1}$ & 2 & 3 & 1 & 2 & 3 & 1 & 2 & 3 \\
\hline SUN42 & orf19.5032 & (2) & -1.5 & -1.5 & -1.6 & -1.9 & -1.3 & -1.9 & -1.2 & 1.1 & 1.1 \\
\hline TAF 25 & orf19.3242 & General RNA polymerase II transcription factor activity & -1.1 & -1.1 & -1.3 & -1.5 & -1.6 & -1.6 & -1.1 & -1.2 & -1.9 \\
\hline$T c a 5 b$ & orf19.2428.2 & -- & -2.3 & 4.1 & 1.0 & -1.6 & 1.8 & -1.7 & -2.2 & -1.7 & -1.7 \\
\hline TEC1 & orf19.5908 & Specific RNA polymerase II transcription factor activity & -1.1 & -1.0 & -1.6 & -2.6 & -3.5 & -2.9 & -1.5 & -1.3 & -2.1 \\
\hline TES12 & orf19.4122 & -- & 1.6 & 1.1 & 1.5 & -2.2 & -1.8 & -1.9 & 1.0 & -1.1 & -1.3 \\
\hline$T P K 2$ & orf19.2277 & cAMP-dependent protein kinase activity & -2.0 & -1.5 & -2.3 & -1.5 & -1.4 & 1.0 & -1.2 & -1.1 & -1.5 \\
\hline$U G A 12.3 f$ & orf19.802 & 4-aminobutyrate transaminase activity & -1.1 & -1.8 & -1.1 & -1.6 & -1.6 & -1.7 & -1.2 & 1.3 & -1.9 \\
\hline UGA12.5f & orf19.802 & 4-aminobutyrate transaminase activity & -1.0 & -1.1 & -1.1 & -1.6 & -1.9 & -2.8 & -1.0 & -1.0 & -1.4 \\
\hline$V C X 1$ & orf19.405 & Calcium ion transporter activity & -2.1 & -1.7 & -1.7 & -2.1 & -1.3 & -1.2 & -1.1 & 1.0 & -1.8 \\
\hline VPS184 & orf19.5543 & Protein binding & 5.1 & 1.5 & -2.8 & -1.8 & 1.1 & -1.3 & -1.6 & -1.6 & -2.3 \\
\hline YHM1 & orf19.685 & Transporter activity & -1.4 & -1.2 & -1.2 & -1.8 & -1.7 & -2.0 & -1.1 & 1.1 & 1.4 \\
\hline YORIO0 & orf19.2599 & Carnitine:acyl carnitine antiporter activity & -1.4 & -1.2 & -1.0 & -2.4 & -1.8 & -2.0 & -1.3 & -1.7 & -1.4 \\
\hline ZRT2 & orf19.1585 & Low affinity zinc ion transporter activity & -2.0 & -2.0 & -2.3 & -3.3 & -1.2 & -1.3 & 1.1 & 1.2 & 1.4 \\
\hline
\end{tabular}

${ }^{a}$ Gene name at CandidaDB (http://genolist.pasteur.fr/CandidaDB/).

${ }^{b}$ orf19 nomenclature according to the Assembly 19 version.

${ }^{c} \mathrm{GO}$ annotation found at CGD (http://www.candidagenome.org/). 
Table B-3. Genes down-regulated in the absence of MRR1.

\begin{tabular}{|c|c|c|c|c|c|c|c|c|c|c|}
\hline \multirow[b]{3}{*}{ CandidaDB Name $^{a}$} & \multirow[b]{3}{*}{ orf19 Name ${ }^{b}$} & \multirow[b]{3}{*}{ GO Annotation (Molecular Function) ${ }^{c}$} & \multicolumn{4}{|c|}{ F5 $\Delta m r r 1$ vs. F5 } & \multicolumn{4}{|c|}{ G5 $\Delta m r r 1$ vs. G5 } \\
\hline & & & \multicolumn{2}{|c|}{ Strain A } & \multicolumn{2}{|c|}{ Strain B } & \multicolumn{2}{|c|}{ Strain A } & \multicolumn{2}{|c|}{ Strain B } \\
\hline & & & 1 & 2 & 1 & 2 & 1 & 2 & 1 & 2 \\
\hline$A A F 1$ & orf19.7436 & 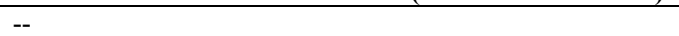 & -3.5 & -3.7 & -2.2 & -2.9 & -5.6 & -1.9 & -4.3 & -1.8 \\
\hline ACOI & orf19.6385 & Aconitate hydratase activity & 1.1 & -1.8 & 1.0 & -1.9 & -2.7 & -1.5 & -2.0 & -1.7 \\
\hline AKL1 & orf19.5357 & Protein serine/threonine kinase activity & -1.6 & -1.8 & -1.6 & -1.7 & -1.6 & -1.9 & -1.6 & -1.5 \\
\hline ALS7 & orf19.7400 & Carbohydrate binding & -1.5 & -2.1 & -1.9 & -1.8 & 1.2 & -1.1 & 1.3 & -1.1 \\
\hline$A M Y G 1$ & orf19.4899 & Alpha-glucosidase activity & -3.8 & -1.9 & -4.7 & -1.7 & 1.1 & -1.7 & 1.1 & 1.7 \\
\hline ARD 8 & orf19.6322 & 2,4-dienoyl-CoA reductase (NADPH) activity & -1.6 & -3.3 & -1.7 & -2.4 & -3.1 & -2.3 & -2.2 & -1.7 \\
\hline$B F R 2$ & orf19.7624 & -- & 1.6 & -4.8 & 2.1 & -2.5 & -2.4 & -1.7 & -2.0 & -2.2 \\
\hline$B M R 1$ & orf19.5604 & Multidrug transporter activity & -21.4 & -47.5 & -35.0 & -83.7 & -168.9 & -149.4 & -143.0 & -43.9 \\
\hline$C D C 1$ & orf19.3083 & -- & 1.1 & -2.1 & -2.1 & -1.8 & -2.4 & -1.6 & -2.6 & -2.4 \\
\hline CDR11.5f & orf19.918 & ATP binding & -4.9 & 1.2 & -2.0 & -1.0 & -1.9 & -1.8 & -2.6 & -1.5 \\
\hline$C D R 3$ & orf19.1313 & ATP binding & -3.3 & -1.7 & -2.0 & -1.8 & -1.0 & 1.3 & -1.3 & 3.6 \\
\hline $\mathrm{CHO}_{2}$ & orf19.169 & Methylene-fatty-acyl-phospholipid synthase activity & -1.2 & -1.5 & -1.2 & -1.8 & -3.7 & -1.5 & -2.0 & -3.2 \\
\hline CHS3.5f & orf19.4937 & Chitin synthase activity & -25.6 & -8.2 & -2.3 & -2.1 & -4.7 & 5.6 & -2.3 & 13.8 \\
\hline CHS4 & orf19.7349 & Enzyme activator activity & -2.0 & -2.9 & -1.8 & -1.5 & -1.7 & 1.0 & -2.3 & -1.5 \\
\hline$C L N 21$ & orf19.6028 & Cyclin-dependent protein kinase regulator activity & -14.4 & -8.8 & -1.6 & -4.6 & -7.8 & 5.2 & -1.2 & 3.6 \\
\hline CNH1 & orf19.367 & Cation:cation antiporter activity & -1.9 & -2.0 & -2.8 & -1.8 & -2.1 & -1.2 & -1.6 & 1.5 \\
\hline $\mathrm{CNHI}$ & orf19.367 & Cation:cation antiporter activity & -3.3 & -2.5 & -10.7 & -3.1 & -2.6 & -2.0 & -1.9 & 1.6 \\
\hline $\mathrm{CPHI}$ & orf19.4433 & Transcription factor activity & -2.0 & -3.0 & -1.8 & -2.0 & -3.4 & -2.0 & -2.6 & 1.6 \\
\hline CTR1 & orf19.3646 & Copper ion binding & -1.3 & -2.2 & -1.4 & -1.7 & -2.8 & -1.6 & -1.7 & -1.7 \\
\hline DHHI & orf19.6197 & Protein binding & -2.9 & -1.8 & -1.5 & -1.6 & -1.4 & 1.0 & -1.7 & 1.4 \\
\hline$D L D 3$ & orf19.5805 & D-lactate dehydrogenase (cytochrome) activity & -1.9 & -4.1 & -2.8 & -2.5 & 1.3 & -1.1 & -1.1 & 1.1 \\
\hline$D N A 2$ & orf19.1192 & ATP-dependent DNA helicase activity & -2.6 & -2.2 & -1.6 & -1.5 & -2.0 & -1.1 & -1.4 & -1.2 \\
\hline DRS1 & orf19.7635 & ATP-dependent RNA helicase activity & 1.0 & -2.2 & 1.1 & -2.6 & -2.4 & -1.8 & -3.2 & -2.4 \\
\hline DRS23 & orf19.323 & Phospholipid-translocating ATPase activity & -1.6 & -2.8 & -1.6 & -2.1 & -2.2 & -1.1 & -1.2 & 1.1 \\
\hline EBP4 & orf19.3433 & NADPH dehydrogenase activity & -2.6 & -1.6 & -1.6 & -1.9 & -1.3 & -2.1 & -1.2 & -1.0 \\
\hline EFG1 & orf19.610 & Transcription factor activity & -2.2 & -2.9 & -1.9 & -1.5 & -3.8 & -2.2 & -2.3 & 1.3 \\
\hline FAS2.53f & orf19.5949 & 3-oxoacyl-[acyl-carrier-protein] reducatase activity & -17.0 & -1.2 & -1.4 & 1.2 & -2.9 & -2.0 & -2.7 & -1.8 \\
\hline FRE30.3f & orf19.6139 & Ferric-chelate reductase activity & -1.8 & -5.5 & -1.8 & -3.8 & -4.3 & -1.9 & -3.0 & -2.5 \\
\hline FRE $30.53 f$ & orf19.6140 & Ferric-chelate reductase activity & -5.0 & -4.3 & -2.1 & -2.5 & -4.3 & -1.7 & -3.4 & -1.7 \\
\hline FRE7 & orf19.7077 & Ferric-chelate reductase activity & -1.5 & -2.2 & -1.8 & -3.3 & -3.0 & -1.6 & -2.4 & -1.7 \\
\hline$G A C 1$ & orf19.7053 & Protein phosphatase type 1 activity & -1.5 & -3.8 & -1.5 & -3.3 & -3.9 & -1.5 & -4.8 & -1.2 \\
\hline GAL1 & orf19.3670 & Galactokinase activity & -1.5 & -1.5 & -20.4 & -4.4 & -1.0 & 1.4 & -1.3 & -1.3 \\
\hline GAP6 & orf19.6659 & Amino acid permease activity & -1.8 & -2.4 & -1.6 & -2.4 & -2.0 & -1.9 & -1.5 & -1.5 \\
\hline$G D H 2$ & orf19.2192 & Glutamate dehydrogenase activity & -1.5 & -2.6 & -1.8 & -2.9 & -1.1 & 1.0 & 1.0 & -1.2 \\
\hline$G P X 1$ & orf19.86 & Glutathione peroxidase activity & -2.8 & -1.1 & -1.6 & -1.4 & -1.8 & -2.0 & -1.6 & -1.7 \\
\hline GRP2 & orf19.4309 & Oxidoreductase activity & -2.1 & -1.9 & -1.8 & -1.8 & -3.7 & -3.7 & -3.5 & -2.9 \\
\hline GTS1 & orf19.6393 & Specific RNA polymerase II transcription factor activity & -2.4 & -1.7 & -1.9 & -1.7 & -1.5 & 1.1 & -1.4 & 1.2 \\
\hline GUT1 & orf19.558 & Glycerol kinase activity & -2.0 & -3.5 & -1.6 & -3.8 & -4.9 & -1.9 & -3.0 & 1.6 \\
\hline HEM12 & orf19.5369 & Uroporphyrinogen decarboxylase activity & -2.0 & -1.7 & -1.7 & -1.6 & -1.6 & -1.2 & -1.2 & 1.4 \\
\hline HGT11 & orf19.4527 & Glucose transporter activity & -4.1 & -9.5 & -3.9 & -2.7 & -8.5 & -3.1 & -5.7 & -6.3 \\
\hline
\end{tabular}


Table B-3 (continued).

\begin{tabular}{|c|c|c|c|c|c|c|c|c|c|c|}
\hline \multirow[b]{3}{*}{ CandidaDB Name $^{a}$} & \multirow[b]{3}{*}{ orf19 $\mathrm{Name}^{b}$} & \multirow[b]{3}{*}{ GO Annotation (Molecular Function) ${ }^{c}$} & \multicolumn{4}{|c|}{ F5 $\Delta m r r 1$ vs. F5 } & \multicolumn{4}{|c|}{ G5Amrr1 vs. G5 } \\
\hline & & & \multicolumn{2}{|c|}{ Strain A } & \multicolumn{2}{|c|}{ Strain B } & \multicolumn{2}{|c|}{ Strain A } & \multicolumn{2}{|c|}{ Strain B } \\
\hline & & & 1 & 2 & 1 & 2 & 1 & 2 & 1 & 2 \\
\hline HGT12 & orf19.3668 & Glucose transporter activity & -4.2 & -2.3 & -4.4 & -2.2 & -12.4 & -5.6 & -6.5 & -4.2 \\
\hline HIK1 & orf19.5181 & Osmosensor activity & -8.1 & -2.2 & -2.1 & -1.8 & -3.7 & -1.5 & -2.5 & 1.9 \\
\hline HSP78.5f & orf19.882 & ATP-dependent peptidase activity & -1.4 & 1.3 & -1.4 & 1.4 & -1.5 & -1.5 & -1.7 & -1.6 \\
\hline IFA17.5f & orf19.4511 & -- & -3.8 & -2.7 & -2.0 & -1.7 & -2.2 & 1.9 & -1.8 & 1.3 \\
\hline IFA18.53f & orf19.4508 & -- & -3.9 & -2.2 & -1.8 & -1.7 & 2.0 & 1.2 & 2.9 & -2.3 \\
\hline IFD2 & orf19.771 & Aryl-alcohol dehydrogenase activity & -4.2 & -2.3 & -3.8 & -2.0 & -2.0 & -4.1 & -1.6 & -3.4 \\
\hline IFD4 & orf19.4477 & Aryl-alcohol dehydrogenase activity & -2.7 & -4.6 & -2.4 & -1.9 & -4.0 & -4.4 & -3.4 & -2.9 \\
\hline IFD5 & orf19.1048 & Aryl-alcohol dehydrogenase activity & -3.3 & -2.1 & -2.6 & -2.6 & -6.1 & -5.2 & -4.5 & -2.9 \\
\hline IFD5 & orf19.1048 & Aryl-alcohol dehydrogenase activity & -15.0 & -12.2 & -15.8 & -9.6 & -4.9 & -4.2 & -4.1 & -2.7 \\
\hline IFD6 & orf19.4476 & Aryl-alcohol dehydrogenase activity & -31.2 & -41.8 & -27.0 & -21.3 & -12.8 & -22.1 & -12.6 & -78.1 \\
\hline IFD7 & orf19.629 & Aryl-alcohol dehydrogenase activity & -10.5 & -4.3 & -5.8 & -6.0 & -8.0 & -8.2 & -9.6 & -6.5 \\
\hline IFK3.3f & orf19.857 & -- & -1.8 & -8.8 & -2.1 & -5.6 & -2.1 & -1.9 & -2.0 & -5.9 \\
\hline$I M P 2$ & orf19.1981 & Peptidase activity & 1.0 & 1.3 & -1.4 & -1.0 & -2.4 & -1.6 & -1.8 & -2.1 \\
\hline IPF10062 & orf19.1086 & -- & -1.9 & -1.9 & -1.5 & -1.6 & -2.0 & -1.3 & -1.5 & 1.4 \\
\hline IPF11006 & orf19.4658 & -- & -1.5 & 2.1 & 1.2 & 1.5 & -2.1 & -1.6 & -3.0 & -2.3 \\
\hline IPF11090.5f & orf19.4622 & -- & -8.4 & -1.3 & -1.2 & 1.1 & -2.4 & -1.5 & -2.1 & -1.6 \\
\hline IPF11569 & orf19.9 & -- & -1.3 & -6.0 & 1.2 & -1.1 & -1.9 & -1.9 & -2.2 & -2.6 \\
\hline IPF11610 & orf19.2146 & $\mathrm{H} 3 / \mathrm{H} 4$ histone acetyltransferase activity & -1.6 & -1.7 & -1.8 & -2.1 & -1.6 & -1.3 & -1.9 & -1.2 \\
\hline IPF11694 & orf19.4355 & Nicotinamide mononucleotide permase activity & -2.2 & -1.6 & -2.6 & -3.0 & -2.4 & -1.9 & -3.5 & 2.6 \\
\hline IPF12105 & orf19.5633 & -- & -1.8 & -2.3 & -1.6 & -1.8 & -2.3 & -2.1 & -2.6 & 1.4 \\
\hline IPF12316 & orf19.5069 & -- & -2.5 & 1.8 & -1.7 & -1.1 & -2.4 & -2.1 & -3.0 & -2.2 \\
\hline IPF12513 & orf19.5605 & -- & -2.7 & -3.2 & -1.7 & -2.7 & -1.5 & -1.2 & -1.2 & 1.1 \\
\hline IPF12606.5eoc & orf19.3288 & -- & -1.5 & -3.7 & -1.7 & -4.4 & -3.6 & -1.4 & -3.4 & -1.2 \\
\hline IPF1264 & orf19.7371 & Transcription factor activity & -1.8 & -3.4 & -1.5 & -2.0 & -6.1 & -1.4 & -8.2 & 1.1 \\
\hline IPF1292 & orf19.7381 & Transcription factor activity & -1.8 & -2.2 & -1.9 & -2.0 & -3.5 & -1.2 & -5.3 & -1.2 \\
\hline IPF13042 & orf19.2410 & -- & -1.4 & 1.1 & 1.7 & 1.6 & -2.1 & -1.6 & -3.5 & -2.0 \\
\hline IPF13116 & orf19.3463 & -- & 1.2 & -3.3 & 1.3 & -2.4 & -3.5 & -1.6 & -2.2 & -2.7 \\
\hline IPF13202 & orf19.215 & -- & -1.7 & -1.6 & -1.5 & -1.9 & -2.3 & -1.1 & -2.9 & 1.6 \\
\hline IPF13361 & orf19.2330 & snoRNA binding & 1.5 & -1.3 & 1.7 & -1.2 & -5.3 & -1.8 & -4.1 & -1.7 \\
\hline IPF13383.53eoc & orf19.1840 & -- & -3.9 & -1.8 & -1.6 & -1.5 & -1.4 & -1.1 & -1.5 & 1.5 \\
\hline IPF13565 & orf19.1609 & -- & -1.7 & -1.9 & -2.6 & -1.8 & 3.4 & 2.8 & 3.3 & 3.2 \\
\hline IPF13756.5f & orf19.4479 & -- & -3.5 & -1.3 & 1.6 & -1.7 & -6.6 & -1.5 & -6.5 & -2.4 \\
\hline IPF1382 & orf19.6444 & -- & -1.0 & -1.3 & -1.2 & -2.0 & -2.3 & -2.0 & -1.6 & -1.8 \\
\hline IPF14888 & orf19.4339 & -- & -1.1 & -1.2 & 1.2 & -1.2 & -5.2 & -2.3 & -4.1 & -1.6 \\
\hline IPF14895 & orf19.93 & -- & 1.2 & -1.6 & -1.2 & -1.8 & -2.6 & -1.6 & -2.5 & -29.4 \\
\hline IPF15013 & orf19.5626 & -- & -1.7 & -2.0 & -1.5 & -2.3 & -1.9 & -1.4 & -2.4 & -2.8 \\
\hline IPF15153 & orf19.5867 & -- & -2.0 & -1.8 & -1.6 & -1.5 & -1.1 & 1.5 & -1.2 & 1.3 \\
\hline IPF15183 & orf19.1806 & -- & -2.3 & -2.0 & -2.0 & -1.5 & -1.2 & -1.2 & -1.2 & 1.6 \\
\hline IPF15273 & orf19.1822 & Transcription factor activity & -3.3 & -12.7 & -1.6 & -3.3 & -1.4 & -1.2 & -1.1 & -4.1 \\
\hline IPF1588 & orf19.972 & -- & -1.5 & -1.8 & -2.5 & -2.1 & -1.7 & -2.9 & -1.1 & -1.7 \\
\hline
\end{tabular}


Table B-3 (continued).

\begin{tabular}{|c|c|c|c|c|c|c|c|c|c|c|}
\hline \multirow[b]{3}{*}{ CandidaDB Name $^{a}$} & \multirow[b]{3}{*}{ orf19 Name ${ }^{b}$} & \multirow[b]{3}{*}{ GO Annotation (Molecular Function) ${ }^{c}$} & \multicolumn{4}{|c|}{ F5 $\Delta m r r 1$ vs. F5 } & \multicolumn{4}{|c|}{ G5 $\Delta m r r 1$ vs. G5 } \\
\hline & & & \multicolumn{2}{|c|}{ Strain A } & \multicolumn{2}{|c|}{ Strain B } & \multicolumn{2}{|c|}{ Strain A } & \multicolumn{2}{|c|}{ Strain B } \\
\hline & & & 1 & 2 & 1 & 2 & 1 & 2 & 1 & 2 \\
\hline IPF16 & orf19.5984 & ( & 1.0 & -1.0 & -1.6 & -1.3 & -2.4 & -1.9 & -1.8 & -1.6 \\
\hline IPF16189.5f & orf19.5576 & -- & -1.1 & 1.1 & 1.2 & 1.1 & -3.2 & -1.6 & -1.6 & -1.7 \\
\hline IPF1634 & orf19.6578 & Inorganic phosphate transporter activity & -1.5 & -2.4 & -2.0 & -3.6 & -5.0 & -1.5 & -4.2 & -2.6 \\
\hline IPF16981 & orf19.2458 & Transcription factor activity & -1.9 & -1.7 & -1.9 & -1.6 & 1.7 & -1.0 & -1.1 & -1.4 \\
\hline IPF17186 & orf19.251 & -- & -10.9 & -11.0 & -8.7 & -6.2 & -5.5 & -9.5 & -5.6 & -7.4 \\
\hline IPF17914.3eoc & orf19.5124 & -- & -2.1 & -1.5 & -2.2 & -1.9 & -1.6 & -2.0 & 1.1 & 1.3 \\
\hline IPF17998 & orf19.3147 & -- & -1.6 & -2.6 & -2.5 & -2.3 & 2.4 & 1.0 & 3.1 & 1.0 \\
\hline IPF18316 & orf19.5145 & -- & -1.9 & -1.8 & -2.1 & -1.7 & -1.7 & -2.1 & 1.2 & -1.0 \\
\hline IPF1837 & orf19.5877 & Alcohol O-acetyltransferase activity & -1.5 & -1.6 & -2.1 & -1.9 & -1.6 & -2.6 & -1.3 & 1.0 \\
\hline IPF18586 & orf19.761 & -- & -1.6 & -2.1 & -1.7 & -2.5 & -7.9 & -2.6 & -3.2 & 1.3 \\
\hline IPF18690.5eoc & orf19.6621 & Structural constituent of cytoskeleton & -136.5 & -2.1 & -1.5 & -1.5 & -1.2 & 1.0 & 1.2 & -1.0 \\
\hline IPF1922 & orf19.7148 & -- & -1.7 & -1.8 & -2.3 & -2.8 & -3.2 & -1.7 & -1.8 & 2.0 \\
\hline IPF19538.3f & orf19.6957.3 & _- & -1.5 & -2.0 & -2.1 & -2.1 & -7.6 & -6.8 & -4.1 & -3.1 \\
\hline IPF1956 & orf19.7159 & -- & -1.6 & 1.8 & -2.2 & -1.5 & -2.9 & -1.5 & -1.5 & -1.7 \\
\hline IPF19664 & orf19.2510 & Chaperone binding & -7.4 & -9.0 & -1.5 & -1.7 & 1.1 & 1.1 & -1.2 & -1.0 \\
\hline IPF19700 & orf19.1600 & -- & -1.5 & -1.6 & -1.6 & -1.5 & -2.0 & 1.1 & -1.8 & 1.0 \\
\hline IPF19810 & orf19.1430 & -- & -2.4 & -1.9 & -2.1 & -2.0 & 1.3 & -1.1 & -1.1 & 12.4 \\
\hline IPF2050 & orf19.5265 & Structural constituent of cytoskeleton & -2.3 & -2.0 & -1.7 & -1.5 & -1.1 & -1.1 & 1.3 & 1.1 \\
\hline IPF2111 & orf19.4106 & 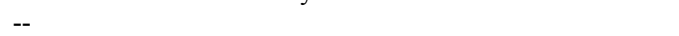 & -2.3 & 1.4 & -1.2 & 1.1 & -1.6 & -2.2 & -1.9 & -3.0 \\
\hline IPF2130 & orf19.7204 & -- & -2.8 & 1.2 & -2.4 & -1.5 & -2.1 & -4.4 & -1.5 & -3.6 \\
\hline IPF 2142 & orf19.7197 & -- & 2.4 & 1.4 & 7.7 & -12.7 & -11.8 & -1.6 & -36.7 & -1.7 \\
\hline IPF2186 & orf19.7166 & -- & -2.9 & -3.5 & -3.7 & -3.4 & -7.0 & -5.1 & -4.8 & -2.7 \\
\hline IPF3080 & orf19.7042 & -- & -1.5 & -1.2 & -1.2 & -2.3 & -10.3 & -6.3 & -9.3 & -2.6 \\
\hline IPF3092 & orf19.4445 & -- & -1.7 & -3.1 & -1.7 & -12.5 & -3.8 & -1.4 & -2.7 & -3.2 \\
\hline IPF3239 & orf19.2875 & Signal recognition particle binding & 1.9 & 2.4 & 2.0 & 2.0 & -1.5 & -2.1 & -1.6 & -2.0 \\
\hline IPF3277 & orf19.4384 & Fructose transporter activity & -3.5 & -4.2 & -2.5 & -2.9 & -2.6 & -1.4 & -3.5 & 1.8 \\
\hline IPF3329 & orf19.7388 & MAP kinase kinase activity & -1.6 & -3.4 & -1.5 & -2.3 & -2.2 & 1.2 & -2.3 & 1.4 \\
\hline IPF3426 & orf19.6173 & -- & -1.3 & -3.7 & -2.0 & -3.0 & -2.7 & -1.6 & -2.5 & -1.6 \\
\hline IPF3448 & orf19.6185 & -- & -4.4 & -18.6 & -2.8 & -2.1 & -2.5 & -2.9 & -2.3 & -1.2 \\
\hline IPF3642 & orf19.6726 & DNA binding & -1.7 & -1.5 & -2.0 & -2.5 & -1.7 & 1.2 & -1.4 & -1.8 \\
\hline IPF3687 & orf19.886 & Cytoskeletal adaptor activity & -1.6 & -1.9 & -2.3 & -1.7 & -2.8 & -1.6 & -2.2 & 1.4 \\
\hline IPF3912 & orf19.6514 & Specific RNA polymerase II transcription factor activity & -1.2 & -1.4 & -1.2 & -1.3 & -2.2 & -1.8 & -1.9 & -2.5 \\
\hline IPF3967 & orf19.673 & NADPH dehydrogenase activity & -1.6 & -1.2 & -3.2 & -1.5 & -1.5 & -1.5 & -1.7 & -3.0 \\
\hline IPF4160 & orf19.5459 & 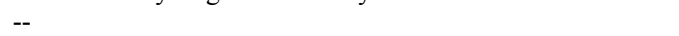 & -1.4 & -3.5 & -2.1 & -1.3 & -2.6 & -1.6 & -1.7 & -1.5 \\
\hline IPF4253 & orf19.1630 & -- & -1.9 & -1.7 & -3.5 & -2.5 & -3.2 & -1.5 & -3.1 & -1.3 \\
\hline IPF4290 & orf19.541 & -- & 1.4 & -2.1 & -1.0 & -2.0 & -1.8 & -1.6 & -2.3 & -2.0 \\
\hline IPF4799 & orf19.3342 & -- & -1.6 & -1.5 & -2.0 & -1.9 & -1.2 & -1.1 & -1.0 & -1.0 \\
\hline IPF486 & orf19.7065 & -- & -1.1 & 1.5 & 1.2 & 1.6 & -2.2 & -1.8 & -1.9 & -2.0 \\
\hline IPF5064 & orf19.804 & Transporter activity & -1.5 & -1.8 & -1.7 & -1.5 & -1.9 & -1.0 & -2.3 & 1.5 \\
\hline IPF5149 & orf19.6530 & Signal recognition particle binding & -1.4 & 1.1 & -1.4 & -1.3 & -4.1 & -2.2 & -2.1 & -2.3 \\
\hline
\end{tabular}


Table B-3 (continued).

\begin{tabular}{|c|c|c|c|c|c|c|c|c|c|c|}
\hline \multirow[b]{3}{*}{ CandidaDB Name ${ }^{a}$} & \multirow[b]{3}{*}{ orf19 Name $^{b}$} & \multirow[b]{3}{*}{ GO Annotation (Molecular Function) ${ }^{c}$} & \multicolumn{4}{|c|}{ F5 $\Delta m r r 1$ vs. F5 } & \multicolumn{4}{|c|}{ 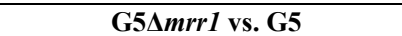 } \\
\hline & & & \multicolumn{2}{|c|}{ Strain A } & \multicolumn{2}{|c|}{ Strain B } & \multicolumn{2}{|c|}{ Strain A } & \multicolumn{2}{|c|}{ Strain B } \\
\hline & & & 1 & 2 & 1 & 2 & 1 & 2 & 1 & 2 \\
\hline IPF5217 & orf19.7270 & ( & -1.2 & -3.0 & -2.1 & -1.9 & -3.2 & -2.3 & -1.6 & -1.7 \\
\hline IPF5228.5 & orf19.7265 & -- & -1.9 & -2.7 & -2.4 & -2.3 & -1.2 & -1.7 & 1.1 & -1.1 \\
\hline IPF525 & orf19.7085 & -- & -2.9 & -17.2 & -2.4 & -2.4 & -1.7 & -2.6 & -1.7 & -3.4 \\
\hline IPF5291 & orf19.3674 & UDP-glucose 4-epimerase activity & -1.3 & -2.1 & -1.5 & 1.1 & -2.7 & -1.9 & -2.6 & -3.9 \\
\hline IPF564 & orf19.7104 & -- & -1.5 & -1.5 & -1.7 & -1.8 & -1.7 & -1.6 & -1.6 & -1.1 \\
\hline IPF5987 & orf19.7306 & -- & -7.4 & -6.4 & -9.0 & -6.9 & -8.6 & -12.5 & -7.4 & -10.5 \\
\hline IPF6011 & orf19.1486 & -- & -2.4 & -10.4 & -1.8 & -2.1 & -5.7 & -1.9 & -3.8 & 1.2 \\
\hline IPF6067 & orf19.6734 & General transcriptional repressor activity & -2.0 & -1.6 & -1.9 & -1.8 & -3.7 & -1.6 & -4.4 & 1.4 \\
\hline IPF6298 & orf19.1964 & -- & 3.0 & -1.1 & 1.5 & 1.3 & -3.7 & -1.8 & -2.1 & -1.8 \\
\hline IPF6700 & orf19.2459 & -- & -1.5 & -2.5 & -2.2 & -1.8 & -1.1 & 1.2 & -1.0 & 1.3 \\
\hline IPF690.5f & orf19.7590 & -- & -4.3 & -1.6 & -1.6 & -2.5 & -1.6 & 1.6 & -2.3 & -1.2 \\
\hline IPF708 & orf19.5370 & -- & -1.5 & -1.6 & -2.3 & -1.5 & -2.6 & -1.6 & -1.1 & 1.8 \\
\hline IPF7493 & orf19.4682 & Glucose transporter activity & -1.5 & -2.3 & -6.0 & -2.4 & -7.5 & -3.4 & -9.1 & -2.6 \\
\hline IPF7666 & orf19.4056 & Transcription factor activity & -4.8 & -8.1 & -2.0 & -1.7 & -8.2 & 1.0 & -8.5 & 2.6 \\
\hline IPF7685 & orf19.1396 & ARF GTPase activator activity & -1.8 & -1.8 & -1.9 & -1.7 & -2.0 & -1.9 & -1.7 & 1.1 \\
\hline IPF7686 & orf19.1395 & Inorganic phosphate transporter activity & -1.7 & -2.0 & -1.7 & -1.5 & 1.6 & 1.3 & 1.3 & 2.7 \\
\hline IPF776 & orf19.5338 & Transcription factor activity & -1.7 & -2.8 & -1.8 & -3.0 & -11.2 & -1.0 & -11.9 & 1.2 \\
\hline IPF7817 & orf19.3131 & NADPH dehydrogenase activity & -3.8 & -4.7 & -5.5 & -3.6 & -3.6 & -2.8 & -3.3 & -2.1 \\
\hline IPF7863 & orf19.1449 & -- & -16.9 & -6.9 & -15.5 & -8.0 & -2.9 & -4.5 & -3.0 & -4.3 \\
\hline IPF7998 & orf19.4191 & -- & -1.4 & -2.1 & 1.4 & -1.7 & -3.2 & -1.6 & -4.7 & -3.2 \\
\hline IPF823 & orf19.7245 & tRNA (5-methylaminomethyl-2-thiouridylate)-methyltransferase activity & 1.4 & -1.1 & 1.1 & -1.7 & -2.2 & -1.9 & -2.0 & -1.5 \\
\hline IPF8405 & orf19.1721 & Carbonate dehydratase activity & 1.5 & 1.0 & 1.2 & -1.3 & -6.1 & -1.6 & -4.1 & -1.6 \\
\hline IPF8464 & orf19.6855 & -- & -1.9 & -9.7 & -2.7 & -3.2 & -2.4 & -1.1 & -3.9 & -7.5 \\
\hline IPF8627 & orf19.3969 & Specific transcriptional repressor activity & -1.9 & -4.0 & -1.8 & -1.5 & -2.2 & 1.1 & -2.0 & 1.3 \\
\hline IPF9063 & orf19.203 & -- & 1.0 & -1.8 & -1.9 & -1.5 & -2.4 & -1.8 & -1.8 & -1.5 \\
\hline IPF9238 & orf19.6143 & -- & -1.7 & -2.5 & -18.5 & -2.7 & -1.6 & -1.9 & -1.2 & -3.6 \\
\hline IPF9538 & orf19.271 & 2,4-dienoyl-CoA reductase (NADPH) activity & -164.3 & -548.1 & -280.0 & -137.2 & -15.3 & -16.6 & -25.5 & -13.3 \\
\hline IPF9560 & orf19.1942 & -- & -1.8 & -1.6 & -1.7 & -1.8 & -1.4 & -1.0 & -1.5 & 1.4 \\
\hline LAT1 & orf19.6561 & Dihydrolipoyllysine-residue acetyltransferase activity & -1.0 & -3.1 & -1.4 & -2.2 & -3.3 & -2.7 & -1.6 & -1.7 \\
\hline LEU42 & orf19.1375 & 2-isopropylmalate synthase activity & -2.0 & -1.6 & -2.0 & -2.2 & 1.5 & -1.1 & 1.8 & 1.5 \\
\hline LSC2.3eoc & orf19.1860 & Succinate-CoA ligase (ADP-forming) activity & -1.0 & -2.1 & -1.3 & -2.1 & -2.6 & -1.5 & -1.7 & -2.2 \\
\hline$M D L 2.3 f$ & orf19.5599 & ATP binding & 1.1 & 1.2 & -1.1 & -1.5 & -1.9 & -1.7 & -1.9 & -1.5 \\
\hline MET3 & orf19.5025 & Sulfate adenylyltransferase (ATP) activity & 2.7 & 2.0 & 1.1 & 1.2 & -2.3 & -1.5 & -2.0 & -2.0 \\
\hline MRP $1.5 f$ & orf19.1662 & Structural constituent of ribosome & 2.0 & 1.5 & -1.2 & -1.8 & -2.0 & -1.5 & -1.7 & -3.8 \\
\hline MRRI & orf19.7372 & RNA polymerase II transcription factor activity & -74.6 & -35.6 & -17.1 & -75.6 & -69.9 & -19.6 & -83.5 & -5.9 \\
\hline MSB1.5eoc & orf19.1133 & -- & -1.9 & -2.4 & -1.6 & -2.8 & -2.5 & -1.1 & -2.2 & 1.8 \\
\hline $\mathrm{NDH1}$ & orf19.339 & NADH dehydrogenase activity & -1.2 & -1.6 & -1.1 & -1.8 & -1.8 & -1.5 & -1.5 & -2.1 \\
\hline NOP4 & orf19.5198 & RNA binding & -1.3 & -2.4 & 1.5 & -1.2 & -3.5 & -1.5 & -4.4 & -2.4 \\
\hline NUC2 & orf19.6531 & -- & 1.1 & -1.6 & -1.2 & -1.6 & -2.8 & -1.8 & -1.6 & -1.6 \\
\hline$O G G 1$ & orf19.7190 & Oxidized purine base lesion DNA N-glycosylase activity & -2.2 & -3.9 & -1.4 & -1.9 & -4.3 & -2.2 & -5.4 & -2.2 \\
\hline
\end{tabular}


Table B-3 (continued).

\begin{tabular}{|c|c|c|c|c|c|c|c|c|c|c|}
\hline \multirow[b]{3}{*}{ CandidaDB Name $^{a}$} & \multirow[b]{3}{*}{ orf19 $\mathrm{Name}^{b}$} & \multirow[b]{3}{*}{ GO Annotation (Molecular Function) ${ }^{c}$} & \multicolumn{4}{|c|}{ F5 $\Delta$ mrr1 vs. F5 } & \multicolumn{4}{|c|}{ G5 $\Delta$ mrr1 vs. G5 } \\
\hline & & & \multicolumn{2}{|c|}{ Strain A } & \multicolumn{2}{|c|}{ Strain B } & \multicolumn{2}{|c|}{ Strain A } & \multicolumn{2}{|c|}{ Strain B } \\
\hline & & & 1 & 2 & 1 & 2 & 1 & 2 & 1 & 2 \\
\hline ORMI & orf19.5751 & ( & -1.3 & -2.2 & -1.3 & -1.5 & -2.3 & -1.5 & -1.6 & -1.6 \\
\hline PEX13 & orf19.7282 & Protein binding & -1.5 & -2.5 & -2.0 & -1.9 & 1.1 & -1.3 & 1.1 & -1.9 \\
\hline PGA9 & orf19.2108 & Copper, zinc superoxide dismutase activity & -1.2 & 1.1 & -1.9 & 1.3 & -2.3 & -1.7 & -2.5 & -1.6 \\
\hline PHR1 & orf19.3829 & 1,3-beta-glucanosyltransferase activity & -2.8 & -7.8 & -2.3 & -1.7 & -2.0 & -1.3 & -2.0 & 1.6 \\
\hline PRP12 & orf19.4379 & Exonuclease activity & -1.7 & -1.8 & -1.9 & -1.7 & 1.3 & -1.4 & 1.1 & 1.1 \\
\hline PRP5 & orf19.6831 & ATP-dependent RNA helicase activity & -1.1 & 1.5 & -1.1 & 1.3 & -2.3 & -1.5 & -1.8 & -1.6 \\
\hline PRY2 & orf19.7218 & -- & -1.8 & -1.6 & -2.6 & -2.7 & -4.0 & -7.0 & -1.6 & 1.4 \\
\hline RBT4 & orf19.6202 & -- & -1.9 & -2.5 & -3.2 & -1.6 & -1.4 & -1.2 & -1.2 & 1.9 \\
\hline RIP1 & orf19.5893 & Ubiquinol-cytochrome-c reductase activity & 1.1 & -1.4 & 1.1 & -1.6 & -1.6 & -1.5 & -1.5 & -1.6 \\
\hline RKII & orf19.1701 & Ribose-5-phosphate isomerase activity & -1.7 & -1.6 & -1.6 & -1.5 & -1.7 & 1.2 & -1.8 & -1.6 \\
\hline RPC31 & orf19.2831 & DNA-directed RNA polymerase activity & 1.2 & -4.1 & 2.3 & -1.6 & -3.4 & -1.6 & -2.4 & -3.3 \\
\hline RPN4 & orf19.1069 & Transcription factor activity & -1.7 & 1.2 & -1.6 & -1.3 & -2.0 & -1.8 & -2.3 & -2.1 \\
\hline SEC 31 & orf19.6217 & -- & -2.0 & -1.8 & -1.8 & -1.6 & -2.8 & -1.6 & -2.3 & 1.5 \\
\hline SEN1 & orf19.5938 & ATP-dependent RNA helicase activity & -2.1 & -3.9 & -1.5 & -2.1 & 1.3 & -1.9 & 3.1 & 2.5 \\
\hline SEN2 & orf19.2735 & tRNA-intron endonuclease activity & -1.5 & -1.5 & -1.6 & -2.1 & -5.1 & -1.6 & -3.8 & 3.2 \\
\hline SIS 2 & orf19.7378 & Protein phosphatase inhibitor activity & -1.8 & -1.9 & -1.8 & -2.1 & 1.1 & -1.7 & -1.2 & -1.5 \\
\hline SNF3 & orf19.5962 & Glucose transporter activity & -1.7 & -3.2 & -2.2 & -2.1 & -3.0 & -2.0 & -2.0 & -1.0 \\
\hline SOD21 & orf19.7111 & Manganese superoxide dismutase activity & -1.9 & -5.2 & -1.5 & -1.5 & -1.8 & -2.0 & 1.5 & -1.0 \\
\hline SOU3 & orf19.732 & 2,4-dienoyl-CoA reductase (NADPH) activity & -1.6 & 1.1 & -2.0 & 1.1 & -1.7 & -1.8 & -1.6 & -1.7 \\
\hline TAF90 & orf19.536 & General RNA polymerase II transcription factor activity & -1.2 & -1.2 & -1.2 & -1.5 & -2.2 & -1.8 & -1.5 & -1.6 \\
\hline TAO3.5f & orf19.1693 & Transcription factor activity & -59.3 & -1.7 & -1.6 & -1.7 & -2.4 & -1.2 & -2.0 & 1.6 \\
\hline TFB2 & orf19.5846 & General RNA polymerase II transcription factor activity & -1.5 & -2.0 & -1.5 & -1.8 & -1.2 & -1.2 & -1.5 & -1.2 \\
\hline TIM22 & orf19.1352 & Protein transporter activity & -1.1 & -3.0 & 1.0 & -2.0 & -2.2 & -2.1 & -2.3 & -3.1 \\
\hline TOF $1.5 f$ & orf19.4136 & -- & -1.7 & 1.0 & -1.7 & 1.2 & -2.4 & -1.5 & -1.7 & -1.9 \\
\hline TRK1.5f & orf19.600 & Chloride transporter activity & -1.8 & -2.6 & -1.7 & -2.4 & -2.1 & 1.4 & -2.1 & 1.6 \\
\hline$U G A 2$ & orf19.345 & Succinate-semialdehyde dehydrogenase activity & -1.8 & -2.7 & -3.0 & -2.4 & -1.4 & 1.2 & -1.1 & 1.5 \\
\hline WHI3 & orf19.6494 & RNA binding & -1.7 & -1.9 & -1.6 & -2.0 & -3.0 & -1.6 & -2.4 & 1.3 \\
\hline YAE1 & orf19.7037 & -- & -1.4 & 2.3 & 1.1 & 1.2 & -1.9 & -2.1 & -1.5 & -1.6 \\
\hline YNK1 & orf19.4311 & Nucleoside diphosphate kinase activity & -1.1 & -1.0 & 1.3 & -1.7 & -2.8 & -1.7 & -1.6 & -1.5 \\
\hline
\end{tabular}

${ }^{a}$ Gene name at CandidaDB (http://genolist.pasteur.fr/CandidaDB/).

${ }^{\boldsymbol{b}}$ orf19 nomenclature according to the Assembly 19 version.

${ }^{c} \mathrm{GO}$ annotation found at CGD (http://www.candidagenome.org/). 
APPENDIX C: UPC2 SUPPLEMENTAL DATA

Table C-1. Genes up-regulated in either resistant isolate S2 or gain-of-function S2 allele in SC5314.

\begin{tabular}{|c|c|c|c|c|c|c|c|c|c|}
\hline \multirow{3}{*}{$\begin{array}{c}\text { CandidaDB } \\
\text { Name }^{a}\end{array}$} & \multirow{3}{*}{$\begin{array}{c}\text { CGD } \\
\text { Name }^{b}\end{array}$} & \multirow{3}{*}{$\begin{array}{c}\text { orf19 } \\
\text { Number }^{c}\end{array}$} & \multirow[b]{3}{*}{ GO Annotation (Molecular Function) ${ }^{d}$} & & & \multicolumn{2}{|c|}{$\mathrm{UPC}^{\mathrm{S2-1}}$ vs. $U P C 2^{\mathrm{S1-1}}$} & \multirow{2}{*}{\multicolumn{2}{|c|}{$\frac{\mathrm{UPC2}^{\mathrm{S} 2-1} \text { vs. } U P C 2^{\mathrm{S} 1-1}}{\text { Strain B }}$}} \\
\hline & & & & \multicolumn{2}{|c|}{ S2vS1 } & \multicolumn{2}{|c|}{ Strain A } & & \\
\hline & & & & 1 & 2 & 1 & 2 & 1 & 2 \\
\hline BMR1 & MDRI & orf19.5604 & Multidrug transporter activity & 2.1 & 3.7 & 5.4 & 2.5 & 3.5 & 5.1 \\
\hline DDR48 & DDR48 & orf19.4082 & -- & 7.1 & 8.0 & 2.4 & 2.4 & 3.4 & 3.8 \\
\hline ECM3 & ECM3 & orf19.1563 & -- & 1.6 & 1.5 & 1.7 & 2.9 & 4.1 & 3.2 \\
\hline ERG1 & ERG1 & orf19.406 & Squalene monooxygenase activity & 2.4 & 1.6 & 1.5 & 1.6 & 1.7 & 1.8 \\
\hline ERG24 & ERG24 & orf19.1598 & Delta 14 -sterol reductase activity & 2.3 & 2.0 & 3.2 & 3.5 & 2.9 & 2.9 \\
\hline ERG26 & ERG26 & orf19.2909 & C-3 sterol dehydrogenase (C-4 sterol decarboxylase) activity & 2.0 & 1.6 & 2.0 & 1.9 & 2.0 & 2.0 \\
\hline ERG27 & ERG27 & orf19.3240 & 3-keto sterol reducatase activity & 2.3 & 2.1 & 2.2 & 3.0 & 3.6 & 2.8 \\
\hline FEN2 & -- & orf19.5535 & Pantothenate transporter activity & 2.2 & 2.2 & 2.3 & 2.7 & 2.7 & 2.8 \\
\hline FTH1 & FTH1 & orf19.4802 & -- & 1.5 & 1.5 & 1.8 & 2.4 & 4.3 & 3.7 \\
\hline IDP2 & IDP2 & orf19.3733 & Isocitrate dehydrogenase $(\mathrm{NADP}+)$ activity & 3.6 & 2.1 & 1.8 & 1.8 & 1.6 & 1.7 \\
\hline IFA24.3 & FGR51 & orf19.156 & -- & 1.8 & 1.9 & 1.7 & 1.6 & 1.7 & 1.7 \\
\hline NCP1 & $N C P 1$ & orf19.2672 & NADPH-hemoprotein reductase activity & 2.5 & 1.5 & 1.9 & 1.7 & 2.2 & 1.8 \\
\hline QRI8 & -- & orf19.7329 & Ubiquitin-protein ligase activity & 1.5 & 2.0 & 1.6 & 1.5 & 1.7 & 1.7 \\
\hline TEF41 & TEF4 & orf19.2652 & Translation elongation factor activity & 4.1 & 3.4 & 18.8 & 19.0 & 28.9 & 14.9 \\
\hline IPF12101 & $P G A 7$ & orf19.5635 & -- & 1.6 & 2.7 & 5.4 & 6.0 & 8.8 & 9.0 \\
\hline IPF1218 & SOD4 & orf19.2062 & Copper, zinc superoxide dismutase activity & 2.3 & 2.3 & 2.2 & 1.8 & 1.7 & 1.6 \\
\hline IPF14119 & $D A G 7$ & orf19.4688 & -- & 1.8 & 1.6 & 1.7 & 1.7 & 1.6 & 1.8 \\
\hline IPF14652 & -- & orf19.2653 & -- & 4.3 & 1.6 & 1.7 & 4.3 & 2.8 & 1.9 \\
\hline IPF18080 & -- & orf19.7357 & -- & 1.9 & 1.6 & 1.5 & 1.6 & 1.8 & 1.6 \\
\hline IPF20010 & -- & orf19.5799 & -- & 2.0 & 2.2 & 2.2 & 1.6 & 2.8 & 2.0 \\
\hline IPF20169 & PGA10 & orf19.5674 & Heme binding & 4.1 & 10.8 & 5.6 & 7.0 & 13.0 & 11.1 \\
\hline IPF2903 & -- & orf19.7456 & -- & 1.8 & 2.3 & 2.0 & 2.2 & 1.7 & 1.6 \\
\hline IPF3352 & -- & orf19.4013 & -- & 2.3 & 2.3 & 2.0 & 1.9 & 2.5 & 2.0 \\
\hline IPF4059 & -- & orf19.1865 & -- & 1.6 & 2.1 & 2.7 & 1.9 & 2.5 & 2.4 \\
\hline IPF4721 & -- & orf19.3737 & -- & 1.5 & 1.6 & 2.3 & 1.8 & 1.5 & 1.6 \\
\hline IPF6298 & -- & orf19.1964 & -- & 3.0 & 1.6 & 1.7 & 2.3 & 1.7 & 1.7 \\
\hline IPF6518 & -- & orf19.1691 & -- & 3.3 & 5.3 & 1.7 & 2.0 & 2.3 & 2.7 \\
\hline IPF7289 & $U P C 2$ & orf19.391 & Transcription factor activity & 1.6 & 2.4 & 2.1 & 2.4 & 2.5 & 2.5 \\
\hline IPF7397 & -- & orf19.1800 & -- & 2.9 & 2.6 & 1.8 & 1.6 & 1.7 & 2.2 \\
\hline IPF7432 & -- & orf19.6219 & -- & 2.0 & 2.3 & 1.9 & 2.1 & 2.4 & 2.2 \\
\hline IPF867 & SET3 & orf19.7221 & NAD-dependent histone deacetylase activity & 2.1 & 1.7 & 3.6 & 3.2 & 2.9 & 2.4 \\
\hline IPF8970 & -- & orf19.496 & -- & 1.9 & 2.5 & 2.2 & 1.6 & 1.9 & 1.8 \\
\hline ALK3 & -- & orf19.7512 & Catalytic activity & 1.5 & 1.7 & 1.0 & -1.1 & 1.5 & -1.1 \\
\hline$A R G 5,6$ & $A R G 5,6$ & orf19.4788 & $\mathrm{N}$-acetyl-gamma-glutamyl-phosphate reductase activity & 7.4 & 12.7 & 1.6 & -1.8 & -1.2 & -1.3 \\
\hline ARP6 & -- & orf19.4904 & -- & 1.7 & 1.6 & 1.1 & -1.3 & -1.1 & -1.4 \\
\hline BET5 & -- & orf19.302 & -- & 1.8 & 1.5 & -1.1 & 1.1 & 1.3 & -1.1 \\
\hline
\end{tabular}


Table C-1 (continued).

\begin{tabular}{|c|c|c|c|c|c|c|c|c|c|}
\hline \multirow{3}{*}{$\begin{array}{l}\text { CandidaDB } \\
\text { Name }^{a}\end{array}$} & \multirow{3}{*}{$\begin{array}{c}\text { CGD } \\
\text { Name }^{b}\end{array}$} & \multirow{3}{*}{$\begin{array}{c}\text { orf19 } \\
\text { Number }^{c}\end{array}$} & \multirow[b]{3}{*}{ GO Annotation (Molecular Function) ${ }^{d}$} & \multirow{2}{*}{\multicolumn{2}{|c|}{ S2vS1 }} & \multicolumn{2}{|c|}{$\frac{\mathrm{UPC}^{\mathrm{S2}-1} \text { vs. } U P C 2^{\mathrm{S1-1}}}{\text { Strain }}$} & \multirow{2}{*}{\multicolumn{2}{|c|}{$\begin{array}{c}\mathrm{UPC2}^{\mathrm{S} 2-1} \text { vs. } U P C 2^{\mathrm{S} 1-1} \\
\text { Strain B }\end{array}$}} \\
\hline & & & & & & & & & \\
\hline & & & & 1 & 2 & 1 & 2 & 1 & 2 \\
\hline CAN2 & CAN2 & orf19.111 & Basic amino acid transporter activity & 1.5 & 1.9 & -2.1 & 1.1 & 3.2 & 2.7 \\
\hline CBPI & $C B P 1$ & orf19.7323 & Steroid binding & 1.5 & 1.6 & 1.3 & 1.4 & 1.9 & 1.4 \\
\hline CDR12 & CDR11 & orf19.918 & ATPase activity & 1.8 & 3.1 & 1.4 & -1.7 & 1.9 & 1.4 \\
\hline Cirt $4 a$ & CIRT4B & orf19.2839 & -- $\quad$ - & 1.7 & 1.6 & -1.0 & 1.1 & 1.9 & 1.7 \\
\hline COQ4 & COQ4 & orf19.3008 & -- & 1.5 & 1.5 & 1.9 & 1.4 & 1.6 & 1.4 \\
\hline CPA2 & -- & orf19.3221 & Carbamoyl-phosphate synthase activity & 3.4 & 2.2 & 1.6 & -1.4 & -1.2 & -1.2 \\
\hline CTAl & CATI & orf19.6229 & Catalase activity & 1.5 & 1.6 & 1.4 & 1.5 & 1.2 & 1.3 \\
\hline DAL51 & $D A L 9$ & orf19.6956 & Allantoate transporter activity & 1.5 & 2.4 & -1.1 & 1.2 & 2.1 & 1.6 \\
\hline$D N L I$ & LIG4 & orf19.5798 & DNA ligase (ATP) activity & 1.6 & 2.2 & 1.3 & 1.7 & 3.0 & 1.9 \\
\hline ECM18 & ECM18 & orf19.6958 & -- & 2.1 & 1.5 & -1.3 & 1.7 & 1.7 & 1.2 \\
\hline ECM21.3 & ECM21 & orf19.4887 & -- & 1.5 & 2.1 & -1.1 & 1.2 & 1.5 & 1.3 \\
\hline ECM42 & ECM42 & orf19.6500 & Amino-acid $\mathrm{N}$-acetyltransferase activity & 1.7 & 2.9 & 1.2 & -2.0 & -1.1 & -1.0 \\
\hline ERG3 & ERG3 & orf19.767 & C-5 sterol desaturase activity & 1.5 & 1.5 & 1.1 & 1.3 & 1.2 & 1.3 \\
\hline ERG4 & ERG4 & orf19.5379 & -- & 1.5 & 1.6 & 1.3 & 1.7 & 1.3 & 1.7 \\
\hline$E R G 7$ & $E R G 7$ & orf19.1570 & Lanosterol synthase activity & 1.5 & 1.9 & 1.6 & 1.4 & 1.9 & 1.4 \\
\hline EXG1 & XOG1 & orf19.2990 & Glucan 1,3-beta-glucosidase activity & 2.1 & 1.6 & 1.2 & -1.3 & -1.0 & -1.0 \\
\hline FET34.3eoc & FET34 & orf19.4215 & Ferroxidase activity & 1.6 & 1.7 & 2.1 & 1.3 & 2.6 & 2.1 \\
\hline FRP1 & -- & orf19.2496 & -- & 2.6 & 2.2 & 1.3 & 1.6 & 1.3 & 1.5 \\
\hline FTR1 & FTR1 & orf19.7219 & Ferrous iron transporter activity & 1.5 & 2.4 & 1.4 & 1.5 & 1.3 & 1.5 \\
\hline FUN34.5eoc & -- & orf19.2496 & -- & 2.7 & 1.7 & 1.4 & 2.3 & 1.7 & 1.5 \\
\hline GAL1 & GAL1 & orf19.3670 & Galactokinase activity & 2.0 & 1.6 & -1.1 & -1.3 & -1.2 & -1.3 \\
\hline GAL10 & GAD1 & orf19.1153 & UDP-glucose 4-epimerase activity & 1.6 & 1.7 & -1.2 & -1.5 & -1.1 & -1.3 \\
\hline$G D H 2$ & GDH2 & orf19.2192 & Glutamate dehydrogenase activity & 2.5 & 1.6 & 1.1 & -1.0 & 1.1 & 1.2 \\
\hline GLK1 & GLK1 & orf19.734 & Glucokinase activity & 1.6 & 1.8 & 1.1 & 1.2 & 1.5 & 1.4 \\
\hline IFA4 & IFA4 & orf19.4510 & -- & 3.0 & 2.3 & -2.4 & 1.4 & -1.2 & -1.2 \\
\hline$I F C 3$ & IFC3 & orf19.3749 & Oligopeptide transporter activity & 1.5 & 2.2 & -1.4 & -1.2 & 2.1 & 1.5 \\
\hline IFK3 & FMO2 & orf19.857 & -- & 1.8 & 2.5 & -1.1 & 1.1 & 1.0 & -1.3 \\
\hline IFR3 & -- & orf19.2908 & -- & 1.7 & 2.1 & 1.1 & 1.7 & 2.1 & 1.1 \\
\hline ILV3 & ILV3 & orf19.4040 & Dihydroxy-acid dehydratase activity & 1.5 & 1.8 & 1.4 & 1.1 & 1.2 & 1.2 \\
\hline LSC2.3eocl & $L S C 2$ & orf19.1860 & Succinate-CoA ligase (ADP-forming) activity & 1.6 & 1.5 & 1.3 & 1.2 & 1.1 & 1.2 \\
\hline LYP1 & LYP1 & orf19.651 & Basic amino acid transmembrane transporter activity & 1.5 & 2.5 & -1.3 & -1.3 & 1.4 & 1.5 \\
\hline MEP3 & $M E P 1$ & orf19.1614 & Ammonium transporter activity & 2.9 & 3.0 & -1.1 & 1.5 & 2.3 & 2.0 \\
\hline MSY1 & -- & orf19.109 & Tyrosine-tRNA ligase activity & 1.7 & 1.5 & -1.4 & -2.2 & -1.5 & -1.8 \\
\hline NAM2 & NAM2 & orf19.5705 & Leucine-tRNA ligase activity & 1.8 & 1.5 & -1.8 & -2.4 & -1.7 & -2.4 \\
\hline NEM1 & -- & orf19.4657 & -- & 3.0 & 1.8 & 1.0 & 1.2 & -1.0 & -1.3 \\
\hline ORMI & -- & orf19.5751 & -- & 1.7 & 1.6 & 1.6 & 1.4 & 1.3 & 1.3 \\
\hline OSM2 & OSM2 & orf19.5005 & Fumarate reductase (NADH) activity & 1.6 & 1.5 & -1.2 & 1.0 & 1.2 & 1.0 \\
\hline PHRI & PHRI & orf19.3829 & 1,3-beta-glucanosyltransferase activity & 1.8 & 2.3 & 1.3 & 1.5 & 1.0 & 1.2 \\
\hline PLB5 & PLB5 & orf19.5102 & Lysophospholipase activity & 1.6 & 1.8 & 1.4 & 1.1 & 1.8 & 1.5 \\
\hline POT14 & ERG10 & orf19.1591 & Acetyl-CoA C-acetyltransferase activity & 1.8 & 1.8 & 1.2 & 1.3 & 1.3 & 1.3 \\
\hline
\end{tabular}


Table C-1 (continued).

\begin{tabular}{|c|c|c|c|c|c|c|c|c|c|}
\hline \multirow{3}{*}{$\begin{array}{l}\text { CandidaDB } \\
\text { Name }^{a}\end{array}$} & \multirow{3}{*}{$\begin{array}{c}\text { CGD } \\
\text { Name }^{b}\end{array}$} & \multirow{3}{*}{$\begin{array}{c}\text { orf19 } \\
\text { Number }^{c}\end{array}$} & \multirow[b]{3}{*}{ GO Annotation (Molecular Function) ${ }^{d}$} & \multirow{2}{*}{\multicolumn{2}{|c|}{ S2vS1 }} & \multirow{2}{*}{\multicolumn{2}{|c|}{$\frac{\mathrm{UPC2}^{\mathrm{S} 2-1} \text { vs. } U P C 2^{\mathrm{S} 1-1}}{\text { Strain A }}$}} & \multirow{2}{*}{\multicolumn{2}{|c|}{$\frac{\mathrm{UPC2}^{\mathrm{S} 2-1} \text { vs. } U P C 2^{\mathrm{S} 1-1}}{\text { Strain } \mathrm{B}}$}} \\
\hline & & & & & & & & & \\
\hline & & & & 1 & 2 & 1 & 2 & 1 & 2 \\
\hline PRP6 & -- & orf19.6356 & RNA splicing factor activity, transesterification mechanism & 2.0 & 1.5 & -1.0 & -1.2 & 1.1 & -3.9 \\
\hline RAD1.3f & RADI & orf19.5318 & Single-stranded DNA specific endodeoxyribonuclease activity & 1.5 & 1.5 & 1.3 & 1.3 & 1.7 & 1.1 \\
\hline$R B T 2$ & FRE10 & orf19.1415 & Ferric-chelate reductase activity & 2.1 & 2.9 & 1.1 & -1.2 & -1.4 & -1.2 \\
\hline RBT4 & RBT4 & orf19.6202 & -- & 1.6 & 1.9 & 1.3 & 1.6 & 1.5 & 1.5 \\
\hline RNH1 & -- & orf19.5614 & Ribonuclease $\mathrm{H}$ activity & 2.4 & 2.0 & -1.0 & -1.1 & -1.1 & -1.2 \\
\hline SAP1 & SAP1 & orf19.5714 & Aspartic-type endopeptidase activity & 1.7 & 1.9 & 1.3 & -1.1 & 1.5 & -1.2 \\
\hline SAPQ & SAPQ & orf19.6928 & Aspartic-type endopeptidase activity & 1.6 & 1.8 & 1.3 & 1.2 & 1.5 & 1.5 \\
\hline SCW11.3eoc & $S C W 11$ & orf19.3893 & Glucan 1,3-beta-glucosidase activity & 1.7 & 1.6 & -1.2 & -1.7 & -1.3 & -1.2 \\
\hline SSU1 & SSU1 & orf19.7313 & Sulfite transporter activity & 1.8 & 1.6 & 1.5 & 1.1 & 1.3 & 1.6 \\
\hline UGA12.3f & UGAI & orf19.802 & 4-aminobutyrate transaminase activity & 2.0 & 1.8 & 1.3 & 1.0 & 1.1 & 1.2 \\
\hline UGA12.5f & UGA1 & orf19.802 & 4-aminobutyrate transaminase activity & 1.8 & 1.6 & 1.5 & 1.4 & 1.2 & 1.0 \\
\hline VPH2 & -- & orf19.5237 & -- & 1.8 & 2.0 & -1.0 & -1.1 & 1.5 & -1.3 \\
\hline IPF10001 & -- & orf19.6194 & -- & 1.6 & 1.8 & -1.3 & -1.0 & 1.5 & -1.3 \\
\hline IPF10431 & -- & orf19.2888 & -- & 1.5 & 2.1 & -1.2 & 1.3 & 1.1 & -1.2 \\
\hline IPF11105 & -- & orf19.2262 & -- & 1.6 & 1.5 & 1.2 & 1.1 & 1.4 & 1.1 \\
\hline IPF11812 & $S E C 3$ & orf19.2911 & -- & 1.7 & 1.5 & 1.2 & -1.1 & 1.1 & -1.3 \\
\hline IPF11849 & -- & orf19.4459 & -- & 3.8 & 3.3 & 2.9 & 1.2 & -2.7 & -4.1 \\
\hline IPF1217 & -- & orf19.2063 & -- & 1.5 & 1.5 & 1.2 & 1.3 & 1.6 & 1.3 \\
\hline IPF13131.3 & HGT20 & orf19.1587 & Glucose transporter activity & 1.6 & 1.9 & -1.2 & 1.8 & 1.5 & 1.6 \\
\hline IPF13176.3 & $A R G 3$ & orf19.5610 & Ornithine carbamoyltransferase activity & 1.8 & 2.4 & -1.2 & -1.9 & 1.2 & -1.1 \\
\hline IPF13423 & -- & orf19.4128 & -- & 1.8 & 2.9 & 1.1 & -1.1 & -1.3 & -1.3 \\
\hline IPF13815 & -- & orf19.4553 & -- & 1.8 & 1.7 & 1.3 & 1.1 & 1.4 & 1.1 \\
\hline IPF13967 & -- & orf19.5205 & -- & 1.6 & 2.3 & -1.0 & 1.3 & 2.1 & 2.0 \\
\hline IPF14109 & PGA45 & orf19.2451 & -- & 5.1 & 3.9 & 1.4 & 1.8 & 1.5 & 1.7 \\
\hline IPF14968 & -- & orf19.3461 & -- & 1.7 & 2.3 & 1.5 & 1.4 & 1.1 & 1.1 \\
\hline IPF15423 & SOD6 & orf19.2108 & Copper, zinc superoxide dismutase activity & 1.5 & 2.5 & -1.3 & 1.2 & 1.3 & 1.6 \\
\hline IPF16948 & -- & orf19.3285 & -- & 1.8 & 3.8 & -1.2 & -1.0 & 1.1 & 1.0 \\
\hline IPF17054 & -- & orf19.5037 & -- & 3.2 & 2.1 & 1.2 & 1.2 & 1.2 & 1.2 \\
\hline IPF17520.53еoc & -- & orf19.1021 & -- & 5.9 & 2.6 & -- & -- & -- & -- \\
\hline IPF18207 & $D A P 1$ & orf19.489 & -- & 1.9 & 1.7 & -1.0 & 1.7 & 2.0 & 1.8 \\
\hline IPF18316.3f & SSP96 & orf19.5145 & -- & 4.8 & 1.6 & -1.4 & -1.2 & -1.1 & 1.0 \\
\hline IPF18418 & -- & orf19.5620 & -- & 2.0 & 2.6 & 1.4 & 1.3 & 1.2 & 1.3 \\
\hline IPF19735 & -- & orf19.3215 & -- & 1.5 & 1.5 & 1.0 & 1.7 & 1.4 & 1.4 \\
\hline IPF19772 & -- & orf19.5602 & -- & 1.7 & 1.7 & -1.1 & 1.0 & 1.1 & -1.0 \\
\hline IPF19908 & -- & orf19.1344 & -- & 2.2 & 3.0 & 2.0 & 1.2 & -1.0 & 1.0 \\
\hline IPF19998 & -- & orf19.1344 & -- & 1.6 & 1.7 & 1.3 & 1.1 & 1.2 & 1.1 \\
\hline IPF2065 & -- & orf19.5274 & -- & 1.7 & 2.5 & 1.4 & 1.2 & 1.2 & 1.1 \\
\hline IPF227 & -- & orf19.3244 & -- & 2.2 & 1.5 & -1.3 & 1.4 & 1.1 & 1.1 \\
\hline IPF2561 & -- & orf19.6624 & -- & 1.5 & 2.2 & 1.1 & -1.4 & 1.1 & -1.0 \\
\hline IPF2884 & -- & orf19.7449 & -- & 5.8 & 1.6 & 1.4 & 1.2 & -1.1 & -1.3 \\
\hline
\end{tabular}


Table C-1 (continued).

\begin{tabular}{|c|c|c|c|c|c|c|c|c|c|}
\hline \multirow{3}{*}{$\begin{array}{c}\text { CandidaDB } \\
\text { Name }^{a}\end{array}$} & \multirow{3}{*}{$\begin{array}{c}\text { CGD } \\
\text { Name }^{b}\end{array}$} & \multirow{3}{*}{$\begin{array}{c}\text { orf19 } \\
\text { Number }^{c}\end{array}$} & \multirow[b]{3}{*}{ GO Annotation (Molecular Function) ${ }^{d}$} & \multirow{2}{*}{\multicolumn{2}{|c|}{ S2vS1 }} & \multirow{2}{*}{\multicolumn{2}{|c|}{$\frac{\mathrm{UPC2}^{\mathrm{S2}-1} \text { vs. } U P C 2^{\mathrm{S1-1}}}{\text { Strain A }}$}} & \multirow{2}{*}{\multicolumn{2}{|c|}{$\frac{\mathrm{UPC2}^{\mathrm{S} 2-1} \text { vs. } U P C 2^{\mathrm{S} 1-1}}{\text { Strain B }}$}} \\
\hline & & & & & & & & & \\
\hline & & & & 1 & 2 & 1 & 2 & 1 & 2 \\
\hline IPF3282 & HXT5 & orf19.4384 & Fructose transporter activity & 1.7 & 2.0 & 1.5 & 2.6 & -1.0 & -1.1 \\
\hline IPF3355 & -- & orf19.4014 & -- & 2.0 & 2.0 & 1.7 & 1.1 & 1.5 & 1.0 \\
\hline IPF3535 & -- & orf19.6840 & -- & 2.1 & 2.8 & 2.8 & 2.2 & 1.7 & 1.4 \\
\hline IPF3598 & -- & orf19.3047 & Transcription cofactor activity & 1.5 & 1.5 & -1.0 & -1.4 & 1.2 & -1.2 \\
\hline IPF4729 & -- & orf19.3743 & -- & 1.5 & 1.6 & -1.4 & 1.5 & 1.7 & 1.1 \\
\hline IPF5723.exon1 & -- & orf19.6329 & -- & 1.9 & 1.6 & 1.0 & 1.3 & 1.3 & 1.2 \\
\hline IPF5972 & $C D G 1$ & orf19.7314 & -- & 2.1 & 1.8 & -1.2 & -1.2 & -1.0 & -1.0 \\
\hline IPF6203 & $Z C F 21$ & orf19.4166 & -- & 1.7 & 1.7 & 1.6 & 1.4 & 1.7 & 1.0 \\
\hline IPF6375 & -- & orf19.520 & -- & 1.9 & 1.5 & 1.1 & -2.4 & -1.5 & -1.4 \\
\hline IPF6649 & -- & orf19.6704 & -- & 1.8 & 1.5 & -1.2 & 1.0 & 2.0 & 1.1 \\
\hline IPF6679 & -- & orf19.1306 & -- & 1.7 & 2.0 & -1.1 & -1.2 & 1.2 & -1.5 \\
\hline IPF6700 & -- & orf19.2459 & -- & 1.6 & 1.5 & 1.2 & -1.2 & -1.3 & -1.5 \\
\hline IPF721.3f & -- & orf19.5365 & -- & 1.5 & 1.6 & 1.8 & -1.3 & -1.3 & -1.6 \\
\hline IPF7260 & -- & orf19.2790 & Histone-lysine N-methyltransferase activity & 1.5 & 1.5 & 1.1 & -1.2 & 1.0 & -1.2 \\
\hline IPF8311 & -- & orf19.6003 & -- & 1.6 & 1.6 & 1.2 & 1.1 & 1.3 & 1.4 \\
\hline IPF8617 & -- & orf19.3705 & Phosphoprotein phosphatase activity & 1.8 & 1.7 & 1.1 & 1.0 & 1.6 & 1.2 \\
\hline IPF8796 & PGA4 & orf19.4035 & -- & 1.6 & 1.6 & -1.0 & 1.2 & 1.2 & 1.3 \\
\hline IPF9425.3 & -- & orf19.6888 & -- & 1.8 & 1.6 & 1.2 & -1.0 & 1.2 & 1.2 \\
\hline IPF9466 & -- & orf19.4066 & -- & 2.0 & 2.0 & -1.3 & 1.4 & -1.3 & -1.9 \\
\hline IPF9483 & -- & orf19.3395 & -- & 1.6 & 1.5 & 1.1 & 1.3 & 1.6 & 1.4 \\
\hline IPF9995 & -- & orf19.2286 & -- & 1.8 & 1.7 & 1.4 & 1.6 & 1.4 & 1.4 \\
\hline IPF9996 & -- & orf19.2285 & -- & 1.8 & 1.8 & 1.1 & 1.0 & 1.9 & 1.8 \\
\hline$A L S 11.5 f$ & $A L S 9$ & orf19.5742 & -- & -1.2 & -1.0 & 1.5 & 4.9 & 2.7 & 1.9 \\
\hline ALS9.5eoc & $A L S 9$ & orf19.5742 & -- & -1.2 & -1.1 & 1.5 & 5.2 & 2.9 & 2.1 \\
\hline$A P C 11$ & -- & orf19.7644 & Protein binding & -1.2 & -1.1 & 1.6 & 2.6 & 1.5 & 1.6 \\
\hline CDR11.5f & $C D R 11$ & orf19.918 & ATP binding & -1.4 & 1.3 & 1.6 & 3.5 & 1.6 & 1.5 \\
\hline CHTI & CHTI & orf19.7517 & Chitinase activity & 1.3 & 1.4 & 1.7 & 1.6 & 1.8 & 1.6 \\
\hline CLN2 & $C L N 3$ & orf19.1960 & Cyclin-dependent protein kinase regulator activity & 2.0 & -1.0 & 1.8 & 2.7 & 2.1 & 2.4 \\
\hline CNH1.3f & $\mathrm{CNH1}$ & orf19.367 & Cation:cation antiporter activity & -1.2 & -1.3 & 1.5 & 3.4 & 2.4 & 1.5 \\
\hline CNH1.5f & CNH1 & orf19.367 & Cation:cation antiporter activity & -1.1 & -1.4 & 2.0 & 9.2 & 1.7 & 1.7 \\
\hline$D A L 1$ & $D A L 1$ & orf19.5454 & Allantoinase activity & -1.9 & 1.0 & 2.2 & 2.3 & 1.5 & 2.0 \\
\hline DSK2 & -- & orf19.5345 & Protein binding & 1.1 & 1.6 & 1.7 & 1.5 & 1.5 & 1.5 \\
\hline$E R G 2$ & $E R G 2$ & orf19.6026 & C-8 sterol isomerase activity & 2.2 & 1.3 & 2.2 & 2.5 & 1.9 & 1.9 \\
\hline ERG5 & ERG5 & orf19.5178 & C-22 sterol desaturase activity & 1.3 & 1.3 & 1.7 & 1.8 & 1.7 & 1.9 \\
\hline ERG6 & ERG6 & orf19.1631 & Sterol 24-C-methyltransferase activity & 1.3 & 1.4 & 1.7 & 1.9 & 1.6 & 1.8 \\
\hline FCY24 & FCY24 & orf19.7331 & Transporter activity & 1.3 & 1.1 & 1.8 & 2.2 & 1.9 & 1.9 \\
\hline FET5 & FET34 & orf19.4215 & Ferroxidase activity & 1.7 & 1.3 & 2.3 & 2.4 & 2.7 & 2.4 \\
\hline FRE5 & FRP1 & orf19.5634 & Ferric-chelate reductase activity & 2.1 & 2.8 & 19.3 & 40.7 & 24.8 & 29.6 \\
\hline FRP4 & -- & orf19.6997 & -- & -1.5 & -5.9 & 1.5 & 2.8 & 3.2 & 2.7 \\
\hline FRP7 & -- & orf19.6995 & -- & -2.6 & 1.2 & 1.6 & 1.7 & 2.2 & 1.5 \\
\hline
\end{tabular}


Table C-1 (continued).

\begin{tabular}{|c|c|c|c|c|c|c|c|c|c|}
\hline \multirow{3}{*}{$\begin{array}{c}\text { CandidaDB } \\
\text { Name }^{a}\end{array}$} & \multirow{3}{*}{$\begin{array}{c}\text { CGD } \\
\text { Name }^{b}\end{array}$} & \multirow{3}{*}{$\begin{array}{c}\text { orf19 } \\
\text { Number }^{c}\end{array}$} & \multirow[b]{3}{*}{ GO Annotation (Molecular Function) ${ }^{d}$} & \multirow{2}{*}{\multicolumn{2}{|c|}{ S2vS1 }} & \multirow{2}{*}{\multicolumn{2}{|c|}{$\mathrm{UPC2}^{\mathrm{S} 2-1}$ vs. $U P C 2^{\mathrm{S} 1-1}$}} & \multirow{2}{*}{\multicolumn{2}{|c|}{$\begin{array}{c}\mathrm{UPC2}^{\mathrm{S} 2-1} \text { vs. } U P C 2^{\mathrm{S1}-1} \\
\text { Strain B }\end{array}$}} \\
\hline & & & & & & & & & \\
\hline & & & & 1 & 2 & 1 & 2 & 1 & 2 \\
\hline GDI1 & -- & orf19.7261 & Rab GDP-dissociation inhibitor activity & 1.1 & 1.4 & 1.5 & 2.4 & 1.7 & 1.8 \\
\hline IFH2 & -- & orf19.6398 & -- & -1.4 & 1.1 & 1.6 & 1.6 & 1.7 & 1.9 \\
\hline IFU5 & IFU5 & orf19.2568 & -- & 1.7 & 1.3 & 1.5 & 2.5 & 2.3 & 2.3 \\
\hline$P I F 2$ & -- & orf 19.7538 & DNA helicase activity & 1.1 & 1.2 & 1.5 & 1.9 & 1.9 & 1.6 \\
\hline RBT5 & RBT5 & orf19.5636 & Heme binding & 1.2 & 1.3 & 2.4 & 3.0 & 3.0 & 3.0 \\
\hline SAP5 & SAP5 & orf19.5585 & Aspartic-type endopeptidase activity & 1.1 & 1.1 & 2.0 & 2.0 & 2.5 & 3.0 \\
\hline SEC16.53f.eoc & -- & orf19.4346 & Structural molecule activity & -1.1 & -1.8 & 1.5 & 2.2 & 2.0 & 1.5 \\
\hline SIT1 & SIT1 & orf19.2179 & Ferrichrome transporter activity & -1.2 & 1.5 & 1.5 & 1.7 & 2.1 & 1.9 \\
\hline WSC4 & WSC4 & orf19.7251 & -- & 1.2 & 1.3 & 1.6 & 1.6 & 1.8 & 2.2 \\
\hline IPF11259 & -- & orf19.5210 & Transcription factor activity & 3.6 & -1.4 & 3.0 & 2.1 & 4.5 & 2.9 \\
\hline IPF11503 & -- & orf19.3902 & -- & -1.3 & -1.4 & 1.9 & 1.5 & 2.5 & 2.3 \\
\hline IPF11601 & NAG6 & orf19.2151 & GTP binding & 1.3 & 1.3 & 1.5 & 1.5 & 1.6 & 1.6 \\
\hline IPF11607 & -- & orf19.2149 & -- & 1.6 & 1.2 & 2.0 & 2.1 & 2.5 & 1.5 \\
\hline IPF12082.3f & -- & orf19.6832 & -- & 1.2 & 1.3 & 1.5 & 1.9 & 2.2 & 1.9 \\
\hline IPF12082.5f & -- & orf19.6832 & -- & 1.2 & -1.4 & 1.7 & 5.9 & 1.6 & 1.9 \\
\hline IPF12262 & $F G R 23$ & orf19.1616 & -- & 1.1 & -1.4 & 2.1 & 2.2 & 2.6 & 2.1 \\
\hline IPF12963 & BUL1 & orf19.5094 & -- & 1.2 & -1.1 & 2.7 & 2.5 & 3.1 & 2.9 \\
\hline IPF13377 & -- & orf19.2350 & -- & 1.2 & 1.4 & 1.8 & 2.4 & 2.2 & 2.2 \\
\hline IPF13723 & -- & orf19.260 & -- & 2.7 & -1.0 & 2.9 & 2.7 & 3.3 & 3.7 \\
\hline IPF14981 & -- & orf19.3483 & -- & 1.1 & 3.6 & 2.1 & 1.8 & 3.0 & 2.2 \\
\hline IPF1514 & -- & orf19.344 & -- & 1.1 & 12.2 & 3.0 & 17.3 & 3.5 & 2.4 \\
\hline IPF1899 & -- & orf19.7131 & -- & 2.0 & -1.1 & 1.9 & 3.8 & 2.0 & 1.9 \\
\hline IPF20157 & -- & orf19.6696 & -- & -21.5 & -1.5 & 1.5 & 4.0 & 3.0 & 1.8 \\
\hline IPF20164 & -- & orf19.5809 & Arylformamidase activity & -1.3 & -1.1 & 1.7 & 1.5 & 1.9 & 1.7 \\
\hline IPF257.3 & -- & orf19.3261 & 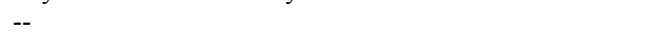 & -1.1 & -1.4 & 2.4 & 2.3 & 3.0 & 2.8 \\
\hline IPF2702 & -- & orf19.2163 & -- & -2.9 & 2.2 & 1.6 & 4.4 & 2.6 & 2.1 \\
\hline IPF3274 & -- & orf19.4383 & -- & -1.4 & -1.1 & 3.0 & 1.5 & 2.1 & 1.9 \\
\hline IPF3444.5f & -- & orf19.6184 & -- & -1.7 & 1.2 & 1.5 & 1.9 & 1.5 & 1.8 \\
\hline IPF3446 & -- & orf19.6186 & -- & -1.3 & 1.3 & 2.5 & 1.8 & 1.8 & 1.5 \\
\hline IPF3468 & -- & orf19.4055 & -- & -1.1 & -1.2 & 1.5 & 3.8 & 2.3 & 1.9 \\
\hline IPF3687 & PAN1 & orf19.886 & Cytoskeletal adaptor activity & -1.1 & 1.0 & 1.5 & 2.1 & 2.2 & 1.7 \\
\hline IPF4055 & -- & orf19.1867 & Malate transmembrane transporter activity & -1.9 & 2.2 & 1.7 & 2.1 & 2.3 & 1.9 \\
\hline IPF4160 & -- & orf19.5459 & -- & -1.2 & 1.1 & 1.5 & 3.1 & 1.5 & 1.7 \\
\hline IPF4649 & -- & orf19.6102 & Specific RNA polymerase II transcription factor activity & -1.2 & 1.4 & 1.7 & 2.0 & 2.0 & 1.5 \\
\hline IPF4724 & $P G A 23$ & orf19.3740 & -- & 1.3 & -1.2 & 1.7 & 2.4 & 2.8 & 2.1 \\
\hline IPF5217 & -- & orf19.7270 & -- & -2.3 & -1.2 & 1.6 & 2.7 & 2.0 & 1.7 \\
\hline IPF5556 & PHO100 & orf19.4424 & Acid phosphatase activity & 1.1 & 1.3 & 4.6 & 2.5 & 3.4 & 3.4 \\
\hline IPF5661 & -- & orf19.7125 & -- & -2.0 & -2.2 & 1.8 & 252.0 & 2.3 & 1.7 \\
\hline IPF6067 & TCC1 & orf19.6734 & General transcriptional repressor activity & -1.2 & 1.1 & 1.7 & 1.6 & 1.7 & 1.5 \\
\hline IPF6678 & -- & orf19.1307 & -- & -1.7 & 1.1 & 1.8 & 1.6 & 2.9 & 2.3 \\
\hline
\end{tabular}


Table C-1 (continued).

\begin{tabular}{|c|c|c|c|c|c|c|c|c|c|}
\hline \multirow{3}{*}{$\begin{array}{c}\text { CandidaDB } \\
\text { Name }^{a}\end{array}$} & \multirow{3}{*}{$\begin{array}{c}\text { CGD } \\
\text { Name }^{b}\end{array}$} & \multirow{3}{*}{$\begin{array}{c}\text { orf19 } \\
\text { Number }^{c}\end{array}$} & \multirow[b]{3}{*}{ GO Annotation (Molecular Function) ${ }^{d}$} & \multirow{2}{*}{\multicolumn{2}{|c|}{ S2vS1 }} & \multirow{2}{*}{\multicolumn{2}{|c|}{$\frac{\mathrm{UPC2}^{\mathrm{S} 2-1} \text { vs. } U P C 2^{\mathrm{S} 1-1}}{\text { Strain A }}$}} & \multirow{2}{*}{\multicolumn{2}{|c|}{$\frac{\mathrm{UPC2}^{\mathrm{S} 2-1} \text { vs. } U P C 2^{\mathrm{S} 1-1}}{\text { Strain B }}$}} \\
\hline & & & & & & & & & \\
\hline & & & & 1 & 2 & 1 & 2 & 1 & 2 \\
\hline IPF708 & -- & orf19.5370 & 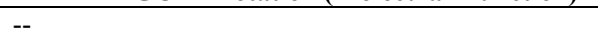 & 1.1 & -1.9 & 1. & 2.0 & 1.6 & 1.5 \\
\hline IPF8904 & -- & orf19.1363 & -- & -2.4 & 1.3 & 2. & 1.5 & 1.8 & 1.7 \\
\hline IPF9370 & -- & orf19.1424 & -- & -1.2 & 1.4 & 1. & 1.6 & 1.7 & 2.0 \\
\hline
\end{tabular}

${ }^{a}$ Gene name at CandidaDB (http://genolist.pasteur.fr/CandidaDB/).

${ }^{b}$ Gene name at CGD (http://www.candidagenome.org/).

${ }^{c}$ orf19 nomenclature according to the Assembly 19 version.

${ }^{d} \mathrm{GO}$ annotation found at CGD (http://www.candidagenome.org/). 
Table C-2. Genes down-regulated in either S2 or gain-of-function S2 allele in SC5314.

\begin{tabular}{|c|c|c|c|c|c|c|c|c|c|}
\hline \multirow{3}{*}{$\begin{array}{c}\text { CandidaDB } \\
\text { Name }^{a}\end{array}$} & \multirow{3}{*}{$\begin{array}{c}\text { CGD } \\
\text { Name }^{b}\end{array}$} & \multirow{3}{*}{$\begin{array}{c}\text { orf19 } \\
\text { Number }^{c}\end{array}$} & \multirow[b]{3}{*}{ GO Annotation (Molecular Function) ${ }^{d}$} & \multirow{2}{*}{\multicolumn{2}{|c|}{ S2vS1 }} & \multirow{2}{*}{\multicolumn{2}{|c|}{$\begin{array}{c}\mathrm{UPC2}^{\mathrm{S} 2-1} \text { vs. } U P C 2^{\mathrm{S} 1-1} \\
\text { Strain } \mathrm{A}\end{array}$}} & \multirow{2}{*}{\multicolumn{2}{|c|}{$\begin{array}{c}\mathrm{UPC2}^{\mathrm{S} 2-1} \text { vs. } U P C 2^{\mathrm{S} 1-1} \\
\text { Strain B }\end{array}$}} \\
\hline & & & & & & & & & \\
\hline & & & & 1 & 2 & 1 & 2 & 1 & 2 \\
\hline COQ1 & -- & orf19.7478 & Trans-hexaprenyltranstransferase activity & -2.8 & -2.9 & -1.5 & -2.5 & -2.4 & -3.8 \\
\hline$M A S 2$ & -- & orf19.6295 & Mitochondrial processing peptidase activity & -1.6 & -1.7 & -1.6 & -2.3 & -2.7 & -2.9 \\
\hline MET14 & MET14 & orf19.946 & Adenylylsulfate kinase activity & -5.6 & -6.9 & -2.3 & -2.9 & -3.5 & -3.7 \\
\hline MET3 & MET3 & orf19.5025 & Sulfate adenylyltransferase (ATP) activity & -4.2 & -3.8 & -3.3 & -4.0 & -3.5 & -5.9 \\
\hline PET127 & -- & orf19.2309 & RNA binding & -1.7 & -1.8 & -2.0 & -4.4 & -7.7 & -5.9 \\
\hline IPF11801 & -- & orf19.2798 & Helicase activity & -1.6 & -1.7 & -2.3 & -2.2 & -2.0 & -1.8 \\
\hline IPF14872 & -- & orf19.4133 & -- & -2.0 & -1.8 & -1.8 & -3.5 & -2.3 & -4.0 \\
\hline IPF20118 & -- & orf19.3846 & -- & -3.8 & -4.4 & -3.6 & -3.1 & -1.7 & -1.6 \\
\hline IPF525 & -- & orf19.7085 & -- & -2.4 & -2.2 & -1.7 & -3.1 & -1.7 & -1.7 \\
\hline IPF6504 & -- & orf19.1544 & -- & -2.1 & -2.0 & -1.8 & -1.5 & -1.7 & -1.6 \\
\hline IPF7334 & -- & orf19.3724 & -- & -2.3 & -2.2 & -1.6 & -3.1 & -1.8 & -3.4 \\
\hline BUL1 & -- & orf19.4629 & -- & -2.0 & -1.7 & -1.8 & -1.1 & -1.2 & -1.1 \\
\hline CDC11 & $C D C 11$ & orf19.5691 & Structural constituent of cytoskeleton & -1.8 & -1.8 & -1.1 & -2.0 & -1.3 & -1.9 \\
\hline CDC62 & -- & orf19.5242 & ATPase activity & -1.6 & -1.7 & 1.9 & 6.7 & -1.3 & 1.6 \\
\hline Cirt3 & -- & orf19.4918 & -- $\quad$ - & -2.5 & -2.5 & -1.2 & -1.4 & 1.3 & -1.2 \\
\hline $\operatorname{COS} 162$ & -- & orf19.4240 & -- & -1.6 & -1.6 & 1.1 & 1.1 & -1.3 & -1.2 \\
\hline CTF8 & CTF8 & orf19.576 & -- & -1.8 & -1.7 & -1.8 & 1.3 & -1.6 & -1.1 \\
\hline CVB1 & -- & orf19.1970 & -- & -1.6 & -1.7 & -1.2 & -1.3 & 1.3 & -1.5 \\
\hline$D A P 2$ & $D A P 2$ & orf19.4322 & Dipeptidyl-peptidase and tripeptidyl-peptidase activity & -1.8 & -2.0 & -1.1 & -1.1 & 1.2 & -1.1 \\
\hline$D C G 1$ & $D C G 1$ & orf19.244 & -- & -1.8 & -1.8 & -2.1 & -1.1 & -1.1 & -1.9 \\
\hline FIP1 & -- & orf19.4258 & -- & -2.2 & -2.4 & 1.3 & 4.5 & -1.7 & -1.1 \\
\hline FOL2 & -- & orf19.3957 & GTP cyclohydrolase I activity & -1.6 & -1.6 & 1.1 & -1.4 & -1.2 & -1.3 \\
\hline GCN2 & GCN2 & orf19.6913 & Eukaryotic translation initiation factor 2alpha kinase activity & -1.6 & -1.6 & -1.1 & 1.3 & -1.2 & -1.4 \\
\hline GLR1 & GLR1 & orf19.4147 & Glutathione-disulfide reductase activity & -1.6 & -1.6 & -1.0 & -1.1 & -1.1 & -1.2 \\
\hline GNA1 & GNA1 & orf19.837 & Glucosamine 6-phosphate N-acetyltransferase activity & -1.7 & -3.1 & -1.0 & 1.6 & -1.2 & -1.0 \\
\hline HNM3 & HNM3 & orf19.2587 & Choline transporter activity & -3.1 & -2.3 & 1.1 & 1.0 & -1.1 & -1.1 \\
\hline HSL1 & HSL1 & orf19.4308 & Protein kinase activity & -2.3 & -1.8 & 1.1 & -1.5 & 1.3 & -1.4 \\
\hline IFG4 & DAOI & orf19.3065 & -- & -1.6 & -1.6 & 1.0 & -1.0 & 1.2 & -1.0 \\
\hline IFN2 & GIT4 & orf19.1980 & Phospholipid transporter activity & -2.0 & -2.4 & -1.2 & -1.7 & 2.2 & 1.7 \\
\hline IFQ3 & RHD1 & orf19.54 & -- & -1.8 & -1.7 & -1.2 & -1.2 & 1.0 & -1.2 \\
\hline IMG1 & -- & orf19.1967 & Structural constituent of ribosome & -2.6 & -1.8 & -1.2 & -1.0 & -1.6 & -2.1 \\
\hline LEU3 & LEU3 & orf19.4225 & Transcription factor activity & -1.7 & -2.1 & 1.4 & -1.3 & -1.1 & -1.3 \\
\hline$M A C 1$ & $M A C 1$ & orf19.7068 & Specific RNA polymerase II transcription factor activity & -1.8 & -1.6 & 1.0 & -1.4 & -1.2 & -1.2 \\
\hline MAK31 & -- & orf19.1968.1 & Amino acid $\mathrm{N}$-acetyltransferase activity & -3.0 & -6.2 & 1.4 & 1.4 & -1.2 & -1.2 \\
\hline MAL31 & MAL31 & orf19.3981 & Alpha-glucoside:hydrogen symporter activity & -2.1 & -1.7 & -1.9 & -1.4 & 1.6 & -1.4 \\
\hline MIG1 & MIG1 & orf19.4318 & Transcription factor activity & -1.6 & -1.7 & -1.1 & -1.4 & -1.5 & -1.8 \\
\hline MNN22 & MNN22 & orf19.3803 & Alpha-1,2-mannosyltransferase activity & -2.2 & -2.3 & 1.1 & -1.8 & 1.5 & 1.0 \\
\hline MRPL36 & -- & orf19.3205 & Structural constituent of ribosome & -2.1 & -1.7 & -1.3 & -1.3 & -2.1 & -2.0 \\
\hline MYO1 & -- & orf19.6294 & Microfilament motor activity & -1.6 & -4.1 & 1.2 & 3.3 & -1.0 & 1.0 \\
\hline NMD2 & -- & orf19.864 & Protein binding & -2.0 & -3.4 & 1.3 & -3.8 & -1.5 & 1.0 \\
\hline
\end{tabular}


Table C-2 (continued).

\begin{tabular}{|c|c|c|c|c|c|c|c|c|c|}
\hline \multirow{3}{*}{$\begin{array}{l}\text { CandidaDB } \\
\text { Name }^{a}\end{array}$} & \multirow{3}{*}{$\begin{array}{l}\text { CGD } \\
\text { Name }^{b}\end{array}$} & \multirow{3}{*}{$\begin{array}{c}\text { orf19 } \\
\text { Number }^{c}\end{array}$} & \multirow[b]{3}{*}{ GO Annotation (Molecular Function) ${ }^{d}$} & \multirow{2}{*}{\multicolumn{2}{|c|}{ S2vS1 }} & \multicolumn{2}{|c|}{$\mathrm{UPC2}^{\mathrm{S} 2-1}$ vs. $U P C 2^{\mathrm{S} 1-1}$} & \multicolumn{2}{|c|}{$\mathrm{UPC2}^{\mathrm{S2-1}}$ vs. $U P C 2^{\mathrm{S1-}}$} \\
\hline & & & & & & & & & \\
\hline & & & & 1 & 2 & 1 & 2 & 1 & 2 \\
\hline NUP2.3eoc & -- & orf19.3583 & Structural molecule activity & -2.0 & -2.4 & 1.0 & -1.6 & 1.2 & -1.2 \\
\hline NUP49 & NUP49 & orf19.4987 & Structural molecule activity & -1.6 & -1.6 & 1.1 & -1.1 & -1.7 & -1.1 \\
\hline$O G G 1$ & -- & orf19.7190 & Oxidized purine base lesion DNA N-glycosylase activity & -1.7 & -1.6 & -1.1 & -2.0 & -1.7 & -2.2 \\
\hline OPT1 & OPT1 & orf19.2602 & Oligopeptide transporter activity & -3.0 & -2.2 & -1.1 & 1.0 & 1.1 & -1.1 \\
\hline PAF1 & -- & orf19.3613 & RNA polymerase II transcription elongation factor activity & -1.6 & -2.3 & 1.3 & -1.0 & -1.4 & 1.1 \\
\hline PAP11 & PAPl & orf19.3197 & RNA binding & -1.6 & -2.9 & 1.1 & -1.1 & 1.2 & -1.1 \\
\hline PEL1 & PEL1 & orf19.7072 & $\begin{array}{l}\text { CDP-diacylglycerol-glycerol-3-phosphate 3-phosphatidyltransferase } \\
\text { activity }\end{array}$ & -1.8 & -2.2 & 1.2 & -2.4 & -2.0 & -2.6 \\
\hline$P R C 2$ & PRC2 & orf19.4135 & Carboxypeptidase $\mathrm{C}$ activity & -3.0 & -2.0 & -1.3 & -1.2 & 1.2 & 1.3 \\
\hline PSD2.3f & -- & orf19.3954 & Phosphatidylserine decarboxylase activity & -1.9 & -2.5 & 1.2 & -1.1 & -1.2 & -1.4 \\
\hline PUS4 & PUS4 & orf19.1954 & Pseudouridylate synthase activity & -1.6 & -2.2 & 1.2 & -1.3 & -1.2 & -1.9 \\
\hline PZF1 & -- & orf19.4125 & RNA polymerase III transcription factor activity & -1.8 & -1.9 & -1.2 & 1.4 & -1.7 & -2.4 \\
\hline RAD51 & RAD51 & orf19.3752 & Recombinase activity & -1.6 & -1.8 & 1.0 & -1.3 & -1.0 & -1.2 \\
\hline RET2 & RET2 & orf19.4236 & Protein binding & -1.8 & -1.6 & -1.1 & -1.3 & -1.0 & -1.2 \\
\hline RMT2 & $R M T 2$ & orf19.920 & -- & -1.6 & -1.9 & 1.3 & -1.6 & -2.2 & -2.3 \\
\hline RPC82 & -- & orf19.2847 & DNA-directed RNA polymerase activity & -2.6 & -4.3 & 1.3 & -1.5 & -1.7 & -2.8 \\
\hline RVS161 & -- & orf19.7124 & Cytoskeletal protein binding & -1.9 & -2.0 & -1.0 & -1.3 & -1.1 & -1.3 \\
\hline SEF11.5eoc & $S E F 2$ & orf19.1926 & -- & -1.7 & -3.3 & 1.1 & -1.4 & -1.3 & -1.7 \\
\hline SMC4 & -- & orf19.964 & ATPase activity & -2.5 & -1.7 & -1.0 & -1.6 & 1.3 & -1.5 \\
\hline SPE2 & SPE2 & orf19.568 & Adenosylmethionine decarboxylase activity & -1.6 & -1.6 & 1.1 & -1.4 & -2.0 & -2.2 \\
\hline SSA4 & HSP70 & orf19.4980 & Unfolded protein binding & -4.2 & -4.8 & -3.4 & -1.3 & 1.5 & -1.1 \\
\hline SSP120 & -- & orf19.3173 & -- & -1.6 & -2.5 & 1.2 & 1.6 & 1.1 & -1.3 \\
\hline SUA5 & -- & orf19.7088 & -- & -1.8 & -2.1 & -1.0 & -1.4 & -4.0 & -3.4 \\
\hline SUL1 & SUL2 & orf19.2738 & Sulfate transporter activity & -2.5 & -5.8 & -1.9 & -1.3 & 1.1 & -1.5 \\
\hline TOF1 & YBL053 & orf19.4136 & -- & -2.2 & -2.9 & 1.1 & -1.6 & -1.2 & -1.8 \\
\hline$T R X 2$ & -- & orf19.1976 & Thiol-disulfide exchange intermediate activity & -1.6 & -2.3 & -1.3 & 1.1 & -1.8 & -1.7 \\
\hline VPS4 & VPS4 & orf19.4339 & ATPase activity & -2.9 & -1.7 & 1.0 & -1.2 & 1.1 & 1.1 \\
\hline IPF1047 & -- & orf19.4563 & -- & -1.6 & -2.1 & 1.2 & -1.2 & -3.1 & -3.0 \\
\hline IPF10521 & -- & orf19.4062 & -- & -1.6 & -2.0 & -1.2 & -1.0 & -1.5 & -1.7 \\
\hline IPF10711 & -- & orf19.3694 & -- & -2.0 & -1.7 & 1.3 & -1.4 & 1.0 & -1.0 \\
\hline IPF10785.exon1 & -- & orf19.5732 & -- & -1.8 & -4.1 & 1.9 & 1.2 & -1.5 & -1.4 \\
\hline IPF10795 & CRZ2 & orf19.2356 & Transcription factor activity & -1.6 & -1.7 & 1.3 & -2.2 & -1.6 & -3.3 \\
\hline IPF11027 & -- & orf19.5722 & -- & -1.9 & -1.7 & -1.1 & -1.5 & -2.1 & -2.7 \\
\hline IPF11090.exon1 & -- & orf19.4622 & -- & -1.7 & -1.7 & 1.3 & 3.1 & 1.2 & -1.4 \\
\hline IPF11161.3 & MTLA1 & orf19.3201 & Transcription factor activity & -2.4 & -3.6 & -1.4 & 1.1 & -1.3 & -1.8 \\
\hline IPF11167 & $O B P A$ & orf19.3198 & Oxysterol binding & -1.6 & -2.3 & -1.1 & -1.3 & -1.1 & -1.5 \\
\hline IPF1129 & -- & orf19.5431 & -- & -2.5 & -1.8 & -1.0 & -1.2 & -1.9 & -3.1 \\
\hline IPF11344 & -- & orf19.4030 & -- & -2.9 & -3.5 & -1.4 & -1.8 & -1.3 & -1.6 \\
\hline IPF11434.5f & -- & orf19.6462 & -- & -2.1 & -1.9 & 1.2 & -1.0 & 1.3 & 1.0 \\
\hline IPF11492 & -- & orf19.2392 & -- & -1.6 & -1.8 & 1.2 & -1.1 & -1.4 & -1.6 \\
\hline
\end{tabular}


Table C-2 (continued).

\begin{tabular}{|c|c|c|c|c|c|c|c|c|c|}
\hline \multirow{3}{*}{$\begin{array}{c}\text { CandidaDB } \\
\text { Name }^{a}\end{array}$} & \multirow{3}{*}{$\begin{array}{c}\text { CGD } \\
\text { Name }^{b}\end{array}$} & \multirow{3}{*}{$\begin{array}{c}\text { orf19 } \\
\text { Number }^{c}\end{array}$} & \multirow[b]{3}{*}{ GO Annotation (Molecular Function) ${ }^{d}$} & \multirow{2}{*}{\multicolumn{2}{|c|}{ S2vS1 }} & \multirow{2}{*}{\multicolumn{2}{|c|}{$\begin{array}{c}\mathrm{UPC2}^{\mathrm{S} 2-1} \text { vs. } U P C 2^{\mathrm{S} 1-1} \\
\text { Strain A }\end{array}$}} & \multirow{2}{*}{\multicolumn{2}{|c|}{$\begin{array}{c}\mathrm{UPC2}^{\mathrm{S} 2-1} \text { vs. } U P C 2^{\mathrm{S} 1-1} \\
\text { Strain B }\end{array}$}} \\
\hline & & & & & & & & & \\
\hline & & & & 1 & 2 & 1 & 2 & 1 & 2 \\
\hline IPF11756 & -- & orf19.7006 & ( & -2.1 & -2.1 & 1.2 & -1.8 & 1.4 & 1.2 \\
\hline IPF11987.3eoc & -- & orf19.692 & -- & -3.9 & -1.7 & 1.2 & 1.1 & 1.6 & -1.4 \\
\hline IPF11989 & -- & orf19.3625 & -- & -1.7 & -2.9 & -1.2 & -12.7 & -1.1 & -1.5 \\
\hline IPF12141 & -- & orf19.1952 & -- & -1.7 & -1.7 & -1.1 & -2.2 & -2.0 & -2.1 \\
\hline IPF12790 & -- & orf19.3180 & Inositol-polyphosphate 5-phosphatase activity & -1.7 & -1.7 & -1.2 & -1.1 & 1.6 & -1.0 \\
\hline IPF12802 & -- & orf19.2513 & -- & -1.6 & -2.0 & 3.6 & -3.0 & 1.5 & -5.0 \\
\hline IPF13142 & -- & orf19.53 & -- & -2.0 & -3.6 & -1.5 & -1.5 & -1.0 & -1.7 \\
\hline IPF13247 & -- & orf19.3376 & -- & -7.4 & -1.6 & -1.1 & 1.2 & 1.4 & 1.2 \\
\hline IPF13407 & -- & orf19.753 & Alpha-1,3-mannosyltransferase activity & -1.6 & -4.2 & -1.1 & 1.4 & 1.2 & -1.1 \\
\hline IPF13683 & -- & orf19.2547 & -- & -1.8 & -1.7 & 1.3 & -1.5 & -6.6 & -3.4 \\
\hline IPF14348.3 & -- & orf19.1207 & -- & -1.6 & -3.3 & 1.4 & 1.0 & 1.0 & -1.2 \\
\hline IPF14487 & -- & orf19.6346 & -- & -2.8 & -2.8 & -1.3 & 2.1 & -1.7 & -1.5 \\
\hline IPF14506 & -- & orf19.1961 & -- & -2.4 & -3.4 & 1.0 & -1.2 & 1.3 & 1.1 \\
\hline IPF14663 & -- & orf19.4316 & -- & -2.1 & -2.0 & 1.1 & -1.2 & -1.2 & -1.1 \\
\hline IPF14743 & -- & orf19.3425 & -- & -1.7 & -1.8 & 1.2 & 1.0 & -1.0 & 1.0 \\
\hline IPF14871 & -- & orf19.4132 & -- & -1.8 & -2.1 & -1.3 & 1.1 & 1.2 & 1.3 \\
\hline IPF1497 & -- & orf19.351 & -- & -1.7 & -1.6 & -1.2 & 1.4 & 1.0 & -1.0 \\
\hline IPF14985 & -- & orf19.4134 & -- & -2.9 & -2.2 & -1.1 & -1.0 & 1.3 & 1.0 \\
\hline IPF1557 & -- & orf19.954 & -- & -1.6 & -1.6 & 1.5 & 1.2 & -1.2 & -1.1 \\
\hline IPF1567 & -- & orf19.961.2 & Structural constituent of ribosome & -1.7 & -1.6 & -1.5 & 1.3 & -1.0 & -1.1 \\
\hline IPF15811 & -- & orf19.4280 & -- & -1.6 & -2.5 & -2.9 & -1.3 & -1.2 & -1.1 \\
\hline IPF15834 & -- & orf19.4675 & -- & -2.2 & -1.9 & -1.2 & -1.3 & -2.7 & -2.1 \\
\hline IPF16141 & -- & orf19.2260 & Transcription factor activity & -2.2 & -2.0 & -1.0 & -2.2 & -1.0 & -2.1 \\
\hline IPF16758 & -- & orf19.5043 & -- & -1.6 & -2.5 & -- & -- & -- & -- \\
\hline IPF16947 & -- & orf19.3286 & -- & -2.9 & -3.5 & -1.2 & 1.2 & 1.6 & -1.1 \\
\hline IPF16988 & -- & orf19.4312 & -- & -2.7 & -8.7 & -- & -- & -- & -- \\
\hline IPF17031 & -- & orf19.2544 & -- & -2.0 & -2.1 & 1.2 & -1.5 & -2.1 & -1.7 \\
\hline IPF17050 & -- & orf19.1049 & -- & -1.5 & -1.6 & -3.1 & -1.4 & -1.7 & -1.8 \\
\hline IPF17186 & -- & orf19.251 & -- & -1.8 & -2.2 & -1.7 & -1.6 & -1.3 & -1.3 \\
\hline IPF17296 & -- & orf19.175 & Transcription factor activity & -3.3 & -1.8 & -12.3 & 1.1 & -7.3 & 1.0 \\
\hline IPF17998 & -- & orf19.3147 & -- & -1.8 & -1.8 & -1.1 & -1.5 & -1.6 & -2.0 \\
\hline IPF18517.5f & -- & orf19.4349 & -- & -3.7 & -33.3 & 1.1 & 1.1 & -1.8 & -1.6 \\
\hline IPF19310.3f & -- & -- - - & -- & -2.2 & -14.6 & -1.9 & \#DIV/0! & 11.0 & -1.9 \\
\hline IPF1956 & -- & orf19.7159 & -- & -2.5 & -1.7 & 1.1 & -1.3 & -1.2 & -2.4 \\
\hline IPF19622 & -- & orf19.115 & -- & -2.7 & -1.7 & -1.1 & -2.2 & -1.5 & 1.1 \\
\hline IPF19775 & -- & orf19.4282 & -- & -2.2 & -1.9 & 1.4 & 1.2 & -1.0 & -1.5 \\
\hline IPF19814 & -- & orf19.7106 & -- & -1.6 & -1.8 & 1.1 & -1.0 & 1.8 & 1.2 \\
\hline IPF19924 & -- & orf19.4324 & -- & -1.6 & -6.7 & -1.1 & 1.6 & -1.8 & -1.3 \\
\hline IPF19937 & -- & orf19.4740 & Aminoacyl-tRNA hydrolase activity & -1.9 & -1.6 & -1.3 & 1.2 & 1.2 & -1.1 \\
\hline IPF2359 & -- & orf19.7022 & -- & -1.7 & -2.1 & -1.5 & -1.7 & -1.2 & -1.8 \\
\hline
\end{tabular}


Table C-2 (continued).

\begin{tabular}{|c|c|c|c|c|c|c|c|c|c|}
\hline \multirow{3}{*}{$\begin{array}{c}\text { CandidaDB } \\
\text { Name }^{a}\end{array}$} & \multirow{3}{*}{$\begin{array}{c}\text { CGD } \\
\text { Name }^{b}\end{array}$} & \multirow{3}{*}{$\begin{array}{c}\text { orf19 } \\
\text { Number }^{c}\end{array}$} & \multirow[b]{3}{*}{ GO Annotation (Molecular Function) ${ }^{d}$} & \multirow{2}{*}{\multicolumn{2}{|c|}{ S2vS1 }} & \multirow{2}{*}{\multicolumn{2}{|c|}{$\begin{array}{c}\mathrm{UPC2}^{\mathrm{S} 2-1} \text { vs. } U P C 2^{\mathrm{S} 1-1} \\
\text { Strain A }\end{array}$}} & \multirow{2}{*}{\multicolumn{2}{|c|}{$\begin{array}{c}\mathrm{UPC2}^{\mathrm{S} 2-1} \text { vs. } U P C 2^{\mathrm{S} 1-1} \\
\text { Strain B }\end{array}$}} \\
\hline & & & & & & & & & \\
\hline & & & & 1 & 2 & 1 & 2 & 1 & 2 \\
\hline IPF2522 & -- & orf19.7460 & ( & -1.7 & -1.6 & -1.1 & -1.0 & -1.2 & 1.3 \\
\hline IPF2929 & -- & orf19.4306 & -- & -1.6 & -1.6 & -1.1 & -1.5 & -1.4 & -1.6 \\
\hline IPF2953 & -- & orf19.4294 & -- & -1.6 & -2.4 & 1.0 & -1.0 & -1.9 & -1.7 \\
\hline IPF2997 & -- & orf19.6983 & -- & -1.8 & -1.8 & 1.1 & 1.1 & 1.3 & -1.4 \\
\hline IPF3087 & MET28 & orf19.7046 & Specific RNA polymerase II transcription factor activity & -1.9 & -1.8 & -1.5 & -2.3 & -2.0 & -2.8 \\
\hline IPF3144 & -- & orf19.4220 & -- & -1.8 & -1.6 & -1.3 & -1.2 & -1.0 & -1.3 \\
\hline IPF3147 & RIX7 & orf19.4219 & Helicase activity & -1.7 & -1.7 & 1.4 & -1.1 & -1.9 & -1.7 \\
\hline IPF3161 & -- & orf19.3161 & -- & -2.0 & -1.9 & -1.1 & -2.4 & -2.3 & -2.6 \\
\hline IPF3178 & -- & orf19.3170 & -- & -2.1 & -1.8 & 1.2 & -2.5 & -2.1 & -3.9 \\
\hline IPF3621 & -- & orf19.6712 & -- & -1.7 & -1.6 & -1.2 & -1.4 & -1.3 & -1.5 \\
\hline IPF3903 & -- & orf19.760 & Structural constituent of ribosome & -1.6 & -1.6 & -1.3 & 1.7 & -1.5 & -1.7 \\
\hline IPF4279 & -- & orf19.547 & Flap endonuclease activity & -1.8 & -1.9 & 1.1 & -3.3 & -2.3 & -2.2 \\
\hline IPF4450 & POL93 & orf19.6078 & -- & -1.9 & -1.7 & -1.3 & -1.2 & -1.6 & -2.4 \\
\hline IPF448 & -- & orf19.7051 & -- & -1.6 & -1.7 & -1.2 & -1.0 & -1.8 & -1.7 \\
\hline IPF4531 & -- & orf19.6989 & rRNA primary transcript binding & -3.0 & -1.7 & -2.7 & -1.1 & -1.8 & 1.1 \\
\hline IPF495 & -- & orf19.7069 & -- & -1.7 & -1.6 & 1.2 & -1.5 & -1.3 & -1.7 \\
\hline IPF511 & -- & orf19.7078 & -- & -1.7 & -1.7 & -1.4 & -1.3 & -1.4 & -1.6 \\
\hline IPF5330 & -- & orf19.3448 & -- & -1.6 & -1.9 & -1.5 & -2.0 & -1.5 & -1.4 \\
\hline IPF539 & -- & orf19.7092 & -- & -2.1 & -1.6 & 1.3 & -1.0 & 1.3 & -1.0 \\
\hline IPF5466 & -- & orf19.5683 & -- & -3.1 & -2.4 & -1.1 & 1.6 & 1.4 & 1.3 \\
\hline IPF560 & -- & orf19.7102 & -- & -1.7 & -2.8 & -1.2 & -1.1 & -1.0 & -1.7 \\
\hline IPF5615 & -- & orf19.7107 & -- & -1.7 & -1.8 & 1.1 & -1.7 & -1.8 & -1.6 \\
\hline IPF5621 & -- & orf19.7111 & -- & -1.8 & -2.1 & -1.3 & -1.1 & 1.1 & -1.3 \\
\hline IPF563 & -- & orf19.7103 & -- & -2.1 & -1.6 & 1.1 & -1.2 & 1.1 & -1.2 \\
\hline IPF5644 & -- & orf19.7116 & RNA polymerase II transcription elongation factor activity & -2.6 & -2.4 & 1.0 & -1.3 & -1.3 & -1.3 \\
\hline IPF5661 & -- & orf19.7125 & -- & -2.0 & -2.2 & 1.8 & 252.0 & 2.3 & 1.7 \\
\hline IPF5818 & -- & orf19.3821 & -- & -1.6 & -2.1 & -1.3 & -1.2 & -1.1 & -1.6 \\
\hline IPF6252 & -- & orf19.1458 & -- & -1.9 & -2.0 & 1.7 & 1.8 & -1.5 & -1.9 \\
\hline IPF6296 & -- & orf19.1966 & -- & -2.8 & -1.8 & 1.6 & -4.6 & -1.3 & -1.9 \\
\hline IPF6396 & -- & orf19.580 & -- & -2.3 & -2.4 & -1.3 & -2.3 & -1.2 & -1.5 \\
\hline IPF6617.repeat 2 & $L D G 3$ & orf19.6486 & -- & -37.3 & -5.1 & 2.3 & 1.4 & 1.3 & 3.7 \\
\hline IPF662 & -- & orf19.7601 & rRNA (uridine-2'-O-)-methyltransferase activity & -1.6 & -1.8 & -1.1 & -1.5 & -2.9 & -2.6 \\
\hline IPF6712.5f & -- & orf19.1414 & -- & -1.6 & -2.2 & 1.2 & -1.1 & 1.0 & -1.0 \\
\hline IPF7088 & -- & orf19.486 & Microtubule binding & -1.8 & -1.6 & 1.1 & -1.6 & 1.4 & 1.4 \\
\hline IPF7204 & PGA26 & orf19.2475 & -- - - - & -4.6 & -3.0 & -1.3 & -1.2 & -1.2 & -1.2 \\
\hline IPF7400 & -- & orf19.1802 & -- & -1.6 & -1.6 & -1.2 & 1.1 & 1.3 & 1.2 \\
\hline IPF7466 & -- & orf19.2051 & -- & -1.7 & -2.5 & 1.5 & -1.0 & -1.4 & -1.4 \\
\hline IPF7616 & -- & orf19.1159 & Homoserine $\mathrm{O}$-acetyltransferase activity & -2.3 & -1.9 & -1.0 & -2.6 & -1.9 & -1.9 \\
\hline IPF7669.3 & -- & orf19.450 & -- & -3.5 & -1.6 & -1.6 & 1.1 & 1.1 & 1.3 \\
\hline IPF7968 & -- & orf19.2693 & Transcription corepressor activity & -1.8 & -1.8 & -1.7 & -1.4 & 1.3 & -1.2 \\
\hline
\end{tabular}


Table C-2 (continued).

\begin{tabular}{|c|c|c|c|c|c|c|c|c|c|}
\hline \multirow{3}{*}{$\begin{array}{l}\text { CandidaDB } \\
\text { Name }^{a}\end{array}$} & \multirow{3}{*}{$\begin{array}{c}\text { CGD } \\
\text { Name }^{b}\end{array}$} & \multirow{3}{*}{$\begin{array}{c}\text { orf19 } \\
\text { Number }^{c}\end{array}$} & \multirow[b]{3}{*}{ GO Annotation (Molecular Function) ${ }^{d}$} & \multirow{2}{*}{\multicolumn{2}{|c|}{ S2vS1 }} & \multicolumn{2}{|c|}{$\mathrm{UPC2}^{\mathrm{S2-1}}$ vs. $U P C 2^{\mathrm{S} 1-1}$} & \multirow{2}{*}{\multicolumn{2}{|c|}{$\frac{\mathrm{UPC2}^{\mathrm{S2}-1} \text { vs. } U P C 2^{\mathrm{S} 1-1}}{\text { Strain B }}$}} \\
\hline & & & & & & & & & \\
\hline & & & & 1 & 2 & 1 & 2 & 1 & 2 \\
\hline IPF8421 & -- & orf19.928 & ( & -1.7 & -1.6 & 1.1 & -1.4 & 1.4 & -1.2 \\
\hline IPF8535 & $P G A 31$ & orf19.5302 & -- & -4.0 & -2.6 & 1.3 & 1.5 & 1.5 & -1.1 \\
\hline IPF8741.5f & RAD9 & orf19.4275 & -- & -1.9 & -2.5 & -1.1 & -1.3 & -1.1 & 1.0 \\
\hline IPF8901 & -- & orf19.3758 & -- & -2.5 & -3.6 & 1.6 & 2.0 & -1.4 & 1.3 \\
\hline IPF8996 & -- & orf19.4634 & -- & -1.9 & -1.8 & -1.0 & 1.1 & -2.0 & -2.1 \\
\hline IPF9417 & СТА & orf19.4775 & Transcription factor activity & -1.7 & -1.8 & 1.8 & -1.7 & -1.9 & -1.4 \\
\hline IPF9522.53f & SPR 28 & orf19.4266 & Structural constituent of cytoskeleton & -2.0 & -1.9 & -1.6 & 7.7 & -1.0 & -1.0 \\
\hline IPF9525 & -- & orf19.4268 & snoRNA binding & -2.0 & -2.4 & 1.1 & -1.3 & -2.7 & -2.1 \\
\hline IPF9529 & -- & orf19.4270 & Alpha-1,3-mannosyltransferase activity & -1.8 & -1.7 & 1.1 & -1.4 & -1.3 & -1.2 \\
\hline IPF9566 & -- & orf19.1939 & -- & -1.8 & -1.6 & 1.3 & 1.9 & -2.0 & -1.0 \\
\hline IPF9568 & -- & orf19.1938 & -- & -1.7 & -2.3 & -1.4 & -1.7 & -1.7 & 1.2 \\
\hline IPF9580 & -- & orf19.687 & -- & -1.7 & -2.1 & 1.1 & 1.3 & -1.1 & -1.6 \\
\hline IPF9841 & -- & orf19.3204 & -- & -1.8 & -2.2 & -2.3 & -1.1 & -3.0 & -2.2 \\
\hline IPF9972.3eoc & -- & orf19.4522 & -- & -1.6 & -1.6 & -1.9 & 1.6 & -2.0 & 1.4 \\
\hline ALS10 & ALS10 & orf19.2355 & Cell adhesion molecule binding & -1.1 & -1.3 & -2.4 & -1.7 & -1.7 & -1.9 \\
\hline ALS3.3eoc & ALS10 & orf19.2355 & Cell adhesion molecule binding & 1.5 & -1.1 & -2.3 & -2.0 & -2.2 & -2.0 \\
\hline ATP11 & -- & orf19.6916 & -- & 1.7 & -2.1 & -1.9 & -2.0 & -3.1 & -2.1 \\
\hline CUS2 & -- & orf19.5767 & RNA binding & -1.2 & -1.2 & -2.1 & -2.0 & -1.7 & -1.6 \\
\hline ECE1 & ECE1 & orf19.3374 & -- & -1.2 & -6.0 & -7.0 & -3.8 & -4.6 & -3.5 \\
\hline FRE7 & -- & orf19.7077 & Ferric-chelate reductase activity & -2.1 & -1.1 & -1.6 & -1.6 & -2.3 & -2.5 \\
\hline GCN5 & -- & orf19.705 & Histone acetyltransferase activity & 1.1 & 1.0 & -1.7 & -1.7 & -1.7 & -2.1 \\
\hline HGT11 & $H G T 1$ & orf19.4527 & Glucose transporter activity & -1.4 & 1.0 & -3.2 & -1.8 & -3.0 & -2.8 \\
\hline$H G T 12$ & HGT2 & orf19.3668 & Glucose transporter activity & -1.1 & 1.1 & -2.0 & -6.6 & -3.0 & -2.7 \\
\hline$H W P 1$ & $H W P 1$ & orf19.1321 & -- & 1.0 & -1.9 & -4.8 & -4.5 & -3.2 & -2.7 \\
\hline MRPL10.3 & MRPL10 & orf19.3532 & Structural constituent of ribosome & 1.5 & -1.2 & -1.5 & -2.2 & -2.1 & -2.6 \\
\hline MRS2 & MRS2 & orf19.2597 & Magnesium ion transporter activity & -1.1 & -1.6 & -1.8 & -1.9 & -1.8 & -2.2 \\
\hline MTF1 & -- & orf19.1528 & Transcription factor activity & -1.5 & -1.4 & -1.6 & -4.0 & -3.7 & -4.9 \\
\hline NAM2 & NAM2 & orf19.5705 & Leucine-tRNA ligase activity & 1.8 & 1.5 & -1.8 & -2.4 & -1.7 & -2.4 \\
\hline NCB2 & -- & orf19.5825 & Transcription corepressor activity & 1.1 & -1.6 & -1.6 & -1.9 & -1.7 & -4.9 \\
\hline PAC10.3 & -- & orf19.5985 & -- & 1.0 & -1.1 & -1.6 & -1.7 & -2.3 & -1.9 \\
\hline PHB2 & -- & orf19.5973 & -- & -1.2 & -1.4 & -1.7 & -2.1 & -1.8 & -1.9 \\
\hline$R R P 45$ & -- & orf19.4078 & 3'-5'-exoribonuclease activity & 1.3 & -3.3 & -1.7 & -1.9 & -3.3 & -2.3 \\
\hline SEC9 & -- & orf19.117 & -- & 1.5 & -1.5 & -1.9 & -2.2 & -1.9 & -1.8 \\
\hline SOU1 & SOU1 & orf19.2896 & 2,4-dienoyl-CoA reductase (NADPH) activity & -1.2 & -1.4 & -3.0 & -2.2 & -2.1 & -2.2 \\
\hline$T L G 1$ & -- & orf19.3898 & t-SNARE activity & 1.6 & -2.0 & -2.3 & -5.7 & -1.6 & -3.3 \\
\hline IPF10399 & -- & orf19.3367 & Structural constituent of ribosome & 1.1 & -1.6 & -1.8 & -1.9 & -2.6 & -3.0 \\
\hline IPF10455 & -- & orf19.104 & -- & -3.2 & -1.6 & -2.0 & -1.8 & -1.8 & -1.9 \\
\hline IPF10716 & -- & orf19.3691 & -- & -1.2 & -1.9 & -1.8 & -2.1 & -2.4 & -1.6 \\
\hline IPF12047 & -- & orf19.3471 & -- & -1.7 & -1.3 & -1.7 & -2.1 & -2.2 & -3.0 \\
\hline IPF1209 & -- & orf19.2068 & -- & -1.5 & 1.2 & -1.7 & -4.4 & -2.2 & -2.6 \\
\hline
\end{tabular}


Table C-2 (continued).

\begin{tabular}{|c|c|c|c|c|c|c|c|c|c|}
\hline \multirow{3}{*}{$\begin{array}{c}\text { CandidaDB } \\
\text { Name }^{a}\end{array}$} & \multirow{3}{*}{$\begin{array}{l}\text { CGD } \\
\text { Name }^{b}\end{array}$} & \multirow{3}{*}{$\begin{array}{c}\text { orf19 } \\
\text { Number }^{c}\end{array}$} & \multirow[b]{3}{*}{ GO Annotation (Molecular Function) $)^{d}$} & \multirow{2}{*}{\multicolumn{2}{|c|}{ S2vS1 }} & \multicolumn{2}{|c|}{$\frac{\mathrm{UPC2}^{\mathrm{S} 2-1} \text { vs. } U P C 2^{\mathrm{S} 1-1}}{\text { Strain }}$} & \multicolumn{2}{|c|}{$\mathrm{UPC2}^{\mathrm{S2-1}}$ vs. $U P C 2^{\mathrm{S1}-}$} \\
\hline & & & & & & & & & \\
\hline & & & & 1 & 2 & 1 & 2 & 1 & 2 \\
\hline IPF12152 & -- & orf19.6236 & ( & -1.1 & -5.0 & -1.7 & -2.0 & -3.2 & -3.3 \\
\hline IPF1242 & -- & orf19.7360 & -- & 1.1 & 1.0 & -1.6 & -2.3 & -2.3 & -2.1 \\
\hline IPF12844 & -- & orf19.2362 & -- & 1.1 & -1.8 & -1.8 & -1.9 & -3.8 & -5.0 \\
\hline IPF13221 & -- & orf19.1697 & -- & 1.7 & -1.7 & -1.8 & -3.7 & -2.5 & -2.5 \\
\hline IPF13402 & -- & orf19.36 & -- & 1.0 & -1.9 & -1.6 & -1.6 & -1.7 & -2.0 \\
\hline IPF13552 & -- & orf19.1300 & -- & -1.2 & -1.2 & -1.6 & -2.7 & -2.3 & -2.7 \\
\hline IPF13613 & -- & orf19.725 & -- & -1.1 & -1.3 & -1.8 & -4.6 & -2.4 & -1.7 \\
\hline IPF13782 & -- & orf19.2314 & -- & 1.9 & -3.0 & -2.0 & -2.4 & -3.4 & -5.1 \\
\hline IPF14574 & -- & orf19.1083 & -- & 1.4 & -1.2 & -1.7 & -1.7 & -1.9 & -2.4 \\
\hline IPF14921 & -- & orf19.1527 & -- & 3.4 & -1.3 & -1.8 & -1.9 & -2.1 & -3.2 \\
\hline IPF2232 & -- & orf19.6899 & -- & 3.9 & -7.0 & -1.5 & -5.6 & -1.9 & -1.8 \\
\hline IPF 2555 & -- & orf19.6628 & Structural constituent of cytoskeleton & -1.2 & -2.9 & -1.9 & -2.0 & -1.8 & -3.6 \\
\hline IPF2839 & -- & orf19.7296 & -- & -1.0 & 1.3 & -2.0 & -3.1 & -3.3 & -2.5 \\
\hline IPF2998 & -- & orf19.6982 & -- & -1.9 & -1.4 & -2.8 & -8.0 & -4.9 & -9.4 \\
\hline IPF3015 & $M O D F$ & orf19.5029 & ATP binding & -1.1 & -1.6 & -1.8 & -2.4 & -1.8 & -2.5 \\
\hline IPF312 & -- & orf19.3154 & -- & 1.0 & -1.1 & -1.6 & -2.4 & -1.6 & -2.0 \\
\hline IPF3857 & -- & orf19.25 & -- & 1.1 & -1.4 & -1.6 & -2.0 & -2.7 & -2.6 \\
\hline IPF4080 & MRP 8 & orf19.3844 & Structural constituent of ribosome & 1.5 & -1.7 & -1.6 & -1.6 & -1.6 & -1.7 \\
\hline IPF4671 & -- & orf19.1849 & -- & 1.6 & -1.7 & -1.8 & -2.2 & -1.7 & -1.7 \\
\hline IPF4880 & -- & orf19.6266 & 3'-5'-exoribonuclease activity & -1.1 & -1.2 & -1.7 & -1.6 & -2.2 & -2.9 \\
\hline IPF4991 & CSP37 & orf19.2531 & -- & 1.1 & -1.5 & -4.3 & -2.4 & -1.6 & -1.9 \\
\hline IPF5149 & -- & orf19.6530 & Signal recognition particle binding & -1.8 & -1.9 & -1.9 & -1.8 & -1.9 & -3.5 \\
\hline IPF61 & -- & orf19.5959 & snoRNA binding & 6.6 & -20.0 & -2.4 & -2.9 & -1.7 & -2.7 \\
\hline IPF6339 & -- & orf19.2724 & -- $\quad$ - n & 1.3 & -1.0 & -1.6 & -2.1 & -1.7 & -2.0 \\
\hline IPF7166 & -- & orf19.1202 & -- & -1.3 & -1.4 & -1.7 & -1.8 & -1.8 & -2.2 \\
\hline IPF8114 & -- & orf19.2939 & -- & -1.2 & -1.6 & -1.6 & -1.7 & -2.2 & -1.5 \\
\hline IPF8422 & -- & orf19.927 & -- & -1.2 & -2.7 & -1.9 & -2.3 & -2.6 & -3.5 \\
\hline IPF8727 & -- & orf19.1794 & -- & -1.8 & -3.2 & -2.0 & -11.5 & -1.6 & -9.8 \\
\hline IPF8854 & -- & orf19.4900 & Alpha-1,3-mannosyltransferase activity & -1.3 & -2.0 & -1.6 & -2.2 & -1.6 & -2.2 \\
\hline
\end{tabular}

${ }^{a}$ Gene name at CandidaDB (http://genolist.pasteur.fr/CandidaDB/).

${ }^{b}$ Gene name at CGD (http://www.candidagenome.org/).

${ }^{c}$ orf19 nomenclature according to the Assembly 19 version.

${ }^{d} \mathrm{GO}$ annotation found at CGD (http://www.candidagenome.org/). 
Table C-3. Genes down-regulated in the absence of UPC2.

\begin{tabular}{|c|c|c|c|c|c|c|c|c|c|c|c|}
\hline \multirow{3}{*}{$\begin{array}{c}\text { CandidaDB } \\
\text { Name }^{a}\end{array}$} & \multirow{3}{*}{$\begin{array}{c}\text { CGD } \\
\text { Name }^{b}\end{array}$} & \multirow{3}{*}{$\begin{array}{c}\text { orf19 } \\
\text { Number }^{c}\end{array}$} & \multirow[b]{3}{*}{ GO Annotation (Molecular Function) ${ }^{d}$} & \multicolumn{4}{|c|}{$\Delta u p c 2$ vs. SC5314 } & \multicolumn{4}{|c|}{$\mathrm{UPC2}^{\mathrm{S} 2-1}$ vs. $U P C 2^{\mathrm{S} 1-1}$} \\
\hline & & & & \multicolumn{2}{|c|}{ Strain A } & \multicolumn{2}{|c|}{ Strain B } & \multicolumn{2}{|c|}{ Strain A } & \multicolumn{2}{|c|}{ Strain B } \\
\hline & & & & 1 & 2 & 1 & 2 & 1 & 2 & 1 & 2 \\
\hline CHA12 & CHAI & orf19.1996 & L-serine ammonia-lyase activity & -1.7 & -1.9 & -1.5 & -1.9 & 1.1 & 1.1 & -1.7 & -1.2 \\
\hline FDH4.3f & -- & orf19.1774 & Formate dehydrogenase activity & -1.7 & -2.5 & -1.7 & -1.8 & -1.1 & -3.3 & -6.4 & -3.5 \\
\hline IPF14026 & -- & orf19.718 & Transcription factor activity & -1.9 & -2.3 & -2.2 & -2.2 & -1.2 & -1.7 & 1.6 & -3.0 \\
\hline IPF15335 & -- & orf19.4970 & -- & -3.0 & -2.0 & -1.6 & -2.0 & -2.8 & 1.1 & -1.4 & 2.4 \\
\hline IPF7289 & $U P C 2$ & orf19.391 & Transcription factor activity & -262.2 & -196.2 & -1573.0 & -164.5 & 2.1 & 2.5 & 2.4 & 2.5 \\
\hline
\end{tabular}

${ }^{a}$ Gene name at CandidaDB (http://genolist.pasteur.fr/CandidaDB/).

${ }^{\boldsymbol{b}}$ Gene name at CGD (http://www.candidagenome.org/).

${ }^{c}$ orf19 nomenclature according to the Assembly 19 version.

${ }^{d} \mathrm{GO}$ annotation found at CGD (http://www.candidagenome.org/). 
Table C-4. Genes up-regulated in the absence of UPC2.

\begin{tabular}{|c|c|c|c|c|c|c|c|c|c|c|c|}
\hline \multirow[b]{3}{*}{ CandidaDB Name ${ }^{a}$} & \multirow[b]{3}{*}{ CGD Name ${ }^{b}$} & \multirow[b]{3}{*}{ orf19 Number ${ }^{c}$} & \multirow[b]{3}{*}{ GO Annotation (Molecular Function) ${ }^{d}$} & \multicolumn{4}{|c|}{ Aupc2 vs. SC5314 } & \multicolumn{4}{|c|}{$\mathrm{UPC2}^{\mathrm{S} 2-1}$ vs. UPC2 ${ }^{\mathrm{S} 1-1}$} \\
\hline & & & & \multicolumn{2}{|c|}{ Strain A } & \multicolumn{2}{|c|}{ Strain B } & \multicolumn{2}{|c|}{ Strain A } & \multicolumn{2}{|c|}{ Strain B } \\
\hline & & & & 1 & 2 & 1 & 2 & 1 & 2 & 1 & 2 \\
\hline AAF1 & AAF1 & orf19.7436 & 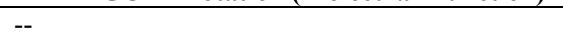 & 1.6 & 1.5 & 1.7 & 1.7 & 1.4 & -1.1 & 1.2 & -1.6 \\
\hline ALS10 & ALS10 & orf19.2355 & Cell adhesion molecule binding & 1.6 & 1.8 & 1.8 & 2.7 & -2.4 & -1.7 & -1.7 & -1.9 \\
\hline ALS3.3eoc & ALS10 & orf19.2355 & Cell adhesion molecule binding & 1.6 & 1.5 & 1.8 & 2.3 & -2.3 & -2.2 & -2.0 & -2.0 \\
\hline ALS3.5eoc & ALS3 & orf19.1816 & Cell adhesion molecule binding & 1.5 & 1.7 & 1.9 & 2.6 & -2.1 & -1.8 & -1.2 & -1.4 \\
\hline ARG1 & ARG1 & orf19.7469 & Argininosuccinate synthase activity & 1.6 & 1.5 & 2.5 & 1.5 & 1.6 & 1.2 & -1.1 & 1.1 \\
\hline CAN2 & CAN2 & orf19.111 & Basic amino acid transporter activity & 1.7 & 1.6 & 2.1 & 1.7 & -2.1 & 3.2 & 1.1 & 2.7 \\
\hline DDR48 & DDR48 & orf19.4082 & -- & 4.4 & 5.2 & 8.2 & 11.5 & 2.4 & 3.4 & 2.4 & 3.8 \\
\hline ECM331 & ECM331 & orf19.4255 & -- & 5.0 & 4.5 & 13.0 & 10.9 & 1.2 & 1.3 & -2.1 & 1.9 \\
\hline FRP2 & -- & orf19.6169 & -- & 1.8 & 1.6 & 1.8 & 1.6 & -1.1 & -1.3 & -1.6 & -1.1 \\
\hline GLG2 & GLG2 & orf19.7434 & Glycogenin glucosyltransferase activity & 1.8 & 1.9 & 2.9 & 1.6 & -1.8 & 1.4 & -1.0 & -2.0 \\
\hline HGT12 & HGT2 & orf19.3668 & Glucose transporter activity & 1.8 & 3.0 & 2.2 & 7.9 & -2.0 & -3.0 & -6.6 & -2.7 \\
\hline IFC4 & OPT4 & orf19.176 & Oligopeptide transporter activity & 1.5 & 1.5 & 1.7 & 1.5 & -1.0 & 4.5 & 2.9 & 4.4 \\
\hline INO1 & INO1 & orf19.7585 & Inositol-3-phosphate synthase activity & 2.0 & 2.5 & 3.4 & 3.6 & 1.7 & 1.3 & 1.2 & 1.3 \\
\hline MIF2 & MIF2 & orf19.5551 & Centromeric DNA binding & 1.7 & 1.6 & 1.5 & 1.6 & -1.1 & -1.4 & -2.4 & -1.9 \\
\hline OSM2 & OSM2 & orf19.5005 & Fumarate reductase (NADH) activity & 1.6 & 1.6 & 1.8 & 1.6 & -1.2 & 1.2 & 1.0 & 1.0 \\
\hline RIM8 & RIM8 & orf19.6091 & -- & 1.8 & 1.6 & 1.8 & 1.9 & 1.1 & 2.5 & 1.3 & -1.4 \\
\hline RTA4 & RTA4 & orf19.6595 & Phospholipid-translocating ATPase activity & 1.5 & 1.6 & 1.8 & 2.4 & -1.5 & 1.2 & 1.1 & 1.1 \\
\hline SNF31 & HGT12 & orf19.7094 & Fructose transmembrane transporter activity & 15.4 & 2.4 & 12.0 & 2.9 & 1.0 & 3.4 & 1.2 & -1.0 \\
\hline YOX1 & -- & orf19.7017 & -- & 1.6 & 2.1 & 1.8 & 1.6 & 1.1 & -2.8 & -1.1 & -1.8 \\
\hline IPF10171.exon3 & HGT9 & orf19.644 & Glucose transmembrane transporter activity & 41.5 & 1.8 & 21.0 & 1.5 & 1.1 & 1.8 & -1.7 & 1.1 \\
\hline IPF11801 & -- & orf19.2798 & Helicase activity & 2.2 & 2.4 & 2.0 & 2.5 & -2.3 & -2.0 & -2.2 & -1.8 \\
\hline IPF11876 & -- & orf19.5295 & -- & 2.0 & 1.8 & 1.7 & 1.5 & 1.4 & -1.2 & -1.3 & 1.5 \\
\hline IPF12312 & -- & orf19.5070 & -- & 1.8 & 1.5 & 1.7 & 1.8 & 1.3 & -1.0 & 1.1 & -1.1 \\
\hline IPF13409 & -- & orf19.751 & -- & 2.3 & 1.8 & 2.3 & 1.9 & -1.9 & -1.1 & 1.0 & -2.3 \\
\hline IPF1341 & PGA13 & orf19.6420 & -- & 1.9 & 2.5 & 2.8 & 4.1 & 1.0 & 1.2 & -1.6 & -1.1 \\
\hline IPF14119 & DAG7 & orf19.4688 & -- & 1.8 & 1.9 & 2.1 & 2.7 & 1.7 & 1.6 & 1.7 & 1.8 \\
\hline IPF14618 & -- & orf19.6079 & -- & 1.6 & 2.0 & 1.8 & 3.0 & -1.3 & -1.2 & 5.3 & -1.1 \\
\hline IPF16253 & -- & orf19.3932 & -- & 1.8 & 1.9 & 2.4 & 2.2 & -1.5 & -2.5 & -1.6 & -1.9 \\
\hline IPF16843 & -- & orf19.2397 & -- & 1.8 & 1.6 & 1.8 & 1.8 & -1.1 & -1.2 & -1.3 & -1.8 \\
\hline IPF18732 & -- & orf19.711 & -- & 1.9 & 2.8 & 2.7 & 4.5 & -1.1 & 1.4 & -1.5 & 1.8 \\
\hline IPF19749 & -- & orf19.94 & -- & 1.5 & 1.6 & 1.5 & 1.6 & -2.2 & -1.5 & -2.0 & -1.4 \\
\hline IPF2839 & -- & orf19.7296 & -- & 3.3 & 2.2 & 4.3 & 3.1 & -2.0 & -3.3 & -3.1 & -2.5 \\
\hline IPF300 & -- & orf19.3282 & -- & 2.0 & 1.9 & 2.4 & 2.2 & 1.0 & 1.2 & -1.2 & 1.0 \\
\hline IPF4443 & -- & orf19.4706 & -- & 1.9 & 2.0 & 2.0 & 2.9 & 1.3 & -1.6 & 1.2 & -1.2 \\
\hline IPF4724 & PGA23 & orf19.3740 & -- & 5.6 & 3.5 & 9.9 & 8.4 & 1.7 & 2.8 & 2.4 & 2.1 \\
\hline IPF7109 & -- & orf19.7350 & -- & 1.5 & 1.6 & 1.6 & 1.7 & -1.1 & 1.1 & 1.1 & 1.0 \\
\hline IPF743 & -- & orf19.5353 & -- & 2.3 & 2.3 & 2.8 & 3.4 & -2.1 & -1.1 & -1.1 & -1.1 \\
\hline IPF7493 & HGT17 & orf19.4682 & Glucose transmembrane transporter activity & 2.0 & 2.1 & 2.3 & 2.7 & 1.3 & -1.2 & 1.1 & -1.1 \\
\hline IPF7666 & GAT2 & orf19.4056 & Transcription factor activity & 1.6 & 1.6 & 1.5 & 1.5 & -1.2 & -1.1 & 1.1 & -1.4 \\
\hline
\end{tabular}


Table C-4 (continued).

\begin{tabular}{|c|c|c|c|c|c|c|c|c|c|c|c|}
\hline \multirow[b]{3}{*}{ CandidaDB Name $^{a}$} & \multirow[b]{3}{*}{ CGD Name ${ }^{b}$} & \multirow[b]{3}{*}{ orf19 Number ${ }^{c}$} & \multirow[b]{3}{*}{ GO Annotation (Molecular Function) ${ }^{d}$} & \multicolumn{4}{|c|}{$\Delta$ upc2 vs. SC5314 } & \multicolumn{4}{|c|}{$\mathrm{UPC2}^{\mathrm{S} 2-1}$ vs. UPC2 ${ }^{\mathrm{S} 1-1}$} \\
\hline & & & & \multicolumn{2}{|c|}{ Strain A } & \multicolumn{2}{|c|}{ Strain B } & \multicolumn{2}{|c|}{ Strain A } & \multicolumn{2}{|c|}{ Strain B } \\
\hline & & & & 1 & 2 & 1 & 2 & 1 & 2 & 1 & 2 \\
\hline IPF9955 & -- & orf19.3499 & -- & 3.2 & 4.2 & 4.6 & 9.7 & -1.3 & 3.0 & 1.5 & 1.9 \\
\hline IPF9996 & -- & orf19.2285 & -- & 6.1 & 1.9 & 4.8 & 2.2 & 1.1 & 1.9 & 1.0 & 1.8 \\
\hline
\end{tabular}

${ }^{a}$ Gene name at CandidaDB (http://genolist.pasteur.fr/CandidaDB/).

${ }^{b}$ Gene name at CGD (http://www.candidagenome.org/).

${ }^{c}$ orf19 nomenclature according to the Assembly 19 version.

${ }^{d} \mathrm{GO}$ annotation found at CGD (http://www.candidagenome.org). 


\section{VITA}

Teresa T. Liu, daughter of Larry and Ginny Liu, was born in Taipei, Taiwan on March 24, 1980. She immigrated to the United States in 1983 and enrolled in the Plano Independent School District in Plano, Texas. After graduating from Plano Senior High School in 1998, she attended Westminster College in Fulton, Missouri, graduating with a Bachelors of Arts in Biology and Computer Science in 2002. She enrolled in the

Interdisciplinary Program at the University of Tennessee Health Science Center in 2002. She anticipates completing her Doctor of Philosophy in May 2008. 